\title{
Measurement Control Workshop Instructional Materials
}

Philip Gibbs/Brookhaven National Laboratory Charles Harvel/Savannah River National Laboratory John Clark/Pacific Northwest National Laboratory/Gregg Protection Services

September 2012

BNL-106050-2012-IR 


\title{
BROOKHGWEN \\ NATIONAL LABORATORY
}

BNL-106050-2012-IR

Measurement Control Workshop Instructional Materials

\author{
Philip Gibbs \\ Brookhaven National Laboratory \\ Charles Harvel \\ Savannah River National Laboratory \\ John Clark \\ Pacific Northwest National Laboratory / Gregg Protection Services
}

September 2012

\section{Nonproliferation and National Security Department Brookhaven National Laboratory}

U.S. Department of Energy

National Nuclear Security Administration

Office of International Material Protection and Cooperation 


\section{DISCLAIMER}

This report was prepared as an account of work sponsored by an agency of the United States Government. Neither the United States Government nor any agency thereof, nor any of their employees, nor any of their contractors, subcontractors, or their employees, makes any warranty, express or implied, or assumes any legal liability or responsibility for the accuracy, completeness, or any third party's use or the results of such use of any information, apparatus, product, or process disclosed, or represents that its use would not infringe privately owned rights. Reference herein to any specific commercial product, process, or service by trade name, trademark, manufacturer, or otherwise, does not necessarily constitute or imply its endorsement, recommendation, or favoring by the United States Government or any agency thereof or its contractors or subcontractors. The views and opinions of authors expressed herein do not necessarily state or reflect those of the United States Government or any agency thereof.

Notice: This manuscript has been authored by employees of Brookhaven Science Associates, LLC under Contract No. DE-AC02-98CH10886 with the U.S. Department of Energy. The publisher by accepting the manuscript for publication acknowledges that the United States Government retains a non-exclusive, paid-up, irrevocable, worldwide license to publish or reproduce the published form of this manuscript, or allow others to do so, for United States Government purposes. 


\section{Executive Summary}

An essential element in an effective nuclear materials control and accountability (MC\&A) program is the measurement of the nuclear material as it is received, moved, processed and shipped. Quality measurement systems and methodologies determine the accuracy of the accountability values. Implementation of a measurement control program is essential to ensure that the measurement systems and methodologies perform as expected. A measurement control program also allows for a determination of the level of confidence in the accounting values.

This report is a compilation of workshop materials consisting of lectures on various aspects of measurement control, including calibration, basic statistics and measurement models, analysis of measurement method qualification data, control charts, inventory difference analysis, and measurement control for specific measurement systems.

The objectives of these materials are to identify, study, and discuss best practices in measurement and measurement control for the accountability of nuclear material. Presentation is envisioned to be through classroom instruction and discussion related to physical and error measurement models, uncertainty estimation, measurement control, and other areas of interest related to measurements. Several practical and hands-on exercises are included for demonstration of the various measurement concepts contained in the lecture/discussion sessions.

\section{Mode of Instruction}

The suggested mode of instruction for these materials is lecture, demonstrations, and participant-led practical exercises.

\section{Participants}

Participants attending this workshop should currently, or in the near future, be responsible for measurements and/or measurement control or material accountability at their facilities. It is suggested that the ideal participant pool consist of several MC\&A "teams" that include MC\&A specialists, measurement experts, and statisticians.

Workshop length - 7 training days 


\section{Definitions:}

3.1 accountability: The determination of quantities of nuclear materials (NM) and current record maintenance associated with receipts; shipments; measured discards; transfers into, out of, or between material balance areas, item control areas, or both; and total material on current inventory.

3.2 accuracy: A measure of the agreement between the measured value and the true (or assigned) value. See bias.

3.3 assigned value: A value assigned to a standard used for calibrating and/or controlling a NM measurement device or system.

3.4 ASTM International: Formerly the acronym for the American Society for Testing and Materials; now used by the Society as its complete name.

3.5 audit: An examination of current activities to assure that they are in compliance with program policies and procedures.

3.6 bias: A systematic error that can be estimated by comparison of the sample mean of a series of measurements with a true or reference value, in which case a correction can be applied to remove the effect of the bias on the measurements.

3.7 calibration: The process of determining the numerical relationship between the observed output of a measurement system and the actual value of the characteristic being measured based upon a certified reference material.

3.8 certified reference material (CRM): A reference material that is certified for the value of one or more of its properties by a technically valid procedure and accompanied by, or traceable to, a certificate or other documentation that is issued by a certifying body.

3.9 chain of custody: A means of assuring that a sample is continually under control to prevent inadvertent or deliberate tampering from the time the sample is taken until its disposal and requiring signed receipts at each point where responsibility for the sample is transferred.

(See also custody.)

3.10 confirmatory measurement: A measurement made to test whether some attribute or characteristic of nuclear material is consistent with the expected response for that material when no significant change in the NM content or concentration has occurred.

3.11 control standards: Standards that are representative of the process material being measured. These standards are measured periodically in order to monitor system reliability and to estimate any bias associated with the measurements of the process material.

3.12 custody: A sample is in custody if it is either: in one's actual physical possession, in one's view after being in one's physical possession, in one's physical possession and subsequently secured so that tampering is excluded, or is kept in a secure area that is restricted to authorized personnel.

\subsection{3 distribution:}

- The relative spatial location of components of a mixture;

- A well-defined universe of possible measurements arising from a property or relationship under study.

3.14 diversion: The unauthorized removal of nuclear material from its approved use or authorized location. 
3.15 documentation: The collection of records that describe the purpose, use, structure, details, and operational requirements of a program, and the performance of activities.

3.16 an estimate: The particular value yielded by a rule or method of estimating a parameter of a parent population.

3.17 homogeneous: A description of a substance that is sufficiently blended to ensure that any sample taken from it is representative of the entire substance.

3.18 Inventory difference (ID): The difference between the quantity of NM on-hand according to accounting records and the quantity of NM on-hand as determined by a physical inventory.

3.19 ISO: The acronym for the International Organization for Standardization.

3.20 limit of error (LE): The boundaries computed as the measured value plus or minus twice its standard deviation (uncertainty). Relative to the uncertainties of the measurement method, the underlying true value of the attribute being determined will lie within such limits for a specified proportion of potential measured values, that is, for approximately 0.95 , or $95 \%$ of them.

3.21 machine-readable: Material (label, tag, etc.) that is capable of being read by an electronic device.

3.22 matrix: The form or composition of a material that best represents the generic physical makeup of the material with regard to impact on measurement response.

3.23 MC\&A: The abbreviation for material control and accountability.

3.24 measured value: A quantitative characteristic, generally with associated uncertainty that has been determined experimentally for a given quantity of material.

3.25 measurement control: The procedures and activities used to ensure that a measurement process generates measurement results of sufficient quality for their intended use, and to determine measurement uncertainty values (or limit-of-error values).

3.26 measurement process: The determination of an attribute, e.g., element concentration, isotopic distribution, and/or bulk quantities.

3.27 NDA: The abbreviation for nondestructive assay.

3.28 net weight: The measured weight (mass) of the contents in a container as determined by subtracting the empty container weight (tare weight) from the gross weight of the container plus contents.

\subsection{9 parameter:}

- A quantity entering into the distribution of a statistic or random variable;

- The quantity being estimated.

3.30 physical inventory: A determination by physical means (visual and measurement) of the quantity of nuclear material on-hand at a specified point in time.

3.31 precision: A quantitative measure of the variability of a set of repeated measurements.

3.32 procedure: A document that specifies or describes how an activity is to be performed

3.33 qualification: Demonstration (through specific test requirements) of adequate knowledge and experience for the performance of a task 
3.34 random error: The specific variation encountered in a single measurement, characterized by the random occurrence of a positive or negative deviation from the mean value of the measurement.

3.35 reference material (RM): A material or substance one or more properties sufficiently well established to be used for the calibration of an apparatus, the assessment of a measurement method, or the assignment of values to materials. Standard RM (SRM) is a reference material distributed and certified by the appropriate national institute for standardization.

3.36 replicate samples: Two or more samples taken independently from the same population.

3.37 shelf life: The time that elapses before a stored material or device is rendered inoperative or unusable for its intended purpose due to age or deterioration.

3.38 special nuclear material (SNM): U-233, uranium enriched in $U-233$ and/or U-235, plutonium, or any combination thereof, and any other material which, pursuant to the provisions of

Section 51 of the Atomic Energy Act of 1954, as amended, has been determined to be special nuclear material, but does not include source material; it also includes any material artificially enriched by any of the foregoing, not including source material as defined by the Atomic Energy Act, Title 42, U. S. Code, Section 2002, et. seq.

3.39 standard deviation: The positive square root of the variance.

3.40 standard error (of a parameter estimate): The standard deviation of the parameter estimate.

3.41 statistical sampling: A statistically valid technique used to select elements from a population, including probability sampling, simple random sampling, systematic sampling, stratified sampling, and cluster sampling.

3.42 systematic error: The mean that would result from an infinite number of measurements of the same measurand carried out under the same conditions of measurement minus a true value of the measurand.

3.43 tare: The weight of a container or wrapper that is deducted from the gross weight to obtain the net weight.

3.44 traceability: The ability to relate individual measurement results to national standards (primary standards) or nationally accepted measurement systems through an unbroken chain of comparisons.

3.45 uncertainty: A concept employed to describe the inability of a measurement process to measure the true value exactly.

3.46 variance: A measure of the dispersion of a set of results.

3.47 variance propagation: The determination of the value to be assigned as the uncertainty of a given quantity using mathematical formulas for the combination of uncertainty components. Variance propagation involves many considerations, and the computational formulas for computing the uncertainty depend upon the functional relationships of the measurement parameters involved.

3.48 verification measurement: A quantitative measurement to verify an existing measured value as previously recorded.

3.49 verisimilitude: A concept applied to standards that represent the material to be analyzed, characterized, or tested as closely as necessary. 


\section{ABBREVIATIONS AND ACRONYMNS:}

ANSI -- American National Standards Institute

BIMP --International Bureau of Weights and Measures

CALEX -- Calorimetry Exchange Program

C -- Celsius

CY -- Calendar year, January to December

DA -- Destructive Analysis

D\&G -- Davies and Gray Titration

DOE --(United States) Department of Energy

DU -- Depleted Uranium $(235 \mathrm{U}<0.3 \mathrm{wt} \%)$

GUM --Guide to the Expression of Uncertainty in Measurement

GSMS --Gas Source Mass Spectrometry

HEU -- High-enriched uranium ( $235 \mathrm{U} 20$ wt \%)

IAEA -- International Atomic Energy Agency

ICPMS -- Inductively Coupled Plasma Mass Spectrometry

ID -- Inventory Difference

IDMS -- Isotope Dilution Mass Spectrometry

INMM -- Institute of Nuclear Materials Management

ISO -- International Organization for Standardization

ITV -- International Target Value

LE -- Limit of Error

LEID -- Limit of Error on Inventory Difference

LEU -- Low-enriched uranium (1wt $\%<235 \mathrm{U}<20$ wt \%)

MBR -- Material Balance Report

MBA -- Material Balance Area

MCP -- Measurement Control Program

ME -- Measurement Evaluation

MUF - Material Unaccounted For

NBL -- New Brunswick Laboratory

NIST -- National Institute of Standards and Technology

NMCC -- Nuclear Material Control Center

NRC -- Nuclear Regulatory Commission

QC -- Quality Control

PMAP -- Process Measurement Assurance Program

RD -- Relative deviation (expressed in percent); also written as \% RD

SC -- Office of Science

SD -- Standard deviation

SME -- Safeguards Measurement Evaluation

SMES -- Safeguards Measurement Evaluation System

SPC -- Statistical Process Control

TUR - Test Uncertainty Ratio

TIMS -- Thermal Ionization Mass Spectrometry

UF6 -- Uranium hexafluoride

UNH -- Uranyl nitrate hydrate (solution)

$\mathrm{UO}_{2}$-- Uranium dioxide $\mathrm{UO}_{3}$ Uranium trioxide $\mathrm{U}_{3} \mathrm{O}_{8}$ Uranium (mixed) oxide

u(c) -- Combined Uncertainty

$\mathbf{u}(\mathbf{r})$-- Random component of uncertainty (indicative of precision) of ITV

u(s) -- Systematic component of uncertainty (indicative of bias) of ITV

VP -- Variance Propagation

VIM -- International Vocabulary of basic and general terms in Metrology

XRF -- X-Ray Fluorescence 


\section{About the Instructors}

John Clark currently works as a consultant, with 50 years of work experience in the nuclear and chemistry fields as chemist, laboratory supervisor, quality assurance engineer, manager and senior fellow technical advisor. His work experience includes special nuclear materials and chemical standards preparation for calibration, training \& testing, QC and inter laboratory sample exchange programs; developing and managing measurement control programs; quality assurance program development and auditing; measurement control manager for MC\&A in safeguards and security; and physical standards calibration and tolerance testing in the field of metrology. John has over 30 publications and/or papers written for national and international technical organizations and participated in writing standards for these groups. As a metrologist and consultant he has helped educate chemists, scientists and engineers in methods for determining and managing measurement errors/uncertainty.

Phil Gibbs has 28 years of project management and subject matter expert experience in Nuclear Safeguards with emphasis in Material Control and Accountability (MC\&A) for the U.S. Department of Energy (DOE) domestic and international programs. Mr. Gibbs currently is working as the Project Lead and MC\&A Subject Matter Expert (SME) for the U.S. MPC\&A program. Prior to moving to international work, Mr. Gibbs served as the Local Area Network Material Accounting System (LANMAS) Project Manager managing the development and implementation of LANMAS, a standardized inventory and control system for nuclear components and inventories among DOE contractors. At the U.S. DOE Savannah River Site, he worked as an MC\&A Manager for Accounting, Technical Support, Procedures, and Training. Prior to that time, Mr. Gibbs worked as a measurement control engineer in the area of mass measurements and process tank calibrations.

Chuck Harvel is a Fellow Scientist at the DOE Savannah River National Laboratory. He has over 20 years experience with MC\&A statistical and software development applications. He has been involved with advisory and technical work in the areas of measurement method qualification and uncertainty estimation for tanks, scales, hydride beds and NDA and analytical methods; development of guidance for accepting measurement methods as qualified for accountability measurements; calculation of limits-of-error (LOEs) and combined limits-of-error (CLOES) for material receipts and shipments; development of guidance for resolving shipper/receiver differences; development of limits-of-error for inventory difference (LEID) models for several material processes; development of guidance for resolving ID problems; technical consulting regarding MC\&A related statistical applications. Chuck has over 30 years experience with software design and development applications. His work in this area includes the design, development and maintenance of software systems for automating the calculation of LEIDs. Chuck has a BS in Mathematics and Computer Science from Eastern New Mexico University (1973), and an MS in Applied Statistics and Computer Science from the University of New Mexico (1976). 


\title{
Measurement Control Workshop Proposed Agenda
}

\author{
Location: Beijing, China
}

Timeframe: September 18-26, 2012

An essential element in an effective nuclear materials control and accountability program is the measurement of the nuclear material as it is received, moved, processed and shipped. Quality measurement systems and methodologies determine the accuracy of the accountability values. Implementation of a measurement control program is essential to ensure that the measurement systems and methodologies perform as expected. A measurement control program also allows for a determination of the level of confidence in the accounting values.

The workshop will consist of lectures on various aspects of measurement control, including calibration, basic statistics and measurement models, analysis of measurement method qualification data, control charts, inventory difference analysis, and measurement control for specific measurement systems.

The objectives of this course are to identify, study, and discuss best practices in measurement and measurement control for the accountability of nuclear material. This will be accomplished through classroom instruction and discussion related to physical and error measurement models, uncertainty estimation, measurement control, and other areas of interest related to measurements. Several practical and hands-on exercises will be used to demonstrate the various measurement concepts discussed during the lecture/discussion sessions.

\section{Mode of Instruction}

The instruction will be accomplished through lecture, demonstrations, and participant-led practical exercises.

\section{Participants}

Participants attending this workshop should currently, or in the near future, be responsible for measurements and/or measurement control or material accountability at their facilities. It is suggested that the participant pool consist of several MC\&A “teams” from different facilities, to include MC\&A specialists, measurement experts, and statisticians from each facility.

Workshop length - 7 training days 


\section{Course Outline}

\section{Day 1}

Welcome and Course Introduction

Expected length: 30 minutes

This section will provide an overview of the week to include a presentation of the general learning objectives, training format, and the types of exercises to be performed.

\section{Module 1: Workshop Overview and Objectives}

\section{Expected length: 2 hours}

This module will outline the topics for the week and the primary elements of measurement control programs.

The role of measurements control in an overall MC\&A program will be discussed, as well as its relationship to the inventory difference. A measurement control program is a good business practice, but it is also driven by regulatory requirements and shaped by international standards.

Learning Objectives

1. To apply results of measurement control programs to determine if Inventory Differences (ID) are significant in the accountability of nuclear material

2. To understand the elements of a measurement control program

3. To understand the DOE requirements for measurement and measurement control programs

4. To understand the facility measurement and measurement control program and its relationship to DOE requirements

5. To understand US and international standards related to measurement control programs and measurement uncertainty [ANSI (1551), ISO(17025), BIPM (GUM), STR (IAEA Target Values), etc.]

\section{$\underline{\text { Basic Statistics }}$}

Expected length: 1 hour

This will be an introduction to basic statistical concepts such as mean, variance, and statistical distributions.

\section{Learning Objectives}

1. Review basic statistical terms and concepts

2. Review Mean/Average and how it is calculated

3. Review the concept of a "Probability Distribution" and the Normal Distribution

4. Review Variance and Standard Deviation and how they are calculated

5. Review the concepts of "Bias" and "Fit for Purpose"

Exercise: Basic Statistics

Expected length: 30 minutes

\section{Learning Objective}

1. Calculate the mean (average), variance, and standard deviation for a group of data by hand or by using Microsoft Excel

\section{Exercise: Height Exercise}

Expected length: 1 hour 30 minutes

This exercise will demonstrate the importance of concepts related to measurement uncertainty. The students will estimate the heights of three people under three sets of conditions. They will estimate the maximum and 
minimum heights (range of uncertainty) that should contain the true heights (that will be determined by measuring each individual with a calibrated tape measure) and the confidence interval (at a 1 standard deviation at $67 \%, 2 \mathrm{SD}$ at $95 \%$ or $3 \mathrm{SD}$ at $99.7 \%$ ). Each student will be given two quarters to demonstrate his/her level of confidence that his/her estimate includes the true value of the heights of the three people. To demonstrate his/her confidence in the estimate, each student will "bet" 0,1 or 2 quarters that the estimated range of uncertainty contains the true values. After the true heights are determined the students will determine if they have won or lost the bets they made. Include discussion of uncertainty (includes random and systematic error/bias).

\section{Learning Objectives}

1. To see measurements are comparisons of unknowns to reference standards.

2. To understand measurements have uncertainty.

3. To understand that reference standards also have uncertainty.

4. To understand measurements need uncertainty estimates with specified confidence intervals to have value.

5. Apply concepts of uncertainty (uncertainty includes random and systematic error/bias). 


\section{Day 2}

Measurement Models

Expected length: 2 hours

This module will include a discussion of statistical measurement models and variance propagation concepts.

\section{Learning Objectives}

1. Review GUM concepts as they relate to measurement models

2. Discuss the statistics used in describing measurement models

3. Understand additive and relative measurement models and be able to establish the type of model for a particular measurement method

4. Understand expectation and variance calculations associated with measurement models and apply them to some example measurement methods

\section{Measurement Models Exercise}

Expected length: 2.5 hours

The students will be given a handout with questions regarding the calculation of variances for single and multiple measurements and functions of measurements and the specification of measurement models for several measurement types.

\section{Learning Objectives}

1. Understand the calculations of variances for functions involving measurements

2. Understand the application to measurement models

\section{Variance Propagation}

Expected length: 1 hour 30 minutes

\section{Learning Objectives}

1. To understand the basic concepts of variance propagation

2. To understand propagation of random variances

3. To understand propagation of systematic variances

4. Be able to use excel to solve variance propagation problems

\section{Measurement Method Qualification Process}

Expected length: 1 hour

Proper evaluation and attestation of measurement methodologies is essential to ensure that the measurement is adequate. This requires a systematic review of measurement procedures, traceability of standards and determination of the accuracy and precision of the methodology. A description of the measurement qualification process and goals and will be provided.

Learning Objectives

1. Understand method selection/qualification process

1. Understand how to document a method qualification

a. Qualification plan

b. Data analysis report

2. Understand the application of a basic variance propagation to a method qualification 
Expected length: 1 hour

The qualification of an actual measurement methodology will be discussed and evaluated. The students will enter the qualification data (for the AWCC) into an Excel spreadsheet, plot the data and conduct an ANOVA. The students will then report on the results.

\section{Learning Objectives}

1. To plan the method qualification process

2. To produce a qualification planning document

3. To collect the measurement data

4. To analyze the data (estimate uncertainties)

5. To produce a qualification document 


\section{$\underline{\text { Day } 3}$}

Proficiency and Sample Exchange Programs

Expected length: 1 hour 30 minutes

Another important aspect of measurement control is the proficiency of the personnel performing the measurements. This module will look at the establishment of qualifications for personnel measuring on nuclear materials and how their proficiency is tested and analyzed through redundant measurements and sample exchange programs.

\section{Learning Objectives}

1. Demonstrate method for qualifying lab personnel

2. Understand the statistical criteria for qualification

3. Study an example of data collected in testing program

4. Illustrate how sequential testing can expedite training

5. Review US DOE proficiency testing program (sample exchange) results for the Davies and Gray Uranium Titration method.

\section{Exercise: Sample analyst qualification training and testing evaluation}

Expected length: 30 minutes

An example of the T\&T data will be provided. Students will determine if a trainee satisfactorily measured 9 unknowns.

Sampling Variability: Solids and Oxides

Expected length: 2 hours

This portion of the course will discuss the various issues and problems associated with sampling a batch of material. Several types of sampling problems will be discussed along with the activities that can be applied to successfully sample a batch of material and reduce sampling errors. An actual sampling problem will be discussed in detail.

\section{Learning Objectives}

1. Understand the issues with sampling

2. Understand the activities that can be done to minimize the problems of heterogeneity within a batch of material

3. Be able to apply correct sampling principles, analyses, tools, and techniques

4. Be able to use Excel functions and the ANOVA capability to evaluate sampling data

Exercise: Sampling Variability

Expected Length:

Learning Objectives:

1. Understand issues with sampling and activities that can be to minimize these

2. Apply Excel functions and the ANOVA capability to determine if selected samples are representative of a batch of material

Q\&A session-

Expected length: 30 minutes 
Students will have the opportunity to discuss materials covered so far as well as any specific questions they would like to discuss. These sessions should be organized every three days to discuss what has been covered so far, and any questions on the material presented.

Accreditation (CIAE)

Expected length: 1 hour 30 minutes

Description to be added 


\section{Day 4}

Measurement Control Charts and Measurement Control

Expected length: 1 hour 30 minutes

Timely identification of out-of-control conditions and/or shifts in the instruments being utilized for measuring the nuclear material is necessary to ensure the accounting values are accurate. Control charts are a tool used to monitor these conditions and shifts. This module will discuss the purpose, use and application of control charts for monitoring measurement system performance.

Learning Objectives

1. Identify the purpose of control charts

2. Identify the six elements of control charts and their purpose

3. Discuss and analyze example control charts

4. Discuss control charts and measurement control

5. Discuss and analyze control charts from actual measurement systems

Performance Calibration Modeling and Data Analysis

Estimated Length: 1.5 hours

Determining the accuracy and precision of various measurement methods requires the modeling of the measurement technique and repeated measurements using known standards. The approach used to model, collect data and analyze the data is important in order to ensure that the variances obtained for the methodology represent actual operation on the shop floor. This module will discuss various calibration models and methods to calculate calibration uncertainties.

Learning Objectives

1. Identify the function of a calibration model

2. Identify the concept of calibration equation

3. Identify the concept of regression analysis and the prediction equation

4. Identify the use of regression analysis for calibration uncertainty

5. Identify the concept of measurement uncertainty in calibration equations

\section{Balance Measurement Control Program}

Expected length: 2 hours

Weight measurements using scales and balances can be a major source for measurement uncertainties. The module will discuss implementation of the measurement control program for scales and balances to include procedures for control, balance calibrations, and use of mass reference standards. The module will specifically address determination and propagation of the weight measurement uncertainties in the metrology laboratory and in operating areas, and how these uncertainties are used to determine the overall weight uncertainty assigned to nuclear material values.

Learning Objectives

1. Review the elements of a MCP for scales or balances

2. Discuss the classes of balances \& mass standards

3. Discuss sources and types of error in weighing

4. Review the GUM's 8 steps for estimating uncertainty

5. Discuss balance calibration program at SRS

Exercise: Selection of appropriate mass standards for balance calibration checks. Expected length: 1 hour

Complete a class exercise on selecting mass standards for evaluating the performance of various scales and balances. 


\section{Day 5}

Tank Sampling and Mixing

Expected length: 30 minutes

Determining and managing the measurement uncertainty when obtaining a representative sample from solutions in tanks or solids from a process requires special consideration. This module will discuss the approaches for determining and managing sampling errors in nuclear processes for liquids and solids.

\section{Learning Objectives}

1. Discuss planning considerations for experiments to determine sampling errors

2. Discuss methodologies for managing and controlling sampling uncertainties

3. Review a tank sampling and mixing case study

\section{Exercise : Balance Calibration}

Expected length: 2 hours

Participants will make a series of measurements on an analytical balance with mass standards calibrated to be within class E2 uncertainty tolerances. The students will use four different balances during the exercise. Data collected will be evaluated to estimate the repeatability of each person; the data collected on the balance will be used to estimate the short-term reproducibility on each balance. All of the data will be evaluated to estimate the reproducibility of mass measurements made on any balance in the laboratory. Five Class E2 calibrated standards will be weighed 10 times to collect data to estimate the uncertainty of measurements at each range tested, that includes the calculated standard deviation, the uncertainty of the mass standard and the average bias. After completion of the exercise, the class will evaluate and discuss the results.

Learning objectives:

1. Calculate the random variation in measurement results

2. Determine estimates of precision due to the variation between analysts and balances

3. Estimate the uncertainty of weight measurements at different levels over the weighing range

4. Learn the importance of knowing the variables included in measurement uncertainty estimates

\section{Analytical Chemistry Measurement Control}

\section{Expected length: 1 hour}

Destructive analysis methods to determine concentration and enrichment are routinely calibrated and controlled with standards having known uncertainties. However, there is still a level of uncertainty associated with these methodologies due to other variables, which include operators, equipment, sample preparation, environmental conditions, etc. This module will describe processes for identifying and estimating measurement uncertainties.

Learning Objectives

1. Understand laboratory measurements must have uncertainty estimates for accountability of NM

2. Review MCP technical \& administrative components of ANSI N15.51

3. Discuss laboratory techniques for estimating error

4. Discuss uses of measurement control data

a. Intro to Laboratory Information Management Systems (LIMS)

\section{Exercise: Pipette Validation}

\section{Expected length: 2 hours}

In this exercise, students will perform volumetric measurements with a pipette to determine the accuracy and precision of their measurements, before and after some of the major sources of error in volumetric measurements and tips for good precision are explained. Multiple pipettes will be used, so the group will be able to compare the average bias and precision estimates obtained from the data generated during the exercise. Finally, all of the data will be evaluated to determine an estimate of the uncertainty of volumetric measurements 
made in that laboratory with the type of equipment used to see if it is within manufacturer's specifications and meets pre determined data quality objectives.

Learning objectives

1. Explain the sources of error in pipette volume measurements

2. Discuss the variation within and between operators

3. Estimate the uncertainty of volumetric measurements

4. Compare calculated uncertainty estimates with manufacturer's specifications. 


\section{Day 6}

Discussion Topic: Equipment Calibration Practices and Lab Exercise Review

Expected length: 1 hour

1. How do calibration practices vary among facilities for different types of equipment?

2. Discuss calibrations vs. tolerance testing

3. What personnel/organizations conduct calibration?

4. Discuss yesterday’s lab exercise

\section{Case Study: Preparation of Uranium Working Standards}

Expected Length: 1 hour 30 minutes

To monitor and control a measurement system's performance a facility must have a working reference material/standard, which is traceable to a national standard (certified reference material) with an uncertainty that is at least $1 / 3$ of the target uncertainty of the measurement system. In many cases it both sound business practice and economical to fabricate working standards for calibrating instrumentation, training personnel and controlling measurement systems. This module will present a case study of how to produce a secondary working standard with traceability back to a national standard and meets or exceeds a predetermined target uncertainty.

Learning objectives

1. Understand how a standard that has an uncertainty of $1 / 3$ or less of the uncertainty of the measurement it is meant to control has been produced

2. Review a case study on the production of a Uranium Nitrate Solution to use as working calibration material (WRM) that is traceable to a Certified Reference Material.

3. Review the statistical tests used in assigning a concentration value with associated estimate of the uncertainty of the WRM that is traceable to the CRM.

\section{NDA Measurement Control Program}

Expected length: 2 hours

Measurement control program for NDA systems (AWCC and general gamma-ray spectroscopy) will be discussed.

Learning Objectives

1. Understand the measurement control programs for AWCC and gamma-ray measurements.

\section{Exercise: NDA Measurement Control}

Expected length: 2 hours

Students will work in two groups. One group will work on gamma-ray spectrometer. One group will work with gamma-ray spectrometer or AWCC 


\section{Day 7}

Inventory Difference Assessment

Expected length: 4 hours

Establishment of a measurement control program provides assurance that the measurement systems perform as expected and allows determination of measurement uncertainties. These uncertainties are important to determine if inventory differences are based on possible measurement variances or other more serious anomalies. This module will discuss the integration of the measurement control data and the analysis of

inventory differences. The discussions will include how the data from the measurement control program is used in the propagation of variance (POV) modeling, and frequency for analyzing and changing POV variances.

Learning Objectives

1. Identify how control limits can be used as a method of evaluating ID

2. Identify how various types of errors contributing to the ID affect the ID mean and the ID standard deviation

3. Calculate the loss detection probabilities

4. Identify the characteristics of historic and variance propagation (VP) ID control limits

5. Calculate the uncertainty of the ID

Course Wrap-up

Expected length: 1 hour 


\section{Equipment required}

\section{Each student should bring a basic calculator (capable of square root function)}

\section{General}

- $\quad$ Student books (CIAE) ${ }^{* *}$ suggestion for CIAE- print some pages full page rather than half.

- 2 Flip charts or dry erase board and colored markers (CIAE)

- $\quad$ Laptop computers: one per group of 4-5 students (CIAE)

- $\quad$ Flash drive or CD pre-loaded with data files required for exercises-one per group of 4-5 students (CIAE)

o measurement qualification exercise.xls,

o balance exercise.xls,

o pipette exercise.xls

\section{Height exercise}

- $\quad$ Tape measure (CIAE and USPT)

- Ruler (USPT)

- Scotch tape (USPT)

- Plain paper (CIAE)

\section{Measurement method qualification exercise}

- Laptops and data files (listed above)

- Microsoft Excel support person; to assist with data plots and ANOVA (analysis of variance) application (CIAE and USPT). This requires Microsoft excel add-in

\section{Balance exercise}

- 1 per group: analytical balances (four decimal places minimum (0.1 mg), at least 100 gram capacity) (CIAE)

- 4-5 1, 10, 50 and 100 gram weights (USPT)

- cotton or insulated gloves- one per student (CIAE)

- $\quad$ tweezers or tongs (one per each mass standard set) (USPT)

- computer and data files (balance linearity.xls and balance repeatability.xls) as listed above

- $\quad$ printer (CIAE)

\section{NDA exercise}

- $\quad$ NDA equipment (CIAE)

\section{Pipette exercise}

- Uncertainty estimate from calibration certificates and date of calibration testing for air thermometer, water thermometer, humidity meter, and barometer.

- Laptop computer and data file (pipette.xls) as listed above

- 1 one gram and 1-ten gram calibrated E2 class mass standards (CIAE)

- $\quad$ cotton or insulated gloves - one per student (CIAE)

- Tweezers or tongs (one per each mass standard set) (USPT)

- One barometer with calibration certificates or manufacturer specifications, in English if possible (+ or - 1\%) (CIAE)

- Two thermometers (at least one capable of reading water) with readability to tenth of a degree (0.1C) with calibration certificates, in English if possible (CIAE)

- Humidity meter with calibration certificate, in English if possible, or manufacturer specifications (CIAE)

- 5 - $1 \mathrm{ml}$ fixed volume air displaced pipettes with calibration certificates, in English if possible, and 5 boxes of disposable tips (CIAE)

- $\quad$ Two liters of water (CIAE)

- Five $50 \mathrm{ml}$ volumetric flask - glass (CIAE)

- Five $250 \mathrm{ml}$ bottles- any glass or plastic bottle/beaker with big opening (CIAE) 


\section{MEASUREMENT CONTROL COURSE \\ STUDENT CRITIQUE SHEET}

Name: (optional)

Date:

We are committed to obtaining information on the quality of the learning experience and will actively encourage participant responses. Please help us improve our training and materials by answering the following questions. Your reactions, comments, and suggestions are needed and appreciated.

\section{Part I - Demographics}

1. Identify your responsibilities (check all that apply).

Material Control and Accounting

Measurement Professional

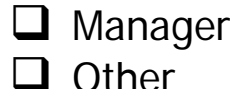

2. Before you attended this training, what level of knowledge did you have in the subject matter?

Expert-level knowledge - I had a complete understanding.

$\square$ Working-level knowledge - I had a good understanding.

$\square$ Familiarity-level knowledge - I only had an understanding of the general concept(s).

$\square$ Little or no knowledge

\section{Part II - Training}

Please use the additional comments section of this form to provide comments and suggestions about items that you have strong opinions, either positive or negative. Indicate the number of each item to which you are referring. Your constructive feedback is appreciated.

\section{Please rate the following:}

3. How well the training met my current/future job needs.

4. Usefulness of the student materials (e.g., notebook and handouts) during the training.

5. Training aides (e.g., slides, videos, models).

6. Materials and equipment

7. Clarity and organization (well structured and easy to follow) of the training.

8. Length of training

9. Pace of the training

10. Sequence of instruction

$\begin{array}{ccccc}\text { Not } & & & & \text { Very } \\ \text { Applicable } & \text { Poor } & \text { Fair } & \text { Good } & \text { Good } \\ \square & 1 & 2 & 3 & 4\end{array}$

$\begin{array}{llll}1 & 2 & 3 & 4\end{array}$

$\begin{array}{llll}1 & 2 & 3 & 4\end{array}$

123

123

Excellent 


\section{Please rate the following:}

11. Exercises/activities reinforced training

12. Organization and preparation of the Instructor(s)

13. Achievement of program goals

14. Adequacy of training facilities

15. Attention to safety

16. Overall quality of the training

\section{Please answer the following:}

17. The material covered was interesting to me.

18. I have learned new skills and techniques in this field.

19. My understanding of concepts and principles in the subject(s) has improved.

20. The instructors explained the relevance of the material to my job.

21. Please provide constructive comments on the instructor(s) (subject knowledge, teaching ability, and skill at working with students):

$\begin{array}{cccccc}\begin{array}{c}\text { Not } \\ \text { Applicable }\end{array} & \text { Poor } & \text { Fair } & \text { Good } & \begin{array}{c}\text { Very } \\ \text { Good }\end{array} & \begin{array}{c}\text { Excellent } \\ \square\end{array} \\ \square & 1 & 2 & 3 & 4 & 5 \\ \square & 1 & 2 & 3 & 4 & 5 \\ \square & 1 & 2 & 3 & 4 & 5 \\ \square & 1 & 2 & 3 & 4 & 5 \\ \square & 1 & 2 & 3 & 4 & 5 \\ \square & 1 & 2 & 3 & 4 & 5\end{array}$

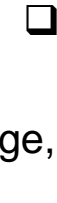


22. What lessons/parts of the training were most useful?

23. How could this training be improved?

24. Additional comments: (use back of this page if more room is needed.) 


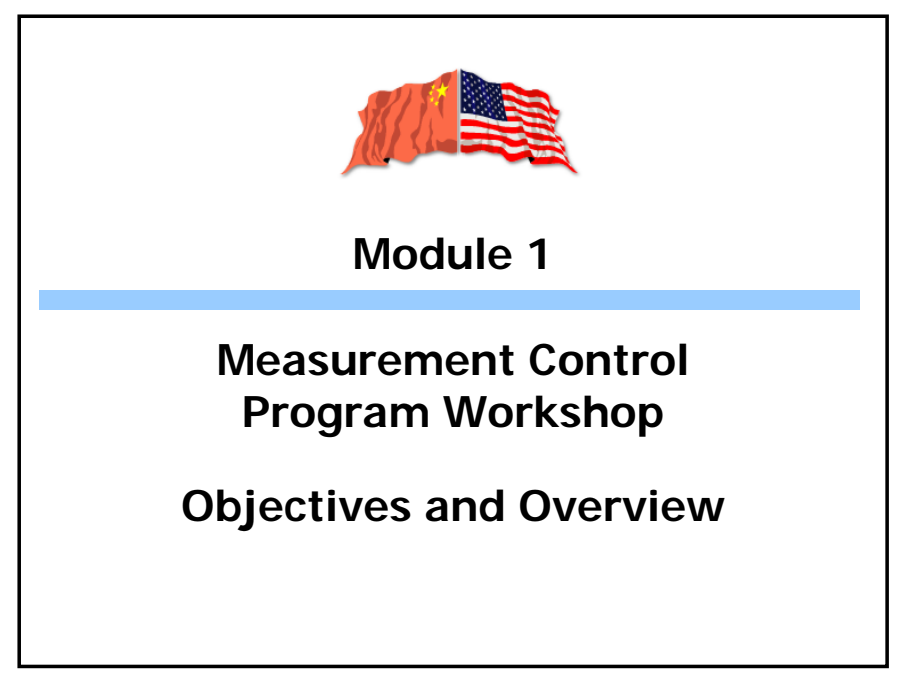

\section{Learning Objectives}

- To apply results of measurement control programs to determine if Inventory Differences (ID) are significant in the accountability of nuclear material

- To understand the elements of a measurement control program

\section{Elements of a Comprehensive Measurement Control Program (MCP)}

1. Data Quality Objectives

2. Measurement Method Selection \& Qualification

3. Traceable Reference Materials (Standards)

4. Physical/Environmental Conditions MC

5. Scales and Balances Program

6. Analytical Quality Control

7. Sampling Verification

8. Statistical Programs \& Statistical Control Limits

9. Proficiency Testing Programs

10. Accreditation by Recognized Accrediting Body

\section{Key Concepts}

- Measurements have uncertainty

- Different types of measurement errors

- Methods for estimating the uncertainty of measurement

- National \& international standards related to measurements and measurement uncertainty

- Metrology vocabulary definitions

- International Vocabulary of Basic and General Terms in Metrology (VIM) 


\section{Ds Are Not Zero Because}

- Measurement uncertainty: measurement system effects

- Location of material

- In calorimeter, on scale, etc.

- For example, heat distribution, weight distribution, etc.

- Calibration of scales

- Fluctuations in air pressure, temperature, etc.

\section{Ds Are Not Zero Because}

- Measurement uncertainty: Non-measurement system effects

- Power fluctuations

- Electronic functioning of equipment

- Non-homogeneity of material being measured

- Statistical nature of radioactive decay

- Improper or incomplete background measurements

\section{Ds Are Not Zero Because}

\section{- Sampling effects}

- Improper or incomplete blending in a destructive analysis sample

- Nondestructive Analysis (NDA) limitations

- Material composition of the NDA standards does not match the material composition of the measured items

- Failure to account for background effects

\section{Ds Are Not Zero Because}

- Accounting system effects

- Better measurement to correct estimates

- Decay, rounding errors, etc.

- Human error

- Clerical mistakes (transcription errors, etc.)

- Failure to follow procedures 


\section{IDs Are Not Zero Because}

- Unmeasured streams or inventories

- Solids entrained in liquid systems settle in tanks

- Holdup can take the form of material associated with specific equipment

- Factors or estimates

\section{MCP Element \# 1 Data Quality Objectives}

- The IAEA's International Target Values (ITVs) for uncertainty components in measurements of nuclear material should be considered

- Maximum Permissible Error VIM 4.26 (5.21) limit of error

Extreme value of measurement error, with respect to a known reference quantity
value, permitted by specifications or regulations for a given measurement, measuring

- NOTE 1 Usually, the term "maximum permissible errors" or "limits of error" is

- NOTE 2 The term "tolerance" should not be used to designate 'maximum
permissible error'

- Target Uncertainty VIM 2.34

- Measurement uncertainty specified as an upper limit and decided on the basis of the intended use of measurement results

\section{MCP Element \#2 \\ Measurement Method Selection and Qualification}

- Method selections are based on target values

- A documented method is used on an identified periodic basis to ensure that a measurement method shall demonstrate acceptable performance before being used for performing accountability measurements

- Verification VIM 2.44

- Provision of objective evidence that a given item fulfills specified requirements

- Validation VIM 2.45

- Verification, where the specified requirements are adequate for an intended use

\section{MCP Element \#3}

\section{Traceable Reference Materials (Standards)}

- Calibration and working standards are traceable, as well as representative of the type and composition of the material being measured when the matrix affects the measured values

- Such standards will have smaller uncertainties associated with their reference values than the uncertainties of the measurement method in which they are used. Should be $<1 / 3$ of the method's uncertainty.

- Metrological traceability VIM 2.41 (6.10)

- Property of a measurement result whereby the result can be related to a reference through a documented unbroken chain of calibrations, each contributing to the measurement uncertainty. 
MCP Element \#4

Physical/ Environmental Conditions Measurements $\&$ Control

- The desired quality of measurements may require adequate control of environmental conditions or correcting measurements to standard conditions.

- The precision and accuracies of volume and density as well as environmental temperatures, pressure, and humidity measurements are determined and assured where applicable.

- Environmental parameters are specified for various measurement method in national standards and guides.

\section{MCP Element \#5}

\section{Scales and Balances Program}

- This program should include:

- Selection of weighing equipment "fit for purpose"

- OIML R 76-1, 3.7.1 "The standard weights or standard masses used for the verification of an instrument shall not have an error greater than $1 / 3$ of the maximum permissible error of the instrument for the applied load"

- Selecting standards with $<=1 / 3$ the uncertainty of the balance load being tested

- Periodic independent calibration \& maintenance

- Accuracy checks prior to and after scales or balances are used for MC\&A measurements and

- Routine statistical evaluation QC data to evaluate performance \& calculate current uncertainty estimates and control limits.

\section{MCP Element \#6}

\section{Analytical Measurement Control Programs}

- Analytical Measurement Control Programs include:

- Standards preparation for:

- Calibration,

- Measurement quality control (QC),

- Analyst training \& testing and

- Method validation.

- MC programs for measurement system verification, (QC standard measurements are within control limits) before making measurements for accountability or process control.

- Data from the assay of known and unknown QC standards samples are used to calculate new uncertainty estimates \& control limits.

\section{MCP Element \#7}

\section{Sampling Verification}

- The uncertainty associated with each sampling method is determined and monitored. Multiple samples are used to validate homogeneity.

- Comparison of density measurements on sequential samples drawn from a tank can assure adequate mixing and representative samples.

- ITVs address sampling uncertainties in the computation of uncertainty of accountability measurements. 


\section{MCP Element \#8}

\section{Statistical Programs and Control Limits}

\section{- Statistical Program}

A documented program for the statistical evaluation of measurement data for determining control limits, calibration limits, and precision and accuracy levels for each measurement system used for accountability. The objective is to ensure the quality of measurement and measurement control data and to provide estimates of uncertainty on inventory and inventory control

\section{- Statistical Control Limits}

Control limits are calculated and monitored with documented procedures in place to address out-of-control conditions for processes and measurement systems

- For example, limits are established so that the measurement system is not used for accountability measurements until the system is demonstrated to be within statistical control

\section{MCP Element \#9 Proficiency Testing}

- Participation in inter-laboratory sample exchange programs provides external validation of measurement system performance.

- Training: Each facility shall have a documented plan for the training of measurement personnel. It shall specify training, qualification, and requalification requirements for each measurement method.

- Qualification program shall ensure measurement personnel demonstrate acceptable levels of proficiency before performing measurements, and are re-qualified according to requirements in the training plan.

\section{MCP Element \#10}

\section{Accreditation}

- Performed by a formal accrediting body to recognized international standards (ISO 17025 General requirements for the competence of testing and calibration laboratories)

- International Laboratory Accreditation Cooperation (ILAC) promotes acceptance of accredited test \& calibration laboratories

- Accreditation bodies must meet the requirements and accepted as signatories to the ILAC Mutual Recognition Arrangement. Each accreditation body that is a signatory to the Arrangement agrees to abide by its terms and conditions and by the ILAC evaluation procedures

\section{Summary}

- Introduced how the results of measurement control programs to determine if Inventory Differences (ID) are significant for accountability of nuclear material

- Introduced the basic elements of a measurement control program 


\section{REFERENCES}

1. ANSI/INMM N15.41-1984 American National Standard for nuclear facilities - derivation of measurement control programs - general principles.

2. *ANSI/INMM N15.51-2007 for Methods of Nuclear Materials Control --Measurement Control Program - Nuclear Materials -Analytical Chemistry Laboratory

3. *BIPM - JCGM 200:2008 - International vocabulary of basic and general terms in metrology, (VIM) Third Ed. 2008.

4. *BIPM - JCGM 100:2008 "Evaluation of measurement data -- Guide to the expression of uncertainty in measurements. (GUM 1995 with minor corrections)

5. *BIPM - JCGM 104:2009 "Evaluation of measurement data - An introduction to the "Guide to the expression uncertainty in measurements" and related documents. July 2009

6. CITAC Guide 1 "International Guide to Quality in Analytical Chemistry--An Aid to Accreditation", ISBN 0948926 090 English First Edition 1995.

7. Clark, J. P. \& C. H. Jackson, "Establishing the Traceability of a Uranyl Nitrate Solution to a Standard Reference Material", AGNS-10-2, Proceedings of the American Nuclear Society Meeting, Williamsburg, VA, 1978.

8. DOE Order 474.2, "Nuclear Material Control and Accountability" Approved: 6-27-2011

9. DOE-STD-1194-2011 "Nuclear Material Control and Accountability", DOE Standard, June 2011

10. EURACHEM / CITAC Guide CG 4, Quantifying Uncertainty in Analytical Measurement, Second edition 2000 ISBN 0948926155

11. *IAEA STR - 368 "International Target Values for Measurement Uncertainties in Safeguarding Nuclear Materials", Vienna, November 2010 (ITVs)

12. International Laboratory Accreditation Cooperation (ILAC) Website http://www.ilac.org/home.html

13. ISBN 0-948926-08-2, "Quantifying Uncertainty in Analytical Measurements, Eurachem English Publication 1995

14. ISO 8655-2 "Piston-operated volumetric apparatus - Part 2: Piston Pipettes

15. *ISO/IEC 17025,"General requirements for the Competence of Testing and Calibration Laboratories", International Organization for Standardization, 2005

16. ISO/TR 20461:2000(E) "Determination of uncertainty for volume measurements using the gravimetric method.

17. Jaech, J. L., Statistical Methods in Nuclear Material Control, Technical Information Center, United States Atomic Energy Commission, 1973

18. Murdock, Cindy, "Assignment of Values to In-House Standard Uf6 Cylinders Using the Fast 4-1 Series", Portsmouth, Ohio, 1988p

19. NIST Technical Note 1297, 1994 Edition, Guidelines for Evaluating and Expressing the Uncertainty of NIST Measurement Results, Taylor \& Kuyatt

20. *NISTIR 6919, Recommended Guide for Determining and Reporting Uncertainties from Balances and Scales. 2002.

21. NISTIR 6969, Selected Laboratory and Measurement Practices and Procedures to Support basic Mass Calibrations. 2003.

22. OIML R 76-2 International Recommendation "Non-automatic weighing instruments Part 2: Test report format", Edition 2007 (E)

23. OIML R111-1-E, International Recommendation, "Weights of classes E 1, E 2, F 1, F 2, M 1, M 1-2, M 2, M 2-3 and M 3 Part 1: Metrological and technical requirements", 2004, http://www.oiml.org/publications/R/R111-1e04.pdf

24. *Pitard, F. F., Pierre Gy's Sampling Theory and Sampling Practice, Volume I Heterogeneity and Sampling, CRC Press, Inc., 1992

25. *Pitard, F. F., Pierre Gy's Sampling Theory and Sampling Practice, Volume II Sampling Correctness and Sampling Practice, CRC Press, Inc., 1989

26. *Rainin Instrument Inc. "Procedure for Evaluating Accuracy and Precision of Rainin Pipettes", http://www.pipette.com/Support/OnlineLecture/Rainin PipetteAccuracyandPrecision.pdf

27. *Ravinovich, Semyon, "Measurement Errors: Theory and Practice", American Institute of Physics, New York, NY ISBN 1-56396-323-X, 1995

28. Ryan, T. P., Statistical Methods for Quality Improvement, Wiley, NY.

29. Smith, P. L., A Primer for Sampling Solids, Liquids, and Gases: Based on the Seven Sampling Errors of Pierre Gy, Society for Industrial and Applied Mathematics (SIAM), 2001

30. Taylor, John Keenan, "Quality Assurance of Chemical Measurements", Lewis Publishers, 1987.

31. Troemner's "Proper Pipetting Techniques \& Tips", http://www.troemner.com/pdf/ProperPipettingTechniques.pdf

32. Yamamura, S. S, Clark, J. P., et al, NUREG-0253, Preparation of Working Calibration Test Materials: Uranyl Nitrate Solution, 1977 


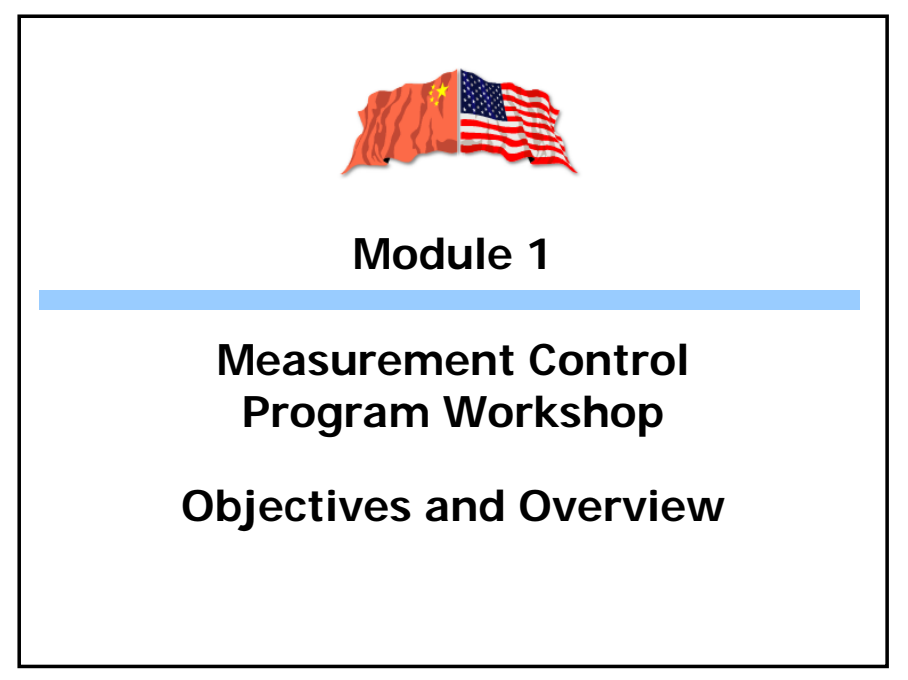

\section{Learning Objectives}

- To apply results of measurement control programs to determine if Inventory Differences (ID) are significant in the accountability of nuclear material

- To understand the elements of a measurement control program

\section{Elements of a Comprehensive Measurement Control Program (MCP)}

1. Data Quality Objectives

2. Measurement Method Selection \& Qualification

3. Traceable Reference Materials (Standards)

4. Physical/Environmental Conditions MC

5. Scales and Balances Program

6. Analytical Quality Control

7. Sampling Verification

8. Statistical Programs \& Statistical Control Limits

9. Proficiency Testing Programs

10. Accreditation by Recognized Accrediting Body

\section{Key Concepts}

- Measurements have uncertainty

- Different types of measurement errors

- Methods for estimating the uncertainty of measurement

- National \& international standards related to measurements and measurement uncertainty

- Metrology vocabulary definitions

- International Vocabulary of Basic and General Terms in Metrology (VIM) 


\section{Ds Are Not Zero Because}

- Measurement uncertainty: measurement system effects

- Location of material

- In calorimeter, on scale, etc.

- For example, heat distribution, weight distribution, etc.

- Calibration of scales

- Fluctuations in air pressure, temperature, etc.

\section{Ds Are Not Zero Because}

- Measurement uncertainty: Non-measurement system effects

- Power fluctuations

- Electronic functioning of equipment

- Non-homogeneity of material being measured

- Statistical nature of radioactive decay

- Improper or incomplete background measurements

\section{Ds Are Not Zero Because}

\section{- Sampling effects}

- Improper or incomplete blending in a destructive analysis sample

- Nondestructive Analysis (NDA) limitations

- Material composition of the NDA standards does not match the material composition of the measured items

- Failure to account for background effects

\section{Ds Are Not Zero Because}

- Accounting system effects

- Better measurement to correct estimates

- Decay, rounding errors, etc.

- Human error

- Clerical mistakes (transcription errors, etc.)

- Failure to follow procedures 


\section{IDs Are Not Zero Because}

- Unmeasured streams or inventories

- Solids entrained in liquid systems settle in tanks

- Holdup can take the form of material associated with specific equipment

- Factors or estimates

\section{MCP Element \# 1 Data Quality Objectives}

- The IAEA's International Target Values (ITVs) for uncertainty components in measurements of nuclear material should be considered

- Maximum Permissible Error VIM 4.26 (5.21) limit of error

Extreme value of measurement error, with respect to a known reference quantity
value, permitted by specifications or regulations for a given measurement, measuring

- NOTE 1 Usually, the term "maximum permissible errors" or "limits of error" is

- NOTE 2 The term "tolerance" should not be used to designate 'maximum
permissible error'

- Target Uncertainty VIM 2.34

- Measurement uncertainty specified as an upper limit and decided on the basis of the intended use of measurement results

\section{MCP Element \#2 \\ Measurement Method Selection and Qualification}

- Method selections are based on target values

- A documented method is used on an identified periodic basis to ensure that a measurement method shall demonstrate acceptable performance before being used for performing accountability measurements

- Verification VIM 2.44

- Provision of objective evidence that a given item fulfills specified requirements

- Validation VIM 2.45

- Verification, where the specified requirements are adequate for an intended use

\section{MCP Element \#3}

\section{Traceable Reference Materials (Standards)}

- Calibration and working standards are traceable, as well as representative of the type and composition of the material being measured when the matrix affects the measured values

- Such standards will have smaller uncertainties associated with their reference values than the uncertainties of the measurement method in which they are used. Should be $<1 / 3$ of the method's uncertainty.

- Metrological traceability VIM 2.41 (6.10)

- Property of a measurement result whereby the result can be related to a reference through a documented unbroken chain of calibrations, each contributing to the measurement uncertainty. 
MCP Element \#4

Physical/ Environmental Conditions Measurements $\&$ Control

- The desired quality of measurements may require adequate control of environmental conditions or correcting measurements to standard conditions.

- The precision and accuracies of volume and density as well as environmental temperatures, pressure, and humidity measurements are determined and assured where applicable.

- Environmental parameters are specified for various measurement method in national standards and guides.

\section{MCP Element \#5}

\section{Scales and Balances Program}

- This program should include:

- Selection of weighing equipment "fit for purpose"

- OIML R 76-1, 3.7.1 "The standard weights or standard masses used for the verification of an instrument shall not have an error greater than $1 / 3$ of the maximum permissible error of the instrument for the applied load"

- Selecting standards with $<=1 / 3$ the uncertainty of the balance load being tested

- Periodic independent calibration \& maintenance

- Accuracy checks prior to and after scales or balances are used for MC\&A measurements and

- Routine statistical evaluation QC data to evaluate performance \& calculate current uncertainty estimates and control limits.

\section{MCP Element \#6}

\section{Analytical Measurement Control Programs}

- Analytical Measurement Control Programs include:

- Standards preparation for:

- Calibration,

- Measurement quality control (QC),

- Analyst training \& testing and

- Method validation.

- MC programs for measurement system verification, (QC standard measurements are within control limits) before making measurements for accountability or process control.

- Data from the assay of known and unknown QC standards samples are used to calculate new uncertainty estimates \& control limits.

\section{MCP Element \#7}

\section{Sampling Verification}

- The uncertainty associated with each sampling method is determined and monitored. Multiple samples are used to validate homogeneity.

- Comparison of density measurements on sequential samples drawn from a tank can assure adequate mixing and representative samples.

- ITVs address sampling uncertainties in the computation of uncertainty of accountability measurements. 


\section{MCP Element \#8}

\section{Statistical Programs and Control Limits}

\section{- Statistical Program}

A documented program for the statistical evaluation of measurement data for determining control limits, calibration limits, and precision and accuracy levels for each measurement system used for accountability. The objective is to ensure the quality of measurement and measurement control data and to provide estimates of uncertainty on inventory and inventory control

\section{- Statistical Control Limits}

Control limits are calculated and monitored with documented procedures in place to address out-of-control conditions for processes and measurement systems

- For example, limits are established so that the measurement system is not used for accountability measurements until the system is demonstrated to be within statistical control

\section{MCP Element \#9 Proficiency Testing}

- Participation in inter-laboratory sample exchange programs provides external validation of measurement system performance.

- Training: Each facility shall have a documented plan for the training of measurement personnel. It shall specify training, qualification, and requalification requirements for each measurement method.

- Qualification program shall ensure measurement personnel demonstrate acceptable levels of proficiency before performing measurements, and are re-qualified according to requirements in the training plan.

\section{MCP Element \#10}

\section{Accreditation}

- Performed by a formal accrediting body to recognized international standards (ISO 17025 General requirements for the competence of testing and calibration laboratories)

- International Laboratory Accreditation Cooperation (ILAC) promotes acceptance of accredited test \& calibration laboratories

- Accreditation bodies must meet the requirements and accepted as signatories to the ILAC Mutual Recognition Arrangement. Each accreditation body that is a signatory to the Arrangement agrees to abide by its terms and conditions and by the ILAC evaluation procedures

\section{Summary}

- Introduced how the results of measurement control programs to determine if Inventory Differences (ID) are significant for accountability of nuclear material

- Introduced the basic elements of a measurement control program 


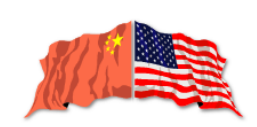

Module 1-2

\section{MCP Guidance, Requirements \& Procedures}

\section{Overview}

- All measurements have an uncertainty associated with the results due to various sources of error.

- The metrological community recognizes the need for standardizing how this "UNCERTAINTY" should be determined and expressed. Many documents have been written that provide essential MCP information.

- Familiarity with the various written national and international standards, guides, publications and orders helps us to see the big picture for MCPs.

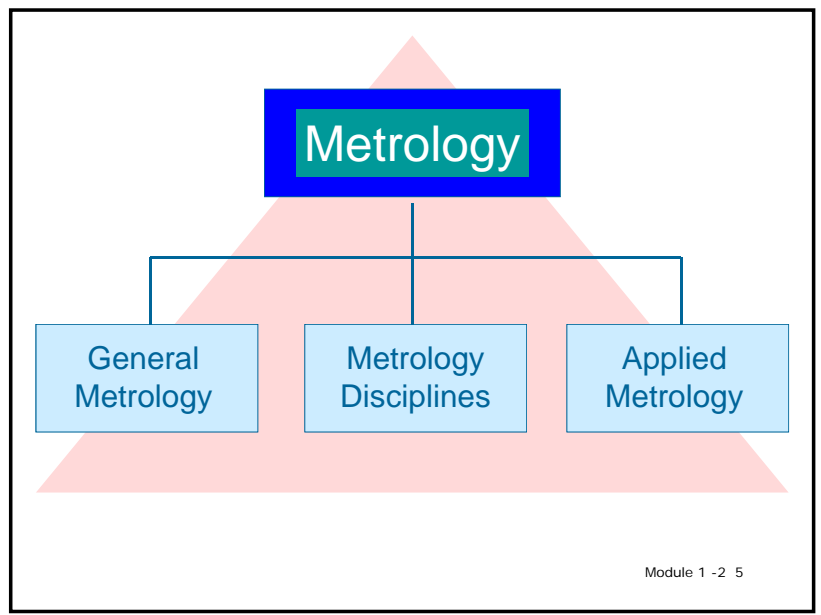

\section{Objectives}

- To understand the DOE requirements for measurement and measurement control programs

- To understand the facility measurement and measurement control program and its relationship to DOE requirements

- To understand US and international standards related to measurement control programs and measurement uncertainty [ANSI (15.51), ISO(17025), BIPM (GUM), STR (IAEA Target Values), etc.]

\section{Metrology is:}
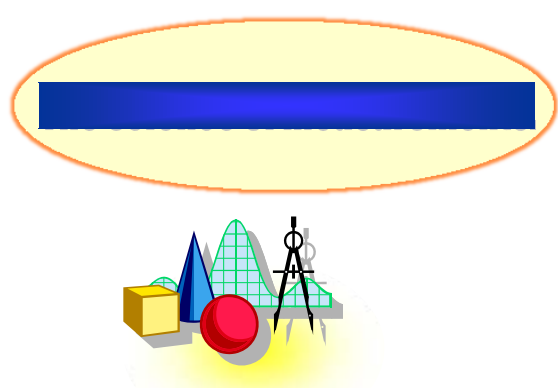

Module 1-2 4

\section{Metrology Organizations}

- National Metrology Labs

- China: National Institute for Metrology (NIM)-9 Divisions

- France: International Bureau des Poids et Mesures (BIPM)

- United Kingdom: National Physical Laboratory(NPL)

- USA: National Institute for Standards \& Technology (NIST)

- Legal Metrology Labs

- Usually state labs responsible for weights, measures, etc.

- Industrial Metrology Laboratories

- Pharmaceutical, Petroleum, Manufacturing, Medical, etc.

- Nuclear: in USA the national laboratories have metrology laboratories 


\section{DOE requirements for measurement \& measurement control programs}

- DOE Order 474.2, approved 6-27-2011 "Nuclear

Material Control and Accountability"

- DOE Standard 1194-2011, approved June 2011

"Nuclear Materials Control and Accountability"

\section{DOE Order 474.2, approved 6-27-2011 "Nuclear Material Control \& Accountability"}

Attachment 1."Contractor Requirements Document DOE 474.2 Nuclear Material Control And Accountability"

(3) Measurement Objectives.

(a) The measurements program must provide measured values with uncertainties sufficient to detect theft or diversion of nuclear material.

(b) The measurement control program must assure the quality of measurements made for MC\&A purposes.

\section{(3) Measurement Objectives. (continued)}

\section{MEASUREMENTS METRICS.}

The measurement program element provides quantitative and qualitative data about nuclear materials for accounting, inventories, and transfers. Measured values are essential for establishing category levels and determining protection requirements for nuclear materials.

a. The quantity of all nuclear material types present on inventory is determined using identified measurement systems, measurement services, technically justified values or accepted shipper's values when approved by the DOE line management.

\section{MEASUREMENTS METRI CS (continued)}

b. Measurements and measurement uncertainties are determined and reported for each measured value using methodologies and statistical terminology accepted by voluntary consensus standards bodies. Other methodologies are only used with sufficient justification and approved in the MC\&A plan.

c. Sources of measurement error that are key contributors to the total measurement limit of error for a material balance period are identified and used to estimate systematic and random errors.

d. Before use (beginning of the work day), the proper functioning of measurement systems is checked against standards.

\section{MEASUREMENTS METRI CS (continued)}

e. Key measurement points are established during construction of or changes to process lines, and documented in project documentation. Key measurement points are included in operational (process and transfer) procedures.

f. The site/facility operator demonstrates that uncertainties remain applicable and are consistent with target values established by the site.

g. Measurement methods are qualified, formally documented, periodically validated, and approved in the MC\&A plan.

\section{MEASUREMENTS METRI CS (continued)}

h. Potential sources of sampling error for bulk measurements are identified and samples are representative of the materials being sampled. If sampling is required to establish accountability measurements, the program describes how the

representativeness/homogeneity is determined and periodically tested or updated.

i. Capability exists to confirm type and quantity of nuclear material present. For each type of nuclear material at the site, measurement methods are identified which are capable of confirming presence of nuclear materials and verifying nuclear material quantities. Nuclear materials not amenable to verification measurement must be identified and documented in the MC\&A Plan. 


\section{MEASUREMENTS METRICS (continued)}

j. Measurements are traceable to the National Institute of Standards and Technology (NIST) or New Brunswick Laboratory (NBL) standards.

k. Measurement systems are calibrated on a defined frequency, including the frequency and methodology for recertifying the measurement standards.

I. The measurement program identifies target values for each MC\&A measurement method, referencing national and international sources as applicable and defines the methodology, including frequency, by which uncertainties are compared to the target values and performance is assessed.

\section{DOE Standard for NMC\&A}

DOE-STD-1194-2011 dated J une 2011.

- The Technical Standard provides an acceptable MC\&A approach commonly or typically used throughout the DOE and NRC.

- In the final analysis, DOE line management reviewer makes a judgment as to whether the site/facility operator can achieve, with high probability, the objectives stated in DOE 0 474.2."

\section{DOE-STD-1194-2011(Continued)}

6.3.1 Specifications [Measurements] (Types) Three types of measurements are used for accountability purposes:

- Accountability measurements shall be used to establish initial values for nuclear materials and to replace existing values with more accurate measured values.

- Verification measurements shall be used to validate the accounting system values when necessary, e.g., at time of physical inventory for non-tamper-indicating items or in response to a security anomaly that could have resulted in a theft or diversion of nuclear material.

- Confirmation measurements shall be used to validate the presence of nuclear material for transfers, and to determine nuclear material presence under anomalous conditions.

\section{MEASUREMENTS METRI CS (continued)}

m. A methodology exists for ensuring that measurement systems are in control when accountability measurements are made, and evidence exists that accounting values are established only when measurement systems are in control.

n. A statistical control system exists that includes establishing control limits, determining out-of-control conditions, returning control to out-of-control measurement systems, and analyzing trends and outliers.

o. The technical basis for the measurement and measurement control program is documented, and the documentation is either included or referenced in the MC\&A Plan.

\section{DOE-STD-1194-2011 (Continued)}

6.1.4.2 Measurements Training

- Training plan that covers personnel who make measurements (e.g., DA, NDA, sampling, process measurements etc.)

- Performance criteria for the personnel making the measurements

- Covers periodic qualification and requalification as part of the measurement control plan for a KMP (key measurement point)

- Personnel shall be knowledgeable of the standards and their values for each measurement system. Where sampling of bulk material is necessary, personnel shall be familiar with the sampling and sub-sampling programs

\section{DOE-STD-1194-2011 (Continued)}

6.3.2 Measurement Methods \& Procedures

- When a weight or mass measurement method is used for accountability purposes, a daily check of both accuracy and linearity shall be performed.

- Accountability measurement methods shall be selected to minimize the uncertainty of the inventory difference, maximize the loss detection measurement results consistent with the consequences of the loss of the material.

- Target values for precision and accuracy, established and approved target values for precision and accuracy, established and approv

- All measurement systems generating accountability values shall be in All measurement systems generating
the measurement control program.

- A measurement control program shall quantify the random and systematic errors in all quantitative measurements used to assess the statistical significance of inventory differences and shipper/receiver
differences. 


\section{DOE-STD-1194-2011(Continued)}

- Target values for precision and accuracy, established and approved by DOE line management, shall be used as performance goals. Performance during qualification shall be documented to validate that the method can be performed with the material types for which the method is qualified. $A$ reference for target values is: International Target Values 2000 for Measurement Uncertainties in Safeguarding Nuclear Materials, IAEA Report STR-327, published April 2001.

- 6.3.4.1 All measurement systems generating accountability values shall be in the measurement control program.

- 6.3.4.2 A measurement control program shall quantify the random and systematic errors in all quantitative measurements used to assess the statistical significance of inventory differences and shipper/receiver differences.

\section{The Origins of GUM}

- The Bureau International des Poids et Mesures (BIPM) Was fo 1875 by the signing of the Convention of the Metre by 17 countries. Currently, there are 54 signatories to the Convention.

- The task of the BIPM is to ensure world-wide uniformity of measurements and their traceability to the International System of

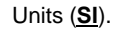

- The BIPM operates through a series of Consultative Committees, whose members are the national metrology laboratories of the Member States of the Convention, and through its own laboratory work.

- The BIPM carries out measurement-related research. It takes part in and organizes, international comparisons of national measurement standards, and it carries out calibrations for Member States.

\section{Facility measurements and MCP relationships to DOE requirements}

- In the DOE complex, facilities are routinely assessed by senior officials and technical experts to evaluate technical and administrative compliance with DOE orders.

- Continuous improvement is emphasized and projects are funded to improve and/or replace measurement having questionable uncertainties.

- Laboratory Information Management Systems (LIMS) are important for MC\&A \& complying with DOE orders.

- Recently an emphasis has been placed on determining measurement uncertainties per the "Guide to the Expression of Uncertainty in Measurement".

- Recently some of the facilities have been getting MC\&A measurement methods accredited to ISO 17025 requirements

\section{The Origins of GUM}

- In 1977 the CIPM asked BIPM to, via collaboration with national metrology institutes:

- Address the problem of the lack of international agreement on expressing uncertainties, and

- Propose a specific recommendation for a solution to the problem.

- BIPM convened the Working Group on the Statement of Uncertainties.

- The Working Group issued Recommendation INC-1 (1980), which is an overview of an agreed-upon approach to measurement uncertainty evaluation.
INC-1: Five Recommendations (as stated in their report)

1.The uncertainty in the result of a measurement generally consists of several components which may be grouped into two categories according to the way in which their numerical value is estimated:

A - those which are evaluated by statistical methods.

$B$ - those which are evaluated by other means.

There is not always a simple correspondence between the classification into categories A or B and the previously used classification into "random" and "systematic" uncertainties. The term "systematic uncertainty" can be misleading and should be avoided.
INC-1: Five Recommendations (as stated in their report) Recommendation 1 (continued)

Any detailed report of the uncertainty should consist of a complete list of the components, specifying for each, the method used to obtain its numerical value.

In common usage, "random "uncertainty is a source of uncertainty (distribution) that can be reduced by replicate measurements, while "systematic" uncertainty is a source of uncertainty (distribution) that is not significantly reduced by replicate measurements. 


\section{NC-1: Five Recommendations (as stated in their report, continued)}

2. The components in category $\mathbf{A}$ are characterized by the estimated variance, $s_{i}{ }^{2}$, (or the estimated "standard deviations" $s_{i}$ ) and the number of degrees of freedom, $v_{i}$ Where appropriate, the estimated covariances should be given.

3. The components in category $\mathbf{B}$ should be characterized by quantities $u^{2}$, which may be considered as approximations to the corresponding variances, the existence of which is assumed. The quantities $u_{j}^{2}$, may be treated like variances and the quantities $u_{j}$ like standard deviations. Where appropriate, the covariance should be treated in a similar way.

\section{The History of the GUM}

- CIPM approved INC-1 in 1981 and reaffirmed it in 1986 via its own recommendations.

- Because INC-1 was a rather broad outline, the CIPM asked the International Organization for Standardization (ISO) to develop a detailed guide.

- ISO was chosen because it could represent the broad interests of industry and commerce.

- ISO Technical Advisory Group on Metrology (TAG 4) was given the responsibility.

\section{The 'modern' GUM}

- In 1997, the seven organizations in ISO/TAG4/WG3 were represented in the BIPM's Joint Committee for Guides in Metrology (JCGM) which was chaired by BIPM.

- The JCGM took over responsibilities for the GUM (and the VIM) from ISO TAG4 with 2 working groups:

- WG1 for the GUM and

- WG2 for the VIM.

- Current documents:

- JCGM 100:2008 is current GUM Guide

- JCGM 104:2009 is an intro to the GUM Guide

- JCGM 200:2008 is the current VIM
INC-1: Five Recommendations (as stated in their report, continued)

4. The combined uncertainty should be characterized by the numerical value obtained by applying the usual method for the combination of variances. The combined uncertainty and its components should be expressed in the form of "standard deviations".

5. if, for particular applications, it is necessary to multiply the combined uncertainty by a factor to obtain an overall uncertainty, the multiplying factor must be stated.

Module $1-2-26$

\section{History of the GUM, continued}

- ISO TAG4 established Working Group 3 in the late ' 80 ' $s$ to develop a guidance document based upon the recommendation of the BIPM WG which was to:

1. Promote full information on how uncertainty statements are arrived at and,

2. Provide a basis for the international comparison of measurement results

- The "Guide to the Expression of Uncertainty in Measurement" (GUM) was published in 1993 by ISO in the name of BIPM, IEC, IFCC, ISO, IUPAC, IUPAP and OIML.

- The Guide (GUM) was amended and corrected in 1995.

\section{The 'modern' GUM, continued}

- Supplement 1 to GUM issued:

- JCGM 101:2008 using Monte Carlo issued

- Other enhancements to the evaluation of measurement data:

- Coming 'soon'

- Role of measurement uncertainty in conformity assessment

- Concepts and basic principles

- Supplement 2 - models with any number of output quantities Coming 'later' :

- Supplement 3 - Modeling

- Applications of the least-squares method 


\section{Uncertainty Requirements Documents} (Continued)

\section{- ANSI/INMM N15.51-2012 for Methods of Nuclear} Materials Control --Measurement Control Program Nuclear Materials -Analytical Chemistry Laboratory

- The latest version of a guide for setting up an analytical laboratory measurement control program

- It was based on ANSI/INMM 15.41-1984 "derivation of measurement control programs - general principle"

- Both standards provide basic principles which address both technical and administrative aspects of measurement processes.

- The administrative aspects correspond to the QA elements and their implementation establishes the QA practices in the MCP.

\section{ANSI / I NMM N15.51-2012 (continued)}

- The standard does not address MCP elements for bulk measurement for processes or specific sampling techniques. It also does not address special analytical techniques such as calorimetry or mass spectrometry.

- The standard references supporting publications and provides annexes containing examples that illustrate the general principles given.

- The 2012 version updates this standard to comply with the GUM concepts of measurement uncertainty.

\section{Uncertainty Requirements Documents}

(Continued)

- ISO 10012 "Measurement Control System"

- 7.3.1 Measurement uncertainty shall be taken into account for each measurement process covered by the measurement control system.

The ISO "Guide to the expression of uncertainty in measurements" (GUM) is referenced for guidance; however, other documented and accepted methods may be used.
ISO/I EC 17025 "General requirements for the competence of testing \& calibration laboratories"

- ISO/IEC Guide 25 'General Requirements for the Competence of Calibration and Testing Laboratories' was published in 1990 as the internationally agreed set of Quality System requirements applicable to laboratories who perform calibration and/or testing services. It was superseded in early 2000 by ISO/IEC 17025 which forms the basis for laboratory accreditation.

- ISO 9001/9002 contains requirements for calibration, inspection and testing. Registration to these standards provides confidence both internally and to the customer that these activities are adequately controlled.

- In 17025, emphasis is placed on the competence of the work as opposed to compliance with procedures. Hence, the the need to quantify measurement quality (uncertainty).

\section{Uncertainty Requirements Documents} (Continued)

- ISO/IEC 17025 "General requirements for the competence of testing \& calibration laboratories"

- 5.4.6.1 A calibration laboratory, or a testing laboratory performing its own calibrations, shall have and shall apply a procedure to estimate the uncertainty of measurement for all calibration and type of calibrations.

The degree of rigor needed in an estimation of uncertainty of measurement depends on factors such as: the test method; the client; the existence of narrow limits on which decisions on conformance to a specification are based.

- Accreditation to ISO/IEC 17025 is far more "competencybased" than registration to ISO 9000 series

\section{Uncertainty Requirements Documents} (Continued)

- American National Standards for Calibration ANSI/NCSL Z540-1-1994 "Calibration Laboratories and Measuring and Test Equipment - General Requirements"

- 10.2.b "The laboratory shall ensure that the calibration uncertainties are sufficiently small so that the adequacy of the measurement is not affected. Well defined and documented measurement assurance techniques or uncertainty analyses may be used to verify the adequacy of a measurement process. If such techniques or analyses are not used then the collective uncertainty of the measurement standards shall not exceed $\mathbf{2 5 \%}$ of the acceptable tolerance for each characteristic of the M\&TE 


\section{Uncertainty Requirements Documents \\ (Continued)}

- IAEA STR - 368 "International Target Values for Measurement Uncertainties in Safeguarding Nuclear Materials", Vienna, November 2010 (ITVs)

- The ITVs are uncertainties to be considered in judging the reliability of analytical techniques applied to industrial nuclear and fissile material, which are subject to safeguards verification.

- The tabulated values represent estimates of the „state of the practice" which should be achievable under routine measurement conditions.

- The most recent standard conventions in representing uncertainty have been considered, while maintaining a format that allows comparison with the previous releases of the ITVs.

\section{AEA STR - 368 (continued)}

- The present (2010) report explains why target values are needed, how the concept evolved and how they relate to the operator's and inspector's measurement systems.

- The ITVs-2010 are intended to be used by plant operators and safeguards organizations, as a reference of the quality of measurements achievable in nuclear material accountancy, and for planning purposes.

- The report suggests that the use of ITVs can be beneficial for statistical inferences regarding the significance of operator-inspector differences whenever valid performance values are not available.

\section{Summary}

- Reviewed the DOE requirements for measurement and measurement control programs

- Discussed the facility measurement and measurement control program and its relationship to DOE requirements

- Reviewed US and international standards related to measurement control programs and measurement uncertainty [ANSI (1551), ISO(17025), BIPM (GUM), STR (IAEA Target Values), etc.] 


\begin{tabular}{|c|c|c|c|c|}
\hline 1) Index & 2) Data & 3) Mean & $\begin{array}{c}\text { 4) Difference between data values } \\
\text { and the mean }\end{array}$ & $\begin{array}{c}\text { 5) The square of } \\
\text { column } 4\end{array}$ \\
\hline $\mathrm{i}$ & $\mathrm{X}_{\mathrm{i}}$ & $\mathrm{Xbar}$ & $\left(\mathrm{X}_{\mathrm{i}}-\mathrm{Xbar}\right)$ & $(\mathrm{Xi}-\mathrm{Xbar})^{2}$ \\
\hline 1 & 1 & 4 & -3 & 9 \\
\hline 2 & 8 & 4 & 4 & 16 \\
\hline 3 & 3 & 4 & -1 & 1 \\
\hline 4 & 6 & 4 & 2 & 4 \\
\hline 5 & 2 & 4 & -2 & 4 \\
\hline
\end{tabular}

Sum of $(X i-X b a r)^{2}$ 


\begin{tabular}{|c|c|c|c|c|}
\hline 1) Index & 2) Data & 3) Mean & $\begin{array}{c}\text { 4) Difference between data values } \\
\text { and the mean }\end{array}$ & $\begin{array}{c}\text { 5) The square of } \\
\text { column 4 }\end{array}$ \\
\hline $\mathrm{i}$ & $\mathrm{X}_{\mathrm{i}}$ & Xbar & $\left(\mathrm{X}_{\mathrm{i}}-\mathrm{Xbar}\right)$ & $(\mathrm{Xi}-\mathrm{Xbar})^{2}$ \\
\hline 1 & & & 0 & 0 \\
\hline 2 & & & 0 & 0 \\
\hline 3 & & & 0 & 0 \\
\hline 4 & & & 0 & 0 \\
\hline 5 & & & 0 & 0 \\
\hline
\end{tabular}




\section{Exercises \\ Introduction to Statistics}

\section{Session Objectives:}

After the session the participants will be able to do the following:

1. Calculate the mean (average), variance, and standard deviation for a group of data by hand or by using Microsoft Excel

\section{Estimated Time:}

The exercises are instructor led so the time is included as part of the presentation time.

\section{Materials Needed:}

1. One computer with Microsoft Excel for each group of 5 students

2. Microsoft Excel workbooks a through c should be loaded on student computers

a. $\quad$ Filename $=$ Module $2-$ Statistical Review.xxls

b. $\quad$ Filename $=$ Module 2 Var and Stddev by hand.xxls

c. Filename $=$ Module 2 - Technician Selection.xxls

3. The students should be familiar with Excel

4. Pencil and paper

\section{Instructions:}

1. There are 3 instructor led exercises within Module 2. Using Microsoft Excel the instructor will lead students through the steps of calculating the mean, variance, and standard deviations for sets of data.

a. Exercise 1 is on Slide 17 - Calculate the mean

b. Exercise 2 is on Slides 30-34 - Calculate the variance and standard deviation without the Excel functions

c. Exercise 3 is on Slide 35 - Calculate the variance and standard deviation with the Excel functions

d. Exercise 4 is on Slide 37 - Calculate the mean, variance, and standard deviation and use that data to contrast the performance of 2 technicians. 


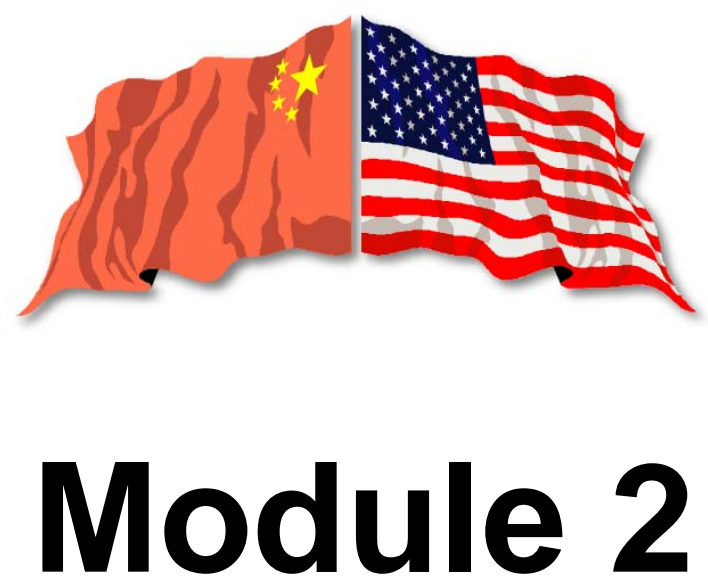

Statistics Review 


\section{Objectives}

- Review basic statistical terms and concepts

- Review Mean/Average and how it is calculated

- Review the concept of a "Probability Distribution" and the Normal Distribution

- Review Variance and Standard Deviation and how they are calculated

- Review the concepts of "Bias" and "Fit for Purpose" 


\section{Populations}

A population is the complete set of elements to be studied.

Examples:

- All measurements made with a particular instrument or method

- All analyses performed by a certain technician

- All uranium destructive analyses made by a certain laboratory over a given time period

- All items created by a particular process 


\section{Parameters}

A parameter is a numeric result that describes the population.

\section{Examples:}

- Concentration of uranium in the process solution

- Ratio of Pu/U in a mixed oxide

- Amount of Pu in a container

Parameters are typically unknown. 


\section{Samples}

- The only way to know the exact value of a parameter is to examine every element in the population.

- Typically it is not feasible to look at every element so samples are taken from the population or an attribute is observed that correlates to the parameter in which we are interested. 


\section{Data}

- The sample is observed or measured in some fashion.

- Data results from observations or measurements. 


\section{Statistics}

- Data is summarized to provide statistics.

- A statistic is a numeric result that describes a sample.

- Statistics are used to estimate unknown parameters. 


\section{Estimation and Confidence I ntervals}

- Calculated statistics will rarely equal the exact value of an unknown parameter so uncertainty is reported with any parameter that is estimated.

- Confidence intervals are a range of values that contain the unknown parameter within a stated confidence level.

- Example: Based on the measurements taken, we are $95 \%$ confident that the uranium content of the item is between 1,500 and 1,900 grams. 


\section{Statistical I nference}

Statistical inference supports the decision making processes

- Is the average weight of an item from a particular process equal to $1.5 \mathrm{~kg}$ (e.g., manufacturing tolerance)?

- Is the inventory difference significantly different from zero (e.g., Safeguards and Safety question)?

- Is a measurement method meeting the accuracy and precision requirements established for it?

- Is a new technician able to meet the job requirements for making uranium analyses? 


\section{Accuracy and Precision are two important characteristics of measurements}

- If the process mean or average is close to the target center, it is said to be accurate.

- If the process has little variation, it is said to be precise. Another term used is dispersion.
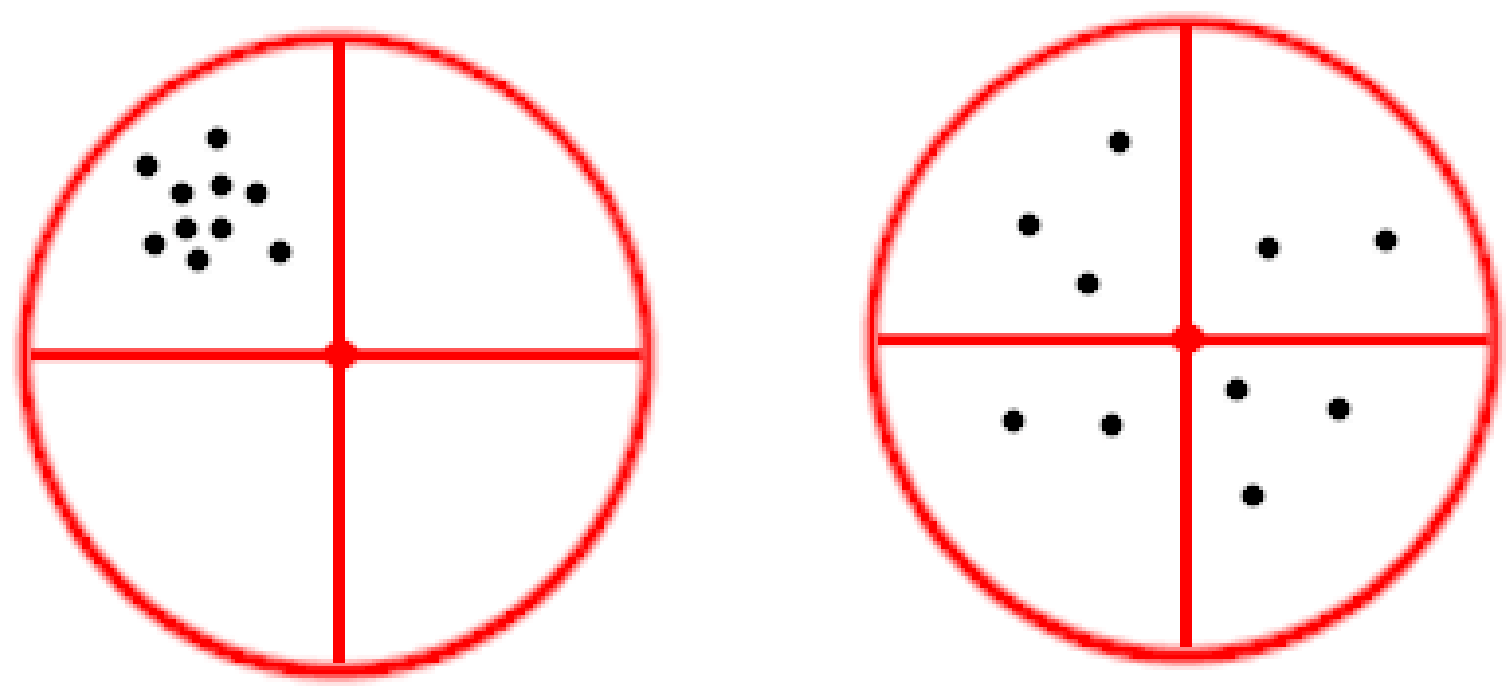


\section{Accuracy and Precision - cont'd.}

- The shooter on the right is the most accurate. Although the shots are widely scattered, the mean or average is close to the target center.

- The shooter on the left has the least variation between shots and is thus more "precise". This shooter's shots are less dispersed.
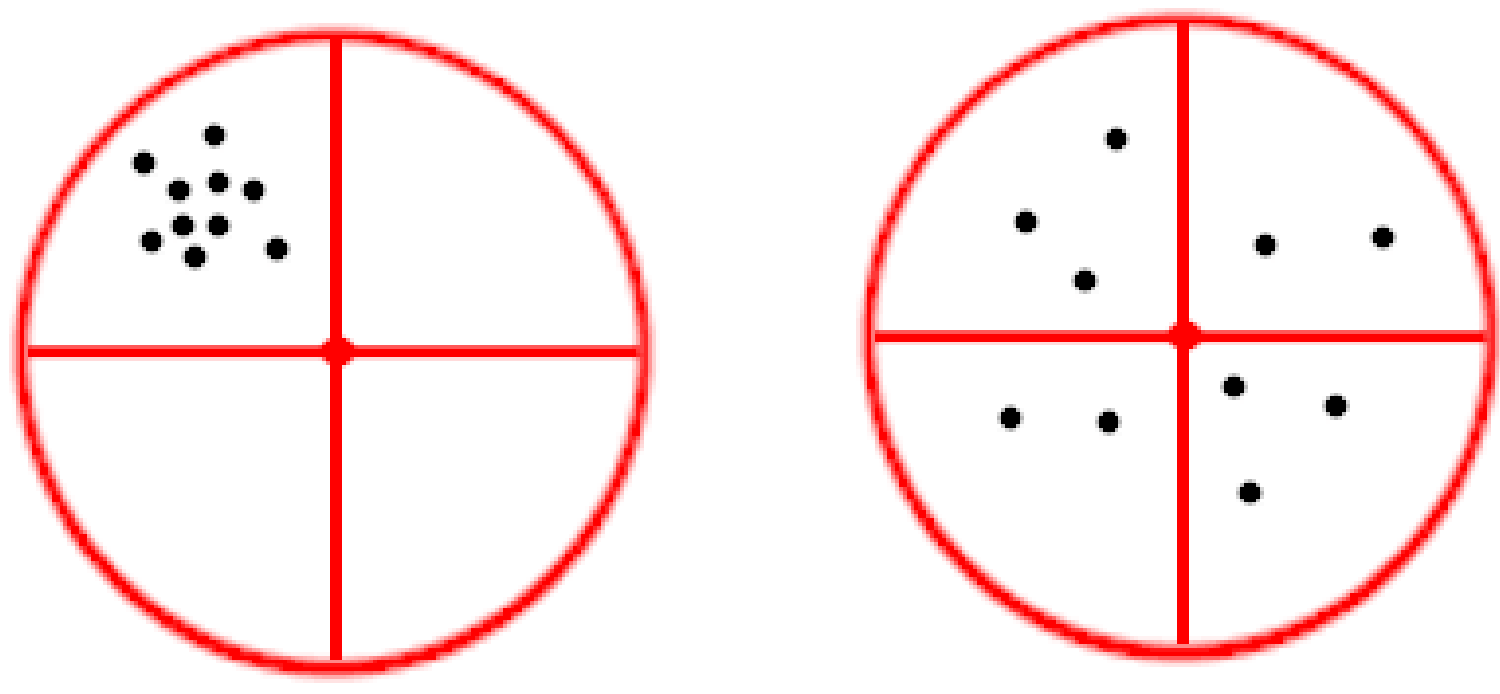


\section{Measures of Central Location (i.e., accuracy)}

\begin{tabular}{|l|l|l|l|}
\hline Measure & Description & Excel Function \\
\hline Mean/Average & $\begin{array}{l}\text { The sum of the sample } \\
\text { values divided by the } \\
\text { sample size. The } \\
\text { sample statistic } x \text { is an } \\
\text { unbiased estimate of } \\
\text { the population } \mu .\end{array}$ & =AVERAGE(date_range) \\
\hline Median & $\begin{array}{l}\text { The middle value after } \\
\text { the sample values have } \\
\text { been sorted into order } \\
\text { by magnitude. If there } \\
\text { are an even number of } \\
\text { values in the sample, } \\
\text { the average of the two } \\
\text { middle values. }\end{array}$ & =MEDIAN(data_range) \\
\hline The most common & & \\
\hline value in the sample. & & \\
\hline Mode & & & \\
\hline
\end{tabular}




\section{Measures of Dispersion (i.e., precision)}

\begin{tabular}{|c|c|c|c|}
\hline Measure & Description & Formula & Excel Function \\
\hline Range & $\begin{array}{l}\text { The difference between the } \\
\text { largest and smallest values in the } \\
\text { sample. }\end{array}$ & & $\begin{array}{l}=\mathrm{MAX}(\text { data_range })- \\
\mathrm{MIN}(\text { data_range) }\end{array}$ \\
\hline Variance & $\begin{array}{l}\text { An estimate of the variation or } \\
\text { dispersion of the process from } \\
\text { which the sample is drawn. The } \\
\text { sample statistic "S2" is an } \\
\text { unbiased estimate of the } \\
\text { population parameter } \sigma^{2} \text {. }\end{array}$ & $s^{2}=\frac{\sum_{i=1}^{i=n}\left(x_{i}-\bar{x}\right)^{2}}{n-1}$ & $=\mathrm{VAR}$ (data_range) \\
\hline $\begin{array}{l}\text { Standard } \\
\text { Deviation }\end{array}$ & $\begin{array}{l}\text { The square root of the variance. } \\
\text { Often preferred as a measure of } \\
\text { the process variation. The } \\
\text { sample statistic "s" is an estimator } \\
\text { of the population parameter } \sigma \text {. } \\
\text { This method of calculating the } \\
\text { standard deviation is known as } \\
\text { the Root Mean Square Error } \\
\text { (RMSE) method. }\end{array}$ & $s=\sqrt{\frac{\sum_{i=1}^{i=n}\left(x_{i}-\bar{x}\right)^{2}}{n-1}}$ & $=\mathrm{STDEV}$ (data_range) \\
\hline
\end{tabular}




\section{Part 2}

Calculating Measures of Central Location (e.g., average or mean) 
Mean or Average - The sum of all values being considered divided by the total number of values in the set.

Calculate the mean of the 5 numbers below:

$$
\begin{array}{lllll}
1 & 8 & 3 & 6 & 2
\end{array}
$$

Formula:

$$
\begin{aligned}
& \text { Xbar (e.g., Mean) }=\frac{X_{1}+X_{2}+X_{3}+\cdots+X n}{n} \\
& \text { OR } \quad \bar{x}=\frac{\sum_{i=1}^{i=n} x_{i}}{n}
\end{aligned}
$$




\section{Mean or Average - The sum of all values being considered divided by the total number of values in the set.}

Calculate the mean of the 5 numbers below:

$$
\begin{array}{lllll}
1 & 8 & 3 & 6 & 2
\end{array}
$$

Answer:

$$
X \mathrm{Bar}=\frac{1+8+3+6+2}{5}=4
$$




\section{Exercise - Calculate the mean}

Our metrologist weighed a $\mathbf{1 0 0}$ gram mass standard $\mathbf{2 0}$ times. The results are shown in the table below. Based on the formulas (or Excel function) presented previously, calculate the mean weight.

$$
\begin{array}{rrrrr}
100.64 & 97.55 & 100.62 & 110.00 & 100.67 \\
99.11 & 102.22 & 107.40 & 112.58 & 101.14 \\
95.41 & 106.86 & 103.77 & 98.53 & 99.59 \\
93.91 & 101.17 & 104.28 & 96.99 & 108.41
\end{array}
$$

Note: Use the "Spreadsheet 1 Module 2" to calculate the average or mean. 
< See Answer Handout > 


\section{Part 3}

Calculating Measures of Dispersion Variance and Standard Deviation

(Random Error) 


\section{How were the weight measurements dispersed from the previous exercise?}

Our metrologist weighed a $\mathbf{1 0 0}$ gram mass standard $\mathbf{2 0}$ times. The results are shown in the table below.

$\begin{array}{rrrrr}100.64 & 97.55 & 100.62 & 110.00 & 100.67 \\ 99.11 & 102.22 & 107.40 & 112.58 & 101.14 \\ 95.41 & 106.86 & 103.77 & 98.53 & 99.59 \\ 93.91 & 101.17 & 104.28 & 96.99 & 108.41\end{array}$

$X-B a r=102.04$ 


\section{Histograms are a way to show variation (precision) graphically}

Histogram of Weight Measurements

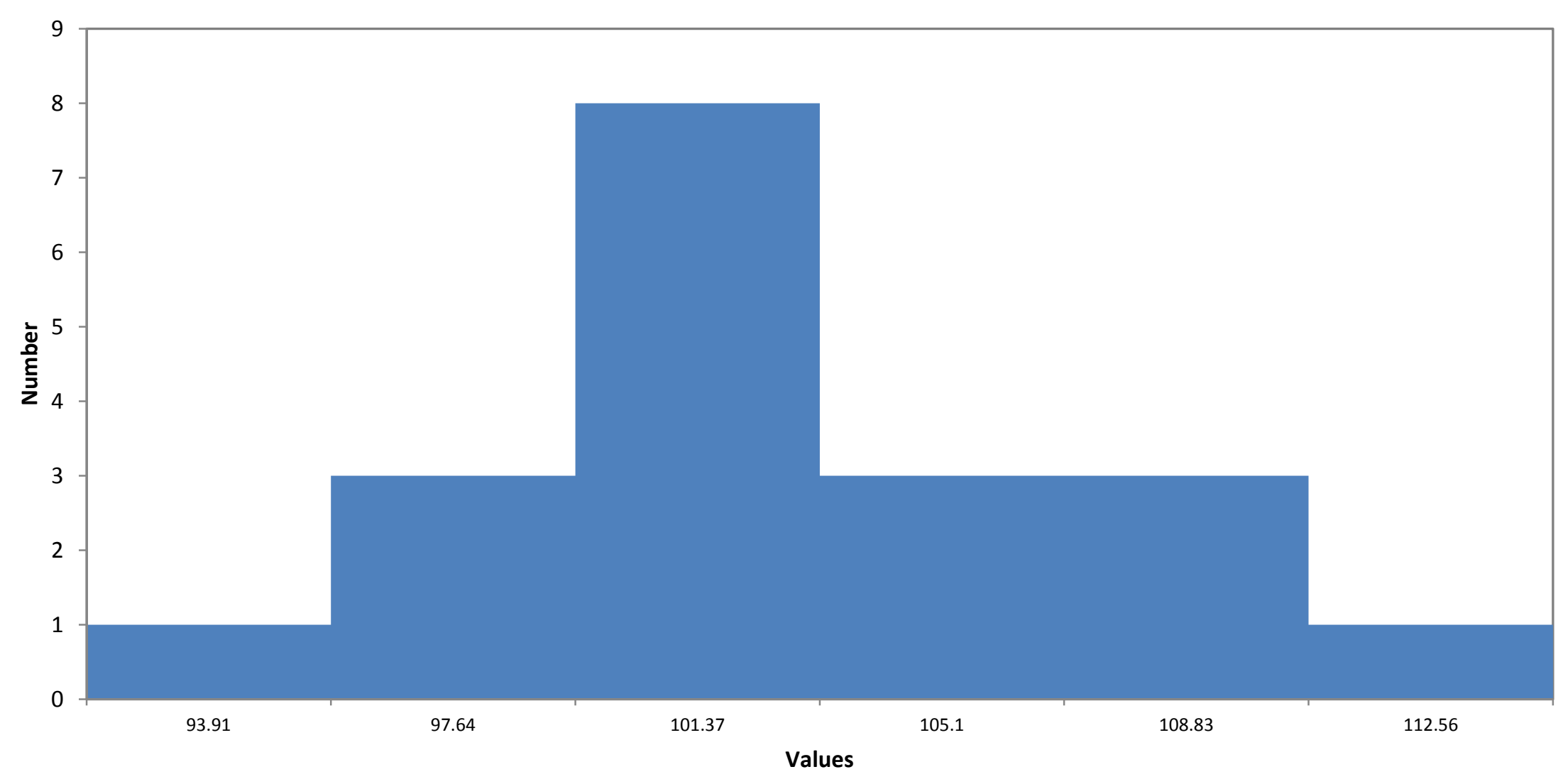




\section{Example histogram/ distribution summing the rolling of 2-5 dice}

Probability Distribution for rolling Dice

- Roll dice 30 times.

- Record the outcomes in column one on the table.

- Record the sum of the two dice in column two.

- Tabulate the frequencies.

- Plot the data on the histogram.

- REPEAT for 3 dice

- REPEAT for 5 dice 


\section{Two Dice}

\section{Probability Distribution for Two Dice}

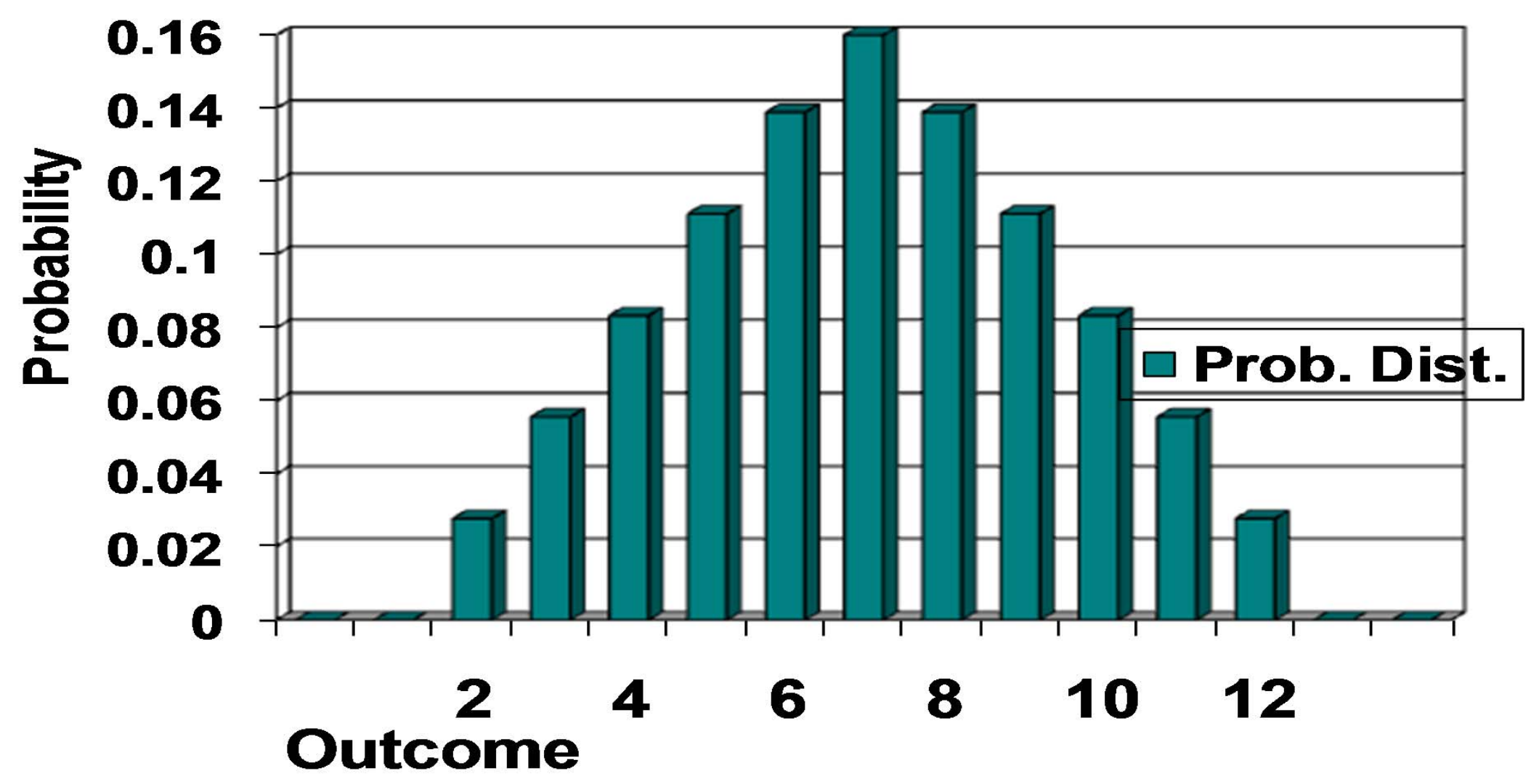




\section{Probability Distribution for Three Dice}
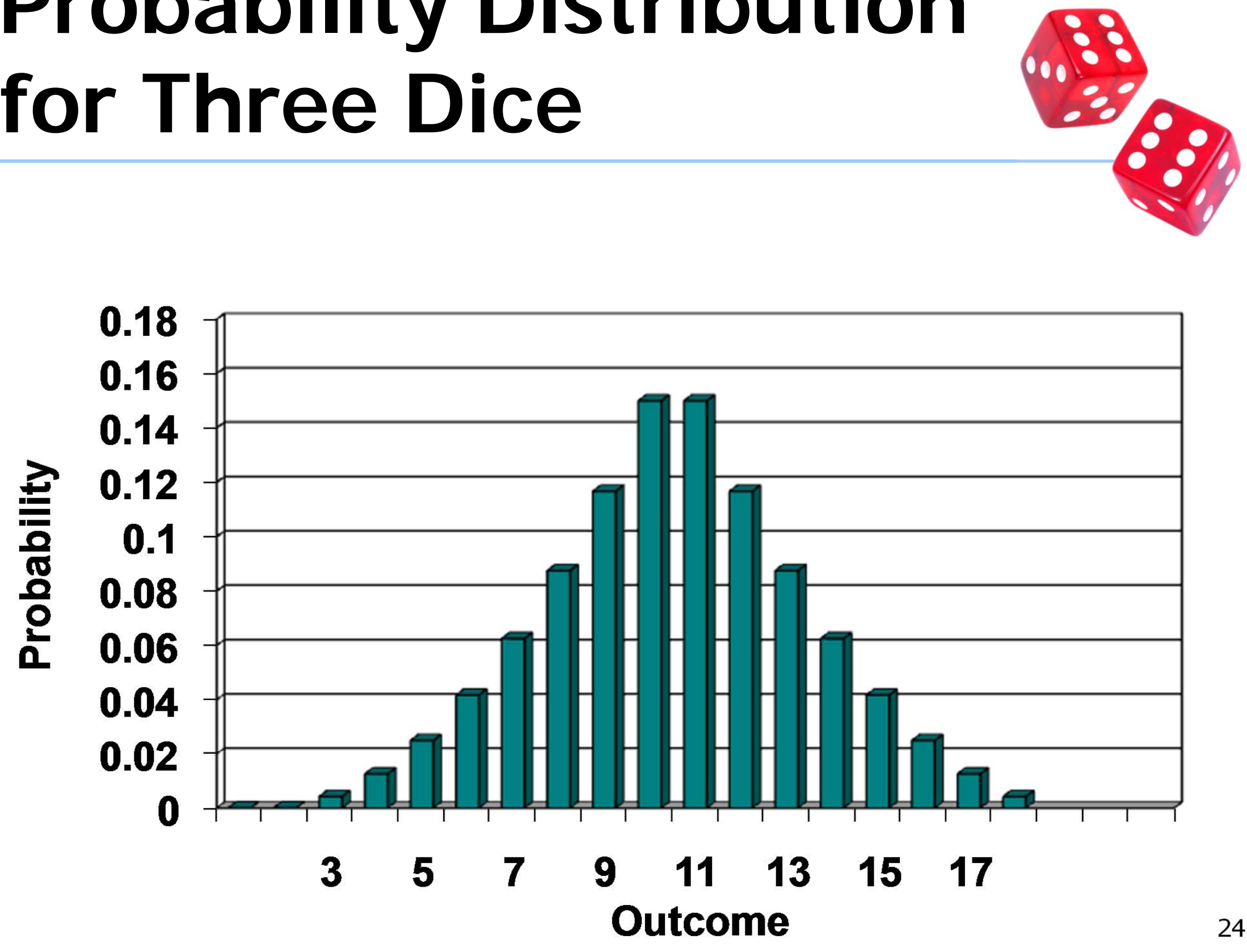


\section{Probability Distribution for Five Dice}
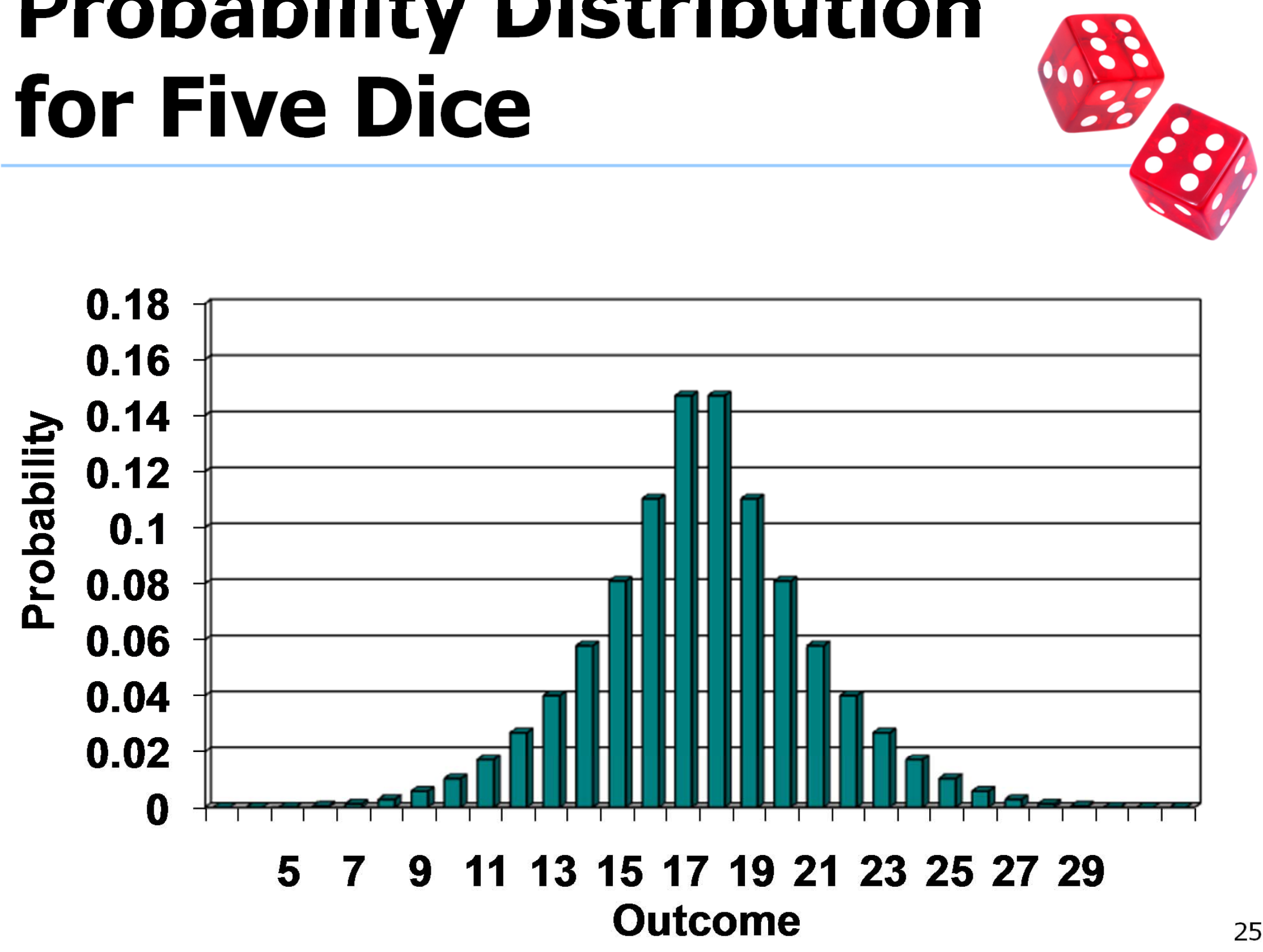
Measurement control requires a "hard number" for variation (precision) to objectively compare and manage measurement processes.

- Variance - An estimate of the variation or dispersion of the process from which the sample is drawn. The sample statistic " $\mathrm{s}^{2}$ " is an unbiased estimate of the population parameter $\sigma^{2}$.

- Standard Deviation - The square root of the variance. Often preferred as a measure of the process variation. The sample statistic "s" is an estimator of the population parameter $\sigma$. This method of calculating the standard deviation is known as the Root Mean Square Error (RMSE) method. 
The Normal Distribution and the math behind it are used to transition the "histogram" into a hard number describing dispersion (precision).

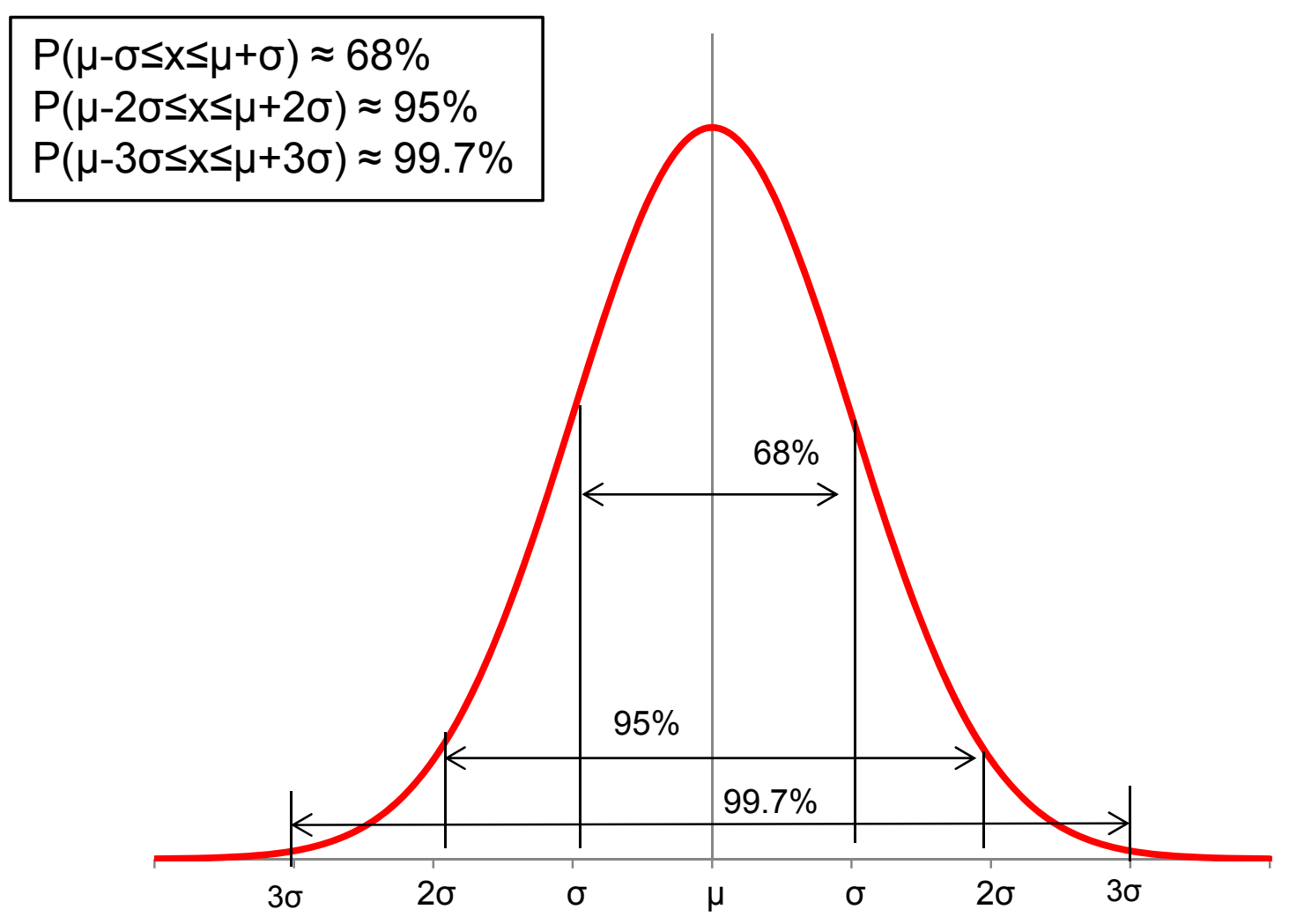




\section{Normal Distribution with different amounts of dispersion (precision).}

Three Normal distributions with the same mean or average, but different levels of precision

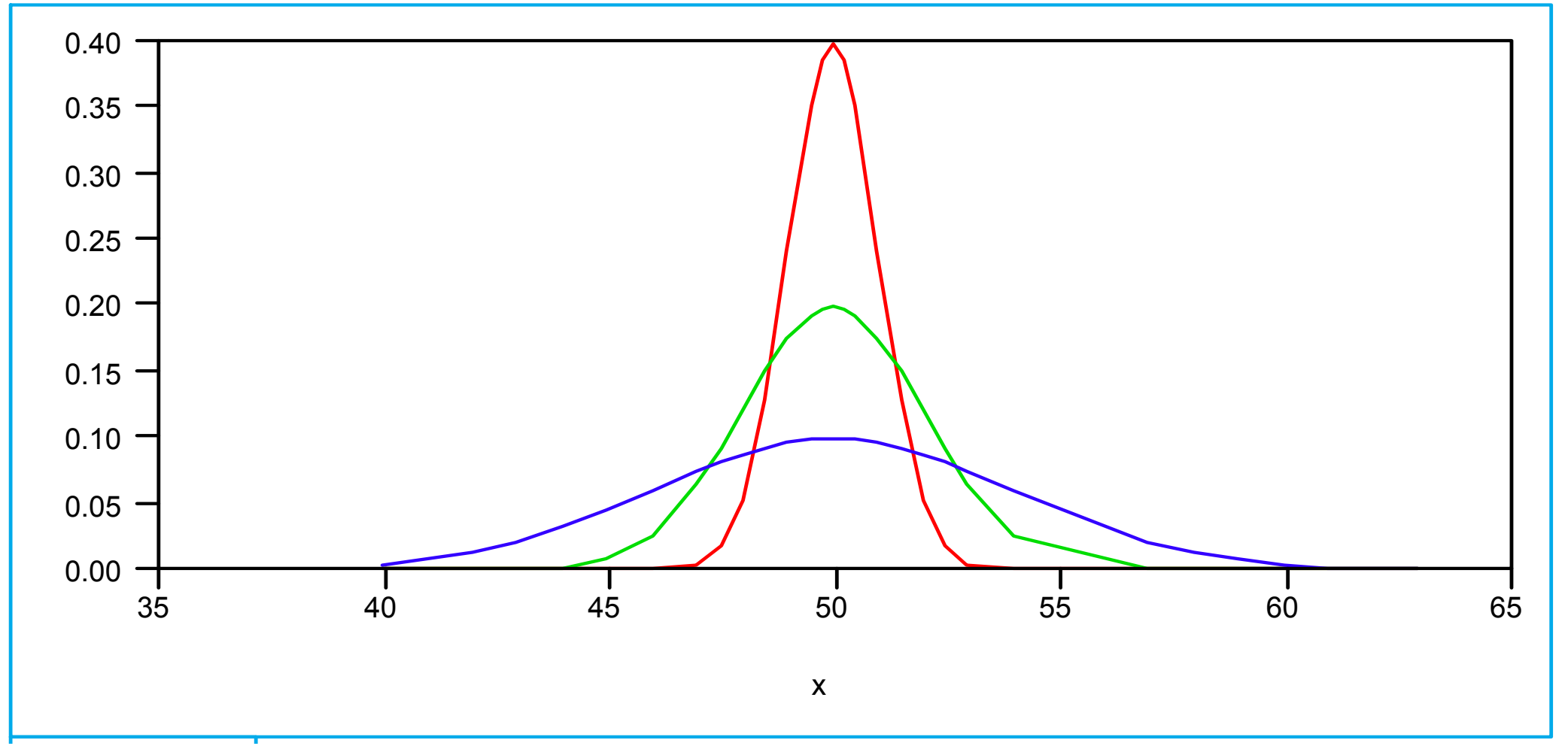

Module 2 - 28 


\section{"Look ahead" to module on Control Charts}

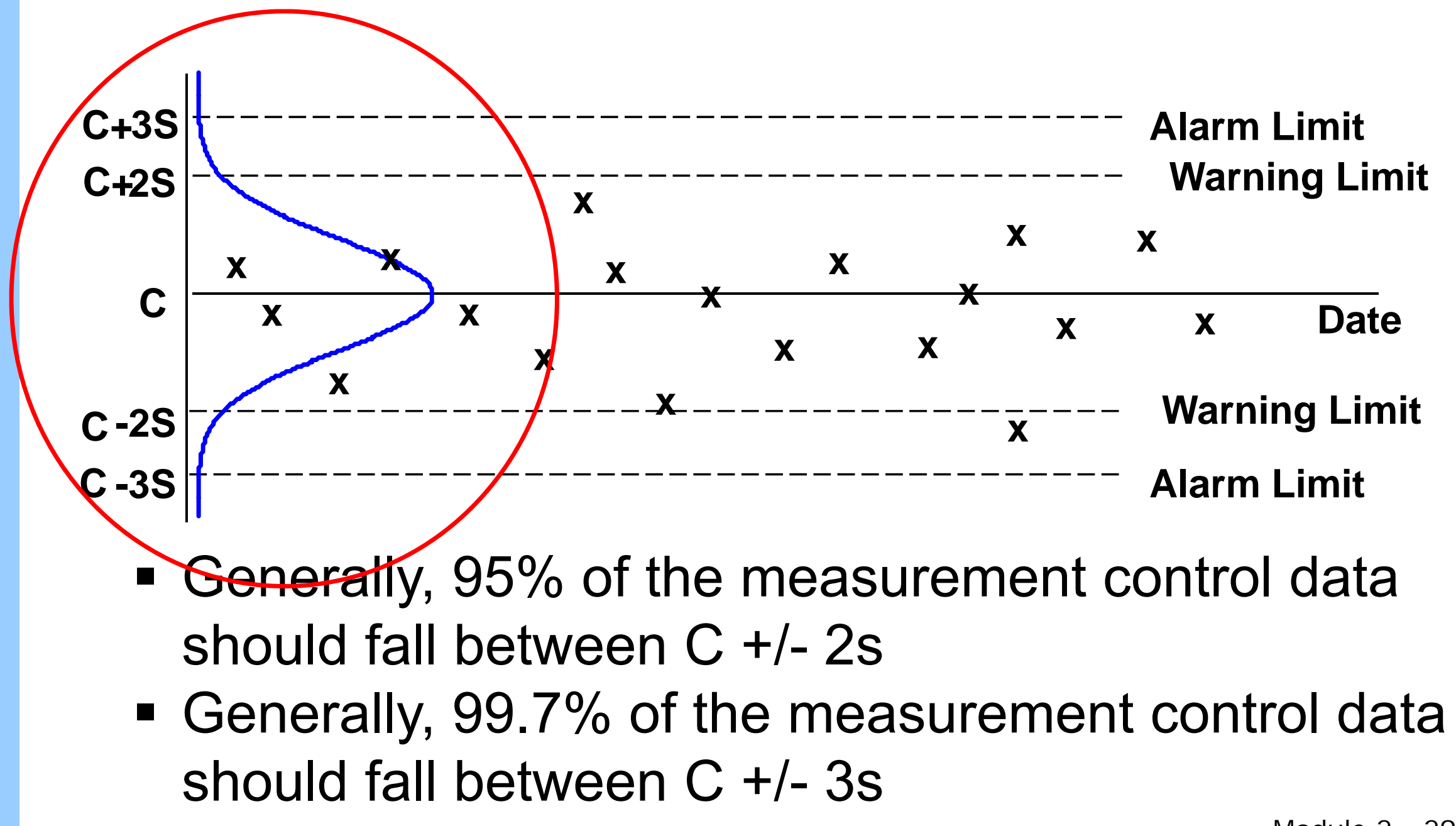




\section{Calculation of the Variance}

Calculate the variance of the 5 numbers below:

$$
\begin{array}{lllll}
1 & 8 & 3 & 6 & 2
\end{array}
$$

Formula:

$$
s^{2}=\frac{\sum_{i=1}^{i=n}\left(x_{i}-\bar{x}\right)^{2}}{n-1}
$$




\section{Calculation of the Variance 1 of 2- cont'd}

\begin{tabular}{|c|c|c|c|c|}
\hline 1) Index & 2) Data & 3) Mean & $\begin{array}{c}\text { 4) Difference between data values and } \\
\text { the mean }\end{array}$ & $\begin{array}{c}\text { 5) The square of } \\
\text { column } 4\end{array}$ \\
\hline $\mathrm{i}$ & $\mathrm{X}_{\mathrm{i}}$ & $\mathrm{Xbar}$ & $\left(\mathrm{X}_{\mathrm{i}}-\mathrm{Xbar}\right)$ & $(\mathrm{Xi}-\mathrm{Xbar})^{2}$ \\
\hline 1 & 1 & 4 & -3 & 9 \\
\hline 2 & 8 & 4 & 4 & 16 \\
\hline 3 & 3 & 4 & -1 & 1 \\
\hline 4 & 6 & 4 & 2 & 4 \\
\hline 5 & 2 & 4 & -2 & 4 \\
\hline
\end{tabular}

$$
\sum_{i=1}^{i=n}\left(x_{i}-\bar{x}\right)^{2}=34
$$

Module 2 - 31 


\section{Calculation of the Variance 2 of 2 - cont'd}

$$
\mathrm{s}^{2}=\frac{\sum_{i=1}^{i=n}\left(x_{i}-\bar{x}\right)^{2}}{n-1}=\frac{\mathbf{3 4}}{\mathbf{5 - 1}}=\mathbf{8 . 5}
$$




\section{Standard Deviation - square root of the Variance}

- The reason the standard deviation is usually preferred because it is in the same units as the original data, not "square units".

- The statistic is represented by " $s$ ". The corresponding process parameter is called " $\sigma$ " (sigma).

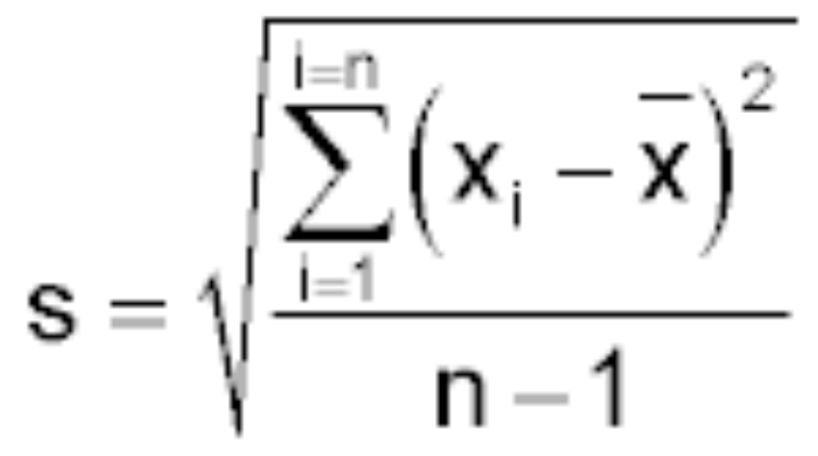




\section{Calculation of the Standard Deviation - cont'd}

$s=\sqrt{\frac{\sum_{i=1}^{i=n}\left(x_{i}-\bar{x}\right)^{2}}{n-1}}=\sqrt{\frac{34}{5-1}}=\sqrt{8.5}=2.915$ 


\section{Calculate the Variance and Standard Deviation of the weight measurements from the previous exercise.}

Our metrologist weighed a $\mathbf{1 0 0}$ gram mass standard 20 times. The results are shown in the table below. Based on the formulas (or Excel Functions) presented previously, calculate the variance and standard deviation.

$\begin{array}{rrrrr}100.64 & 97.55 & 100.62 & 110.00 & 100.67 \\ 99.11 & 102.22 & 107.40 & 112.58 & 101.14 \\ 95.41 & 106.86 & 103.77 & 98.53 & 99.59 \\ 93.91 & 101.17 & 104.28 & 96.99 & 108.41\end{array}$

Note: Use the "Spreadsheet 1 Module 2" to calculate and add the variance and standard deviation. Same spreadsheet as used for the mean exercise. 
< See Answer Handout > 


\section{Exercise - Using measurements and statistics to select a new technician}

Two technicians are being considered for a position in the laboratory. Each is given $\mathbf{1 0 0}$ gram weight and they weigh it $\mathbf{1 6}$ times. The results are shown below. Who is best for the job?

Technician 1:

$\begin{array}{llllllll}100 & 200 & 102 & 105 & 106 & 107 & 108 & 108 \\ 104 & 105 & 107 & 104 & 103 & 105 & 107 & 105\end{array}$

Technician 2:

$\begin{array}{llllllll}95 & 95 & 99 & 102 & 97 & 107 & 98 & 103 \\ 101 & 103 & 109 & 100 & 100 & 91 & 98 & 96\end{array}$


< See Answer Handout > 
Bias (Systematic Error)- the difference between the sample mean and the target mean (or true value)

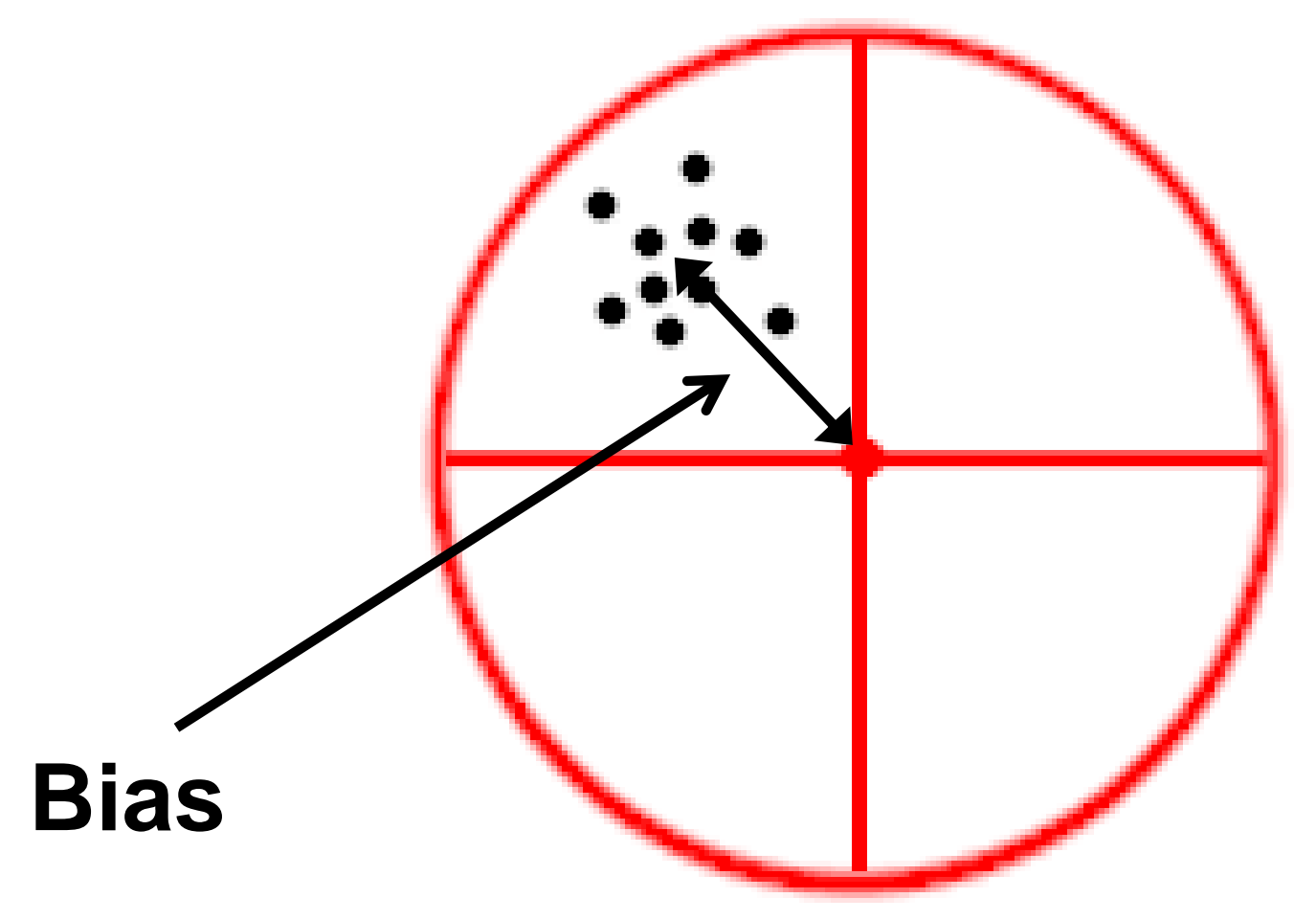




\section{Bias - the difference between the sample mean and the target mean (or true value)}

Technician 1 and Technician 2 both measured a 100 gram weight.

\begin{tabular}{|l|l|l|}
\hline Mean & Technician 1 & Technician 2 \\
\hline Variance & 105.19 & 99.63 \\
\hline $\begin{array}{l}\text { Standard } \\
\text { Deviation }\end{array}$ & 3.63 & 21.05 \\
\hline Bias & 1.91 & 4.59 \\
& $105.19-100=$ & $\begin{array}{l}99.63-100= \\
-0.37\end{array}$
\end{tabular}




\section{"Fit for Purpose" is a phrase used during the workshop}

Simply stated it means that the measurement has the accuracy and precision to meet the engineering requirements that have been established for that particular measurement (i.e., defined in process and/or MC\&A measurement plans).

Fit for Purpose

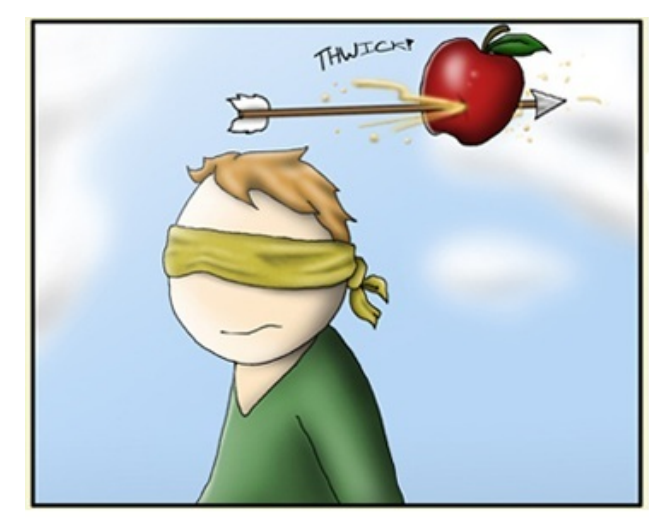

Not Fit for Purpose

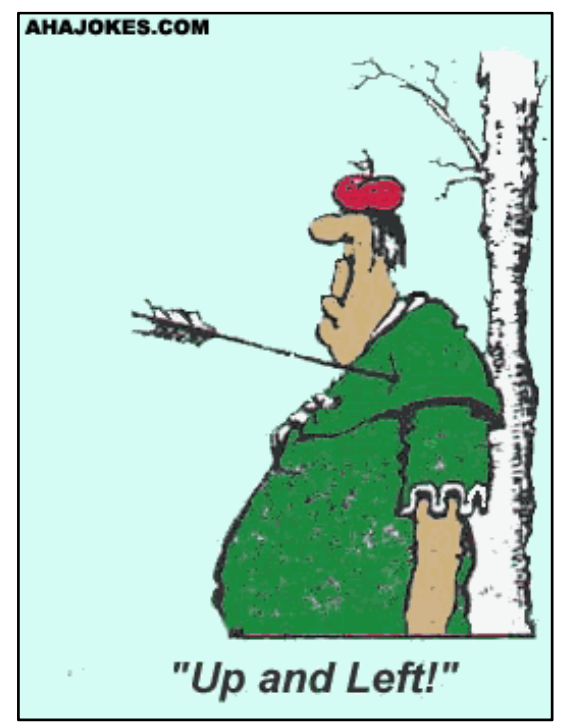




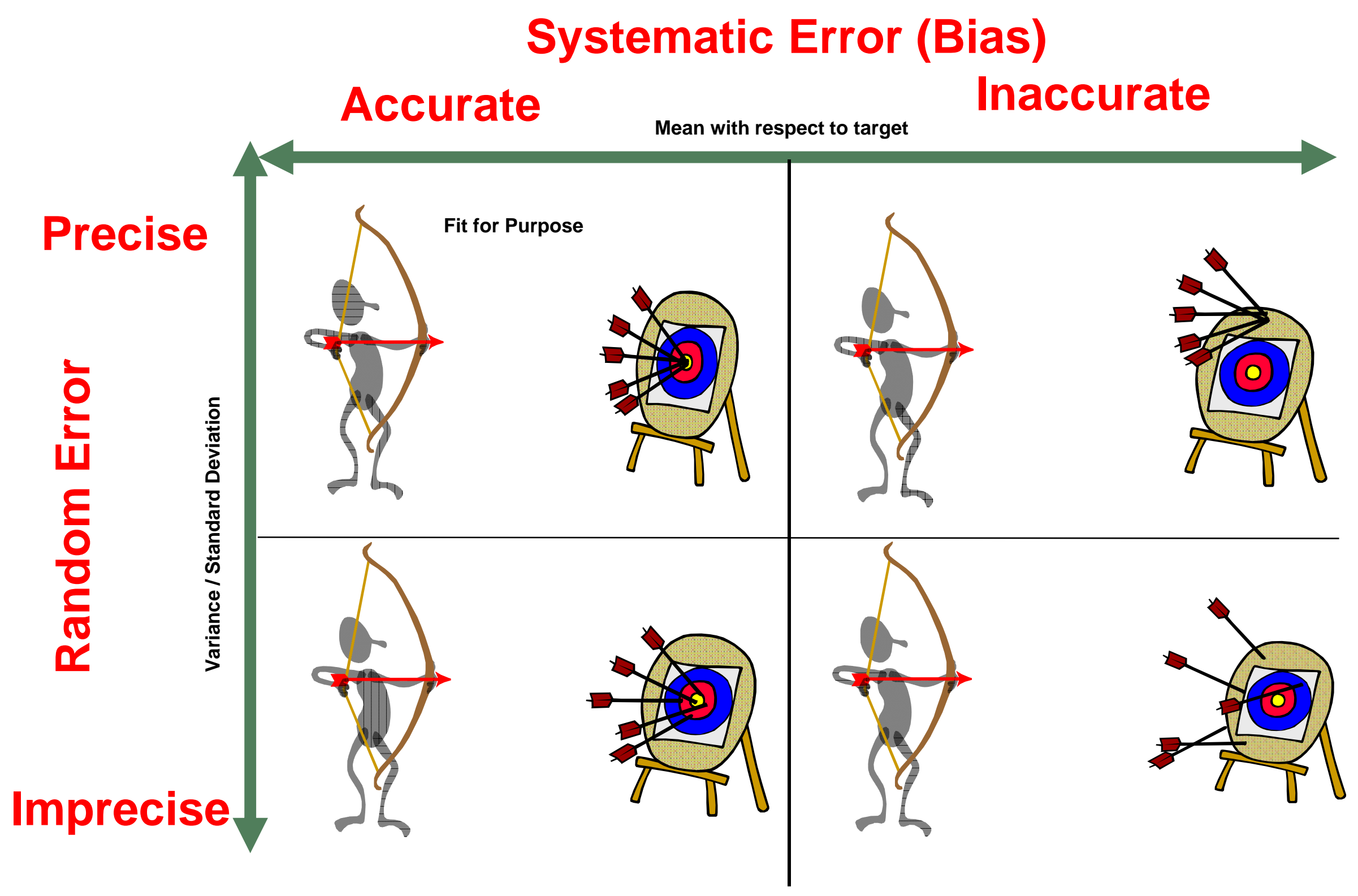

Module 2 - 42 


\section{Summary}

- Reviewed basic statistical terms and concepts

- Reviewed Mean/Average and how it is calculated

- Reviewed the concept of a "Probability Distribution" and the Normal Distribution

- Reviewed Variance and Standard Deviation and how they are calculated

- Reviewed the concepts of "Bias" and "Fit for Purpose" 


\begin{tabular}{|c|c|c|}
\hline Observation & Technician 1 & Technician 2 \\
\hline 1 & 105 & 95 \\
\hline 2 & 102 & 95 \\
\hline 3 & 102 & 99 \\
\hline 4 & 105 & 102 \\
\hline 5 & 106 & 97 \\
\hline 6 & 107 & 107 \\
\hline 7 & 108 & 98 \\
\hline 8 & 108 & 103 \\
\hline 9 & 104 & 101 \\
\hline 10 & 105 & 103 \\
\hline 11 & 107 & 109 \\
\hline 12 & 104 & 100 \\
\hline 13 & 103 & 100 \\
\hline 14 & 105 & 91 \\
\hline 15 & 107 & 98 \\
\hline 16 & 105 & 96 \\
\hline Mean/Average & & \\
\hline Variance & & \\
\hline Standard Deviation & & \\
\hline BIAS (target = 100) & & \\
\hline
\end{tabular}

Note: Insert average Excel Function in the cel Note: Insert Variance Excel Function in the ce Note: Insert Standard Deviation Excel Functio Note: Value for BIAS = target (100 grams) - "I 
Is to the left (e.g., B18 and C18 selecting data in rows 2-17 for columns B and C respectively)

IIs to the left (e.g., B19 and C19 selecting data in rows 2-17 for columns $B$ and $C$ respectively)

$n$ in the cell to the left (e.g., B2O and C2O selecting data in rows 2-17 for columns $B$ and $C$ respectively)

Vean/Average" 


\begin{tabular}{|c|c|}
\hline $\begin{array}{c}\text { Observation } \\
\text { Weighing Number: }\end{array}$ & Measurement Value \\
\hline 1 & 100.64 \\
\hline 2 & 99.11 \\
\hline 3 & 95.41 \\
\hline 4 & 93.91 \\
\hline 5 & 97.55 \\
\hline 6 & 102.22 \\
\hline 7 & 106.86 \\
\hline 8 & 101.17 \\
\hline 9 & 100.62 \\
\hline 10 & 107.40 \\
\hline 11 & 103.77 \\
\hline 12 & 104.28 \\
\hline 13 & 110.00 \\
\hline 14 & 112.58 \\
\hline 15 & 98.53 \\
\hline 16 & 96.99 \\
\hline 17 & 100.67 \\
\hline 18 & 101.14 \\
\hline 19 & 99.59 \\
\hline 20 & 108.41 \\
\hline \multicolumn{2}{|l|}{ Average } \\
\hline \multicolumn{2}{|l|}{ Variance } \\
\hline Standard Deviation & \\
\hline
\end{tabular}

Note: Insert average Excel Function in the cell to the left $f$ Note: Insert Variance Excel Function in the cell to the left. Note: Insert Standard Deviation Excel Function in the cell 
'or data in rows B2 through B21 (reference slide 2-17)

for data in rows $B 2$ through B21 (reference slide 2-31)

to the left for data in rows B2 through B21 (reference slide 2-31) 


\section{Answer for Module 2 Slide 17 Exercise - Calculate the mean}

Our metrologist weighed a $\mathbf{1 0 0}$ gram mass standard $\mathbf{2 0}$ times. The results are shown in the table below. Based on the formulas presented previously, calculate the mean weight.

$\begin{array}{rrrrr}100.64 & 97.55 & 100.62 & 110.00 & 100.67 \\ 99.11 & 102.22 & 107.40 & 112.58 & 101.14 \\ 95.41 & 106.86 & 103.77 & 98.53 & 99.59 \\ 93.91 & 101.17 & 104.28 & 96.99 & 108.41\end{array}$

Answer: X-Bar $=102.04$ 


\section{Answer for Slide Module 2 Slide 35}

\begin{tabular}{|c|c|c|}
\hline $\mathrm{i}$ & $X_{i}$ & $(\mathrm{Xi}-\mathrm{Xbar})^{2}$ \\
\hline 1 & 100.64 & 1.97 \\
\hline 2 & 99.11 & 8.60 \\
\hline 3 & 95.41 & 43.99 \\
\hline 4 & 93.91 & 66.14 \\
\hline 5 & 97.55 & 20.18 \\
\hline 6 & 102.22 & 0.03 \\
\hline 7 & 106.86 & 23.21 \\
\hline 8 & 101.17 & 0.76 \\
\hline 9 & 100.62 & 2.02 \\
\hline 10 & 107.40 & 28.70 \\
\hline 11 & 103.77 & 2.98 \\
\hline 12 & 104.28 & 5.01 \\
\hline 13 & 110.00 & 63.32 \\
\hline 14 & 112.58 & 111.04 \\
\hline 15 & 98.53 & 12.34 \\
\hline 16 & 96.99 & 25.53 \\
\hline 17 & 100.67 & 1.88 \\
\hline 18 & 101.14 & 0.81 \\
\hline 19 & 99.59 & 6.01 \\
\hline 20 & 108.41 & 40.55 \\
\hline \multicolumn{2}{|r|}{ Variance } & 465.08 \\
\hline & Std. Dev. & 21.57 \\
\hline
\end{tabular}




\section{Answers for Module 2 Slide 37}

Calculate the mean, variance, and standard deviation for the Technician 1 and Technician 2

\begin{tabular}{|l|l|l|}
\hline Mean & Technician 1 & Technician 2 \\
\hline Variance & 105.19 & 99.63 \\
\hline $\begin{array}{l}\text { Standard } \\
\text { Deviation }\end{array}$ & 3.63 & 21.05 \\
\hline
\end{tabular}




\section{Answers for Module 2 Slide 37}

Technician 1 is the least accurate but most precise so potentially the better candidate. This is assuming you can quantify and correct their measurement bias.

Notes:

- Typically it is easier to correct and/or manage bias in a process than to reduce the variability of Technician 2.

- Regardless a review of each technician's technique compared to a qualified technician will typically provide insight into the cause of each technician's errors. 
Rev-May 30, 2012

\section{Exercise 1 \\ Height Measurement Exercise}

\section{Session Objectives:}

After the session the participants will be able to do the following:

1. To see measurements are comparisons of unknowns to reference standards.

2. To understand measurements have uncertainty.

3. To understand that reference standards also have uncertainty.

4. To understand measurements need uncertainty estimates with specified confidence intervals to have value.

5. Apply concepts of uncertainty (uncertainty includes random and systematic error/bias).

\section{Estimated Time:}

+. 75 hours completing exercise

+.25 hours in large group discussion

1.0 hours total

\section{Materials needed:}

1. Work Sheets for each participant

2. 3 individuals with varying heights to serves as volunteer measurement objects

3. 1 individual to serve as the working standard

4. 1 calibrated length measurement standard (tape measure)

5. 1 ruler or paint stick

6. I marker or pencil

7. 1 roll of tape

8. Several pieces of plain paper

\section{Instructions:}

1. Write your name on the worksheet.

2. Write the name of the three class members chosen for this exercise in the 3 blocks on line one of your work sheet as the instructor writes the names on the board.

3. Write your $1^{\text {st }}$ estimated measurement for each person's height on the worksheet in the $2^{\text {nd }}$ row marked "First Estimate" as they sequentially stand and give their name. 
4. After all 3 individuals stand together in the front of the room; make your second estimated measurement of each person's height and record it in the row 3, marked "second estimate". (If you are confident that your first estimate is accurate, then write it down again for your second estimate.)

5. After a fourth person joins the first 3 and states his height with a $+/$ - uncertainty. (It should be in the same units being used by the class, if not convert it.) Compare the heights of the 3 people to the known height of the $4^{\text {th }}$ person. Record these estimated measurements on the worksheet on line 4marked "Third Estimate".

6. If your $3^{\text {rd }}$ estimate is different from your second estimate, you made a "bias" correction after you had a "working standard" to use for your third "measurement". Determine the difference between the second and third "measurements" by subtracting the $2^{\text {nd }}$ estimate from the $3^{\text {rd }}$ estimate and record the results on line 5, marked Bias. These are the "bias” corrections you applied.

7. Since you do not know the "true height" of the people you have "measured" there is uncertainty in your measurements. Estimate a $+/$ - interval of maximum and minimum heights for each person that should contain their "true heights". Subtract the minimum value from the maximum value for each person and record the difference. These ranges will be the Measurement Uncertainty estimates for your final measurements. For example $+/-2 \mathrm{~cm}$. Record these uncertainty estimates on line 6.

8. How much confidence do you have in the "uncertainty" estimates listed on line 6? Select one of the following probabilities: $67 \%, 95 \%, 99.7 \%$ and write answer on line 7.

9. On line 8, enter 0,1 or 2 quarters will you bet that all three of your "uncertainty estimates" on line 6 contain the true heights? (Your confidence interval should be considered.)

10. Have 4 new (e.g., different) class members independently use a calibrated tape measure per the steps below to measure the heights of the 3 people and record their measurements respectively on worksheet lines (9a, 9b, 9c and 9d). (Note: The person used as the reference standard is no longer needed.)

a. Tape enough white papers on the wall that will cover the range of the subject's heights.

b. Have each person stand with their back against the wall.

c. Place the ruler or paint stick level on their head and touch the wall.

d. Draw a line on the wall and write the person's name beside it.

e. With the end of the tape on the floor, extend it to one of the marks and read the corresponding mark from the scale on the ruler. Repeat for the other two people.

11. Calculate the average and standard deviation for the measured heights of each person.

12. Calculate the bias of each of your estimates in (Line 4 - Line 10) and record them on Line 12.

13. Evaluate the accuracy of your measurements by seeing if the values in Line 10 are less than the corresponding uncertainty estimates in line 5. If all are within the $+/$ - range you WIN, if not you loose your bet. 
14. The total uncertainty can be estimated by adding the bias to the $1 / 2$ the range you selected to contain the true height of your "measurement " of each person. For example $1 / 2$ of

\begin{tabular}{|c|c|c|c|c|}
\hline & Your Name: & & & \\
\hline Line & & & & \\
\hline & Subject's Number & 1 & 2 & 3 \\
\hline 1 & Subject's Name & & & \\
\hline 2 & First Estimate of Height after each stands independently & & & \\
\hline 3 & Second Estimate of Helght after seelng 3 people side by side & & & \\
\hline 4 & $\begin{array}{l}\text { Third Estimate of Height after seeing each person } \\
\text { standing beside someone of known height }\end{array}$ & & & \\
\hline 5 & Bias (3rd estimate - 2nd estimate) & & & \\
\hline 6 & Estimate + $j$ - U Range Containing True Height & & & \\
\hline 7 & State U probability level; $67 \%, 95 \%$ or $99.7 \%$ & & & \\
\hline 8 & Your bet that line $\mathbf{5}$ includes "true values" & & & \\
\hline $9 a$ & First Technician Measurement with tape measure & & & \\
\hline gb & Second Technician Measurement with tape measure & & & \\
\hline $9 \mathrm{c}$ & Third Technician Measurement with tape meașure & & & \\
\hline 9d & Fourth Technician Measurement with tape measure & & & \\
\hline 10 & Calculate the Average of each person's 4 measurements & & & \\
\hline 11 & Calculate the Standard Deviation of the 4 meașurements & & & \\
\hline 12 & Calculated Corrected "Bias"(Line 10-Line 4) & & & \\
\hline 13 & Win if within +l- range (line 6) or Loose if putside & & & \\
\hline 14 & Final estimate of uncertainty ( $1 / 2$ range in line $6+A B S$ (line 12)) & & & \\
\hline
\end{tabular}

\section{Discussion Questionns}

1 What may have caused the "bias" in your estimates?

2 Which measurement made with the tape measure was the most accurate? Why?

3 How can the variability between the tape measurements be reduced?

4 Record the largest \& smallest uncertainty estimates that included the most accurate "estimates Largest uncertainty estimates: Smallest uncertainty estimates:

5 How do uncertainty estimates help define that quality of measurements?

6 How significant was bias in estimating the uncertainty in line 14? 


\begin{tabular}{|c|c|c|c|c|}
\hline & Your Name: & & & \\
\hline Line & & & & \\
\hline & Subject's Number & 1 & 2 & 3 \\
\hline 1 & Subject's Name & & & \\
\hline 2 & First Estimate of Height after each stands independently & & & \\
\hline 3 & Second Estimate of Height after seeing 3 people side by side & & & \\
\hline 4 & $\begin{array}{l}\text { Third Estimate of Height after seeing each person standing } \\
\text { beside someone of known height }\end{array}$ & & & \\
\hline 5 & Bias (3rd estimate - 2nd estimate) & & & \\
\hline 6 & Estimate +l- U Range Containing True Height & & & \\
\hline 7 & State U probability level; $67 \%, 95 \%$ or $99.7 \%$ & & & \\
\hline 8 & Your bet that line 5 includes "true values" & & & \\
\hline $9 a$ & First Technician Measurement with tape measure & & & \\
\hline $9 b$ & Second Technician Measurement with tape measure & & & \\
\hline 9c & Third Technician Measurement with tape measure & & & \\
\hline 9d & Fourth Technician Measurement with tape measure & & & \\
\hline 10 & Calculate the Average of each person's 4 measurements & & & \\
\hline 11 & Calculate the Standard Deviation of the 4 measurements & & & \\
\hline 12 & Calculated Corrected "Bias"(Line 10-Line 4) & & & \\
\hline 13 & Win if within $+l-$ range (line 6 ) or Loose if outside & & & \\
\hline 14 & Final estimate of uncertainty (1/2 range in line $6+A B S($ line 12$))$ & & & \\
\hline
\end{tabular}

\section{Discussion Questions}

1 What may have caused the "bias" in your estimates?

2 Which measurement made with the tape measure was the most accurate? Why?

3 How can the variability between the tape measurements be reduced?

4 Record the largest \& smallest uncertainty estimates that included the most accurate "estimates" Largest uncertainty estimates: Smallest uncertainty estimates:

5 How do uncertainty estimates help define that quality of measurements?

6 How significant was bias in estimating the uncertainty in line 14? 


\begin{tabular}{|c|c|c|c|c|}
\hline & Your Name: & & & \\
\hline Line & & & & \\
\hline & Subject's Number & 1 & 2 & 3 \\
\hline 1 & Subject's Name & & & \\
\hline 2 & First Estimate of Height after each stands independently & & & \\
\hline 3 & Second Estimate of Height after seeing 3 people side by side & & & \\
\hline 4 & $\begin{array}{l}\text { Third Estimate of Height after seeing each person standing } \\
\text { beside someone of known height }\end{array}$ & & & \\
\hline 5 & Bias (3rd estimate - 2nd estimate) & & & \\
\hline 6 & Estimate +l- U Range Containing True Height & & & \\
\hline 7 & State U probability level; $67 \%, 95 \%$ or $99.7 \%$ & & & \\
\hline 8 & Your bet that line 5 includes "true values" & & & \\
\hline $9 a$ & First Technician Measurement with tape measure & & & \\
\hline $9 b$ & Second Technician Measurement with tape measure & & & \\
\hline 9c & Third Technician Measurement with tape measure & & & \\
\hline 9d & Fourth Technician Measurement with tape measure & & & \\
\hline 10 & Calculate the Average of each person's 4 measurements & & & \\
\hline 11 & Calculate the Standard Deviation of the 4 measurements & & & \\
\hline 12 & Calculated Corrected "Bias"(Line 10-Line 4) & & & \\
\hline 13 & Win if within $+l-$ range (line 6 ) or Loose if outside & & & \\
\hline 14 & Final estimate of uncertainty (1/2 range in line $6+A B S($ line 12$))$ & & & \\
\hline
\end{tabular}

\section{Discussion Questions}

1 What may have caused the "bias" in your estimates?

2 Which measurement made with the tape measure was the most accurate? Why?

3 How can the variability between the tape measurements be reduced?

4 Record the largest \& smallest uncertainty estimates that included the most accurate "estimates" Largest uncertainty estimates: Smallest uncertainty estimates:

5 How do uncertainty estimates help define that quality of measurements?

6 How significant was bias in estimating the uncertainty in line 14? 


\section{Exercises \\ Measurement Models}

\section{Session Objectives:}

After the session the participants will be able to do the following:

1. Understand the calculations of variances for functions involving measurements

2. Understand the application to measurement models

\section{Estimated Time:}

There are six (6) exercises in this module. The completion times are as follows:

1. Exercise \#1 - 30 minutes

2. Exercise $\# 2-20$ minutes

3. Exercise \#3 - 15 minutes

4. Exercise \#4-20 minutes

5. Exercise $\# 5-45$ minutes

6. Exercise \#6 - 30 minutes

These exercises will require 160 minutes or approximately 2.5 hours to complete.

\section{Materials Needed:}

1. One computer with Microsoft Excel for each group of 4 to 5 students

2. The students must be familiar with the use of Excel

3. Pencil and paper

4. Spreadsheet support personnel

\section{Instructions:}

1. There are six (6) exercises in this module

2. Students can work individually or in groups

3. The exercises are included in the presentation

4. Students will be requested to complete a particular exercise

5. Each exercise will then be discussed 


\section{$\underline{\text { Exercise \#1 }}$}

Let $\mathrm{x}_{1}, \ldots, \mathrm{x}_{\mathrm{n}}$ be independent, identically distributed (iid) $\mathrm{N}\left(\mu, \sigma^{2}\right)$.

Compute the following:

1. $\mathbf{E}\left(\mathbf{x}_{1}-\mathbf{x}_{2}\right)=\mathbf{0}$

2. $E\left(x_{1}+2 x_{2}-x_{3}-x_{4}+5\right)=\mu+5$

3. $E\left(x_{1}+\ldots+x_{n}\right)=n \mu$

4. $E\left(\left(x_{1}+\ldots+x_{n}\right) / n\right)$ or $E(\bar{x})=\mu$

5. $V\left(x_{1}-2 x_{2}-5\right)=5 \sigma^{2}$

6. $V\left(x_{1}+\ldots+x_{n}\right)=n \sigma^{2}$

7. $V\left(\left(x_{1}+\ldots+x_{n}\right) / n\right)=V(\bar{x})=\sigma^{2} / n$

\section{$\underline{\text { Exercise \#2 }}$}

Questions related to measurement models:

1. How are the model terms different between the additive and relative models?

- Additive terms are in the same units as the measurement, while relative terms are fractions or percentages

2. How would a plot of the absolute differences look for an additive model?

- The absolute differences should have the same variance or spread over the measurement range

3. How would a plot of the measurement values look for a relative model?

- The measurement values should have an increasing variance over the measurement range

4. How would a plot of the relative differences look for a relative model?

- The absolute differences should have the same variance or spread over the measurement range

\section{$\underline{\text { Exercise \#3 }}$}

Specify the measurement models for the following types of measurement:

1. Temperature - additive model

2. Neutron Coincidence Counter - relative model because of counting

3. Calorimeter - either a mixed or additive model

4. Pressure - additive model

5. Tank volume - additive model, however, the systematic variance tends to relative for the regression parameter estimates, while the random variance is additive

6. Density - additive model 
7. Weight - additive model

\section{$\underline{\text { Exercise \#4 }}$}

Consider an analytical method for concentration involving dilutions, such as Davies-Gray Titration. Process measurements are as follows: two samples, two analysts and two measurements per sample.

1. Specify a reasonable model for this measurement scenario.

- Model is $y_{i j k}=\mu\left(1+\eta_{i}+\theta_{j}+\varepsilon_{i j k}\right)$, where $\eta_{i}$ is the random effect for the $i^{\text {th }}$ sample, $\theta_{j}$ is the random effect for the $j$ th analyst and $\varepsilon_{i j k}$ is the random effect for the $i^{\text {th }}$ sample, the jth analyst and the $k^{\text {th }}$ measurement.

2. What kind of effects are the samples and analysts?

- These are short-term systematic effects

3. Are the sample and analyst effects different from random measurement effects?

- Yes, these effects are held constant for certain measurements

4. What would a significant analyst variance or effect represent?

- This analyst might need additional training or coaching. Some investigation would be needed to find the problem. It could be the chemicals being used as opposed to preparation technique

5. How could an analyst effect be used?

- As indicated in \#4 above, this effect could be used to uncover issues or problems with one or more analyst's technique or with the chemicals used by the analysts.

\section{$\underline{\text { Exercise \#5 }}$}

Suppose $\mathrm{y}_{\mathrm{i}}=\mu\left(1+\eta+\varepsilon_{\mathrm{i}}\right)$, where $\eta$ is $\mathrm{N}\left(0, \sigma_{\eta}^{2}\right)$ and $\varepsilon_{\mathrm{i}}$ are iid $\mathrm{N}\left(0, \sigma_{\varepsilon}^{2}\right)$.

Compute the following by using the defined model for $\mathrm{y}_{\mathrm{i}}$ :

1. $\mathbf{E}\left(\mathbf{y}_{1}\right)=\mu$

2. $E\left(y_{1}+y_{2}\right)=E\left(y_{1}\right)+E\left(y_{2}\right)=2 \mu$

3. $E\left(y_{1}-y_{2}\right)=E\left(y_{1}\right)-E\left(y_{2}\right)=0$

4. $\mathrm{E}(\bar{y})=n \mu / n=\mu$

5. $\mathrm{V}\left(\mathrm{y}_{1}\right)=\mu^{2}\left(\sigma_{\eta}^{2}+\sigma_{\varepsilon}^{2}\right)$

6. $V\left(y_{1}+y_{2}\right)=4 \mu^{2} \sigma_{\eta}^{2}+2 \mu^{2} \sigma_{\varepsilon}^{2}$

7. $V\left(y_{1}-y_{2}\right)=2 \mu^{2} \sigma_{\varepsilon}^{2}$

8. $V(\bar{y})=\mu^{2} \sigma_{\eta}^{2}+\mu^{2} \sigma_{\varepsilon}^{2} / n$

9. Are $\mathrm{y}_{1}$ and $\mathrm{y}_{2}$ independent?

a. If $y_{1}$ and $y_{2}$ are independent, then $V\left(y_{1}+y_{2}\right)=V\left(y_{1}\right)+V\left(y_{2}\right)$ 
b. The $V\left(y_{1}\right)=\mu^{2}\left(\sigma_{\eta}^{2}+\sigma_{\varepsilon}^{2}\right)$, and similarly $V\left(y_{2}\right)=\mu^{2}\left(\sigma_{\eta}^{2}+\sigma_{\varepsilon}^{2}\right)$. And $V\left(y_{1}\right)+V\left(y_{2}\right)=2 \mu^{2}$ $\sigma_{\eta}^{2}+2 \mu^{2} \sigma_{\varepsilon}^{2}$

c. From Exercise \#5 Question 6, V $\left(y_{1}+y_{2}\right)=4 \mu^{2} \sigma_{\eta}^{2}+2 \mu^{2} \sigma_{\varepsilon}^{2}$

d. Therefore $y_{1}$ and $y_{2}$ are not independent. These measurements are correlated through the long-term systematic effect $\eta$.

10. What is $\operatorname{Cov}\left(\mathrm{y}_{1}, \mathrm{y}_{2}\right)$ ?

a. Remember, $\mathbf{V}\left(\mathbf{y}_{1}+\mathbf{y}_{2}\right)=V\left(\mathbf{y}_{1}\right)+V\left(\mathbf{y}_{2}\right)+2 \operatorname{Cov}\left(\mathbf{y}_{1}, \mathbf{y}_{2}\right)$

b. Substituting from above, $4 \mu^{2} \sigma_{\eta}^{2}+2 \mu^{2} \sigma_{\varepsilon}^{2}=2 \mu^{2} \sigma_{\eta}^{2}+2 \mu^{2} \sigma_{\varepsilon}^{2}+2 \operatorname{Cov}\left(\mathbf{y}_{1}, \mathbf{y}_{2}\right)$

c. $2 \operatorname{Cov}\left(\mathbf{y}_{1}, \mathbf{y}_{2}\right)=2 \mu^{2} \sigma_{\eta}^{2}$

d. $\operatorname{Cov}\left(\mathbf{y}_{1}, \mathbf{y}_{2}\right)=\mu^{2} \sigma_{\eta}^{2}$

\section{$\underline{\text { Exercise \#6 }}$}

Suppose $\mathrm{y}_{\mathrm{i}}=\mu_{\mathrm{i}}\left(1+\eta+\varepsilon_{\mathrm{i}}\right)$, where $\eta$ is $\mathrm{N}\left(0, \sigma_{\eta}^{2}\right)$ and $\varepsilon_{\mathrm{i}}$ are iid $\mathrm{N}\left(0, \sigma_{\varepsilon}^{2}\right)$.

Compute and answer the following:

1. $\mathrm{V}\left(\mathrm{y}_{1}+\ldots+\mathrm{y}_{10}\right)=\sigma_{\eta}^{2}\left(\Sigma_{\mathrm{i}} \mu_{\mathrm{i}}\right)^{2}+\boldsymbol{\sigma}_{\varepsilon}^{2} \Sigma_{\mathrm{i}} \mu_{i}^{2}$, for $\mathrm{i}=1$ to 10

2. Discuss the differences in the affects of systematic and random variances on the variance for a sum of measurements.

- Systematic effects can propagate with a much larger effect. The coefficients are larger in magnitude for systematic effects as compared to random effects.

3. In general, what type of variance is most important to control?

- Need to control the systematic variance 


\section{Exercises \\ Measurement Models}

\section{Session Objectives:}

After the session the participants will:

1. Understand the calculations of variances for functions involving measurements

2. Understand the application to measurement models

\section{Estimated Time:}

There are six (6) exercises in this module. The completion times are as follows:

1. Exercise \#1 - 30 minutes

2. Exercise $\# 2-20$ minutes

3. Exercise \#3 - 15 minutes

4. Exercise \#4 - 20 minutes

5. Exercise \#5 - 45 minutes

6. Exercise \#6 - 30 minutes

These exercises will require 160 minutes or approximately 2.5 hours to complete.

\section{Materials Needed:}

1. One computer with Microsoft Excel for each group of 4 to 5 students

2. The students must be familiar with the use of Excel

3. Pencil and paper

4. Spreadsheet support personnel

\section{Instructions:}

1. There are six (6) exercises in this module

2. Students can work individually or in groups

3. The exercises are included in the presentation

4. Students will be requested to complete a particular exercise

5. Each exercise will then be discussed 


\section{Exercise \#1}

Let $\mathrm{x}_{1}, \ldots, \mathrm{x}_{\mathrm{n}}$ be independent, identically distributed (iid) $\mathrm{N}\left(\mu, \sigma^{2}\right)$.

Compute the following:

1. $E\left(x_{1}-x_{2}\right)=$

2. $E\left(x_{1}+2 x_{2}-x_{3}-x_{4}+5\right)=$

3. $E\left(x_{1}+\ldots+x_{n}\right)=$

4. $\mathrm{E}\left(\left(\mathrm{x}_{1}+\ldots+\mathrm{x}_{\mathrm{n}}\right) / \mathrm{n}\right)$ or $\mathrm{E}(\bar{x})=$

5. $V\left(x_{1}-2 x_{2}-5\right)=$

6. $V\left(x_{1}+\ldots+x_{n}\right)=$

7. $\mathrm{V}\left(\left(\mathrm{x}_{1}+\ldots+\mathrm{x}_{\mathrm{n}}\right) / \mathrm{n}\right)=\mathrm{V}(\bar{x})=$

\section{Exercise \#2}

Questions related to measurement models:

1. How are the model terms different between the additive and relative models?

2. How would a plot of the absolute differences look for an additive model?

3. How would a plot of the measurement values look for a relative model?

4. How would a plot of the relative differences look for a relative model?

\section{Exercise \#3}

Specify the measurement models for the following types of measurement:

1. Temperature -

2. Neutron Coincidence Counter-

3. Calorimeter -

4. Pressure -

5. Tank volume-

6. Density -

7. Weight- 


\section{Exercise \#4}

Consider an analytical method for concentration involving dilutions, such as Davies-Gray Titration. Process measurements are as follows: two samples, two analysts and two measurements per sample.

1. Specify a reasonable model for this measurement scenario.

2. What kind of effects are the samples and analysts?

3. Are the sample and analyst effects different from random measurement effects?

4. What would a significant analyst variance or effect represent?

5. How could an analyst effect be used?

\section{$\underline{\text { Exercise \#5 }}$}

Suppose $\mathrm{y}_{\mathrm{i}}=\mu\left(1+\eta+\varepsilon_{\mathrm{i}}\right)$, where $\eta$ is $\mathrm{N}\left(0, \sigma_{\eta}^{2}\right)$ and $\varepsilon_{\mathrm{i}}$ are iid $\mathrm{N}\left(0, \sigma_{\varepsilon}^{2}\right)$.

Compute the following by using the defined model for $\mathrm{y}_{\mathrm{i}}$ :

1. $\mathbf{E}\left(\mathbf{y}_{1}\right)=$

2. $E\left(y_{1}+y_{2}\right)=E\left(y_{1}\right)+E\left(y_{2}\right)=$

3. $E\left(y_{1}-y_{2}\right)=E\left(y_{1}\right)-E\left(y_{2}\right)=$

4. $\mathrm{E}(\bar{y})=n \mu / n=$

5. $\mathrm{V}\left(\mathrm{y}_{1}\right)=$

6. $\quad \mathrm{V}\left(\mathrm{y}_{1}+\mathrm{y}_{2}\right)=$

7. $\mathbf{V}\left(\mathbf{y}_{1}-\mathbf{y}_{2}\right)=$

8. $\mathrm{V}(\overline{\boldsymbol{y}})=$

9. Are $y_{1}$ and $y_{2}$ independent? 
10. What is $\operatorname{Cov}\left(\mathrm{y}_{1}, \mathrm{y}_{2}\right)$ ?

\section{Exercise \#6}

Suppose $\mathrm{y}_{\mathrm{i}}=\mu_{\mathrm{i}}\left(1+\eta+\varepsilon_{\mathrm{i}}\right)$, where $\eta$ is $\mathrm{N}\left(0, \sigma_{\eta}^{2}\right)$ and $\varepsilon_{\mathrm{i}}$ are iid $\mathrm{N}\left(0, \sigma_{\varepsilon}^{2}\right)$.

Compute and answer the following:

1. $\mathrm{V}\left(\mathrm{y}_{1}+\ldots+\mathrm{y}_{10}\right)=$

2. Discuss the differences in the effects of systematic and random variances on the variance for a sum of measurements.

3. In general, what type of variance is most important to control? 


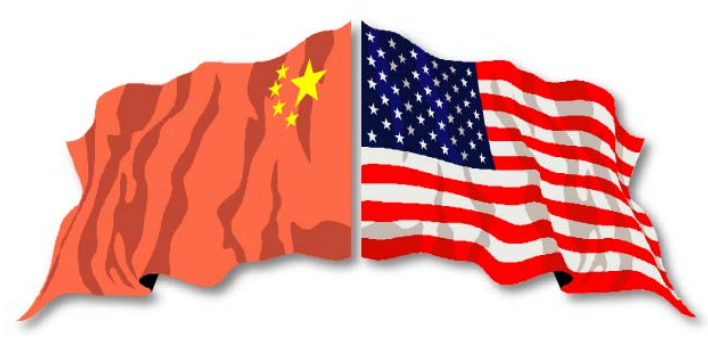

Module 3

Measurement Models 


\section{Objectives}

- Review GUM concepts as they relate to measurement models

- Discuss the statistics used in describing measurement models

- Understand additive and relative measurement models and be able to establish the type of model for a particular measurement method

- Understand expectation and variance calculations associated with measurement models and apply them to some example measurement methods 


\section{Measurement Models and GUM}

GUM recommendations related to measurement models:

- In general, the use of measurement models results in Type A uncertainties. Type $A$ uncertainties are derived by statistical methods.

- Uncertainties derived by statistical methods, such as measurement models, are characterized by estimated variances, $s_{i}{ }^{2}$, and the appropriate degrees of freedom, $v_{i}$. This is exactly the nature of ANOVA techniques.

- GUM recommends that combined uncertainty is derived by adding the variance components and then expressing the combined uncertainty as a standard deviation or a one-sigma value. This is a direct result of using a statistical measurement model. 


\section{Normal Distribution}

Note: Also called the Gaussian distribution

- It is a bell-shaped, symmetric and continuous distribution

- A random variable, $\mathrm{x}$, with a normal distribution can assume any value along a continuum of possible values

- Important uses of the Normal distribution are for hypothesis testing, the derivation of confidence intervals and the distribution of error terms for measurement models 


\section{Normal Distribution}

- The equation for the probability density function (pdf) of $x$ is given by:

$$
\mathrm{f}(\mathrm{x})=\frac{1}{\sigma \sqrt{2 \pi}} \mathrm{e}^{-(\mathrm{x}-\mu)^{2} / 2 \sigma^{2}} \quad-\infty<\mathrm{x}<\infty
$$

where $\mu$ represents the mean and $\sigma$ represents the standard deviation, and $-\infty<\mathrm{x}<\infty$ indicates that the random variable $x$ can assume any real number.

- The expected value or mean represents the location or theoretical average of the distribution, while the variance represents the spread of the distribution 


\section{Normal Distribution: Examples}

- Two Normal distributions with different means, but the same standard deviation

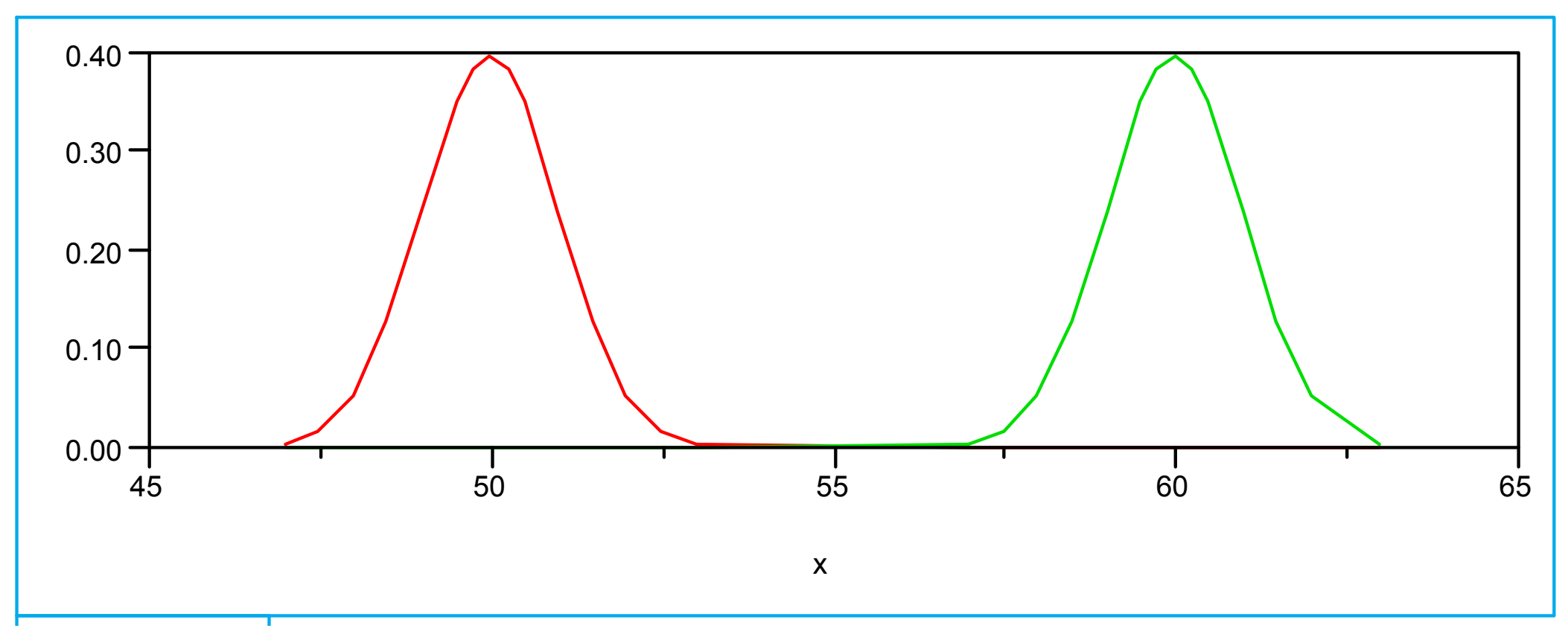




\section{Normal Distribution: Examples}

- Three Normal distributions with the same mean, but different standard deviations

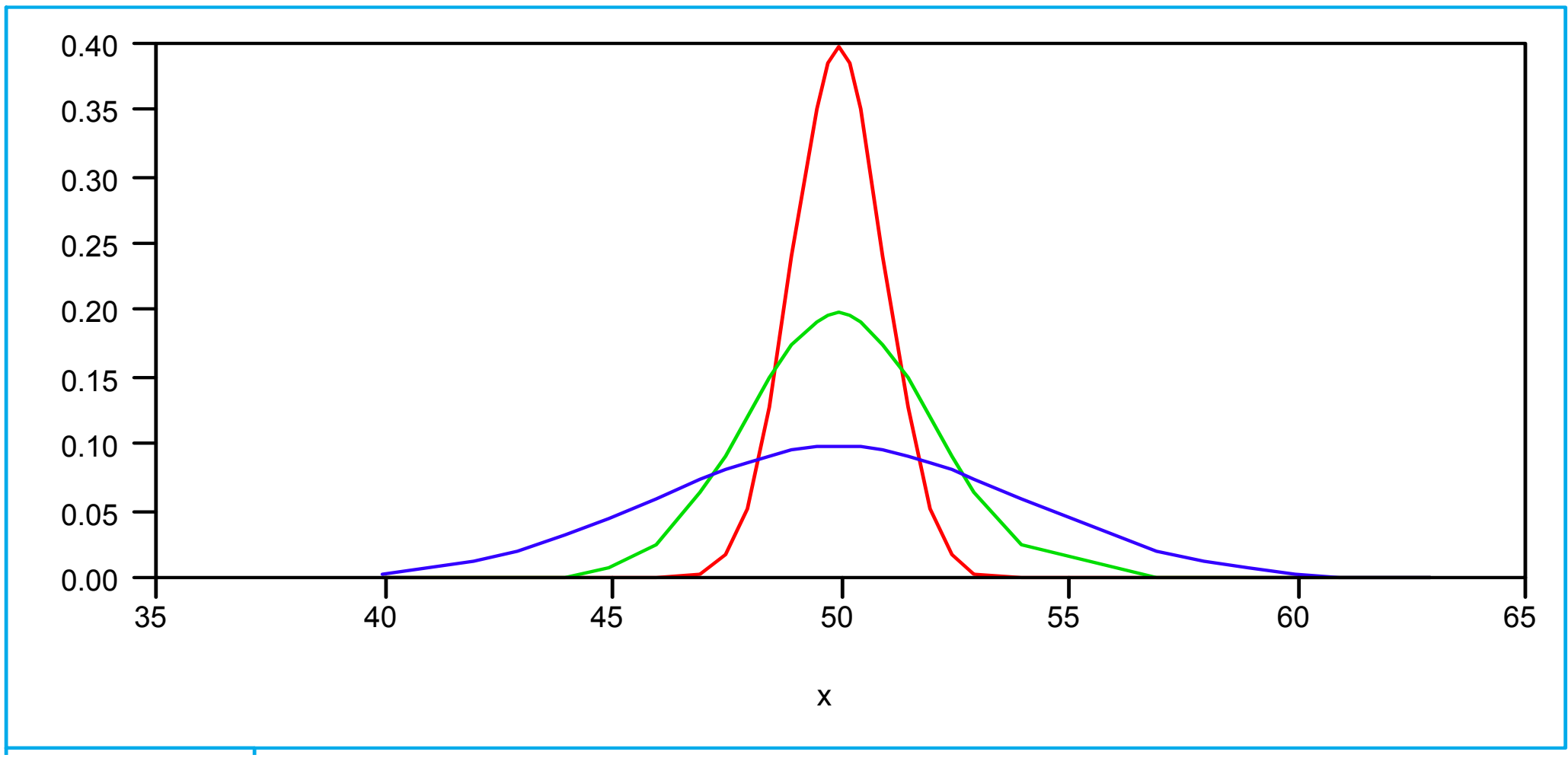




\section{Normal Distribution Properties}

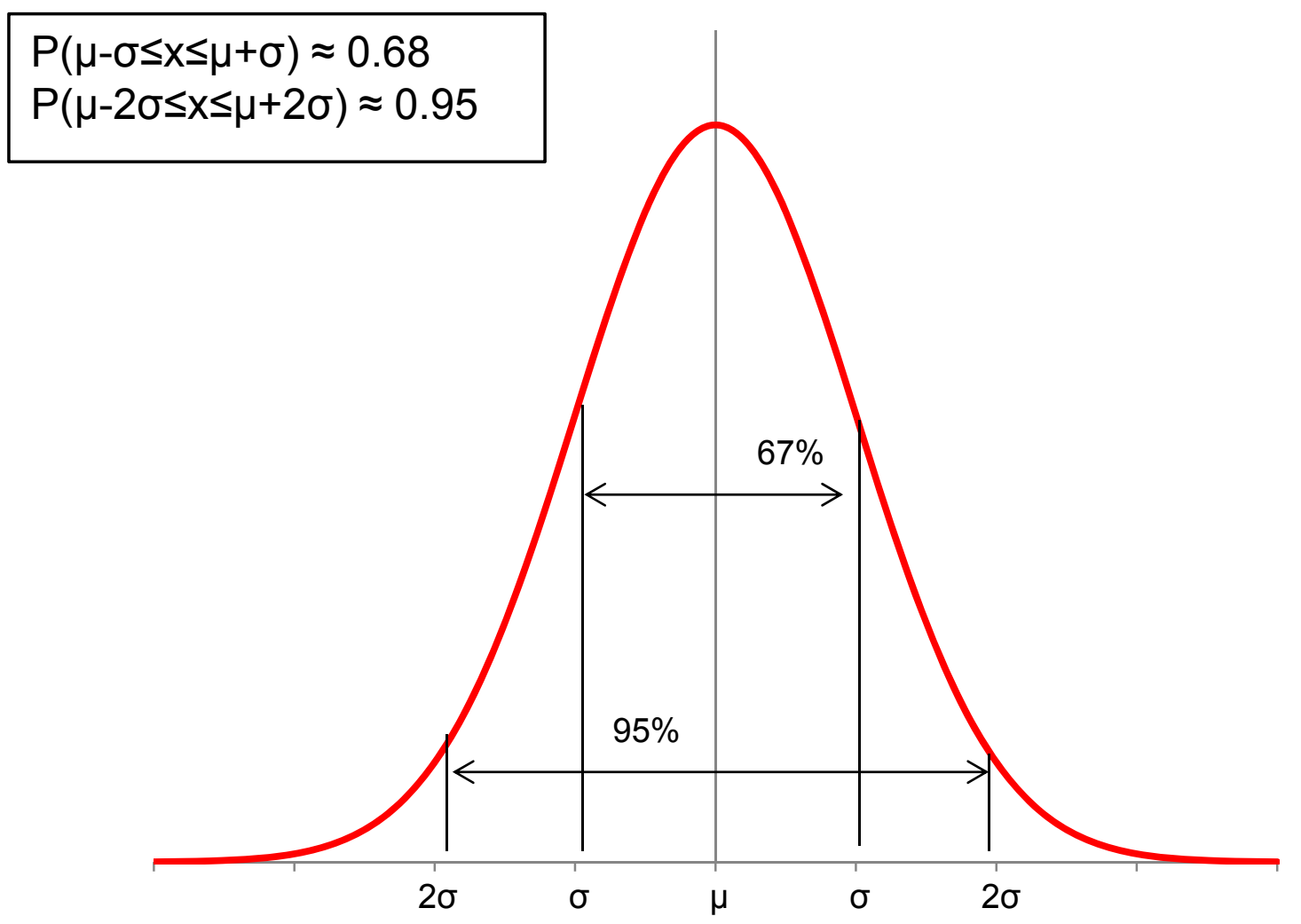




\section{Normal Distribution Properties}

Expectation, Variance and Covariance

Let $x \sim N\left(\mu_{x}, \sigma_{x}^{2}\right)$, then

The expected value of $x$ or $E(x)=\mu_{x}$, and

$E(a x+b)=a \mu_{x}+b$, where $a$ and $b$ are constants

The variance of $x$ or $V(x)=\sigma_{x}{ }^{2}$, and

$$
\mathrm{V}(\mathrm{ax}+\mathrm{b})=\mathrm{a}^{2} \sigma_{\mathrm{x}}^{2}
$$

Let $y \sim N\left(\mu_{y}, \sigma_{y}{ }^{2}\right)$, then

$$
E(x+y)=E(x)+E(y)=\mu_{x}+\mu_{y}
$$

If $x$ and $y$ are independent, then

$$
\mathrm{V}(\mathrm{x}+\mathrm{y})=\mathrm{V}(\mathrm{x})+\mathrm{V}(\mathrm{y})=\sigma_{\mathrm{x}}{ }^{2}+\sigma_{\mathrm{y}}{ }^{2}
$$




\section{Normal Distribution Properties}

Expectation, Variance and Covariance

If $x$ and $y$ are not independent, then

$V(x+y)=V(x)+V(y)+2 \operatorname{Cov}(x, y)$, where,

Covariance of $x$ and $y=\operatorname{Cov}(x, y)=E(x y)-E(x) E(y)$

- The concept of covariance is related to systematic measurement effects

- An example of covariance will be discussed later in this module 


\section{Normal Distribution Properties}

Standard Normal Distribution

- If $x \sim N\left(\mu, \sigma^{2}\right)$, that is, $x$ is distributed as a normal random variable with mean $\mu$ and variance $\sigma^{2}$ and

$$
Z=\frac{x-\mu}{\sigma}
$$

then $Z$ is said to have the standard normal distribution.

- Note than the mean of $Z$ is zero and its standard deviation is 1 .

- In statistical text books tables are available for Z 


\section{Normal Distribution Properties}

One of the most important properties of the Normal distribution is as follows:

- Let $x_{1}$ and $x_{2}$ be independent and identically distributed (iid) $\mathrm{N}\left(\mu, \sigma^{2}\right)$, then $\left(x_{1}+x_{2}\right)$ has a normal distribution with a mean of $2 \mu$ and variance of $2 \sigma^{2}$

- In other words, the sum of normally distributed random variables is also normally distributed

- In general, let $x_{1}, \ldots, x_{n}$ be iid $N\left(\mu, \sigma^{2}\right)$, then $\left(a_{1} x_{1}+\ldots+a_{n} x_{n}\right) \sim N\left(\mu \Sigma_{i} a_{i}, \sigma^{2} \Sigma_{i} a_{i}^{2}\right)$, where $a_{1}, \ldots, a_{n}$ are constants 


\section{Normal Distribution: Parameter Estimation}

Estimation of $\mu$ and $\sigma^{2}$ parameters of the Normal distribution

- Let $x_{1}, \ldots, x_{n}$ be iid $N\left(\mu, \sigma^{2}\right)$

- The parameter $\mu$ represents the mean value or location of the distribution, while $\sigma^{2}$ represents the variance or spread in the distribution

- As expected, the best point estimate of $\mu$ is $\bar{x}$ or $\frac{1}{n} \Sigma_{\mathrm{i}} \mathrm{x}_{\mathrm{i}}$

- The best point estimate of $\sigma^{2}$ is $s^{2}=\frac{1}{n-1} \Sigma_{i}\left(x_{i}-\bar{x}\right)^{2}$

- $\sigma$ is then estimated by $s$ or $+\sqrt{s^{2}}$ 


\section{Exercise \# 1}

Let $\mathrm{x}_{1}, \ldots, \mathrm{x}_{\mathrm{n}}$ be iid $\mathrm{N}\left(\mu, \sigma^{2}\right)$.

Compute the following:

1. $E\left(x_{1}-x_{2}\right)$

2. $E\left(x_{1}+2 x_{2}-x_{3}-x_{4}+5\right)$

3. $E\left(x_{1}+\ldots+x_{n}\right)$

4. $\mathrm{E}\left(\left(\mathrm{x}_{1}+\ldots+\mathrm{x}_{\mathrm{n}}\right) / \mathrm{n}\right)$ or $\mathrm{E}(\bar{x})$

5. $V\left(x_{1}-2 x_{2}-5\right)$

6. $V\left(x_{1}+\ldots+x_{n}\right)$

7. $V\left(\left(x_{1}+\ldots+x_{n}\right) / n\right)$ or $V(\bar{x})$ 


\section{Measurement Models}

- Two main types:

- Absolute

- Also called additive

- Examples would be length, temperature, weight and density measurements

- Relative

- Also called multiplicative

- Examples would be analytical methods that require dilutions, such as Davies-Gray Titration, and neutron coincidence counting

- Mixed Models also possible

- Measurement type determines appropriate model

- Data plots can assist with model determination 


\section{Additive or Absolute Model}

Consider the following simple additive model with only a random effects term:

$$
Y_{i j}=\mu_{i}+\varphi_{i j}
$$

Where,

$Y_{i j}=$ the $j^{\text {th }}$ measurement for $\mu_{i}$

$\mu_{i}=$ true value for standard $\mu_{i}$

$\varphi_{i j}=$ random measurement effect for the $j^{\text {th }}$ measurement for $\mu_{\mathrm{i}}$

The $\varphi_{\mathrm{ij}}$ are iid $\mathrm{N}\left(0, \sigma_{\varphi}{ }^{2}\right)$. 


\section{Distribution of Model Error Effects}

Distribution for additive random effects $\varphi_{\mathrm{ij}}$

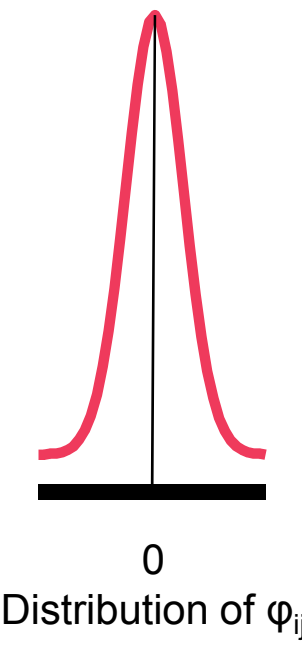

The variance term, $\sigma_{\varphi}{ }^{2}$ is unknown and must be estimated.

The units for this effect are in relative or percentage terms. 


\section{Additive Model: Length Measurements}

Consider a model for length measurements using a tape measure.

The uncertainty associated with length measurements tends to be constant over the measurement range of the tape measure.

A reasonable model is as follows:

$$
Y_{i j}=\mu_{i}+\varphi_{i j}
$$

Where,

$Y_{i j}=j^{\text {th }}$ length measurement for $\mu_{i}$

$\mu_{i}=$ true length for the $i^{\text {th }}$ standard length

$\varphi_{\mathrm{ij}}=$ random measurement effect for the $\mathrm{j}^{\text {th }}$ length measurement for $\mu_{i},\left(\varphi_{i j}\right.$ are iid $\left.N\left(0, \sigma_{\varphi}{ }^{2}\right)\right)$ 


\section{Additive Model: Evaluation}

In general, data plots are used to determine the nature or model associated with a particular measurement process.

Two plots will be presented for evaluating the nature of weight measurements:

1. Plot of length measurements by the reference values

2. Plot of the absolute differences $\left(Y_{i j}-\mu_{i}\right)$ by the reference values 


\section{Additive Model: Data Plots}

Consider a plot of the length measurements by the reference values:

Plot of Length Results by Reference Values

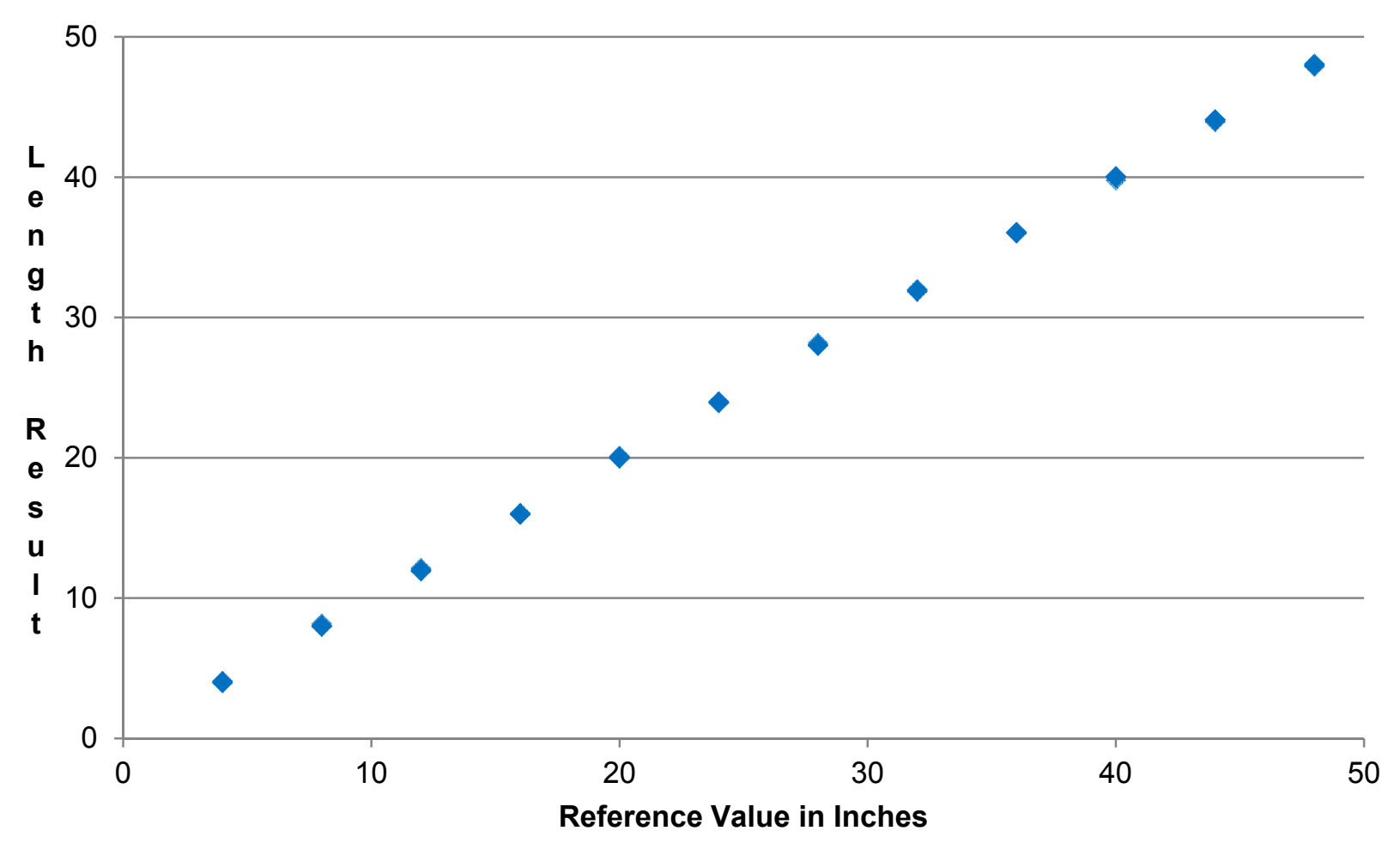




\section{Additive Model: Data Plots}

In many cases, a plot of the length measurements by the reference values does not have enough resolution to evaluate the nature of the measurements.

However, this kind of plot does demonstrate the expected linear relationship between the length measurement and the reference values.

Now, consider the plot of the absolute differences by the reference values. 


\section{Additive Model: Data Plots}

Data plot of the absolute differences $\left(Y_{i j}-\mu_{i}\right)$ by the reference values:

Plot of Absolute Differences by Reference Values

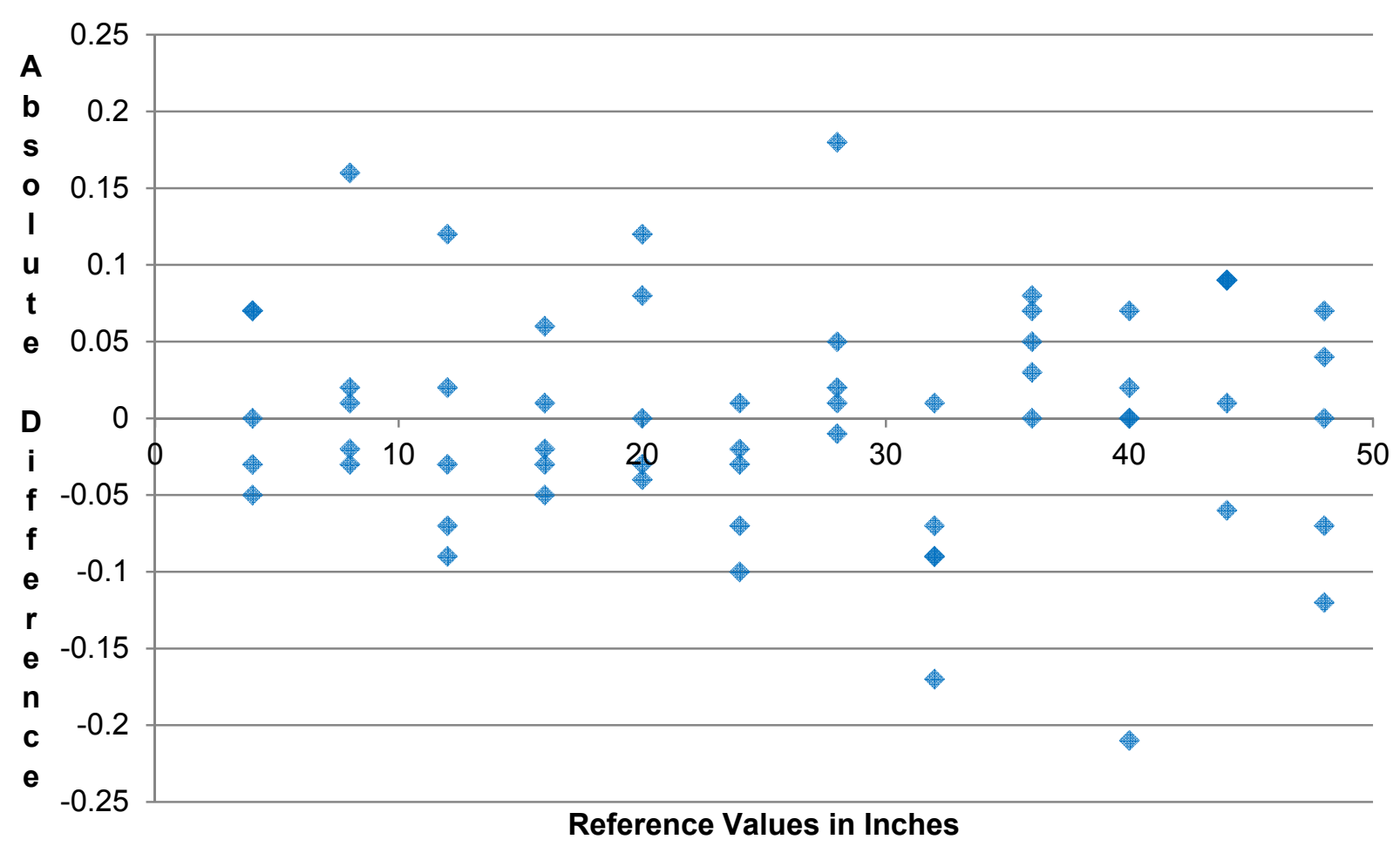




\section{Additive Model: Conclusion}

A data plot of the absolute differences by the reference

values is the best technique for evaluating the appropriateness of an additive model for a particular measurement method.

For an additive model, the variability of the absolute differences should be approximately constant over the measurement range of the method. 


\section{Additive Model}

Depending on the situation, a more complex additive model may be needed. For example:

$$
Y_{i j k l}=\mu_{i}+\theta_{j}+\xi_{k}+\varphi_{i j k l}
$$

Where,

$\mathrm{Y}_{\mathrm{ijkl}}=$ measurement value

$\mu_{\mathrm{i}} \quad=$ true value for $\mu_{\mathrm{i}}$ (may not be a standard)

$\theta_{\mathrm{j}}=$ systematic measurement effect for the $\mathrm{j}^{\text {th }}$ analyst

$\xi_{\mathrm{k}}=$ systematic measurement effect for the $\mathrm{k}^{\text {th }}$ sample

$\varphi_{\mathrm{ijkl}}=$ random measurement effect for the Ith measurement for $\mu_{i}$, analyst $j$ and sample $k$

The $\theta_{\mathrm{j}}$ are iid $\mathrm{N}\left(0, \sigma_{\theta}{ }^{2}\right)$, the $\xi_{\mathrm{k}}$ are iid $\mathrm{N}\left(0, \sigma_{\xi}{ }^{2}\right)$, and the $\varphi_{\mathrm{ijk} k}$ are iid $\mathbf{N}\left(0, \sigma_{\varphi}{ }^{2}\right)$. In addition, the $\theta_{\mathrm{j}}$, $\xi_{\mathrm{k}}$, and $\varphi_{\mathrm{ijkl}}$ are independent. 


\section{Multiplicative or Relative Model}

Consider the following relative model with systematic and random effects terms:

$$
Y_{i j}=\mu_{i}\left(1+\eta+\varepsilon_{i j}\right)
$$

Where,

$Y_{i j}=$ the $j^{\text {th }}$ measurement for $\mu_{i}$

$\mu_{\mathrm{i}} \quad=$ true value for standard $\mu_{\mathrm{i}}$

$\eta \quad=$ systematic measurement effect for a single analyst

$\varepsilon_{\mathrm{ij}} \quad=$ random measurement effect for the $\mathrm{j}^{\text {th }}$ measurement for $\mu_{i}$

The $\eta$ is $N\left(0, \sigma_{\eta}{ }^{2}\right)$ and the $\varepsilon_{i j}$ are iid $N\left(0, \sigma_{\varepsilon}{ }^{2}\right)$. In addition, $\eta$ and the $\varepsilon_{\mathrm{ij}}$ are independent. 


\section{Distribution of Model Error Effects}

Distribution for relative random effects $\eta$ and $\varepsilon_{\mathrm{ij}}$
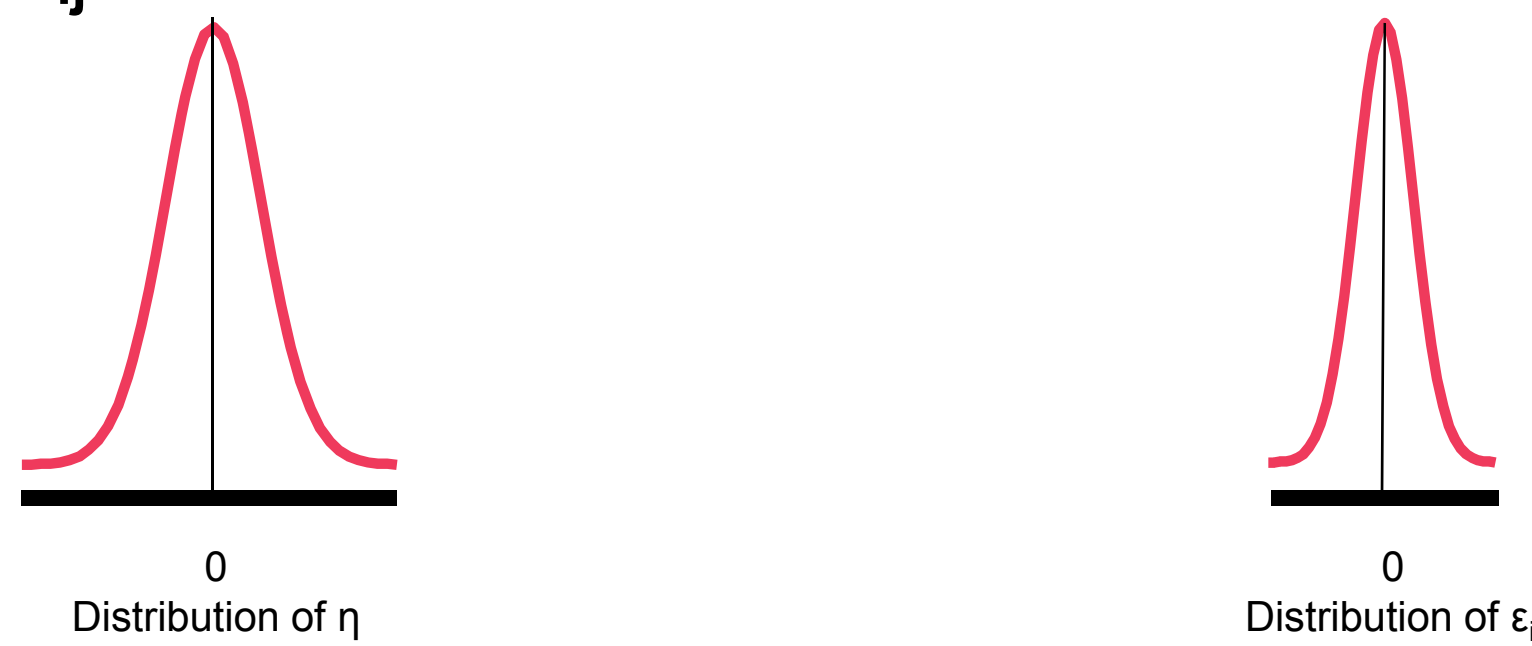

The variance terms, $\sigma_{\eta}{ }^{2}$ and $\sigma_{\varepsilon}{ }^{2}$ are unknown and must be estimated.

The units for these effects are in relative or percentage terms. 


\section{Relative Model: Davies-Gray Titration}

Consider a relative model for Davies-Gray Uranium concentration measurements.

Davies-Gray involves dilutions and, because of this, the uncertainty in the concentration measurements is not constant over the measurement range of the method.

A reasonable model for the Davies-Gray method is as follows:

$$
Y_{i j}=\mu_{i} \times\left(1+\eta+\varepsilon_{i j}\right)
$$




\section{Relative Model: Davies-Gray Titration}

Where,

$Y_{i j}=j^{\text {th }}$ concentration measurement for $\mu_{i}$

$\mu_{i}=$ true concentration for standard $\mu_{i}$

$\eta \quad=$ systematic measurement effect due to a single analyst performing the method

$\varepsilon_{i j}=$ random measurement effect for the $j^{\text {th }}$ concentration measurement of $\mu_{i}$

The $n$ effect is $N\left(0, \sigma_{\eta}{ }^{2}\right)$ and the $\varepsilon_{i j}$ are iid $N\left(0, \sigma_{\varepsilon}{ }^{2}\right)$. In addition, $\eta$ and the $\varepsilon_{i j}$ are independent. 


\section{Relative Model: Evaluation}

In general, data plots are used to determine the nature or model associated with a particular measurement process.

Three plots will be presented for evaluating the nature of Davies-Gray concentration measurements:

1. Plot of the concentration measurements by the concentration standards

2. Plot of the absolute differences $\left(Y_{i j}-\mu_{i}\right)$ by the concentration standards

3. Plot of the relative differences $\left(Y_{i j}-\mu_{i}\right) / \mu_{i}$ by the concentration standards 


\section{Relative Model: Data Plots}

Consider a plot of the concentration measurements by the concentration standards:

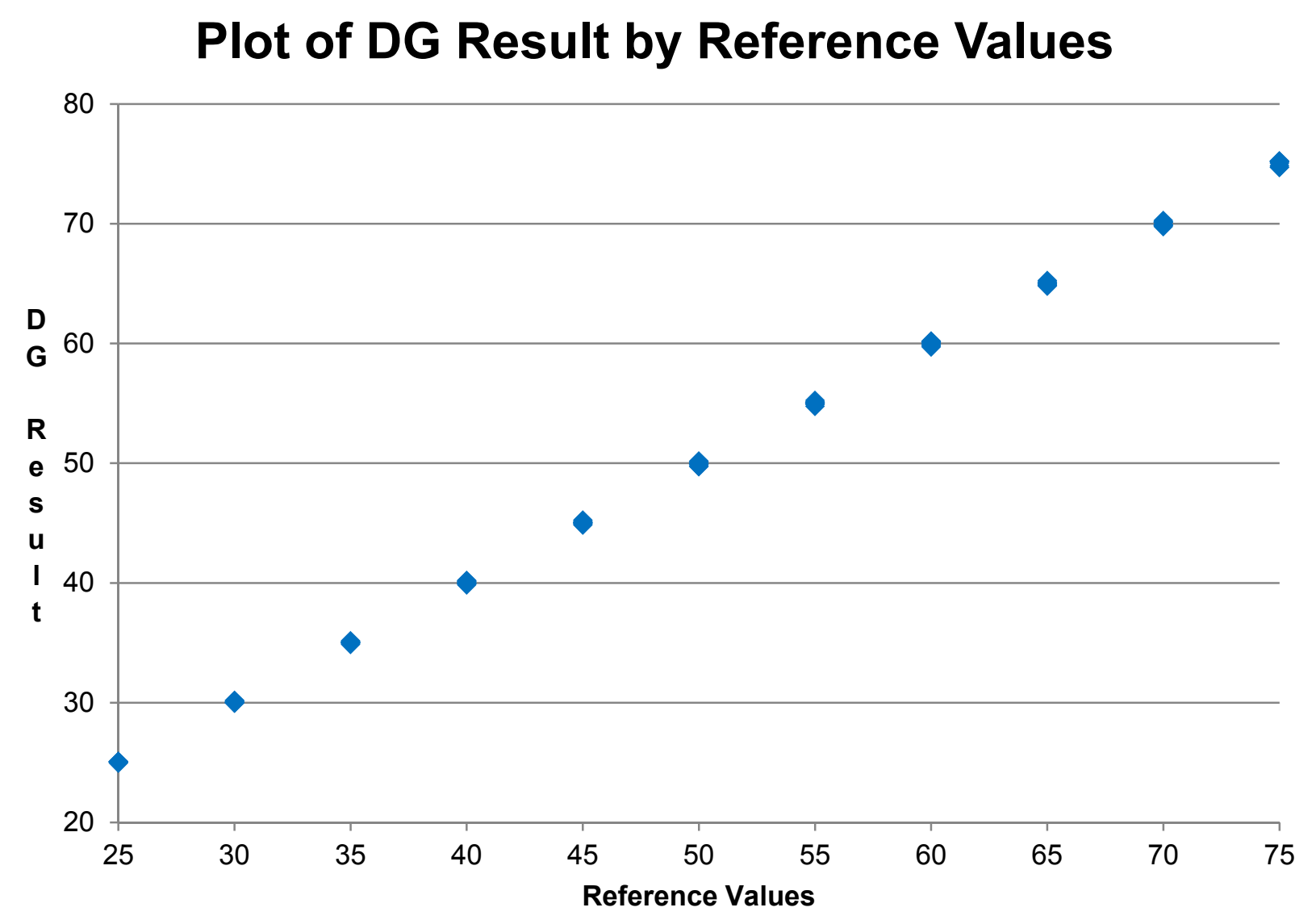




\section{Relative Model: Data Plots}

A plot of the concentration measurements by the standard values does not have enough resolution to evaluate the nature of the measurement model.

However, this plot does demonstrate the expected linear relationship between the method results and the reference values.

Next, consider a plot of the absolute differences, $\left(Y_{i j}-\mu_{i}\right)$, by the standard values. 


\section{Relative Model: Data Plots}

Data plot of the absolute differences, $\left(Y_{i j}-\mu_{i}\right)$, by the standard values:

Plot of Absolute Differences by the Reference Values

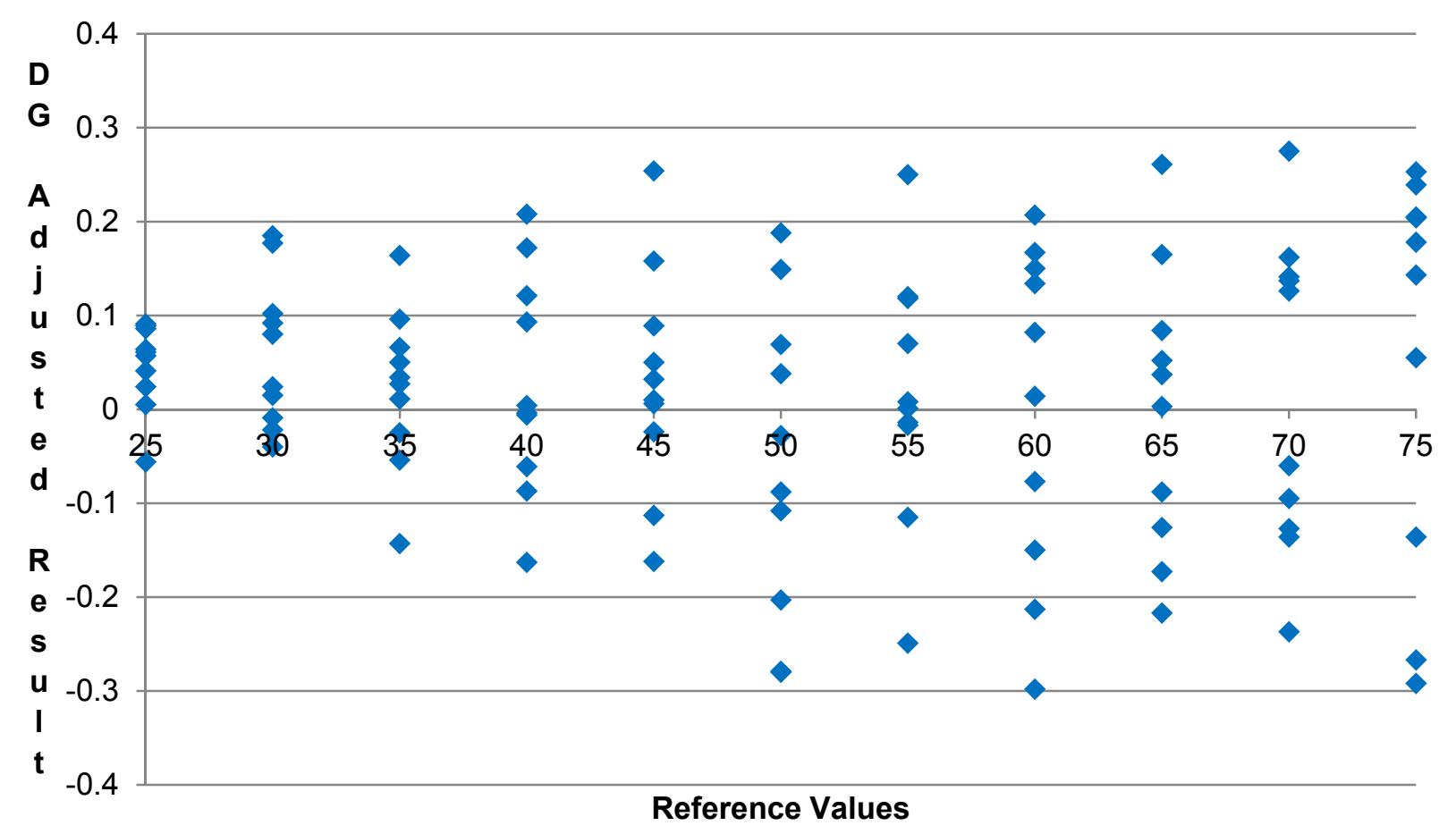




\section{Relative Model: Data Plots}

The data plot of the absolute differences, $\left(Y_{i j}-\mu_{i}\right)$, by the standard values shows the variability is not constant over the measurement range of the DaviesGray method. The variability tends to increase as the standard values increase. This is the expected behavior for a relative model.

Finally, consider a plot of the relative differences, $\left(Y_{i j}-\mu_{i}\right) / \mu_{i}$, by the standard values. 


\section{Relative Model: Data Plots}

Data plot of the relative differences $\left(\left(Y_{i j}-\mu_{i}\right) / \mu_{i}\right)$ by the standard values:

Plot of Relative Differences by Reference Values

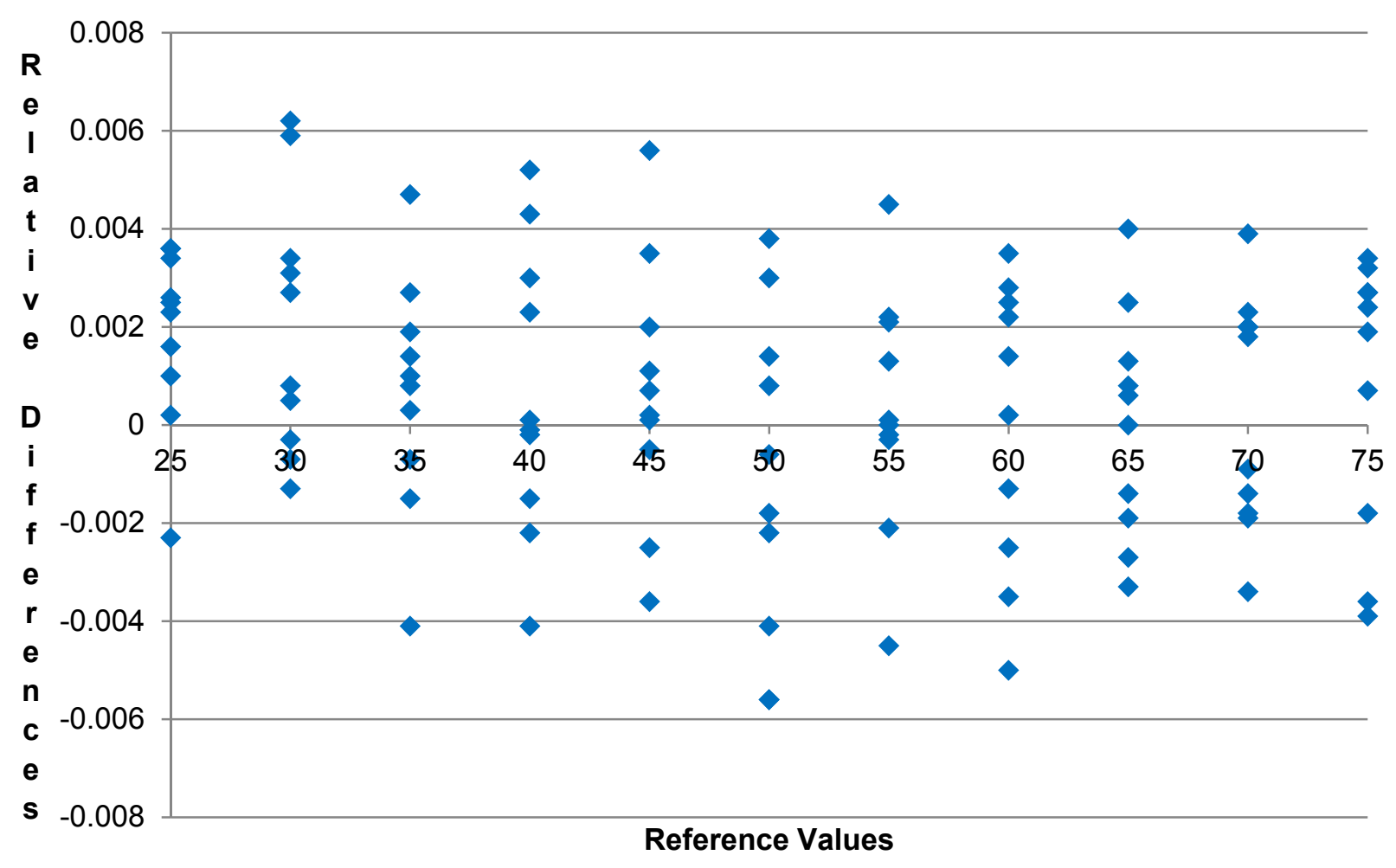




\section{Relative Model: Conclusion}

A data plot of the relative differences by the reference

values is the best technique for evaluating the appropriateness of a relative model for a particular measurement method.

For a relative model, the variability of the relative differences should be approximately constant over the measurement range of the method. 


\section{Relative Model}

Again, depending on the situation, a more complex relative model may be needed. For example:

$$
Y_{i j k l}=\mu_{i}\left(1+\theta_{j}+\xi_{k}+\varphi_{i j k l}\right) \text {, }
$$

where the terms $\theta_{\mathrm{j}}, \xi_{\mathrm{k}}$ and $\varphi_{\mathrm{ijkl}}$ could be similar to the terms described for the additive model discussed earlier. The random effects terms used in the relative model would reflect the measurement situation being described or modeled. 


\section{The Nature of a Measurement}

- The basis for a particular measurement is the assumed statistical model for that measurement

- A measurement is observed or realized as the result of an actual measurement method or process

- A measurement results when systematic and random effects associated with the measurement model are drawn or sampled from their respective Normal distributions

- The frequency with which an effect is sampled depends on the nature of the effect 


\section{The Nature of a Measurement}

- Systematic effects are sampled and held constant for the duration of the effect. For example, during the period of time that a certain standard is used, the systematic effect is sampled and held constant while the standard is in use. When the standard changes, then another systematic effect is sampled and held constant while the new standard is in use.

- Random effects are sampled for each measurement

- The actual values of the systematic and random effects are not known, but the measurement data provides information regarding the uncertainties associated with the systematic and random effects 


\section{Exercise \#2}

Measurement Models

1. How are the model terms different between the additive and multiplicative models?

2. How would a plot of the absolute differences look for an additive model?

3. How would a plot of the measurement values look for a relative model?

4. How would a plot of the relative differences look for a relative model? 


\section{Exercise \#3}

Specify the measurement models for the following types of measurement:

- Temperature

- Neutron Coincidence Counter

- Calorimeter

- Pressure

- Tank volume

- Density

- Weight 


\section{Exercise \#4}

Consider an analytical method for concentration involving dilutions, such as Davies-Gray Titration. Process measurements are as follows: two samples, two analysts, and two measurements per sample.

1. Specify a reasonable model for this measurement scenario.

2. What kind of effects are the samples and analysts?

3. Are sample and analyst effects different from random measurement effects?

4. What would a significant analyst variance or effect represent?

5. How would an analyst effect be addressed? 


\section{Exercise \#5}

Suppose $y_{i}=\mu\left(1+\eta+\varepsilon_{i}\right)$ and $\eta$ is $N\left(0, \sigma_{\eta}{ }^{2}\right)$ and $\varepsilon_{i}$ are iid $\mathrm{N}\left(0, \sigma_{\varepsilon}{ }^{2}\right)$. Compute the following:

1. $E\left(y_{1}\right)$

2. $E\left(y_{1}+y_{2}\right)$

3. $E\left(y_{1}-y_{2}\right)$

4. $E(\bar{y})$

5. $\mathrm{V}\left(\mathrm{y}_{1}\right)$

6. $V\left(y_{1}+y_{2}\right)$

7. $V\left(y_{1}-y_{2}\right)$

8. $\mathrm{V}(\bar{y})$ 


\section{Exercise \#5 (cont'd)}

9. Are $y_{1}$ and $y_{2}$ independent?

$$
\text { Is } \mathrm{V}\left(\mathrm{y}_{1}+\mathrm{y}_{2}\right)=\mathrm{V}\left(\mathrm{y}_{1}\right)+\mathrm{V}\left(\mathrm{y}_{2}\right) \text { ? }
$$

10. If not, what is $\operatorname{Cov}\left(y_{1}, y_{2}\right)$ ? 


\section{Exercise \#6}

Suppose $y_{i}=\mu_{i}\left(1+\eta+\varepsilon_{i}\right)$ and $\eta$ is $N\left(0, \sigma_{\eta}{ }^{2}\right)$ and $\varepsilon_{i}$ are iid $\mathrm{N}\left(0, \sigma_{\varepsilon}{ }^{2}\right)$. Compute the following:

1. $V\left(y_{1}+\ldots+y_{10}\right)$ ?

2. Discuss the differences in the affects of systematic and random variances on the variance for a sum of measurements.

3. What type of variance is most important to control? 


\section{Summary}

- Reviewed GUM concepts as they relate to measurement models

- Discussed the statistics used in describing measurement models

- Introduced additive and relative measurement models and discussed how to establish the type of model for a particular measurement method

- Introduced expectation and variance calculations associated with measurement models and applied them to some example measurement methods 


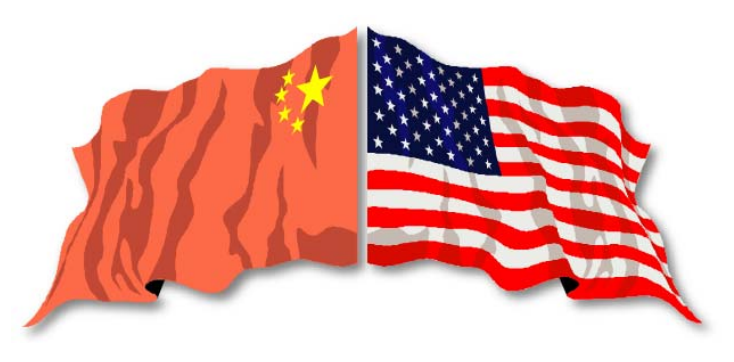

\section{Module 4}

\section{I ntroduction to}

Variance Propagation Techniques 


\section{Objectives}

- Understand the basic concepts of variance propagation

- Understand the propagation of random variances

- Understand the propagation of systematic variances

- Be able to use Excel for solving variance propagation problems 


\section{The GUM “Connection”}

- Recommendation INC-1 (1980) is an overview of an agreed-upon approach to measurement uncertainty evaluation

- INC-1 relied on the General Law of "Variance Propagation." This law is based on partial derivatives of Taylor Series expansions for a given functional relationship

- The partial derivatives are called sensitivity coefficients 


\section{Variance Propagation}

- Often, measurement data are processed through multiplication, addition, or other functional manipulation to arrive at a derived number of more immediate interest.

- The values that are produced by these processing steps will be distributed in a way that is dependent on both the original distribution and the types of operations carried out. 


\section{Combining Random Variables (linear combinations)}

The determination of the expected value and variance of linear combinations of random variables has been reviewed.

Specifically,

Let $X$ and $Y$ be random variables and let $R=a \cdot X+b \cdot Y+c$.

Then

$$
E(R)=a \cdot E(X)+b \cdot E(Y)+c
$$

And

$$
V(R)=a^{2} \cdot V(X)+b^{2} \cdot V(Y)+2 \cdot a \cdot b \cdot \operatorname{Cov}(X, Y)
$$

What if $X$ and $Y$ are uncorrelated?

Specifically, for $R=X+Y$ or $R=X-Y$, if $X$ and $Y$ are uncorrelated, then

$$
\sigma_{R}=\sqrt{\sigma_{X}^{2}+\sigma_{Y}^{2}}
$$




\section{Sum or Differences of Counts}

A common application of this situation arises when counts resulting from a radioactive source must be corrected by subtracting an appropriate background count. If we assume equal counting times and note the independence of the two counts, then net counts $=$ total counts - background counts

Or

$$
r=x-y
$$

Let $x=1071$ and $y=521$. Then $r=1071-521=550$.

In addition, the estimators of the standard deviations of $x$ and $y$ are the square roots of the measured counts. Thus, $\sigma_{x}$ is estimated by $\sqrt{ } \mathbf{x}$ and $\sigma_{y}$ is estimated by $\sqrt{ }_{y}$. Then, $\sigma_{r}$ is estimated by

$$
\sigma_{\mathrm{r}}=\sqrt{\sigma_{\mathrm{x}}^{2}+\sigma_{\mathrm{y}}^{2}} \rightarrow \hat{\sigma}_{\mathrm{r}}=\sqrt{1071+521}=\sqrt{1592}=39.9
$$




\section{Multiplication or Division by a Constant}

A familiar example of this case is the calculation of a counting rate. If $x$ counts are recorded over a time $t$, then

$$
\text { Count Rate }=r=x / t
$$

Let $x=1120$ counts and $t=5 \mathrm{~s}$ (assumed to be measured with very small uncertainty).

Then $r=1120$ counts $/(5 s)=224$ counts $/ s$.

The associated standard deviation (in counts per second) is, where it is noted that the estimated variance of $x$ is 1120 .

$$
\sigma_{\mathrm{r}}=\sqrt{\frac{\sigma_{\mathrm{X}}^{2}}{\mathrm{t}^{2}}}=\frac{\sigma_{\mathrm{X}}}{\mathrm{t}} \rightarrow \hat{\sigma}_{\mathrm{r}}=\frac{\sqrt{1120}}{5}=6.7
$$




\section{Mean Value of Multiple I ndependent Counts}

Suppose we have recorded $\mathrm{n}$ repeated counts from the same source for equal counting times. Let the results of these counts be designated $x_{1}, x_{2}, \ldots, x_{n}$ and let the sum be represented as

$$
\operatorname{SUM}_{x}=x_{1}+x_{2}+\cdots+x_{n}
$$

Then the variance of $\operatorname{SUM}_{x}$ (assuming that all of the counts $x_{i}$ are independent) is given by

$$
\sigma_{\operatorname{SUM}_{\mathrm{X}}}^{2}=\sigma_{\mathrm{x}_{1}}^{2}+\sigma_{\mathrm{x}_{2}}^{2}+\cdots+\sigma_{\mathrm{x}_{\mathrm{n}}}^{2}
$$

And since $\sigma_{x_{i}}=\sqrt{ } x_{i}$ for each independent count,

$$
\sigma_{\operatorname{SUM}_{\mathrm{X}}}=\sqrt{\mathrm{x}_{1}+\mathrm{x}_{2}+\cdots+\mathrm{x}_{\mathrm{n}}}
$$




\section{Mean Value of Multiple I ndependent Counts}

Now calculate a sample mean from the $\mathbf{n}$ independent counts

$$
\overline{\mathrm{x}}=\frac{\mathrm{SUM}_{\mathrm{X}}}{\mathrm{n}}
$$

The standard deviation of the sample mean is given by

$$
\sigma_{\overline{\mathrm{X}}}=\frac{\sigma_{\mathrm{SUM}_{\mathrm{X}}}}{\mathrm{n}} \Rightarrow \hat{\sigma}_{\overline{\mathrm{X}}}=\frac{\sqrt{\mathrm{SUM}_{\mathrm{X}}}}{\mathrm{n}}=\frac{\sqrt{\mathrm{n} \overline{\mathrm{x}}}}{\mathrm{n}}=\sqrt{\frac{\overline{\mathrm{x}}}{\mathrm{n}}}
$$

A general conclusion is that, to improve the statistical uncertainty of a given measurement by a factor of 2 , the number of counts must increase by a factor of 4 . 


\section{A More General Case}

What about the variance of a value $r$ calculated as a general function of any number of random variable?

$$
r=f(x, y, z, \ldots)
$$

The variance of $r$ is estimated using a variance propagation formula that is based on a Taylor series expansion of the function $f(x, y, z, \ldots)$.

$$
\begin{aligned}
\sigma_{\mathrm{r}}^{2}= & \left(\frac{\partial \mathrm{f}}{\partial \mathrm{x}}\right)^{2} \sigma_{\mathrm{x}}^{2}+\left(\frac{\partial \mathrm{f}}{\partial \mathrm{y}}\right)^{2} \sigma_{\mathrm{y}}^{2}+\left(\frac{\partial \mathrm{f}}{\partial \mathrm{z}}\right)^{2} \sigma_{\mathrm{z}}^{2}+\ldots+ \\
& 2\left(\frac{\partial \mathrm{f}}{\partial \mathrm{x}}\right)\left(\frac{\partial \mathrm{f}}{\partial \mathrm{y}}\right) \operatorname{Cov}(\mathrm{x}, \mathrm{y})+2\left(\frac{\partial \mathrm{f}}{\partial \mathrm{x}}\right)\left(\frac{\partial \mathrm{f}}{\partial \mathrm{z}}\right) \operatorname{Cov}(\mathrm{x}, \mathrm{z})+\ldots
\end{aligned}
$$

It should be noted that not all of the variables $x, y, z$, etc. will be correlated. 


\section{A More General Case}

The $\frac{\partial f}{\partial x}$ represents the partial of $f$ with respect to $x$ and $\sigma_{\mathrm{x}}^{2}$ represents the variance of $x$ and so on.

If the variables $x, y, z, \ldots$ are uncorrelated, then the covariance terms are zero and the variance of $r$ becomes:

$$
\sigma_{\mathrm{r}}^{2}=\left(\frac{\partial \mathrm{f}}{\partial \mathrm{x}}\right)^{2} \sigma_{\mathrm{x}}^{2}+\left(\frac{\partial \mathrm{f}}{\partial \mathrm{y}}\right)^{2} \sigma_{\mathrm{y}}^{2}+\left(\frac{\partial \mathrm{f}}{\partial \mathrm{z}}\right)^{2} \sigma_{\mathrm{z}}^{2}+\ldots+
$$




\section{Product of Two Measurements}

Consider a Pu gram amount determination that is calculated as follows:

$$
\begin{aligned}
G & =V \times C, \text { where } \\
G & =\text { Pu gram amount } \\
V & =\text { Measured tank volume } \\
C & =\text { Measured concentration from a sample } \\
\sigma_{V} & =\text { Random uncertainty for volume } \\
\sigma_{C} & =\text { Random uncertainty for concentration }
\end{aligned}
$$

Assume additive models for both $\mathrm{V}$ and $\mathrm{C}$ and that $\mathrm{V}$ and $\mathrm{C}$ are not correlated.

What is the variance of $G$ or $V(G)$ ? 


\section{Product of Two Measurements}

The partial derivatives are as follows:

$$
\begin{aligned}
\sigma_{\mathrm{G}}^{2} & =\left(\frac{\partial \mathrm{G}}{\partial \mathrm{V}}\right)^{2} \sigma_{\mathrm{V}}^{2}+\left(\frac{\partial \mathrm{G}}{\partial \mathrm{C}}\right)^{2} \sigma_{\mathrm{C}}^{2} \\
& =\mathrm{C}^{2} \sigma_{\mathrm{V}}^{2}+\mathrm{V}^{2} \sigma_{\mathrm{C}}^{2}
\end{aligned}
$$

If $\sigma_{\mathrm{V}}$ and $\sigma_{\mathrm{C}}$ are relative uncertainties, then $\mathrm{V}(\mathrm{G})$ is as follows:

$$
\begin{aligned}
\sigma_{G}^{2} & =C^{2} V^{2} \sigma_{V}^{2}+V^{2} C^{2} \sigma_{C}^{2} \\
& =(V C)^{2} \sigma_{V}^{2}+(V C)^{2} \sigma_{C}^{2} \\
& =G^{2} \sigma_{V}^{2}+G^{2} \sigma_{C}^{2}
\end{aligned}
$$

In practice, the terms $\sigma_{V}^{2}$ and $\sigma_{C}^{2}$ would be estimated by $s_{V}^{2}$ and $s_{C}^{2}$. 


\section{Sums of Products}

Consider a Pu gram amount determination that is calculated as follows:

$$
\begin{aligned}
G & =V_{1} C_{1}+V_{2} C_{2}, \text { where } \\
G & =\text { Pu gram amount } \\
V_{i} & =\text { Measured volume for tank } k_{i} \\
C_{i} & =\text { Measured concentration for tank } k_{i} \\
\sigma_{V_{i}} & =\text { Volume random uncertainty for tank } k_{i} \\
\sigma_{\beta_{C}} & =\text { Concentration systematic uncertainty } \\
\sigma_{C} & =\text { Concentration random uncertainty }
\end{aligned}
$$

Assume $\sigma_{V_{i}}, \sigma_{\beta_{c}}$ and $\sigma_{C}$ are relative uncertainties.

What is $V(G)$ ? 


\section{Sums of Products}

Since the uncertainties are relative, the $V(G)$ will be relative to the $G_{1}$ and $G_{2}$ gram amounts. The $V(G)$ calculation is as follows:

$$
\begin{aligned}
\sigma_{G}^{2}= & G_{1}^{2} \sigma_{V_{1}}^{2}+G_{1}^{2} \sigma_{\beta_{c}}^{2}+G_{1}^{2} \sigma_{C}^{2}+G_{2}^{2} \sigma_{V_{2}}^{2}+G_{2}^{2} \sigma_{\beta_{c}}^{2}+G_{2}^{2} \sigma_{C}^{2}+ \\
& 2 G_{1} G_{2} \sigma_{\beta_{c}}^{2} \\
= & G_{1}^{2} \sigma_{V_{1}}^{2}+G_{2}^{2} \sigma_{V_{2}}^{2}+\left(G_{1}^{2}+G_{2}^{2}\right) \sigma_{C}^{2}+ \\
& \left(G_{1}^{2}+G_{2}^{2}\right) \sigma_{\beta_{c}}^{2}+2 G_{1} G_{2} \sigma_{\beta_{c}}^{2} \\
= & G_{1}^{2} \sigma_{V_{1}}^{2}+G_{2}^{2} \sigma_{V_{2}}^{2}+\left(G_{1}^{2}+G_{2}^{2}\right) \sigma_{C}^{2}+\left(G_{1}+G_{2}\right)^{2} \sigma_{\beta_{c}}^{2}
\end{aligned}
$$

Again, the terms $\sigma_{V_{1}}^{2}, \sigma_{V_{2}}^{2}, \sigma_{C}^{2}$ and $\sigma_{\beta_{c}}^{2}$ would be estimated by $s_{V_{1}}^{2}, s_{V_{2}}^{2}, s_{C}^{2}$ and $s_{\beta_{c}}^{2}$. 


\section{Exercise \# 1}

Background Information:

After Pu oxide has been calcined, the material is loaded to a mixer/blending device where the material is blended for a set amount of time. After blending, four random samples are taken and sent to the laboratory for analysis. The final Pu weight \% value is based on the average of the four analyses. A single container is loaded with $500 \mathrm{~g}$ of $\mathrm{PuO}_{2}$ from the blender. A blender batch holds enough material to load five containers. The amount of $\mathrm{Pu}$ in each container is determined by weight and the Pu weight $\%$ measurement from the laboratory. The readability of the scale is $0.1 \mathrm{~g}$.

Over a particular inventory period, five blender batches of material are loaded to containers. 


\section{Exercise \# 1}

The total inventory of $\mathrm{Pu}$ from the five blender batches is computed as follows:

$$
\begin{gathered}
\mathrm{G}=\sum_{i j} \mathrm{~W}_{\mathrm{ij}} \mathrm{C}_{\mathrm{ij}}, \text { for } \mathrm{i}=1 \text { to } 5 \text { and } \mathrm{j}=1 \text { to } 5 \text {, where, } \\
\mathrm{G}=\text { Total } \text { Pu grams } \\
\mathrm{W}_{\mathrm{ij}}=\mathrm{PuO}_{2} \text { weight for the } \mathrm{i}^{\text {th }} \text { batch } \& \mathrm{j}^{\text {th }} \text { container } \\
\mathrm{C}_{\mathrm{ij}}=\mathrm{Pu} \text { weight } \% \text { for the } \mathrm{i}^{\text {th }} \text { batch } \& \mathrm{j}^{\text {th }} \text { container } \\
\sigma_{W}=\mathrm{PuO}_{2} \text { weight random uncertainty }=0.02 \% \\
\quad\left(\sigma_{W} \text { is derived as } 100 \times(0.1 \mathrm{~g} / 500 \mathrm{~g})=0.02 \%\right) \\
\sigma_{C}=\text { Pu weight percent random uncertainty }=0.25 \%
\end{gathered}
$$

For each container, assume the $W_{i j}$ are equal to $500 \mathrm{~g}$ and the $C_{i j}$ are equal to $85 \%$ or 0.85 . 


\section{Exercise \# 1}

$$
\mathrm{G}=\sum_{i j} \mathrm{~W}_{\mathrm{ij}} \mathrm{C}_{\mathrm{ij}} \text {, for } \mathrm{i}=1 \text { to } 5 \text { and } \mathrm{j}=1 \text { to } 5
$$

Develop an Excel spreadsheet to answer the following:

- What is G?

- What is the random variance portion of $\mathrm{V}(\mathrm{G})$ ?

- Is there a systematic variance?

- If so, what is the systematic variance portion of $V(G)$ ?

- What is $V(G)$ ?

- What is the total uncertainty? 


\section{Exercise \#2}

Background Information:

Same situation as Exercise \#1, except that now one sample is taken from each container and sent to the laboratory for analysis. A single analysis is conducted and the resulting weight percent measurement is used to determine the Pu inventory for that container.

For each container, assume the $\mathrm{W}_{\mathrm{ij}}$ are equal to $500 \mathrm{~g}$ and the $C_{i j}$ are equal to $85 \%$ or 0.85 . Also, the scale and weight percent random uncertainties are the same as Exercise \#1. 


\section{Exercise \#2}

$$
\mathrm{G}=\sum_{i j} \mathrm{~W}_{\mathrm{ij}} \mathrm{C}_{\mathrm{ij}} \text {, for } \mathrm{i}=1 \text { to } 5 \text { and } \mathrm{j}=1 \text { to } 5
$$

Develop an Excel spreadsheet to answer the following:

- What is G?

- What is the random variance portion of $\mathrm{V}(\mathrm{G})$ ?

- Is there a systematic variance?

- If so, what is the systematic variance portion of $V(G)$ ?

- What is $V(G)$ ?

- What is the total uncertainty? 


\section{Summary}

- Discussed the basic concepts of variance propagation

- Reviewed the propagation of random variances

- Reviewed the propagation of systematic variances

- Used Excel for solving variance propagation problems 


\begin{tabular}{|l|r|}
\hline \multicolumn{2}{|c|}{ Variance Propagation Module } \\
\hline \multicolumn{2}{|c|}{ Exercise \#2 - One Sample from each Container } \\
\hline Scale Uncertainty & 0.0002 \\
\hline Weight \% Uncertainty & 0.0025 \\
\hline & 5 \\
\hline Number of batches & 5 \\
\hline Number of containers & 500 \\
\hline Pu Oxide in one container & 0.85 \\
\hline Weight \% for each container & 1 \\
\hline Samples analyzed & 425 \\
\hline Pu in one container & 10,625 \\
\hline & 0.00 \\
\hline Total Pu & 0.00 \\
\hline Systematic Variance & 28.40 \\
\hline Systematic Uncertainty & 5.33 \\
\hline Random Variance & \\
\hline Random Uncertainty & 28.40 \\
\hline & 5.33 \\
\hline Total Variance & \\
\hline Total Uncertainty & reight and weight \% applied to each container \\
\hline
\end{tabular}




\begin{tabular}{|l|r|}
\hline \multicolumn{2}{|c|}{ Variance Propagation Module } \\
\hline Exercise \#1 - Average of 4 Samples from each Blender \\
Batch \\
\hline \multicolumn{2}{|c|}{ ' } \\
\hline Scale Uncertainty & 0.0002 \\
\hline Weight \% Uncertainty & 0.0025 \\
\hline Number of batches & 5 \\
\hline Number of containers & 500 \\
\hline Pu Oxide in one container & 0.85 \\
\hline Weight \% for each container & 4 \\
\hline Samples analyzed & 425 \\
\hline Pu in one container & \\
\hline & \\
\hline Total Pu & \\
\hline Systematic Variance & \\
\hline Systematic Uncertainty & \\
\hline Random Variance & \\
\hline Random Uncertainty & \\
\hline & \\
\hline Total Variance & \\
\hline Total Uncertainty & \\
\hline & \\
\hline
\end{tabular}




\section{Exercise \\ Variance Propagation}

\section{Session Objectives:}

After the session the participants will be able to do the following:

1. Understand the basic concepts of variance propagation

2. Understand the propagation of random variances

3. Understand the propagation of systematic variances

4. Be able to use Excel to solve variance propagation problems

\section{Estimated Time:}

There are two exercises in this module. The completion times are as follows:

1. Exercise \#1 - 30 minutes

2. Exercise \#2 - 15 minutes

A total of 45 minutes will be required to complete the exercises.

\section{Materials Needed:}

1. One computer with Microsoft Excel for each group of four or five students

2. This problem can be solved with pencil and paper or with Excel

3. Students need to have a good working knowledge of Microsoft Excel

4. Spreadsheet support person

\section{Instructions:}

1. Students should work in groups of four or five

2. The exercise information is presented in the Student Copy Excel spreadsheet for this module

3. Students will use Excel to perform the needed calculations

4. All results will be discussed 


\section{Exercise \#1}

Background Information:

After Pu oxide has been calcined, the material is loaded into a mixer/blending device where the material is blended for a set amount of time. After blending, four random samples are taken and sent to the laboratory for analysis. The final Pu weight \% value is based on the average of the four analyses. Four containers are loaded with 500g of Pu from the blender. The amount of Pu in each container is determined by weight and the Pu weight \% measurement from the laboratory. The readability of the scale is $0.1 \mathrm{~g}$.

The total inventory of $\mathrm{Pu}$ is computed as follows:

$$
\begin{aligned}
& \mathrm{G}=\sum_{i j} \mathrm{WijC}_{\mathrm{ij}} \text {, for } \mathrm{i}=1 \text { to } 5 \text { and } \mathrm{j}=1 \text { to } 5 \text {, where, } \\
& \mathrm{G}=\text { Total Pu grams } \\
& \mathrm{W}_{\mathrm{ij}}=\mathrm{PuO}_{2} \text { weight for the } \mathrm{i}^{\text {th }} \text { batch and } \mathrm{j}^{\text {th }} \text { container } \\
& \mathrm{C}_{\mathrm{ij}}=\mathrm{Pu} \text { weight } \% \text { for the } \mathrm{i}^{\text {th }} \text { batch and } \mathrm{j}^{\text {th }} \text { container } \\
& \sigma_{W}=\mathrm{PuO}_{2} \text { weight random uncertainty }=0.02 \% \\
& \quad\left(\sigma_{W} \text { is derived as } 100 \times(0.1 \mathrm{~g} / 500 \mathrm{~g})=0.02 \%\right) \\
& \sigma_{C}=\mathrm{Pu} \text { weight percent random uncertainty }=0.25 \%
\end{aligned}
$$

For each container, assume the $\mathrm{W}_{\mathrm{ij}}$ are equal to $500 \mathrm{~g}$ and the $\mathrm{C}_{\mathrm{ij}}$ are equal to $85 \%$ or 0.85 .

Use Excel and the information provided to answer the following questions:

1. What is $G$ ?

2. What is the random variance portion of $\mathrm{V}(\mathrm{G})$ ?

3. Is there a systematic variance?

- If so, what is the systematic variance portion of $V(G)$ ?

4. What is $V(G)$ ?

5. Total uncertainty is

\section{Exercise \#2}

Background Information:

Everything is the same as for Exercise \#1. The only difference is that no samples are taken from the blender. One sample is taken from each container and sent to the laboratory for analysis. A single weight percent measurement along with a weight measurement is used to determine the Pu inventory for a particular container.

Use Excel and the information provided to answer the following questions:

1. What is $G$ ?

2. What is the random variance portion of $V(G)$ ?

3. Is there a systematic variance?

- If so, what is the systematic variance portion of $V(G)$ ?

4. What is $\mathrm{V}(\mathrm{G})$ ?

5. What is the total uncertainty? 


\section{Exercise \\ Variance Propagation}

\section{Session Objectives:}

After the session the participants will be able to do the following:

1. Understand the basic concepts of variance propagation

2. Understand the propagation of random variances

3. Understand the propagation of systematic variances

4. Be able to use Excel to solve variance propagation problems

\section{Estimated Time:}

There are two exercises in this module. The completion times are as follows:

1. Exercise \#1 - 30 minutes

2. Exercise \#2 - 15 minutes

A total of 45 minutes will be required to complete the exercises.

\section{Materials Needed:}

1. One computer with Microsoft Excel for each group of four or five students

2. This problem can be solved with pencil and paper or with Excel

3. Students need to have a good working knowledge of Microsoft Excel

4. Spreadsheet support person

\section{Instructions:}

1. Students should work in groups of four or five

2. The exercise information is presented in the Student Copy Excel spreadsheet for this module

3. Students will use Excel to perform the needed calculations

4. All results will be discussed 


\section{Exercise \#1}

Background Information:

After Pu oxide has been calcined, the material is loaded into a mixer/blending device where the material is blended for a set amount of time. After blending, four random samples are taken and sent to the laboratory for analysis. The final Pu weight \% value is based on the average of the four analyses. Four containers are loaded with 500g of Pu from the blender. The amount of Pu in each container is determined by weight and the Pu weight \% measurement from the laboratory. The readability of the scale is $0.1 \mathrm{~g}$.

The total inventory of $\mathrm{Pu}$ is computed as follows:

$$
\begin{aligned}
& \mathrm{G}=\sum_{i j} \mathrm{WijC}_{\mathrm{ij}} \text {, for } \mathrm{i}=1 \text { to } 5 \text { and } \mathrm{j}=1 \text { to } 5 \text {, where, } \\
& \mathrm{G}=\text { Total Pu grams } \\
& \mathrm{W}_{\mathrm{ij}}=\mathrm{PuO}_{2} \text { weight for the } \mathrm{i}^{\text {th }} \text { batch and } \mathrm{j}^{\text {th }} \text { container } \\
& \mathrm{C}_{\mathrm{ij}}=\mathrm{Pu} \text { weight } \% \text { for the } \mathrm{i}^{\text {th }} \text { batch and } \mathrm{j}^{\text {th }} \text { container } \\
& \sigma_{W}=\mathrm{PuO}_{2} \text { weight random uncertainty }=0.02 \% \\
& \quad\left(\sigma_{W} \text { is derived as } 100 \times(0.1 \mathrm{~g} / 500 \mathrm{~g})=0.02 \%\right) \\
& \sigma_{C}=\mathrm{Pu} \text { weight percent random uncertainty }=0.25 \%
\end{aligned}
$$

For each container, assume the $\mathrm{W}_{\mathrm{ij}}$ are equal to $500 \mathrm{~g}$ and the $\mathrm{C}_{\mathrm{ij}}$ are equal to $85 \%$ or 0.85 .

Use Excel and the information provided to answer the following questions:

\section{What is $G$ ? 10,625 grams}

2. What is the random variance portion of $V(G)$ ? $\mathbf{0 . 1 8} \mathbf{g}^{2}$

3. Is there a systematic variance? Yes

- If so, what is the systematic variance portion of $V(G) ? 35.28 \mathbf{g}^{2}$

4. What is $V(G)$ ? $35.46 \mathbf{g}^{2}$

5. Total uncertainty is $\mathbf{5 . 9 5 g}$

\section{Exercise \#2}

Background Information:

Everything is the same as for Exercise \#1. The only difference is that no samples are taken from the blender. One sample is taken from each container and sent to the laboratory for analysis. A single weight percent measurement along with a weight measurement is used to determine the Pu inventory for a particular container.

Use Excel and the information provided to answer the following questions:

1. What is $G$ ? 10,625 grams

2. What is the random variance portion of $V(G)$ ? $\mathbf{2 8 . 4 0 \mathbf { g } ^ { 2 }}$

3. Is there a systematic variance? No

- If so, what is the systematic variance portion of $V(G)$ ? NA

4. What is $V(G)$ ? $\mathbf{2 8 . 4 0}^{2}$

5. What is the total uncertainty? 5.33g 


\section{Exercise \\ Measurement Method Qualification}

\section{Session Objectives:}

After the session the participants will be able to do the following:

1. Plan the method qualification process

2. Produce a qualification planning document

3. Collect the measurement data

4. Analyze the data (estimate uncertainties)

5. Produce a qualification document

\section{Estimated Time:}

This module contains five (5) exercises. The exercise completion times are as follows:

1. Exercise \#1 - 20 minutes

2. Exercise \#2 - 15 minutes

3. Exercise \#3 - 45 minutes

4. Exercise \#4 - 15 minutes

5. Exercise $\# 5-45$ minutes

These exercises will require 2 hours and 20 minutes to complete.

\section{Materials Needed:}

1. One computer with Microsoft Excel for each group of 5 students

2. Excel needs to have the Stat Package add-in loaded

3. The students must be familiar with Excel

4. Pencil and paper

5. Spreadsheet support person

\section{Instructions:}

1. There are five (5) exercises in this module

2. Students should work in groups of four or five

3. The exercises are included in the presentation

4. The students will be requested to complete a particular exercise

5. Excel spreadsheets are provided for Exercises \#3, \#4 and \#5

6. Exercises will be instructor led and discussed 


\section{Exercise \#1}

Method Qualification Process

1. Where should the qualification plan originate?

- Typically, the facility that will be using the instrument for accountability measurements will originate the document. The facility may have their own MC\&A personnel, who will work with site MC\&A to qualify the instrument.

2. What personnel should be involved with designing the data collection process?

- The facility measurement personnel will work with site MC\&A and the MC\&A statistician to design a measurement plan.

3. What protocol should be followed when collecting the measurements for the qualification process?

- Measurement procedures should be the same for qualification and process measurements

4. What should be documented in the qualification report?

- At a minimum, the estimated uncertainty components and the applicable control limits

5. What personnel should receive the qualification report?

- Facility and site MC\&A personnel and the appropriate MC\&A statistician

\section{Exercise \#2}

HBL Scale Qualification Process

1. Can this scale be used for weight measurements?

- This scale can be used by making bias correction adjustments using the estimated calibration equation

2. If so, how would the weight measurements be adjusted?

- Invert the calibration equation. This will adjust the measured value to the reference value.

3. Discuss methods or actions for improving the measurements for this scale.

\section{Exercise \#3}

AWCC Qualification Process

1. Outline the steps for completing a method qualification:

- Selection process (target accuracy and precision values)

- Develop a qualification plan 
- Ensure proper training and qualifications of measurement personnel

- Collect the measurement data

- Conduct the data analysis

- Produce final qualification report

- If applicable, submit final report for approvals

2. Use the Excel spreadsheet for the AWCC one-way ANOVA

\section{Exercise \#4}

AWCC Qualification Process

Use the AWCC model assumptions and uncertainty estimates to calculate the bias uncertainty. The key is using the calibration uncertainty as a short-term systematic effect.

$\mathrm{V}($ Bias $)=75^{*} 0.13^{2}+15^{*} 0.08^{2}$ and the Bias Uncertainty $= \pm 7.69 \%$.

\section{Listing of HBL AWCC Pu Qualification Data}

\begin{tabular}{|c|c|c|c|c|}
\hline File \# & $\begin{array}{l}\text { Standard } \\
\text { Id }\end{array}$ & $\begin{array}{c}\text { True } \\
\text { Pu mass } \\
\text { (g) }\end{array}$ & $\begin{array}{l}\text { Measured } \\
\text { Pu mass } \\
\text { (g) }\end{array}$ & $\begin{array}{l}\text { Relative } \\
\text { Difference }\end{array}$ \\
\hline $162 \mathrm{~K} 0410$ & SGB-30 & 29.989 & 36.2 & 0.207 \\
\hline $162 \mid 1040$ & SGB-30 & 29.989 & 33.8 & 0.127 \\
\hline 162M1846 & SGB-30 & 29.989 & 35.5 & 0.184 \\
\hline 162N2438 & SGB-30 & 29.989 & 35.3 & 0.177 \\
\hline 16203955 & SGB-30 & 29.989 & 34.8 & 0.160 \\
\hline 15UI3558 & SGB-100 & 99.964 & 99.7 & -0.003 \\
\hline 15 UJ4120 & SGB-100 & 99.964 & 89.6 & -0.104 \\
\hline 15UM0935 & SGB-100 & 99.964 & 81.7 & -0.183 \\
\hline $15 \mathrm{UO} 2454$ & SGB-100 & 99.964 & 88.2 & -0.118 \\
\hline $15 \cup 2943$ & SGB-100 & 99.964 & 93.7 & -0.063 \\
\hline $15 \mathrm{VO5519}$ & SGB-200 & 199.927 & 232.7 & 0.164 \\
\hline 15VQ0230 & SGB-200 & 199.927 & 176.1 & -0.119 \\
\hline 161J0906 & SGB-200 & 199.927 & 184.5 & -0.077 \\
\hline $161 \mathrm{~K} 3809$ & SGB-200 & 199.927 & 207.1 & 0.036 \\
\hline 16100600 & SGB-200 & 199.927 & 213 & 0.065 \\
\hline
\end{tabular}




\section{Plot of Relative Differences from HBL AWCC Pu Qualification Data}

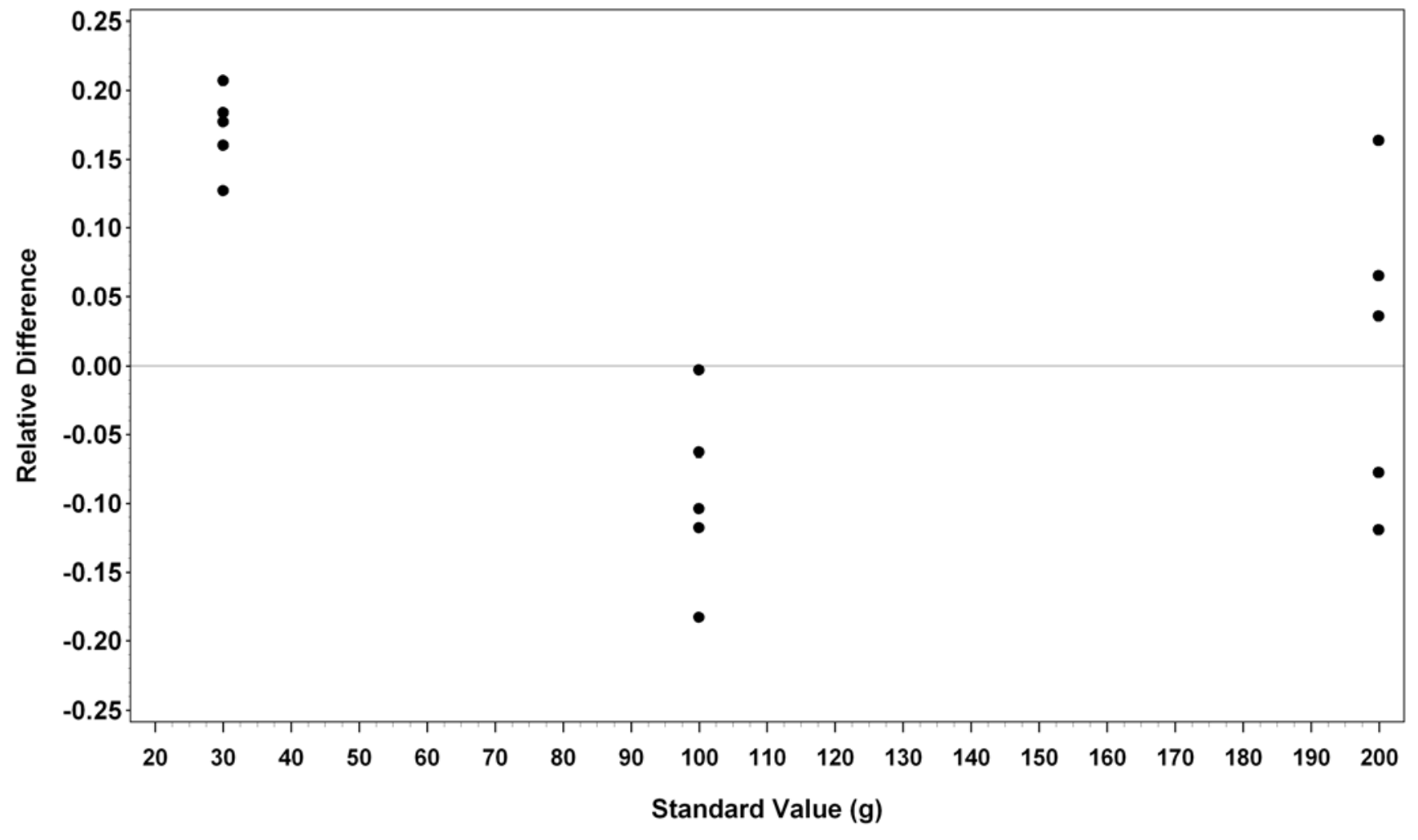




\title{
ANOVA Results for HBL AWCC Calibration and Random Uncertainty Analysis
}

\author{
The GLM Procedure \\ Class Level Information \\ Class Levels Values \\ std_id 3 SGB-100 SGB-200 SGB-30 \\ Number of observations 15 \\ The GLM Procedure
}

Dependent Variable: reldiff

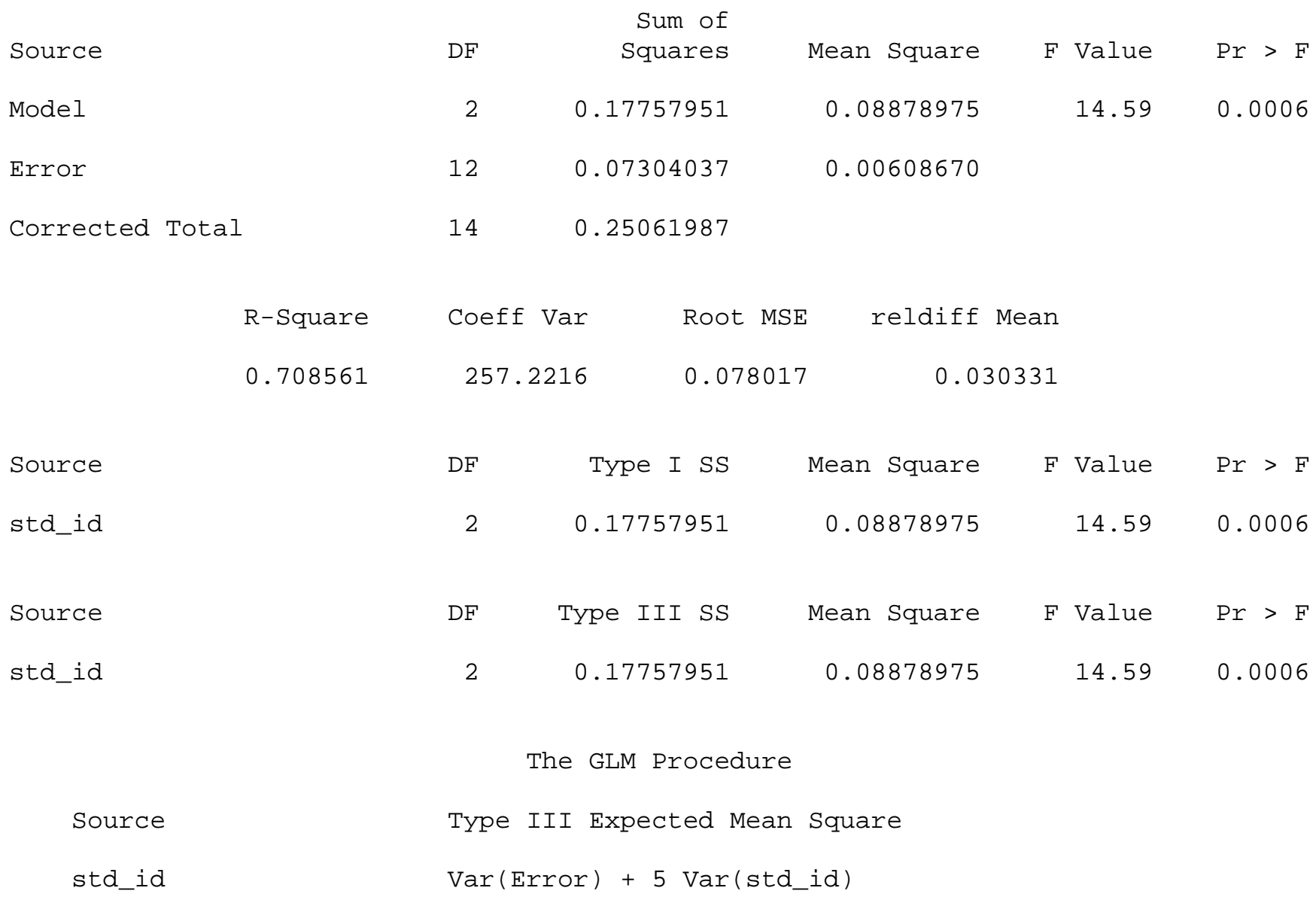




\section{HBL AWCC Pu Uncertainty Components}

\begin{tabular}{|c|c|c|c|}
\hline Bias & $\begin{array}{c}\text { Bias } \\
\text { Uncertainty }\end{array}$ & $\begin{array}{c}\text { Calibration } \\
\text { Uncertainty }\end{array}$ & $\begin{array}{c}\text { Random } \\
\text { Uncertainty }\end{array}$ \\
\hline $3.03 \%$ & $\pm 7.69 \%$ & $\pm 12.86 \%$ & $\pm 7.80 \%$ \\
\hline
\end{tabular}

\section{Exercise \#5}

Uncertainty Estimation for the Davies-Gray Concentration Method

1. A Student Worksheet has been provided for the students. There is also an Instructor Worksheet with the analysis results. The students will need to rearrange the data by measurement date in order to run the ANOVA. 


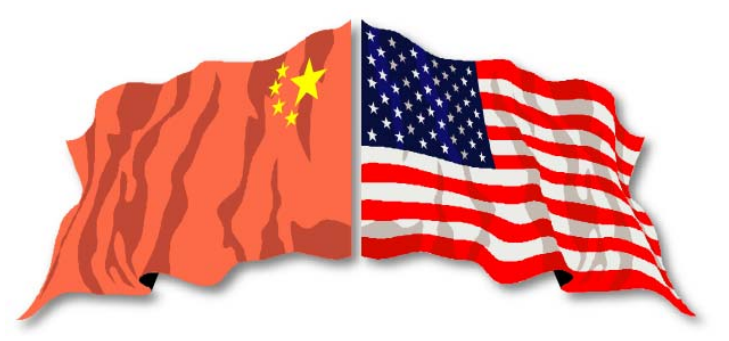

\section{Module 5}

\section{Measurement Method Qualification}




\section{Objectives}

- Understand method selection/qualification process

- Understand how to document a method qualification

- Qualification plan

- Data analysis report

- Understand the application of a basic variance propagation to a method qualification 


\section{Control of Measurement Systems}

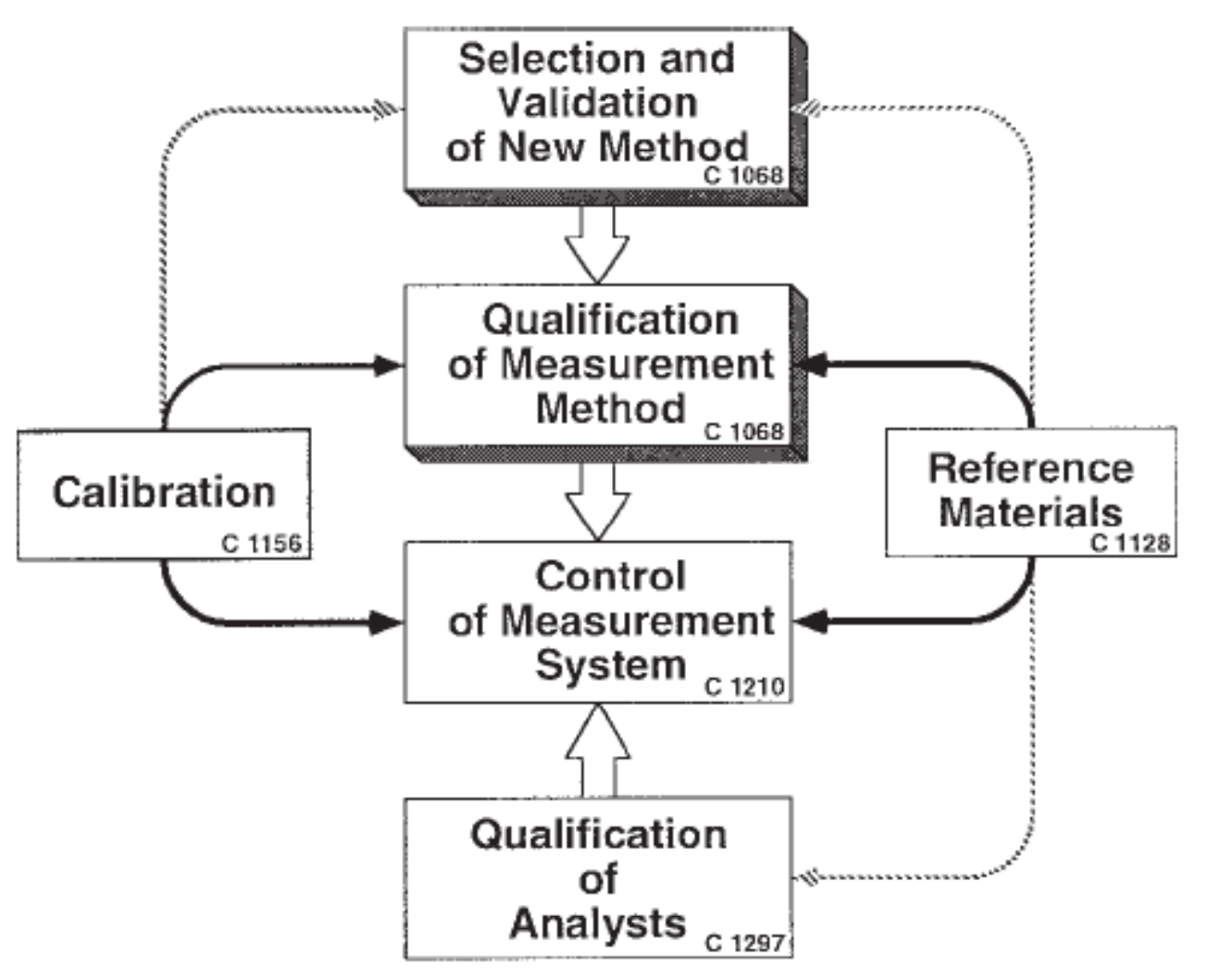

of Measurement Methods by a Laboratory within the Nuclear Industry 


\section{Method Qualification Process}

- Measurement methods are selected and qualified based on the material type and the method uncertainty.

- The qualification process characterizes or estimates the uncertainty components for a measurement method.

- The selection/qualification process proceeds as follows:

1. The appropriate method for the required measurement is selected (precision and accuracy)

2. Facility personnel produce a qualification plan for the method

3. A measurement plan for method evaluation is developed

4. Evaluation data are collected

5. Data are analyzed by MC\&A statistician

6. Statistician produces a method qualification report

7. Qualification report is submitted to DOE MC\&A for approval 


\section{Method Qualification Process cont'd}

- Laboratory QC data are typically used for method qualification

- In some cases, experimental designs that utilize laboratory QC data may be needed for uncertainty estimation

- Analysis of Variance (ANOVA) techniques are typically used to estimate method variance components

- For a multiplicative model, relative differences are analyzed

- For an additive model, absolute differences are analyzed 


\section{Measurement Method Qualification Plan}

- Procedures

- Method Performance Requirements

- Test materials

- Major Constituents

- Impurities

- Qualification Requirements
- Bias

- Precision

- Range

- Reporting Data

- Failure

- Requalification

- Documentation

- Laboratory Records control and approval 


\section{Measurement Method Selection}

Selection of measurement method is necessary to assure the method is adequate for the application. Selection Criteria:

- Technical basis

- Proven laboratory and instrumental techniques are used

- National or international standards

- Accepted for a specific application based on wide use

- Interferences

- Knowledge of limitations and composition of material

- Range of applicability

- Method adequately responds over range of concentrations

- Reliability of method

- Must meet accuracy/precision goals in expected conditions 


\section{Measurement Method Validation}

- To investigate the applicability of a method to a particular use.

- New or unique application

- Ensure qualification effort would be successful

- Usually performed under controlled conditions

- Investigate selection criteria

- Identify modifications to method that are needed 


\section{Exercise \#1}

Method Qualification Process:

1. Where should the qualification plan originate?

2. What personnel should be involved with designing the data collection process?

3. What protocol should be followed when conducting the measurements for the qualification process?

4. What should be documented in the qualification report?

5. What personnel should receive a copy of the qualification report? 


\section{Method Qualification Process}

Scale Qualification for the

Savannah River Site

HB-Line Facility 


\section{HB-Line Scale HBL-08: Qualification Plan}

10/11/04 10:52 FAX

\section{SRS}

WESTINGHOUSE SAVANNAH RIVER COMPANY
INTEROFFICE MEMORANDUM

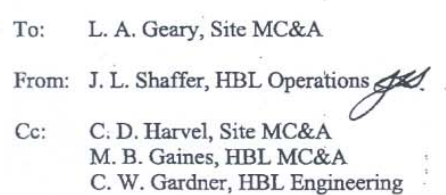

HB-Line (HBL) requests the qualification of a Mettler-Toledo balance (Model: PG8 balance to be qualified is identified as HBL-08 (Serial \#: 1125170974). This balanc MonoBloc TM weighing cell technology and has a range of $0-8100$ grams. The bala display with a readability of 0.1 grams.

2. Reason for Qualification

The balance will be used to perform weighing of Special Nuclear Material (SNM) in This SNM may be plutonim, uranium andor neptnium. The measurements involv \& Security inventory of material being processed or repackaged in HBL Phase I. If also be able to replace the HBL Phase II Mechanical Line or Operating Area 5 Balar

Additionally, this balance may be used during performance of HBL vessel calibratio of spanning solution during these calibrations. The calibration/spanning will ultimat of spanning solution during these calibrations. The calibration/spanning
vessel level instrumentation as MC\&A qualified level instrumentation.

3. Process and Product Specifications

SNM to be handled in the HBL Phase I facility will be weighed prior to processing or repackaging. The SNM to be handled is normally between 100 and 4000 grams. The balance will be set up to perform measurements in the Phase I gloveboxes and/or supporting areas.

When used for vessel calibration/spanning, the balance will be used to measure the weight of a poly bottle

Bounding environmental conditions for all these locations are 5 to 40 degrees $\mathrm{C}$ and $10 \%$ to $85 \%$ relativc humidity.
4. Composition of Materials Used for Qualification Process

NIST traceable weight standards will be used for balance calibrations and baseline measurements. The weight set designated for qualification and use of this balance is HW-986. The weight standards included in this standards. These standards are intended to bracket the expected weight of the items to be weighed by the balance, while also covering the overall range of the balance.

5. Amount and Description of Test Data to be Generated

Baseline data will be generated over various shift time periods in HBL with approved procedures that implement the random weighing plan, which will be supplied by MC\&A.

6. Qualification Acceptance Criteria

Intial calibration of the balance and weight standards has been performed by the Savannah River Standard Lab (SRL), and this calibration data will be supplied to Site MC\&A. After baseline data is collected in

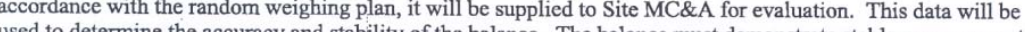
characteristics during acuracy and stability of the balance. The balance must demonstrate stable measurement arget values and control limits (waming and alam limits) by Site MC\&A. Site MC\&A will determine the Qualification Report.

7. Approval of Qualification Plan

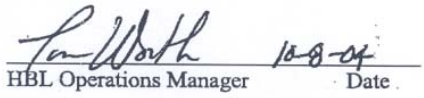

$\begin{array}{cc}\text { Caszer ex } & 10 / 864 \\ \text { HBL Engineering } & \text { Date } \\ \text { Site MC\&A } & \text { Date }\end{array}$

Site MC\&A Date 


\section{HB-Line Scale HBL-08: Qualification Data}

\begin{tabular}{|c|c|c|c|}
\hline $\begin{array}{l}\text { Result } \\
\text { in } \\
\text { grams }\end{array}$ & $\begin{array}{l}\text { Reference } \\
\text { Value } \\
\text { in } \\
\text { grams }\end{array}$ & $\begin{array}{l}\text { Absolute } \\
\text { Difference }\end{array}$ & $\begin{array}{l}\text { Relative } \\
\text { Difference }\end{array}$ \\
\hline 1000 & 1000 & 0 & 0 \\
\hline 3999.9 & 4000 & -0.1 & -0.000025 \\
\hline 5999.8 & 6000 & -0.2 & -0.000033 \\
\hline 500.1 & 500 & 0.1 & 0.0002 \\
\hline 2000 & 2000 & 0 & 0 \\
\hline 7999.7 & 8000 & -0.3 & -0.000038 \\
\hline 500 & 500 & 0 & 0 \\
\hline 2000 & 2000 & 0 & 0 \\
\hline 7999.7 & 8000 & -0.3 & -0.000038 \\
\hline 6999.7 & 4000 & 2999.7 & 0.749925 \\
\hline 1000.1 & 1000 & 0.1 & 0.0001 \\
\hline 500.1 & 500 & 0.1 & 0.0002 \\
\hline 2000 & 2000 & 0 & 0 \\
\hline 7999.8 & 8000 & -0.2 & -0.000025 \\
\hline 5999.9 & 6000 & -0.1 & -0.000017 \\
\hline 4000 & 4000 & 0 & 0 \\
\hline 3999.9 & 4000 & -0.1 & -0.000025 \\
\hline 500.1 & 500 & 0.1 & 0.0002 \\
\hline$\ldots$ & $\ldots$ & $\ldots$ & $\ldots$ \\
\hline
\end{tabular}

Module $5-12$ 


\section{HB-Line Scale HBL-08: Plot of Qualification Data}

(Value of plot symbol indicates number of dbsenations)

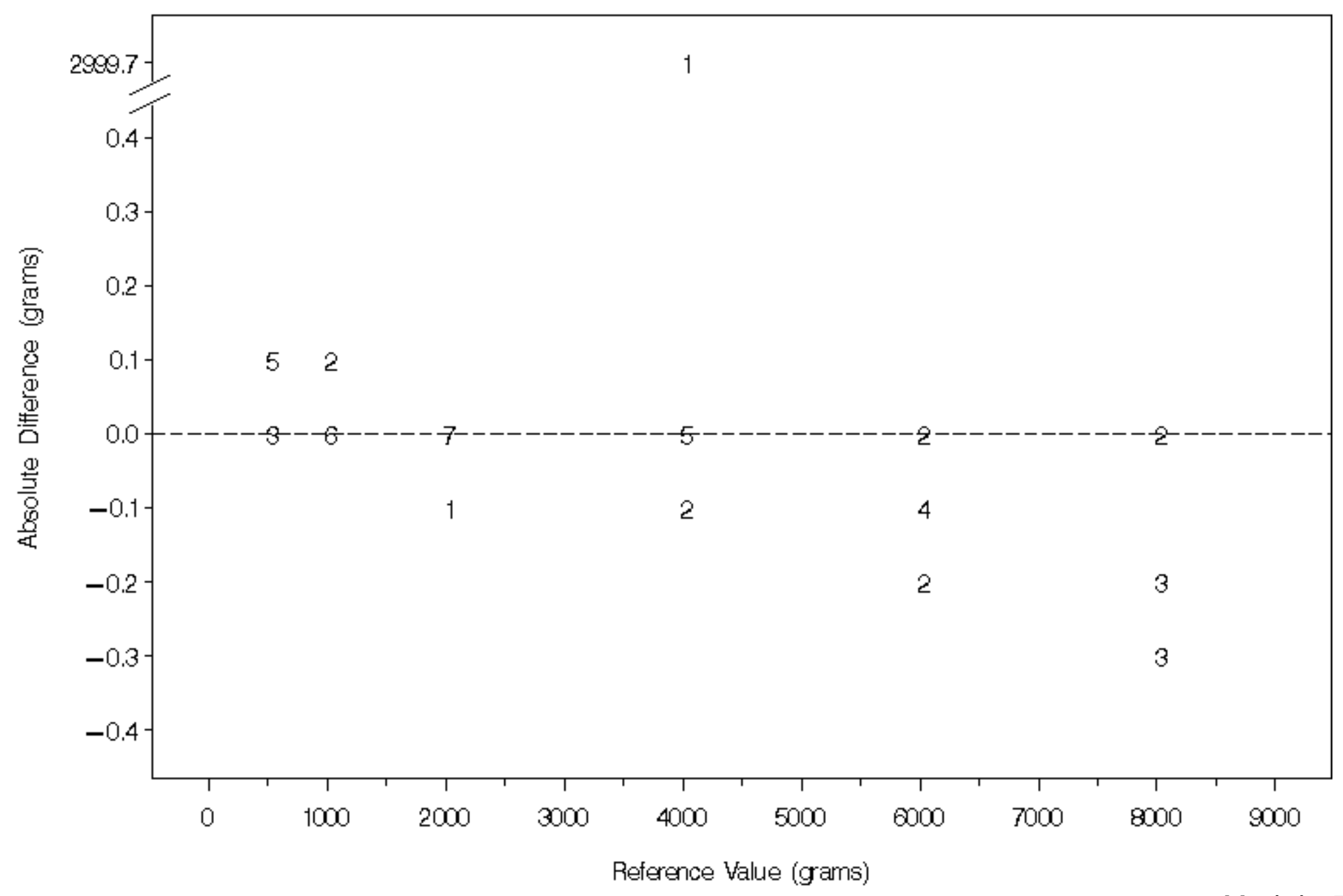

Module $5-13$ 


\section{HB-Line Scale HBL-08: Estimation of Calibration Equation}

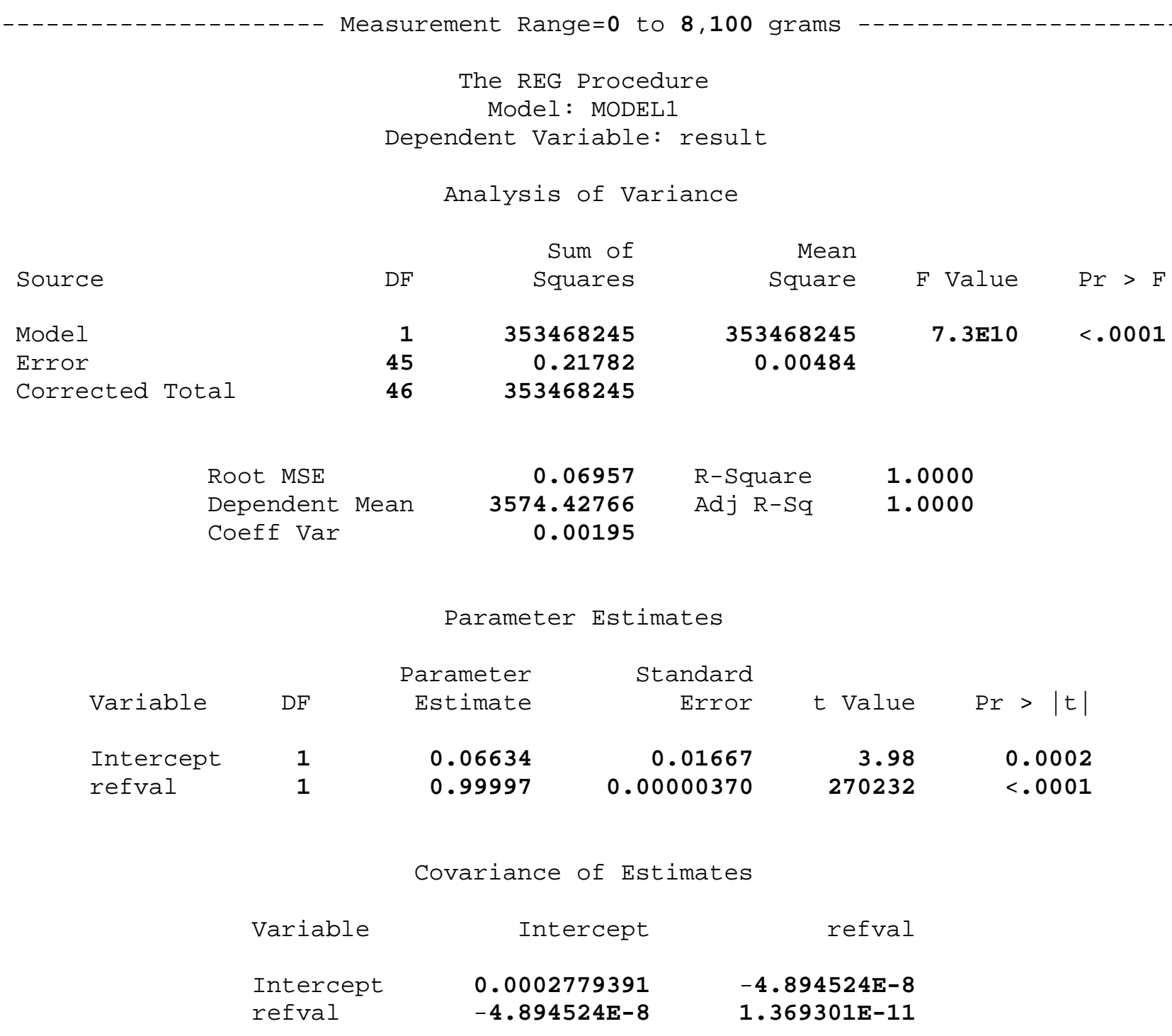

$\begin{array}{lrrrrr}\text { Variable } & \text { DF } & \begin{array}{r}\text { Parameter } \\ \text { Estimate }\end{array} & \begin{array}{r}\text { Standard } \\ \text { Error }\end{array} & \text { t value } & \operatorname{Pr}>|t| \\ \text { Intercept } & 1 & 0.06634 & 0.01667 & 3.98 & 0.0002 \\ \text { refval } & 1 & 0.99997 & 0.00000370 & 270232 & <.0001\end{array}$

Covariance of Estimates

$\begin{array}{lrr}\text { Variable } & \text { Intercept } & \text { refval } \\ & & \\ \text { Intercept } & \mathbf{0 . 0 0 0 2 7 7 9 3 9 1} & \mathbf{- 4 . 8 9 4 5 2 4 E - 8} \\ \text { refval } & -\mathbf{4 . 8 9 4 5 2 4 E - 8} & \mathbf{1 . 3 6 9 3 0 1 E - 1 1}\end{array}$




\section{Exercise \#2}

Scale Qualification Process

1. Can this scale be used for weight measurements?

2. If so, how would these measurements be adjusted?

3. Discuss methods or actions for improving the measurements for this scale 


\section{AWCC: Neutron Measurement Basics}

Most nuclear materials emit neutrons. An Active Well

Coincidence Counter (AWCC) is a non-destructive assay (NDA) device used to measure neutrons. The amount of neutrons measured can be correlated to the amount of nuclear material.

An AWCC has two modes of operation:

-Passive Mode - Plutonium

- Plutonium fissions spontaneously

-Active Mode - Uranium

- Uranium measurements are done by using an external source (active) of neutrons to excite the U235 


\section{AWCC: Measurement Process}

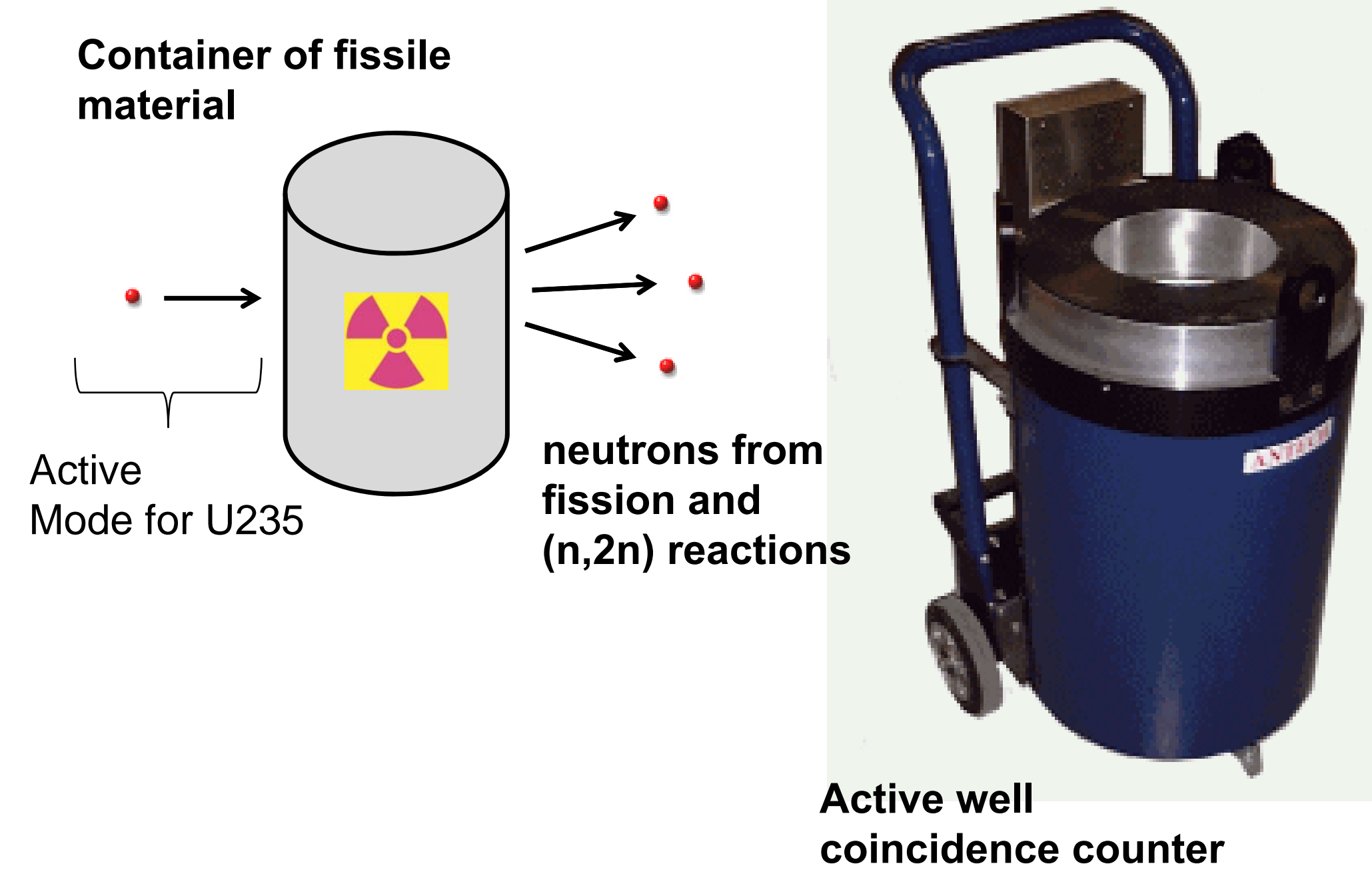

Module 5 - 17 


\section{Exercise \#3}

Active Well Coincidence Counter (AWCC)

Qualification Process

Purpose: Qualify an Active Well Coincidence Counter for Pu Accountability Measurements

- Outline the steps for conducting the AWCC method qualification

- Input the AWCC qualification data (two slides down) into an Excel spreadsheet

- Use the "Standard ID" column and the relative differences column only to expedite the analysis 


\section{Exercise \#3 (continued)}

Active Well Coincidence Counter (AWCC)

Qualification Process

- The following four slides represent the analysis conducted at the Savannah River Site

- Compare the Excel results to the SRS analysis 


\section{AWCC Qualification: SRS Analysis}

\section{Listing of HBL AWCC Pu Qualification Data}

\begin{tabular}{|c|c|c|c|c|}
\hline File \# & $\begin{array}{l}\text { Standard } \\
\text { Id }\end{array}$ & $\begin{array}{c}\text { True } \\
\text { Pu mass } \\
\text { (g) }\end{array}$ & $\begin{array}{c}\text { Measured } \\
\text { Pu mass } \\
\text { (g) }\end{array}$ & $\begin{array}{l}\text { Relative } \\
\text { Difference }\end{array}$ \\
\hline $162 \mathrm{~K} 0410$ & SGB-30 & 29.989 & 36.2 & 0.207 \\
\hline $162 \mid 1040$ & SGB-30 & 29.989 & 33.8 & 0.127 \\
\hline $162 \mathrm{M} 1846$ & SGB-30 & 29.989 & 35.5 & 0.184 \\
\hline 162 N2438 & SGB-30 & 29.989 & 35.3 & 0.177 \\
\hline 16203955 & SGB-30 & 29.989 & 34.8 & 0.160 \\
\hline 15UI3558 & SGB-100 & 99.964 & 99.7 & -0.003 \\
\hline 15 UJ4120 & SGB-100 & 99.964 & 89.6 & -0.104 \\
\hline 15UM0935 & SGB-100 & 99.964 & 81.7 & -0.183 \\
\hline $15 \cup O 2454$ & SGB-100 & 99.964 & 88.2 & -0.118 \\
\hline $15 \cup 2943$ & SGB-100 & 99.964 & 93.7 & -0.063 \\
\hline 15VO5519 & SGB-200 & 199.927 & 232.7 & 0.164 \\
\hline 15VQ0230 & SGB-200 & 199.927 & 176.1 & -0.119 \\
\hline $161 J 0906$ & SGB-200 & 199.927 & 184.5 & -0.077 \\
\hline $161 K 3809$ & SGB-200 & 199.927 & 207.1 & 0.036 \\
\hline 16100600 & SGB-200 & 199.927 & 213 & 0.065 \\
\hline
\end{tabular}




\section{AWCC Qualification: SRS Analysis}

Plot of Relative Differences from HBL AWCC Pu Qualification Data

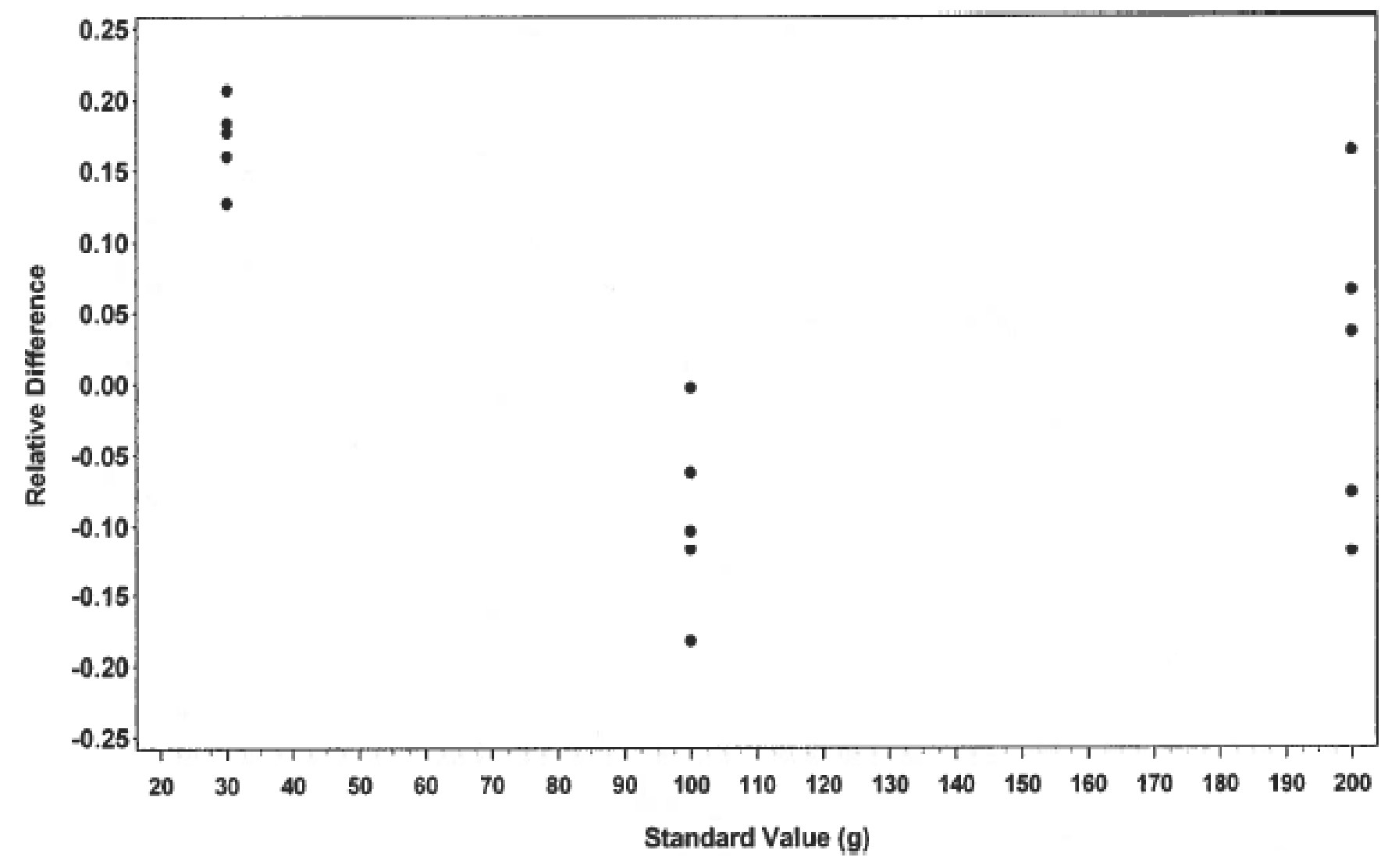

Module $5-21$ 


\title{
AWCC Qualification: SRS Analysis
}

ANOVA Results for HBL AWCC Calibration and Random Uncertainty Analysis

\author{
The GLM Procedure \\ Class Level Information \\ Class Levels Values \\ std_id 3 SGB-100 SGB-200 SGB-30
}

Number of observations 15

The GLM Procedure

Dependent Variable: reldiff

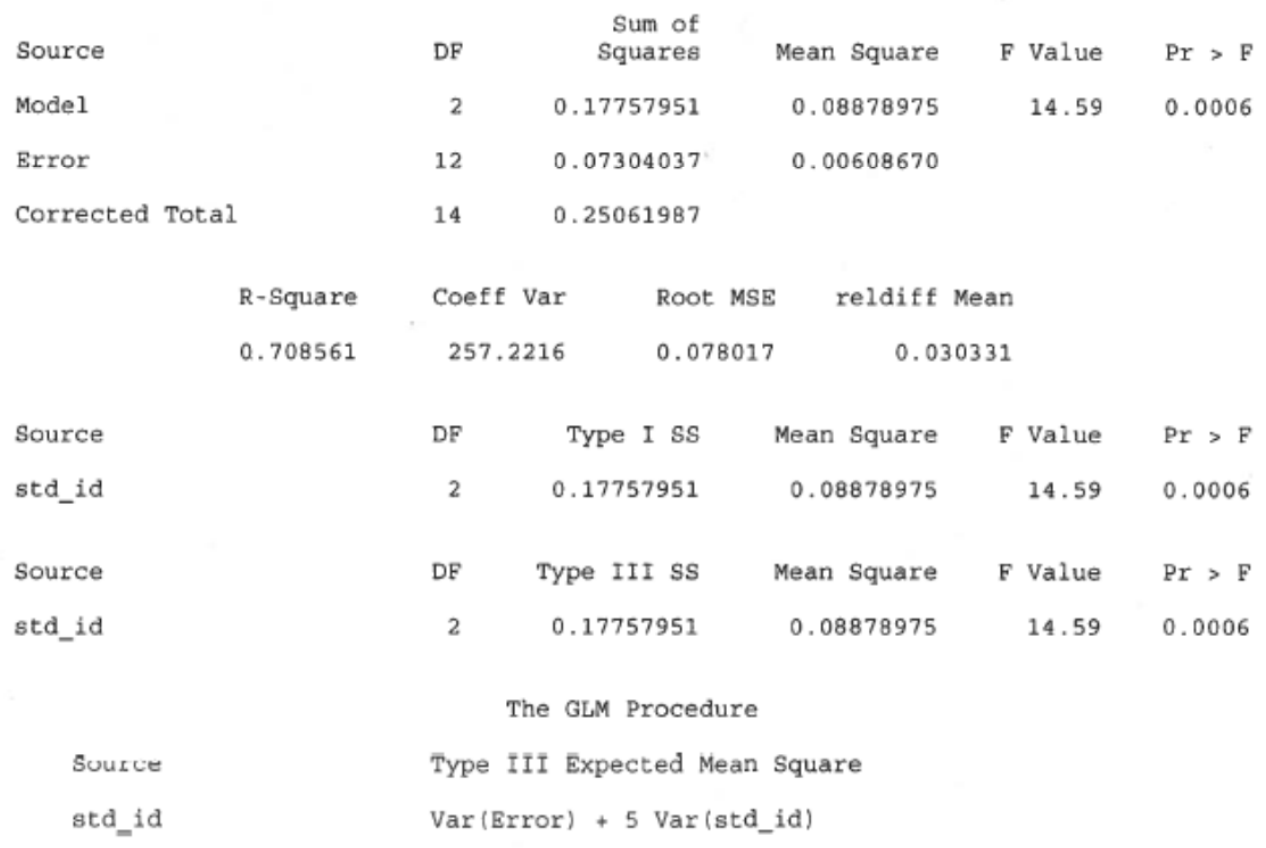




\section{AWCC Qualification: SRS Analysis}

HBL AWCC Pu Uncertainty Components

\begin{tabular}{|c|c|c|c|}
\hline Bias & $\begin{array}{c}\text { Bias } \\
\text { Uncertainty }\end{array}$ & $\begin{array}{c}\text { Calibration } \\
\text { Uncertainty }\end{array}$ & $\begin{array}{c}\text { Random } \\
\text { Uncertainty }\end{array}$ \\
\hline $3.03 \%$ & $\pm ?$ & $\pm 12.86 \%$ & $\pm 7.80 \%$ \\
\hline
\end{tabular}




\section{Exercise \#4: AWCC Qualification Process}

Assume the following relative model for the AWCC measurement method:

$y_{i j}=\mu_{i}\left(1+\eta_{i j}+\varepsilon_{i j}\right)$, where the $\eta_{i}$ are iid $N\left(0,0.13^{2}\right)$ and the $\varepsilon_{\mathrm{ij}}$ are iid $\mathrm{N}\left(0,0.08^{2}\right)$

The bias is the average of the $\left(y_{i j}-\mu_{i}\right) / \mu_{i}$ relative differences and is equal to 0.03 or $3 \%$.

- Using this information, derive V(Bias)

- Use the $\eta_{i}$ as short-term systematic effects Note: This is actually a simple variance propagation problem! 


\section{Exercise \#5: Davies-Gray Titration}

Uncertainty Estimation for the Davies-Gray Titration Method

Purpose: To review the uncertainty estimates for the Davies-Gray concentration method. This methodology is used for Uranium accountability measurements.

- An Excel spreadsheet has been provided with the measurement data for the students

- Students will need to rearrange the data in order to run the ANOVA

- Arrange the relative differences by measurement date in different columns starting in column 1 


\section{Summary}

- Discussed method selection/qualification process

- Discussed documenting a method qualification

- Qualification plan

- Data analysis report

- Applied a basic variance propagation in the qualification of 3 measurement methods (physical, DA, NDA) 


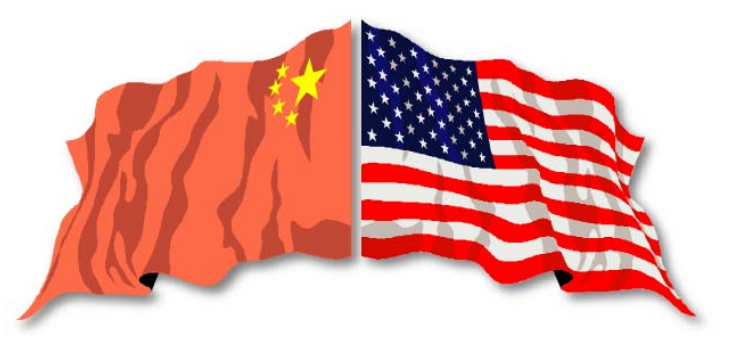

\section{Module 5}

\section{Measurement Method Qualification}




\section{Objectives}

- Understand method selection/qualification process

- Understand how to document a method qualification

- Qualification plan

- Data analysis report

- Understand the application of a basic variance propagation to a method qualification 


\section{Control of Measurement Systems}

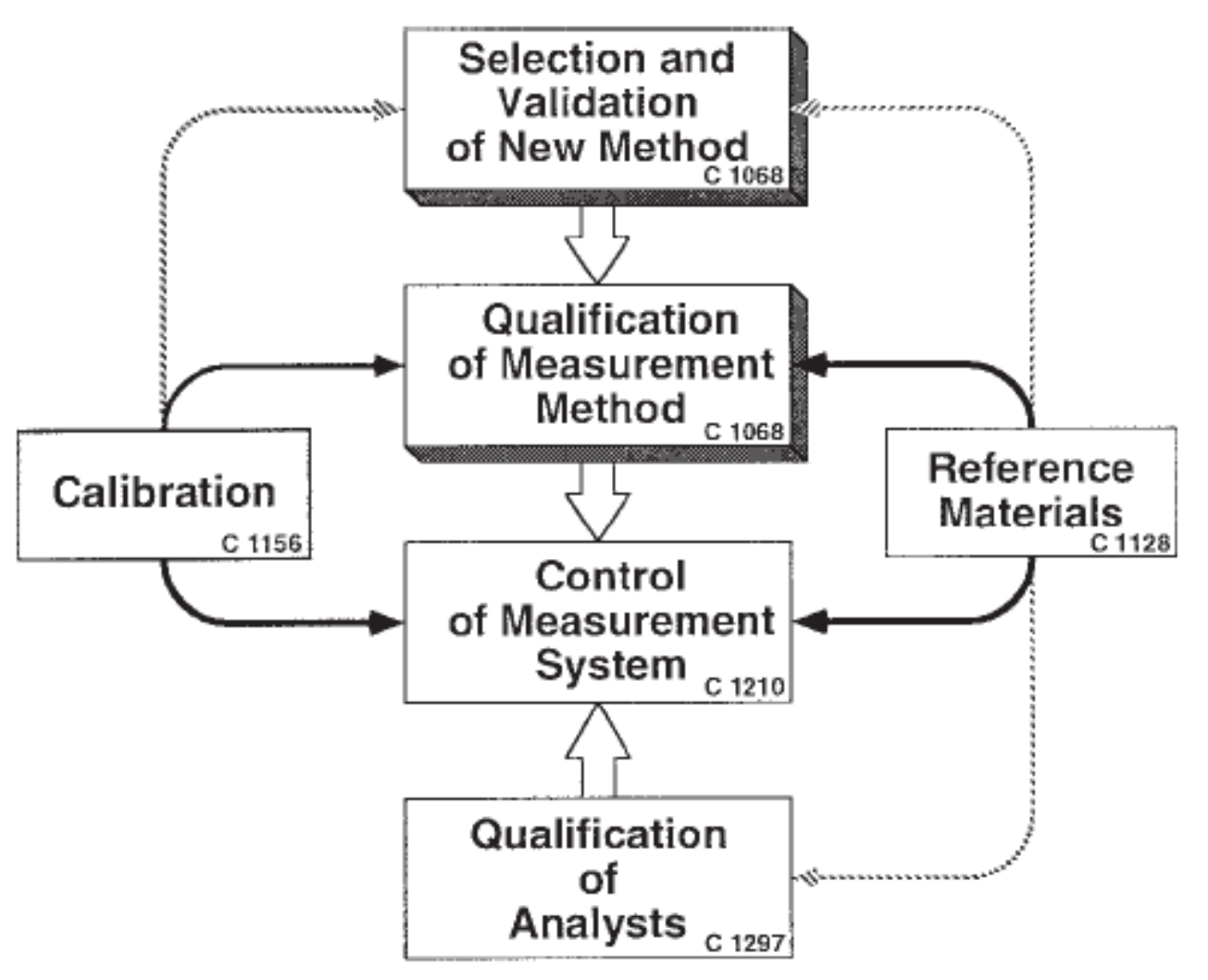

of Measurement Methods by a Laboratory within the Nuclear Industry 


\section{Method Qualification Process}

- Measurement methods are selected and qualified based on the material type and the method uncertainty.

- The qualification process characterizes or estimates the uncertainty components for a measurement method.

- The selection/qualification process proceeds as follows:

1. The appropriate method for the required measurement is selected (precision and accuracy)

2. Facility personnel produce a qualification plan for the method

3. A measurement plan for method evaluation is developed

4. Evaluation data are collected

5. Data are analyzed by MC\&A statistician

6. Statistician produces a method qualification report

7. Qualification report is submitted to DOE MC\&A for approval 


\section{Method Qualification Process cont'd}

- Laboratory QC data are typically used for method qualification

- In some cases, experimental designs that utilize laboratory QC data may be needed for uncertainty estimation

- Analysis of Variance (ANOVA) techniques are typically used to estimate method variance components

- For a multiplicative model, relative differences are analyzed

- For an additive model, absolute differences are analyzed 


\section{Measurement Method Qualification Plan}

- Procedures

- Method Performance Requirements

- Test materials

- Major Constituents

- Impurities

- Qualification Requirements
- Bias

- Precision

- Range

- Reporting Data

- Failure

- Requalification

- Documentation

- Laboratory Records control and approval 


\section{Measurement Method Selection}

Selection of measurement method is necessary to assure the method is adequate for the application. Selection Criteria:

- Technical basis

- Proven laboratory and instrumental techniques are used

- National or international standards

- Accepted for a specific application based on wide use

- Interferences

- Knowledge of limitations and composition of material

- Range of applicability

- Method adequately responds over range of concentrations

- Reliability of method

- Must meet accuracy/precision goals in expected conditions 


\section{Measurement Method Validation}

- To investigate the applicability of a method to a particular use.

- New or unique application

- Ensure qualification effort would be successful

- Usually performed under controlled conditions

- Investigate selection criteria

- Identify modifications to method that are needed 


\section{Exercise \#1}

Method Qualification Process:

1. Where should the qualification plan originate?

2. What personnel should be involved with designing the data collection process?

3. What protocol should be followed when conducting the measurements for the qualification process?

4. What should be documented in the qualification report?

5. What personnel should receive a copy of the qualification report? 


\section{Method Qualification Process}

Scale Qualification for the

Savannah River Site

HB-Line Facility 


\section{HB-Line Scale HBL-08: Qualification Plan}

10/11/04 10:52 FAX

\section{SRS}

WESTINGHOUSE SAVANNAH RIVER COMPANY
INTEROFFICE MEMORANDUM

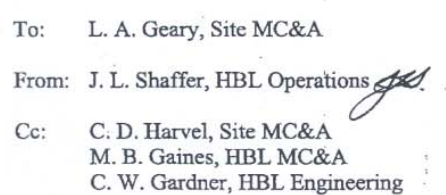

HB-Line (HBL) requests the qualification of a Mettler-Toledo balance (Model: PG8 balance to be qualified is identified as HBL-08 (Serial \#: 1125170974). This balanc MonoBloc TM weighing cell technology and has a range of $0-8100$ grams. The bala display with a readability of 0.1 grams.

2. Reason for Qualification

The balance will be used to perform weighing of Special Nuclear Material (SNM) in This SNM may be plutonim, uranium andor neptnium. The measurements involv \& Security inventory of material being processed or repackaged in HBL Phase I. If also be able to replace the HBL Phase II Mechanical Line or Operating Area 5 Balar

Additionally, this balance may be used during performance of HBL vessel calibratio of spanning solution during these calibrations. The calibration/spanning will ultimat of spanning solution during these calibrations. The calibration/spanning
vessel level instrumentation as MC\&A qualified level instrumentation.

3. Process and Product Specifications

SNM to be handled in the HBL Phase I facility will be weighed prior to processing or repackaging. The SNM to be handled is normally between 100 and 4000 grams. The balance will be set up to perform measurements in the Phase I gloveboxes and/or supporting areas.

When used for vessel calibration/spanning, the balance will be used to measure the weight of a poly bottle

Bounding environmental conditions for all these locations are 5 to 40 degrees $\mathrm{C}$ and $10 \%$ to $85 \%$ relativc humidity.
4. Composition of Materials Used for Qualification Process

NIST traceable weight standards will be used for balance calibrations and baseline measurements. The weight set designated for qualification and use of this balance is HW-986. The weight standards included in this standards. These standards are intended to bracket the expected weight of the items to be weighed by the balance, while also covering the overall range of the balance.

5. Amount and Description of Test Data to be Generated

Baseline data will be generated over various shift time periods in HBL with approved procedures that implement the random weighing plan, which will be supplied by MC\&A.

6. Qualification Acceptance Criteria

Intial calibration of the balance and weight standards has been performed by the Savannah River Standard Lab (SRL), and this calibration data will be supplied to Site MC\&A. After baseline data is collected in

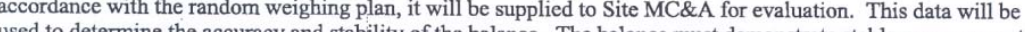
characteristics during acuracy and stability of the balance. The balance must demonstrate stable measurement arget values and control limits (waming and alam limits) by Site MC\&A. Site MC\&A will determine the Qualification Report.

7. Approval of Qualification Plan

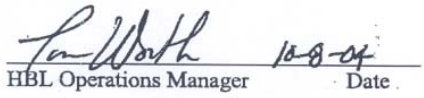

$\begin{array}{cc}\text { Caszer ex } & 10 / 864 \\ \text { HBL Engineering } & \text { Date } \\ \text { Site MC\&A } & \text { Date }\end{array}$

Site MC\&A Date 


\section{HB-Line Scale HBL-08: Qualification Data}

\begin{tabular}{|c|c|c|c|}
\hline $\begin{array}{l}\text { Result } \\
\text { in } \\
\text { grams }\end{array}$ & $\begin{array}{l}\text { Reference } \\
\text { Value } \\
\text { in } \\
\text { grams }\end{array}$ & $\begin{array}{l}\text { Absolute } \\
\text { Difference }\end{array}$ & $\begin{array}{l}\text { Relative } \\
\text { Difference }\end{array}$ \\
\hline 1000 & 1000 & 0 & 0 \\
\hline 3999.9 & 4000 & -0.1 & -0.000025 \\
\hline 5999.8 & 6000 & -0.2 & -0.000033 \\
\hline 500.1 & 500 & 0.1 & 0.0002 \\
\hline 2000 & 2000 & 0 & 0 \\
\hline 7999.7 & 8000 & -0.3 & -0.000038 \\
\hline 500 & 500 & 0 & 0 \\
\hline 2000 & 2000 & 0 & 0 \\
\hline 7999.7 & 8000 & -0.3 & -0.000038 \\
\hline 6999.7 & 4000 & 2999.7 & 0.749925 \\
\hline 1000.1 & 1000 & 0.1 & 0.0001 \\
\hline 500.1 & 500 & 0.1 & 0.0002 \\
\hline 2000 & 2000 & 0 & 0 \\
\hline 7999.8 & 8000 & -0.2 & -0.000025 \\
\hline 5999.9 & 6000 & -0.1 & -0.000017 \\
\hline 4000 & 4000 & 0 & 0 \\
\hline 3999.9 & 4000 & -0.1 & -0.000025 \\
\hline 500.1 & 500 & 0.1 & 0.0002 \\
\hline$\ldots$ & $\ldots$ & $\ldots$ & $\ldots$ \\
\hline
\end{tabular}

Module $5-12$ 


\section{HB-Line Scale HBL-08: Plot of Qualification Data}

(Value of plot symbol indicates number of dbsenations)

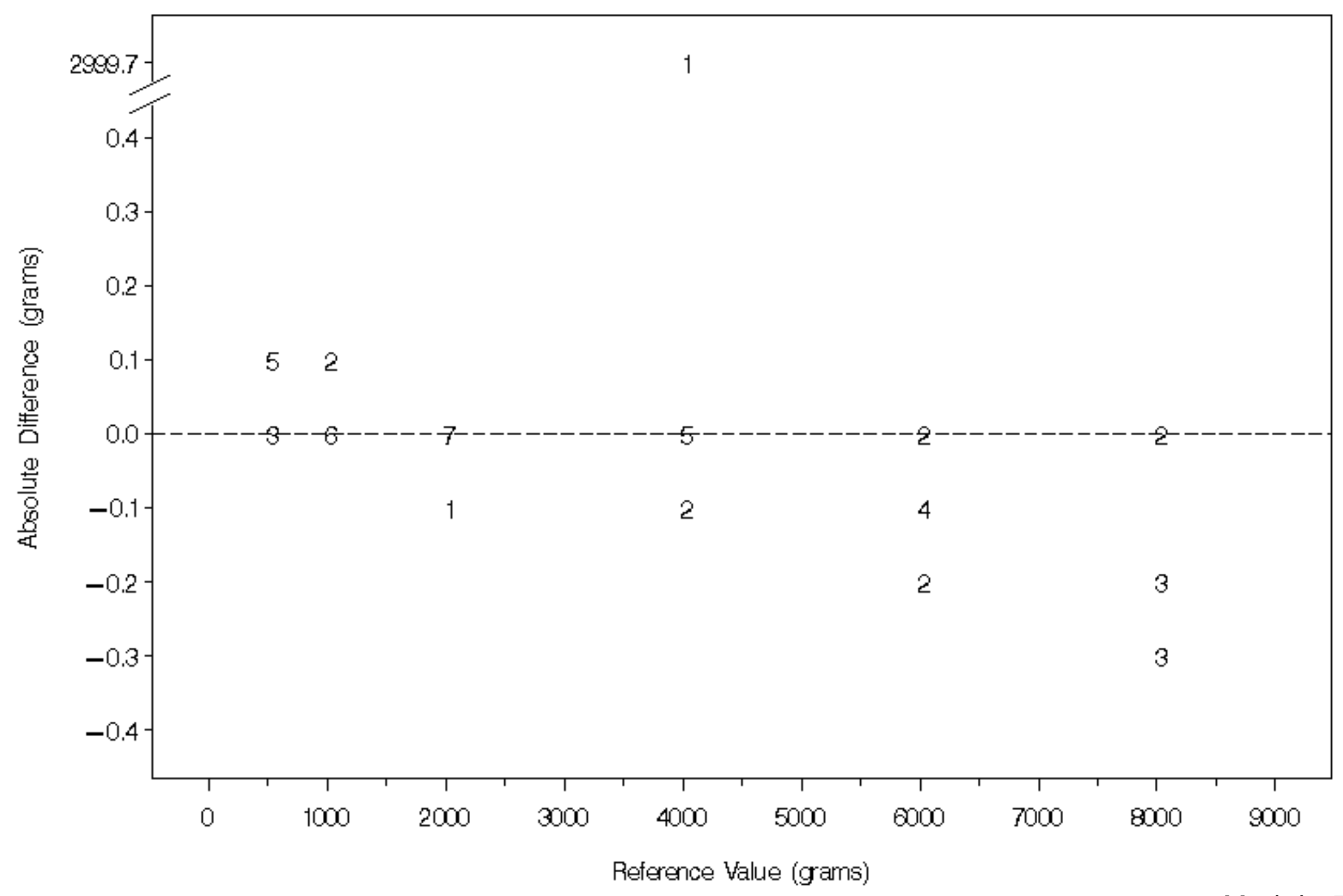

Module $5-13$ 


\section{HB-Line Scale HBL-08: Estimation of Calibration Equation}

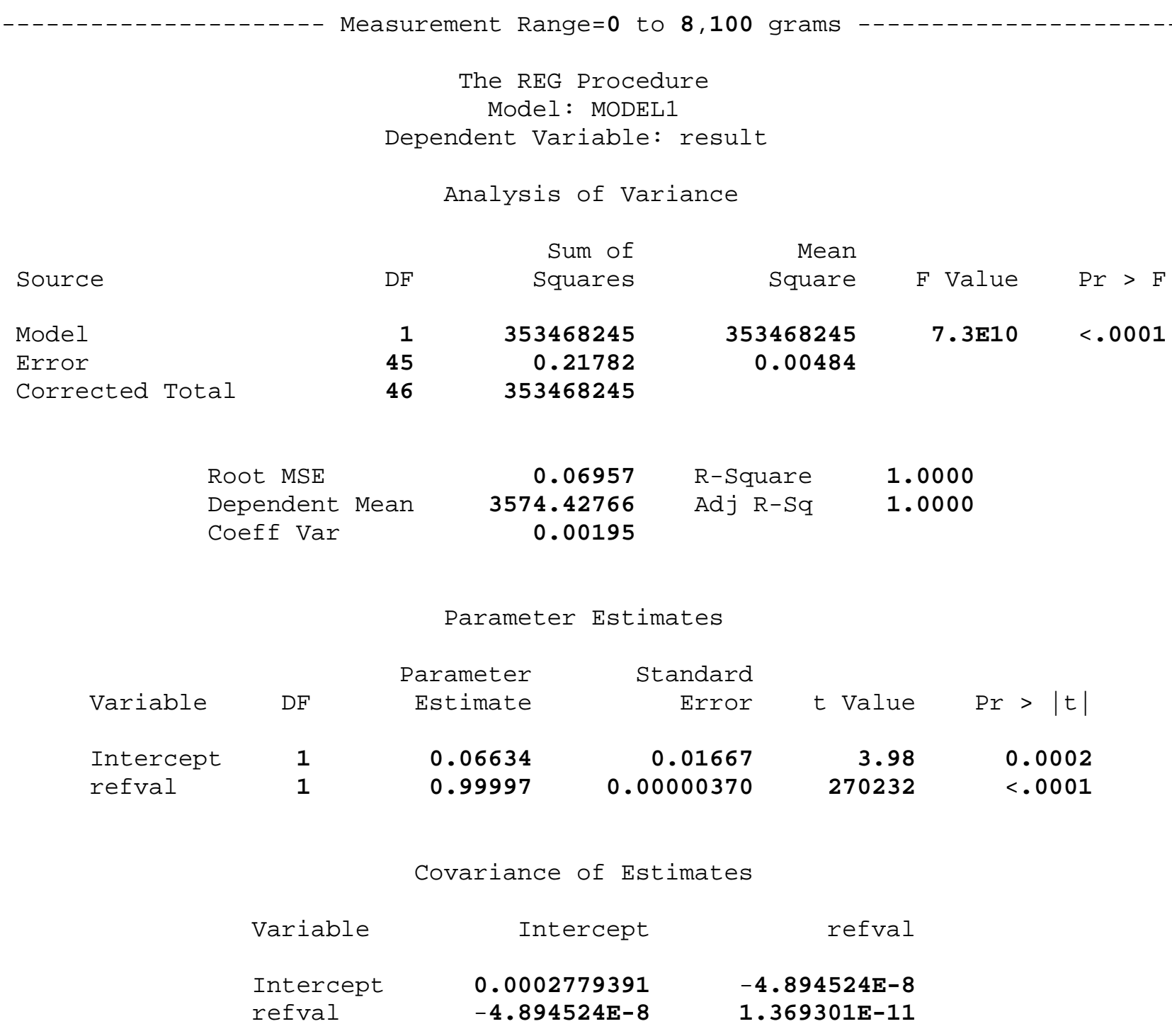

$\begin{array}{lrrrrr}\text { Variable } & \text { DF } & \begin{array}{r}\text { Parameter } \\ \text { Estimate }\end{array} & \begin{array}{r}\text { Standard } \\ \text { Error }\end{array} & \text { t value } & \operatorname{Pr}>|t| \\ \text { Intercept } & 1 & 0.06634 & 0.01667 & 3.98 & 0.0002 \\ \text { refval } & 1 & 0.99997 & 0.00000370 & 270232 & <.0001\end{array}$

Covariance of Estimates

$\begin{array}{lrr}\text { Variable } & \text { Intercept } & \text { refval } \\ & & \\ \text { Intercept } & \mathbf{0 . 0 0 0 2 7 7 9 3 9 1} & \mathbf{- 4 . 8 9 4 5 2 4 E - 8} \\ \text { refval } & -\mathbf{4 . 8 9 4 5 2 4 E - 8} & \mathbf{1 . 3 6 9 3 0 1 E - 1 1}\end{array}$




\section{Exercise \#2}

Scale Qualification Process

1. Can this scale be used for weight measurements?

2. If so, how would these measurements be adjusted?

3. Discuss methods or actions for improving the measurements for this scale 


\section{AWCC: Neutron Measurement Basics}

Most nuclear materials emit neutrons. An Active Well

Coincidence Counter (AWCC) is a non-destructive assay (NDA) device used to measure neutrons. The amount of neutrons measured can be correlated to the amount of nuclear material.

An AWCC has two modes of operation:

-Passive Mode - Plutonium

- Plutonium fissions spontaneously

-Active Mode - Uranium

- Uranium measurements are done by using an external source (active) of neutrons to excite the U235 


\section{AWCC: Measurement Process}

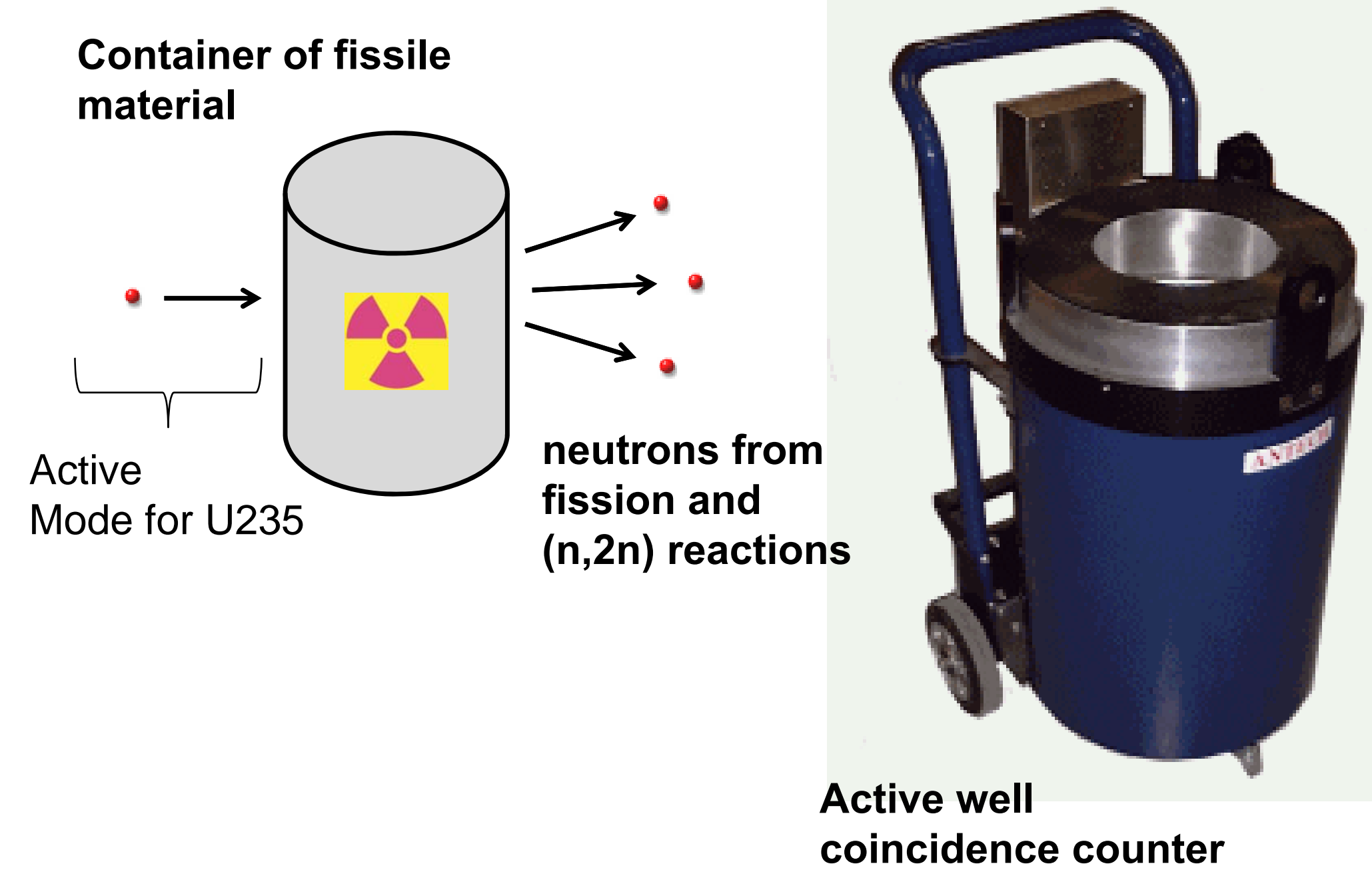

Module 5 - 17 


\section{Exercise \#3}

Active Well Coincidence Counter (AWCC)

Qualification Process

Purpose: Qualify an Active Well Coincidence Counter for Pu Accountability Measurements

- Discuss the steps for conducting the AWCC method qualification

- Bring up the Excel Student Worksheet for Exercises \#3 and \#4

- Use the Excel one-way ANOVA to estimate the AWCC calibration and random uncertainties 


\section{Exercise \#3 (continued)}

Active Well Coincidence Counter (AWCC)

Qualification Process

- The following four slides represent the analysis conducted at the Savannah River Site

- Compare the Excel results to the SRS analysis 


\section{AWCC Qualification: SRS Analysis}

\section{Listing of HBL AWCC Pu Qualification Data}

\begin{tabular}{|c|c|c|c|c|}
\hline File \# & $\begin{array}{l}\text { Standard } \\
\text { Id }\end{array}$ & $\begin{array}{c}\text { True } \\
\text { Pu mass } \\
\text { (g) }\end{array}$ & $\begin{array}{c}\text { Measured } \\
\text { Pu mass } \\
\text { (g) }\end{array}$ & $\begin{array}{l}\text { Relative } \\
\text { Difference }\end{array}$ \\
\hline $162 \mathrm{~K} 0410$ & SGB-30 & 29.989 & 36.2 & 0.207 \\
\hline $162 \mid 1040$ & SGB-30 & 29.989 & 33.8 & 0.127 \\
\hline $162 \mathrm{M} 1846$ & SGB-30 & 29.989 & 35.5 & 0.184 \\
\hline 162 N2438 & SGB-30 & 29.989 & 35.3 & 0.177 \\
\hline 16203955 & SGB-30 & 29.989 & 34.8 & 0.160 \\
\hline 15UI3558 & SGB-100 & 99.964 & 99.7 & -0.003 \\
\hline 15 UJ4120 & SGB-100 & 99.964 & 89.6 & -0.104 \\
\hline 15UM0935 & SGB-100 & 99.964 & 81.7 & -0.183 \\
\hline $15 \cup O 2454$ & SGB-100 & 99.964 & 88.2 & -0.118 \\
\hline $15 \cup 2943$ & SGB-100 & 99.964 & 93.7 & -0.063 \\
\hline 15VO5519 & SGB-200 & 199.927 & 232.7 & 0.164 \\
\hline 15VQ0230 & SGB-200 & 199.927 & 176.1 & -0.119 \\
\hline $161 J 0906$ & SGB-200 & 199.927 & 184.5 & -0.077 \\
\hline $161 K 3809$ & SGB-200 & 199.927 & 207.1 & 0.036 \\
\hline 16100600 & SGB-200 & 199.927 & 213 & 0.065 \\
\hline
\end{tabular}




\section{AWCC Qualification: SRS Analysis}

Plot of Relative Differences from HBL AWCC Pu Qualification Data

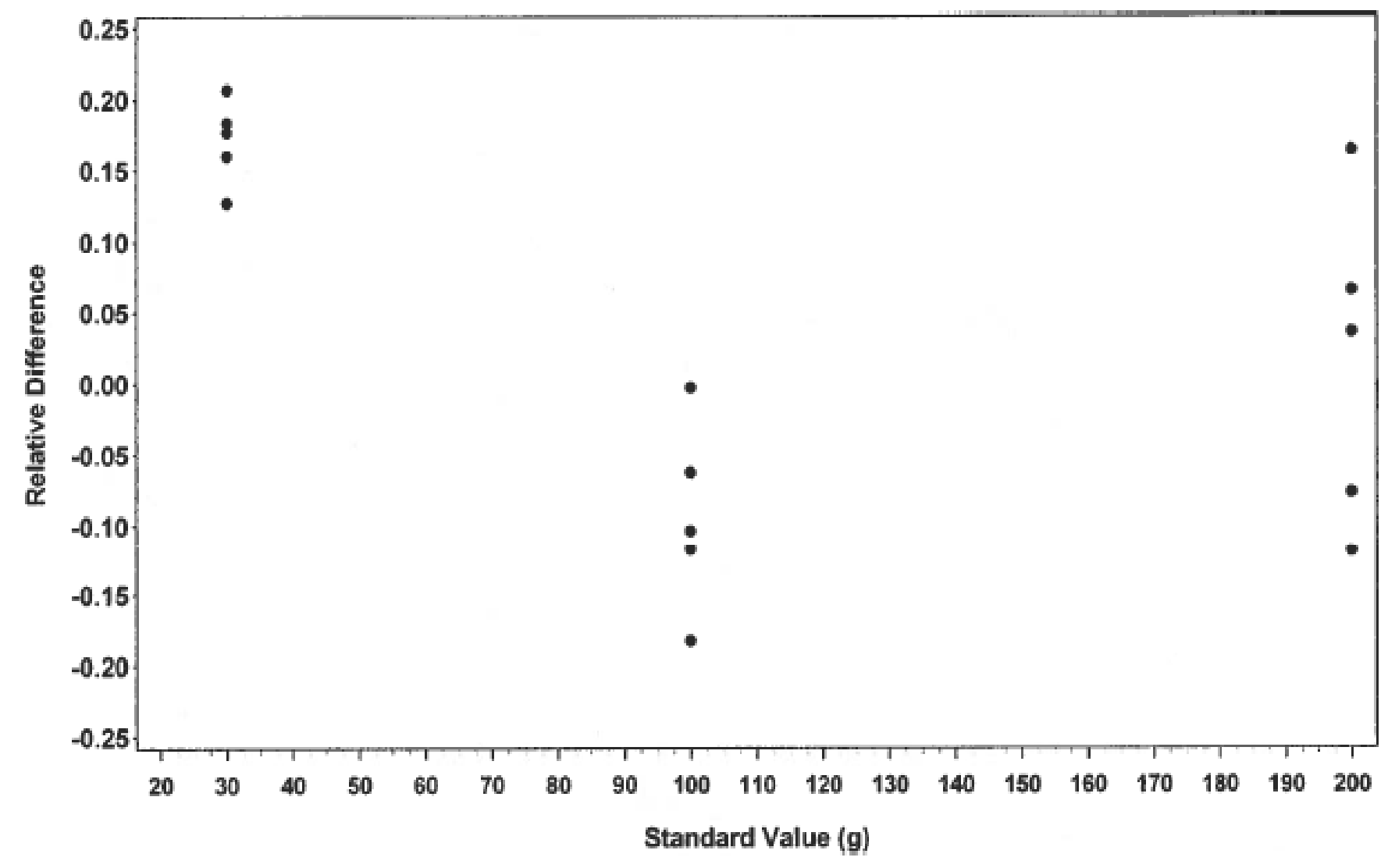

Module $5-21$ 


\title{
AWCC Qualification: SRS Analysis
}

ANOVA Results for HBL AWCC Calibration and Random Uncertainty Analysis

\author{
The GLM Procedure \\ Class Level Information \\ Class Levels Values \\ std_id 3 SGB-100 SGB-200 SGB-30
}

Number of observations 15

The GLM Procedure

Dependent Variable: reldiff

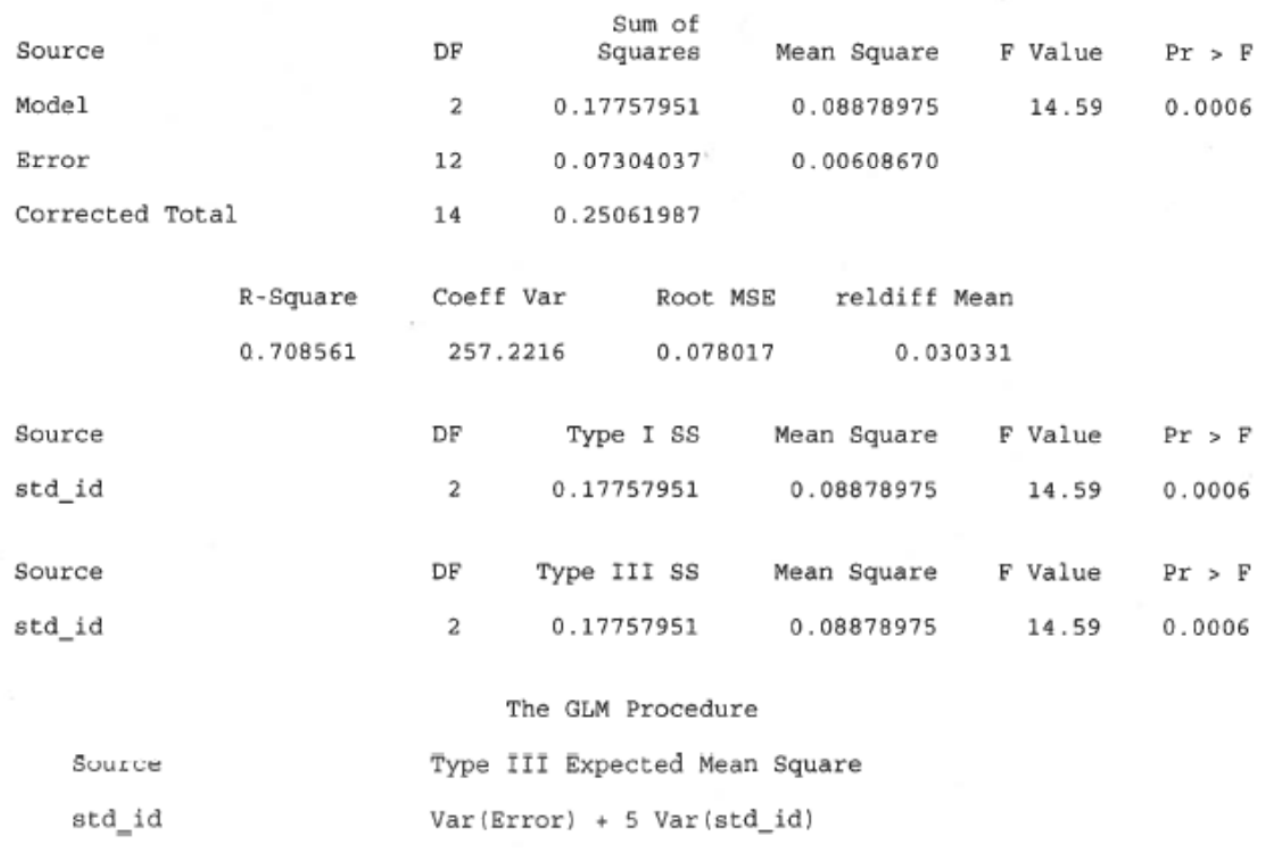




\section{AWCC Qualification: SRS Analysis}

HBL AWCC Pu Uncertainty Components

\begin{tabular}{|c|c|c|c|}
\hline Bias & $\begin{array}{c}\text { Bias } \\
\text { Uncertainty }\end{array}$ & $\begin{array}{c}\text { Calibration } \\
\text { Uncertainty }\end{array}$ & $\begin{array}{c}\text { Random } \\
\text { Uncertainty }\end{array}$ \\
\hline $3.03 \%$ & $\pm ?$ & $\pm 12.86 \%$ & $\pm 7.80 \%$ \\
\hline
\end{tabular}




\section{Exercise \#4: AWCC Qualification Process}

Assume the following relative model for the AWCC measurement method:

$y_{i j}=\mu_{i}\left(1+\eta_{i j}+\varepsilon_{i j}\right)$, where the $\eta_{i}$ are iid $N\left(0,0.13^{2}\right)$ and the $\varepsilon_{\mathrm{ij}}$ are iid $\mathrm{N}\left(0,0.08^{2}\right)$

The bias is the average of the $\left(y_{i j}-\mu_{i}\right) / \mu_{i}$ relative differences and is equal to 0.03 or $3 \%$.

- Using this information, derive V(Bias)

- Use the $\eta_{i}$ as short-term systematic effects Note: This is actually a simple variance propagation problem! 


\section{Exercise \#5: Davies-Gray Titration}

Uncertainty Estimation for the Davies-Gray Titration Method

Purpose: To review the uncertainty estimates for the Davies-Gray concentration method. This methodology is used for Uranium accountability measurements.

- Bring up the Student Worksheet for Exercise \#5

- Students will need to rearrange the data in order to run the ANOVA

- Arrange the relative differences by measurement date in different columns starting in column 1 


\section{Summary}

- Discussed method selection/qualification process

- Discussed documenting a method qualification

- Qualification plan

- Data analysis report

- Applied a basic variance propagation in the qualification of 3 measurement methods (physical, DA, NDA) 
Chinese Measurement Control Workshop Measurement Method Qualification Module

AWCC Qualification Data

Exercise \#3

\begin{tabular}{|c|c|c|}
\hline SGB30 & SGB100 & SGB200 \\
\hline 0.207 & -0.003 & 0.164 \\
\hline 0.127 & -0.104 & -0.119 \\
\hline 0.184 & -0.183 & -0.077 \\
\hline 0.177 & -0.118 & 0.036 \\
\hline 0.16 & -0.063 & 0.065 \\
\hline
\end{tabular}

Anova: Single Factor

SUMMARY

\begin{tabular}{lrrrr}
\hline \multicolumn{1}{c}{ Groups } & Count & \multicolumn{1}{c}{ Sum } & Average & Variance \\
\hline Column 1 & 5 & 0.855 & 0.171 & 0.000889 \\
Column 2 & 5 & -0.471 & -0.0942 & 0.00446 \\
Column 3 & 5 & 0.069 & 0.0138 & 0.012889 \\
\hline
\end{tabular}

ANOVA

\begin{tabular}{lcrcccc}
\hline Source of Variation & SS & $d f$ & MS & $F$ & P-value & F crit \\
\hline Between Groups & 0.177845 & 2 & 0.088922 & 14.62708 & 0.000606 & 3.885294 \\
Within Groups & 0.072952 & 12 & 0.006079 & & & \\
& & & & & & \\
Total & 0.250796 & 14 & 0.017914 & & & \\
\hline
\end{tabular}

\begin{tabular}{|r|r|}
\hline Bias $=$ & $3.02 \%$ \\
\hline Bias Uncertainty $=$ & $7.70 \%$ \\
\hline Calibration Uncertainty $=$ & $12.87 \%$ \\
\hline Random Uncertainty $=$ & $7.80 \%$ \\
\hline
\end{tabular}

Note the following:

$\mathrm{E}($ Between Groups MS $)=\mathrm{V}($ Random $)+5 \mathrm{~V}($ Calibration $)$

$\mathrm{V}($ Random $)=($ Within Groups MS $)$

Therefore, $\mathrm{V}($ Calibration $)=(($ Between Groups MS $)-\mathrm{V}($ Random $)) / 5$ 
Chinese Measurement Control Workshop Measurement Method Qualification Module AWCC Qualification Data

\section{Exercise \#3}

\begin{tabular}{|c|c|c|}
\hline SGB30 & SGB100 & SGB200 \\
\hline 0.207 & -0.003 & 0.164 \\
\hline 0.127 & -0.104 & -0.119 \\
\hline 0.184 & -0.183 & -0.077 \\
\hline 0.177 & -0.118 & 0.036 \\
\hline 0.16 & -0.063 & 0.065 \\
\hline
\end{tabular}

\begin{tabular}{|r|r|}
\hline Bias $=$ & \\
\hline Bias Uncertainty $=$ & \\
\hline Calibration Uncertainty $=$ & \\
\hline Random Uncertainty $=$ & \\
\hline
\end{tabular}

\section{Note the following:}

$\mathrm{E}($ Between Groups MS $)=\mathrm{V}($ Random $)+5 \mathrm{~V}($ Calibration $)$

$\mathrm{V}($ Random $)=($ Within Groups MS $)$

Therefore, $\mathrm{V}($ Calibration $)=(($ Between Groups MS $)-\mathrm{V}($ Random $)) / 5$ 
Chinese Measurement Control Workshop Measurement Method Qualification Module Davies-Gray Concentration Measurements

\section{Exercise \#5}

\begin{tabular}{|r|r|r|r|r|}
\hline & & Result & Standard & \\
\hline & Measurement & in & in & Relative \\
\hline Sample ID & Date & \multicolumn{1}{c|}{$\mathbf{~ g / g}$} & $\mathbf{~ m g / g}$ & \multicolumn{1}{c|}{ Difference } \\
\hline & & & & \\
\hline 200158371 & 12-Jun-01 & 54.0506 & 54.0705 & -0.0004 \\
\hline 200158373 & 12-Jun-01 & 53.9417 & 54.0705 & -0.0024 \\
\hline 200158374 & 12-Jun-01 & 53.9998 & 54.0705 & -0.0013 \\
\hline 200158375 & 12-Jun-01 & 53.9956 & 54.0705 & -0.0014 \\
\hline 200161089 & 19-Jun-01 & 54.0748 & 54.0705 & 0.0001 \\
\hline 200162439 & 19-Jun-01 & 54.1235 & 54.0705 & 0.001 \\
\hline 200161086 & 19-Jun-01 & 53.9037 & 54.0705 & -0.0031 \\
\hline 200161088 & 19-Jun-01 & 54.2321 & 54.0705 & 0.003 \\
\hline 200161092 & 19-Jun-01 & 54.2349 & 54.0705 & 0.003 \\
\hline 200158379 & 20-Jun-01 & 54.1849 & 54.0705 & 0.0021 \\
\hline 200163495 & 28-Jun-01 & 54.0767 & 54.0705 & 0.0001 \\
\hline 200162441 & 28-Jun-01 & 54.0088 & 54.0705 & -0.0011 \\
\hline 200162458 & 23-Jul-01 & 54.26 & 54.0705 & 0.0035 \\
\hline 200162460 & 25-Jul-01 & 54.1456 & 54.0705 & 0.0014 \\
\hline 200162469 & 1-Aug-01 & 54.105 & 54.0705 & 0.0006 \\
\hline 200162466 & 1-Aug-01 & 54.0617 & 54.0705 & -0.0002 \\
\hline 200162468 & 1-Aug-01 & 54.0518 & 54.0705 & -0.0003 \\
\hline 200162471 & 11-Aug-01 & 54.1523 & 54.0705 & 0.0015 \\
\hline 200163507 & 20-Aug-01 & 54.1328 & 54.0705 & 0.0012 \\
\hline
\end{tabular}

\begin{tabular}{|c|c|c|c|c|c|c|c|c|}
\hline \multicolumn{9}{|c|}{ Relative Differences for Analysis } \\
\hline-0.0004 & 0.0001 & 0.0021 & 0.0001 & 0.0035 & 0.0014 & 0.0006 & 0.0015 & 0.0012 \\
\hline-0.0024 & 0.001 & & -0.0011 & & & -0.0002 & & \\
\hline-0.0013 & -0.0031 & & & & & -0.0003 & & \\
\hline-0.0014 & 0.003 & & & & & & & \\
\hline & 0.003 & & & & & & & \\
\hline
\end{tabular}

Anova: Single Factor

\begin{tabular}{lrrrr} 
SUMMARY & \multicolumn{1}{c}{ Count } & \multicolumn{1}{l}{ Sum } & \multicolumn{1}{c}{ Average } & Variance \\
\hline \multicolumn{1}{c}{ Groups } & 4 & -0.0055 & -0.001375 & $6.69 \mathrm{E}-07$ \\
Column 1 & 5 & 0.004 & 0.0008 & $6.36 \mathrm{E}-06$ \\
Column 2 & 1 & 0.0021 & 0.0021 & \#DIV/0! \\
Column 3 & 2 & -0.001 & -0.0005 & $7.2 \mathrm{E}-07$ \\
Column 4 & 1 & 0.0035 & 0.0035 & \#DIV/0! \\
Column 5 & 1 & 0.0014 & 0.0014 & \#DIV/0! \\
Column 6 & 3 & 0.0001 & $3.33333 \mathrm{E}-05$ & $2.43 \mathrm{E}-07$ \\
Column 7 & 1 & 0.0015 & 0.0015 & \#DIV/0! \\
Column 8 & 1 & 0.0012 & 0.0012 & \#DIV/0! \\
Column 9 & & & &
\end{tabular}

\begin{tabular}{|l|r|}
\hline \multicolumn{2}{|c|}{ Uncertainty Estimates } \\
\hline & \\
\hline Bias & $0.038 \%$ \\
\hline Systematic & $0.068 \%$ \\
\hline Random & $0.169 \%$ \\
\hline
\end{tabular}

\begin{tabular}{|c|c|c|c|c|c|c|}
\hline Source of Variatior & SS & $d f$ & $M S$ & $F$ & $P$-value & $F$ crit \\
\hline Between Groups & $3.08 \mathrm{E}-05$ & 8 & 3.84639E-06 & 1.343286 & 0.324694 & 3.071658 \\
\hline Within Groups & 2.86E-05 & 10 & 2.86342E-06 & & & \\
\hline Total & 5.94E-05 & 18 & 3.30029E-06 & & & \\
\hline
\end{tabular}

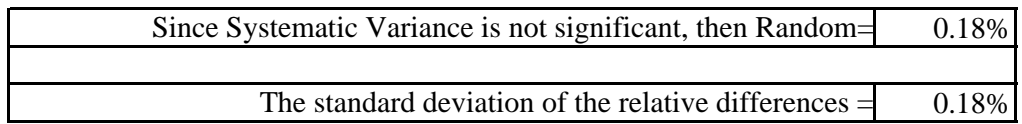


Measurement Method Qualification

Davies-Gray Concentration Measurements

Result Standard

\begin{tabular}{rrrrr} 
Sample ID & $\begin{array}{c}\text { Measurement } \\
\text { Date }\end{array}$ & \multicolumn{1}{c}{ in } & in & \multicolumn{1}{c}{$\begin{array}{c}\text { Relative } \\
\text { Difference }\end{array}$} \\
200158371 & 12-Jun-01 & 54.0506 & 54.0705 & -0.0004 \\
200158373 & 12-Jun-01 & 53.9417 & 54.0705 & -0.0024 \\
200158374 & 12-Jun-01 & 53.9998 & 54.0705 & -0.0013 \\
200158375 & 12-Jun-01 & 53.9956 & 54.0705 & -0.0014 \\
200161089 & 19-Jun-01 & 54.0748 & 54.0705 & 0.0001 \\
200162439 & 19-Jun-01 & 54.1235 & 54.0705 & 0.001 \\
200161086 & 19-Jun-01 & 53.9037 & 54.0705 & -0.0031 \\
200161088 & 19-Jun-01 & 54.2321 & 54.0705 & 0.003 \\
200161092 & 19-Jun-01 & 54.2349 & 54.0705 & 0.003 \\
200158379 & 20-Jun-01 & 54.1849 & 54.0705 & 0.0021 \\
200163495 & 28-Jun-01 & 54.0767 & 54.0705 & 0.0001 \\
200162441 & 28-Jun-01 & 54.0088 & 54.0705 & -0.0011 \\
200162458 & 23-Jul-01 & 54.26 & 54.0705 & 0.0035 \\
200162460 & 25-Jul-01 & 54.1456 & 54.0705 & 0.0014 \\
200162469 & 1-Aug-01 & 54.105 & 54.0705 & 0.0006 \\
200162466 & 1-Aug-01 & 54.0617 & 54.0705 & -0.0002 \\
200162468 & 1-Aug-01 & 54.0518 & 54.0705 & -0.0003 \\
200162471 & 11-Aug-01 & 54.1523 & 54.0705 & 0.0015 \\
200163507 & 20-Aug-01 & 54.1328 & 54.0705 & 0.0012
\end{tabular}




\section{Exercise \\ Measurement Method Qualification}

\section{Session Objectives:}

After the session the participants will be able to do the following:

1. Plan the method qualification process

2. Produce a qualification planning document

3. Collect the measurement data

4. Analyze the data (estimate uncertainties)

5. Produce a qualification document

\section{Estimated Time:}

This module contains five (5) exercises. The exercise completion times are as follows:

1. Exercise \#1 - 20 minutes

2. Exercise \#2 - 15 minutes

3. Exercise \#3 - 45 minutes

4. Exercise \#4 - 15 minutes

5. Exercise $\# 5-45$ minutes

These exercises will require 2 hours and 20 minutes to complete.

\section{Materials Needed:}

1. One computer with Microsoft Excel for each group of 5 students

2. Excel needs to have the Stat Package add-in loaded

3. The students must be familiar with Excel

4. Pencil and paper

5. Spreadsheet support person

\section{Instructions:}

1. There are five (5) exercises in this module

2. Students should work in groups of four or five

3. The exercises are included in the presentation

4. The students will be requested to complete a particular exercise

5. Excel spreadsheets are provided for Exercises \#3 and \#5

6. Exercises will be instructor led and discussed 


\section{Exercise \#1}

Method Qualification Process

1. Where should the qualification plan originate?

- Typically, the facility that will be using the instrument for accountability measurements will originate the document. The facility may have their own MC\&A personnel, who will work with site MC\&A to qualify the instrument.

2. What personnel should be involved with designing the data collection process?

- The facility measurement personnel will work with site MC\&A and the MC\&A statistician to design a measurement plan.

3. What protocol should be followed when collecting the measurements for the qualification process?

- Measurement procedures should be the same for qualification and process measurements

4. What should be documented in the qualification report?

- At a minimum, the estimated uncertainty components and the applicable control limits

5. What personnel should receive the qualification report?

- Facility and site MC\&A personnel and the appropriate MC\&A statistician

\section{Exercise \#2}

HBL Scale Qualification Process

1. Can this scale be used for weight measurements?

- This scale can be used by making bias correction adjustments using the estimated calibration equation

2. If so, how would the weight measurements be adjusted?

- Invert the calibration equation. This will adjust the measured value to the reference value.

3. Discuss methods or actions for improving the measurements for this scale.

\section{Exercise \#3}

AWCC Qualification Process

1. Outline the steps for completing a method qualification:

- Selection process (target accuracy and precision values)

- Develop a qualification plan 
- Ensure proper training and qualifications of measurement personnel

- Collect the measurement data

- Conduct the data analysis

- Produce final qualification report

- If applicable, submit final report for approvals

2. Use the Excel spreadsheet for the AWCC one-way ANOVA

\section{Exercise \#4}

AWCC Qualification Process

Use the AWCC model assumptions and uncertainty estimates to calculate the bias uncertainty. The key is using the calibration uncertainty as a short-term systematic effect.

$\mathrm{V}($ Bias $)=75^{*} 0.13^{2}+15^{*} 0.08^{2}$ and the Bias Uncertainty $= \pm 7.69 \%$.

\section{Listing of HBL AWCC Pu Qualification Data}

\begin{tabular}{|c|c|c|c|c|}
\hline File \# & $\begin{array}{l}\text { Standard } \\
\text { Id }\end{array}$ & $\begin{array}{c}\text { True } \\
\text { Pu mass } \\
(\mathrm{g})\end{array}$ & $\begin{array}{c}\text { Measured } \\
\text { Pu mass } \\
\text { (g) }\end{array}$ & $\begin{array}{l}\text { Relative } \\
\text { Difference }\end{array}$ \\
\hline $162 \mathrm{~K} 0410$ & SGB-30 & 29.989 & 36.2 & 0.207 \\
\hline $162 \mid 1040$ & SGB-30 & 29.989 & 33.8 & 0.127 \\
\hline $162 \mathrm{M} 1846$ & SGB-30 & 29.989 & 35.5 & 0.184 \\
\hline 162N2438 & SGB-30 & 29.989 & 35.3 & 0.177 \\
\hline 16203955 & SGB-30 & 29.989 & 34.8 & 0.160 \\
\hline 15UI3558 & SGB-100 & 99.964 & 99.7 & -0.003 \\
\hline 15 UJ4120 & SGB-100 & 99.964 & 89.6 & -0.104 \\
\hline 15UM0935 & SGB-100 & 99.964 & 81.7 & -0.183 \\
\hline $15 \mathrm{UO} 2454$ & SGB-100 & 99.964 & 88.2 & -0.118 \\
\hline $15 U 2943$ & SGB-100 & 99.964 & 93.7 & -0.063 \\
\hline $15 V 05519$ & SGB-200 & 199.927 & 232.7 & 0.164 \\
\hline 15VQ0230 & SGB-200 & 199.927 & 176.1 & -0.119 \\
\hline 161J0906 & SGB-200 & 199.927 & 184.5 & -0.077 \\
\hline 161K3809 & SGB-200 & 199.927 & 207.1 & 0.036 \\
\hline 16100600 & SGB-200 & 199.927 & 213 & 0.065 \\
\hline
\end{tabular}




\section{Plot of Relative Differences from HBL AWCC Pu Qualification Data}

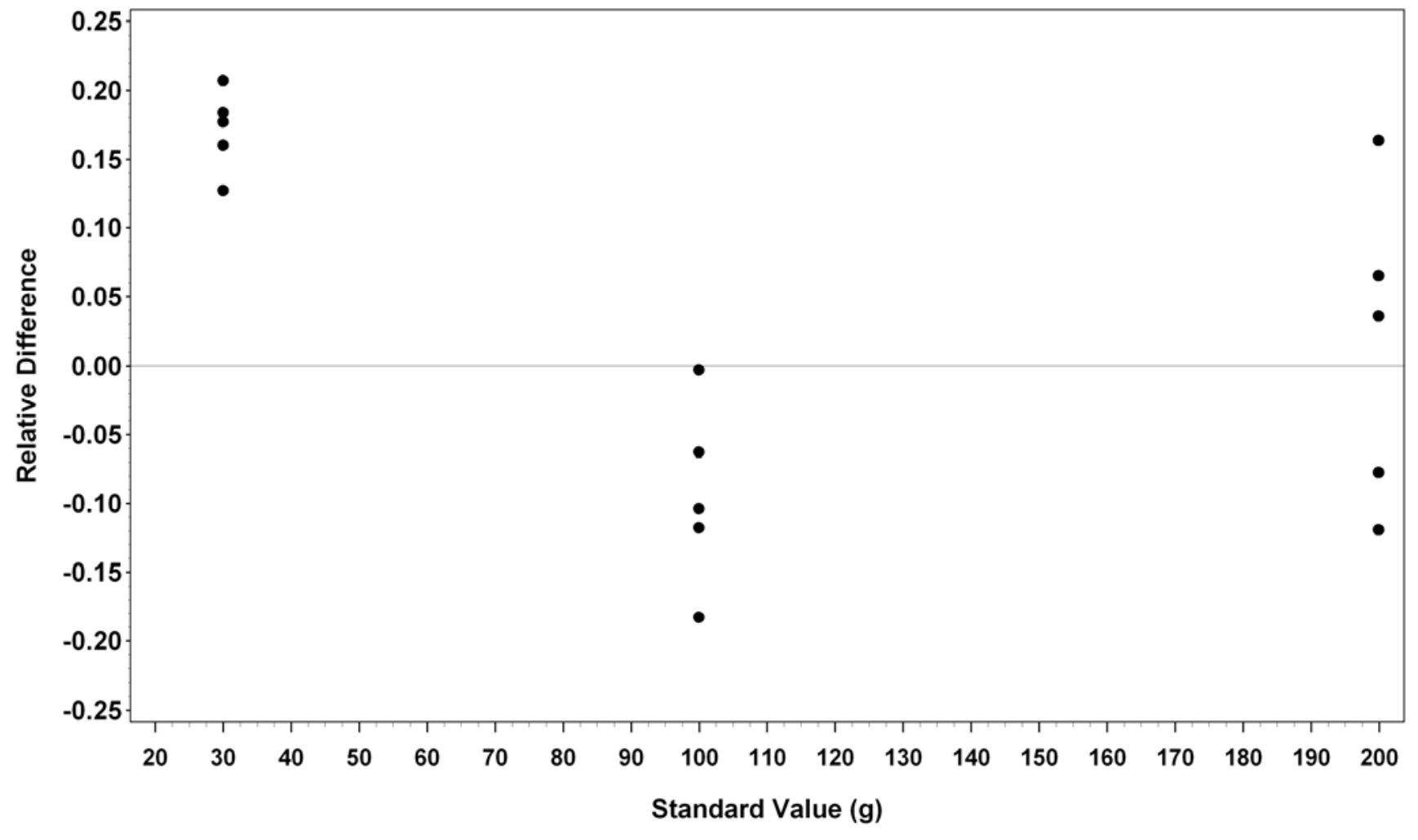




\title{
ANOVA Results for HBL AWCC Calibration and Random Uncertainty Analysis
}

\author{
The GLM Procedure \\ Class Level Information \\ Class Levels Values \\ std_id 3 SGB-100 SGB-200 SGB-30 \\ Number of observations 15 \\ The GLM Procedure
}

Dependent Variable: reldiff

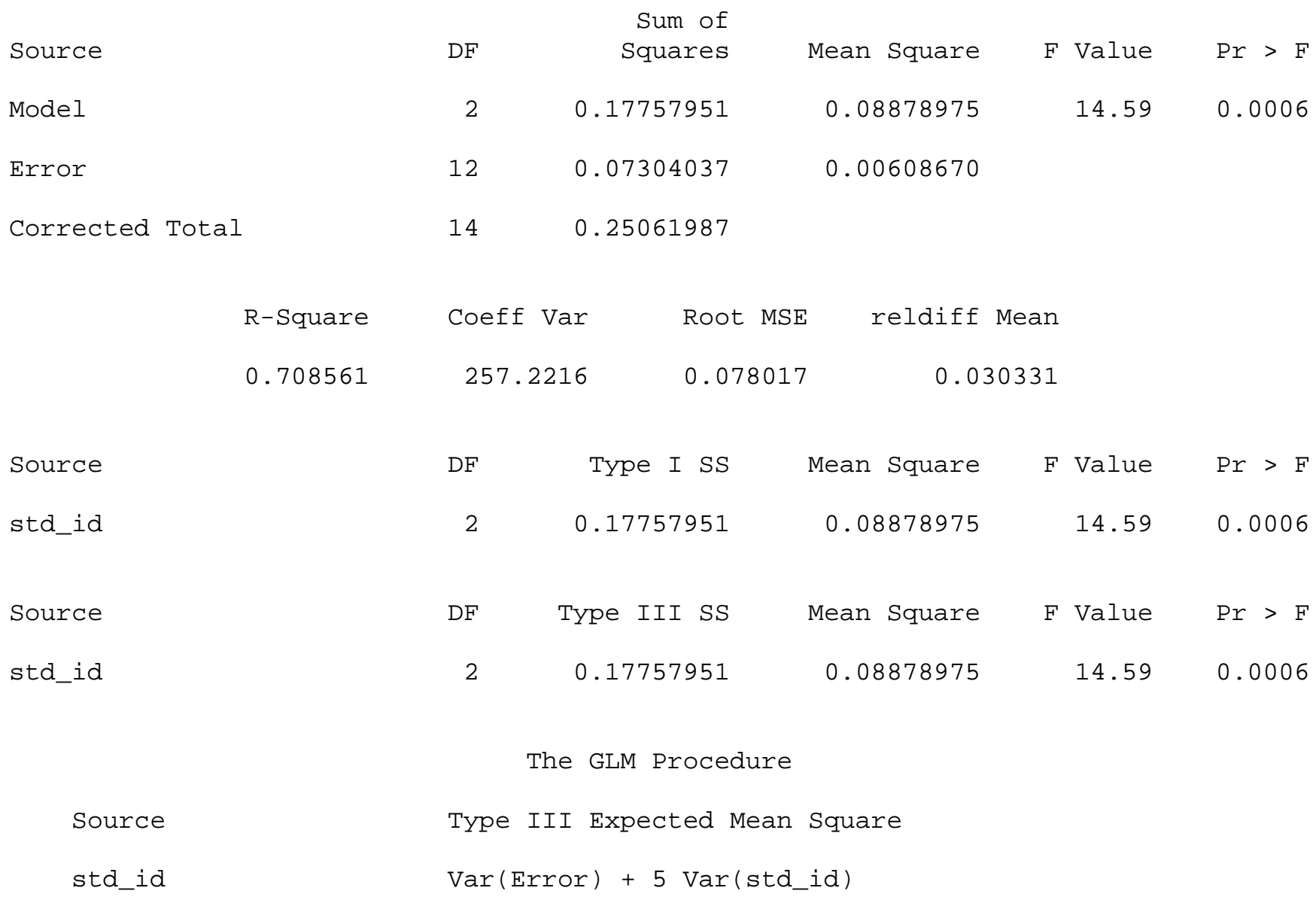




\section{HBL AWCC Pu Uncertainty Components}

\begin{tabular}{|c|c|c|c|}
\hline Bias & $\begin{array}{c}\text { Bias } \\
\text { Uncertainty }\end{array}$ & $\begin{array}{c}\text { Calibration } \\
\text { Uncertainty }\end{array}$ & $\begin{array}{c}\text { Random } \\
\text { Uncertainty }\end{array}$ \\
\hline $3.03 \%$ & $\pm 7.69 \%$ & $\pm 12.86 \%$ & $\pm 7.80 \%$ \\
\hline
\end{tabular}

\section{Exercise \#5}

Uncertainty Estimation for the Davies-Gray Concentration Method

1. A Student Worksheet has been provided for the students. There is also an Instructor Worksheet with the analysis results. The students will need to rearrange the data by measurement date in order to run the ANOVA. 


\section{Exercise \\ Measurement Method Qualification}

\section{Session Objectives:}

After the session the participants will be able to do the following:

1. Plan the method qualification process

2. Produce a qualification planning document

3. Collect the measurement data

4. Analyze the data (estimate uncertainties)

5. Produce a qualification document

\section{Estimated Time:}

This module contains five (5) exercises. The exercise completion times are as follows:

1. Exercise \#1 - 20 minutes

2. Exercise \#2 - 15 minutes

3. Exercise \#3 - 45 minutes

4. Exercise \#4 - 15 minutes

5. Exercise $\# 5-45$ minutes

These exercises will require 2 hours and 20 minutes to complete.

\section{Materials Needed:}

1. One computer with Microsoft Excel for each group of 5 students

2. Excel needs to have the Stat Package add-in loaded

3. The students must be familiar with Excel

4. Pencil and paper

5. Spreadsheet support person

\section{Instructions:}

1. There are five (5) exercises in this module

2. Students should work in groups of four or five

3. The exercises are included in the presentation

4. The students will be requested to complete a particular exercise

5. Excel spreadsheets are provided for Exercises \#3 and \#5

6. Exercises will be instructor led and discussed 


\section{Exercise \#1}

Method Qualification Process

1. Where should the qualification plan originate?

2. What personnel should be involved with designing the data collection process?

3. What protocol should be followed when collecting the measurements for the qualification process?

4. What should be documented in the qualification report?

5. What personnel should receive the qualification report?

\section{Exercise \#2}

HBL Scale Qualification Process

1. Can this scale be used for weight measurements?

2. If so, how would the weight measurements be adjusted?

3. Discuss methods or actions for improving the measurements for this scale. 


\section{Exercise \#3}

AWCC Qualification Process

1. Outline the steps for completing a method qualification:

2. Use the Excel spreadsheet for the AWCC one-way ANOVA

\section{Exercise \#4}

\section{AWCC Qualification Process}

Use the AWCC model assumptions and uncertainty estimates to calculate the bias uncertainty. The key is using the calibration uncertainty as a short-term systematic effect.

\section{Listing of HBL AWCC Pu Qualification Data}

\begin{tabular}{|c|c|c|c|c|}
\hline File \# & $\begin{array}{c}\text { Standard } \\
\text { Id }\end{array}$ & $\begin{array}{c}\text { True } \\
\text { Pu mass } \\
(\mathbf{g})\end{array}$ & $\begin{array}{l}\text { Measured } \\
\text { Pu mass } \\
\text { (g) }\end{array}$ & $\begin{array}{c}\text { Relative } \\
\text { Difference }\end{array}$ \\
\hline $162 \mathrm{~K} 0410$ & SGB-30 & 29.989 & 36.2 & 0.207 \\
\hline $162 \mid 1040$ & SGB-30 & 29.989 & 33.8 & 0.127 \\
\hline $162 \mathrm{M} 1846$ & SGB-30 & 29.989 & 35.5 & 0.184 \\
\hline 162N2438 & SGB-30 & 29.989 & 35.3 & 0.177 \\
\hline 16203955 & SGB-30 & 29.989 & 34.8 & 0.160 \\
\hline 15UI3558 & SGB-100 & 99.964 & 99.7 & -0.003 \\
\hline 15 UJ4120 & SGB-100 & 99.964 & 89.6 & -0.104 \\
\hline 15UM0935 & SGB-100 & 99.964 & 81.7 & -0.183 \\
\hline 15UO2454 & SGB-100 & 99.964 & 88.2 & -0.118 \\
\hline $15 U 2943$ & SGB-100 & 99.964 & 93.7 & -0.063 \\
\hline 15VO5519 & SGB-200 & 199.927 & 232.7 & 0.164 \\
\hline 15VQ0230 & SGB-200 & 199.927 & 176.1 & -0.119 \\
\hline $161 J 0906$ & SGB-200 & 199.927 & 184.5 & -0.077 \\
\hline 161K3809 & SGB-200 & 199.927 & 207.1 & 0.036 \\
\hline 16100600 & SGB-200 & 199.927 & 213 & 0.065 \\
\hline
\end{tabular}




\section{Plot of Relative Differences from HBL AWCC Pu Qualification Data}

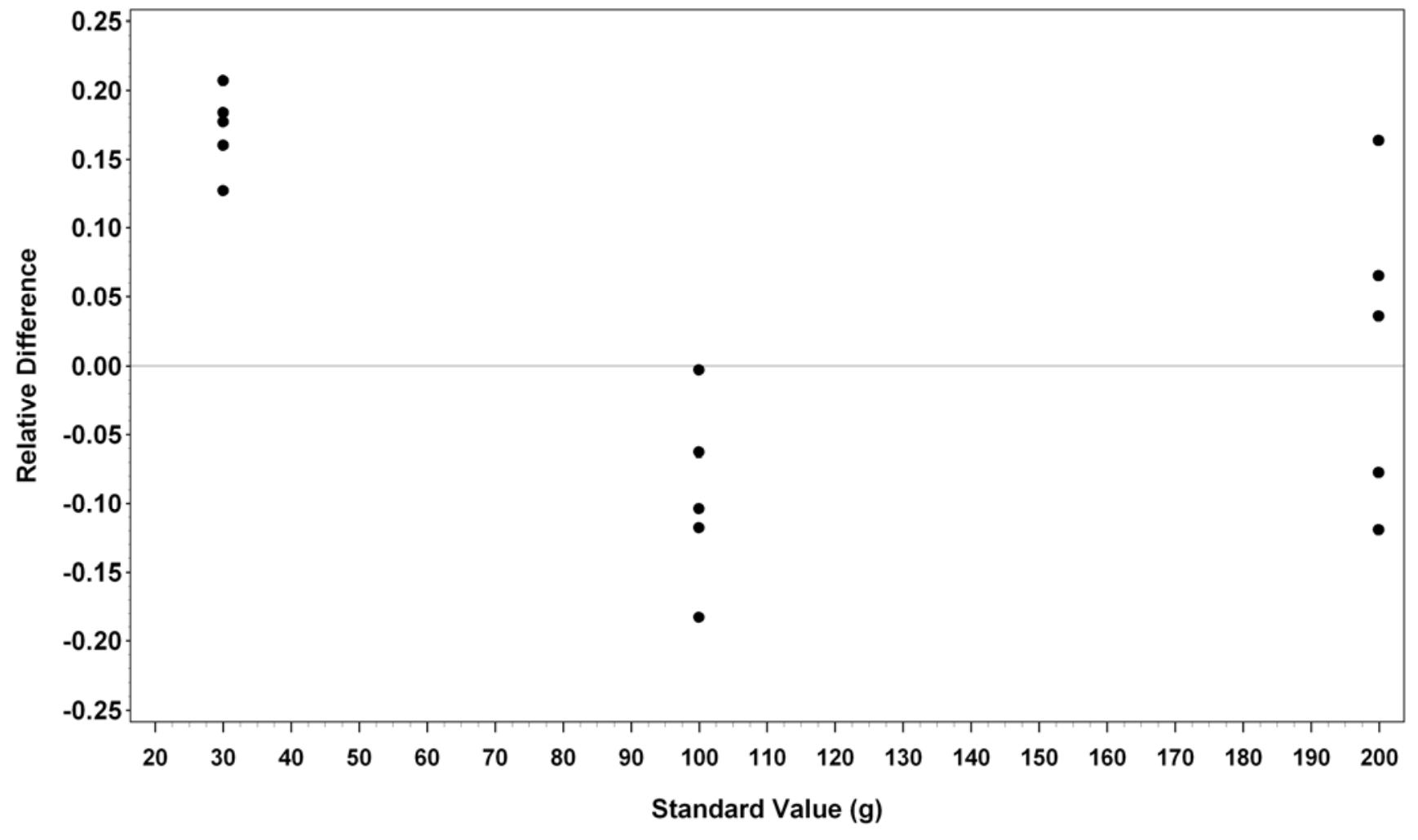




\title{
ANOVA Results for HBL AWCC Calibration and Random Uncertainty Analysis
}

\author{
The GLM Procedure \\ Class Level Information \\ Class Levels Values \\ std_id 3 SGB-100 SGB-200 SGB-30 \\ Number of observations 15 \\ The GLM Procedure
}

Dependent Variable: reldiff

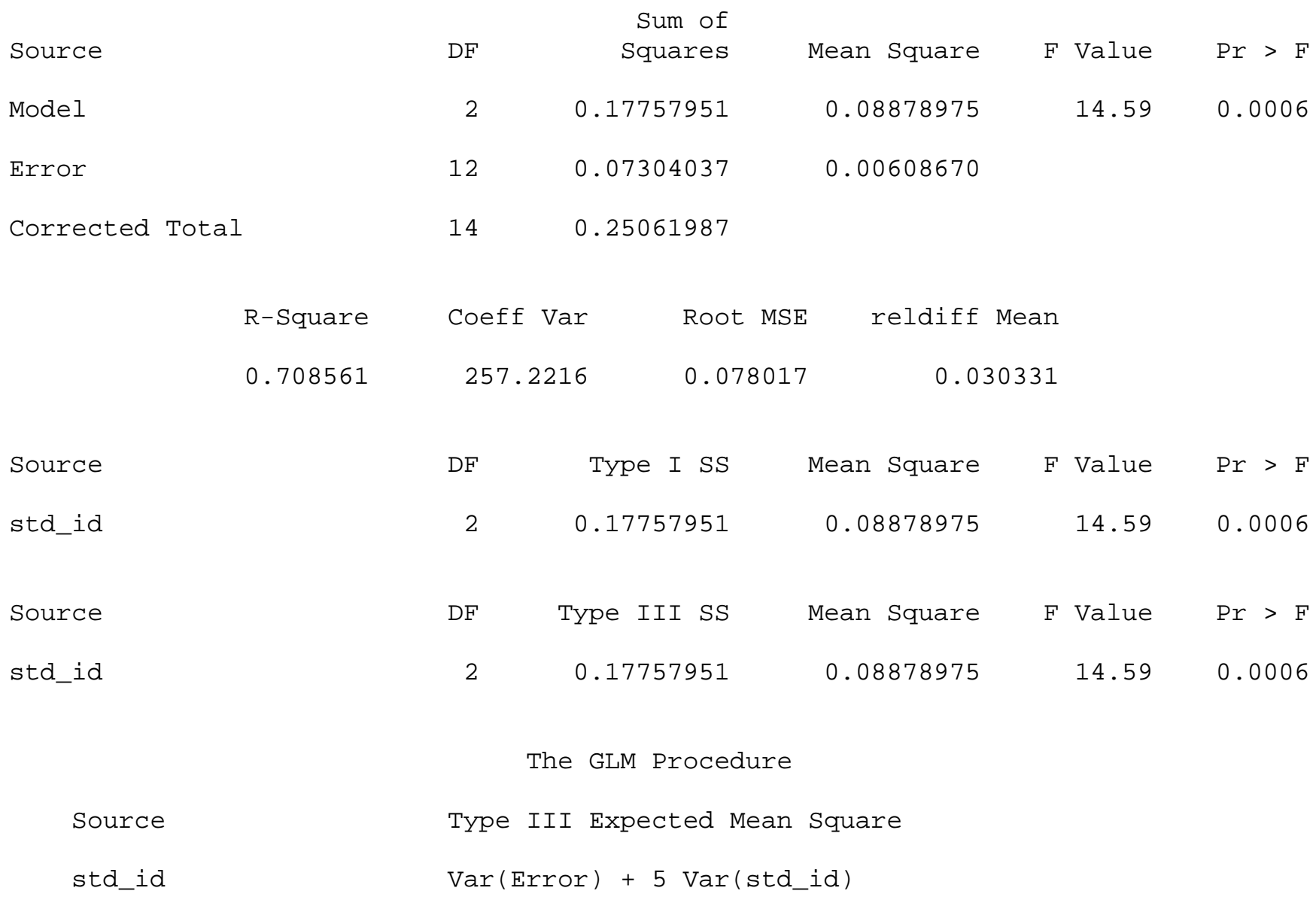




\section{HBL AWCC Pu Uncertainty Components}

\begin{tabular}{|c|c|c|c|}
\hline Bias & $\begin{array}{c}\text { Bias } \\
\text { Uncertainty }\end{array}$ & $\begin{array}{c}\text { Calibration } \\
\text { Uncertainty }\end{array}$ & $\begin{array}{c}\text { Random } \\
\text { Uncertainty }\end{array}$ \\
\hline $3.03 \%$ & $\pm 7.69 \%$ & $\pm 12.86 \%$ & $\pm 7.80 \%$ \\
\hline
\end{tabular}

\section{Exercise \#5}

Uncertainty Estimation for the Davies-Gray Concentration Method

1. A Student Worksheet has been provided for the students. There is also an Instructor Worksheet with the analysis results. The students will need to rearrange the data by measurement date in order to run the ANOVA. 
Chinese Measurement Control Workshop Measurement Method Qualification Module AWCC Qualification Data

Instructor Worksheet for Exercises \#3 and \#4

\begin{tabular}{|c|c|c|}
\hline SGB30 & SGB100 & SGB200 \\
\hline 0.207 & -0.003 & 0.164 \\
\hline 0.127 & -0.104 & -0.119 \\
\hline 0.184 & -0.183 & -0.077 \\
\hline 0.177 & -0.118 & 0.036 \\
\hline 0.16 & -0.063 & 0.065 \\
\hline
\end{tabular}

Anova: Single Factor

SUMMARY

\begin{tabular}{lrrrr}
\hline \multicolumn{1}{c}{ Groups } & Count & \multicolumn{1}{c}{ Sum } & Average & Variance \\
\hline Column 1 & 5 & 0.855 & 0.171 & 0.000889 \\
Column 2 & 5 & -0.471 & -0.0942 & 0.00446 \\
Column 3 & 5 & 0.069 & 0.0138 & 0.012889 \\
\hline
\end{tabular}

ANOVA

\begin{tabular}{lcrcccc}
\hline Source of Variation & SS & $d f$ & MS & $F$ & P-value & F crit \\
\hline Between Groups & 0.177845 & 2 & 0.088922 & 14.62708 & 0.000606 & 3.885294 \\
Within Groups & 0.072952 & 12 & 0.006079 & & & \\
& & & & & & \\
Total & 0.250796 & 14 & 0.017914 & & & \\
\hline
\end{tabular}

\begin{tabular}{|r|r|}
\hline Bias $=$ & $3.02 \%$ \\
\hline Bias Uncertainty $=$ & $7.70 \%$ \\
\hline Calibration Uncertainty $=$ & $12.87 \%$ \\
\hline Random Uncertainty $=$ & $7.80 \%$ \\
\hline
\end{tabular}

Note the following:

$\mathrm{E}($ Between Groups MS $)=\mathrm{V}($ Random $)+5 \mathrm{~V}($ Calibration $)$

$\mathrm{V}($ Random $)=($ Within Groups MS $)$

Therefore, $\mathrm{V}($ Calibration $)=(($ Between Groups MS $)-\mathrm{V}($ Random $)) / 5$ 
Chinese Measurement Control Workshop Measurement Method Qualification Module AWCC Qualification Data

Student Worksheet for Exercises \#3 and \#4

\begin{tabular}{|c|c|c|}
\hline SGB30 & SGB100 & SGB200 \\
\hline 0.207 & -0.003 & 0.164 \\
\hline 0.127 & -0.104 & -0.119 \\
\hline 0.184 & -0.183 & -0.077 \\
\hline 0.177 & -0.118 & 0.036 \\
\hline 0.16 & -0.063 & 0.065 \\
\hline
\end{tabular}

\begin{tabular}{|r|r|}
\hline Bias $=$ & \\
\hline Bias Uncertainty $=$ & \\
\hline Calibration Uncertainty $=$ & \\
\hline Random Uncertainty $=$ & \\
\hline
\end{tabular}

Note the following:

$\mathrm{E}($ Between Groups MS $)=\mathrm{V}($ Random $)+5 \mathrm{~V}($ Calibration $)$

$\mathrm{V}($ Random $)=($ Within Groups MS $)$

Therefore, $\mathrm{V}($ Calibration $)=(($ Between Groups MS $)-\mathrm{V}($ Random $)) / 5$ 
Chinese Measurement Control Workshop

Measurement Method Qualification Module

Davies-Gray Concentration Measurements

Instructor Worksheet for Exercise \#5

\begin{tabular}{|r|r|r|r|r|}
\hline & & Result & Standard & \\
\hline & Measurement & in & in & Relative \\
\hline Sample ID & Date & \multicolumn{1}{c|}{$\mathbf{~ m g / g}$} & mg/g & Difference \\
\hline & & & & \\
\hline 200158371 & 12-Jun-01 & 54.0506 & 54.0705 & -0.0004 \\
\hline 200158373 & 12-Jun-01 & 53.9417 & 54.0705 & -0.0024 \\
\hline 200158374 & 12-Jun-01 & 53.9998 & 54.0705 & -0.0013 \\
\hline 200158375 & 12-Jun-01 & 53.9956 & 54.0705 & -0.0014 \\
\hline 200161089 & 19-Jun-01 & 54.0748 & 54.0705 & 0.0001 \\
\hline 200162439 & 19-Jun-01 & 54.1235 & 54.0705 & 0.001 \\
\hline 200161086 & 19-Jun-01 & 53.9037 & 54.0705 & -0.0031 \\
\hline 200161088 & 19-Jun-01 & 54.2321 & 54.0705 & 0.003 \\
\hline 200161092 & 19-Jun-01 & 54.2349 & 54.0705 & 0.003 \\
\hline 200158379 & 20-Jun-01 & 54.1849 & 54.0705 & 0.0021 \\
\hline 200163495 & 28-Jun-01 & 54.0767 & 54.0705 & 0.0001 \\
\hline 200162441 & 28-Jun-01 & 54.0088 & 54.0705 & -0.0011 \\
\hline 200162458 & 23-Jul-01 & 54.26 & 54.0705 & 0.0035 \\
\hline 200162460 & 25-Jul-01 & 54.1456 & 54.0705 & 0.0014 \\
\hline 200162469 & 1-Aug-01 & 54.105 & 54.0705 & 0.0006 \\
\hline 200162466 & 1-Aug-01 & 54.0617 & 54.0705 & -0.0002 \\
\hline 200162468 & 1-Aug-01 & 54.0518 & 54.0705 & -0.0003 \\
\hline 200162471 & 11-Aug-01 & 54.1523 & 54.0705 & 0.0015 \\
\hline 200163507 & 20-Aug-01 & 54.1328 & 54.0705 & 0.0012 \\
\hline
\end{tabular}

\begin{tabular}{|c|c|c|c|c|c|c|c|c|}
\hline \multicolumn{9}{|c|}{ Relative Differences for Analysis } \\
\hline-0.0004 & 0.0001 & 0.0021 & 0.0001 & 0.0035 & 0.0014 & 0.0006 & 0.0015 & 0.0012 \\
\hline-0.0024 & 0.001 & & -0.0011 & & & -0.0002 & & \\
\hline-0.0013 & -0.0031 & & & & & -0.0003 & & \\
\hline-0.0014 & 0.003 & & & & & & & \\
\hline & 0.003 & & & & & & & \\
\hline
\end{tabular}

Anova: Single Factor

\begin{tabular}{lrrrc} 
SUMMARY & \multicolumn{1}{c}{ Count } & \multicolumn{1}{c}{ Sum } & \multicolumn{1}{c}{ Average } & Variance \\
\hline Groups & 4 & -0.0055 & -0.001375 & $6.69 \mathrm{E}-07$ \\
Column 1 & 5 & 0.004 & 0.0008 & $6.36 \mathrm{E}-06$ \\
Column 2 & 1 & 0.0021 & 0.0021 & \#DIV/0! \\
Column 3 & 2 & -0.001 & -0.0005 & $7.2 \mathrm{E}-07$ \\
Column 4 & 1 & 0.0035 & 0.0035 & \#DIV/0! \\
Column 5 & 1 & 0.0014 & 0.0014 & \#DIV/0! \\
Column 6 & 3 & 0.0001 & $3.33333 \mathrm{E}-05$ & $2.43 \mathrm{E}-07$ \\
Column 7 & 1 & 0.0015 & 0.0015 & \#DIV/0! \\
Column 8 & 1 & 0.0012 & 0.0012 & \#DIV/0! \\
Column 9 &
\end{tabular}

\begin{tabular}{|l|r|}
\hline \multicolumn{2}{|c|}{ Uncertainty Estimates } \\
\hline & \\
\hline Bias & $0.038 \%$ \\
\hline Systematic & $0.068 \%$ \\
\hline Random & $0.169 \%$ \\
\hline
\end{tabular}

\begin{tabular}{|c|c|c|c|c|c|c|}
\hline Source of Variatior & SS & $d f$ & $M S$ & $F$ & P-value & F crit \\
\hline Between Groups & $3.08 \mathrm{E}-05$ & 8 & 3.84639E-06 & 1.343286 & 0.324694 & 3.071658 \\
\hline Within Groups & 2.86E-05 & 10 & 2.86342E-06 & & & \\
\hline Total & 5.94E-05 & 18 & 3.30029E-06 & & & \\
\hline
\end{tabular}

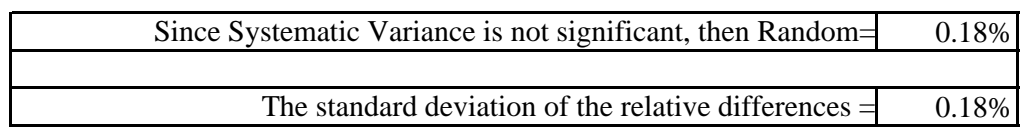


Chinese Measurement Control Workshop Measurement Method Qualification Module

Davies-Gray Concentration Measurements Student Worksheet for Exercise \#5

\begin{tabular}{|r|r|r|r|r|}
\hline & & \multicolumn{1}{c|}{ Result } & Standard & \\
\hline & Measurement & in & in & \multicolumn{1}{c|}{ Relative } \\
\hline Sample ID & Date & \multicolumn{1}{c|}{$\mathbf{~} \mathbf{g} / \mathbf{g}$} & \multicolumn{1}{c|}{$\mathbf{~ g / g}$} & \multicolumn{1}{c|}{ Difference } \\
\hline & & & & \\
\hline 200158371 & 12-Jun-01 & 54.0506 & 54.0705 & -0.0004 \\
\hline 200158373 & 12-Jun-01 & 53.9417 & 54.0705 & -0.0024 \\
\hline 200158374 & 12-Jun-01 & 53.9998 & 54.0705 & -0.0013 \\
\hline 200158375 & 12-Jun-01 & 53.9956 & 54.0705 & -0.0014 \\
\hline 200161089 & 19-Jun-01 & 54.0748 & 54.0705 & 0.0001 \\
\hline 200162439 & 19-Jun-01 & 54.1235 & 54.0705 & 0.001 \\
\hline 200161086 & 19-Jun-01 & 53.9037 & 54.0705 & -0.0031 \\
\hline 200161088 & 19-Jun-01 & 54.2321 & 54.0705 & 0.003 \\
\hline 200161092 & 19-Jun-01 & 54.2349 & 54.0705 & 0.003 \\
\hline 200158379 & 20-Jun-01 & 54.1849 & 54.0705 & 0.0021 \\
\hline 200163495 & 28-Jun-01 & 54.0767 & 54.0705 & 0.0001 \\
\hline 200162441 & 28-Jun-01 & 54.0088 & 54.0705 & -0.0011 \\
\hline 200162458 & 23-Jul-01 & 54.26 & 54.0705 & 0.0035 \\
\hline 200162460 & 25-Jul-01 & 54.1456 & 54.0705 & 0.0014 \\
\hline 200162469 & 1-Aug-01 & 54.105 & 54.0705 & 0.0006 \\
\hline 200162466 & 1-Aug-01 & 54.0617 & 54.0705 & -0.0002 \\
\hline 200162468 & 1-Aug-01 & 54.0518 & 54.0705 & -0.0003 \\
\hline 200162471 & 11-Aug-01 & 54.1523 & 54.0705 & 0.0015 \\
\hline 200163507 & 20-Aug-01 & 54.1328 & 54.0705 & 0.0012 \\
\hline
\end{tabular}

\begin{tabular}{|l|l|l|l|l|l|l|l|l|}
\hline \multicolumn{9}{|c|}{ Relative Differences for Analysis } \\
\hline
\end{tabular}

Start ANOVA Here

\begin{tabular}{|l|r|}
\hline \multicolumn{2}{|c|}{ Uncertainty Estimates } \\
\hline & $0.000 \%$ \\
\hline Bias & $0.000 \%$ \\
\hline Systematic & $0.000 \%$ \\
\hline Random & \\
\hline
\end{tabular}

If Systematic Uncertainty is not significant, then Random Uncertainty $=20.00 \%$

The standard deviation of the relative differences $=\quad 0.00 \%$ 


\section{Completed T\&T Exercise Worksheet}

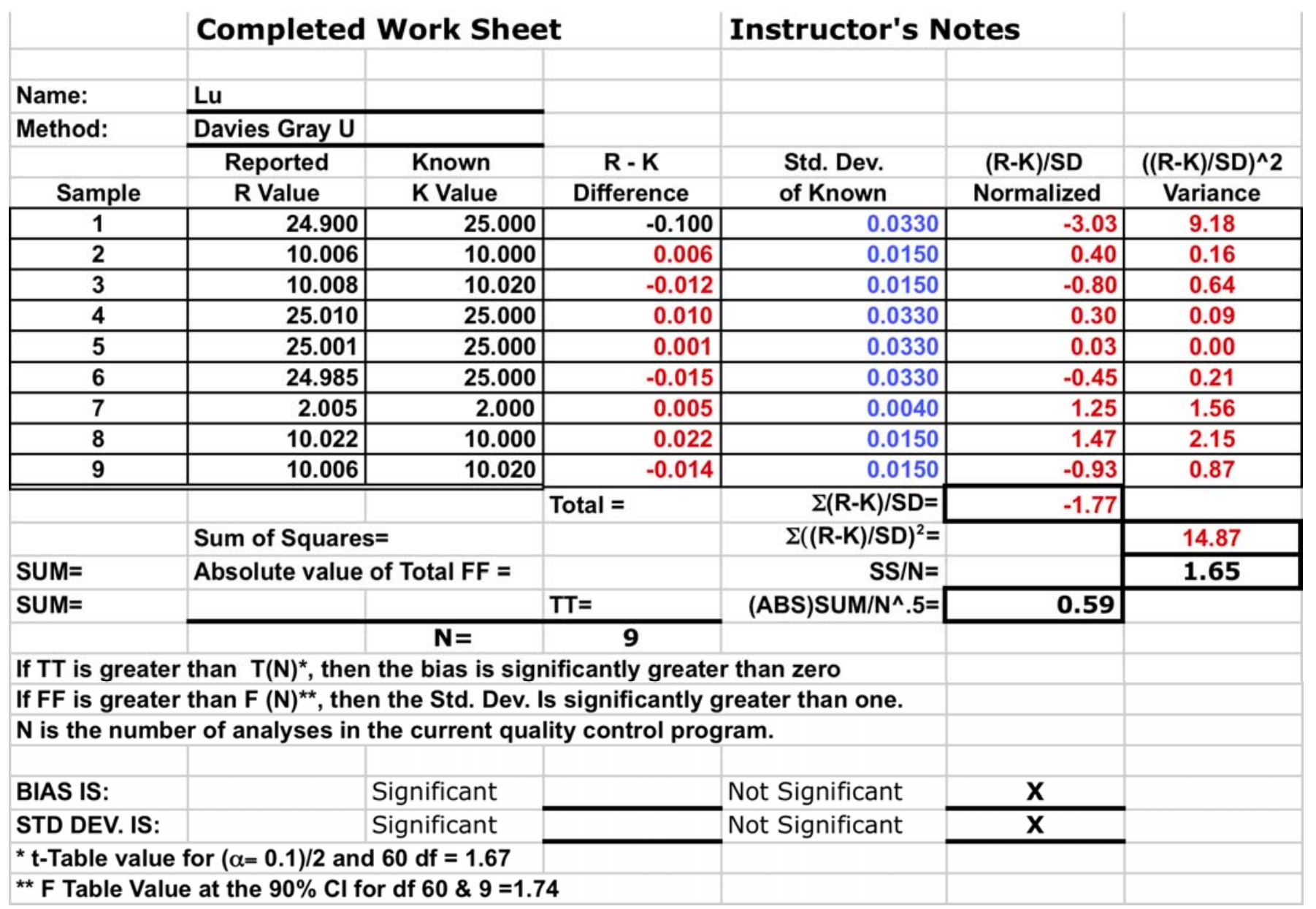




\section{Module 6}

\section{Proficiency Training \&}

Testing Program $\&$

Sample Exchange Programs 


\section{Learning Objectives}

- Demonstrate method for qualifying lab personnel

- Understand the statistical criteria for qualification

- Study an example of data collected in testing program

- Illustrate how sequential testing can expedite training

- Review US DOE proficiency testing program (sample exchange) 


\section{Department of Energy Order Training Plan Requirements}

- Training: Each facility shall have a documented plan for the training of measurement personnel. It shall specify training, qualification, and requalification requirements for each measurement method

- Qualification program shall ensure measurement personnel demonstrate acceptable levels of proficiency before performing measurements, and are re-qualified according to requirements in the training plan

- For destructive analysis of nuclear material, this proficiency shall be demonstrated, at a minimum, once per day for each method used that day 


\section{Elements of Training Program}

- Academic training: completion of high school or above, depending on responsibility required for the job

- Applicable experience

- On-the-job training in an analytical chemistry laboratory

- Special courses: seminars, factory instrumentation classes, or company training courses, shall be used to update and improve skills 


\section{Statistical Criteria for Testing}

- QC program's method standard deviation for each standard is used to normalize the trainee's difference between the measured \& known values

- A modified student's t-test is used to compare the trainee' $s$ absolute average bias with the table value at $90 \%$ confidence interval with 60 degrees of freedom

- The trainee's and method's average random errors are compared using an $F$ test

- Testing is done at the $90 \%$ confidence level for testing the trainee's bias and reproducibility

- 60 degrees of freedom for t-test value of 1.67 and

- 60 and 9 degrees of freedom for F-test value of 1.74 are used as the critical limits for their bias and precision. 


\section{Overview of Training Program}

- Method is demonstrated to Analyst

- Procedure is read \& applied

- Known standards are run until confident

- Testing involves analyzing 9 unknowns over 3 days

- Testing data evaluated against method's uncertainty 


\section{T\&T Evaluation Form:}

\section{Record trainee's measurands of unknown QC standards}

\begin{tabular}{|c|c|c|c|c|c|}
\hline \multirow{2}{*}{$\begin{array}{l}\text { Name: } \\
\text { Method: }\end{array}$} & \multicolumn{2}{|l|}{ George } & & & \\
\hline & \multicolumn{5}{|l|}{ Davies \& Gray U } \\
\hline & Reported & Known & $\mathbf{R}-\mathbf{K}$ & Std. Dev. & $(\mathrm{R}-\mathrm{K}) / \mathrm{SD}$ \\
\hline Sample & R Value & K Value & Difference & of Known & Normalized \\
\hline 1 & 1.952 & & & & \\
\hline 2 & 10.006 & & & & \\
\hline 3 & 10.008 & & & & \\
\hline 4 & 25.010 & & & & \\
\hline 5 & 25.001 & & & & \\
\hline 6 & 24.985 & & & & \\
\hline 7 & 2.005 & & & & \\
\hline 8 & 10.000 & & & & \\
\hline \multirow[t]{3}{*}{9} & 10.006 & & & & \\
\hline & & & Total $=$ & $\Sigma(\mathrm{R}-\mathrm{K}) / \mathrm{SD}=$ & \\
\hline & \multicolumn{2}{|c|}{ Sum of Squares= } & & $\Sigma((\mathrm{R}-\mathrm{K}) / \mathrm{SD})^{2}=$ & \\
\hline SUM= & \multicolumn{2}{|c|}{ Absolute value of Total FF $=$} & & $\mathrm{SS} / \mathrm{N}=$ & \\
\hline SUM= & & & $\mathrm{TT}=$ & SUM(ABS)N^.5= & \\
\hline \multirow{2}{*}{\multicolumn{6}{|c|}{$\begin{array}{l}\text { If TT is greater than } T(N)^{*} \text {, then the bias is significantly greater than zero } \\
\text { If } F F \text { is greater than } F(N)^{* *} \text {, then the Std. Dev. Is significantly greater than one. }\end{array}$}} \\
\hline & & & & & \\
\hline \multicolumn{6}{|c|}{$\mathrm{N}$ is the number of analyses in the current quality control program. } \\
\hline BIAS IS: & & Significant & & Not Significant & \\
\hline STD DEV. & IS: & Significant & & Not Significant & \\
\hline \multicolumn{4}{|c|}{${ }^{*} t-T a b l e ~ v a l u e ~ f o r ~(\alpha=0.1) / 2$ and $60 \mathrm{df}=1.67$} & & \\
\hline
\end{tabular}




\section{QC Standards Used for Training \& Testing}

\begin{tabular}{|c|c|c|}
\hline U Standards & $\begin{array}{c}\text { Standard } \\
\text { Uncertainty (U) }\end{array}$ & $\begin{array}{c}\text { Relative } \\
\text { Standard U }\end{array}$ \\
\hline Known & Std Dev & \% Std Dev \\
\hline 2.000 & 0.0040 & $\mathbf{0 . 2 0 \%}$ \\
\hline 10.000 & 0.0120 & $\mathbf{0 . 1 2} \%$ \\
\hline 10.010 & 0.0120 & $\mathbf{0 . 1 2} \%$ \\
\hline 25.000 & 0.0250 & $\mathbf{0 . 1 0 \%}$ \\
\hline
\end{tabular}

Module 6 


\section{T\&T Data Evaluation Form:}

\section{+ known values \& their standard uncertainties}

\begin{tabular}{|c|c|c|c|c|c|}
\hline \multirow{2}{*}{$\begin{array}{l}\text { Name: } \\
\text { Method: }\end{array}$} & \multicolumn{2}{|l|}{ George } & & & \multirow[b]{3}{*}{ (R-K)/SD } \\
\hline & \multicolumn{2}{|l|}{ Davies \& Gray U } & \multirow[b]{2}{*}{$\mathbf{R}-\mathbf{K}$} & \multirow{3}{*}{$\begin{array}{l}\text { Std. Dev. } \\
\text { of Known }\end{array}$} & \\
\hline & Reported & Known & & & \\
\hline Sample & R Value & K Value & Difference & & Normalized \\
\hline 1 & 1.992 & 2.000 & & 0.004 & \\
\hline 2 & 10.006 & 10.000 & & 0.012 & \\
\hline 3 & 10.008 & 10.010 & & 0.012 & \\
\hline 4 & 25.010 & 25.000 & & 0.025 & \\
\hline 5 & 25.001 & 25.000 & & 0.025 & \\
\hline 6 & 24.985 & 25.000 & & 0.025 & \\
\hline 7 & 2.005 & 2.000 & & 0.004 & \\
\hline 8 & 10.000 & 10.010 & & 0.012 & \\
\hline \multirow[t]{3}{*}{9} & 10.006 & 10.000 & & 0.012 & \\
\hline & & & Total $=$ & $\Sigma($ R-K)/SD= & \\
\hline & \multicolumn{2}{|c|}{ Sum of Squares= } & & $\Sigma((\mathrm{R}-\mathrm{K}) / \mathrm{SD})^{2}=$ & \\
\hline SUM= & \multicolumn{2}{|c|}{ Absolute value of Total FF = } & & $\mathbf{S S} / \mathbf{N}=$ & \\
\hline SUM= & & & $\mathrm{TT}=$ & ABS(SUM)/N ${ }^{\cdot 5}=$ & \\
\hline \multicolumn{6}{|c|}{ If TT is greater than $\mathbf{T}(\mathbf{N})^{*}$, then the bias is significantly greater than zero } \\
\hline \multicolumn{6}{|c|}{ If $F F$ is greater than $F(N)^{\star *}$, then the Std. Dev. Is significantly greater than one. } \\
\hline \multicolumn{5}{|c|}{$\mathbf{N}$ is the number of analyses in the current quality control program. } & \\
\hline BIAS IS: & & Significant & & Not Significant & \\
\hline \multicolumn{2}{|c|}{ STD DEV. IS: } & Significant & & Not Significant & \\
\hline \multirow{2}{*}{\multicolumn{4}{|c|}{$\begin{array}{l}{ }^{*} \mathrm{t}-\text { Table value for }(\alpha=0.1) / 2 \text { and } 60 \mathrm{df}=1.67 \\
{ }^{* *} \mathrm{~F} \text { Table Value at the } 90 \% \mathrm{Cl} \text { for df } 60 \& 9=\end{array}$}} & & \\
\hline & & & & & \\
\hline
\end{tabular}




\section{T\&T Data Evaluation Form: \\ Reported - known values = bias (difference)}

\begin{tabular}{|c|c|c|c|c|c|}
\hline Name: & George & & & & \\
\hline Method: & Davies \& Gray U & & Bias & & \\
\hline & Reported & Known & $\mathbf{R}-\mathbf{K}$ & Std. Dev. & $(R-K) / S D$ \\
\hline Sample & R Value & K Value & Difference & of Known & Normalized \\
\hline 1 & 1.992 & 2.000 & -0.008 & 0.0040 & \\
\hline 2 & 10.006 & 10.000 & 0.006 & 0.0120 & \\
\hline 3 & 10.008 & 10.010 & -0.002 & 0.0120 & \\
\hline 4 & 25.010 & 25.000 & 0.010 & 0.0250 & \\
\hline 5 & 25.001 & 25.000 & 0.001 & 0.0250 & \\
\hline 6 & 24.985 & 25.000 & -0.015 & 0.0250 & \\
\hline 7 & 2.005 & 2.000 & 0.005 & 0.0040 & \\
\hline 8 & 10.000 & 10.010 & -0.010 & 0.0120 & \\
\hline 9 & 10.006 & 10.000 & 0.006 & 0.0120 & \\
\hline & & & Total $=$ & $\Sigma(\mathrm{R}-\mathrm{K}) / \mathrm{SD}=$ & \\
\hline & Sum of Squares= & & & $\Sigma((\mathrm{R}-\mathrm{K}) / \mathrm{SD})^{2}=$ & \\
\hline SUM= & Absolute value of & f Total FF = & & $\mathbf{S S} / \mathbf{N}=$ & \\
\hline SUM= & & & TT $=$ & ABS(SUM)/N $\mathbf{N}^{\cdot 3}=$ & \\
\hline If $\mathrm{TT}$ is $\mathrm{gr}$ & ater than $T(N)^{*}$, th & hen the bias is & ignificantly gr & ter than zero & \\
\hline If $F F$ is $\mathbf{g r}$ & ater than $F(N)^{* *}, t$ & then the Std. De & v. Is significan & greater than ol & \\
\hline $\mathrm{N}$ is the $\mathrm{n}$ & mber of analyses & in the current $q$ & uality control pr & ogram. & \\
\hline BIAS IS: & & Significant & & Not Significant & \\
\hline STD DEV. & & Significant & & Not Significant & \\
\hline${ }^{*}$ t-Table $v$ & lue for $(\alpha=0.1) / 2$ & and $60 \mathrm{df}=1.67$ & & & \\
\hline${ }^{* *}$ F Table & Value at the $90 \% \mathrm{C}$ & I for df $60 \& 9=$ & .74 & & \\
\hline
\end{tabular}




\section{T\&T Data Evaluation Form: Bias values normalized by dividing by SD of U Stds.}

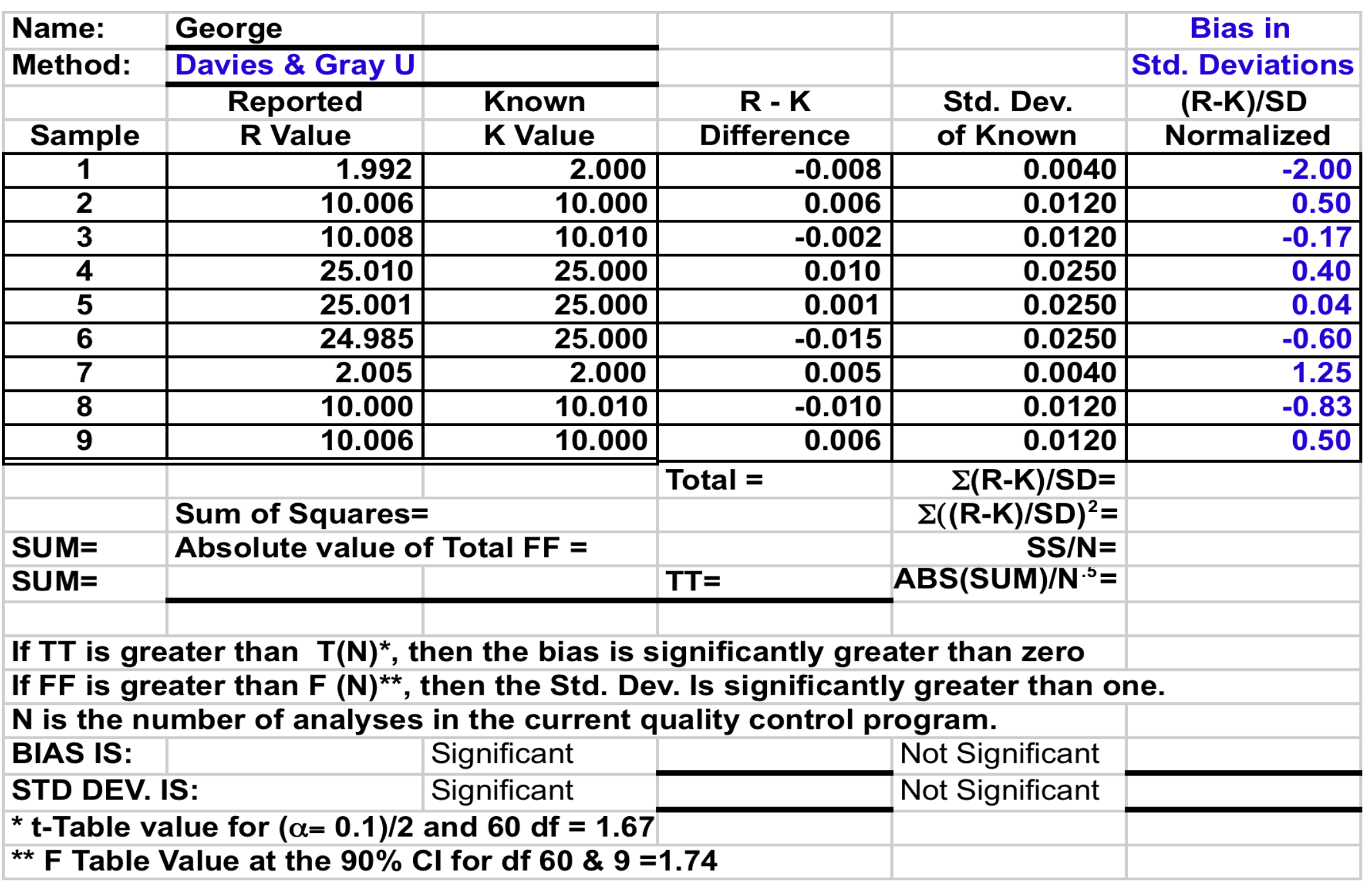




\section{T\&T Data Evaluation Form: Bias t-tested \& variance ratio F tested (Pass)}

\begin{tabular}{|c|c|c|c|c|c|}
\hline \multirow{3}{*}{$\begin{array}{l}\text { Name: } \\
\text { Method: } \\
\text { Sample }\end{array}$} & \multicolumn{2}{|l|}{ Lu } & \multirow[b]{3}{*}{$\begin{array}{c}\mathbf{R}-\mathbf{K} \\
\text { Difference }\end{array}$} & \multirow[b]{3}{*}{$\begin{array}{l}\text { Std. Dev. } \\
\text { of Known }\end{array}$} & \multirow[b]{3}{*}{$\begin{array}{l}\text { (R-K)/SD } \\
\text { Normalized }\end{array}$} \\
\hline & \multicolumn{2}{|l|}{ Davies Gray U } & & & \\
\hline & $\begin{array}{c}\text { Reported } \\
\text { R Value }\end{array}$ & $\begin{array}{l}\text { Known } \\
\text { K Value }\end{array}$ & & & \\
\hline 1 & 24.900 & 25.000 & -0.100 & 0.0330 & -3.03 \\
\hline 2 & 10.006 & 10.000 & 0.006 & 0.0150 & 0.40 \\
\hline 3 & 10.008 & 10.020 & -0.012 & 0.0150 & -0.80 \\
\hline 4 & 25.010 & 25.000 & 0.010 & 0.0330 & 0.30 \\
\hline 5 & 25.001 & 25.000 & 0.001 & 0.0330 & 0.03 \\
\hline 6 & 24.985 & 25.000 & -0.015 & 0.0330 & -0.45 \\
\hline 7 & 2.005 & 2.000 & 0.005 & 0.0040 & 1.25 \\
\hline 8 & 10.022 & 10.000 & 0.022 & 0.0150 & 1.46 \\
\hline 9 & 10.006 & 10.020 & -0.014 & 0.0150 & -0.93 \\
\hline $\begin{array}{l}\text { SUM= } \\
\text { SUM= }\end{array}$ & $\begin{array}{l}\text { Sum of Square } \\
\text { Absolute value }\end{array}$ & Total FF = & Total $=$ & $\begin{array}{r}\Sigma(\mathrm{R}-\mathrm{K}) / \mathrm{SD}= \\
\Sigma((\mathrm{R}-\mathrm{K}) / \mathrm{SD})^{2}= \\
\mathrm{SS} / \mathrm{N}= \\
(\mathrm{ABS}) \mathrm{SU} \mathbf{M N}^{\cdot 5}=\end{array}$ & $\begin{array}{r}-1.77 \\
14.86 \\
1.65 \\
0.59\end{array}$ \\
\hline
\end{tabular}

If TT is greater than $T(N)^{*}$, then the bias is significantly greater than zero If $F F$ is greater than $F(N)^{* *}$, then the Std. Dev. Is significantly greater than one.

$\mathrm{N}$ is the number of analyses in the current quality control program.

BIAS IS:

STD DEV. IS:

Significant

* $t$-Table value for $(\alpha=0.1) / 2$ and $60 \mathrm{df}=1.6 \overline{7}$

** $F$ Table Value at the $90 \% \mathrm{Cl}$ for df $60 \& 9=1.74$
Not Significant

Not Significant

$(-0.91<1.67)$

$(0.3<1.74)$

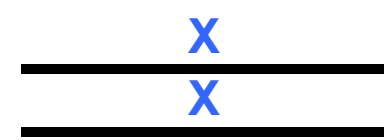

Module 6- 12 


\begin{tabular}{|c|c|c|c|c|c|}
\hline \multirow{2}{*}{$\begin{array}{l}\text { Name: } \\
\text { Method: }\end{array}$} & George & & & & \\
\hline & \multicolumn{5}{|l|}{ Davies \& Gray U } \\
\hline & Reported & Known & $\mathbf{R}-\mathbf{K}$ & Std. Dev. & (R-K)/SD \\
\hline Sample & R Value & K Value & Difference & of Known & Normalized \\
\hline 1 & 1.992 & 2.000 & -0.008 & 0.0040 & -2.00 \\
\hline 2 & 10.006 & 10.000 & 0.006 & 0.0120 & 0.50 \\
\hline 3 & 10.008 & 10.010 & -0.002 & 0.0120 & -0.17 \\
\hline 4 & 25.010 & 25.000 & 0.010 & 0.0250 & 0.40 \\
\hline 5 & 25.001 & 25.000 & 0.001 & 0.0250 & 0.04 \\
\hline 6 & 24.985 & 25.000 & -0.015 & 0.0250 & -0.60 \\
\hline 7 & 1.990 & 2.000 & -0.010 & 0.0040 & -2.50 \\
\hline 8 & 9.988 & 10.010 & -0.022 & 0.0120 & -1.83 \\
\hline \multirow[t]{3}{*}{9} & 10.006 & 10.000 & 0.006 & 0.0120 & 0.50 \\
\hline & & & Total $=$ & $\Sigma(\mathrm{R}-\mathrm{K}) / \mathrm{SD}=$ & -5.66 \\
\hline & \multicolumn{2}{|c|}{ Sum of Squares $=$} & & $\Sigma((\mathrm{R}-\mathrm{K}) / \mathrm{SD})^{2}=$ & 14.65 \\
\hline SUM= & \multicolumn{2}{|c|}{ Absolute value of Total FF = } & & $\mathbf{S S} / \mathbf{N}=$ & 1.63 \\
\hline \multirow{2}{*}{ SUM= } & & & TT $=$ & ABS(SUM)/N $\mathbf{N}^{5}=$ & 1.89 \\
\hline & \multicolumn{3}{|c|}{ Tecnician must retest before qualified. } & & \\
\hline \multicolumn{6}{|c|}{ If TT is greater than $T(N)^{*}$, then the bias is significantly greater than zero } \\
\hline \multicolumn{6}{|c|}{ If $F F$ is greater than $F(N)^{\star *}$, then the Std. Dev. Is significantly greater than one. } \\
\hline \multicolumn{6}{|c|}{$\mathrm{N}$ is the number of analyses in the current quality control program. } \\
\hline BIAS IS: & & Significant & $\mathbf{X}$ & \multirow{3}{*}{$\begin{array}{r}\text { Not Significant } \\
\text { Not Significant } \\
(1.89>1.67)\end{array}$} & \\
\hline \multirow{2}{*}{\multicolumn{3}{|c|}{ 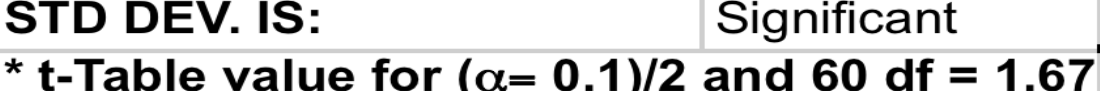 }} & & & $\overline{\mathbf{X}}$ \\
\hline & & & & & \\
\hline \multicolumn{4}{|c|}{ ** F Table Value at the $90 \% \mathrm{Cl}$ for df $60 \& 9=1.74$} & $(1.63<1.74)$ & \\
\hline
\end{tabular}




\section{Class Exercise in Evaluating Testing Data}

- The next slide has the results of a trainee listed.

- Use your calculator to evaluate his results and determine if he has adequate skill in making D\&G measurements to be qualified by doing the following:

- In the R-K column subtract the known value from the reported value to get the deviation between the two values

- In the (R-K)/SD column divide the difference by the SD to get all of the differences expressed as SDs.

- Square each of these values \& record them in the last column

- Next sum the last two columns to get the total bias \& variance

- By dividing the $\Sigma(R-K) / S D$ by $9^{\wedge} .5$ gives an average deviation or bias whose absolute value is compared to a t-table value

- By dividing the SS by 9 an average variance is calculated that can be compared to a $F$ table value for a precision test 


\section{T\&T Qualification Exercise Calculate: Use your calculator to solve}

\begin{tabular}{|c|c|c|c|c|c|c|}
\hline Name: & Lu & & & & & \\
\hline Method: & Davies Gray U & & & & & \\
\hline Sample & $\begin{array}{c}\text { Reported } \\
\text { R Value }\end{array}$ & $\begin{array}{l}\text { Known } \\
\text { K Value }\end{array}$ & $\begin{array}{c}\text { R - K } \\
\text { Difference }\end{array}$ & $\begin{array}{l}\text { Std. Dev. } \\
\text { of Known }\end{array}$ & $\begin{array}{c}\text { (R-K)/SD } \\
\text { Normalized }\end{array}$ & $\begin{array}{c}((\mathrm{R}-\mathrm{K}) / \mathrm{SD})^{\wedge} \mathbf{2} \\
\text { Variance }\end{array}$ \\
\hline 1 & 24.900 & 25.000 & & 0.0330 & & \\
\hline 2 & 10.006 & 10.000 & & 0.0150 & & \\
\hline 3 & 10.008 & 10.020 & & 0.0150 & & \\
\hline 4 & 25.010 & 25.000 & & 0.0330 & & \\
\hline 5 & 25.001 & 25.000 & & 0.0330 & & \\
\hline 6 & 24.985 & 25.000 & & 0.0330 & & \\
\hline 7 & 2.005 & 2.000 & & 0.0040 & & \\
\hline 8 & 10.022 & 10.000 & & 0.0150 & & \\
\hline 9 & 10.006 & 10.020 & & 0.0150 & & \\
\hline & & & Total = & $\Sigma(\mathrm{R}-\mathrm{K}) / \mathrm{SD}=$ & & \\
\hline & Sum of Square & & & $\Sigma((\mathrm{R}-\mathrm{K}) / \mathrm{SD})^{2}=$ & & \\
\hline SUM= & Absolute value & Total FF = & & $\mathrm{SS} / \mathrm{N}=$ & & \\
\hline SUM= & & & $\mathbf{T T}=$ & $(\mathrm{ABS}) \mathrm{SUM} / \mathrm{N}^{\wedge} .5=$ & & \\
\hline
\end{tabular}

If $T T$ is greater than $T(N)^{*}$, then the bias is significantly greater than zero

If $F F$ is greater than $F(N)^{* *}$, then the Std. Dev. Is significantly greater than one.

$\mathrm{N}$ is the number of analyses in the current quality control program.

BIAS IS:

STD DEV. IS:
Significant

Significant
Not Significant Not Significant 


\section{Performance Testing Required Before Qualification Attained}

- Technician qualified if his $t$ and $F$ values are less than the statistical limits established (slide 5)

- Technician must re-test if either value exceeds the limits

- If a manual system is used in the testing program, significant time can lapse between the time the trainee submits his testing data and the time it is evaluated and returned to management

- An automated program can be used to evaluate testing data and greatly reduce the training and testing time 


\section{Sequential Tests of Trainee Precision and Accuracy-1}

- Why Sequential Testing?

- A sequential test can be more efficient (in terms of number of required tests) than a preset number of samples

- When is a Sequential Test Preferred over a preset number of samples?

- If the technician precision and accuracy is generally much better or much worse than the acceptable levels of precision and accuracy, a sequential test generally is preferred to a preset number of samples, because an early decision is likely

- If a decision cannot be easily and quickly made between successive stages (measurements), then a preset number of samples is preferable 


\section{Sequential Tests of Trainee Precision and Accuracy-2}

- Preset number of samples

- A preset number of measurements must be completed. Then a rule is applied to decide between a hypothesis (A) that the technician has acceptable precision and accuracy and a hypothesis (B) that the technician does not have acceptable precision or accuracy

- Sequential Test

- The sample size is not preset. The sample size varies from application to application. After each measurement a rule is defined to decide whether $(A)$ that the technician has acceptable precision and accuracy, a hypothesis $(B)$ that the technician does not have acceptable precision or accuracy, or $(C)$ insufficient information exists to make a determination between $(A)$ and $(B)$ 


\section{Sequential Tests of Trainee Precision and Accuracy-3}

- Can a sequential test fail to terminate?

- No, but the number of measurement could be very large in some of the applications

- This is not an issue with a truncated sequential test. A truncated sequential test is forced to terminate after a preset maximum

- What is the technical basis for the technician accuracy and precision tests?

- The technician accuracy and precision tests are based on a truncated sequential probability ratio tests (SPRT). An SPRT minimizes the expected sample size under the null and alternative hypotheses for stated probabilities of false positives and false negatives 


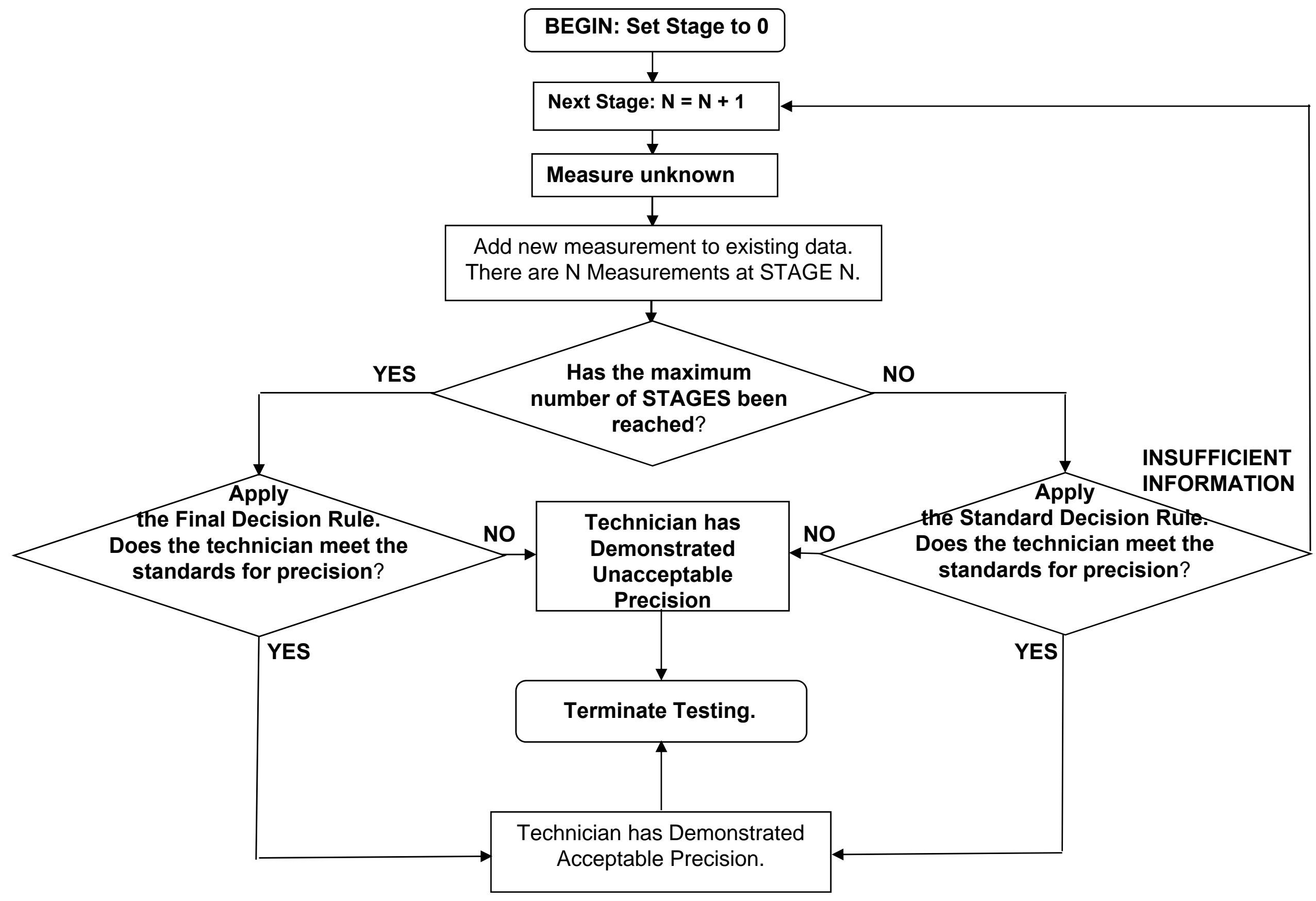

Module 6 - 20 


\section{Graphical Procedure for Sequential Testing}

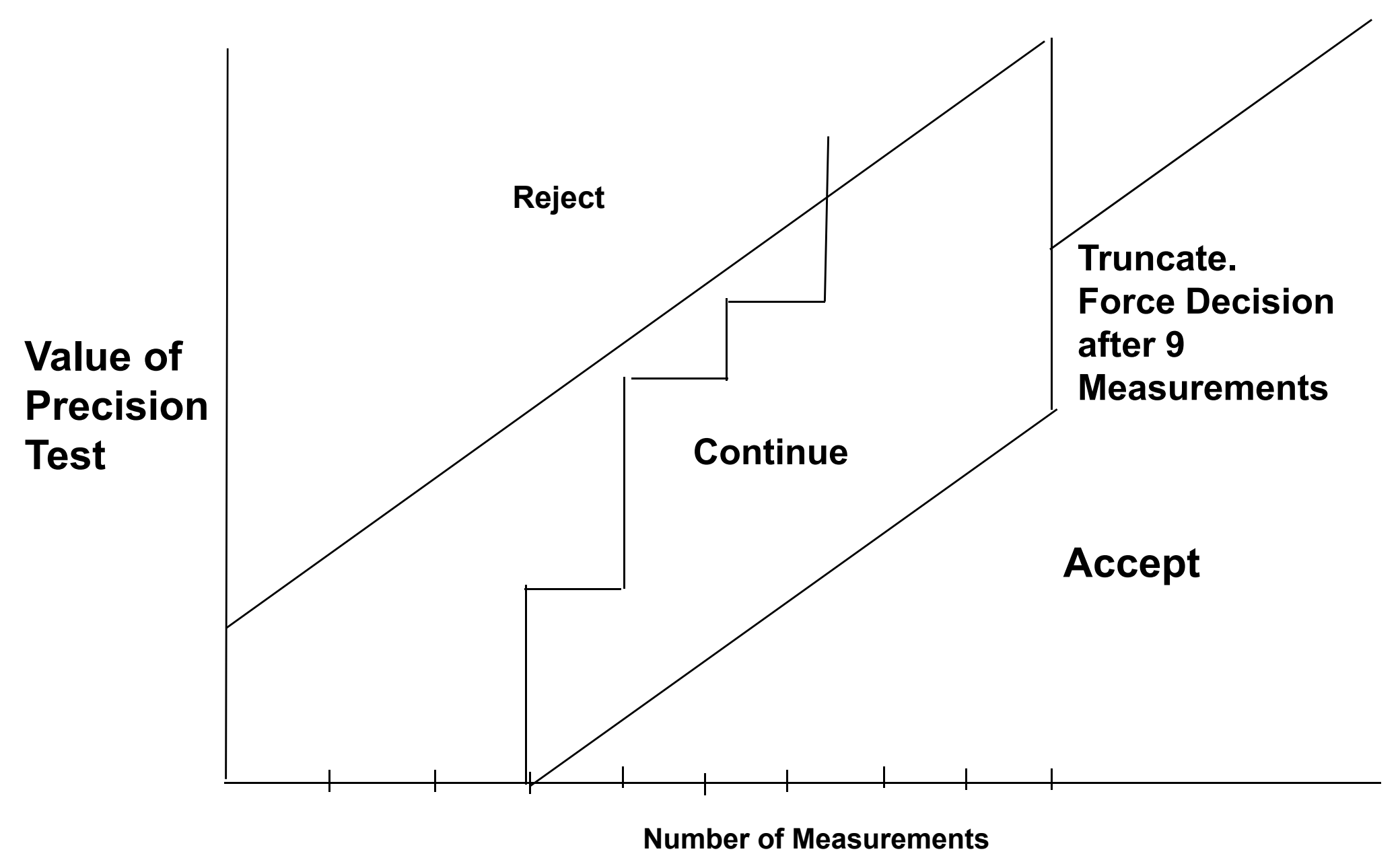

Module 6 - 21 


\section{Current T\&T Program}

- Training and practice is the same

- A computer program evaluates the testing data as it is collected

- If a value exceeds a critical limit they must start over

- If the trainee demonstrates excellent results on the first 6 samples they qualify

- This improvement significantly reduces training time and allows the lab to use the trainee to provide analytical support sooner 


\section{Training \& Testing Program Summary}

- Trainees must perform multiple measurements within specified bias and precision limits to demonstrate their measurement proficiency

- Manual testing systems often delay qualifying trainees and waste time in qualifying them to make routine laboratory measurements

- Automated evaluation of testing data using sequential testing enhances the training and testing qualification process 


\section{Sample Exchange Programs}

- ITVs utilized data from 3 international programs:

- Safeguards Measurement Evaluation Program (SMEP)

- Regular European Interlaboratory Measurement Evaluation Program (REIMEP)

- Evaluation de la Qualité des Resultats d'Analyses dans I' Industrie Nucléaire, (EQRAIN)

- ANSI N15.51-2007 - Measurement Control Program - Analytical Chemistry Laboratory *4.4.6 Measurement of Interlaboratory Comparison Program Samples.

- Data from participation in appropriate interlaboratory comparison programs should be used to provide independent verification of internal analytical quality control 


\section{US Department of Energy's (DOE) New Brunswick Laboratory (NBL)}

- NBL manages interlaboratory measurement evaluation programs to provide independent validation of facility nuclear material measurement quality/capabilities.

- The program includes:

- preparation, characterization, packaging and distribution of samples to participating laboratories

- The receipt, recording, statistical evaluation and reporting of the measurement data from the participating laboratories are the products of the program

- Most laboratories perform analyses on a quarterly or semiannual frequency. No charges to DOE labs. Non-DOE labs participate on a cost-recovery basis 


\section{NBL Sample Exchange Programs}

- NBL has conducted interlaboratory measurement comparison programs involving more than 50 laboratories from more than 15 countries

- Providing independent oversight of the effectiveness of measurement systems used for safeguards materials accountability and of the quality of measured values

- Specific evaluation programs have also involved the preparation of materials and evaluation of analyses by commercial analytical laboratories in establishing control programs for U-235 in fuel loadings 


\section{Two Proficiency Testing Programs}

- The destructive analyses results are evaluated in the Safeguards Measurement Evaluation (SME) Program

- The non-destructive analyses results in the Calorimetric Exchange (CALEX) Program

- Test samples of uranium and plutonium bearing materials, sent by NBL, are analyzed in safeguards laboratories by procedures routinely used in material accountability measurements

- The results are evaluated by NBL for accuracy, precision, day-to-day variation, and compliance to method/material specific International Target Values (ITVs). Reports \& recommendations are sent to labs 


\section{Elemental Uranium in UNH Test Samples by Davies \& Gray Titration}

< Results from 6 participants to be shown on next 3 slides > 


\section{Plot of Participant's Random Error \& ITV Target Values for U in UNH by D\&G Method}

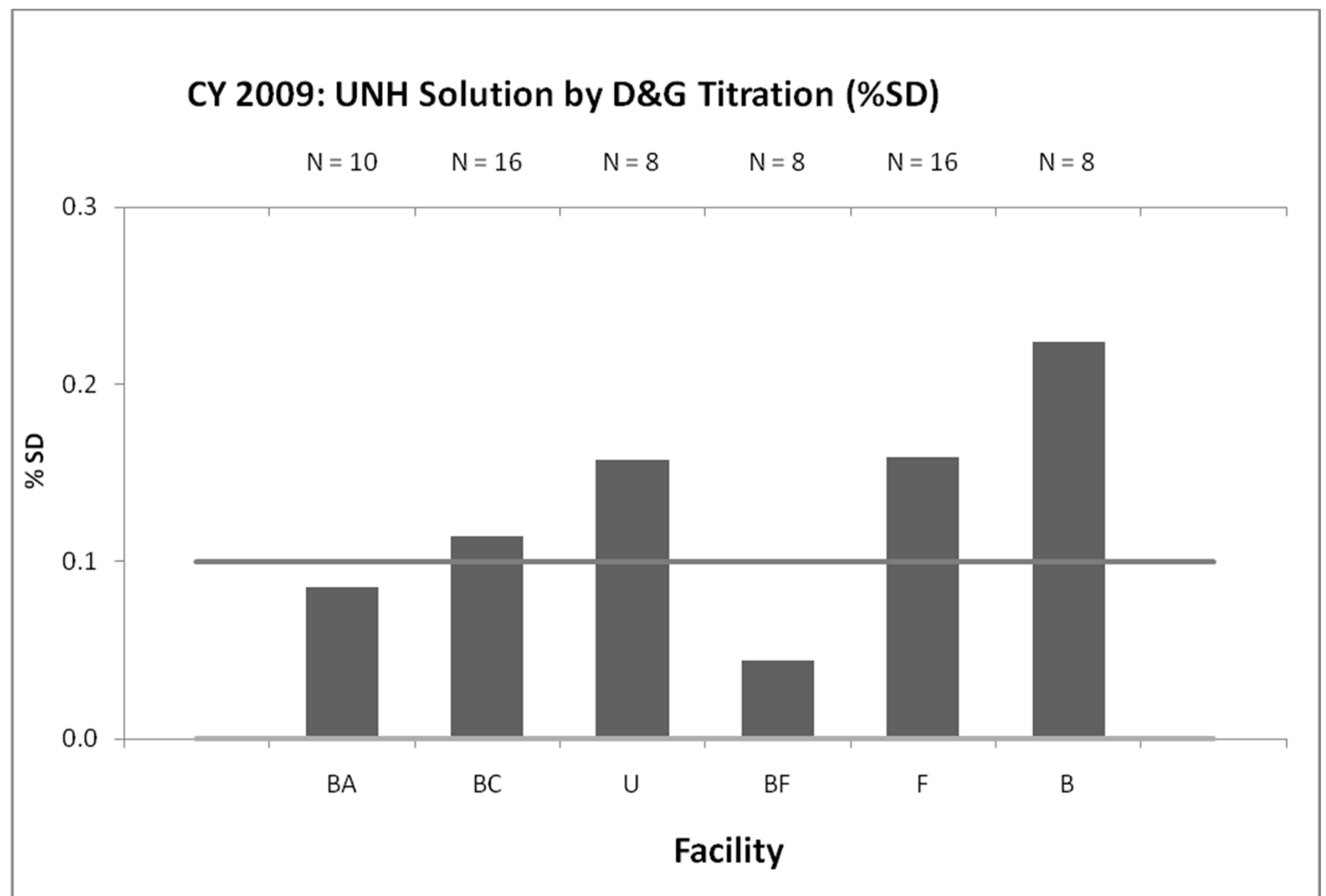




\section{Elemental Uranium in UNH by D\&G}

\begin{tabular}{cccccc}
\hline Lab Code & Mean\% RD & SD & N & \multicolumn{2}{c}{ ITV Compliance } \\
\hline BA & & & & $\mathrm{U}(\mathbf{s})=0.1$ & $\mathrm{U}(\mathbf{r})=\mathbf{0 . 1}$ \\
BC & -0.130 & 0.085 & 10 & No & Yes \\
U & -0.117 & 0.158 & 8 & No & No \\
BF & 0.064 & 0.044 & 8 & Yes & Yes \\
F & -0.75 & 0.159 & 16 & Yes & No \\
B & -0.018 & 0.224 & 8 & Yes & No \\
\hline
\end{tabular}




\section{Mean \% RD in elemental uranium Determination in UNH test samples by D\&G titration compared to I AEA I TVs}

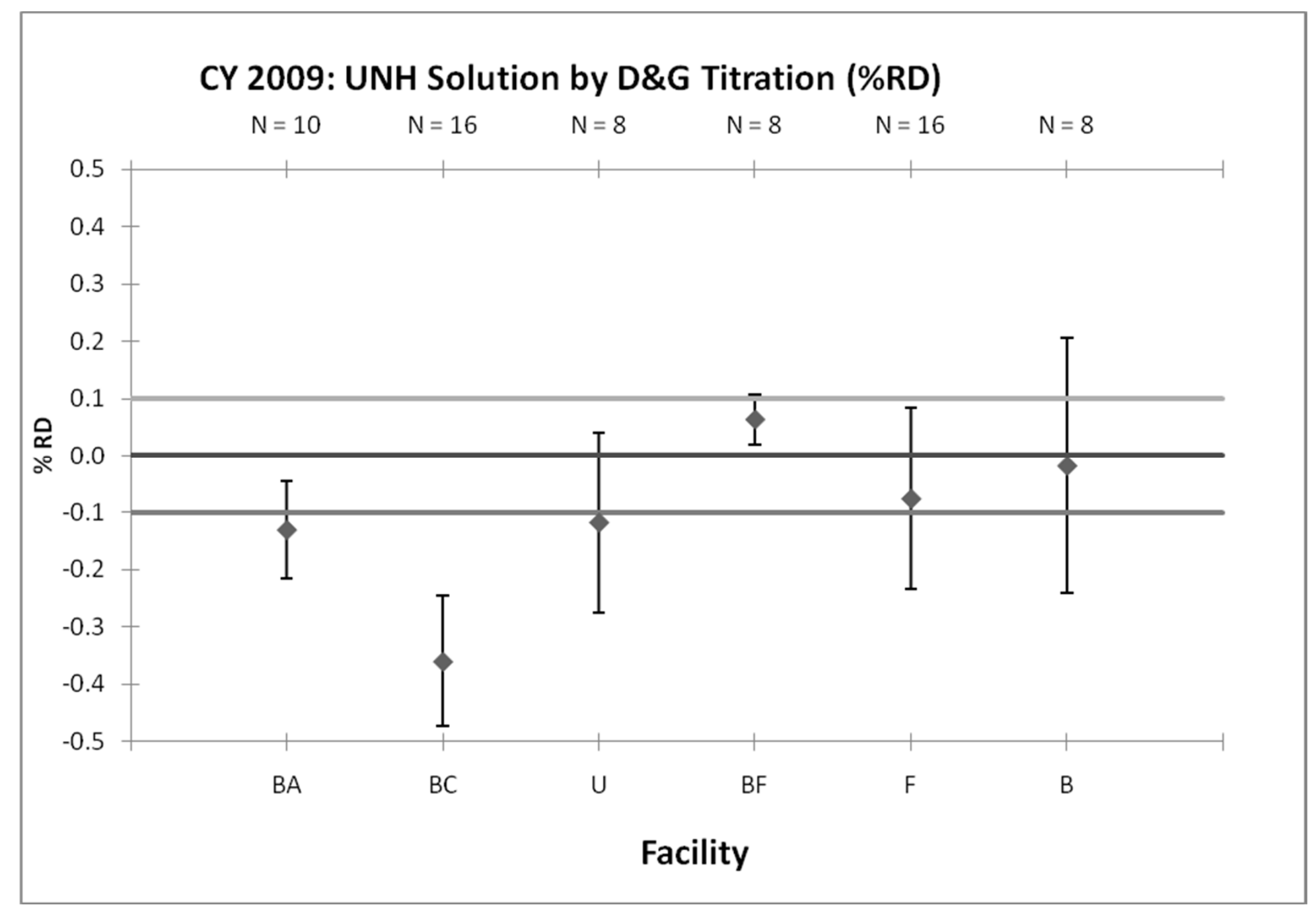

Module 6 - 31 


\section{Certified Reference Materials at NBL}

The following materials are typically available for the indicated analyses:

- Uranyl nitrate solutions for $U$ concentration

- Uranyl nitrate solutions for 235U enrichment

- UO2 pellets for U concentration and enrichment

- UO3 powder for U concentration

- UF6 (normal or low-enriched) solid for U concentration

- UF6 (low-enriched) solid for 235U enrichment

- Plutonium sulfate for isotopic abundances and IDMS 


\section{Completed T\&T Exercise Worksheet}

\section{See solution}




\section{Discussion Topic: Measurement Quality of the Laboratory and the trainee Mr. Lu.}

Review the slides to see:

- what the relative uncertainty values each standard had for the standards of the U D\&G method and

- the $t$ and $F$ values for Mr. Lu's Training and Testing results.

The International Target Values for U D\&G values for Bias and precision (SD) is $0.1 \%$.

Does Mr. Lu bias or precision indicate that he could make measurements that would meet these ITVs?

Why? 


\section{Summary}

- Demonstrated method for qualifying lab personnel

- Described the statistical criteria for qualification

- Reviewed an example of data collected in testing program

- Illustrated how sequential testing can expedite training

- Reviewed US DOE proficiency testing program (sample exchange) 
June 13, 2012 Revision

\section{Technician Training and Testing Results Evaluation Exercise:}

Evaluate the data below as follows:

1. Subtract the known value from the reported value and record in column R-K

2. Divide these values by the corresponding Standard Deviation (SD) and record in the last column.

3. Sum the values in column (R-K)/SD and record in first cell below the column as $\Sigma(\mathbf{R}-\mathbf{K}) / \mathbf{S D}=$ below.

4. Square each value in the (R-K)/SD column and sum and record them as $\Sigma(\mathbf{R}-\mathbf{K}) / \mathbf{S D})^{2}=$ below .

5. Divide the total from line 4 above by 9 , which is the number of test samples and record as SS/N.

6. Divide the absolute value in step 3 above by the square root of 9 and record as (ABS)SUM(ABS)N^.5=

7. Test average bias ((ABS)SUM(ABS)N^.5=) to see if it is less the t-table value of 1.67 and Pass or Fail.

8. Test the precision (SS/N) to if it is less the F-table value of 1.74 and Pass or Fail.

Name: $\quad$ Lu

Method: $\quad$ Davies Gray U

\begin{tabular}{|c|c|c|c|c|c|c|}
\hline Sample & $\begin{array}{l}\text { Reported } \\
\text { R Value }\end{array}$ & $\begin{array}{l}\text { Known } \\
\text { K Value }\end{array}$ & $\begin{array}{c}\mathbf{R}-\mathbf{K} \\
\text { Difference }\end{array}$ & $\begin{array}{l}\text { Std. Dev. } \\
\text { of Known }\end{array}$ & $\begin{array}{c}(\mathrm{R}-\mathrm{K}) / \mathrm{SD} \\
\text { Normalized }\end{array}$ & $\begin{array}{c}((\mathrm{R}-\mathrm{K}) / \mathrm{SD})^{\wedge} 2 \\
\text { Variance }\end{array}$ \\
\hline 1 & 24.900 & 25.000 & & 0.0330 & & \\
\hline 2 & 10.006 & 10.000 & & 0.0150 & & \\
\hline 3 & 10.008 & 10.020 & & 0.0150 & & \\
\hline 4 & 25.010 & 25.000 & & 0.0330 & & \\
\hline 5 & 25.001 & 25.000 & & 0.0330 & & \\
\hline 6 & 24.985 & 25.000 & & 0.0330 & & \\
\hline 7 & 2.005 & 2.000 & & 0.0040 & & \\
\hline 8 & 10.022 & 10.000 & & 0.0015 & & \\
\hline 9 & 10.006 & 10.020 & & 0.0015 & & \\
\hline \multirow{4}{*}{$\begin{array}{l}\text { SUM= } \\
\text { SUM= }\end{array}$} & & & Total $=$ & $\Sigma(\mathrm{R}-\mathrm{K}) / \mathrm{SD}=$ & & \\
\hline & \multicolumn{2}{|c|}{ Sum of Squares= } & & $\Sigma((\mathrm{R}-\mathrm{K}) / \mathrm{SD})^{2}=$ & & \\
\hline & \multicolumn{2}{|c|}{ Absolute value of Total FF = } & & $\mathrm{SS} / \mathrm{N}=$ & & \\
\hline & & & $\mathrm{TT}=$ & $(A B S) S U M / N^{\wedge} .5=$ & & \\
\hline
\end{tabular}

If $\mathrm{TT}$ is greater than $\mathrm{T}(\mathrm{N})^{*}$, then the bias is significantly greater than zero

If $F F$ is greater than $F(N)^{* *}$, then the Std. Dev. Is significantly greater than one.

$\mathrm{N}$ is the number of analyses in the current quality control program.

BIAS IS:

STD DEV.

IS:

* $t$-Table value for $(\alpha=0.1) / 2$ and $60 \mathrm{df}=1.67$

** $\mathrm{F}$ Table Value at the $90 \% \mathrm{Cl}$ for df $60 \& 9=1.74$

Significant

Significant
Standards

1

2

3

4
Not Significant

Not Significant

$\mathrm{mgU} / \mathrm{gSol}$

Concentration

2.000

10.000

10.020

25.000
Method RSD Method SD

$0.20 \% \quad 0.004$

$0.15 \% \quad 0.015$

$0.15 \% \quad 0.015$

$0.13 \% \quad 0.033$ 
June 13, 2012 Revision

Completed Worksheet for Instructor

\section{Completed Work Sheet I nstructor's Notes}

\begin{tabular}{|c|c|c|c|c|c|c|}
\hline \multirow{2}{*}{$\begin{array}{l}\text { Name: } \\
\text { Method: }\end{array}$} & \multicolumn{2}{|l|}{ Lu } & \multirow[b]{3}{*}{$\begin{array}{c}\mathbf{R}-\mathrm{K} \\
\text { Difference }\end{array}$} & \multirow[b]{3}{*}{$\begin{array}{l}\text { Std. Dev. } \\
\text { of Known }\end{array}$} & \multirow[b]{3}{*}{$\begin{array}{c}\text { (R-K)/SD } \\
\text { Normalized }\end{array}$} & \multirow[b]{3}{*}{$\begin{array}{c}((\mathrm{R}-\mathrm{K}) / \mathrm{SD})^{\wedge} 2 \\
\text { Variance }\end{array}$} \\
\hline & \multicolumn{2}{|l|}{ Davies Gray U } & & & & \\
\hline Sample & $\begin{array}{l}\text { Reported } \\
\text { R Value }\end{array}$ & $\begin{array}{l}\text { Known } \\
\text { K Value }\end{array}$ & & & & \\
\hline 1 & 24.900 & 25.000 & -0.100 & 0.0330 & -3.03 & 9.18 \\
\hline 2 & 10.006 & 10.000 & 0.006 & 0.0150 & 0.40 & 0.16 \\
\hline 3 & 10.008 & 10.020 & -0.012 & 0.0150 & -0.80 & 0.64 \\
\hline 4 & 25.010 & 25.000 & 0.010 & 0.0330 & 0.30 & 0.09 \\
\hline 5 & 25.001 & 25.000 & 0.001 & 0.0330 & 0.03 & 0.00 \\
\hline 6 & 24.985 & 25.000 & -0.015 & 0.0330 & -0.45 & 0.21 \\
\hline 7 & 2.005 & 2.000 & 0.005 & 0.0040 & 1.25 & 1.56 \\
\hline 8 & 10.022 & 10.000 & 0.022 & 0.0150 & 1.47 & 2.15 \\
\hline 9 & 10.006 & 10.020 & -0.014 & 0.0150 & -0.93 & 0.87 \\
\hline \multirow{4}{*}{$\begin{array}{l}\text { SUM= } \\
\text { SUM= }\end{array}$} & & Total $=$ & $\Sigma(\mathrm{R}-\mathrm{K}) / \mathrm{SD}=$ & -1.77 & \\
\hline & \multirow{3}{*}{\multicolumn{2}{|c|}{$\begin{array}{l}\text { Sum of Squares= } \\
\text { Absolute value of Total FF = }\end{array}$}} & & $\Sigma((\mathrm{R}-\mathrm{K}) / \mathrm{SD})^{2}=$ & & 14.87 \\
\hline & & & $F F=$ & $\mathrm{SS} / \mathrm{N}=$ & & 1.65 \\
\hline & & & $\mathrm{TT}=$ & (ABS)SUM/N^.5= & 0.59 & \\
\hline
\end{tabular}

If $T T$ is greater than $T(N)^{*}$, then the bias is significantly greater than zero If $\mathrm{FF}$ is greater than $\mathrm{F}(\mathrm{N})^{* *}$, then the Std. Dev. Is significantly greater than one. $\mathrm{N}$ is the number of analyses in the current quality control program.

\section{BIAS IS:}

STD DEV. IS:

* $\mathrm{t}$-Table value for $(\alpha=0.1) / 2$ and $60 \mathrm{df}=1.67$

Significant

Significant

** F Table Value at the $90 \% \mathrm{Cl}$ for df $60 \& 9=1.74$
Not Significant Not Significant

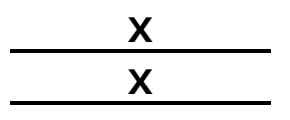

Ave SD $=\quad=(1.65) \wedge .5$ 1.29

The Excel worksheet does the calculations for this exercise.

Note the Normalized deviations to see how accurate the measurements were. It appears the first sample analyzed was out by 3 standard deviations. However, after that Mr. Lu did much better and all the rest of the samples were within two standard deviations, which is to be expected in a normal distribution. 
June 13, 2012 Revision

\section{Technician Training and Testing Results Evaluation Exercise:}

Evaluate the data below as follows:

1. Subtract the known value from the reported value and record in column R-K

2. Divide these values by the corresponding Standard Deviation (SD) and record in the last column.

3. Sum the values in column (R-K)/SD and record in first cell below the column as $\Sigma(\mathbf{R}-\mathbf{K}) / \mathbf{S D}=$ below.

4. Square each value in the (R-K)/SD column and sum and record them as $\Sigma(\mathbf{R}-\mathbf{K}) / \mathbf{S D})^{2}=$ below .

5. Divide the total from line 4 above by 9 , which is the number of test samples and record as SS/N.

6. Divide the absolute value in step 3 above by the square root of 9 and record as (ABS)SUM(ABS)N^.5=

7. Test average bias ((ABS)SUM(ABS)N^.5=) to see if it is less the t-table value of 1.67 and Pass or Fail.

8. Test the precision (SS/N) to if it is less the F-table value of 1.74 and Pass or Fail.

Name: $\quad$ Lu

Method: $\quad$ Davies Gray U

\begin{tabular}{|c|c|c|c|c|c|c|}
\hline Sample & $\begin{array}{l}\text { Reported } \\
\text { R Value }\end{array}$ & $\begin{array}{l}\text { Known } \\
\text { K Value }\end{array}$ & $\begin{array}{c}\mathbf{R}-\mathbf{K} \\
\text { Difference }\end{array}$ & $\begin{array}{l}\text { Std. Dev. } \\
\text { of Known }\end{array}$ & $\begin{array}{c}(\mathrm{R}-\mathrm{K}) / \mathrm{SD} \\
\text { Normalized }\end{array}$ & $\begin{array}{c}((\mathrm{R}-\mathrm{K}) / \mathrm{SD})^{\wedge} 2 \\
\text { Variance }\end{array}$ \\
\hline 1 & 24.900 & 25.000 & & 0.0330 & & \\
\hline 2 & 10.006 & 10.000 & & 0.0150 & & \\
\hline 3 & 10.008 & 10.020 & & 0.0150 & & \\
\hline 4 & 25.010 & 25.000 & & 0.0330 & & \\
\hline 5 & 25.001 & 25.000 & & 0.0330 & & \\
\hline 6 & 24.985 & 25.000 & & 0.0330 & & \\
\hline 7 & 2.005 & 2.000 & & 0.0040 & & \\
\hline 8 & 10.022 & 10.000 & & 0.0015 & & \\
\hline 9 & 10.006 & 10.020 & & 0.0015 & & \\
\hline \multirow{4}{*}{$\begin{array}{l}\text { SUM= } \\
\text { SUM= }\end{array}$} & & & Total $=$ & $\Sigma(\mathrm{R}-\mathrm{K}) / \mathrm{SD}=$ & & \\
\hline & \multicolumn{2}{|c|}{ Sum of Squares= } & & $\Sigma((\mathrm{R}-\mathrm{K}) / \mathrm{SD})^{2}=$ & & \\
\hline & \multicolumn{2}{|c|}{ Absolute value of Total FF = } & & $\mathrm{SS} / \mathrm{N}=$ & & \\
\hline & & & $\mathrm{TT}=$ & $(A B S) S U M / N^{\wedge} .5=$ & & \\
\hline
\end{tabular}

If $\mathrm{TT}$ is greater than $\mathrm{T}(\mathrm{N})^{*}$, then the bias is significantly greater than zero

If $F F$ is greater than $F(N)^{* *}$, then the Std. Dev. Is significantly greater than one.

$\mathrm{N}$ is the number of analyses in the current quality control program.

BIAS IS:

STD DEV.

IS:

* $t$-Table value for $(\alpha=0.1) / 2$ and $60 \mathrm{df}=1.67$

** $\mathrm{F}$ Table Value at the $90 \% \mathrm{Cl}$ for df $60 \& 9=1.74$

Significant

Significant
Standards

1

2

3

4
Not Significant

Not Significant

$\mathrm{mgU} / \mathrm{gSol}$

Concentration

2.000

10.000

10.020

25.000
Method RSD Method SD

$0.20 \% \quad 0.004$

$0.15 \% \quad 0.015$

$0.15 \% \quad 0.015$

$0.13 \% \quad 0.033$ 


\section{Module 7}

\section{Sampling Variability Solids and Oxides}




\section{Objectives}

- Understand the types of variability associated with sampling

- Understand the nature of heterogeneity for oxide materials

- Understand the activities that can be done to minimize the problems of heterogeneity within a batch of material

- Be able to apply correct sampling principles, analyses, tools, and techniques

- Be able to use Excel functions and the ANOVA capability to evaluate data 


\section{Sampling Variability}

- Batch of material resulting from some process

- Material needs to be sampled

- Material is not homogeneous

- How do you sample this material? 


\section{Population versus Sample}

- A population is the totality of those entities about which we desire to establish a certain property or characteristic.

- Examples

- Number of TIDs improperly applied on containers in a particular vault.

- The number of containers in the correct location in a particular vault. 


\section{Population versus Sample}

- A sample of a population is a part of that population. It is a group of observations taken from a population.

- The objective of sampling is to obtain samples that are representative of the population from which they are drawn. 


\section{Types of Samples}

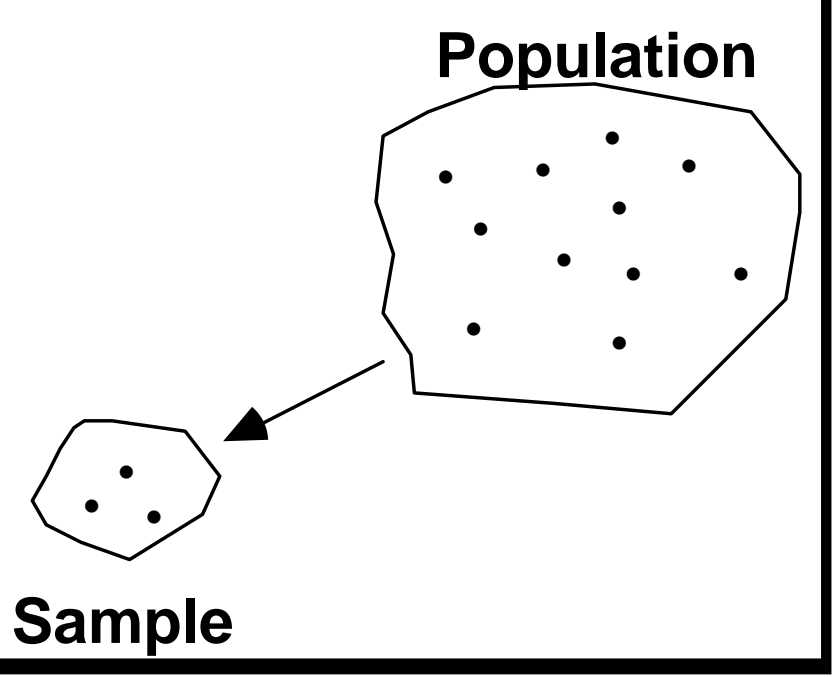

A simple random sample of size $n$ is one in which every possible sample of size $n$ has the same probability of being selected.

A convenience sample is one that is chosen simply by taking observations that are easily or inexpensively obtained.

Which approach do you think is more likely to meet our objective for sampling? 


\section{Goal of Good Sampling}

The goal of good sampling is to follow a sampling protocol that produces a sample whose chemical or physical measurements of interest are:

1. Representative of the entire lot

The key is random sampling which enables an unbiased estimate and an estimate of the precision

2. As consistent as possible with other samples that would be obtained if the entire sampling protocol could be repeated 


\section{Representative and Consistent Samples}

- Getting representative samples requires using physical sampling techniques that have as little bias as possible (Accuracy)

- Getting consistent samples means reducing sampling-tosampling variation (Precision)

- Accuracy and precision (reproducibility) should be agreed upon by the customer and supplier

- Sampling errors can lead to unnecessary process changes, the analysis of additional samples, or the release of off-spec material 


\section{Proper Separation of Variation}

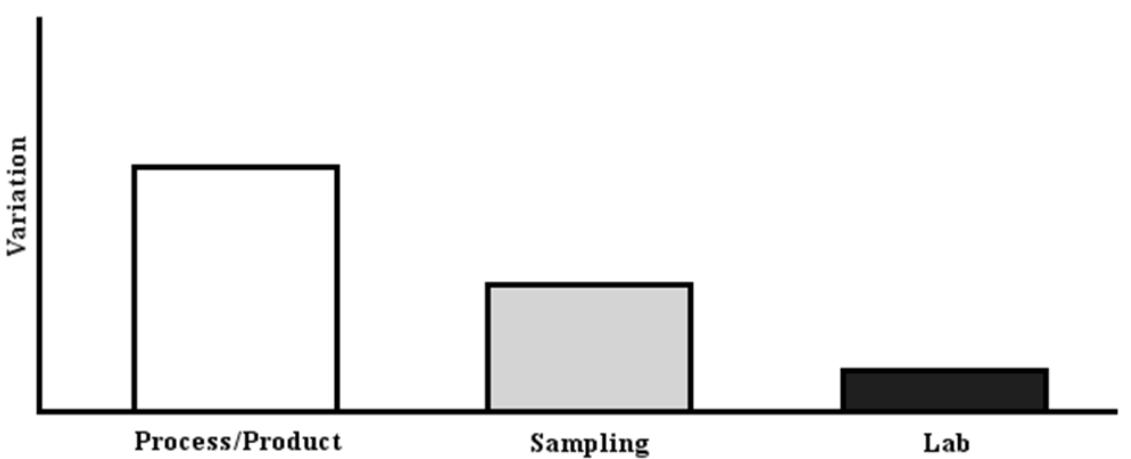

Process, sampling, and lab variation separated

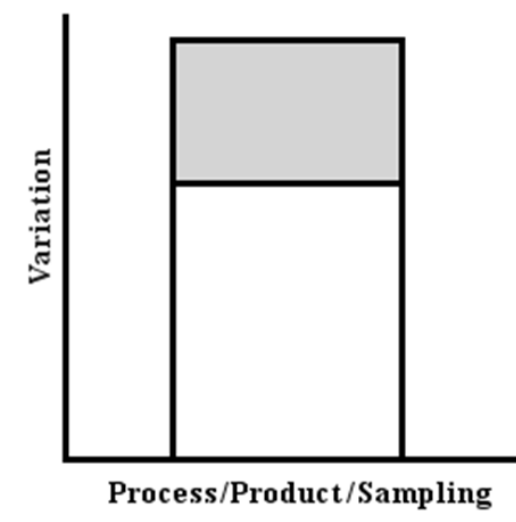

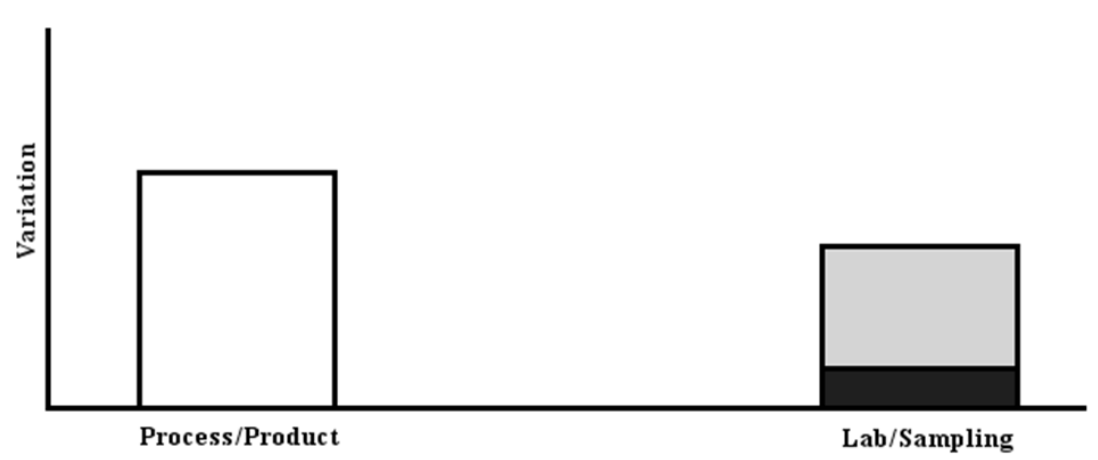

Sampling and lab variation not separated.

Sampling and process variation not separated 


\section{Material Variation}

- Error 1: Fundamental Error (FE)

Heterogeneity of solids is influenced by particle size, shape, density, chemical composition, and other physical properties. This error is usually large for solids and negligible for liquids and gases.

- Error 2: Grouping and Segregation Error

Heterogeneity caused by the spatial distribution of the constituents and shape of the lot. Many solids are known to settle or stratify. Sampling from the bottom versus the top can generate different samples. 


\section{Process Variation}

- Error 3: Long-range non-periodic heterogeneity fluctuation

- Processes change over time, sometimes in short intervals and sometimes over a longer time span. Samples taken at different times can produce different results. Should determine trends and how they behave.

- Error 4: Long-range periodic heterogeneity fluctuation

Processes can experience periodic changes over time. For example, the process may be effected by day and night temperature cycles. Systematic sampling that has the same frequency as the cycles will not reveal the entire variation of the process and produce biased results. 


\section{Tools and Techniques}

- Error 5: Delimitation error

Nonrandom samples, such as judgmental and spot samples, are useful but probability and random samples are fundamental to obtaining unbiased estimates. A delimitation error occurs when not every part of the lot has an equal chance of being in the sample. For example, scooping off the top of a large solid pile can produce samples with misleading results.

- Error 6: Extraction Error

An extraction error occurs when a sample that has been identified cannot be obtained. Extraction error is typically due to the equipment used. For example, in sampling a container, a thief probe may produce an extraction error because it may not be able to extract material at the very bottom. 


\section{Sample Handling}

- Error 7: Sample handling, sample integrity, or sample preservation

Samples can change between the time they are taken and the time they were analyzed. 


\section{Error Sources}

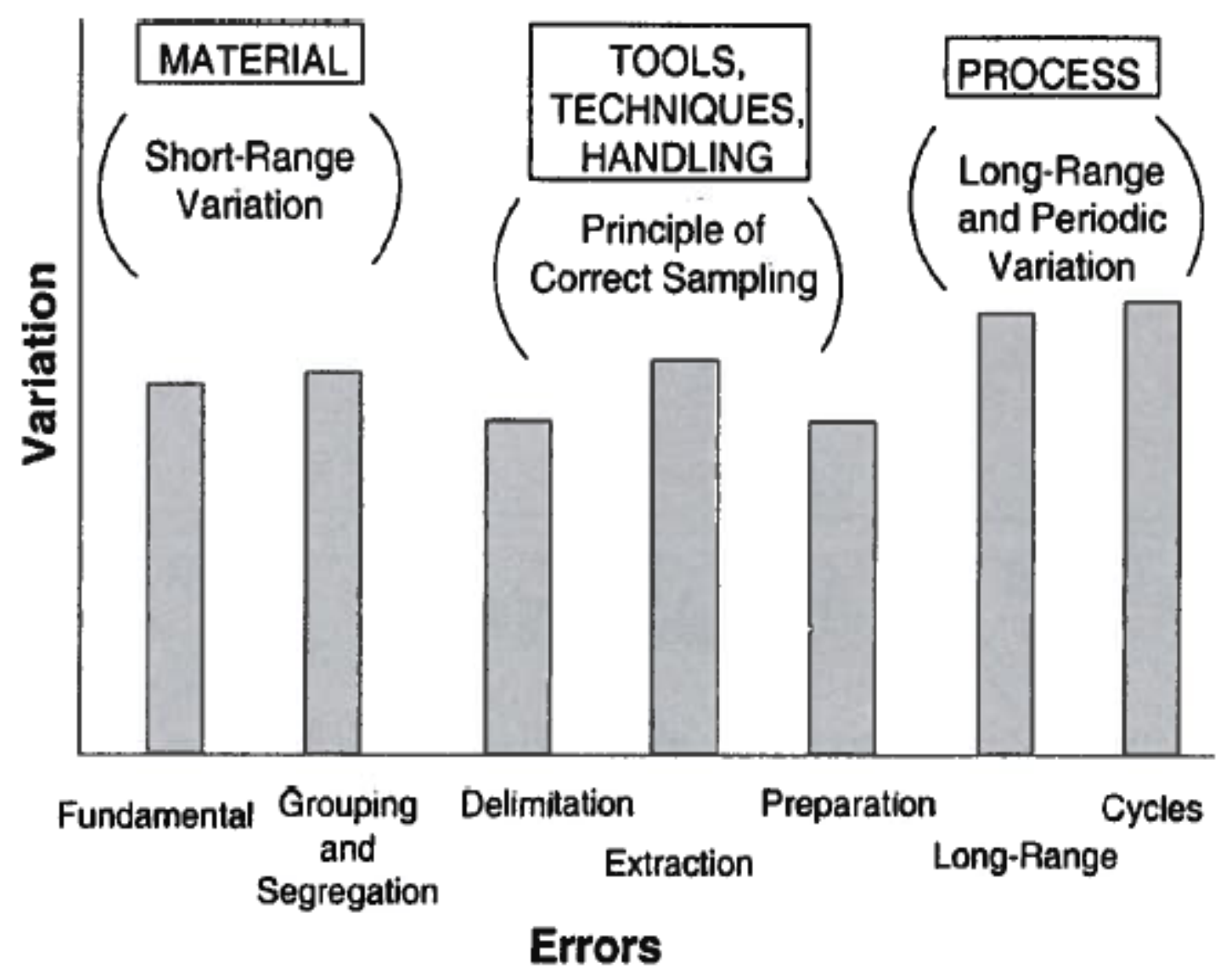

Example bar graph of variation vs. errors for sampling.

(Actual variation depends on the situation.) 


\section{Heterogeneity}

\section{Heterogeneous:}

Consisting of dissimilar ingredients or constituents "not all the same" "not uniform throughout" or "different."

There are two types of material heterogeneity: constituent and distribution.

1. Constituent heterogeneity $(\mathrm{CH})$ : Differences in constitution or makeup of material (particles or molecules)

2. Distribution heterogeneity (DH): How the material is distributed or mixed due to density, particle size, etc.

Both give rise to sampling error. 


\section{I llustrations of Heterogeneity}

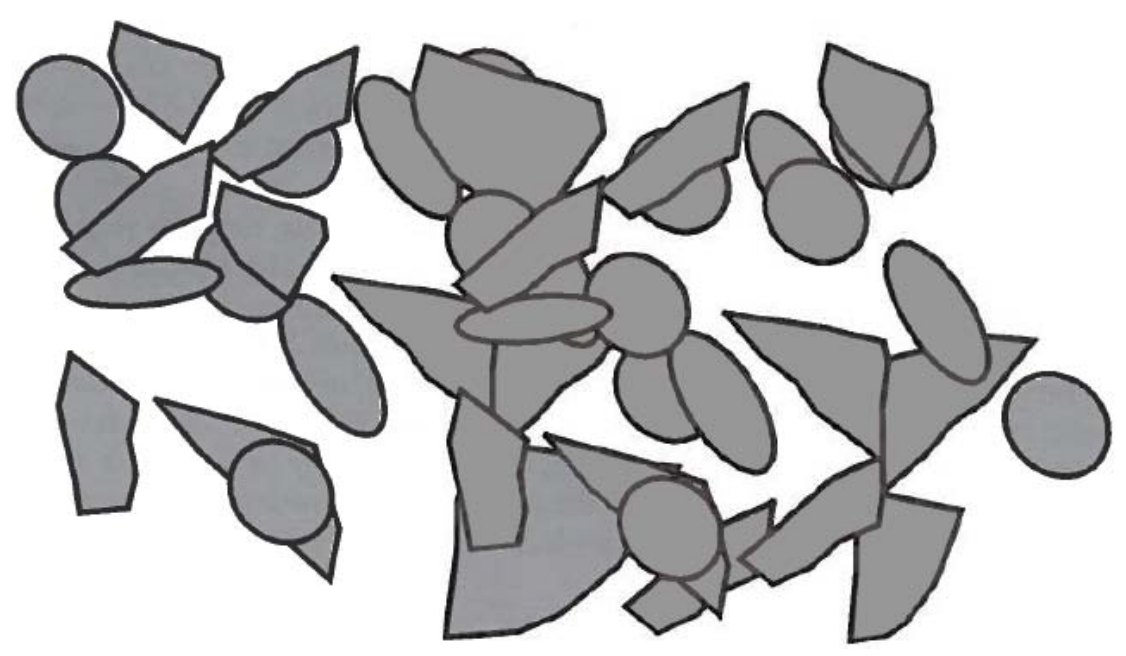

Constitution heterogeneity $(\mathrm{CH})$. The particles are not uniform.

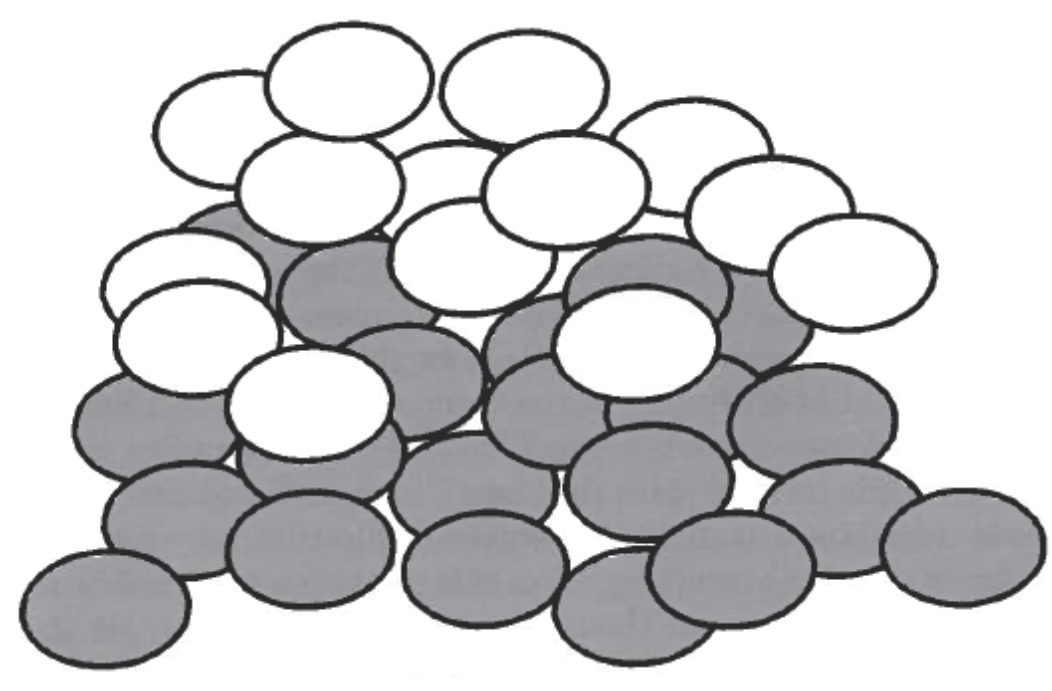

Distribution heterogeneity (DH) for solids. The particles are not distributed uniformly. 


\section{Fundamental Error}

Fundamental Error is the difference between the sample amount and the lot content relative to the lot as a whole.

- Reduce the Fundamental Error: With random sampling, increasing the quantity of material in the sample-> Reduced Variability

- Decreasing the individual particle size of material (e.g., By Grinding) in the lot before sampling-> Reduced Variability

Must preserve the integrity of the samples so that the components of interest are not changed

Sample Size:

- Statisticians think of the number of units in the sample

- Chemists think of mass, weight, or volume of the sample 


\section{Reducing the Fundamental Error}

In statistical sampling, under independence it is well known that:

$$
\sigma_{\bar{x}}=\sigma_{\text {Population }} / \sqrt{n}
$$

- For a fixed particle size, increasing the number of units in the sample is comparable to increasing the sample weight of a chemical sample.

- For a fixed sample weight, decreasing the particle size of material in the lot before sampling has the effect of increasing the number of sampling units. 


\section{Reducing Segregation Error}

- To reduce the effect of segregation, mix the entire lot if possible. Other alternatives are necessary for large immobile lots (e.g., waste piles, ship cargo)

- Solid particles that differ in size, density, and shape are susceptible to poor mixing. After mixing, solid granules may re-segregate during handling and storage.

- Mixing studies could be performed to measure the effectiveness of mixing over time and space.

- Good practice is to take small increments randomly and combine (composite) them to get a sample when estimating the average. 


\section{Composite Sampling}

In compositing, drawing the sample and the physical mixing procedure may change the physical characteristics of the material (e.g.: the particle size distribution could change).

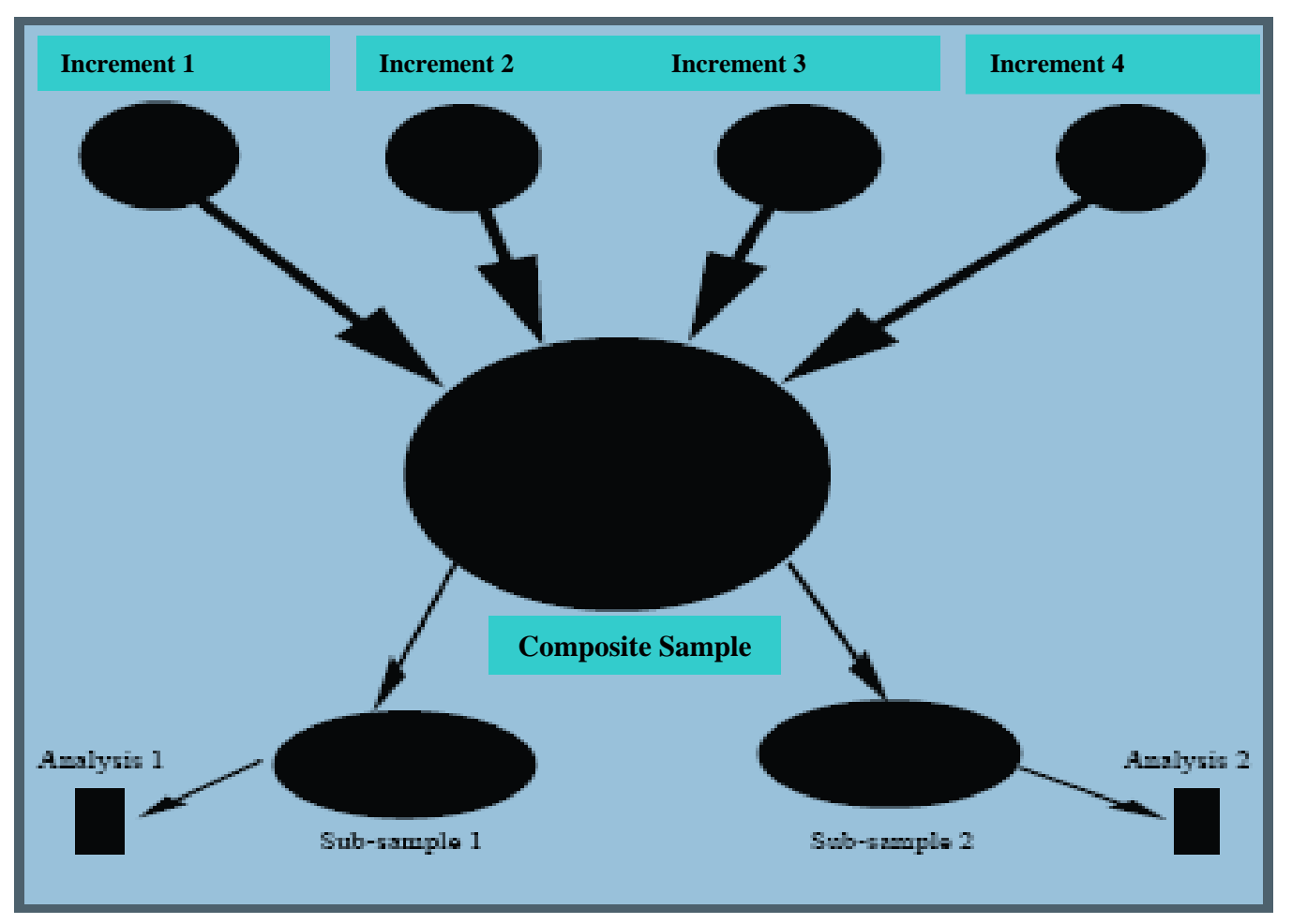

- Useful for reducing cost when the samples (increments) being combined are similar.

- Compositing is not useful when looking for hot spots or segregating material.

- Have sufficient material from each increment for discrete samples to be analyzed. 


\section{Good Sampling Practices}

- Increase the mass of the total physical sample

- Mix and grind the material before sampling

- Collect several random samples or increments and combine them to form the sample

- Use a container that will not react with the sample

- Handle samples properly

- Sampling method must preserve the integrity of the sample 


\section{Correct Sampling for Bulk Solids}

Guiding principals for random sampling of heterogeneous material

- Every part of the lot has an equal chance of being in the sample.

- Define and physically obtain the sample.

- In Grab Sampling, certain parts of the lot have no chance of being in the sample.

- The integrity of the sample is preserved during and after sampling. Between the time it is taken and analyzed, oxidation, abrasion, and evaporation may take place.

- Contamination (Clean your device after every use)

- Loss

- Mistakes (Use proper labeling) 


\section{Correct Sampling or Not?}

Grab from a conveyor belt.

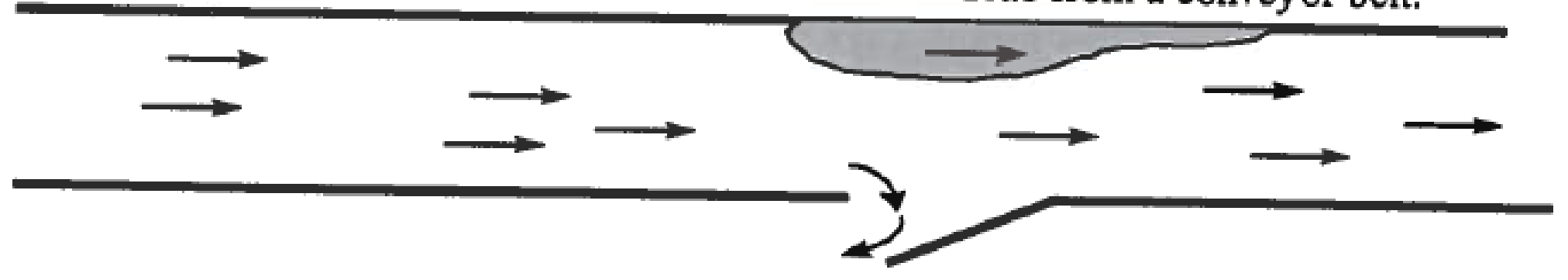

Grab from the bottom of a pipe.

Examples of grab sampling from the side of a conveyor belt or from the bottom of a pipe. 


\section{Zero Dimensional Sampling}

- Blocks numbered individually.

- All blocks are accessible.

- Every part of the lot has an equal chance of being in the sample.

- No difficulty extracting randomly selected blocks.

- No sample handling issues.

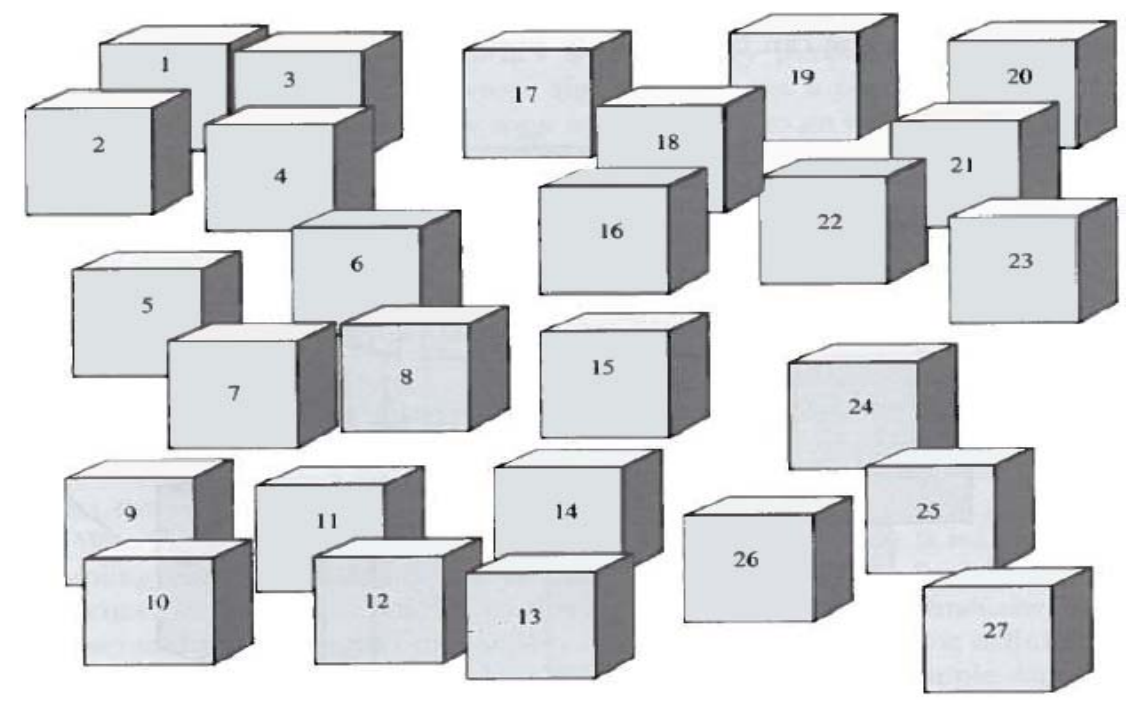




\section{Three Dimensional Sampling}

- The 27 blocks are numbered as before.

- Selected blocks may be in the middle or bottom layers.

- Pulling out the blocks could be a major problem for a larger number of stacked blocks.
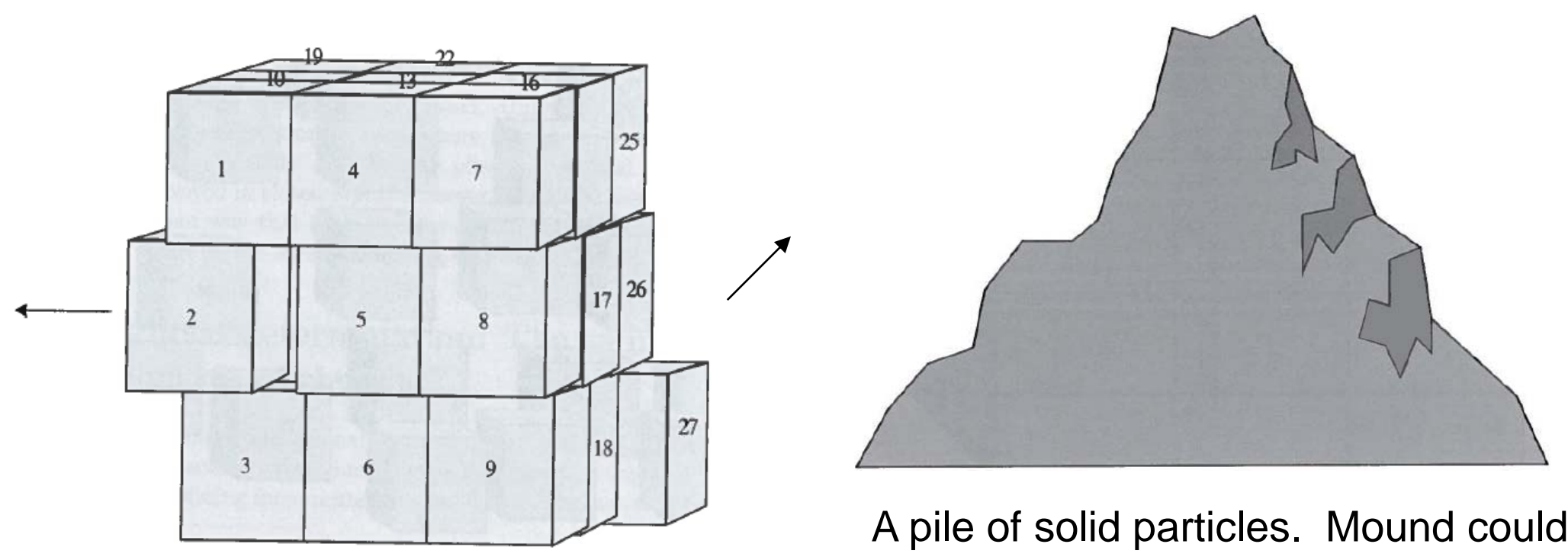

A pile of solid particles. Mound could collapse as soon as sampling starts. 


\section{Two Dimensional Sampling}

- 27 blocks arranged in 9 numbered stacks. Now there are 9 rather than 27 sampling units.

- The sampling unit is a vertical stack of three blocks.

- Vertical sampling core should be a cylinder ensuring an equal amount of material from the top, middle and bottom.
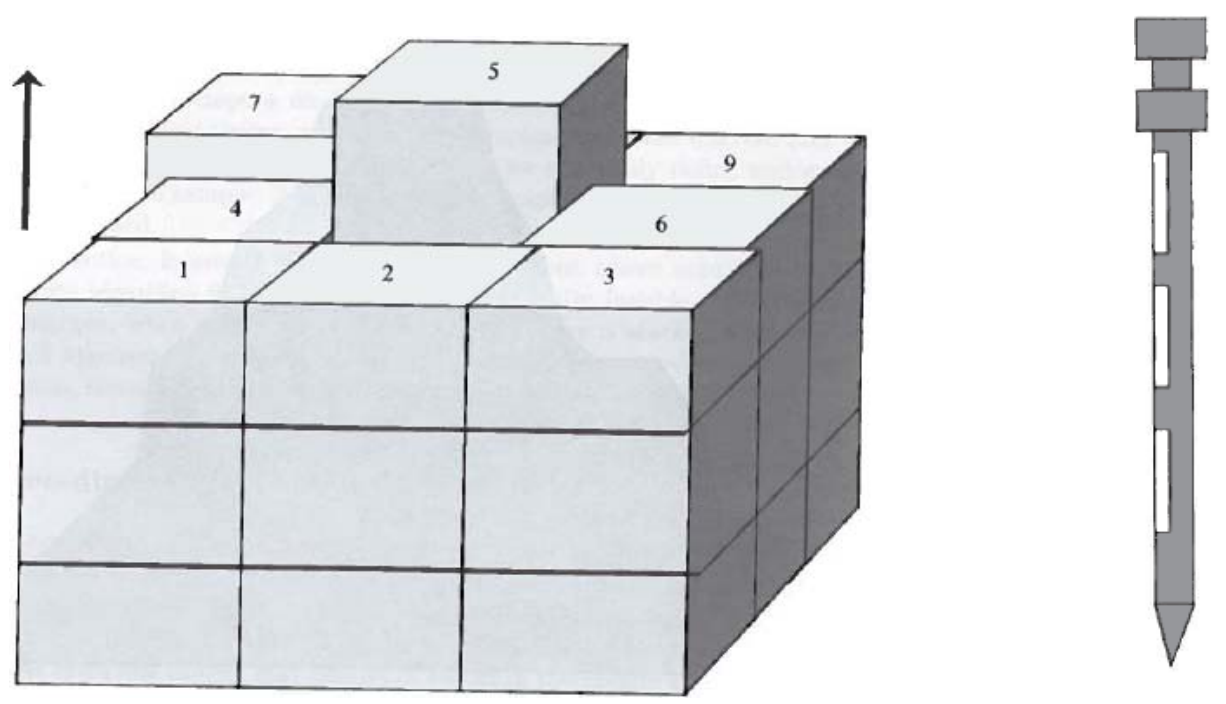

Thief probe for solids sampling. 


\section{Slot Sampler/ Powder Thief}

- Designed for volume sampling of several points

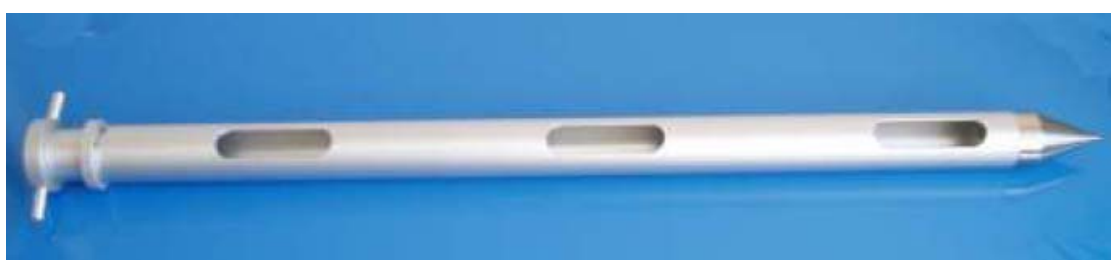

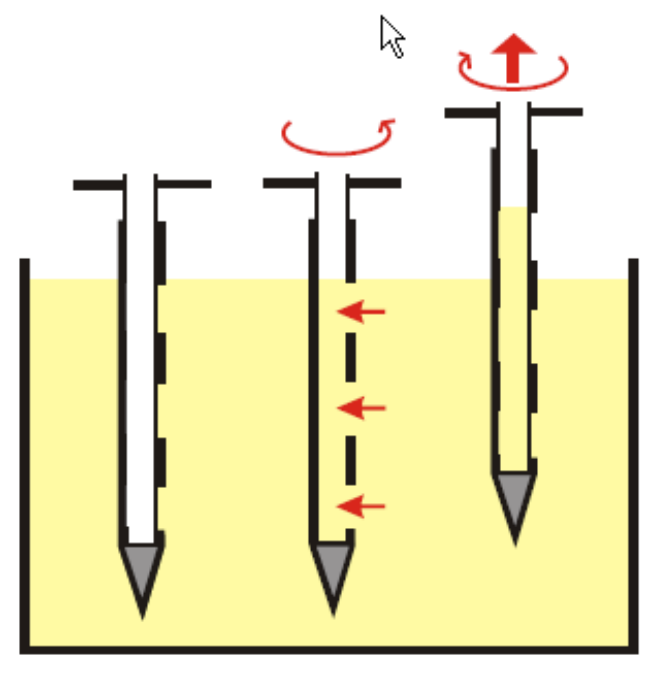

Multi-Level Sampling

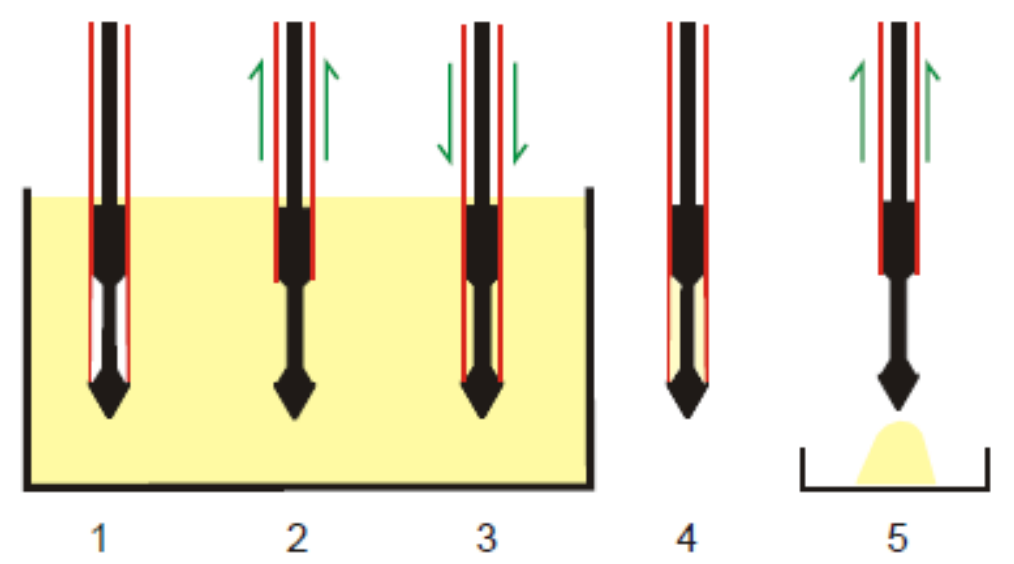

Single Level Sampling 


\section{One Dimensional Sampling}

- Frequently, one dimensional sampling is practical in sampling solids.

- Group in 2 dimensions and sample across the $3^{\text {rd }}$ dimension.

- Defining the sampling unit as 9 blocks in a plane giving 3 consecutive stacks of 3

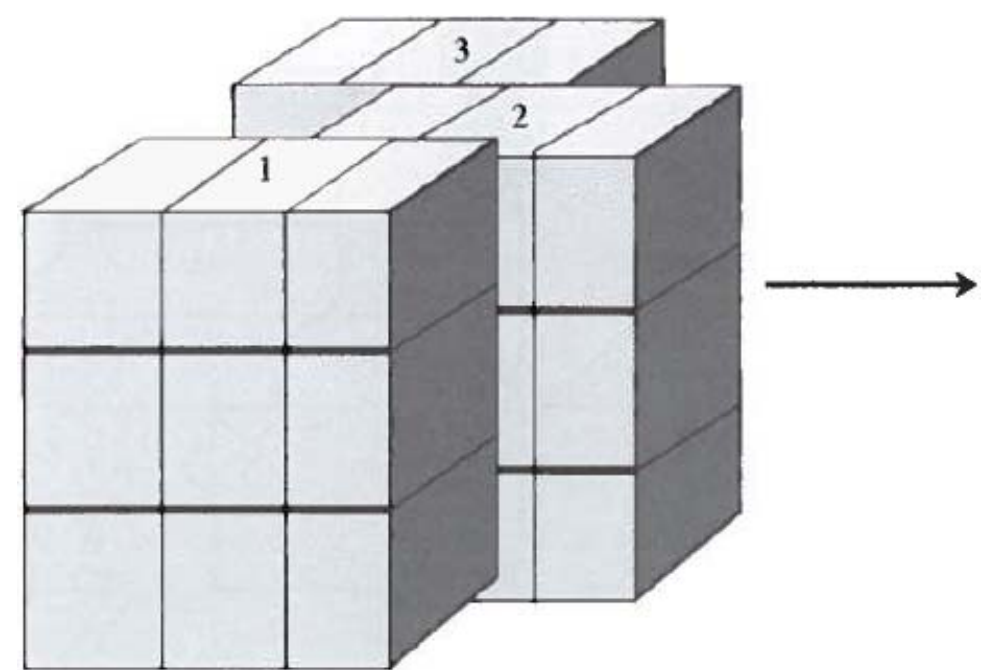

27 blocks arranged in 3 numbered planes

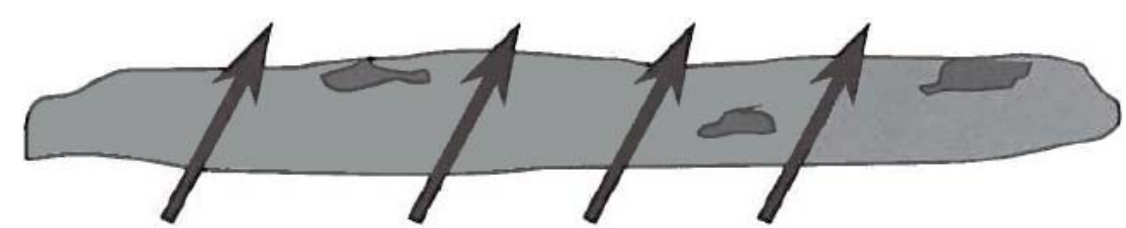

Slicing across a fairly flat pile. May be prone to extraction error, but can be minimized by using a proper tool. 


\section{One vs. Three Dimensional Sampling}

- May not be able to take a one-dimensional slice across a three dimensional lot because the material may be in a container.

- Taking a cross-stream sample from a flowing stream before it becomes a stationary lot. Could be material moving along a conveyer belt.

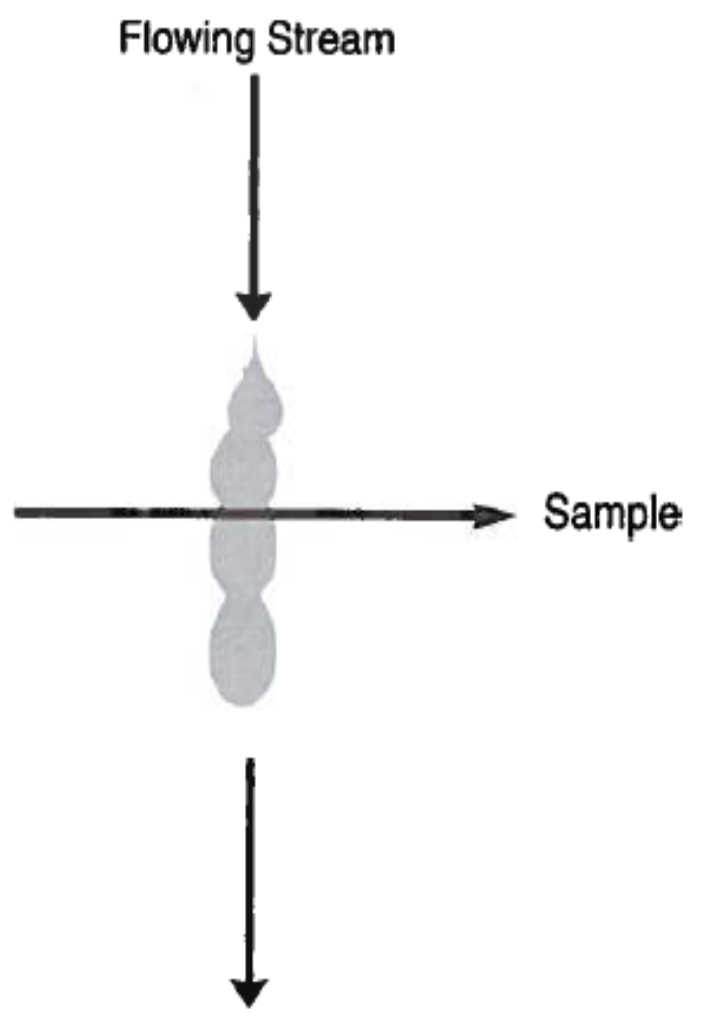




\section{Extracting the Sample}

- The error in slicing across a pile can be minimized if the proper sampling tool is used.

- Must be large enough to hold all the material in the slice.

- Should have sides perpendicular to the bottom.

- Should be cleaned between samples.

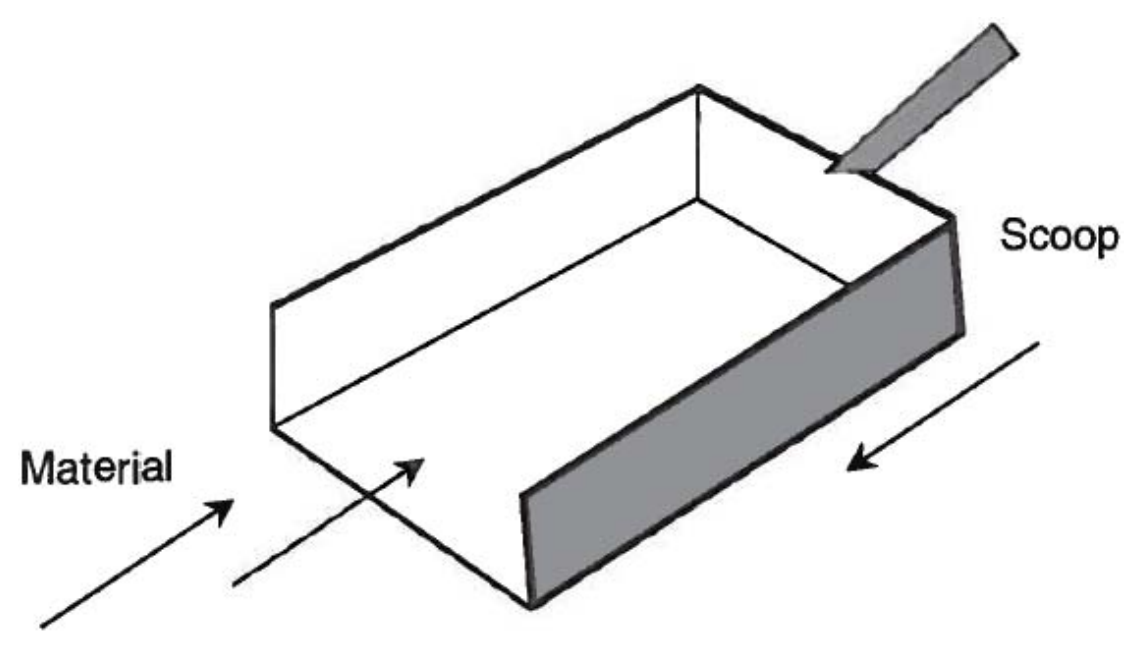




\section{Extracting the Sample (continued)}

Typical riffle splitter (rifflers) - Rifflers are used for mixing and subsampling solid particles. Roughly half of the material is collected in each pan. The procedure can be repeated to get sub-samples by randomly selecting one of the pans. Spinning rifflers that generally produce better samples are also used.

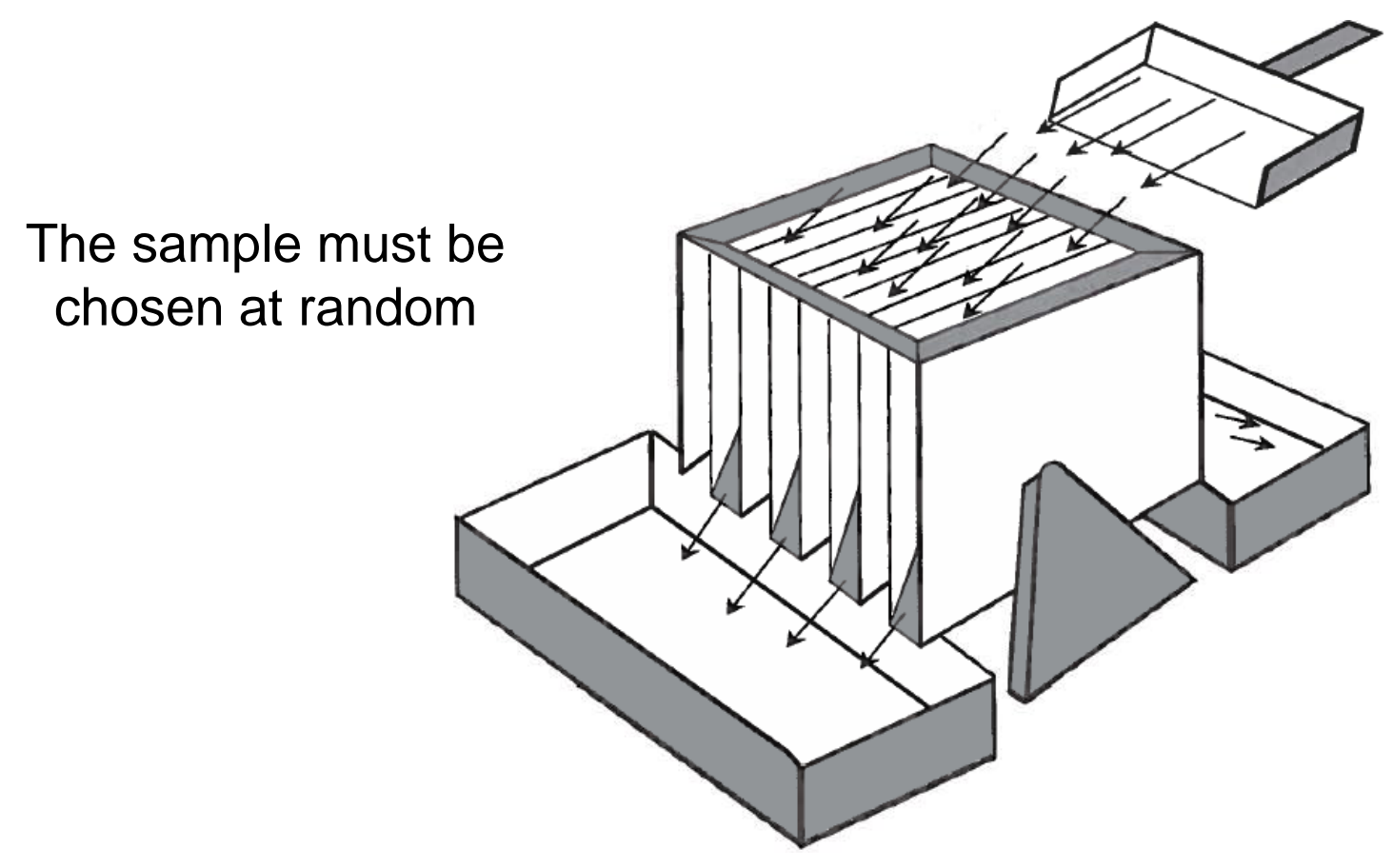




\section{Spinning Riffler}

By attaching a sieve to the top of the hopper, samples can be obtained which are representative of the initial sample while excluding all particles larger than the selected sieve size.

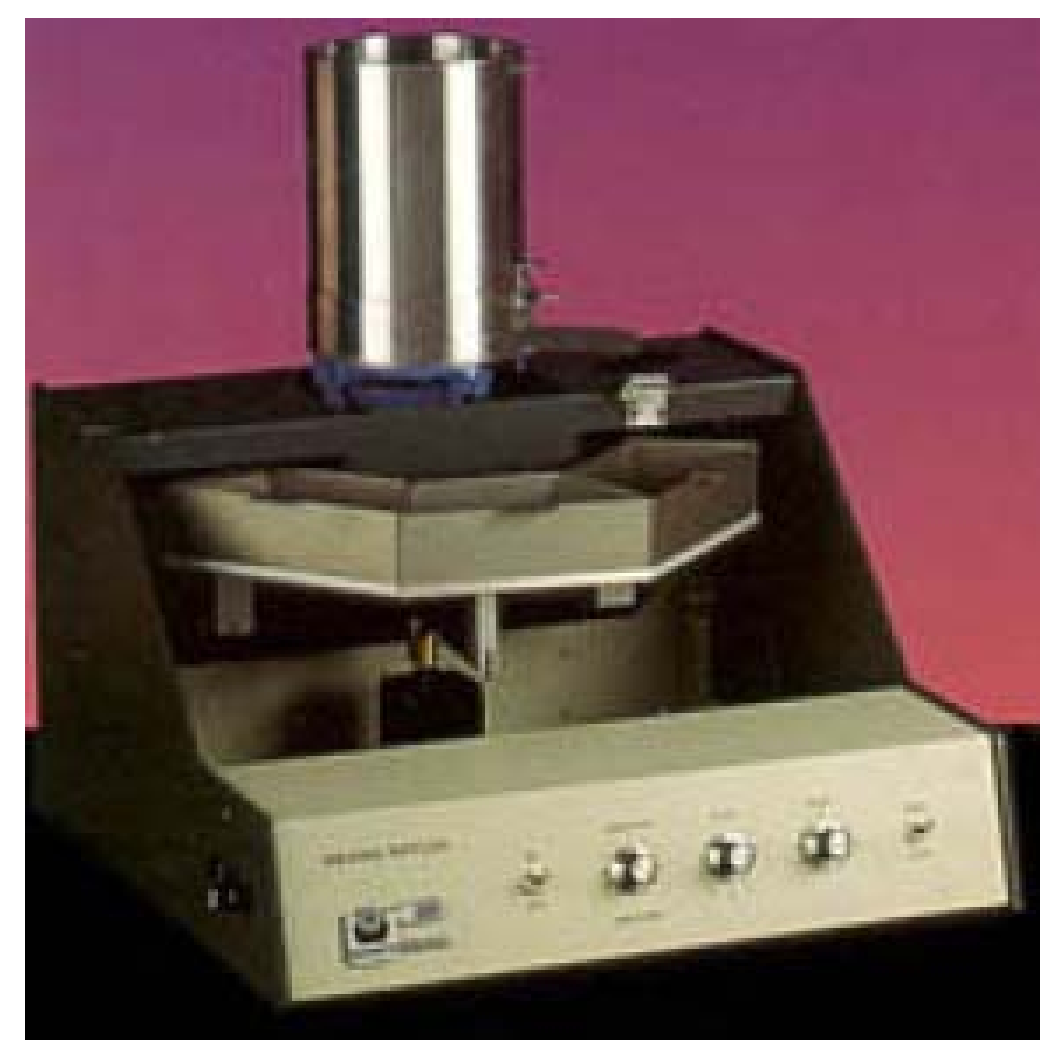




\section{Exercises - Process Stability Over Time}

Goal - Sample batches to determine if the process is consistently mixing/blending product. This will be done by taking random samples from multiple batches and statistically analyzing the results. Three different exercises will be used for evaluating batch differences and for estimating sampling variability.

- Exercise \#1 - Determine if several batches of material are consistent from a weight percent perspective from data set 1

- Exercise \#2 - Determine if several batches of material are consistent from a weight percent perspective from data set 2

- Exercise \#3 - Estimate the sampling and random measurement uncertainties for the batches of material based on weight percent data set 3 


\section{Exercise \#1}

Background and Sampling approach:

Four oxide samples are taken from a large tray of calcined material after manually mixing the material for a set period of time. The tray is divided into four quadrants and each quadrant is divided into four equal sub-quadrants. For each quadrant, a random sub-quadrant is chosen and sampled. The four samples are sent to the laboratory and are analyzed individually.

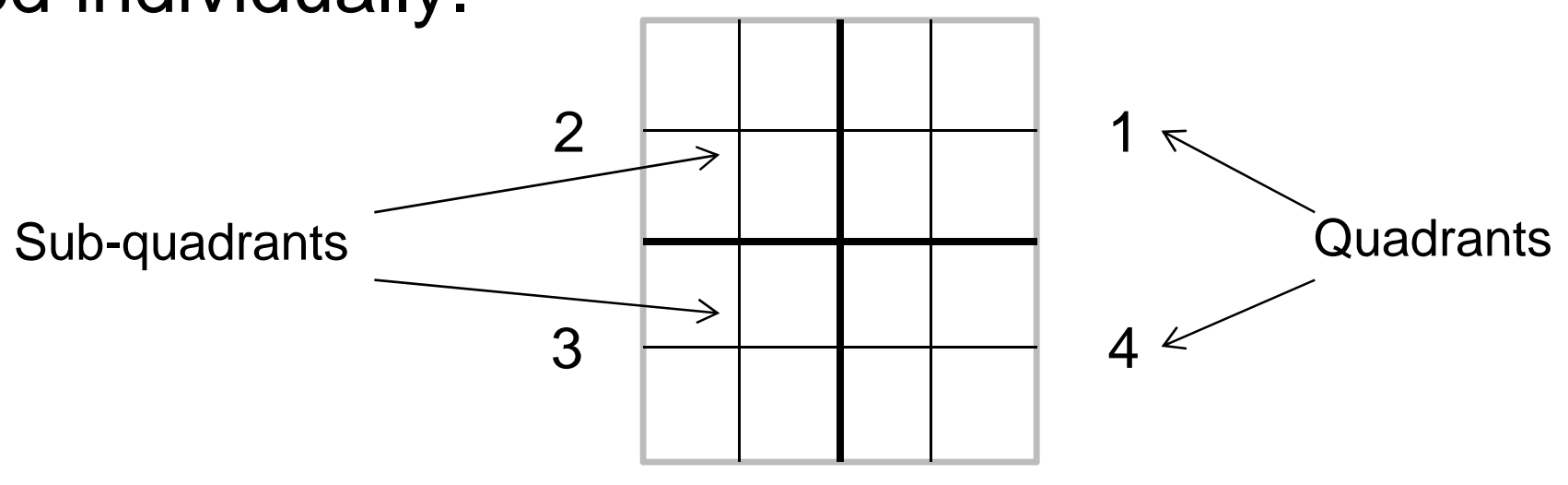




\section{Exercise \# 1}

Discussion topics and questions related to exercise:

1. Discuss methods for manually mixing the material.

2. How should the material be distributed prior to sampling?

3. Discuss methods for obtaining the four samples.

4. Bring up the Student Worksheet for Exercise \#1.

5. Following the instructor's lead, use the Excel one-way ANOVA to determine if several batches of material are consistent from a weight percent perspective.

6. What is the result of the ANOVA evaluation?

7. What is the estimate for the weight percent random uncertainty? 


\section{Exercise \#2}

Instructor led analysis of sampling results:

1. Bring up the Student Worksheet for Exercise \#2.

2. Use the Excel one-way ANOVA to determine if several batches of material are consistent from a weight percent perspective.

3. What is the result of the ANOVA evaluation?

4. What is the estimate for the weight percent random uncertainty? 


\section{Exercise \#3}

Discussion topics and Questions:

1. Bring up the Student Worksheet for Exercise \#3. The instructor will briefly discuss the data for this exercise.

2. Following the instructor's lead, the students will use the Excel AVERAGE, DEVSQ and SUM functions to estimate the sampling and random uncertainties for the batches of material based on weight percent.

3. What is the estimate for the weight percent sampling uncertainty?

4. What is the estimate for the weight percent random uncertainty? 


\section{Summary}

- Discussed the types of variability associated with sampling

- Discussed the nature of heterogeneity for oxide materials

- Discussed activities that can be done to minimize the problems of heterogeneity within a batch of material

- Discussed correct sampling principles, analyses, tools, and techniques

- Used Excel functions and the ANOVA capability to evaluate data from several batch sampling problems 


\section{Chinese Measurement Control Workshop Sampling Oxides Module Instructor Worksheet}

\begin{tabular}{|c|c|c|c|c|c|c|c|c|c|c|}
\hline Exercise 1 & & & & & & & & & & \\
\hline & \multicolumn{10}{|c|}{ Original Data } \\
\hline & Batch & & & & & & & & & \\
\hline Sample & 1 & 2 & 3 & 4 & 5 & 6 & 7 & 8 & 9 & 10 \\
\hline 1 & 0.8443 & 0.8441 & 0.849 & 0.8455 & 0.8481 & 0.8444 & 0.8463 & 0.8455 & 0.8446 & 0.8422 \\
\hline 2 & 0.8464 & 0.8454 & 0.8429 & 0.8467 & 0.8457 & 0.8408 & 0.8439 & 0.8421 & 0.8476 & 0.845 \\
\hline 3 & 0.8481 & 0.8401 & 0.8481 & 0.8408 & 0.8449 & 0.8453 & 0.8455 & 0.8447 & 0.8447 & 0.8474 \\
\hline 4 & 0.8442 & 0.8485 & 0.8465 & 0.8422 & 0.8462 & 0.8434 & 0.8464 & 0.844 & 0.8469 & 0.8463 \\
\hline & & & & & & & & & & \\
\hline & & & & & & & & \multicolumn{2}{|c|}{ Sample Mean = } & 0.8451 \\
\hline & \multicolumn{10}{|c|}{ Relative Differences } \\
\hline & Batch & & & & & & & & & \\
\hline Sample & 1 & 2 & 3 & 4 & 5 & 6 & 7 & 8 & 9 & 10 \\
\hline 1 & -0.00097 & -0.0012 & 0.004594 & 0.000453 & 0.003529 & -0.00085 & 0.001399 & 0.000453 & -0.00061 & -0.00345 \\
\hline 2 & 0.001518 & 0.000334 & -0.00262 & 0.001873 & 0.000689 & -0.00511 & -0.00144 & -0.00357 & 0.002937 & -0.00014 \\
\hline 3 & 0.003529 & -0.00594 & 0.003529 & -0.00511 & -0.00026 & 0.000216 & 0.000453 & -0.00049 & -0.00049 & 0.002701 \\
\hline 4 & -0.00109 & 0.004002 & 0.001636 & -0.00345 & 0.001281 & -0.00203 & 0.001518 & -0.00132 & 0.002109 & 0.001399 \\
\hline
\end{tabular}

Anova: Single Factor

SUMMARY

\begin{tabular}{lrrrr}
\hline \multicolumn{1}{c}{ Groups } & Count & \multicolumn{1}{c}{ Sum } & \multicolumn{1}{c}{ Average } & Variance \\
\hline Column 1 & 4 & 0.002994 & 0.000748 & $4.88 \mathrm{E}-06$ \\
Column 2 & 4 & -0.0028 & -0.0007 & $1.7 \mathrm{E}-05$ \\
Column 3 & 4 & 0.007135 & 0.001784 & $1.01 \mathrm{E}-05$ \\
Column 4 & 4 & -0.00624 & -0.00156 & $1.07 \mathrm{E}-05$ \\
Column 5 & 4 & 0.005242 & 0.00131 & $2.59 \mathrm{E}-06$ \\
Column 6 & 4 & -0.00777 & -0.00194 & $5.3 \mathrm{E}-06$ \\
Column 7 & 4 & 0.001929 & 0.000482 & $1.87 \mathrm{E}-06$ \\
Column 8 & 4 & -0.00493 & -0.00123 & $2.95 \mathrm{E}-06$ \\
Column 9 & 4 & 0.00394 & 0.000985 & $3.27 \mathrm{E}-06$ \\
Column 10 & 4 & 0.000509 & 0.000127 & $7.04 \mathrm{E}-06$ \\
\hline
\end{tabular}

ANOVA

\begin{tabular}{|c|c|c|c|c|c|c|}
\hline Source of Variation & SS & $d f$ & $M S$ & $F$ & P-value & F crit \\
\hline Between Groups & $5.96 \mathrm{E}-05$ & 9 & $6.62 \mathrm{E}-06$ & 1.008613 & 0.454998 & 2.210697 \\
\hline Within Groups & 0.000197 & 30 & 6.57E-06 & \multirow{2}{*}{\multicolumn{2}{|c|}{$\sigma($ Weight Percent $)=$}} & \\
\hline & & & & & & $0.26 \%$ \\
\hline Total & 0.000257 & 39 & & & & \\
\hline
\end{tabular}


Chinese Measurement Control Workshop

Sampling Variability (Oxides) Module

Student Worksheet

\begin{tabular}{|c|c|c|c|c|c|c|c|c|}
\hline \multicolumn{2}{|c|}{ Exercise 3} & & & & & & & \\
\hline & \multicolumn{8}{|c|}{ Original Data } \\
\hline & Samples & & & & & & & \\
\hline & 1 & & 2 & & 3 & & 4 & \\
\hline & Repeats & & & & & & & \\
\hline Batch & 1 & 2 & 1 & 2 & 1 & 2 & 1 & 2 \\
\hline 1 & 0.8468 & 0.8467 & 0.8438 & 0.8488 & 0.8464 & 0.8418 & 0.8418 & 0.8436 \\
\hline 2 & 0.8446 & 0.8445 & 0.8495 & 0.8463 & 0.8468 & 0.8431 & 0.8479 & 0.8439 \\
\hline 3 & 0.8454 & 0.8437 & 0.8419 & 0.8448 & 0.8449 & 0.8448 & 0.846 & 0.8417 \\
\hline 4 & 0.8482 & 0.842 & 0.844 & 0.8439 & 0.8483 & 0.8494 & 0.8424 & 0.8472 \\
\hline 5 & 0.8426 & 0.8441 & 0.8453 & 0.8424 & 0.8447 & 0.8415 & 0.846 & 0.8481 \\
\hline 6 & 0.8488 & 0.8447 & 0.8444 & 0.8445 & 0.8414 & 0.8466 & 0.8468 & 0.8498 \\
\hline 7 & 0.8453 & 0.8476 & 0.8402 & 0.8407 & 0.8442 & 0.8462 & 0.8474 & 0.8493 \\
\hline 8 & 0.8456 & 0.8452 & 0.8403 & 0.8441 & 0.8486 & 0.848 & 0.8445 & 0.8453 \\
\hline 9 & 0.8455 & 0.8464 & 0.8453 & 0.8408 & 0.845 & 0.8481 & 0.8461 & 0.8506 \\
\hline 10 & 0.8408 & 0.8418 & 0.8446 & 0.8458 & 0.8489 & 0.8482 & 0.8461 & 0.8478 \\
\hline & & & & & & Sample & ean $=$ & \\
\hline
\end{tabular}

\begin{tabular}{|r|r|r|r|r|r|r|r|r|}
\hline & \multicolumn{9}{|c|}{ Relative Differences } \\
\hline & Samples & & & & & & \\
\hline \\
\hline
\end{tabular}

$\mathrm{E}($ Between Samples MSE $)=($ Within Samples MSE $)+2 \mathrm{~V}($ Sampling $)$

Total SS

Degrees of Freedom

MSE

Uncertainty

\begin{tabular}{|c|c|c|}
\hline & & Total \\
\hline Within & Between & Within \\
\hline Samples & Samples & Batch \\
\hline Sum of Squares & Sum of Squares & Sum of Squares \\
\hline & & \\
\hline & & \\
\hline & & \\
\hline & & \\
\hline & & \\
\hline & & \\
\hline & & \\
\hline & & \\
\hline & & \\
\hline & & \\
\hline & & \\
\hline & & \\
\hline & & \\
\hline & & \\
\hline & & \\
\hline
\end{tabular}

The sampling uncertainty $=$ SQRT $(\mathrm{V}($ Sampling $))$ 


\section{Exercises \\ Sampling Variability (Oxides)}

\section{Session Objectives:}

After the session the participants will be able to do the following:

1. Understand issues with sampling and activities that can be to minimize these

2. Apply Excel functions and the ANOVA capability to determine if selected samples are representative of a batch of material

\section{Estimated Time:}

There are three exercises in this module:

- Exercises \#1 and \#2 will require 45 minutes to complete.

- Exercise \#3 will require 45 to complete.

- A total of 90 minutes will be required to complete the exercises.

\section{Materials Needed:}

1. One computer with Microsoft Excel for each group of four or five students

2. The problems will be solved using the ANOVA and various function capabilities within Excel

3. Students need to have a good working knowledge of Microsoft Excel

4. Spreadsheet support person

\section{Instructions:}

1. The students will be provided with a single spreadsheet for all three exercises

2. Exercises \#1 and \#2 are one-way ANOVAs for evaluating the differences of weight percent values between several batches of material

3. Exercise \#3 is a set of data for evaluating sampling differences. A sampling uncertainty will be estimated in this exercise. For this exercise, the students will need to use Excel statistical formulas to derive various sums of squares and MSEs for estimating a sampling uncertainty and a weight percent random measurement uncertainty.

4. The exercises will be instructor led

5. The results will be discussed 


\section{$\underline{\text { Exercise \#1 }}$}

Background:

Four oxide samples are taken from a large tray of calcined material after manually mixing the material for a set period of time. The tray is divided into four quadrants and each quadrant is divided into four equal sub-quadrants. For each quadrant, a random sub-quadrant is chosen and sampled. The four samples are sent to the laboratory and are analyzed individually.

Discussion topics and Questions:

1. Discuss methods for manually mixing the material.

2. How should the material be distributed prior to sampling?

a) The material should be uniformly distributed over the sampling tray

3. Discuss methods for obtaining the four samples.

a) Once a sub-quadrant is chosen, may want to draw the sample from the middle portion of the sub-quadrant

b) The sample should be taken over the full depth of the sub-quadrant location

4. Use the Excel data for Exercise \#1 and the Excel ANOVA to determine if several batches of material are consistent from a weight percent perspective.

a) Relative differences do not need to be calculated. The data can be analyzed as is to determine if there are differences between the batches

5. What is the result of the ANOVA evaluation?

a) The differences are not significant between the batches of samples

6. What is the estimate of the weight percent random uncertainty?

a) If the original data is analyzed, the weight percent random uncertainty is calculated as SQRT(Within Groups MS) / AVERAGE(Applicable data range)

b) The weight percent random uncertainty is $0.26 \%$. The data was generated with a random uncertainty of $0.25 \%$.

\section{$\underline{\text { Exercise \#2 }}$}

Background:

Same situation as Exercise \#1 but different data set

Discussion topics and Questions:

1. Use the Excel data for Exercise \#2 and the Excel ANOVA to determine if several batches of material are consistent from a weight percent perspective.

a) Again, relative differences do not need to be calculated. The data can be analyzed as is to determine if there are differences between the batches 
2. What is the result of the ANOVA evaluation?

a) The differences are significant between the batches of samples

3. What is the estimate of the weight percent random uncertainty?

a) If the original data is analyzed, the weight percent random uncertainty is calculated as SQRT(Within Groups MS) / AVERAGE(Applicable data range)

b) The weight percent random uncertainty is $0.23 \%$. The data was generated with a random uncertainty of $0.25 \%$.

4. Discuss how or why these differences could have occurred.

a) A change in the production process

b) A change in the analytical measurement method

$\underline{\text { Exercise \#3 }}$

Background:

This is a similar situation to Exercises \#1 and \#2 with a new data set. In this example repeated measurements have been made for each sample in order to estimate a sampling uncertainty. The students will need to use the Excel AVERAGE, DEVSQ and SUM functions to estimate a sampling uncertainty and a weight percent random uncertainty.

Based on the measurement plan in the spreadsheet, the sampling and weight percent variances are calculated as follows:

$\mathrm{E}(\operatorname{MSE}($ Sampling $))=\mathrm{V}($ Weight Percent $)+2 \times \mathrm{V}($ Sampling $)$, where

$$
\begin{aligned}
\text { MSE }(\text { Sampling }) & =(\text { Pooled Between Samples Sum of Squares }) / 30 \\
\mathrm{~V}(\text { Weight Percent }) & =(\text { Pooled Within Samples Sum of Squares }) / 40 \\
& =\text { MSE }(\text { Weight Percent })
\end{aligned}
$$

Discussion topics and Questions:

1. Use the spreadsheet for Exercise \#3 and the Excel AVEAGE, DEVSQ, and SUM functions to estimate a sampling uncertainty with respect to weight percent.

2. What is the sampling uncertainty estimate?

a) Sampling uncertainty is 0.00201 or $0.201 \%$.

3. What is the weight percent random uncertainty estimate?

a) Weight percent random uncertainty is 0.00246 or $0.246 \%$.

4. Discuss the details for estimating the sampling uncertainty.

a) The data was generated using a sampling uncertainty of $0.20 \%$ and a weight percent random uncertainty of $0.25 \%$. The population mean value for weight percent was 0.8450 . 


\section{Exercises \\ Sampling Variability (Oxides)}

\section{Session Objectives:}

After the session the participants will be able to do the following:

1. Understand issues with sampling and activities that can be to minimize these issues

2. Apply Excel functions and the ANOVA capability to determine if selected samples are representative of a batch of material

\section{Estimated Time:}

There are three exercises in this module:

- Exercises \#1 and \#2 will require 45 minutes to complete.

- Exercise \#3 will require 45 to complete.

- A total of 90 minutes will be required to complete the exercises.

\section{Materials Needed:}

1. One computer with Microsoft Excel for each group of four or five students

2. The problems will be solved using the ANOVA and various function capabilities within Excel

3. Students need to have a good working knowledge of Microsoft Excel

4. Spreadsheet support person

\section{Instructions:}

1. The students will be provided with a single spreadsheet for all three exercises

2. Exercises \#1 and \#2 are one-way ANOVAs for evaluating the differences of weight percent values between several batches of material

3. Exercise \#3 is a set of data for evaluating sampling differences. A sampling uncertainty will be estimated in this exercise. For this exercise, the students will need to use Excel statistical formulas to derive various sums of squares and MSEs for estimating a sampling uncertainty and a weight percent random measurement uncertainty.

4. The exercises will be instructor led

5. The results will be discussed 


\section{Exercise \#1}

Background:

Four oxide samples are taken from a large tray of calcined material after manually mixing the material for a set period of time. The tray is divided into four quadrants and each quadrant is divided into four equal sub-quadrants. For each quadrant, a random sub-quadrant is chosen and sampled. The four samples are sent to the laboratory and are analyzed individually.

Discussion topics and Questions:

1. Discuss methods for manually mixing the material.

2. How should the material be distributed prior to sampling?

3. Discuss methods for obtaining the four samples.

4. Use the Excel data for Exercise \#1 and the Excel ANOVA to determine if several batches of material are consistent from a weight percent perspective.

5. What is the result of the ANOVA evaluation?

6. What is the estimate of the weight percent random uncertainty?

\section{Exercise \#2}

Background:

Same situation as Exercise \#1 but different data set

Discussion topics and Questions:

1. Use the Excel data for Exercise \#2 and the Excel ANOVA to determine if several batches of material are consistent from a weight percent perspective. 
2. What is the result of the ANOVA evaluation?

3. What is the estimate of the weight percent random uncertainty?

4. Discuss how or why these differences could have occurred.

\section{$\underline{\text { Exercise \#3 }}$}

Background:

This is a similar situation to Exercises \#1 and \#2 with a new data set. In this example repeated measurements have been made for each sample in order to estimate a sampling uncertainty. The students will need to use the Excel AVERAGE, DEVSQ and SUM functions to estimate a sampling uncertainty and a weight percent random uncertainty.

Based on the measurement plan in the spreadsheet, the sampling and weight percent variances are calculated as follows:

$$
\begin{aligned}
& \mathrm{E}(\mathrm{MSE}(\text { Sampling }))=\mathrm{V}(\text { Weight Percent })+2 \times \mathrm{V}(\text { Sampling }), \text { where } \\
&\text { MSE(Sampling })=\text { (Pooled Between Samples Sum of Squares }) / 30 \\
& \mathrm{~V}(\text { Weight Percent })=\text { (Pooled Within Samples Sum of Squares }) / 40 \\
&=\text { MSE(Weight Percent })
\end{aligned}
$$

Discussion topics and Questions:

1. Use the spreadsheet for Exercise \#3 and the Excel AVEAGE, DEVSQ, and SUM functions to estimate a sampling uncertainty with respect to weight percent.

2. What is the sampling uncertainty estimate?

3. What is the weight percent random uncertainty estimate?

4. Discuss the details for estimating the sampling uncertainty. 


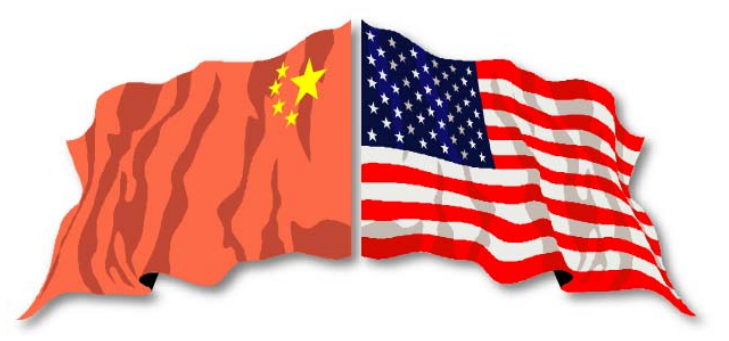

\section{Module 8}

\section{Control Charts}

and

Measurement Control 


\section{Objectives}

- Identify the purpose of control charts

- Identify the six elements of control charts and their purpose

- Discuss and analyze example control charts

- Discuss control charts and measurement control

- Discuss and analyze control charts from actual measurement systems 


\section{Purpose of Control Charts}

- To obtain a clear picture of the performance of the process

- Method

- Inventory

- To indicate if process is under control and, if not, to indicate extent of departure from control

- To indicate capability of process when system is in control 


\section{Reasons for Monitoring Control Charts}

1. Assure stability

2. Quantify quality

3. Measure improvement 


\section{To Assure Measurement Process Stability}

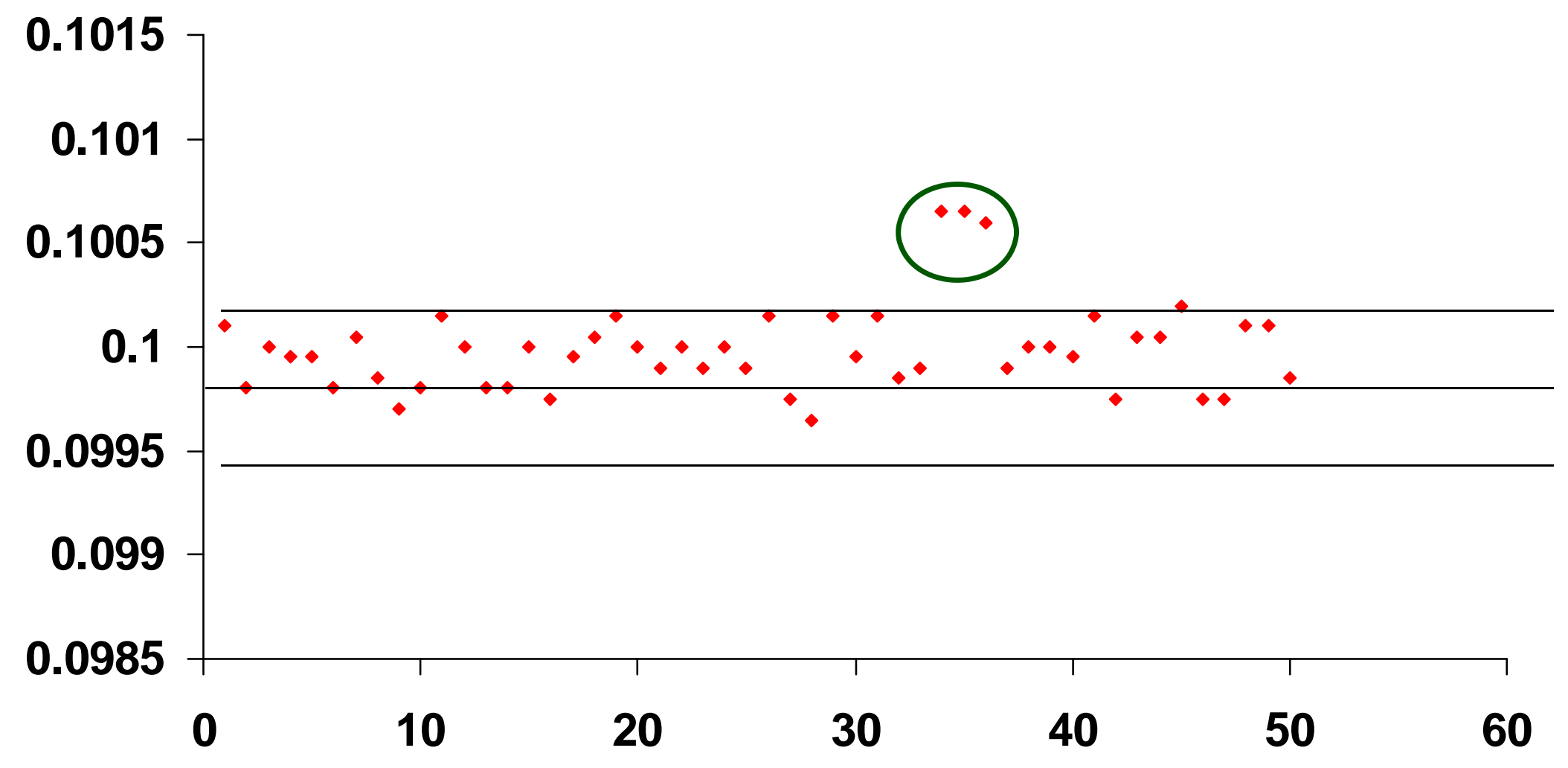




\section{To Determine Measurement Quality}

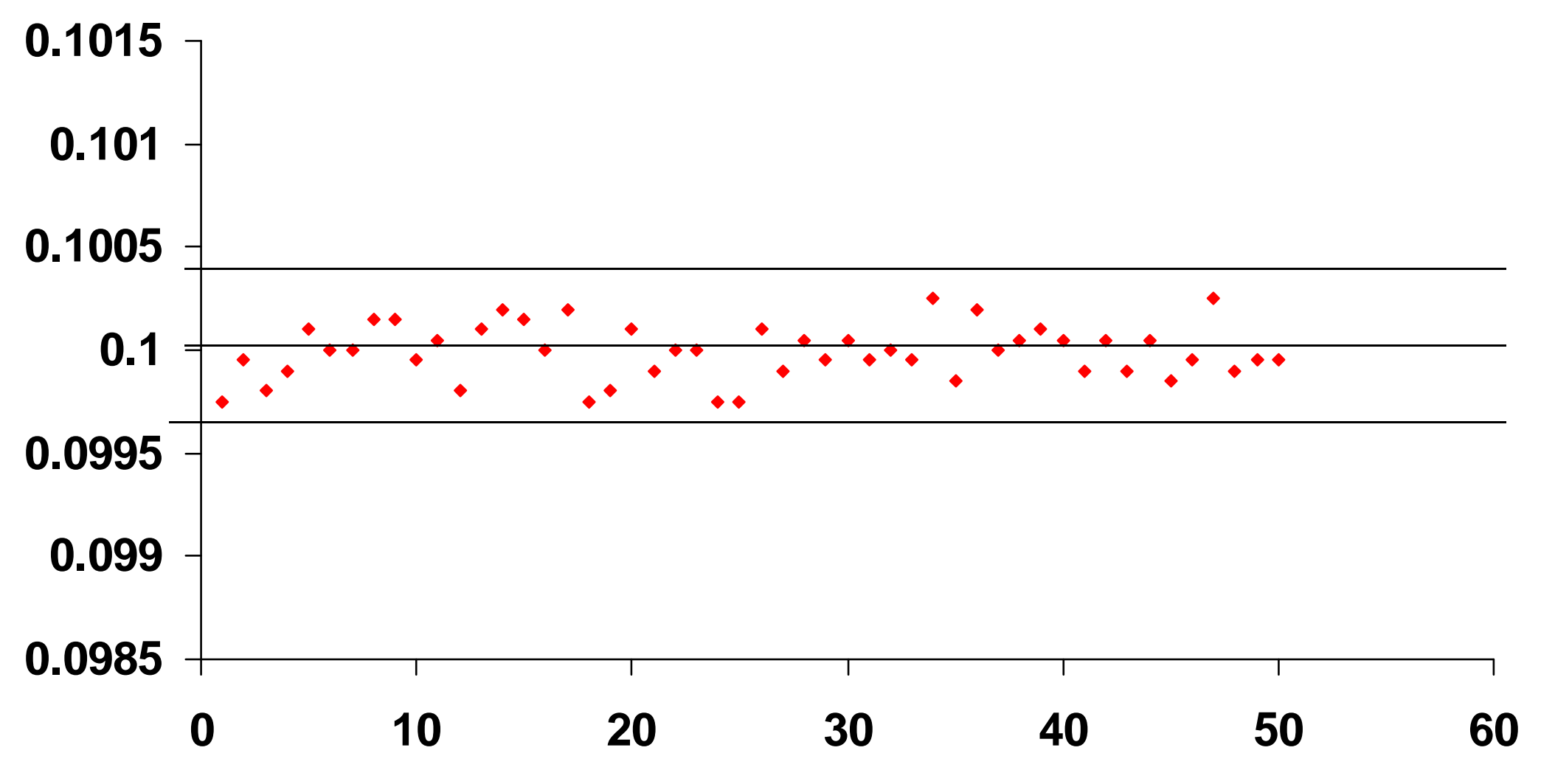

Mean is 0.1 Standard deviation is $\approx .0002$ Module 8 - 6 


\section{To Provide a Process for Measuring I mprovement}

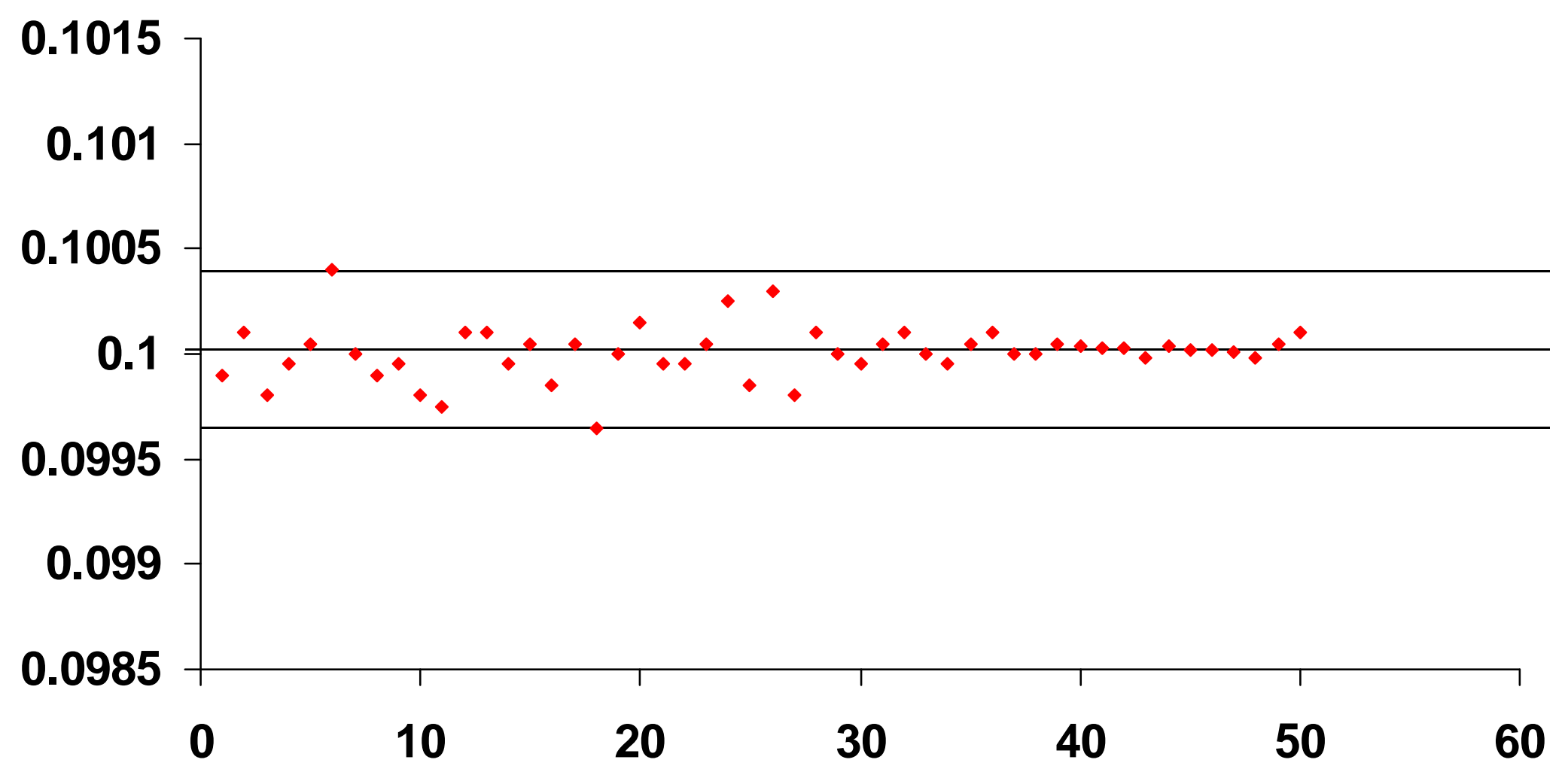




\section{Construction of a Control Chart}

- Order is of the greatest importance:

- Data points must be plotted in the order (by date or time) in which they are taken

- Control limits are usually at the mean \pm 2 sigma or \pm 3 -sigma units apart 


\section{Construction of a Control Chart}

Center Line could be any of the following:

- A target value

- Standard value

- Overall mean computed from the data points after specified time period 


\section{Six Elements of a Control Chart}

(2) Measured Value Axis

(6) Upper Control Limits

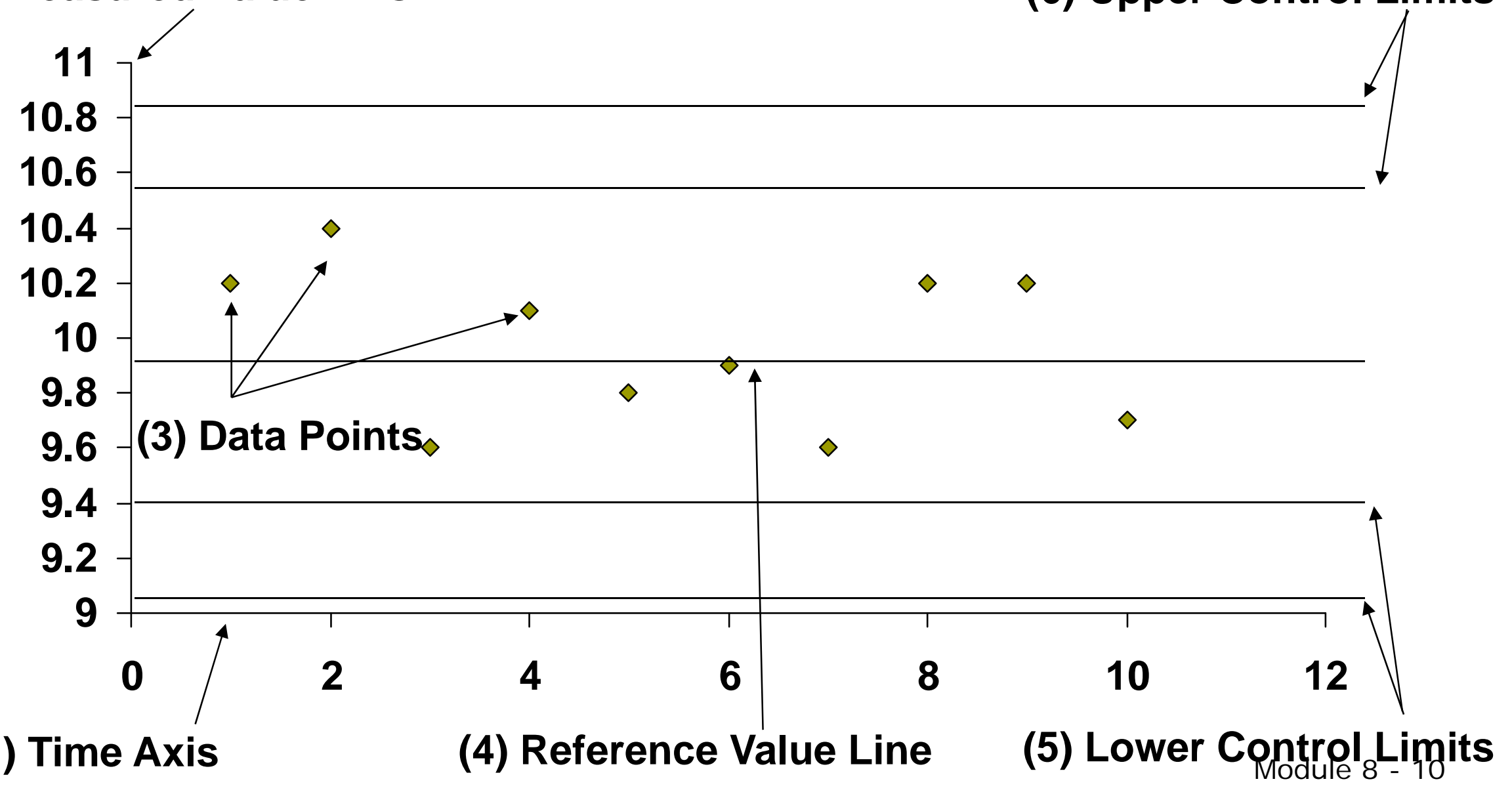




\section{Control Charts Simplified}

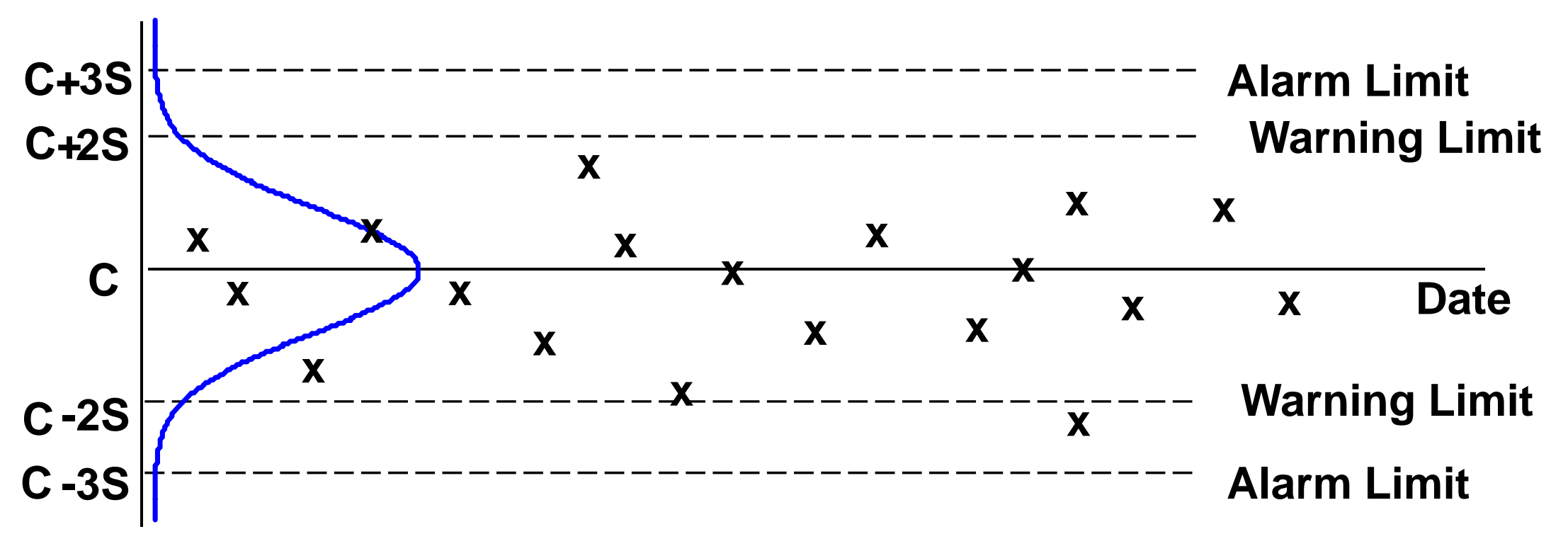

- Centerline $\mathrm{C}$ would generally be based on a known or estimated target value $T$

- $(\bar{X}-\mathrm{T})$ is called bias (or "systematic error")

- Variability about $\bar{X}$ indicates "random errors" 


\section{Control Charts Simplified}

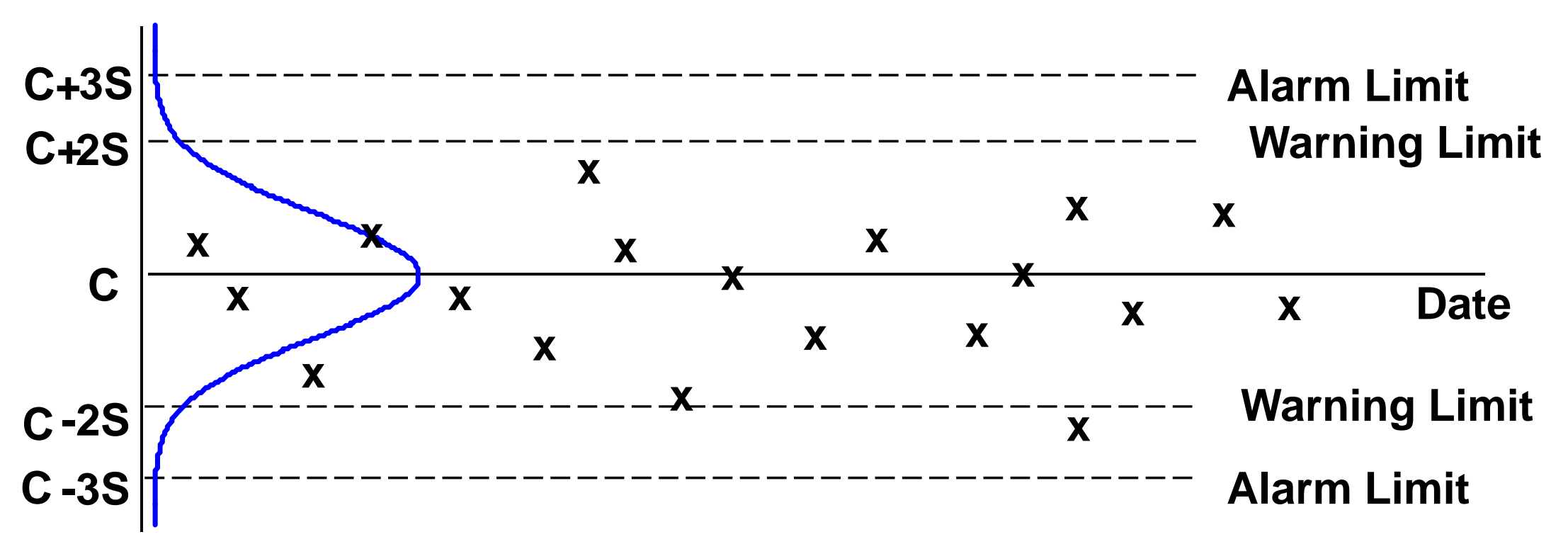

- Generally, $95 \%$ of the measurement control data should fall between $\mathrm{C}+/-2 \mathrm{~s}$

- Generally, $99.7 \%$ of the measurement control data should fall between $\mathrm{C}+/-3 \mathrm{~s}$ 


\section{Analyzing Control Charts}

- Trends

- Trends in data are non-random occurrences

- Outlier

- An outlier is an observation that is so far removed from the remaining data that it suggests either of the following:

- A mistake in measurement occurred

- It came from a different population 


\section{Indications of Potential Problems}

- One point outside the 3 s line

- Two out of three points outside the 2s line

- Eight consecutive points all above or below the centerline

- Six consecutive points trending upward or downward 


\section{Indications of Potential Problems}

- Fifteen consecutive points alternating above and below the centerline

- Each situation is a possible indication of an out-of-control process

- A measurement control program should adopt rules to identify out-of-control situations 


\section{Control Chart Example}

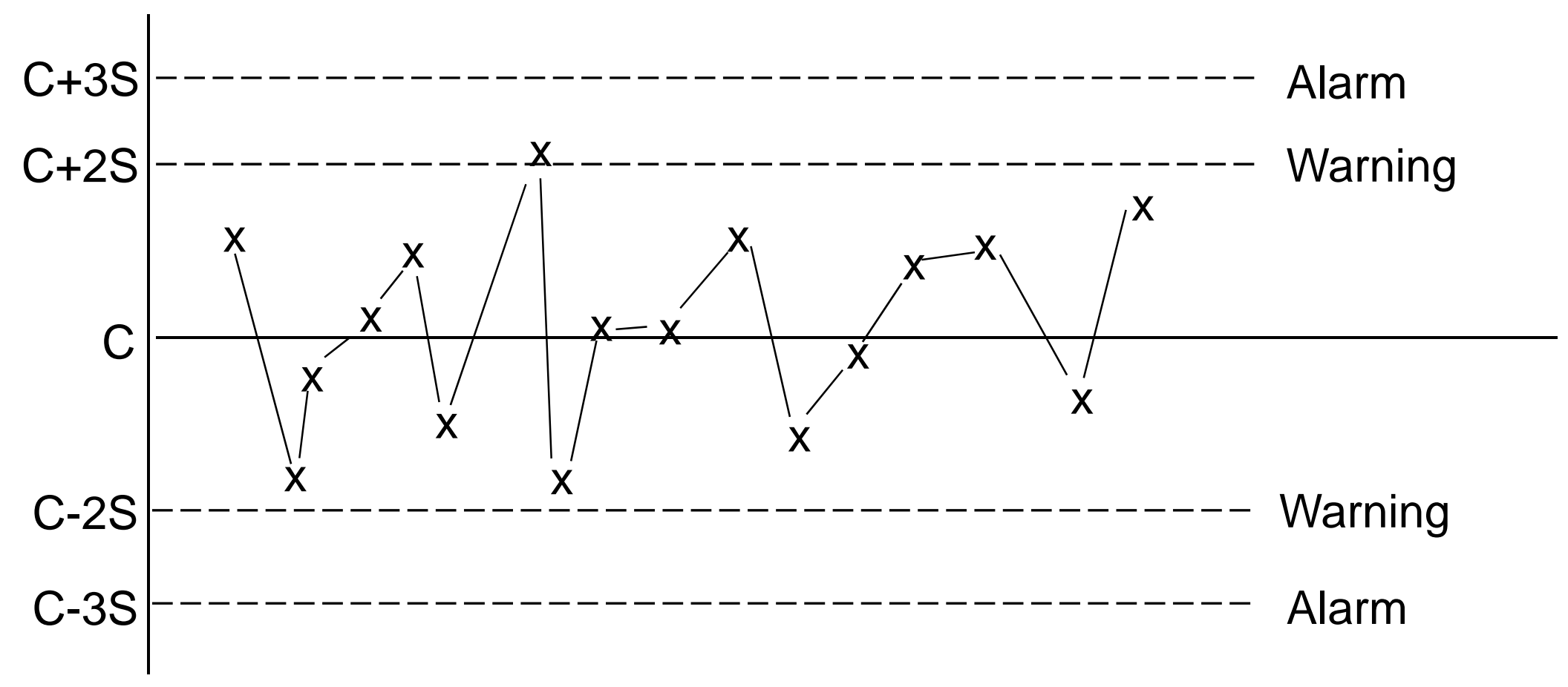

Module 8 - 16 


\section{Control Chart Example}

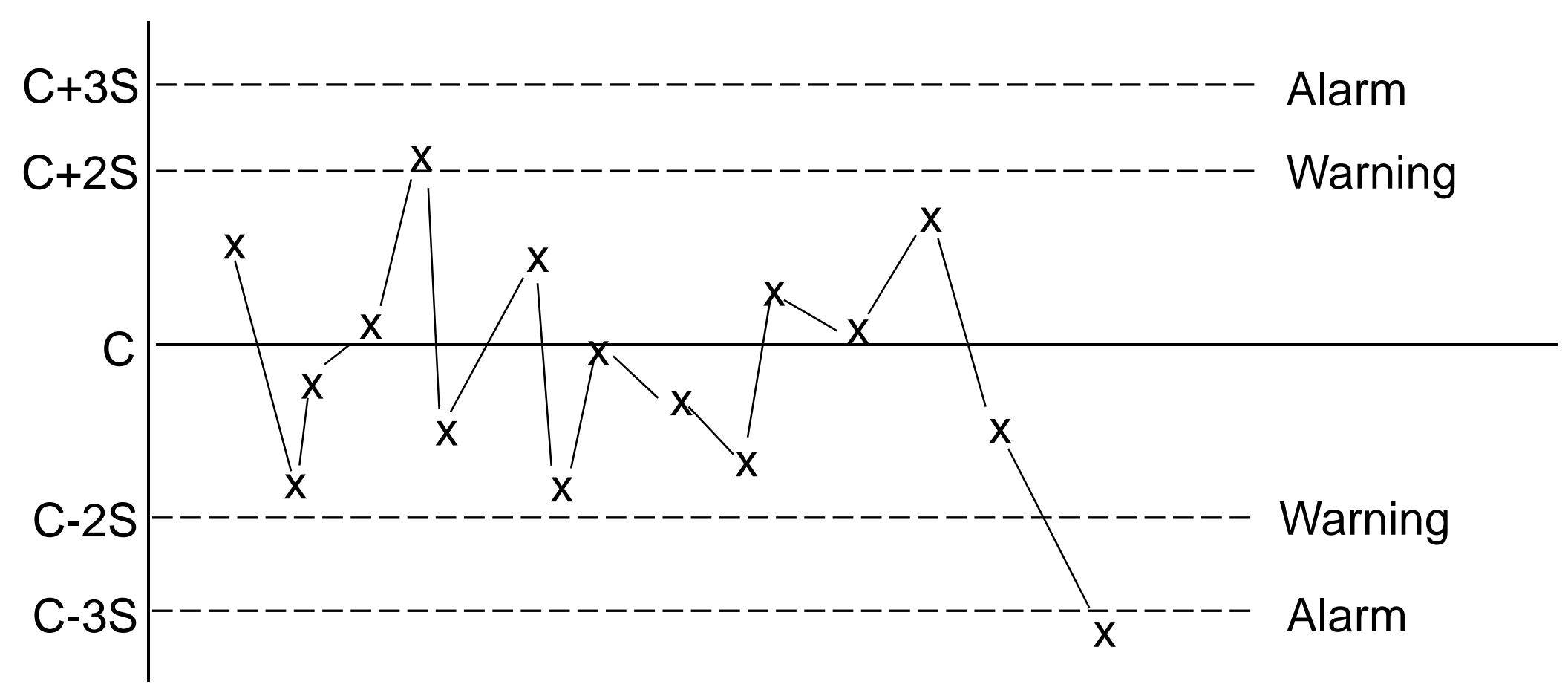

Module 8 - 17 


\section{Control Chart Example}

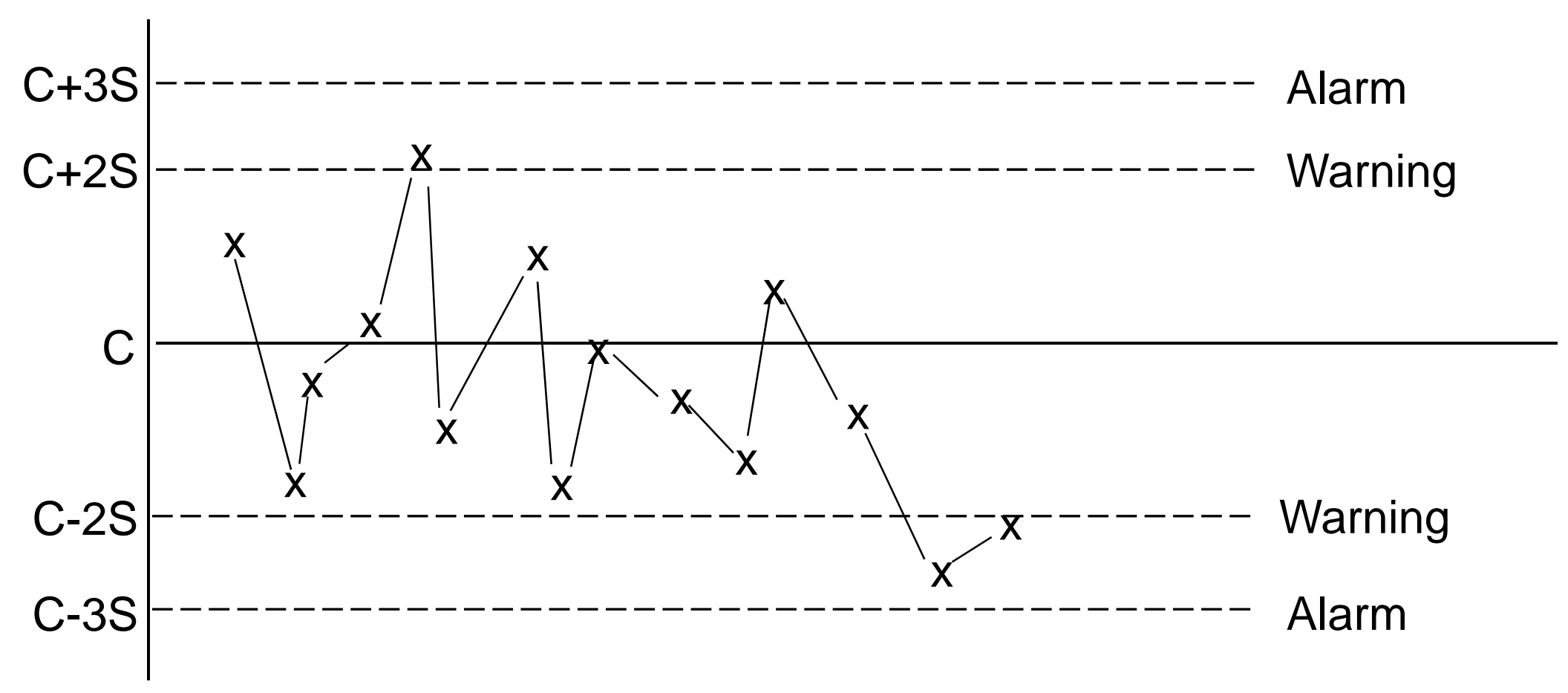

Module 8 - 18 


\section{Control Chart Example}

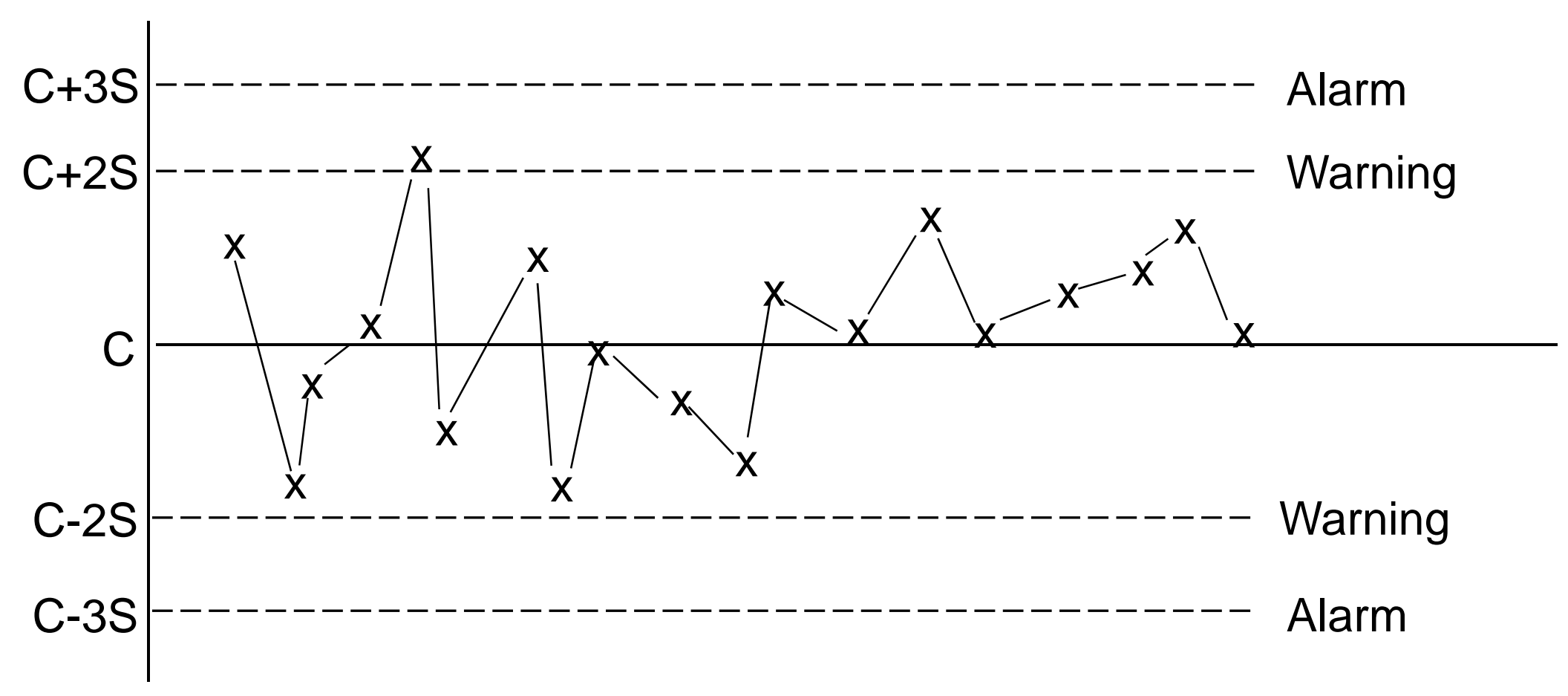

Module 8 - 19 


\section{Control Chart Example}

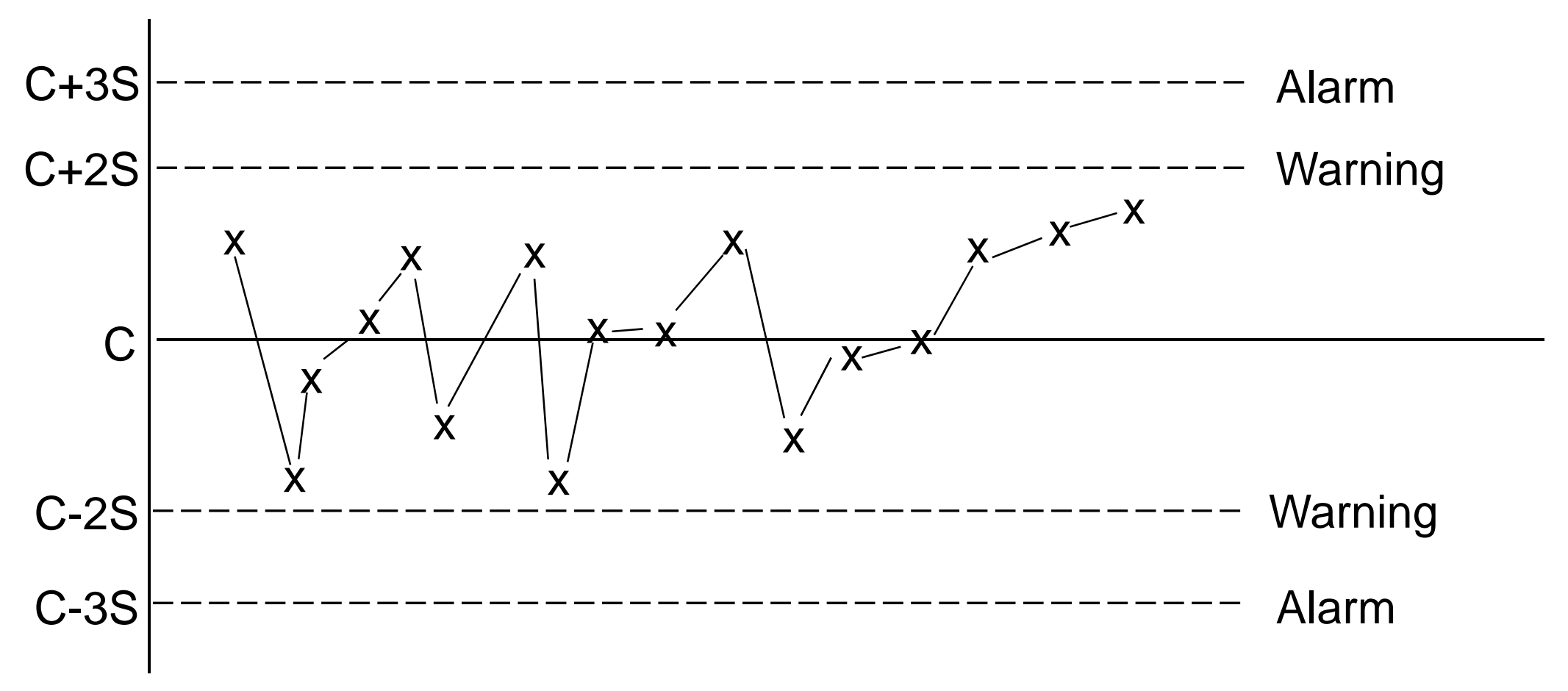

Module 8 - 20 


\section{Control Chart Example}

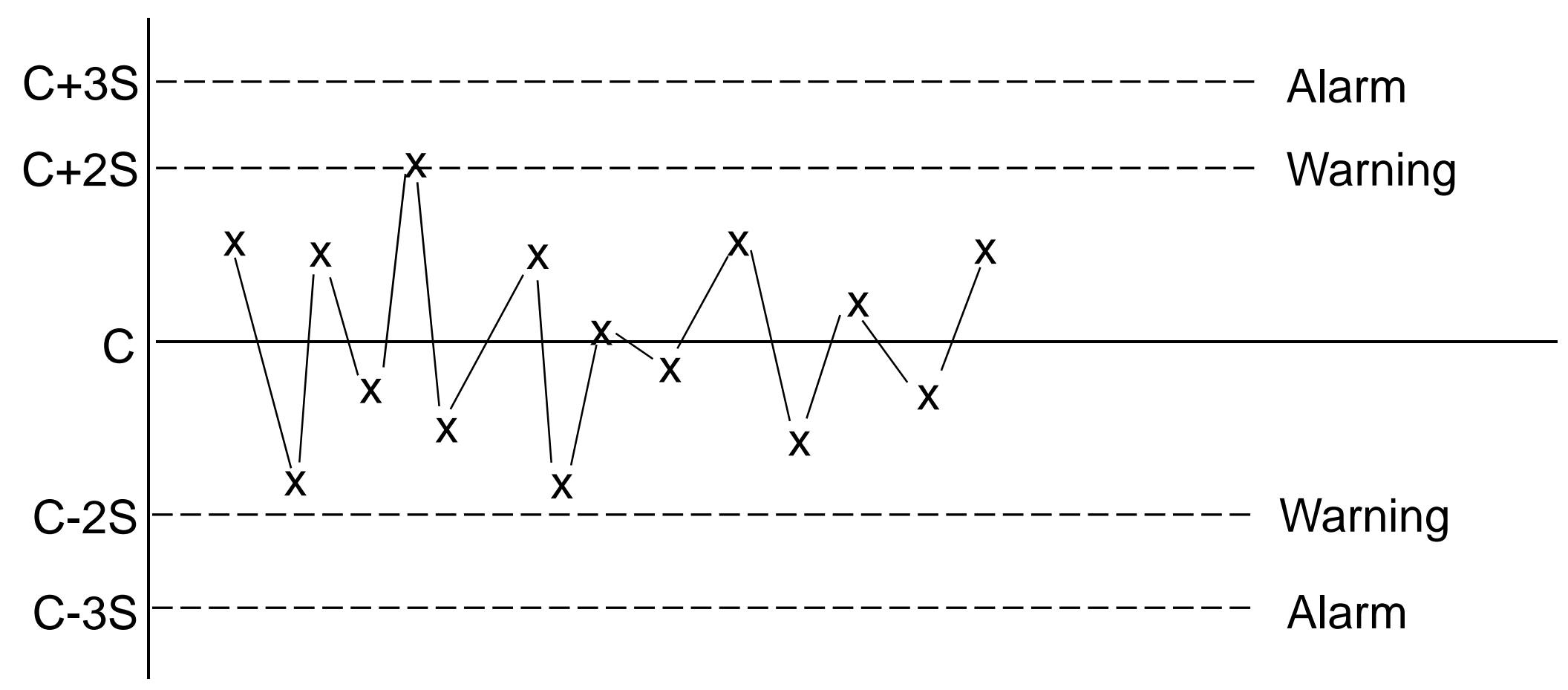

Module 8 - 21 


\section{Other Potential Problems}

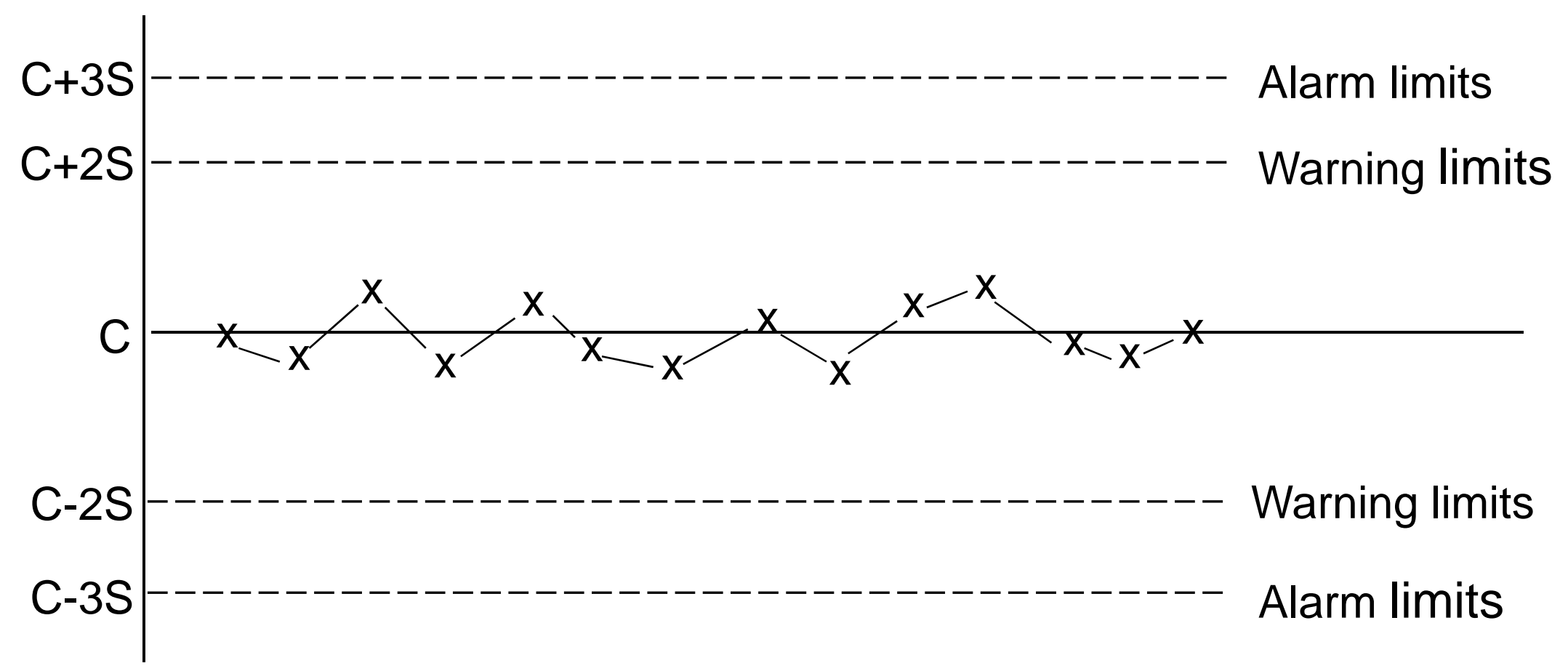




\section{Other Potential Problems}

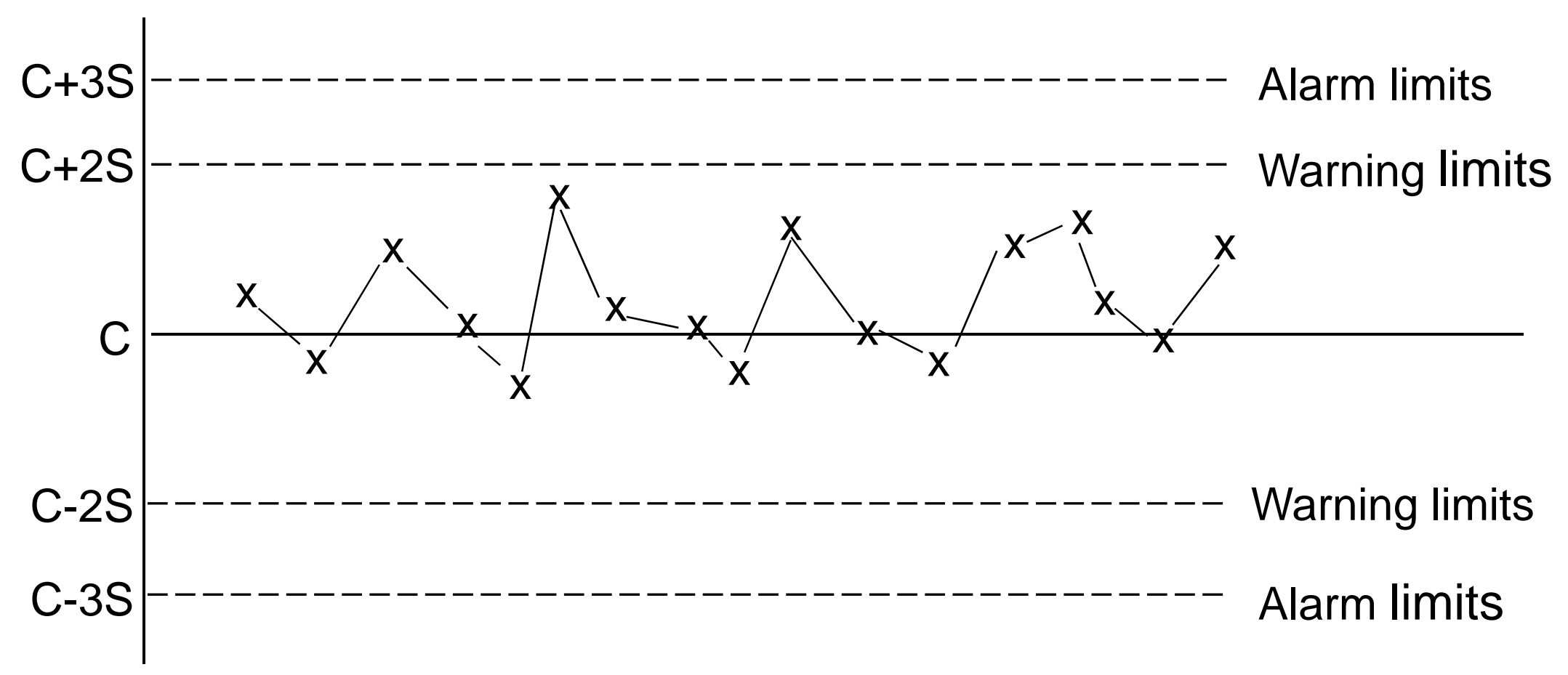

Module 8 - 23 


\section{Other Potential Problems}

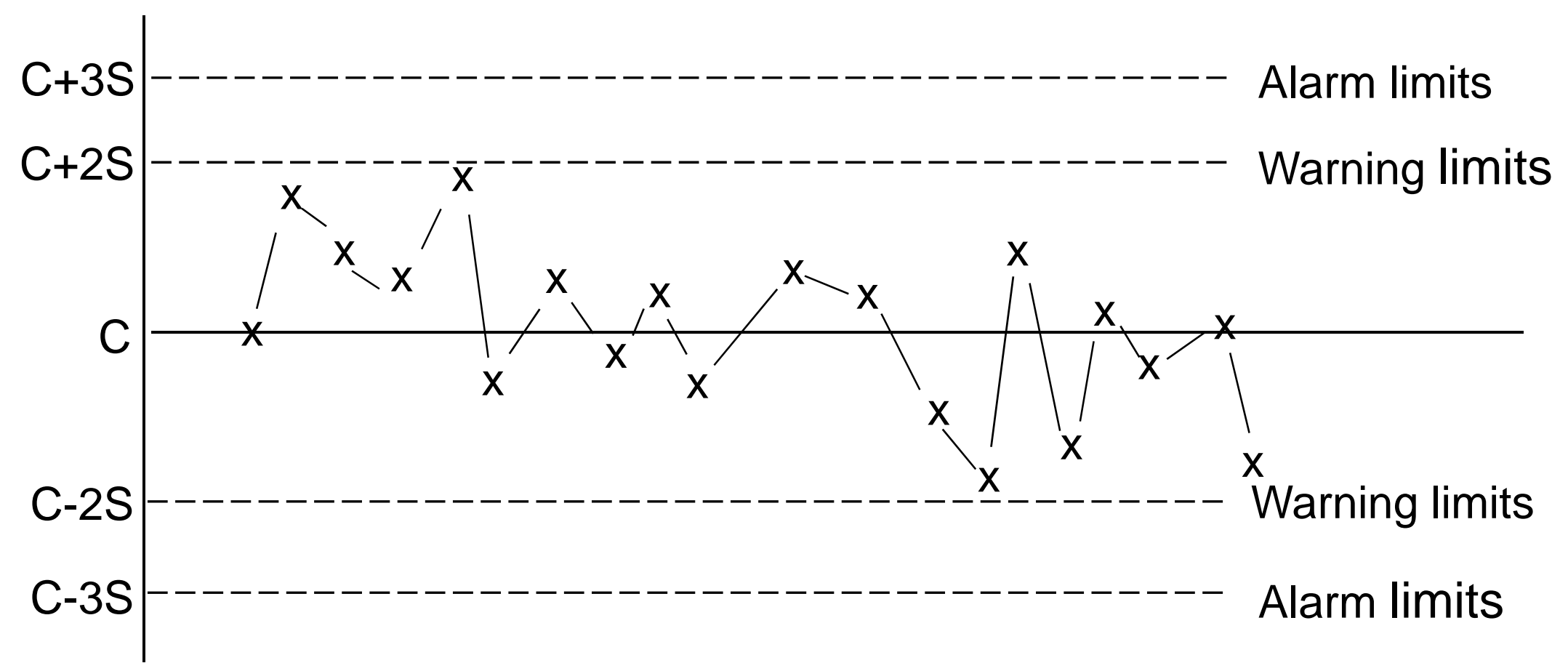

Module 8 - 24 


\section{Excluding Data and Outliers}

- When data is analyzed, examine the data for "special causes" or "assignable causes" that are used to exclude data that does not belong

- For example, a review of raw data for an analytical result identified a significant weighing error 


\section{Excluding Data and Outliers}

- When analyzing data, one or more data points do not seem to "belong" and could be considered outliers

- Tests for outliers exist

- Visual picture may serve as an indicator but formal tests should be applied

- Statistical Tests (e.g., Grubbs' T Test) 


\section{Part 2}

\section{Measurement Control Process}

and

\section{Control Charts}




\section{Measurement Control Process}

- The purpose of measurement control is to ensure the quality of a measurement

- This results in a measured value and an uncertainty for that value

- Sample or process measurements involve the determination of an unknown value

- Without measurement control, a measured value has very little, if any, meaning 


\section{Measurement Control Process}

- Quality control (QC) measurements must be made along with the process sample measurements

- This process cannot guarantee that process measurements are always correct

- However, if

- the QC measurements are done the same way as the process measurements

- and

- the QC samples are in control

- Then there is a high level of assurance that the process measurements are valid and meaningful 


\section{Measurement Control Process}

- Process sample measurements should be bracketed or contained within QC sample measurements

- The measurement process should proceed as follows:

1. Make an opening $\mathrm{QC}$ measurement

2. If $\mathrm{QC}$ is in control, then proceed

3. Make one or more process measurements

4. Make a closing QC measurement

5. If $\mathrm{QC}$ is in control, accept process measurements

6. If more process measurements are required, then return to step 3 


\section{Measurement Control Process}

\section{Bracket measurements of items with those of}

standards of similar weight

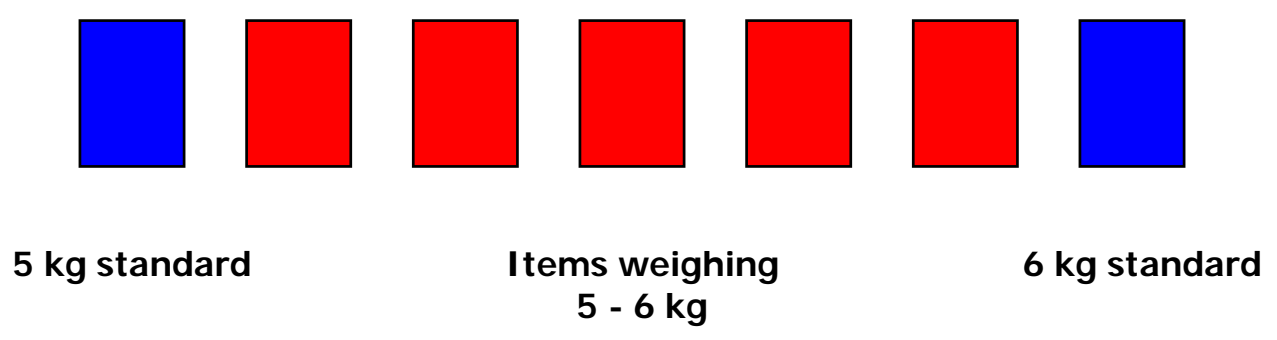

Standards within limits $\rightarrow$ accept measurements 


\section{Control Limits and Control Charts}

- Measurement control limits are derived and documented in the Method Qualification Report

- Control Limits are calculated at the 2 and 3 sigma levels

- The 2 sigma limits are warning limits

- The 3 sigma limits are alarm limits

- Control chart center line is set to zero or to the appropriate reference or historical value

- For additive models, limits are expressed in the same units as the measurement

- For multiplicative models, limits are expressed in relative or percent relative terms 


\section{Control Limits and Control Charts}

- Control limits can be calculated with or without bias corrections

- Control charts should be developed for each method

- In some cases, a particular method may have several control charts

- These charts are a critical tool for measurement control 


\section{Measurement Control Process}

- A control chart can be used to monitor the state of a measurement system

- A measurement system is considered out-of-control when

- 2 out of 3 consecutive QC results are outside warning limits

- or

- 1 QC result is outside of alarm limits

- A measurement system declared as out-of-control cannot be used for accountability measurements

- Actions must be taken to resolve the out-of-control condition 


\section{Measurement Control Process}

- The measurement system must demonstrate incontrol capability before measurements can resume

- This capability is demonstrated by having

- 3 consecutive QC results within warning limits

- and

- a result on each side of the center line

- If an opening QC measurement is out-of-control, then process measurements cannot proceed until the measurement system is shown to be in-control

- If a closing QC measurement is out-of-control, then all process measurements since the last opening QC must be re-measured 


\section{Measurement Control Process Discussion Topics}

1. What has happened if an opening $Q C$ is out-ofcontrol?

2. How can a closing $Q C$ be out-of-control?

3. What action should be taken if an opening $Q C$ value exceeds a warning limit?

4. When can a closing $Q C$ also be an opening $Q C$ ?

5. Discuss strategies for bracketing process measurements.

6. In an out-of-control situation, are re-measurements always feasible? 


\section{Actual Control Charts}

The next six slides are examples of actual control charts used at the Savannah River Site

Review and discuss each of these charts 


\section{Davies-Gray U Concentration}

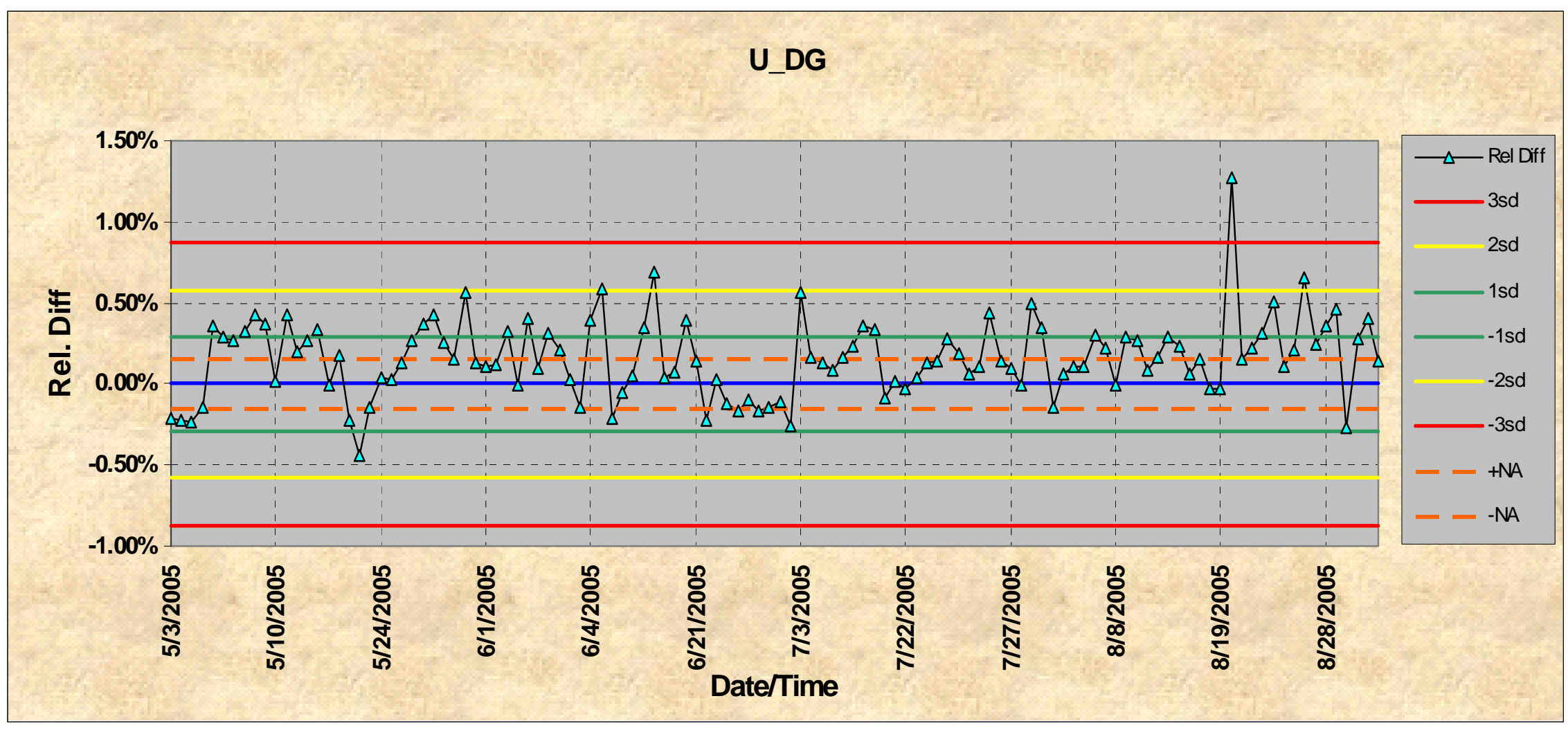

Module 8 - 38 


\section{Davies-Gray U Concentration}

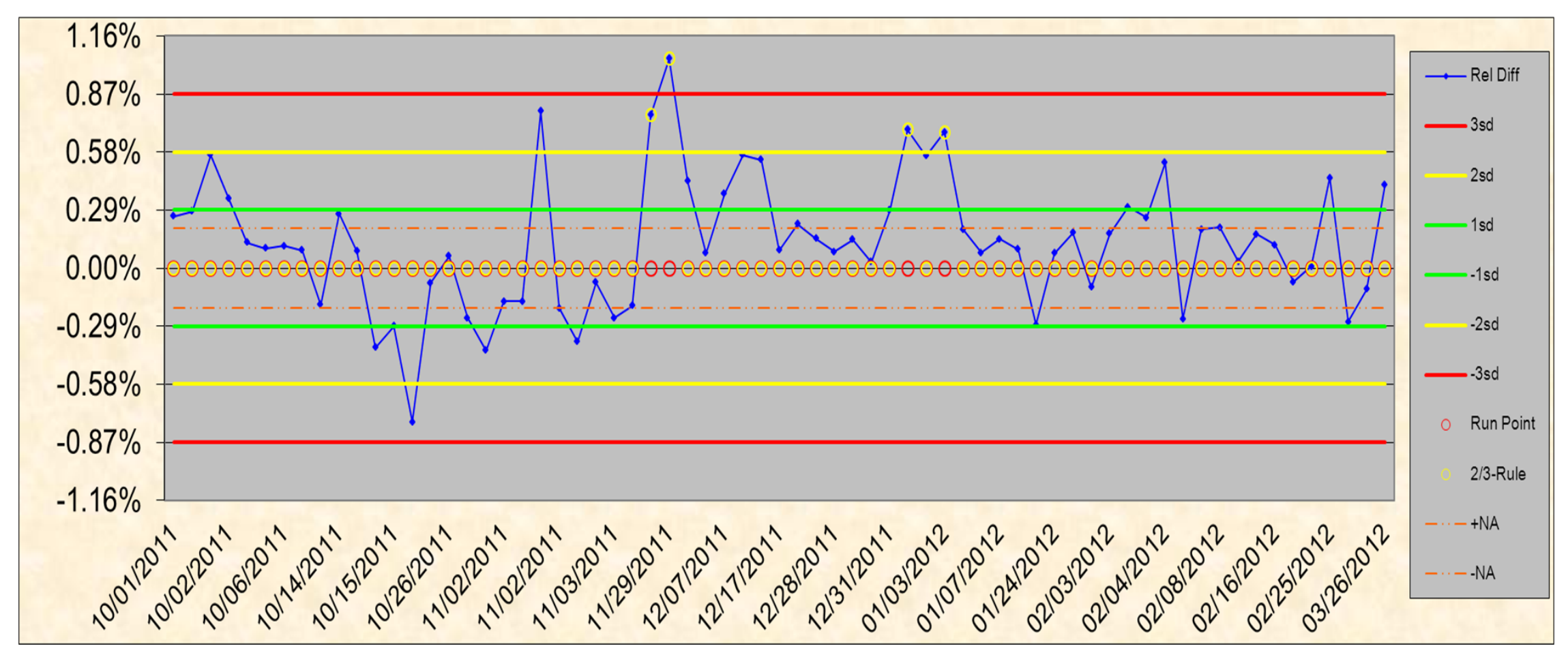

Module 8 - 39 


\section{Pu I DMS : Pu Concentration}

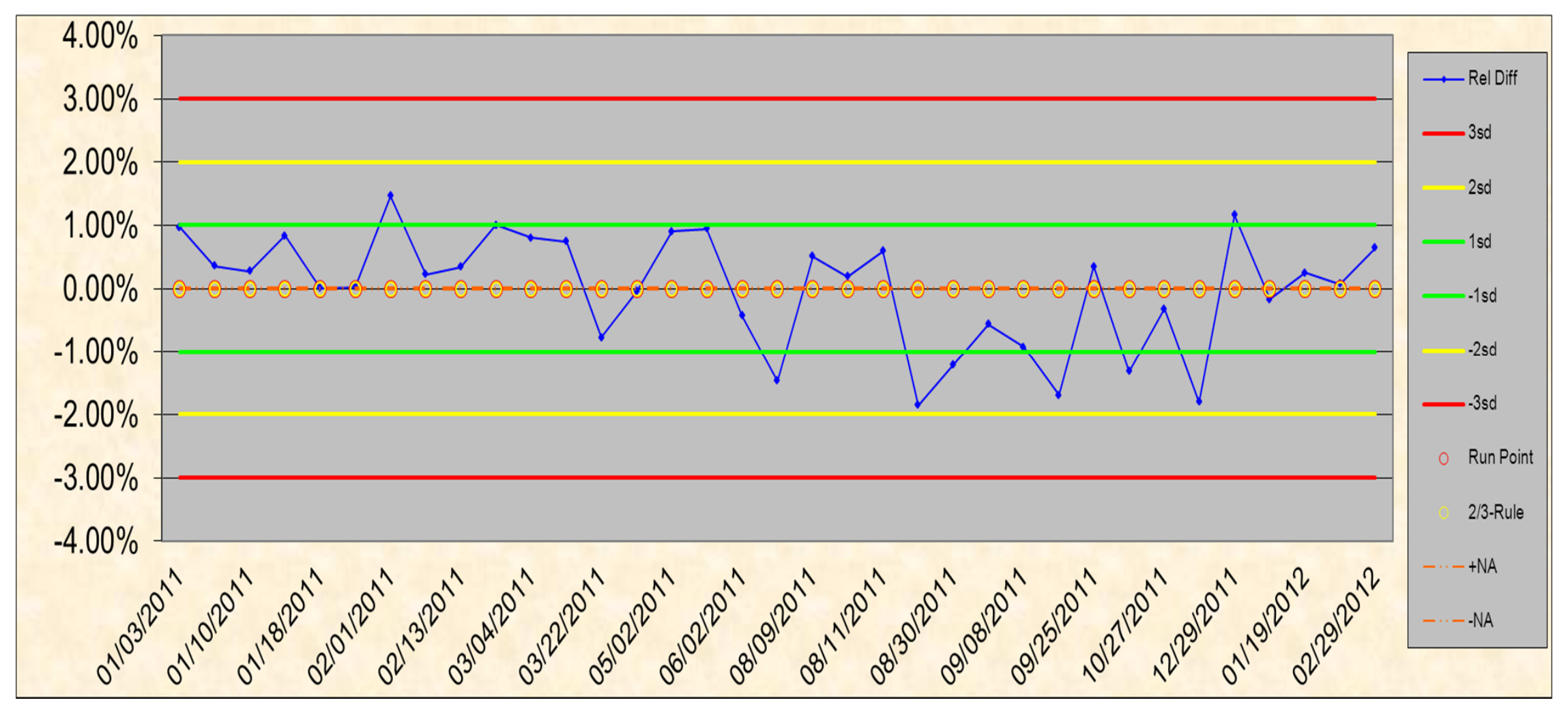




\section{Mass Spec Pu I sotopics}

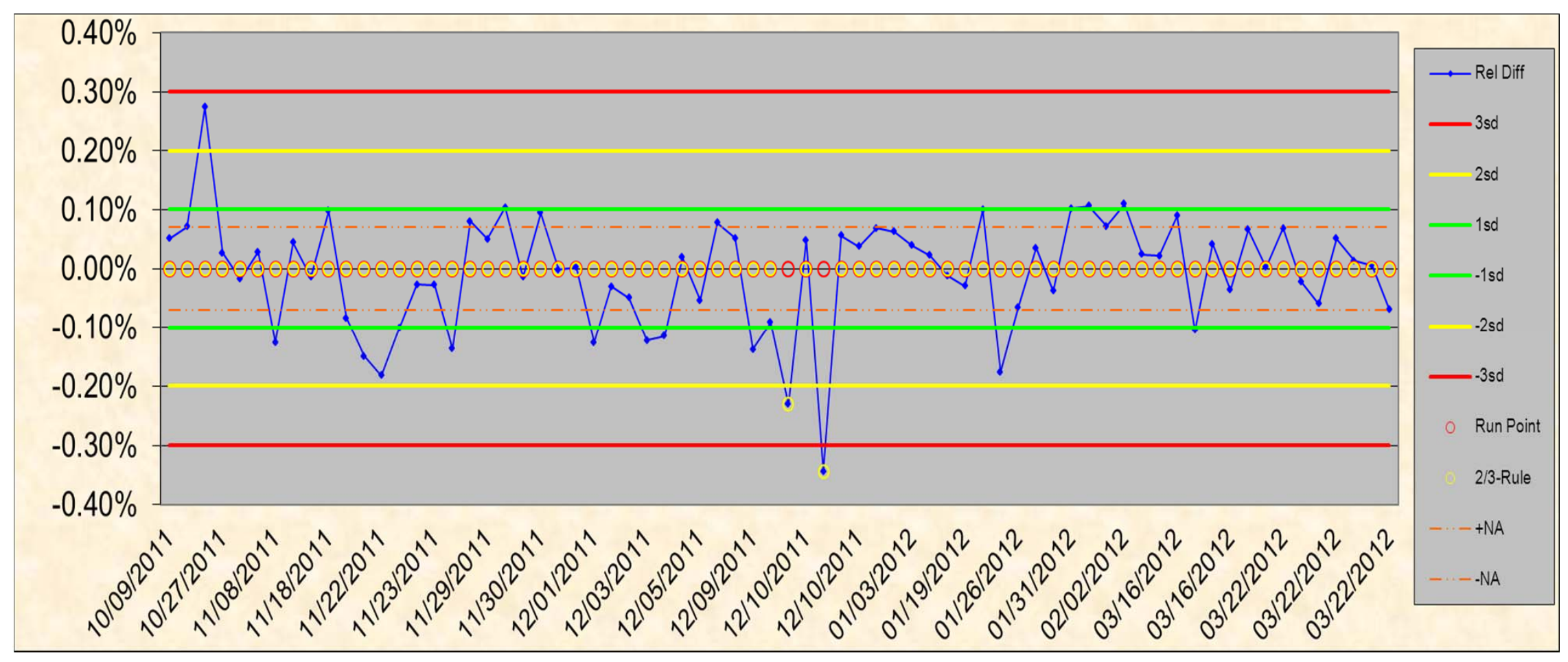




\section{U I DMS : U Concentration}

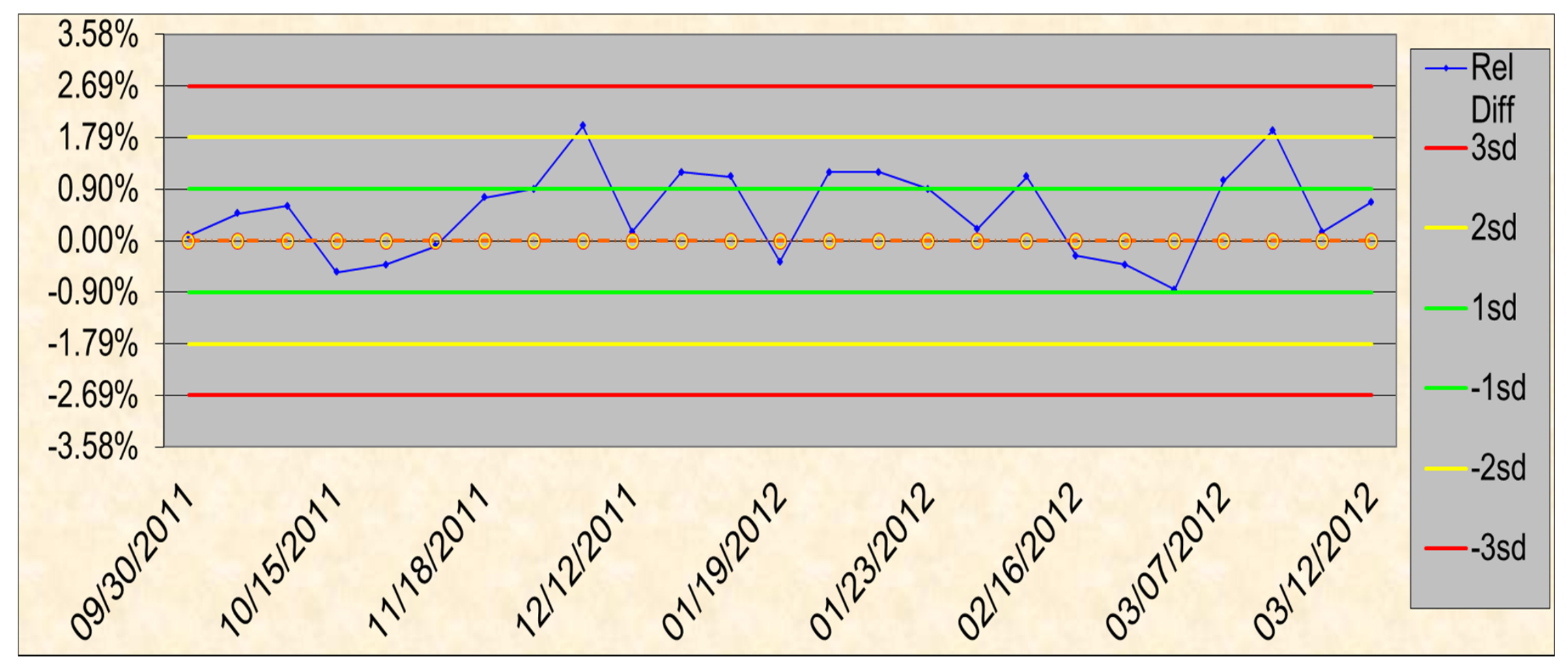




\section{Mass Spec U-235 I sotopic}

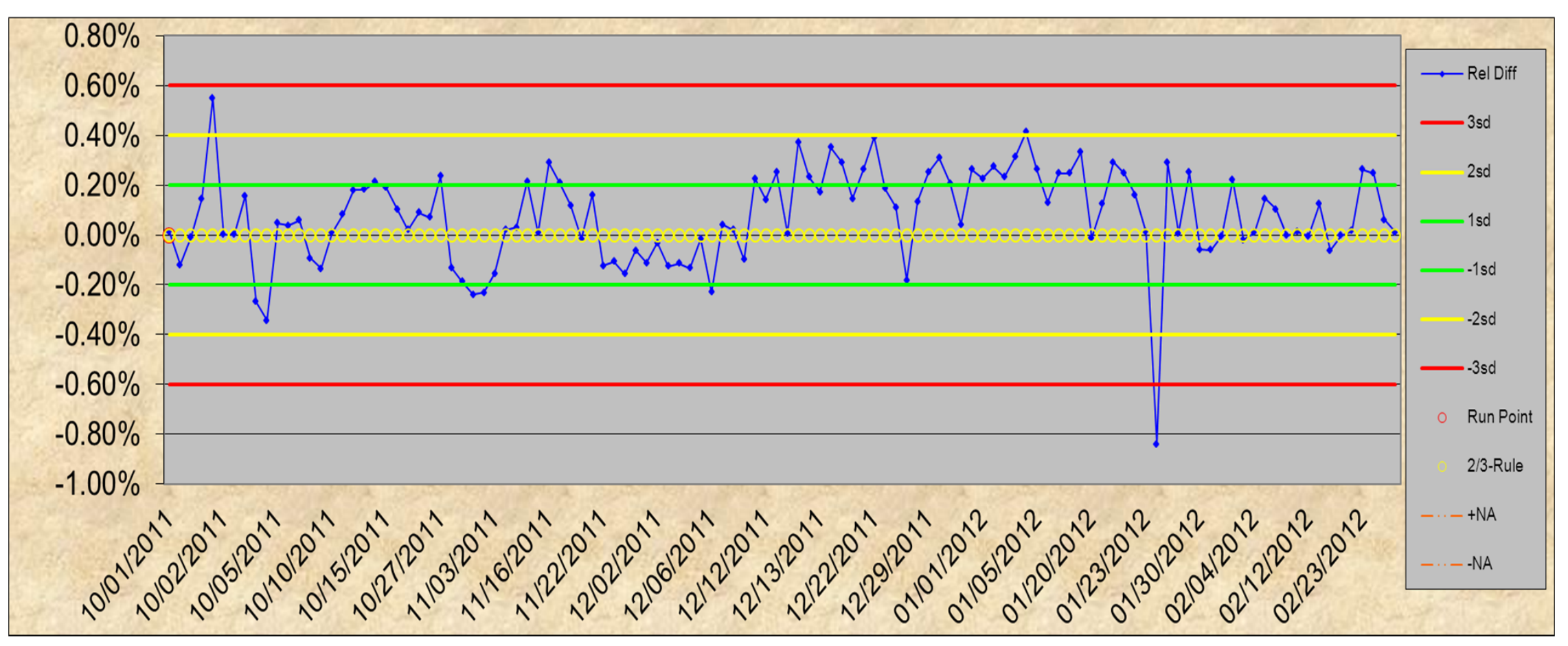




\section{Runs Rule Criteria Applied}

- A runs rule is designed to detect an abrupt or slow change to a measurement system that results in a consistent bias

- This bias would result in QC measurements that are consistently high or low

- A runs rule violation, considered an "adverse condition," occurs when 8 consecutive QC values are on the same side of the control chart center line

- An adverse condition is handled as follows:

1. Investigate, and if possible, correct the adverse condition

- An acceptable action is to continue with measurements while monitoring the system

2. Document the adverse condition and any corrective actions taken 


\section{Runs Rule Criteria - Discussion Topics}

1. Could a method runs rule problem affect actual process samples?

2. What should be done if 5 consecutive $Q C$ values show a constant bias? 


\section{Runs Rule Control Charts}

Review and discuss the following actual control charts used at the Savannah River Site 


\section{Diode Array U Concentration}

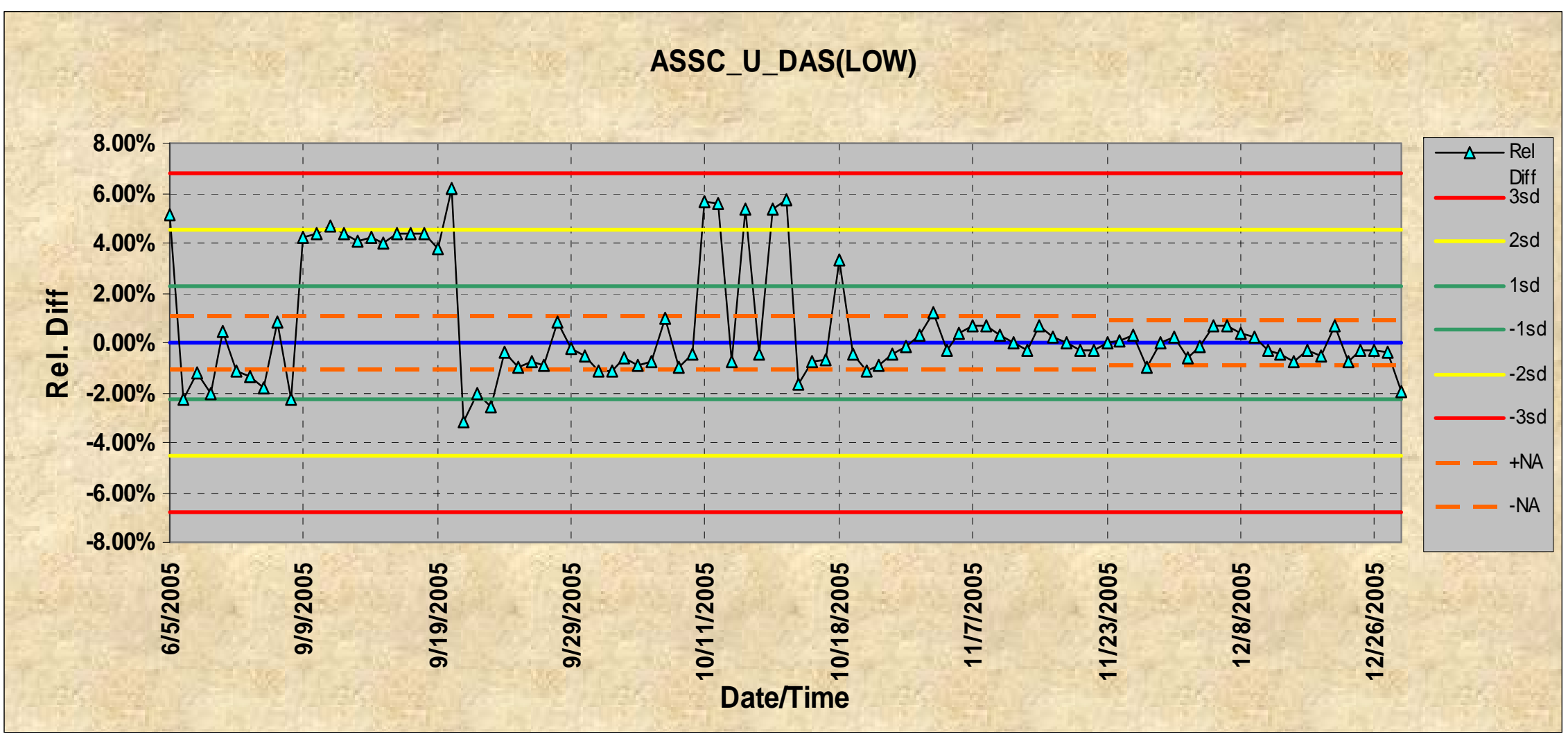

Module 8 - 47 


\section{ChemChek : Low U Concentration}

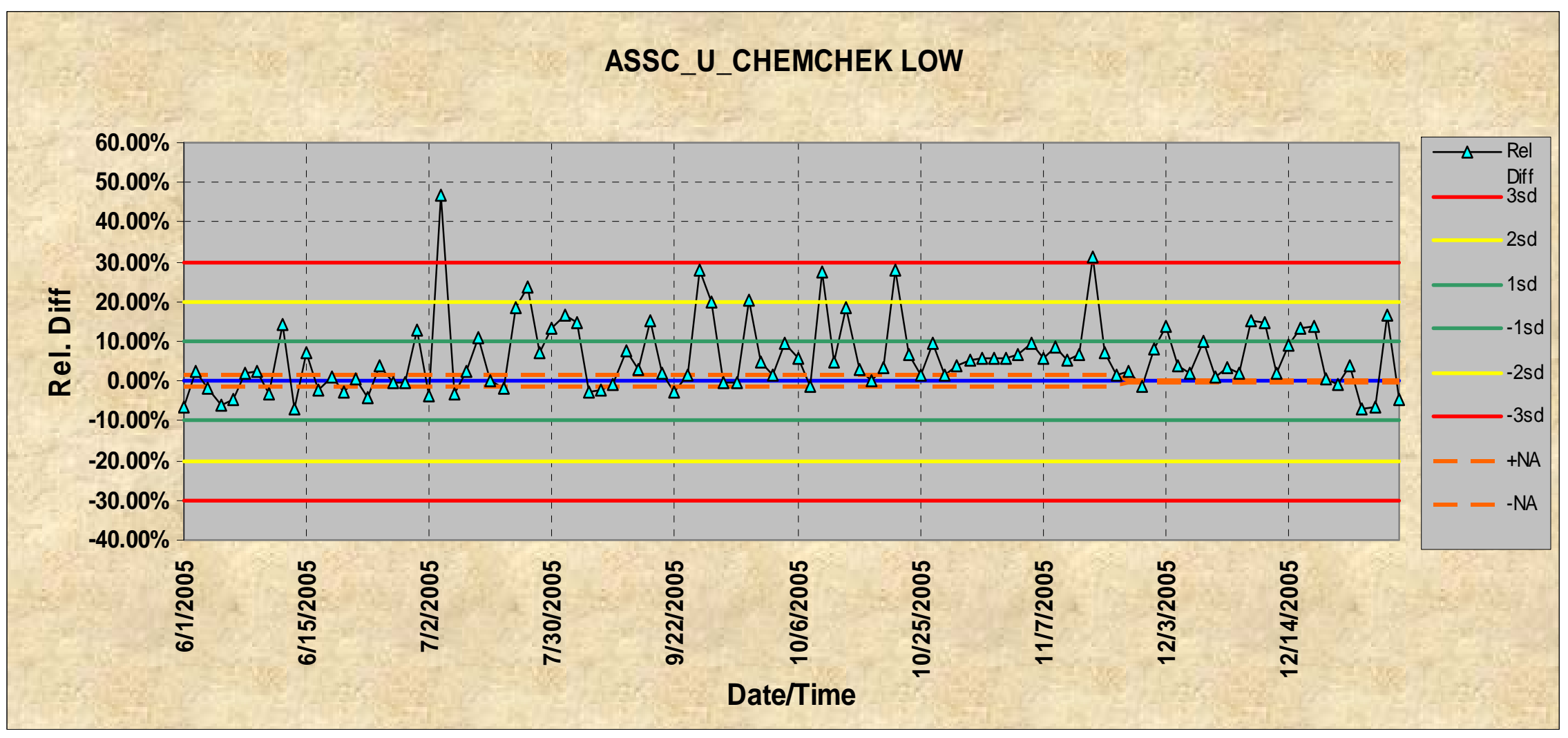

Module 8 - 48 


\section{Pu TEVA : Low Concentration}

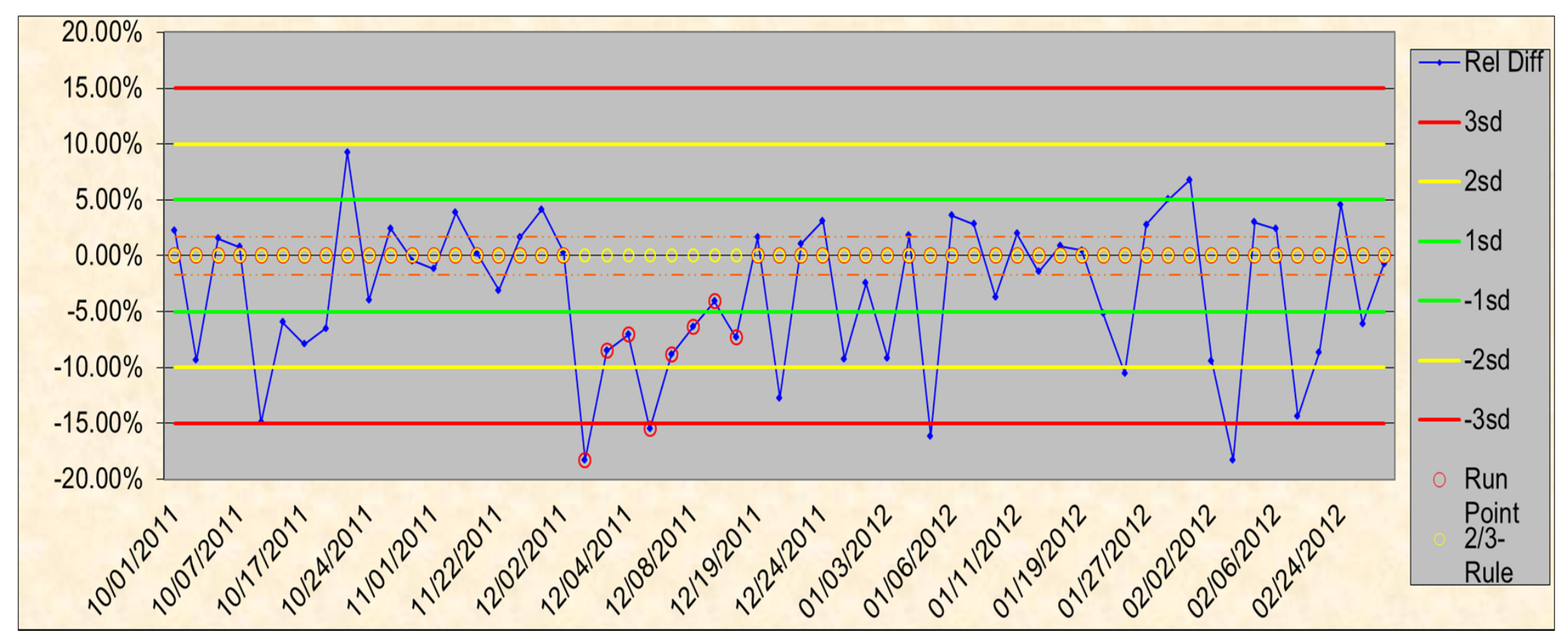

Module 8 - 49 


\section{Lesson Summary}

- Identified the purpose of control charts

- Identified the six elements of control charts and their purpose

- Discussed and analyzed control charts

- Discussed control charts and measurement control

- Discussed and analyzed control charts from actual measurement systems 


\section{Exercise \\ Calibration Module}

\section{Session Objectives:}

After the session the participants will be able to do the following:

1. Understand the function of a calibration model

2. Understand the concept of a calibration equation

3. Understand the concept of regression analysis and the prediction equation

4. Understand the use of regression analysis for calibration uncertainty

5. Understand the concept of measurement uncertainty in calibration and prediction equations

6. Understand the derivation of the prediction equation random and systematic uncertainties

\section{Estimated Time:}

60 minutes to complete Exercise \#1

\section{Materials Needed:}

1. One computer with Microsoft Excel for each group of four or five students

2. Excel needs to have the Stat Package add-in loaded

3. Students need to have a good working knowledge of Microsoft Excel

4. Spreadsheet support person

\section{Instructions:}

1. Students should work in groups of four or five

2. The tank calibration data will be supplied to the students in an Excel spreadsheet

3. Students will use the Excel Regression and other functions to compute uncertainties

4. The exercise will be instructor led

5. Discussion will occur at each step in the exercise emphasizing session objectives 


\section{$\underline{\text { Exercise \#1 }}$}

The students, with instructor assistance, will complete the following activities:

1. Use the Excel Scatter Plot to produce a plot of the tank calibration data

2. Use the Regression Analysis function to estimate the calibration equation parameters, the parameter uncertainties and the residual mean square or variance

3. Invert the calibration equation to derive the prediction equation

4. Use variance propagation techniques to compute the systematic and random uncertainties for the prediction equation for level values of $0.3,0.6$ and 0.9 volts. 


\section{Exercise \\ Calibration Module}

\section{Session Objectives:}

After the session the participants will be able to do the following:

1. Understand the function of a calibration model

2. Understand the concept of a calibration equation

3. Understand the concept of regression analysis and the prediction equation

4. Understand the use of regression analysis for calibration uncertainty

5. Understand the concept of measurement uncertainty in calibration and prediction equations

6. Understand the derivation of the prediction equation random and systematic uncertainties

\section{Estimated Time:}

60 minutes to complete Exercise \#1

\section{Materials Needed:}

1. One computer with Microsoft Excel for each group of four or five students

2. Excel needs to have the Stat Package add-in loaded

3. Students need to have a good working knowledge of Microsoft Excel

4. Spreadsheet support person

\section{Instructions:}

1. Students should work in groups of four or five

2. The tank calibration data will be supplied to the students in an Excel spreadsheet

3. Students will use the Excel Regression and other functions to compute uncertainties

4. The exercise will be instructor led

5. Discussion will occur at each step in the exercise emphasizing session objectives 


\section{$\underline{\text { Exercise \#1 }}$}

The students, with instructor assistance, will complete the following activities:

1. Use the Excel Scatter Plot to produce a plot of the tank calibration data

2. Use the Regression Analysis function to estimate the calibration equation parameters, the parameter uncertainties and the residual mean square or variance

- $\quad$ The Intercept $=\mathbf{- 0 . 2 9}$ and the $\mathrm{V}($ Intercept $)=0.0049^{2}$

- $\quad$ The Slope $=0.0017$ and the $\mathrm{V}($ Slope $)=(9.53 \mathrm{E}-6)^{2}$

- The Cov(Intercept, Slope $)=-4.51 E-8$ (not from Excel Regression Analysis)

- The Residual Mean Square (RMS) $=3.51 E-5$ Volts $^{2}$

3. Invert the calibration equation to derive the prediction equation

- Prediction equation or Volume $=($ Level +0.29$) / 0.0017$

4. Use variance propagation techniques to compute the systematic and random uncertainties for the prediction equation for level values of $0.3,0.6$ and 0.9 volts.

- At 0.3 Volts: Predicted Volume $=347 \mathrm{~L}$, Systematic Uncertainty $=1.0 \mathrm{~L}$

- At 0.6 Volts: Predicted Volume $=523 \mathrm{~L}$, Systematic Uncertainty $=0.6 \mathrm{~L}$

- At 0.9 Volts: Predicted Volume $=699$ L, Systematic Uncertainty $=1.3 \mathrm{~L}$

- Over Entire Voltage Range: Random Uncertainty $=3.5 \mathrm{~L}$ 


\section{Chinese Measurement Control Workshop}

Calibration Module

Exercise \#1

\begin{tabular}{|r|r|}
\hline Volume & \multicolumn{1}{|l|}{ Level } \\
\hline Liters & \multicolumn{1}{|l|}{ Volts } \\
\hline & \\
\hline 330 & 0.28 \\
\hline 332 & 0.28 \\
\hline 333 & 0.27 \\
\hline 340 & 0.29 \\
\hline 370 & 0.33 \\
\hline 375 & 0.34 \\
\hline 377 & 0.36 \\
\hline 390 & 0.37 \\
\hline 415 & 0.42 \\
\hline 418 & 0.43 \\
\hline 425 & 0.43 \\
\hline 430 & 0.45 \\
\hline 465 & 0.5 \\
\hline 470 & 0.52 \\
\hline 475 & 0.52 \\
\hline 480 & 0.52 \\
\hline 500 & 0.57 \\
\hline 515 & 0.59 \\
\hline 520 & 0.6 \\
\hline 525 & 0.6 \\
\hline 550 & 0.64 \\
\hline 565 & 0.67 \\
\hline 570 & 0.69 \\
\hline 575 & 0.7 \\
\hline 620 & 0.77 \\
\hline 625 & 0.77 \\
\hline 625 & 0.77 \\
\hline 630 & 0.77 \\
\hline 655 & 0.82 \\
\hline 655 & 0.82 \\
\hline 670 & 0.84 \\
\hline 675 & 0.86 \\
\hline & \\
\hline
\end{tabular}

\begin{tabular}{|c|c|c|c|}
\hline \multicolumn{5}{|c|}{ Predicted Volumes and Uncertainties } \\
\hline & & Systematic Uncertainty & Random Uncertainty \\
\hline Volume in Liters & Level in Volts & in liters & in liters \\
\hline & & & \\
\hline & 0.300 & & \\
\hline & 0.600 & & \\
\hline & 0.900 & & \\
\hline
\end{tabular}

Regression Level Uncertainty =

\begin{tabular}{c|c} 
Simulation Level Uncertainty $=$ & 0.005
\end{tabular} 


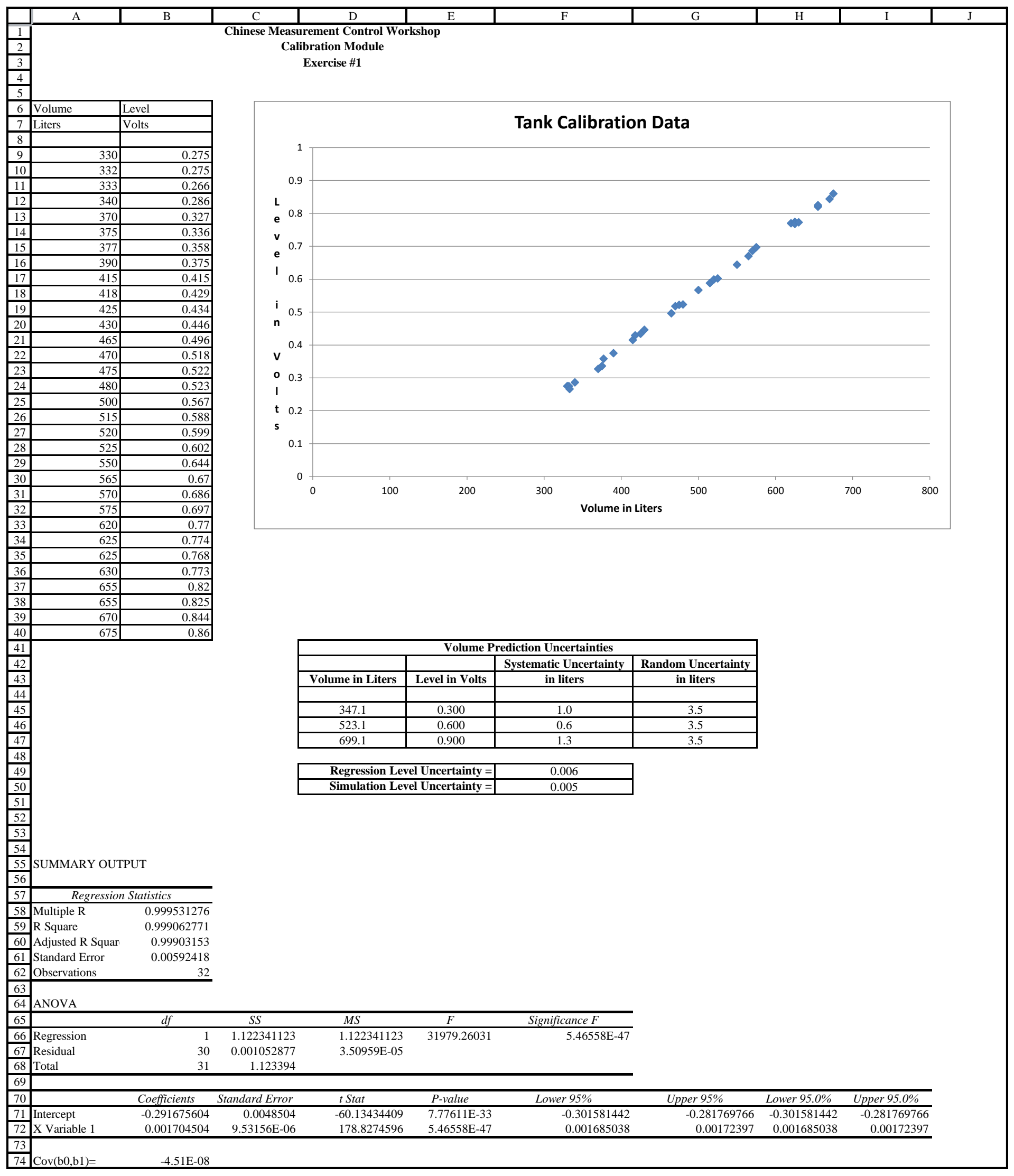




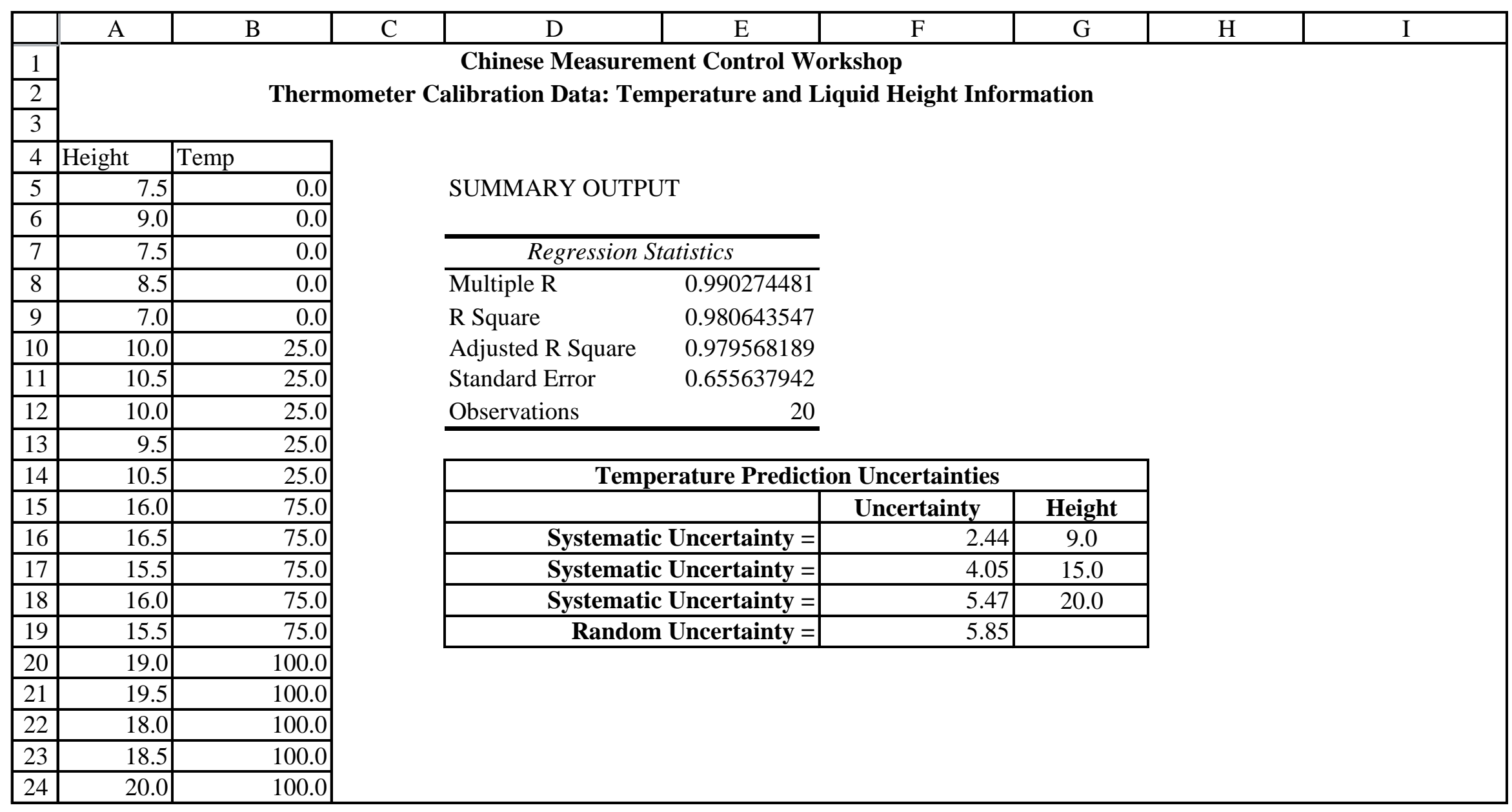




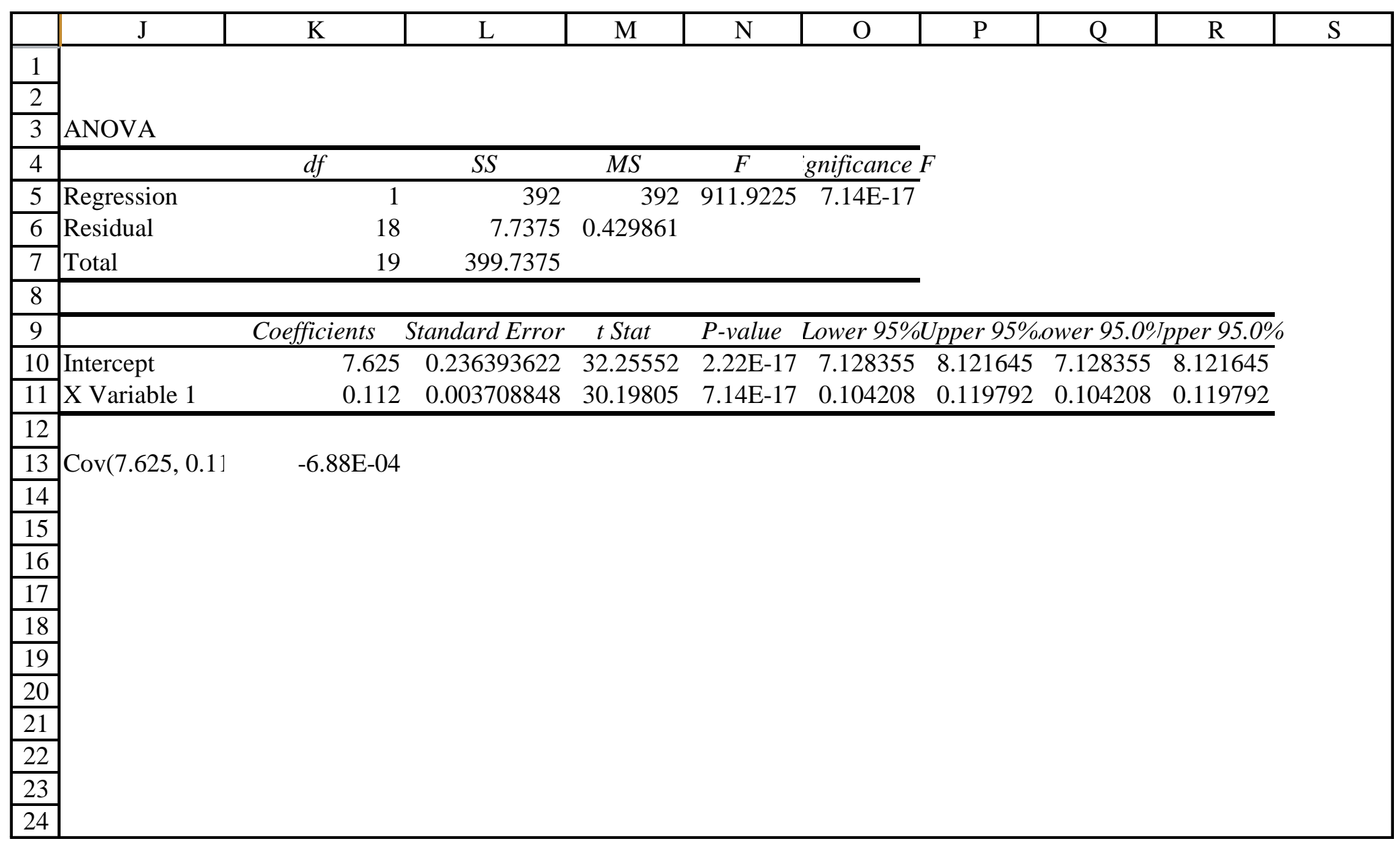




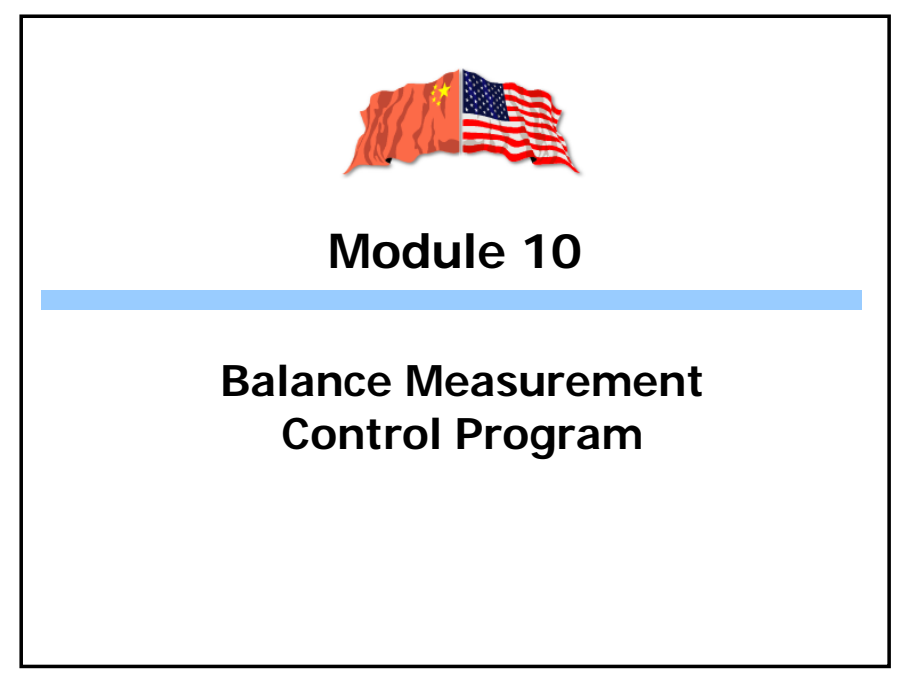

\section{Objectives}

- Review the elements of a MCP for scales or balances

- Discuss the classes of balances \& mass standards

- Exercise on classes of balances and weights

- Review method for determining weighing uncertainty

- Discuss the Savannah River site balance calibration/verification program

- Discuss sources and types of error in weighing

\section{Balance MCP Elements}

- Selection of appropriate equipment \& standards

- Training personnel in care and use of equipment

- Controls to verify balances are in control before use

- Procedures for verification prior to use

- Test the range of use (maximum \& minimum if $<75 \%$ )

- May use calibrated artifact or appropriate mass standard

- Repeat validation if environmental conditions are changing

- Good to end weighing sequence with check standard

- Data collection and control charting: use computers

- Statistical procedures for evaluating control data

- Periodic updating of uncertainty estimates \& control limits

\section{Good Weight Measurements Require:}

- Choosing the appropriate weighing equipment

- Choosing appropriate mass standards

- Proper handling and use of standards \& balances

- Routine calibration/verification

- Administrative procedures for measurement control

- Verify balance is "in control" before measuring unknowns using appropriate check standards

- Reliable uncertainty estimates for weighing systems 


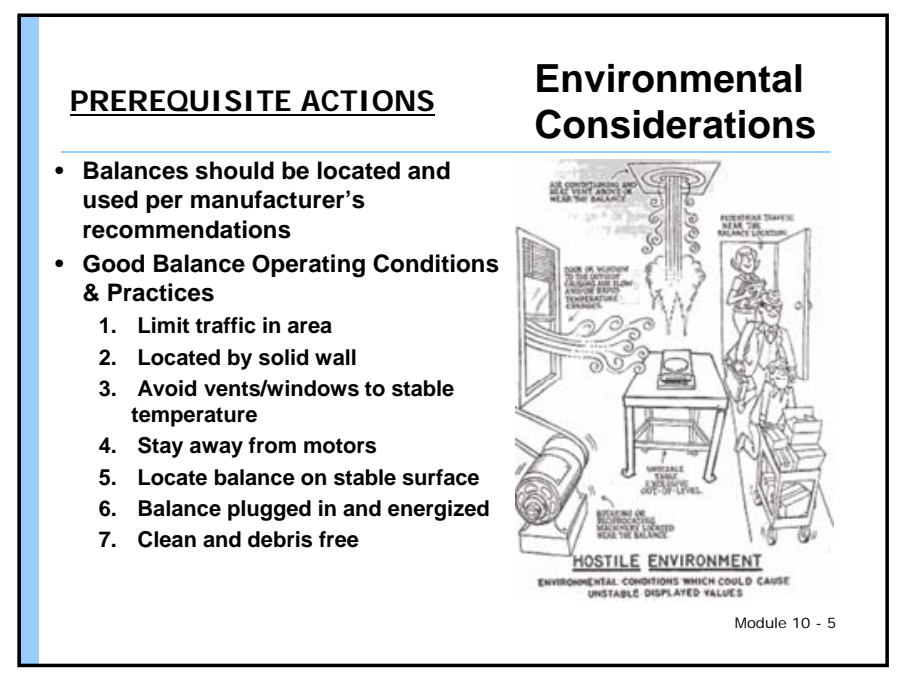

\section{Factors that influence weight readings}

- Design

- Installation

- Staff \& Procedures

- Standards

- Facility (Environment/Location)

- Method of Use

\section{Four classes of scales and balances}

Parameters for Accuracy Classes

\begin{tabular}{|c|c|c|c|}
\hline \multirow{2}{*}{ Class } & $\begin{array}{c}\text { Value of the Verification Scale } \\
\text { Division }\end{array}$ & $\begin{array}{c}\text { Number of Scale Divisions } \\
\text { (n) }\end{array}$ \\
\cline { 2 - 4 } & \multicolumn{3}{|c|}{ SI Units } \\
\hline \multicolumn{3}{|c|}{$\begin{array}{c}\text { Minimu } \\
\text { m }\end{array}$} & Maximum \\
\hline I & equal to or greater than 1 mg & 50000 & 20000000 \\
\hline II & 1 to $50 \mathrm{mg}$, inclusive & 100 & 100000 \\
\hline & equal to or greater than $100 \mathrm{mg}$ & 5000 & 100000 \\
\hline III & 0.1 to $2 \mathrm{~g}$ inclusive & 100 & 10000 \\
\hline & equal to or greater than $5 \mathrm{~g}$ & 500 & 10000 \\
\hline IIII & equal to or greater than $5 \mathrm{~g}$ & 100 & 1200 \\
\hline
\end{tabular}




\section{OI ML Weight Classes (continued)}

- Class F1 weights - appropriate for calibrating highprecision top loading balances with readability as low as $0.01 \mathrm{~g}$ to $0.001 \mathrm{~g}$

- can also be used to calibrate Class F2 weights

- Class F2 weights can be used to calibrate weighing instruments for important commercial transactions like gold and precious stones.

- For calibration of semi-analytical balances \& student use

- They can also be used to calibrate Class M2 weights

\section{OI ML Weight Classes cont'd}

- Class M1 weights are used to:

- validate M2 weights and

- validate class III balances

- Class M2 weights are used to:

- validate M3 weights and

- validate accuracy class III balances

- They are brass weights most commonly used for educational purposes

- Class M3 weights are used on weighing class IIII instruments.

- M Class weights are economical weights for general laboratory, industrial, commercial, technical and educational use

\section{Background}

\section{ISO/IEC 17025, "General Requirements of the} Competence of Testing and Calibration Laboratories"

1. States that a calibration or testing laboratory shall have, and shall apply, a procedure to estimate the uncertainty of measurement for all calibrations/measurements

2. Requires calibration reports shall contain the measurement results and measurement uncertainty statement and

3. Requires measurement results be traceable to a national standard through an unbroken chain of calibrations or comparisons, each having a stated uncertainty

\section{NI STI R 6919 Overview}

- Recommended Guide for Determining and Reporting Uncertainties for Balances and Scales

- Chapter 1 gives purpose and background

- Chapter 2 presents general concepts \& GUM 8 step method

- Chapters 3, 4 and 5 address specific processes.

- Each chapter addresses some special issues that are typically encountered in those calibration processes

- Instructions are provided for the most appropriate method of calculating a reasonable uncertainty for a weighing device in each situation

- Chapter 6 contains sample calculations and includes the rationale that might be used in calculating and evaluating the resulting uncertainties 


\section{NI STI R6919 (continued)}

This guide provides the necessary tools to:

- evaluate the calibration process being used

- identify uncertainty contributors for the measurements made

- quantify the impact of the uncertainty contributors on the measurement results

- combine the uncertainty contributions in a standardized manner

- obtain and evaluate an expanded uncertainty, and

- report the measurement results with a properly computed, properly documented, uncertainty statement

\section{Eight Basic Steps in Determining} Measurement Uncertainty Estimates

1. Specify the process and equation:

2. Identify and characterize the uncertainty sources

3. Quantify the resulting uncertainty components

4. Convert the influences of the uncertainty components on the measurement to standard deviation equivalents

5. Calculate the combined standard uncertainty $\left(\underline{u}_{\underline{c}}\right)$

6. Calculate the expanded uncertainty $(\mathrm{U})$

7. Evaluate $U$ for appropriateness

8. Report the uncertainty

\section{Uncertainty Sources}

- The calibration engineer must be capable of identifying those measurement influences that affect the measurement result and be able to estimate how each influence affects the balance or scale indication (Type B error sources)

- These estimated quantities are then combined according to a documented procedure and reported as the uncertainty of the balance or scale calibration process

\section{Sources of Uncertainty in Weighing}

1. Uncertainty or tolerance of the applied load

2. Repeatability of the weighing system

3. Readability

4. Reproducibility of the weighing system, and

5. Effects of:

- temperature changes

- drafts or wind

- off center loading

- indicator drift

- electrical noise and variation

- vibration

Note: This list is not all inclusive 


\section{Savannah River Site Balance Calibration Program}

\section{- Savannah River Standards Laboratory}

- Accredited to Requirements of ISO 17025

- Calibrates over 500 scales \& balances on site

- Calibrates over $\mathbf{5 0 0 0}$ mass standards

- Most balance calibrations are done on site in place

- Most balance are calibrated when received \& when moved

- Most balances are calibrated annually. Some quarterly

\section{Balance Calibration/ Validation}

- All calibration/validation tests report both "As Found" and "As Left" conditions.

- Many analytical 5 place analytical balances have built in calibration systems.

- (Balances with this feature are recommended)

- These balances compensate for local gravity \& elevation in force compensation weighing systems

- Many of these balances have auto calibration features, which will recalibrate the balance when the room temperature change by $1^{\circ} \mathrm{C}$

- These built in calibration feature do not nullify the calibration/verification done by the standards organization

\section{Typical Balance Calibration Ovenview}

- Perform visual inspections \& verify functionality

- Exercise balance high range weight and determine if the "load" test is within the QA limits.

- Measure the respective test weights at the high, mid, low \& sensitivity points after zeroing balance.

- Repeat 4 more times to test reproducibility

- Perform corner loading test

- Evaluate automated Calibration Report Analyses

- As needed adjust and/or calibrate balance per manufacturer's procedure. Repeat "As Left" Calibration verification tests as needed.

\section{Calibration/ Verification I ncludes:}

- Record environmental conditions at time of test.

- Function Testing includes:

- Verify the balance has been energized and is working properly. NOTE: The balance should be turned on for at least $\mathbf{3 0}$ minutes before starting the performance testing.

- The display is readable and complete.

- The zero feature is verified by depressing the tare bar and observing zero on the display.

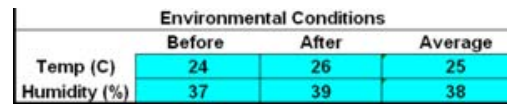




\section{Calibration/ Verification I ncludes: (continued)}

- The load function is tested:

- first by placing the full capacity test weight on the scale twice to exercise the balance and observing the value after zeroing between loading the weighing pan.

The balance is zeroed and the high range weight is weighed 3 more times and the results record in the "Span Load Test" blocks If the average value does not fall within QA precision tolerance the balance fails the load test.

\begin{tabular}{|c|c|} 
High Wt & Span Load Test \\
\cline { 2 - 2 } 1 & 9999.983 \\
\cline { 2 - 2 } 2 & 9999.999 \\
\cline { 2 - 2 } 3 & 10000.032 \\
\cline { 2 - 2 } Ave $=$ & 10000.005 \\
\hline
\end{tabular}

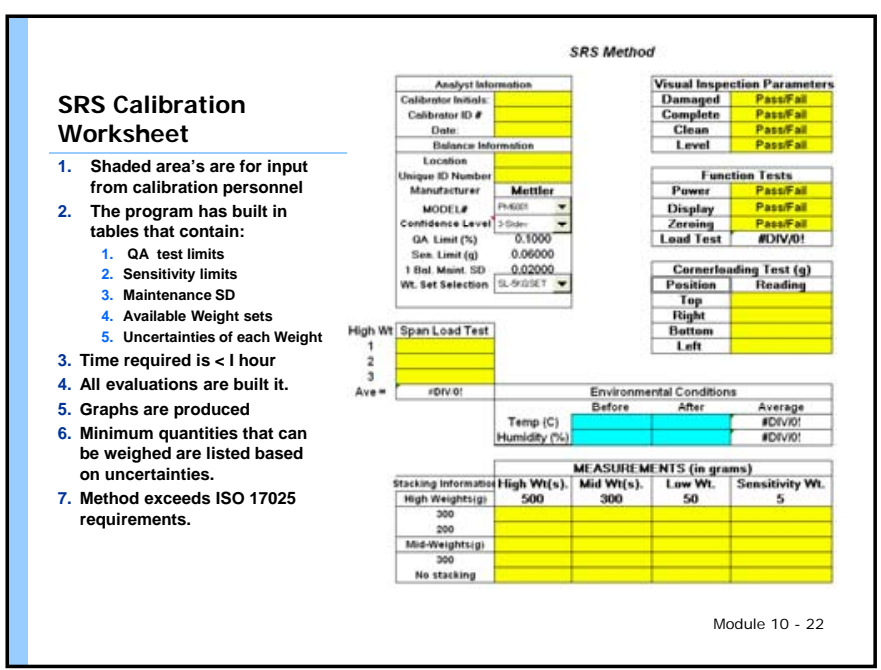

\section{Statistical Evaluation of the performance testing data}

\begin{tabular}{ccccc}
\hline & & \multicolumn{3}{c}{ Statistics } \\
\hline AVERAGE & High Wh(s). & Mid Wl(s). & Low Wt. & Minimal Wl. \\
Bias (abs) & 9999.9996 & 5000.0326 & 2000.0136 & 200.002000 \\
\% Bias & 0.0026 & -0.0036 & -0.0006 & 0.000180 \\
\% RSD & 0.0000 & -0.0001 & 0.0000 & 0.000090 \\
SD(abs) & 0.0000 & 0.0000 & 0.0000 & 0.000354 \\
Max SD & 0.0011 & 0.0009 & 0.0009 & 0.000707 \\
Calculated t & 0.0023 & 0.0022 & 0.0020 & 0.002001 \\
\hline Pooled Abs. Std. Dev.. 16 df $=$ & 0.00092 & Tab. Chi Sq $=$ & 32.0 \\
\hline Tabled t. 16 dt (99\% Cont.) $=$ & 2.921 & Cal. Chi Sq $=$ & 3.4 \\
\hline Tabled F. 2, 16 dt (99\% Cont.)= & 6.23 & F Statistic $=$ & Not Significant \\
& & & & \\
& & & & \\
& & & & \\
\end{tabular}




\section{Graph Showing Variation of Deviations}

- Data plots of the deviations over the balance's range show all results were within +/- 3 SD

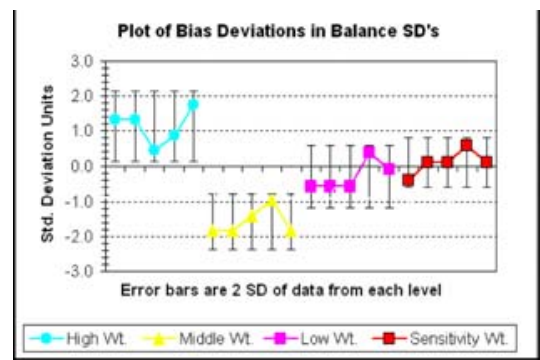

\section{Graph of Biases, Stdev's \& Error Bars}

- Results of tests over the balance's range show the balance performs with specified control limits

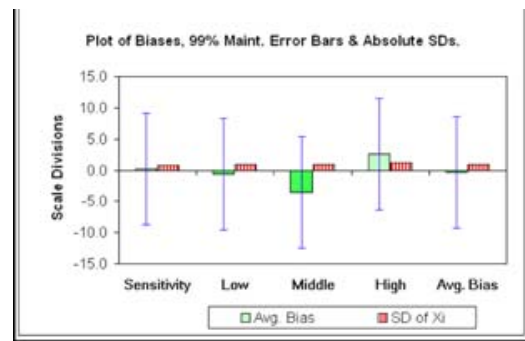

\section{Results of Corner Load Tests}

- The deviation of each measurement from the average of the mid range weight is divided by the greater of the maintenance $\mathrm{SD}$ or the pooled SD of all the measurements or the maintenance SD plus the RSS of the weight(s) uncertainty.

- The balance fails corner loading if any deviation from the average reading in the center is $>3 \mathrm{SD}$. The next slide shows this balance failed the corner loading tests.

\begin{tabular}{|c|c|}
\hline Cornerloading(g) & $\mathbf{5 0 0 0}$ \\
\hline Position & Reading \\
\hline Top & 5000.024 \\
\hline Right & 5000.042 \\
\hline Bottom & 5000.044 \\
\hline Left & 5000.024 \\
\hline
\end{tabular}

\section{Results of Corner Load Tests}

- Plot shows corner loading errors outside of control limits. A warning will be given on the report.

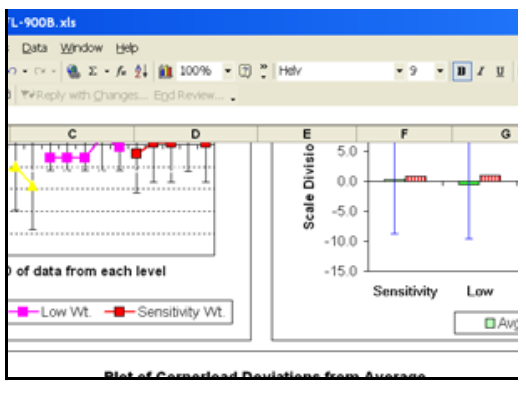




\section{I llustration of Final Evaluation of Tests}

- The evaluation below shows there may be a corner loading problem.

- This is not uncommon, so a label should be attached that states "CENTER ALL OBJECTS"

- The evaluation provides the minimum quantities that can be weighed to stay within given control limits

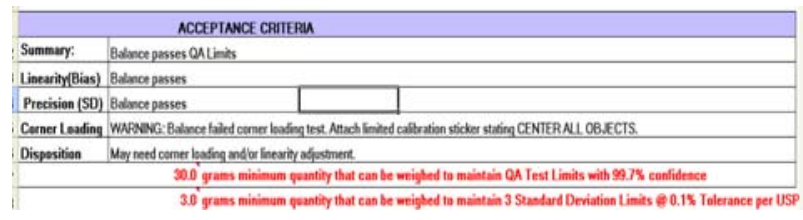

\section{Random \& Systematic Errors in Weight Measurement}

- VIM Definitions of these types of error will be studied

- At the end of this module weighing exercises will be conducted to collect data that will be used to estimate errors affecting precision and accuracy errors in weighing

- Also these errors will be determined over the operating range on an analytical balance

\section{Measurement Precision vim 2.19 (3.13)}

Precision is closeness of agreement between indications or measured quantity values obtained by replicate measurements on the same or similar objects under specified conditions

NOTE 1 - Measurement precision is usually expressed numerically by measures of imprecision, such as standard deviation, variance, or coefficient of variation under the specified conditions of measurement

NOTE 2 - The 'specified conditions' can be, for example,

- repeatability conditions of measurement

- intermediate precision conditions of measurement, or

- reproducibility conditions of measurement (see ISO 57253:1994)

\section{Random Measurement Error vм 2.19 (3.13)}

Random error is a component of measurement error that in replicate measurements varies in an unpredictable manner

NOTE 1 - A reference quantity value for a random measurement error is the average that would ensue from an infinite number of replicate measurements of the same measurand

NOTE 2 - Random measurement errors of a set of replicate measurements form a distribution that can be summarized by its expectation, which is generally assumed to be zero, and its variance

NOTE 3 - Random measurement error equals measurement error minus systematic measurement error 


\subsection{0 repeatability condition of measurement}

- condition of measurement, out of a set of conditions that includes the same measurement procedure, same operators, same measuring system, same operating conditions and same location, and replicate measurements on the same or similar objects over a short period of time

- NOTE 1 A condition of measurement is a repeatability condition only with respect to a specified set of repeatability conditions.

\section{I ntermediate Precision Condition vı 2.22}

Condition of measurement, out of a set of conditions that includes the same measurement procedure, same location, and replicate measurements on the same or similar objects over an extended period of time, but may include other conditions involving changes

NOTE 1 - The changes can include new calibrations, calibrators, operators, and measuring systems

NOTE 2 - A specification for the conditions should contain the conditions changed and unchanged, to the extent practical

\section{Summary}

- Reviewed the elements of a MCP for scales or balances

- Discussed the classes of balances \& mass standards

- Completed and Exercise on classes of Balances \& Standards

- Reviewed method for determining weighing uncertainty

- Discussed the Savannah River site balance calibration/verification program

- Discussed sources and types of error in weighing

\section{Reproducibility Condition of Measurement vм 2.24 (3.7, Note 2)}

Condition of measurement, out of a set of conditions that includes different locations, operators, measuring systems, and replicate measurements on the same or similar objects

NOTE 1 - The different measuring systems may use different measurement procedures.

NOTE 2 - A specification should give the conditions to the extent practical 


\section{I nstrumental Bias viм 4.20 (5.25)}

Average of replicate indications minus a reference quantity value

\section{Balance Exercise Objectives:}

- Determine uncertainty estimates for a balance

- By using technical information from specifications

- By experiment using calibrated weights

- Evaluate balance accuracy and precision errors (exercise)

- Determine what effect multiple operators and balances will have on random error estimates in weight measurements 
Revision 6-04-2012

\section{Exercise 1 \\ Balance Module Exercise}

\section{Session Objectives:}

After the session the participants will be able to do the following:

1. To see measurements are comparisons of unknowns to reference standards.

2. To understand measurements have uncertainty.

3. To understand that reference standards also have uncertainty.

4. To understand measurements need uncertainty estimates with specified confidence intervals to have value.

\section{Estimated Time:}

+. 5 hours completing exercise

+.5 hours in large group discussion

hours total

\section{Materials need:}

1. Work Sheets for each participant

2. Class Workbook with slides

3. Tables showing scale \& weight classes (copies included in 3 page work sheet)

4. Pen or pencil

5. Calculators may be used, but are not required

\section{Instructions:}

1. Write your name on the worksheet.

There are four classes of accuracy for weighing equipment. There are 7 classes of OIML weights. The lowest class weight is M3 and is usually the least expensive. It also has the highest uncertainty. List the accuracy class and choose the appropriate class of weights having uncertainties "fit for purpose" to test the accuracy of each of the 10 weighing instruments listed below.

You may refer to the tables given below for help in determining your answers to the 10 questions below. Fill in the blanks. 
1. What class would a bathroom scale be if it has a $150 \mathrm{~kg}$ capacity and reads to $0.1 \mathrm{~kg}$ ? and could be calibrated with a Class weight.

2. What is the accuracy class of a 4-place analytical balance that has a $200 \mathrm{~g}$ capacity and reads to $0.1 \mathrm{mg}$ ? Class and could be calibrated with a Class weight.

3. What is the accuracy class of a scale in the chemical make up area of a plant that has a $500 \mathrm{~kg}$ capacity and reads to $0.5 \mathrm{~kg}$ ? Class and could be calibrated with a Class weight.

4. What is the accuracy class of an industrial scale that has a $2500 \mathrm{~kg}$ capacity and reads to $0.1 \mathrm{~kg}$ ? Class and could be calibrated with a Class weight.

5. What is the accuracy class of a top loading balance that has a $15 \mathrm{~kg}$ capacity and reads to $1 \mathrm{mg}$ and is used in a production line? Class and could be calibrated with a Class weight.

6. What is the accuracy class of a truck scale that has a capacity of $25000 \mathrm{~kg}$ and reads to $1 \mathrm{~kg}$ ? Class and could be calibrated with a Class weight.

7. What is the accuracy class of a scale in the store that has a capacity of $10 \mathrm{~kg}$ and reads to $100 \mathrm{~g}$ ? Class and could be calibrated with a Class weight.

8. What is the accuracy class of a Jeweler's $500 \mathrm{~g}$ capacity scale that reads to 0.1 g? Class and could be calibrated with a Class weight.

9. What is the accuracy class of a student's top loading balance that has a $1000 \mathrm{~g}$ capacity and has $10 \mathrm{mg}$ readability? Class and could be calibrated with a Class weight.

10. What is the accuracy class of a microbalance that reads to $1 \mu \mathrm{g}$ with a 5 -gram capacity? Class and could be calibrated with a Class weight.

Information on the classes of balances and weights are listed below. 
Revision 6-04-2012

TROEMNER PI

Metric Weight Tolerances - OIML

The table below is a listing of the tolerances of various classes of masses. The weight value can deviate above of below the nominal value by the tolerance defined. To determine the tolerance of a mass, look at the denomination or nominal value of the weight and the appropriate class you need.

\begin{tabular}{|c|c|c|c|c|c|c|c|c|}
\hline \multirow[b]{3}{*}{$\begin{array}{l}\text { Denomination } \\
\text { Metric }\end{array}$} & \multicolumn{7}{|c|}{$\begin{array}{c}\text { International Organization of } \\
\text { Legal Metrology Rermmmendation R111* }\end{array}$} & \multirow[b]{3}{*}{$\begin{array}{c}\text { Denomination } \\
\text { Metric }\end{array}$} \\
\hline & E1 & $E 2$ & $F 1$ & $\mathrm{~F} 2$ & $M 1$ & M2 & $M 3$ & \\
\hline & $\mathrm{mg}$ & $\mathrm{mg}$ & $\mathrm{mg}$ & $\mathrm{mg}$ & $\mathrm{mg}$ & $\mathrm{mg}$ & $\mathrm{mg}$ & \\
\hline $5000 \mathrm{~kg}$ & & & 25000 & 80000 & 250000 & 800000 & 2500000 & $5000 \mathrm{~kg}$ \\
\hline $3000 \mathrm{~kg}$ & & & & & & & & $3000 \mathrm{~kg}$ \\
\hline $2000 \mathrm{~kg}$ & & & 10000 & 30000 & 100000 & 300000 & 1000000 & $2000 \mathrm{~kg}$ \\
\hline $1000 \mathrm{~kg}$ & & 1600 & 5000 & 16000 & 50000 & 160000 & 500000 & $1000 \mathrm{~kg}$ \\
\hline $500 \mathrm{~kg}$ & & 800 & 2500 & 8000 & 25000 & 80000 & 250000 & $500 \mathrm{~kg}$ \\
\hline $300 \mathrm{~kg}$ & & & & & & & & $300 \mathrm{~kg}$ \\
\hline $200 \mathrm{~kg}$ & & 300 & 1000 & 3000 & 10000 & 30000 & 100000 & $200 \mathrm{~kg}$ \\
\hline $100 \mathrm{~kg}$ & & 160 & 500 & 1600 & 5000 & 16000 & 50000 & $100 \mathrm{~kg}$ \\
\hline $50 \mathrm{~kg}$ & 25 & 80 & 250 & 800 & 2500 & 8000 & 25000 & $50 \mathrm{~kg}$ \\
\hline $30 \mathrm{~kg}$ & & & & & & & & $30 \mathrm{~kg}$ \\
\hline $25 \mathrm{~kg}$ & & & & & & & & $25 \mathrm{~kg}$ \\
\hline $20 \mathrm{~kg}$ & 10 & 30 & 100 & 300 & 1000 & 3000 & 10000 & $20 \mathrm{~kg}$ \\
\hline $10 \mathrm{~kg}$ & 5.0 & 16 & 50 & 160 & 500 & 1600 & 5000 & $10 \mathrm{~kg}$ \\
\hline $5 \mathrm{~kg}$ & 2.5 & 8.0 & 25 & 80 & 250 & 800 & 2500 & $5 \mathrm{~kg}$ \\
\hline $3 \mathrm{~kg}$ & & & & & & & & $3 \mathrm{~kg}$ \\
\hline $2 \mathrm{~kg}$ & 1.0 & 3.0 & 10 & 30 & 100 & 300 & 1000 & $2 \mathrm{~kg}$ \\
\hline $1 \mathrm{~kg}$ & 0.5 & 1.6 & 5.0 & 16 & 50 & 160 & 500 & $1 \mathrm{~kg}$ \\
\hline $500 \mathrm{~g}$ & 0.25 & 0.8 & 2.5 & 8.0 & 25 & 80 & 250 & $500 \mathrm{~g}$ \\
\hline $300 \mathrm{~g}$ & & & & & & & & $300 \mathrm{~g}$ \\
\hline $200 \mathrm{~g}$ & 0.10 & 0.3 & 1.0 & 3.0 & 10 & 30 & 100 & $200 \mathrm{~g}$ \\
\hline $100 \mathrm{~g}$ & 0.05 & 0.16 & 0.5 & 1.6 & 5.0 & 16 & 50 & $100 \mathrm{~g}$ \\
\hline $50 \mathrm{~g}$ & 0.03 & 0.10 & 0.30 & 1.0 & 3.0 & 10 & 30 & $50 \mathrm{~g}$ \\
\hline $30 \mathrm{~g}$ & & & & & & & & $30 \mathrm{~g}$ \\
\hline $20 \mathrm{~g}$ & 0.025 & 0.08 & 0.25 & 0.8 & 2.5 & 8.0 & 25 & $20 \mathrm{~g}$ \\
\hline $10 \mathrm{~g}$ & 0.020 & 0.06 & 0.20 & 0.6 & 2.0 & 6.0 & 20 & $10 \mathrm{~g}$ \\
\hline 59 & 0.016 & 0.05 & 0.16 & 0.5 & 1.6 & 5.0 & 16 & $5 \mathrm{~g}$ \\
\hline $3 \mathrm{~g}$ & & & & & & & & $3 \mathrm{~g}$ \\
\hline $2 g$ & 0.012 & 0.04 & 0.12 & 0.4 & 12 & 4.0 & 12 & $2 g$ \\
\hline 19 & 0.010 & 0.03 & 0.10 & 0.3 & 1.0 & 3.0 & 10 & 19 \\
\hline $500 \mathrm{mg}$ & 0.008 & 0.025 & 0.08 & 0.25 & 0.8 & 2.5 & & $500 \mathrm{mg}$ \\
\hline $300 \mathrm{mg}$ & & & & & & & & $300 \mathrm{mg}$ \\
\hline $200 \mathrm{mg}$ & 0.006 & 0.020 & 0.06 & 0.20 & 0.6 & 2.0 & & $200 \mathrm{mg}$ \\
\hline $100 \mathrm{mg}$ & 0.005 & 0.016 & 0.05 & 0.16 & 0.5 & 1.6 & & $100 \mathrm{mg}$ \\
\hline $50 \mathrm{mg}$ & 0.004 & 0.012 & 0.04 & 0.12 & 0.4 & & & $50 \mathrm{mg}$ \\
\hline $30 \mathrm{mg}$ & & & & & & & & $30 \mathrm{mg}$ \\
\hline $20 \mathrm{mg}$ & 0.003 & 0.010 & 0.03 & 0.10 & 0.3 & & & $20 \mathrm{mg}$ \\
\hline $10 \mathrm{mg}$ & 0.003 & 0.008 & 0.025 & 0.08 & 0.25 & & & $10 \mathrm{mg}$ \\
\hline $5 \mathrm{mg}$ & 0.003 & 0.006 & 0.020 & 0.06 & 0.20 & & & $5 \mathrm{mg}$ \\
\hline $3 \mathrm{mg}$ & & & & & & & & $3 \mathrm{mg}$ \\
\hline $2 \mathrm{mg}$ & 0.003 & 0.006 & 0.020 & 0.06 & 0.20 & & & $2 \mathrm{mg}$ \\
\hline $1 \mathrm{mg}$ & 0.003 & 0.006 & 0.020 & 0.06 & 0.20 & & & $1 \mathrm{mg}$ \\
\hline $0.5 \mathrm{mg}$ & & & & & & & & $0.5 \mathrm{mg}$ \\
\hline $0.3 \mathrm{mg}$ & & & & & & & & $0.3 \mathrm{mg}$ \\
\hline $0.2 \mathrm{mg}$ & & & & & & & & $0.2 \mathrm{mg}$ \\
\hline $0.1 \mathrm{mg}$ & & & & & & & & $0.1 \mathrm{mg}$ \\
\hline $0.05 \mathrm{mg}$ & & & & & & & & $0.05 \mathrm{mg}$ \\
\hline
\end{tabular}

" OIML- International Recommendation R111 replaces International Recommendation numbers 1, 2, 20, 52. 
Revision 6-04-2012

\begin{tabular}{|c|c|c|c|}
\hline \multirow[b]{2}{*}{ Class } & \multirow[t]{2}{*}{ Value of the Verification Scale Division } & \multicolumn{2}{|c|}{ Number of Scale Divisions (n) } \\
\hline & & Minimum & Maximum \\
\hline \multicolumn{4}{|c|}{ SI Units } \\
\hline$I$ & Equal to or greater than $1 \mathrm{mg}$ & 50000 & 20000000 \\
\hline II & 1 to $50 \mathrm{mg}$, inclusive & 100 & 100000 \\
\hline & Equal to or greater than $100 \mathrm{mg}$ & 5000 & 100000 \\
\hline III & 0.1 to $2 \mathrm{~g}$ inclusive & 100 & 10000 \\
\hline & Equal to or greater than $5 \mathrm{~g}$ & 500 & 10000 \\
\hline IIII & Equal to or greater than $5 \mathrm{~g}$ & 100 & 1200 \\
\hline
\end{tabular}




\section{Balance Module Exercise:}

Fill in the blank. You may refer to your copy of the slides used in the "Balance Measurement Control Program Module" in determining your answers.

There are four classes of accuracy for weighing equipment. There are 7 classes of OIML weights. The lowest class weight is M3 and is usually the least expensive. It also has the highest uncertainty. List the accuracy class and choose the appropriate class of weights having uncertainties "fit for purpose" to test the accuracy of each of the 10 weighing instruments listed below.

1. What class would a bathroom scale be if it has a $150 \mathrm{~kg}$ capacity and reads to $0.1 \mathrm{~kg}$ ? IIII and could be calibrated with a M3 Class weight.

2. What class would a 4-place analytical balance be if it has a $200 \mathrm{~g}$ capacity and reads to $0.1 \mathrm{mg}$ ? and could be calibrated with a E2 Class weight.

3. What class is a scale in the chemical make up area of a plant that has a 500 $\mathrm{kg}$ capacity and reads to $0.5 \mathrm{~kg}$ IIII and could be calibrated with a M3__ Class weight?

4. What Class is an industrial scale that has a $3000 \mathrm{~kg}$ capacity and reads to 0.5 $\mathrm{kg}$ ? III__ and could be calibrated with a M2 Class weight.

5. What class is a top loading balance that has a $15 \mathrm{~kg}$ capacity and reads to 1 $\mathrm{mg}$ and is used in a production line? and could be calibrated with a F1 Class weight.

6. What class is a truck scale that has a capacity of $25000 \mathrm{~kg}$ and reads to $1 \mathrm{~kg}$ ? II__ and could be calibrated with a M1 Class weight.

7. What class is a scale in the store that has a capacity of $10 \mathrm{~kg}$ and reads to $100 \mathrm{~g}$ ? IIII and could be calibrated with a M3 Class weight.

8. What class is a Jeweler's $500 \mathrm{~g}$ capacity scale that reads to $0.1 \mathrm{~g}$ ? III and could be calibrated with a F1 Class weight.

9. What class is a student's top loading balance that has a $1000 \mathrm{~g}$ capacity and has $10 \mathrm{mg}$ readability? II___ and could be calibrated with a F1 Class weight.

10. What class is a microbalance that reads to $1 \mu \mathrm{g}$ with a 5 -gram capacity? ? ___ and could be calibrated with a E2 Class weight. 


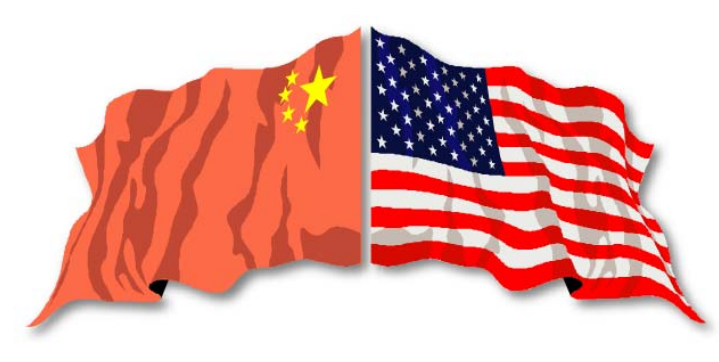

\section{Module 11}

Tank Sampling \& Mixing Study for Liquids 


\section{Objectives}

- Discuss planning considerations for experiments to determine sampling errors

- Discuss methodologies for managing and controlling sampling uncertainties

- Review a tank sampling and mixing case study 


\section{Tank Sampling \& Mixing}

- Sampling and mixing studies have been conducted for many of the input/output accountability tanks at Savannah River Site

- The purpose of these studies is to establish a mixing time and to estimate a sampling uncertainty

- Sampling uncertainties are typically associated with solution concentration measurements

- If a sampling study has not been conducted, sampling uncertainties can be estimated using process sample analytical results 


\section{Study Planning}

- Some planning considerations are as follows:

- Need to coordinate with the facility

- What material will be used for the study?

- How long will the tank be mixed?

- At what time intervals will samples be pulled?

- How many samples will be pulled at each time interval?

- How many analyses per sample? 


\section{Tank Sampling \& Mixing Study}

Background: A particular process was experiencing ID problems. The investigation suggested a sampling/mixing study on the main input accountability tank. This was to ensure the solution was adequately mixed when accountability samples were taken. The tank was at process heel prior to the beginning of the study. Acid was added to the tank to reduce the concentration by approximately $50 \%$. Eventually, process solution from another location was transferred into the tank. The sampling study was then continued. This tank uses air sparging for mixing, but has a recirculation pump as a backup system.

Using the information on the following slides, discuss the details of this sampling and mixing study 


\section{Tank Sampling \& Mixing Study (see handout)}

HB-Line Tank JT-71

Sampling and Mixing Study

The Analysis of Np Concentration Data

\begin{tabular}{|c|c|c|c|c|c|c|}
\hline Sample Desc & $\begin{array}{l}\text { Mixing } \\
\text { Time } \\
\text { Hours }\end{array}$ & $\begin{array}{l}\text { Group } \\
\text { No. }\end{array}$ & Aliquot & $\begin{array}{c}\mathrm{Np} \\
\text { Concentration } \\
\mathrm{mg} / \mathrm{g}\end{array}$ & $\begin{array}{l}\text { Density } \\
\mathrm{g} / \mathrm{ml}\end{array}$ & $\begin{array}{c}\mathrm{Np} \\
\text { Concentration } \\
\mathrm{g} / \mathrm{l}\end{array}$ \\
\hline JT71 - 007 - ACCT - 1 - HEEL & 0.0 & 1 & 1 & 6.8839 & 1.2094 & 8.3254 \\
\hline JT7 1-007-ACCT-1-HEEL & 0.0 & 1 & 2 & 6.8729 & 1.2094 & 8.3121 \\
\hline JT71-007-ACCT-1-HEEL & 0.0 & 1 & 1 & 6.8719 & 1.2094 & 8.3109 \\
\hline JT71-007-ACCT-1-HEEL & 0.0 & 1 & 2 & 6.8669 & 1.2094 & 8.3048 \\
\hline JT71-007-ACCT - HEEL & 0.0 & 2 & 1 & 6.8631 & 1.2095 & 8.3009 \\
\hline JT71-007-ACCT - HEEL & 0.0 & 2 & 1 & 6.8744 & 1.2095 & 8.3146 \\
\hline JT71-007-ACCT-HEEL & 0.0 & 2 & 2 & 6.8726 & 1.2095 & 8.3124 \\
\hline JT71-007-00 & 0.0 & 3 & 1 & 11.4207 & 1.2377 & 14.1354 \\
\hline JT71-007-00 & 0.0 & 3 & 2 & 11.4472 & 1.2377 & 14.1682 \\
\hline JT71-007-02 & 2.0 & 4 & 1 & 11.9467 & 1.2386 & 14.7972 \\
\hline JT71-007-02 & 2.0 & 4 & 2 & 11.9335 & 1.2386 & 14.7808 \\
\hline JT71-007-04 & 4.0 & 5 & 1 & 11.9092 & 1.2385 & 14.7495 \\
\hline JT71-007-04 & 4.0 & 5 & 2 & 11.9082 & 1.2385 & 14.7483 \\
\hline JT71-007-06 & 6.0 & 6 & 1 & 11.9574 & 1.2388 & 14.8128 \\
\hline JT71-007-06 & 6.0 & 6 & 2 & 11.9164 & 1.2388 & 14.7620 \\
\hline JT71-007-08 & 8.0 & 7 & 1 & 11.9049 & 1.2385 & 14.7442 \\
\hline JT71-007-08 & 8.0 & 7 & 2 & 11.8943 & 1.2385 & 14.7311 \\
\hline JT71-007-10 & 10.0 & 8 & 1 & 11.8886 & 1.2386 & 14.7252 \\
\hline JT71-007-10 & 10.0 & 8 & 2 & 11.8845 & 1.2386 & 14.7201 \\
\hline JT71-007-12 & 12.0 & 9 & 1 & 11.9006 & 1.2390 & 14.7448 \\
\hline JT71-007-12 & 12.0 & 9 & 2 & 11.9240 & 1.2390 & 14.7738 \\
\hline JT71-007-14 & 14.0 & 10 & 1 & 11.8904 & 1.2389 & 14.7310 \\
\hline JT71-007-14 & 14.0 & 10 & 2 & 11.9042 & 1.2389 & 14.7481 \\
\hline JT71-007-ACCT & 16.0 & 11 & 1 & 11.9846 & 1.2388 & 14.8465 \\
\hline JT71-007-ACCT & 16.0 & 11 & 2 & 11.9478 & 1.2388 & 14.8009 \\
\hline JT71-007-ACCT & 16.0 & 11 & 1 & 11.8961 & 1.2388 & 14.7369 \\
\hline JT71-007-ACCT & 16.0 & 11 & 2 & 11.9355 & 1.2388 & 14.7857 \\
\hline JT71-007-АCСТ - 1 & 16.0 & 12 & 1 & 11.9292 & 1.2388 & 14.7779 \\
\hline JT71-007-ACCT-1 & 16.0 & 12 & 2 & 11.9790 & 1.2388 & 14.8396 \\
\hline JT71-007-ACCT-1 & 16.0 & 12 & 3 & 11.9186 & 1.2388 & 14.7648 \\
\hline JT71-007-ACCT - 1 & 16.0 & 12 & 1 & 11.9165 & 1.2388 & 14.7622 \\
\hline JT71-007-ACCT-1 & 16.0 & 12 & 2 & 11.8965 & 1.2388 & 14.7374 \\
\hline JT71-007-P001 & 17.0 & 13 & 1 & 11.8834 & 1.2387 & 14.7200 \\
\hline JT71-007-P001 & 17.0 & 13 & 2 & 11.9100 & 1.2387 & 14.7529 \\
\hline JT71-007-P002 & 18.0 & 14 & 1 & 11.9157 & 1.2388 & 14.7612 \\
\hline JT71-007-P002 & 18.0 & 14 & 2 & 11.9229 & 1.2388 & 14.7701 \\
\hline JT71-007-P003 & 18.5 & 15 & 1 & 11.9518 & 1.2386 & 14.8035 \\
\hline JT71-007-P003 & 18.5 & 15 & 2 & 11.8895 & 1.2386 & 14.7263 \\
\hline JT71-007-P003 & 18.5 & 15 & 3 & 11.8639 & 1.2386 & 14.6946 \\
\hline
\end{tabular}

Module $11-6$ 


\section{Tank Sampling \& Mixing Study (see handout)}

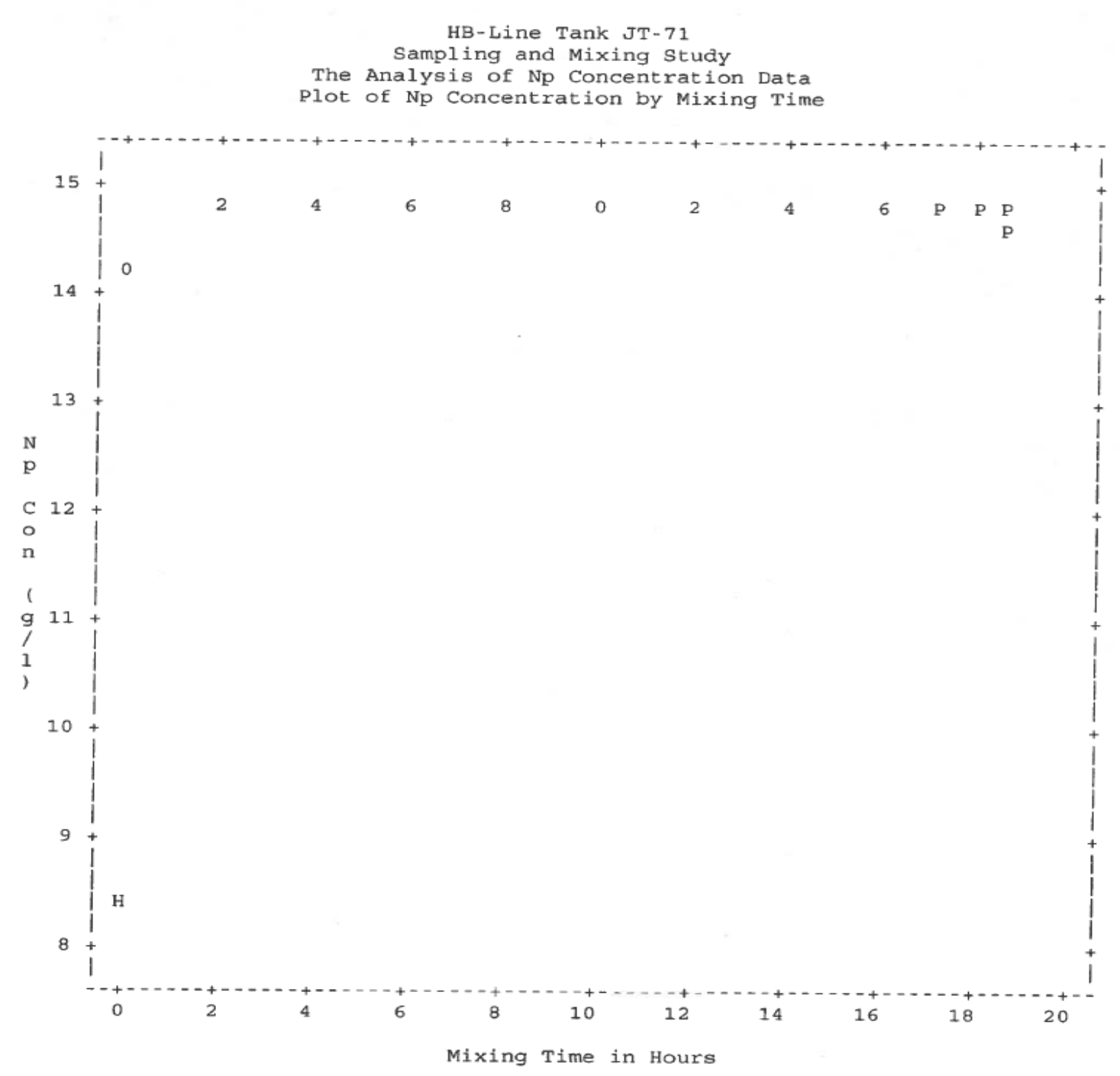

Module $11-7$ 


\section{Tank Sampling \& Mixing Study (see handout)}

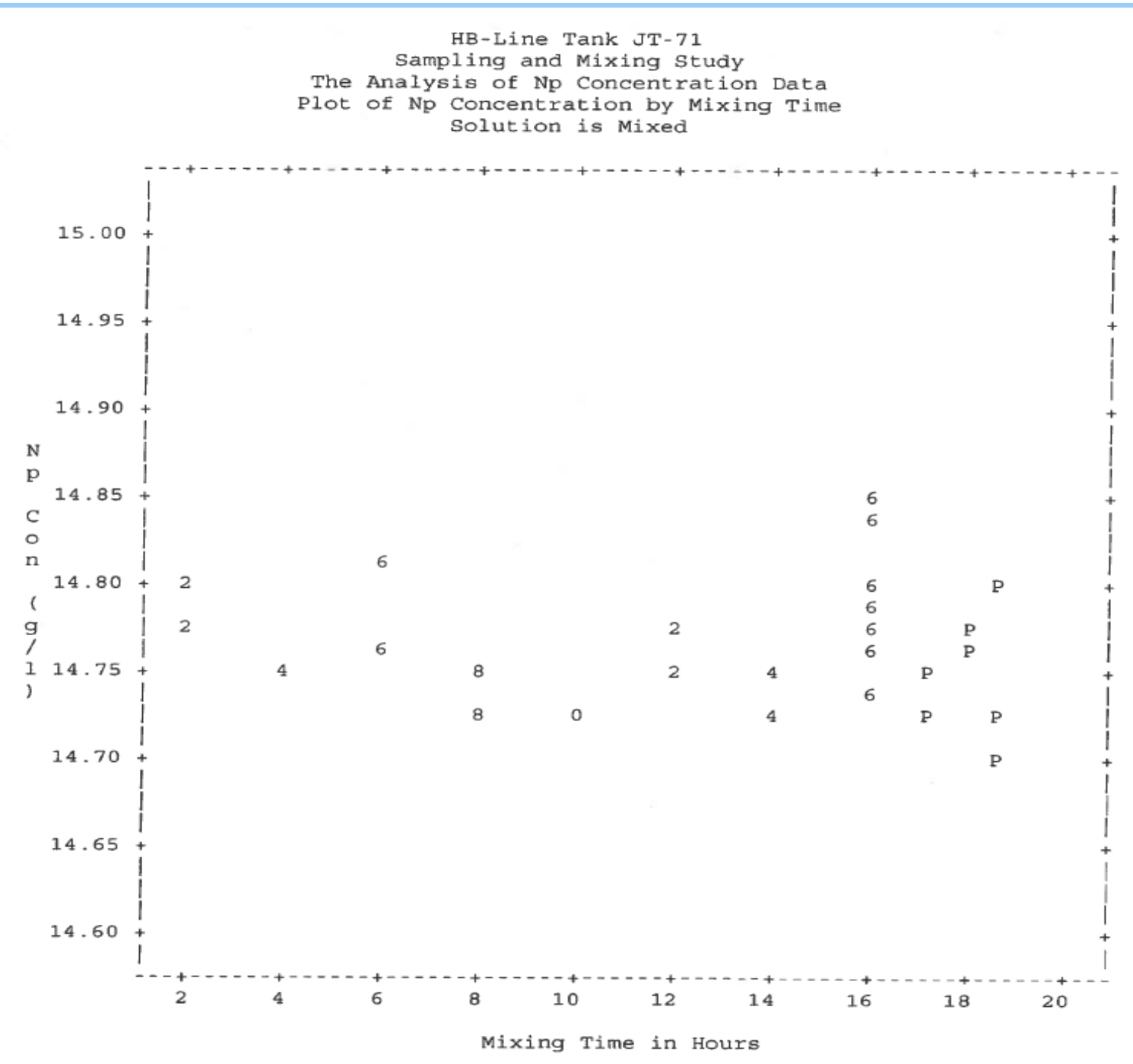

NOTE : 4 obs hidden. 9 obs out of range.

Module $11-8$ 


\section{Tank Sampling \& Mixing Study (see handout)}

HB-Line Tank JT-71

Sampling and Mixing study

The Analysis of Np Concentration Data

The Estimation of Sampling and Analytical Method Uncertainty Components

The GLM Procedure

Dependent Variable: npgl Np Concentration $g / 1$

\begin{tabular}{|c|c|c|c|c|c|c|c|c|c|}
\hline \multicolumn{2}{|l|}{ Source } & DF & & $\begin{array}{l}n \text { of } \\
\text { reses }\end{array}$ & Mean S & Square & $F$ & Value & $\operatorname{Pr}>F$ \\
\hline Model & & 11 & 0.0 & & 0.001 & 143075 & & 1.23 & 0.3360 \\
\hline Error & & 18 & 0.0 & 2694 & 0.001 & 116261 & & & \\
\hline \multirow[t]{3}{*}{ Corrected } & Total & 29 & 0.0 & & & & & & \\
\hline & R-Square & Coeff & Var & Root & MSE & npgl & Mean & & \\
\hline & 0.429242 & 0.230 & 0984 & 0.034 & 4097 & 14.7 & 76165 & & \\
\hline
\end{tabular}

$\begin{array}{lccccc}\text { Source } & \text { DF } & \text { Type I SS } & \text { Mean Squarc F Value Pr F } \\ \text { sampleid } & 11 & 0.01573826 & 0.00143075 & 1.23 & 0.3360 \\ \text { Source } & \text { DF } & \text { Type III SS } & \text { Mean Square F Value Pr F } \\ \text { sampleid } & 11 & 0.01573826 & 0.00143075 & 1.23 & 0.3360\end{array}$

The estimation of the sampling uncertainty is as follows:

Source

Type III Expected Mean Square

sampleid

Var(Error) + 2.4667 Var(sampleid)

Module $11-9$ 


\section{Tank Sampling \& Mixing Study (see handout)}

HB-Line Tank JT-71

Sampling and Mixing Study

The Analysis of Np Concentration Data

The Estimation of Sampling and Analytical Method Uncertainty Components

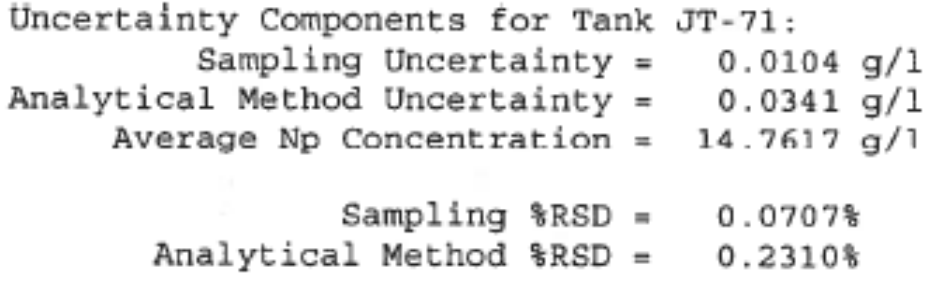




\section{Summary}

- Reviewed the planning considerations for tank sampling and mixing study

- Discussed approach for collecting and analyzing samples

- Discussed how to determine when a tank solution is mixed

- Reviewed the documentation of the results for an example case study 


\section{Discussion Exercise Tank Sampling \& Mixing Study for Liquids}

\section{Session Objectives:}

After the session the participants will be able to do the following:

1. Plan a sampling/mixing study

2. Collect the measurement data

3. Analyze the data

4. Determine when a tank solution is mixed

5. Document results

\section{Estimated Time:}

There are 10 discussion topics for this module.

These topics will require 30 minutes to complete.

\section{Materials Needed:}

1. This exercise will be a discussion

2. The instructor will lead the discussion while viewing the slides

\section{Instructions:}

1. Cover the discussion topics while viewing the slides

2. A listing of the data, data plots and an ANOVA will be on the slides or in the student booklet 


\section{Background Information:}

A particular process was experiencing ID problems. The investigation suggested a sampling/mixing study on the main input accountability tank. This was to ensure the solution was adequately mixed when accountability samples were taken. The tank was at process heel prior to the beginning of the study. Acid was added to the tank to reduce the concentration by approximately 50\%. Eventually, process solution from another location was transferred into the tank. The sampling study was then continued.

This tank uses air sparging for mixing, but has a recirculation pump as a backup system.

\section{Discussion Topics}

1. What material was used for this study?

a. Actual process material was used for the sampling study

2. What needs to be done before the study can begin?

a. The existing material was diluted with nitric acid

b. Additional material was added with a much higher concentration

c. Two initial samples were taken and analyzed

3. When did the sampling study actually begin?

a. The study began with sample descriptor JT71-007-00

4. How many samples were pulled at each time interval?

a. Two to three samples were pulled every 1 to 2 hours

b. Also, there was 5 to 10 minutes of mixing between samples

5. Current mixing time is 8 hours. When is this tank mixed?

a. This tank is mixed after 2 hours

6. How long was this mixing study conducted?

a. This study was conducted over 18.5 hours

7. How can the sampling uncertainty be estimated?

a. By analyzing some of the samples at least 2 times, the sampling variability can be separated from the measurement method random variability

8. What is an estimate of the sampling uncertainty?

a. The sampling uncertainty is $\mathbf{0 . 0 7 \%}$ and the measurement method random uncertainty is $0.23 \%$

9. What is a possible statistical model for this study?

a. A reasonable model is $y_{i}=\mu\left(1+\varepsilon_{i}\right)$, where $\mu$ is the true concentration and $\varepsilon_{i}$ are random measurement effects that are iid $N\left(0, \sigma^{2}\right)$ 
10. Explore other options for pulling and analyzing samples for a mixing study.

a. Samples can be pulled over shorter or longer time intervals

b. Each sample does not require two analytical measurements. However, the sampling design should ensure a good estimate of the analytical method random uncertainty. This means a reasonably large number of duplicate measurements are conducted. 
HB-Line Tank JT-71

Sampling and Mixing Study

The Analysis of Np Concentration Data

\begin{tabular}{|c|c|c|c|c|c|c|}
\hline Sample Desc & $\begin{array}{l}\text { Mixing } \\
\text { Time } \\
\text { Hours }\end{array}$ & $\begin{array}{c}\text { Group } \\
\text { No. }\end{array}$ & Aliquot & $\begin{array}{c}\mathrm{Np} \\
\text { Concentration } \\
\mathrm{mg} / \mathrm{g}\end{array}$ & $\begin{array}{l}\text { Density } \\
\mathrm{g} / \mathrm{ml}\end{array}$ & $\begin{array}{c}\mathrm{Np} \\
\text { Concentration } \\
\mathrm{g} / \mathrm{l}\end{array}$ \\
\hline JT71- $\odot \odot 7-A C C T-1-H E E L$ & 0.0 & 1 & 1 & 6.8839 & 1.2094 & 8.3254 \\
\hline JT71-๑๑7-ACCT - 1- HEEL & $\odot .0$ & 1 & 2 & 6.8729 & 1.2094 & 8.3121 \\
\hline JT71- $\odot \odot 7$ - АCCT - 1 - HEEL & 0.0 & 1 & 1 & 6.8719 & 1.2094 & 8.3109 \\
\hline JT71-๑๑7-ACCT - 1- HEEL & 0.0 & 1 & 2 & 6.8669 & 1.2094 & 8.3048 \\
\hline JT71-๑९7 - ACCT - HEEL & 0.0 & 2 & 1 & 6.8631 & 1.2095 & 8.3009 \\
\hline JT71-๑९7 - АCCT - HEEL & 0.0 & 2 & 1 & 6.8744 & 1.2095 & 8.3146 \\
\hline JT71-๑९7 - ACCT - HEEL & 0.0 & 2 & 2 & 6.8726 & 1.2095 & 8.3124 \\
\hline 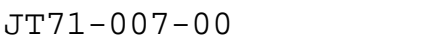 & 0.0 & 3 & 1 & 11.4207 & 1.2377 & 14.1354 \\
\hline 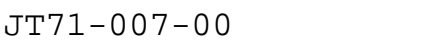 & 0.0 & 3 & 2 & 11.4472 & 1.2377 & 14.1682 \\
\hline 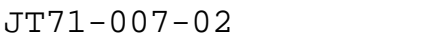 & 2.0 & 4 & 1 & 11.9467 & 1.2386 & 14.7972 \\
\hline 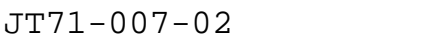 & 2.0 & 4 & 2 & 11.9335 & 1.2386 & 14.7808 \\
\hline 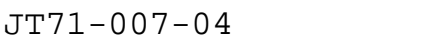 & 4.0 & 5 & 1 & 11.9092 & 1.2385 & 14.7495 \\
\hline 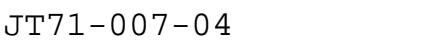 & 4.0 & 5 & 2 & 11.9082 & 1.2385 & 14.7483 \\
\hline JT71-๑०7-๑6 & 6.0 & 6 & 1 & 11.9574 & 1.2388 & 14.8128 \\
\hline 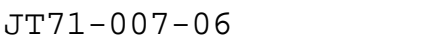 & 6.0 & 6 & 2 & 11.9164 & 1.2388 & 14.7620 \\
\hline JT71-๑९7- ๑8 & 8.0 & 7 & 1 & 11.9049 & 1.2385 & 14.7442 \\
\hline JT71-๑९7- ๑8 & 8.0 & 7 & 2 & 11.8943 & 1.2385 & 14.7311 \\
\hline JT71- -๑7 - $1 \odot$ & 10.0 & 8 & 1 & 11.8886 & 1.2386 & 14.7252 \\
\hline JT71- ๑०7 - $1 \odot$ & 10.0 & 8 & 2 & 11.8845 & 1.2386 & 14.7201 \\
\hline JT71-๑๑7-12 & 12.0 & 9 & 1 & 11.9006 & 1.2390 & 14.7448 \\
\hline JT71-๑०7- 12 & 12.0 & 9 & 2 & 11.9240 & 1.2390 & 14.7738 \\
\hline JT71-๑०7-14 & 14.0 & 10 & 1 & 11.8904 & 1.2389 & 14.7310 \\
\hline JT71-๑९7-14 & 14.0 & 10 & 2 & 11.9042 & 1.2389 & 14.7481 \\
\hline 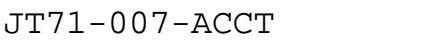 & 16.0 & 11 & 1 & 11.9846 & 1.2388 & 14.8465 \\
\hline JT71-๑๑7 - АCCT & 16.0 & 11 & 2 & 11.9478 & 1.2388 & 14.8009 \\
\hline JT71-๑๑7-АCСТ & 16.0 & 11 & 1 & 11.8961 & 1.2388 & 14.7369 \\
\hline JT71-๑๑7-АCCT & 16.0 & 11 & 2 & 11.9355 & 1.2388 & 14.7857 \\
\hline JT71- ๑९7-АCCT - 1 & 16.0 & 12 & 1 & 11.9292 & 1.2388 & 14.7779 \\
\hline JT71- ๑९7-АCCT - 1 & 16.0 & 12 & 2 & 11.9790 & 1.2388 & 14.8396 \\
\hline JT71- ๑९7-АCCT - 1 & 16.0 & 12 & 3 & 11.9186 & 1.2388 & 14.7648 \\
\hline JT71-๑९7-АCCT - 1 & 16.0 & 12 & 1 & 11.9165 & 1.2388 & 14.7622 \\
\hline JT71-๑०7-АCCT - 1 & 16.0 & 12 & 2 & 11.8965 & 1.2388 & 14.7374 \\
\hline JT71-๑९7-P०९1 & 17.0 & 13 & 1 & 11.8834 & 1.2387 & 14.7200 \\
\hline 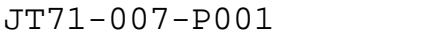 & 17.0 & 13 & 2 & 11.9100 & 1.2387 & 14.7529 \\
\hline 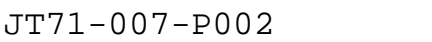 & 18.0 & 14 & 1 & 11.9157 & 1.2388 & 14.7612 \\
\hline 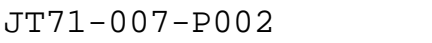 & 18.0 & 14 & 2 & 11.9229 & 1.2388 & 14.7701 \\
\hline 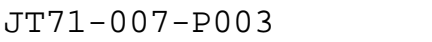 & 18.5 & 15 & 1 & 11.9518 & 1.2386 & 14.8035 \\
\hline 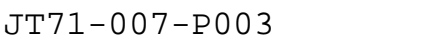 & 18.5 & 15 & 2 & 11.8895 & 1.2386 & 14.7263 \\
\hline 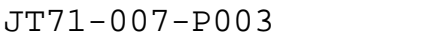 & 18.5 & 15 & 3 & 11.8639 & 1.2386 & 14.6946 \\
\hline
\end{tabular}


HB-Line Tank JT-71

Sampling and Mixing Study

The Analysis of Np Concentration Data

Plot of Np Concentration by Mixing Time

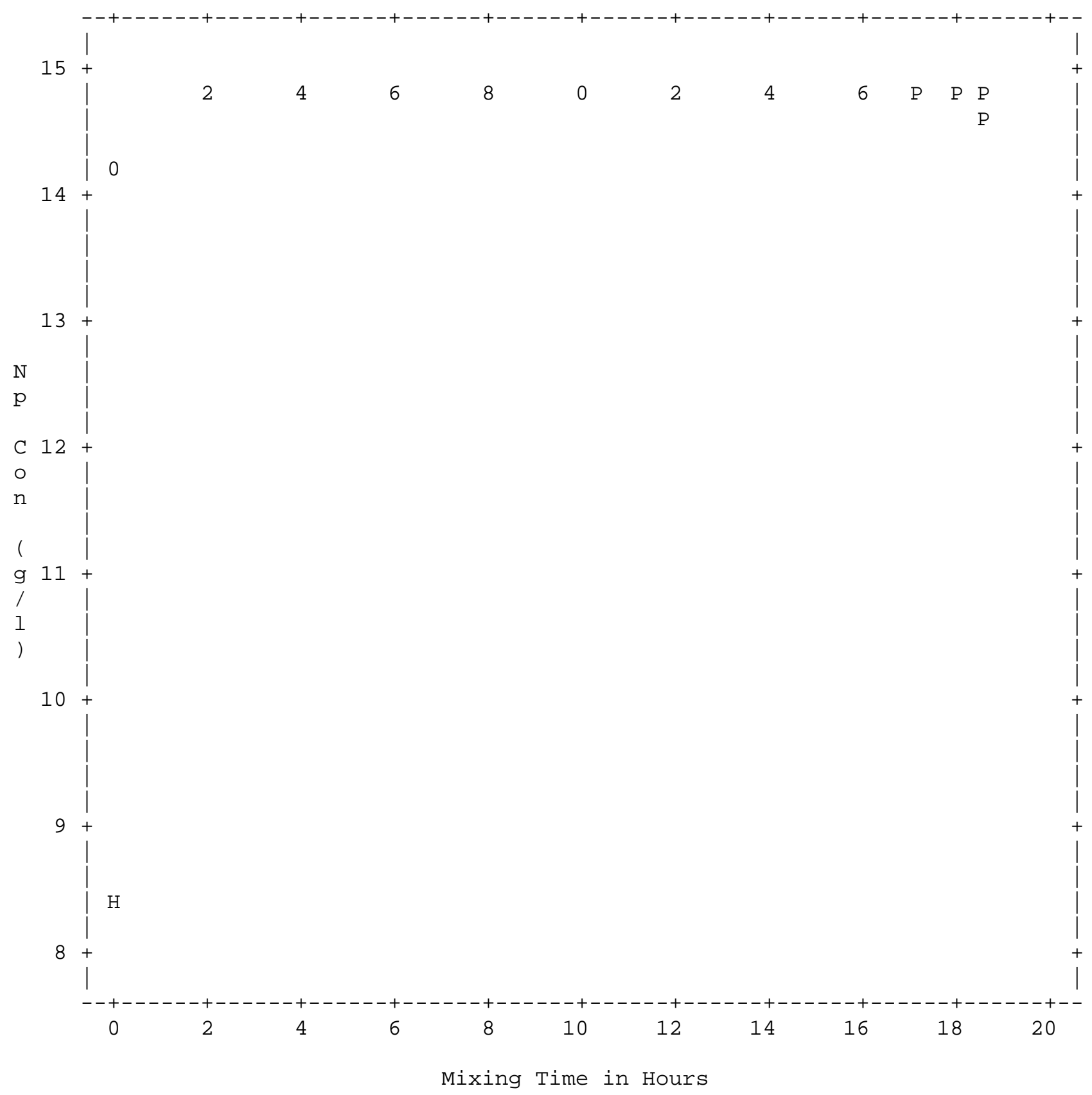

NOTE: 25 obs hidden. 
HB-Line Tank JT-71

Sampling and Mixing Study

The Analysis of Np Concentration Data

Plot of Np Concentration by Mixing Time

Solution is Mixed

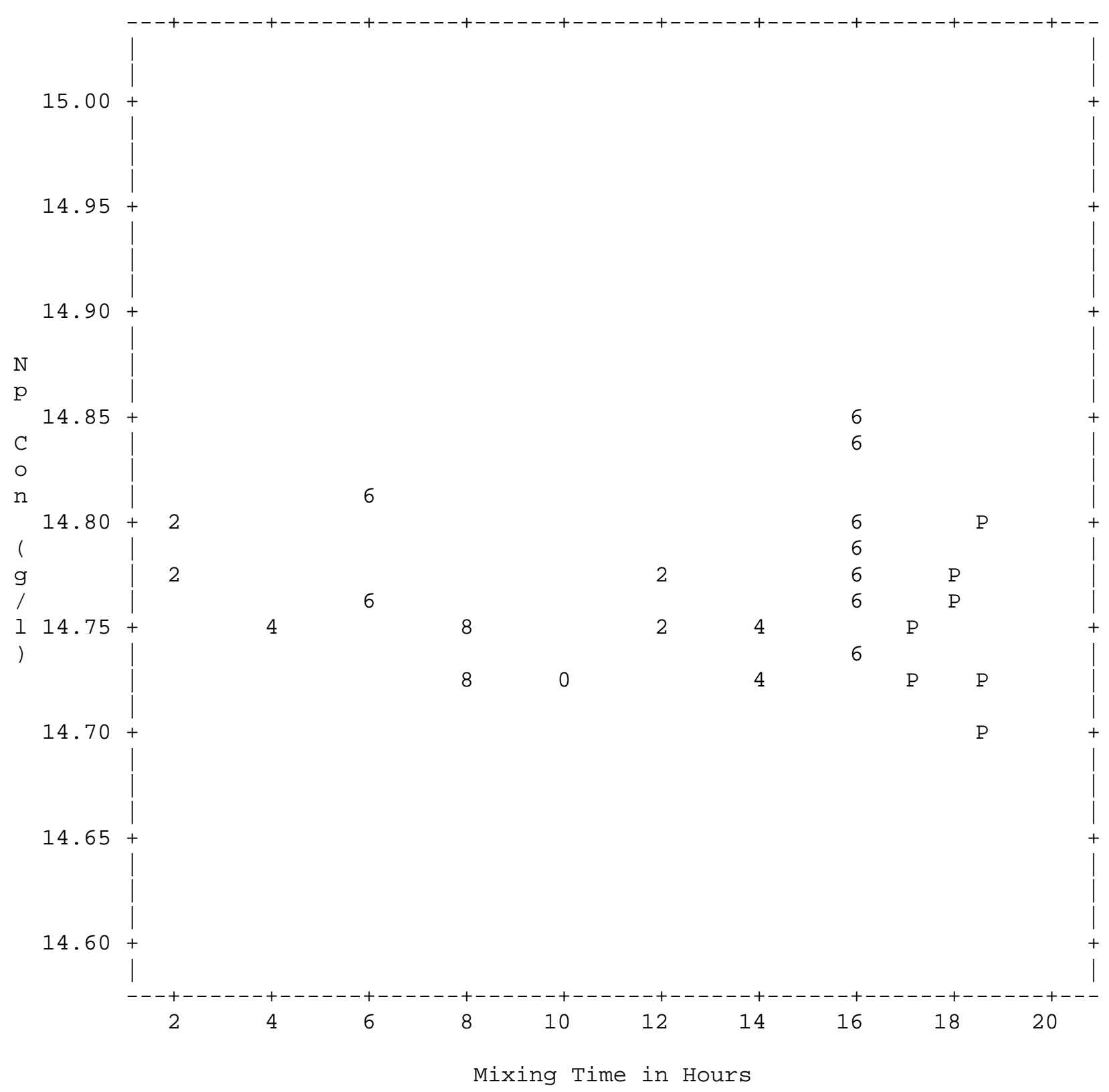

NOTE: 4 obs hidden. 9 obs out of range. 
HB-Line Tank JT-71

Sampling and Mixing Study

The Analysis of Np Concentration Data

The Estimation of Sampling and Analytical Method Uncertainty Components

The GLM Procedure

Dependent Variable: npgl Np Concentration $\mathrm{g} / \mathrm{l}$

$\begin{array}{lrrrrr}\text { Source } & \text { DF } & \begin{array}{r}\text { Sum of } \\ \text { Squares }\end{array} & \text { Mean Square } & \text { F value } & \text { Pr }>\text { F } \\ \text { Model } & 11 & 0.01573826 & 0.00143075 & 1.23 & 0.3360 \\ \text { Error } & 18 & 0.02092694 & 0.00116261 & & \\ \text { Corrected Total } & 29 & 0.03666520 & & \end{array}$

$\begin{array}{lrrr}\text { R-Square } & \text { Coeff Var } & \text { Root MSE } & \text { npgl Mean } \\ 0.429242 & 0.230984 & 0.034097 & 14.76165\end{array}$

$\begin{array}{lrrrrr}\text { Source } & \text { DF } & \text { Type I SS } & \text { Mean Square } & \text { F Value } & \text { Pr }>\text { F } \\ \text { sampleid } & 11 & 0.01573826 & 0.00143075 & 1.23 & 0.3360 \\ \text { Source } & \text { DF } & \text { Type III SS } & \text { Mean Square } & \text { F Value } & \text { Pr }>\text { F } \\ \text { sampleid } & 11 & 0.01573826 & 0.00143075 & 1.23 & 0.3360\end{array}$

The estimation of the sampling uncertainty is as follows:

Source

sampleid
Type III Expected Mean Square

$\operatorname{Var}($ Error $)+2.4667 \operatorname{Var}($ sampleid $)$ 
HB-Line Tank JT-71

Sampling and Mixing Study

The Analysis of Np Concentration Data

The Estimation of Sampling and Analytical Method Uncertainty Components

$$
\begin{array}{rr}
\text { Uncertainty Components for Tank } & \text { JT-71: } \\
\text { Sampling Uncertainty }= & 0.0104 \mathrm{~g} / 1 \\
\text { Analytical Method Uncertainty }= & 0.0341 \mathrm{~g} / 1 \\
\text { Average Np Concentration }= & 14.7617 \mathrm{~g} / 1 \\
\text { Sampling \%RSD }= & 0.0707 \% \\
\text { Analytical Method \%RSD }= & 0.2310 \%
\end{array}
$$




\section{Discussion Exercise Tank Sampling \& Mixing Study for Liquids}

\section{Session Objectives:}

After the session the participants will be able to do the following:

1. Plan a sampling/mixing study

2. Collect the measurement data

3. Analyze the data

4. Determine when a tank solution is mixed

5. Document results

\section{Estimated Time:}

There are 10 discussion topics for this module.

These topics will require 30 minutes to complete.

\section{Materials Needed:}

1. This exercise will be a discussion

2. The instructor will lead the discussion while viewing the slides

\section{Instructions:}

1. Cover the discussion topics while viewing the slides

2. A listing of the data, data plots and an ANOVA will be on the slides or in the student booklet 


\section{Background Information:}

A particular process was experiencing ID problems. The investigation suggested a sampling/mixing study on the main input accountability tank. This was to ensure the solution was adequately mixed when accountability samples were taken. The tank was at process heel prior to the beginning of the study. Acid was added to the tank to reduce the concentration by approximately $50 \%$. Eventually, process solution from another location was transferred into the tank. The sampling study was then continued.

This tank uses air sparging for mixing, but has a recirculation pump as a backup system.

\section{Discussion Topics}

1. What material was used for this study?

2. What needs to be done before the study can begin?

3. When did the sampling study actually begin?

4. How many samples were pulled at each time interval?

5. Current mixing time is 8 hours. When is this tank mixed?

6. How long was this mixing study conducted?

7. How can the sampling uncertainty be estimated?

8. What is an estimate of the sampling uncertainty?

9. What is a possible statistical model for this study? 
10. Explore other options for pulling and analyzing samples for a mixing study. 
HB-Line Tank JT-71

Sampling and Mixing Study

The Analysis of Np Concentration Data

\begin{tabular}{|c|c|c|c|c|c|c|}
\hline Sample Desc & $\begin{array}{l}\text { Mixing } \\
\text { Time } \\
\text { Hours }\end{array}$ & $\begin{array}{c}\text { Group } \\
\text { No. }\end{array}$ & Aliquot & $\begin{array}{c}\mathrm{Np} \\
\text { Concentration } \\
\mathrm{mg} / \mathrm{g}\end{array}$ & $\begin{array}{l}\text { Density } \\
\mathrm{g} / \mathrm{ml}\end{array}$ & $\begin{array}{c}\mathrm{Np} \\
\text { Concentration } \\
\mathrm{g} / \mathrm{l}\end{array}$ \\
\hline JT71- $\odot \odot 7-A C C T-1-H E E L$ & 0.0 & 1 & 1 & 6.8839 & 1.2094 & 8.3254 \\
\hline JT71-๑๑7-ACCT - 1- HEEL & $\odot .0$ & 1 & 2 & 6.8729 & 1.2094 & 8.3121 \\
\hline JT71- $\odot \odot 7$ - АCCT - 1 - HEEL & 0.0 & 1 & 1 & 6.8719 & 1.2094 & 8.3109 \\
\hline JT71-๑๑7-ACCT - 1- HEEL & 0.0 & 1 & 2 & 6.8669 & 1.2094 & 8.3048 \\
\hline JT71-๑९7 - ACCT - HEEL & 0.0 & 2 & 1 & 6.8631 & 1.2095 & 8.3009 \\
\hline JT71-๑९7 - АCCT - HEEL & 0.0 & 2 & 1 & 6.8744 & 1.2095 & 8.3146 \\
\hline JT71-๑९7 - ACCT - HEEL & 0.0 & 2 & 2 & 6.8726 & 1.2095 & 8.3124 \\
\hline 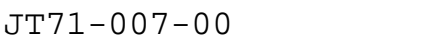 & 0.0 & 3 & 1 & 11.4207 & 1.2377 & 14.1354 \\
\hline 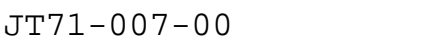 & 0.0 & 3 & 2 & 11.4472 & 1.2377 & 14.1682 \\
\hline 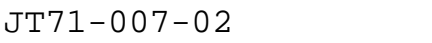 & 2.0 & 4 & 1 & 11.9467 & 1.2386 & 14.7972 \\
\hline 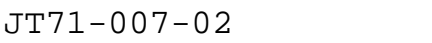 & 2.0 & 4 & 2 & 11.9335 & 1.2386 & 14.7808 \\
\hline 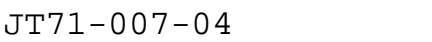 & 4.0 & 5 & 1 & 11.9092 & 1.2385 & 14.7495 \\
\hline 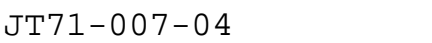 & 4.0 & 5 & 2 & 11.9082 & 1.2385 & 14.7483 \\
\hline JT71-๑०7-๑6 & 6.0 & 6 & 1 & 11.9574 & 1.2388 & 14.8128 \\
\hline 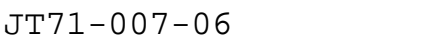 & 6.0 & 6 & 2 & 11.9164 & 1.2388 & 14.7620 \\
\hline JT71-๑९7- ๑8 & 8.0 & 7 & 1 & 11.9049 & 1.2385 & 14.7442 \\
\hline JT71-๑९7- ๑8 & 8.0 & 7 & 2 & 11.8943 & 1.2385 & 14.7311 \\
\hline JT71- -๑7 - $1 \odot$ & 10.0 & 8 & 1 & 11.8886 & 1.2386 & 14.7252 \\
\hline JT71- ๑०7 - $1 \odot$ & 10.0 & 8 & 2 & 11.8845 & 1.2386 & 14.7201 \\
\hline JT71-๑๑7-12 & 12.0 & 9 & 1 & 11.9006 & 1.2390 & 14.7448 \\
\hline JT71-๑०7- 12 & 12.0 & 9 & 2 & 11.9240 & 1.2390 & 14.7738 \\
\hline JT71-๑०7-14 & 14.0 & 10 & 1 & 11.8904 & 1.2389 & 14.7310 \\
\hline JT71-๑९7-14 & 14.0 & 10 & 2 & 11.9042 & 1.2389 & 14.7481 \\
\hline 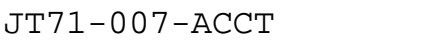 & 16.0 & 11 & 1 & 11.9846 & 1.2388 & 14.8465 \\
\hline JT71-๑๑7 - АCCT & 16.0 & 11 & 2 & 11.9478 & 1.2388 & 14.8009 \\
\hline JT71-๑๑7-АCСТ & 16.0 & 11 & 1 & 11.8961 & 1.2388 & 14.7369 \\
\hline JT71-๑๑7-АCCT & 16.0 & 11 & 2 & 11.9355 & 1.2388 & 14.7857 \\
\hline JT71- ๑९7-АCCT - 1 & 16.0 & 12 & 1 & 11.9292 & 1.2388 & 14.7779 \\
\hline JT71- ๑९7-АCCT - 1 & 16.0 & 12 & 2 & 11.9790 & 1.2388 & 14.8396 \\
\hline JT71- ๑९7-АCCT - 1 & 16.0 & 12 & 3 & 11.9186 & 1.2388 & 14.7648 \\
\hline JT71-๑९7-АCCT - 1 & 16.0 & 12 & 1 & 11.9165 & 1.2388 & 14.7622 \\
\hline JT71-๑०7-АCCT - 1 & 16.0 & 12 & 2 & 11.8965 & 1.2388 & 14.7374 \\
\hline JT71-๑९7-P०९1 & 17.0 & 13 & 1 & 11.8834 & 1.2387 & 14.7200 \\
\hline 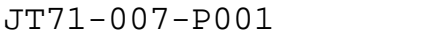 & 17.0 & 13 & 2 & 11.9100 & 1.2387 & 14.7529 \\
\hline 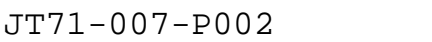 & 18.0 & 14 & 1 & 11.9157 & 1.2388 & 14.7612 \\
\hline 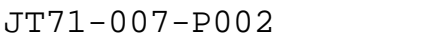 & 18.0 & 14 & 2 & 11.9229 & 1.2388 & 14.7701 \\
\hline 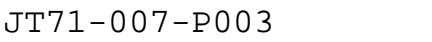 & 18.5 & 15 & 1 & 11.9518 & 1.2386 & 14.8035 \\
\hline 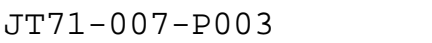 & 18.5 & 15 & 2 & 11.8895 & 1.2386 & 14.7263 \\
\hline 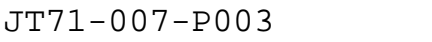 & 18.5 & 15 & 3 & 11.8639 & 1.2386 & 14.6946 \\
\hline
\end{tabular}


HB-Line Tank JT-71

Sampling and Mixing Study

The Analysis of Np Concentration Data

Plot of Np Concentration by Mixing Time

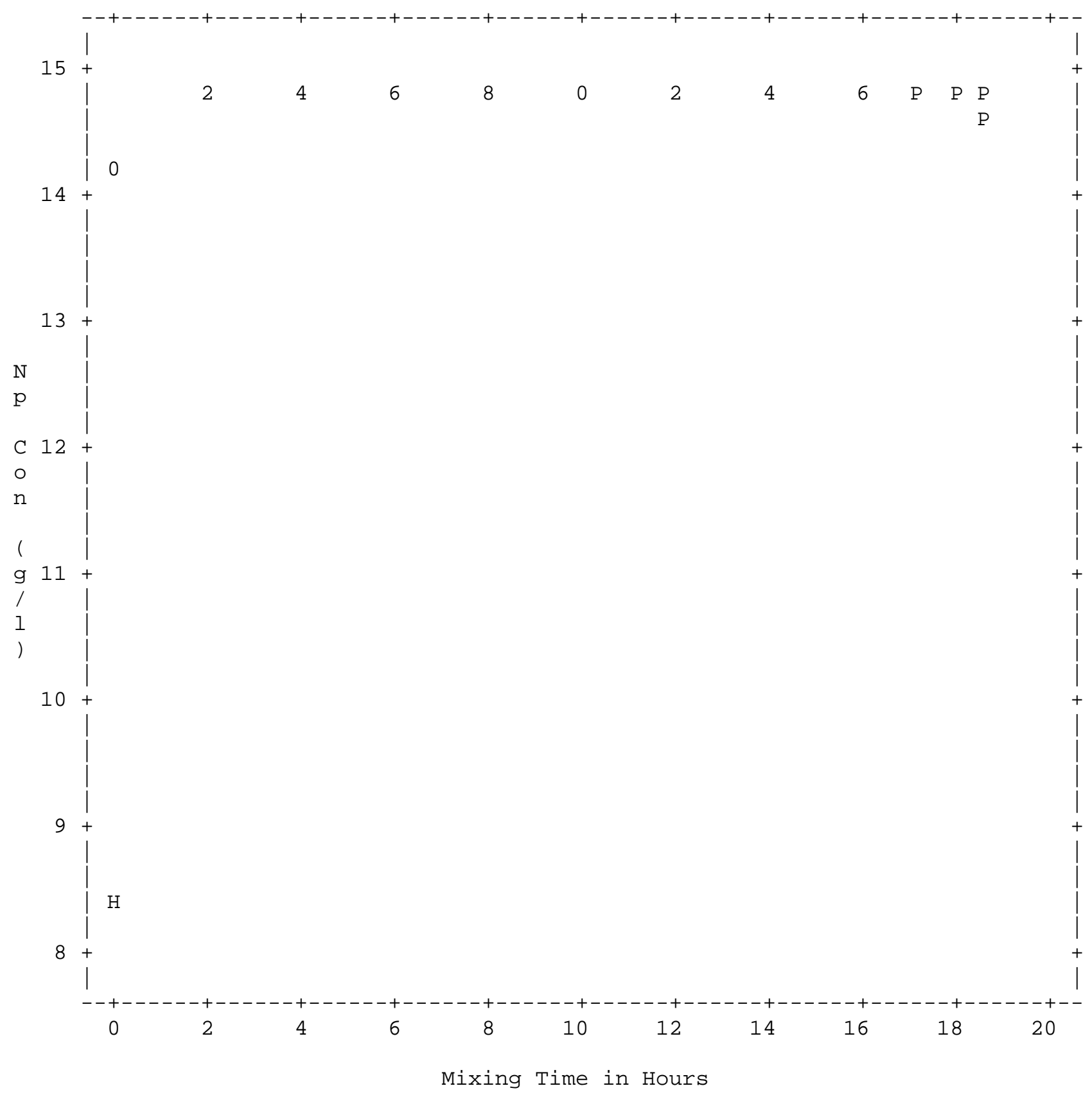

NOTE: 25 obs hidden. 
HB-Line Tank JT-71

Sampling and Mixing Study

The Analysis of Np Concentration Data

Plot of Np Concentration by Mixing Time

Solution is Mixed

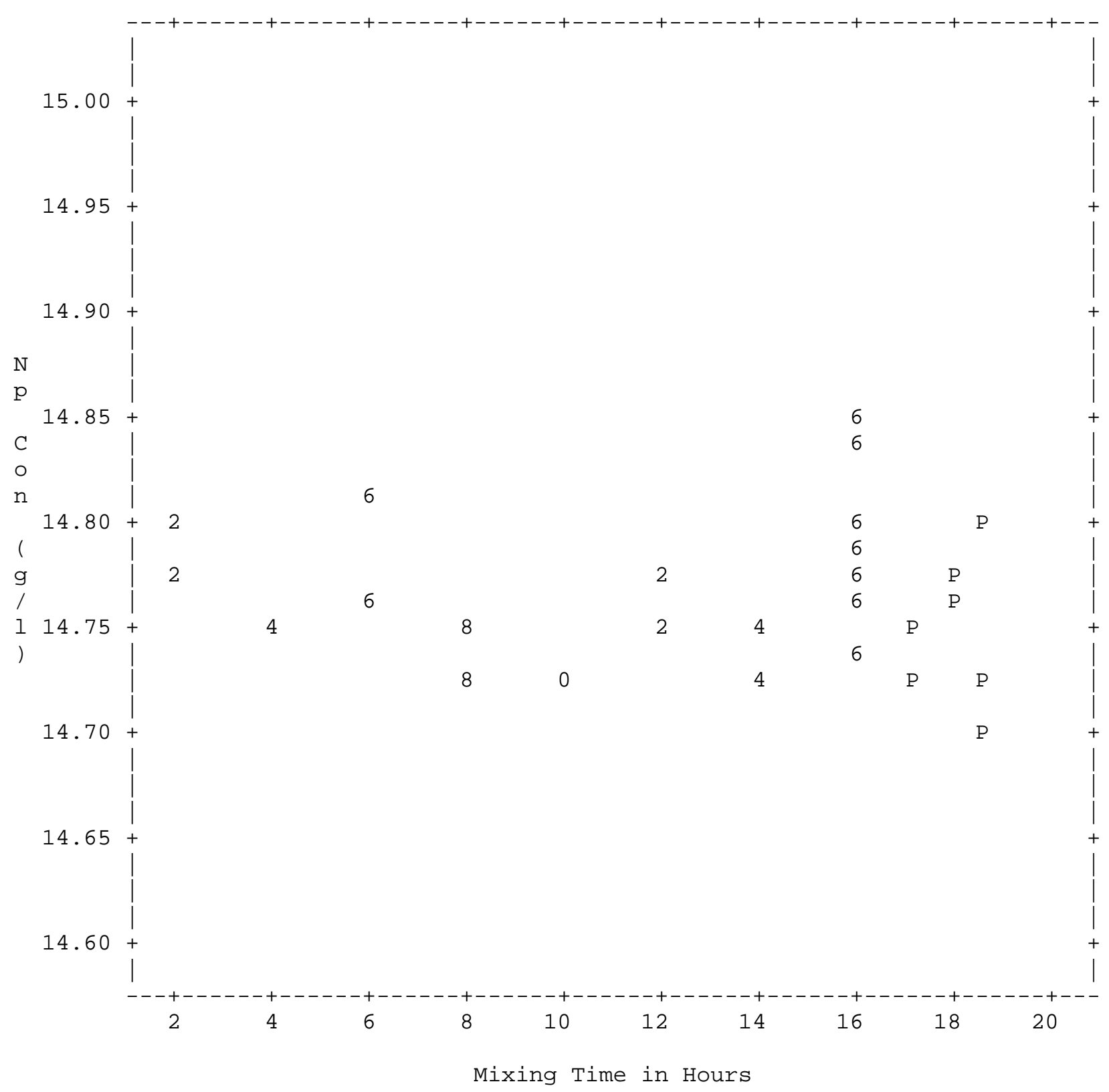

NOTE: 4 obs hidden. 9 obs out of range. 
HB-Line Tank JT-71

Sampling and Mixing Study

The Analysis of Np Concentration Data

The Estimation of Sampling and Analytical Method Uncertainty Components

The GLM Procedure

Dependent Variable: npgl Np Concentration $\mathrm{g} / \mathrm{l}$

$\begin{array}{lrrrrr}\text { Source } & \text { DF } & \begin{array}{r}\text { Sum of } \\ \text { Squares }\end{array} & \text { Mean Square } & \text { F value } & \text { Pr }>\text { F } \\ \text { Model } & 11 & 0.01573826 & 0.00143075 & 1.23 & 0.3360 \\ \text { Error } & 18 & 0.02092694 & 0.00116261 & & \\ \text { Corrected Total } & 29 & 0.03666520 & & \end{array}$

$\begin{array}{lrrr}\text { R-Square } & \text { Coeff Var } & \text { Root MSE } & \text { npgl Mean } \\ 0.429242 & 0.230984 & 0.034097 & 14.76165\end{array}$

$\begin{array}{lrrrrr}\text { Source } & \text { DF } & \text { Type I SS } & \text { Mean Square } & \text { F Value } & \text { Pr }>\text { F } \\ \text { sampleid } & 11 & 0.01573826 & 0.00143075 & 1.23 & 0.3360 \\ \text { Source } & \text { DF } & \text { Type III SS } & \text { Mean Square } & \text { F Value } & \text { Pr }>\text { F } \\ \text { sampleid } & 11 & 0.01573826 & 0.00143075 & 1.23 & 0.3360\end{array}$

The estimation of the sampling uncertainty is as follows:

Source

sampleid
Type III Expected Mean Square

$\operatorname{Var}($ Error $)+2.4667 \operatorname{Var}($ sampleid $)$ 
HB-Line Tank JT-71

Sampling and Mixing Study

The Analysis of Np Concentration Data

The Estimation of Sampling and Analytical Method Uncertainty Components

$$
\begin{array}{rr}
\text { Uncertainty Components for Tank } & \text { JT-71: } \\
\text { Sampling Uncertainty }= & 0.0104 \mathrm{~g} / 1 \\
\text { Analytical Method Uncertainty }= & 0.0341 \mathrm{~g} / 1 \\
\text { Average Np Concentration }= & 14.7617 \mathrm{~g} / 1 \\
\text { Sampling \%RSD }= & 0.0707 \% \\
\text { Analytical Method \%RSD }= & 0.2310 \%
\end{array}
$$


Revision 06-13-2012

Balance Exercise

Analytical Balance Precision, Accuracy and Uncertainty Determination Exercise

\section{Exercise Objectives:}

1. Determine the variation in making weight measurements

2. Determine estimates of precision due to the variation between analysts and balances

3. Estimate the uncertainty of weight measurements at different levels over the weighing range.

4. Learn the importance of knowing the variable included in measurement precision estimates.

\section{Estimated Time:}

+1.00 hours completing exercise

+0.45 hours in large group discussion

1.75 hours total

\section{Materials needed:}

1. Four Laptop computers

2. Four zip or flash drives for use in transferring data between teams

3. Work sheets for each person and the certificate with the calibration values \& uncertainties.

4. One barometer with calibration certificate or manufacturer specifications in both Chinese and English

5. One thermometer with readability to tenth of a degree (0.1C) must have calibration certificate or manufacturers specification.

6. Humidity meter with calibration certificate or manufacturer specifications in both Chinese and English

7. Four analytical balances (four places minimum)

8. Four sets of calibrated E2 mass standards (1-100 gram sets) with calibration certificates

9. Four sets of tweezers or tongs for handling weights.

10. At least 24 pair of cotton or insulated gloves.

11. Flip chart or dry board and colored markers

12. A printer for at least one of the computers to print out the results of the exercises. 


\section{Instructions:}

\section{Exercise 1a (Repeatability)}

\section{Perform the following steps.}

1. On a team work sheet record the environmental conditions; weight ID, weight's conventional value \& uncertainty from the calibration report, date and the name of each team member.

2. Make sure the balance is level.

3. Exercise the balance by placing a $100 \mathrm{~g}$ weight on the pan and removing it 3 times using tweezers. Keep hands off the weights!

4. Zero the balance.

5. Using tweezers, place the $100 \mathrm{~g}$ weight on center of the pan.

6. Record the first stable reading.

7. Remove the weight using tweezers.

8. Repeat steps 4 through 7 nine more times. Do not Zero.

9. Use the Excel spread sheet to record your data and calculate the average bias (Ub), standard deviation (Usd), the standard's standard deviation (Ustd), uncertainty \& relative uncertainty. 10. Print 5 copies of the spreadsheet after you have accurately input the correct information. 11. Give copies to the other teams and instructors, after your team has studied them.

\section{Discussion Questions:}

1. How do the bias, standard deviation and uncertainty estimates differ between team members?

2. What is the largest source of uncertainty that is used to calculate the uncertainty of weight measurements made by your balance?

3. Did the uncertainty of the standard contribute significantly to the total uncertainty?

4. How much of the total uncertainty was contributed by the standard used?

5. This balance will be used in another exercise to calibrate pipettes.

6. Is it fit for that purpose? Why? 


\section{Exercise 1b (Intermediate Precision)}

This exercise uses all of the measurements made by each team to compute an intermediate precision estimate. All conditions have been held constant, except for the operators. The average and standard deviation of all the data are calculated in the last column of the Excel spreadsheet and an uncertainty estimate has been calculated and reported in the bottom line.

\section{Discussion Questions:}

1. Is the team estimate different than the estimates of the individuals?

2. What is the major source of uncertainty in this exercise?

3. Save a copy of your Excel spreadsheet and change the uncertainty of each weight by multiplying it by 3 and recalculate the uncertainty estimates.

4. What affect did this have on the total uncertainty?

5. How can the uncertainties be reduced in Weighing? 


\section{Exercise 1c (Reproducibility)}

This exercise requires each team to get copies of the other teams exercise and use the information to determine the best estimate of uncertainty for weight measurements made by anyone in the class on any of the balances. Be ready to discuss how you determined the estimate with the rest of the class.

\section{Discussion Questions:}

1. After reviewing the precision data and uncertainty estimates from all teams, which team has the smallest uncertainty? .

2. Is there a significant difference in the uncertainty estimates of the various balances?

3. Is there a significant difference in biases calculated for each of the balances?

\section{Summary Points for Exercises 1a through 1c:}

1. The exercises should demonstrate that the more variables a measurement system has, the larger the uncertainty estimate. Was this the case for this exercise?

2. Standards used for calibration and validation must have uncertainties $<1 / 3$ of the measurement instrument's uncertainty. Did the standards used for this exercise have small uncertainties?

3. Reproducibility conditions must be stated to have a meaningful estimate of the random error associated with weight measurements. 


\section{Exercise 2}

\section{Balance Linearity Testing and Uncertainty Estimations}

This exercise involves testing the linearity of a balance at 5 points over the range and using the data to estimate the uncertainty of measurements made at the different ranges. Have one person from your team make 10 measurements with each of the 5 weights provided.

1. Record your name, the date \& time, environmental conditions, balance and weight information. This includes the certificate conventional weight and uncertainty for each standard

2. Zero the balance then, place the $1 \mathrm{~g}$ weight in the center of the pan, record the first stable reading,

3. Remove, then weight the $10 \mathrm{~g}$ weight, record,

4. Remove, then weigh the $50 \mathrm{~g}$ weight, record,

5. Remove, then weigh the 100 weight, record,

6. Remove, and then weigh the 150 combined weights.

7. Repeat steps $2-6$ nine more times. Only zero the balance before weighing the series of 5 weights.

8. Then use the Exercise 2 Excel spreadsheet to calculate estimates of uncertainty at each level.

9. Print 5 copies of the spreadsheet after you have accurately input the correct information.

\section{Discussion Questions:}

1. How do the relative uncertainties differ for each level?

2. How do you characterize the linearity error of the balance? Is it significant?

3. What uncertainty error would you assign for weight measurements made with your balance?

4. Will the balance contribute significantly in weight measurements made for accountability? 
Revision 06-13-2012

Balance Exercise 1a Repeatability Test \& Uncertainty Estimates Balance Exercise 1 b I ntermediate Precision \& Uncertainty Estimate

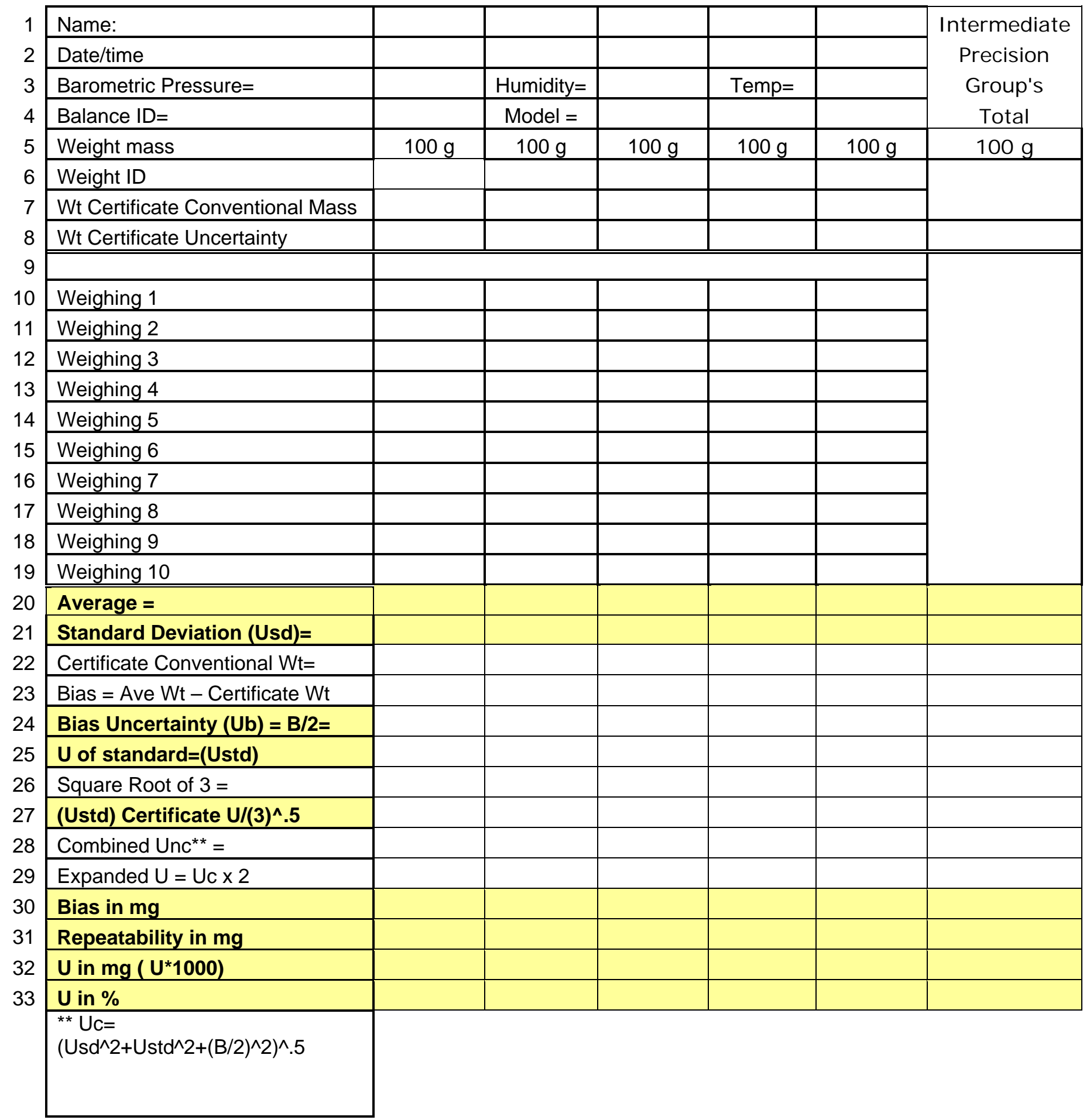


Revision 06-13-2012

\section{Balance Linearity Test Exercise 2}

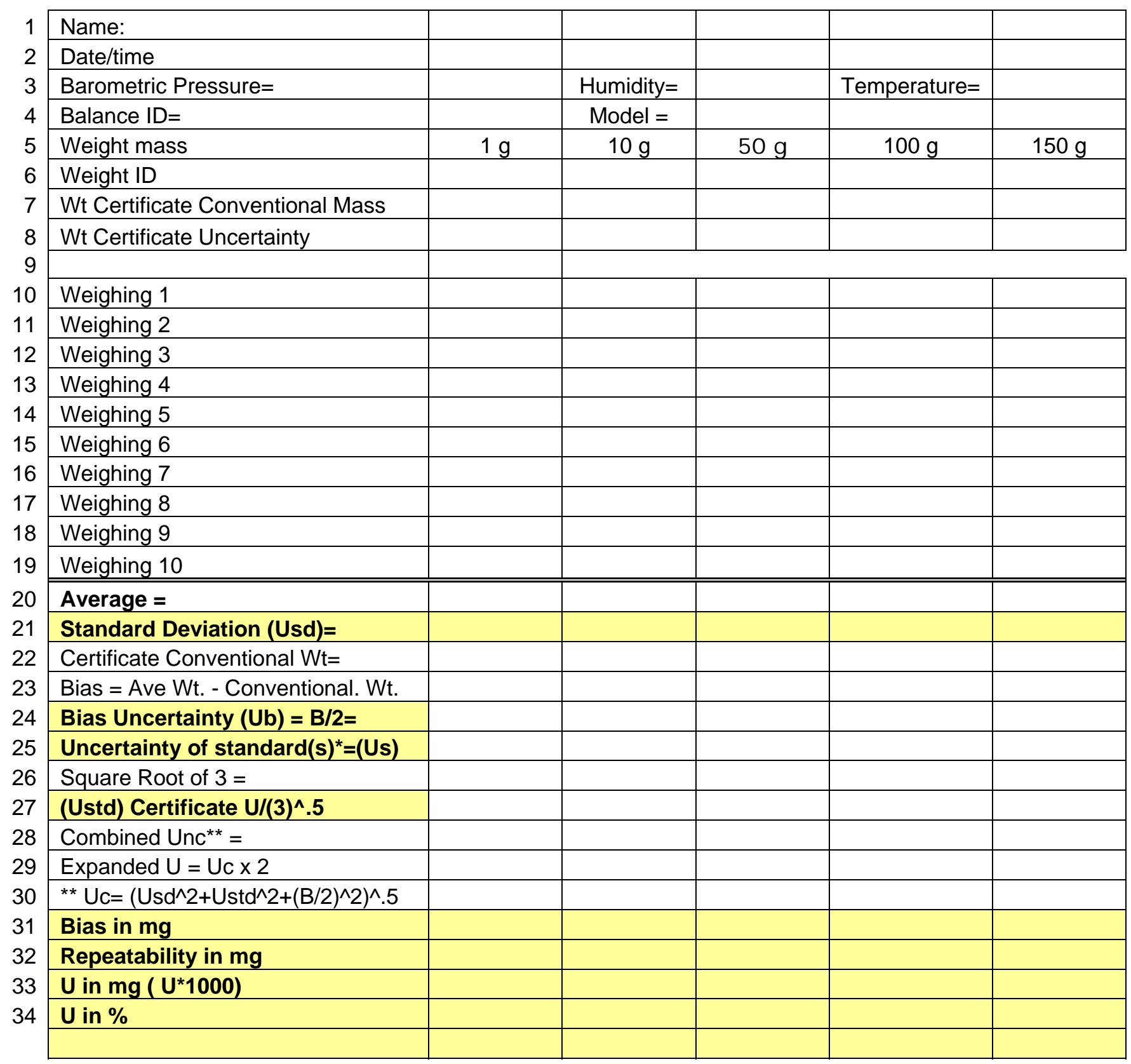


Revision 06-13-2012

Calibration Certificate Values for Weights done in 2011 by Troemner for 50 \& $100 \mathrm{~g}$ Weights and SRS Standard Lab for 1 and $10 \mathrm{~g}$ weights.

\begin{tabular}{|c|c|c|c|c|c|c|}
\hline Weight Set & Mass & Marking & Conventional Wt & & $\begin{array}{l}\text { Uncertainty } \\
\qquad \mathrm{K}=\mathbf{2}\end{array}$ & $\begin{array}{c}\text { Tolerrance } \\
\text { mg }\end{array}$ \\
\hline \multirow[t]{5}{*}{$\overline{8}$} & $1 \mathrm{~g}$ & $T$ & 1.00002 & $\bar{g}$ & 0.0045 & 0.054 \\
\hline & $10 \mathrm{~g}$ & 10 & 10.00001 & g & 0.015 & 0.074 \\
\hline & $50 \mathrm{~g}$ & + & 49.98410 & $\mathbf{g}$ & 0.12 & 0.12 \\
\hline & $100 \mathrm{~g}$ & + & 99.97173 & $\mathbf{g}$ & 0.45 & 0.25 \\
\hline & $50+100 \mathrm{~g}$ & $*$ & 149.95583 & $\mathbf{g}$ & $0.4 \mathrm{BB}$ & 0.277 \\
\hline
\end{tabular}

\begin{tabular}{|c|c|c|c|c|c|c|}
\hline \multirow[t]{5}{*}{$\bar{A}$} & $1 \mathrm{~g}$ & $\mathbf{A}$ & 1.00003 & $g$ & 0.00145 & 0.0 .24 \\
\hline & $10 \mathrm{~g}$ & $\mathbf{A}$ & 10.00004 & $g$ & 0.015 & 0.074 \\
\hline & $50 \mathrm{~g}$ & $*$ & 49.99996 & $\mathbf{g}$ & 0.12 & 0.12 \\
\hline & $100 \mathrm{~g}$ & + & 99.95392 & $\mathbf{g}$ & 0.45 & 0.25 \\
\hline & $50+100 \mathrm{~g}$ & $*$ & 149.95389 & $\mathbf{g}$ & $0.4 \mathrm{Bb}$ & 0.277 \\
\hline \multirow[t]{5}{*}{$\bar{B}$} & $1 \mathrm{~g}$ & $\bar{B}$ & 1.00015 & $g$ & 0.00145 & 0.024 \\
\hline & $10 \mathrm{~g}$ & $\mathrm{~B}$ & 10.00149 & $g$ & 0.015 & 0.074 \\
\hline & $50 \mathrm{~g}$ & $+n$ & 50.00058 & $\mathbf{g}$ & 0.12 & 0.12 \\
\hline & $100 \mathrm{~g}$ & 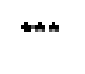 & 99.92643 & $\mathbf{g}$ & 0.45 & 0.25 \\
\hline & $50+100 \mathrm{~g}$ & $\leftrightarrow n$ & 149.92700 & $\mathbf{g}$ & $0.4 \mathrm{Bb}$ & 0.277 \\
\hline \multirow[t]{5}{*}{$\bar{C}$} & $1 \mathrm{~g}$ & $\mathrm{C}$ & 1.00001 & $g$ & 0.00145 & 0.024 \\
\hline & $10 \mathrm{~g}$ & c & 10.00009 & $g$ & 0.015 & 0.074 \\
\hline & $50 \mathrm{~g}$ & that & 49.99816 & $\mathbf{g}$ & 0.12 & 0.12 \\
\hline & $100 \mathrm{~g}$ & 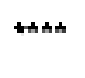 & 99.99340 & $\mathbf{g}$ & 0.45 & 0.25 \\
\hline & $50+100 \mathrm{~g}$ & that & 149.99156 & $\mathbf{g}$ & $0.4 \mathrm{BB}$ & 0.277 \\
\hline
\end{tabular}

Environment Conditions are in the Original Calibration Reports

Page 8 of 8 
Balance Exercise 1a Repeatability Test Uncertainty Estimates Balance Exercise 1b I ntermediate Precision Uncertainty Estimate

\begin{tabular}{|c|c|c|c|c|c|c|}
\hline $\begin{array}{l}\text { 姓名 : } \\
\text { Name }\end{array}$ & & & & & & $\begin{array}{c}\text { 中间 } \\
\text { I ntermediate }\end{array}$ \\
\hline $\begin{array}{l}\text { 日期/时间： } \\
\text { Date/Time }\end{array}$ & & & & & & $\begin{array}{c}\text { 精度 } \\
\text { Precision }\end{array}$ \\
\hline $\begin{array}{l}\text { 气压： } \\
\text { Barometric Pressure }\end{array}$ & & $\begin{array}{l}\text { 湿度= } \\
\text { Humidity }\end{array}$ & & $\begin{array}{l}\text { 温度= } \\
\text { Temp }\end{array}$ & & \\
\hline $\begin{array}{l}\text { 天平 ID= } \\
\text { Balance ID }\end{array}$ & & $\begin{array}{l}\text { 型号= } \\
\text { Model }\end{array}$ & & & & $\begin{array}{l}\text { 总计 } \\
\text { Total }\end{array}$ \\
\hline $\begin{array}{l}\text { 砝码质量 } \\
\text { Weight Mass }\end{array}$ & $100 \mathrm{~g}$ & $100 \mathrm{~g}$ & $100 \mathrm{~g}$ & $100 \mathrm{~g}$ & $100 \mathrm{~g}$ & $100 \mathrm{~g}$ \\
\hline $\begin{array}{l}\text { 砝码ID } \\
\text { Weight ID }\end{array}$ & & & & & & \\
\hline $\begin{array}{l}\text { 砝码证书常规质量 } \\
\text { Wt Certificate Conventional M }\end{array}$ & & & & & & \\
\hline $\begin{array}{l}\text { 砝码证书不确定性 } \\
\text { Wt Certificate Uncertainty }\end{array}$ & & & & & & \\
\hline 称重 (Weighing) 1 & & & & & & \#\#\# \\
\hline 称重 (Weighing) 2 & & & & & & \#\#\# \\
\hline 称重 (Weighing) 3 & & & & & & \#\#\# \\
\hline 称重 (Weighing) 4 & & & & & & \#\#\# \\
\hline 称重 (Weighing) 5 & & & & & & \#\#\# \\
\hline 称重 (Weighing) 6 & & & & & & \#\#\# \\
\hline 称重 (Weighing) 7 & & & & & & \#\#\# \\
\hline 称重 (Weighing) 8 & & & & & & \#\#\# \\
\hline 称重 (Weighing) 9 & & & & & & \#\#\# \\
\hline 称重 (Weighing) 10 & & & & & & \#\#\# \\
\hline $\begin{array}{l}\text { 平均 }= \\
\text { Average }\end{array}$ & \#DIV/O! & \#DIV/O! & \#DIV/O! & \#DIV/0! & \#DIV/O! & \#DIVI0! \\
\hline $\begin{array}{l}\text { 标准偏移 (Usd)= } \\
\text { Standard Deviation }\end{array}$ & \#DIV/O! & \#DIV/O! & \#DIV/O! & \#DIV/O! & \#DIV/O! & \#DIVI0! \\
\hline $\begin{array}{l}\text { 证书常规重量 }= \\
\text { Certificate Conventional Wt. }\end{array}$ & & & & & & 0.000000 \\
\hline $\begin{array}{l}\text { 偏差 = 平均重量 }- \text { 证书重量 } \\
\text { Bias = Avg Wt - Convent. Wt. }\end{array}$ & \#DIV/O! & \#DIV/O! & \#DIV/O! & \#DIV/0! & \#DIV/O! & \#DIVI0! \\
\hline $\begin{array}{l}\text { 偏差不确定性 }(\mathrm{Ub})=\mathrm{B} / 2= \\
\text { Bias Uncertainty }\end{array}$ & \#DIV/O! & \#DIV/O! & \#DIV/0! & \#DIV/O! & \#DIV/0! & \#DIVIO! \\
\hline $\begin{array}{l}\text { 标样不确定性 =(Ustd) } \\
\text { Uncertainty of Standard(s) }\end{array}$ & 0.00000 & & & & & \\
\hline $\begin{array}{l}3 \text { 的均方根 }= \\
\text { Square Root of } 3\end{array}$ & 1.73 & 1.73 & 1.73 & 1.73 & 1.73 & 1.73 \\
\hline $\begin{array}{l}\text { (标样不确定性) 证书 U/(3)^.5 } \\
(\text { Ustd)Certificate U/(3)^.5 }\end{array}$ & 0.00000 & \#VALUE! & \#VALUE! & \#VALUE! & \#VALUE! & \#VALUE! \\
\hline $\begin{array}{l}\text { 合并 Unc } \\
\text { Combined Unc** }\end{array}$ & \#DIV/O! & \#DIV/O! & \#DIV/O! & \#DIV/O! & \#DIV/O! & \#DIVI0! \\
\hline $\begin{array}{l}\text { 扩展不确定性= Uc } \times 2 \\
\text { Expanded U }\end{array}$ & \#DIV/O! & \#DIV/O! & \#DIV/O! & \#DIV/O! & \#DIV/O! & \#DIVI0! \\
\hline $\begin{array}{l}\text { 偏差(mg) } \\
\text { Bias in mg }\end{array}$ & \#DIVI0! & \#DIVI0! & \#DIVI0! & \#DIVI0! & \#DIVI0! & \#DIVI0! \\
\hline $\begin{array}{l}\text { 可重复性(mg) } \\
\text { Repeatability in mg }\end{array}$ & \#DIVI0! & \#DIVIO! & \#DIVI0! & \#DIVI0! & \#DIVI0! & \#DIVI0! \\
\hline $\begin{array}{l}\text { 不确定性mg (U*1000) } \\
\text { U in mg }\end{array}$ & \#DIVIO! & \#DIVIO! & \#DIVI0! & \#DIVI0! & \#DIVI0! & \#DIVI0! \\
\hline $\begin{array}{l}\text { 不确定性 \% } \\
\text { U in \% }\end{array}$ & \# \#\#\# \# & \# \# \#\# \# & \#\#\#＃） & \#\# \# \# \# & \#\#\#\#\# & \#DIV/ 0 ! \\
\hline
\end{tabular}

** $U c=\left(U s d^{\wedge} 2+U s t d^{\wedge} 2+(B / 2)^{\wedge} 2\right)^{\wedge} .5$ 


\section{Mass Calibration Data Summary}

Issued By

Savannah River Standards Laboratory

Savannah River National Laboratory, Building 736-A

Aiken, SC 29808

\section{Calibration Serial No. \\ ONEnTEN-2011001}

Tolerance Class

$$
\text { ASTM } 2
$$

\section{Comments:}

TOLERANCE REQ: NONE

Environmental Conditions

$$
\text { Barometric Pressure }( \pm 1 \mathrm{mmHg})
$$

Relative Humidity $( \pm 10 \%)$

Room Temperature $\left( \pm 1.0^{\circ} \mathrm{C}\right)$

M\&TE No.

ONEnTEN

Metrologist

$\begin{array}{ccc}\text { Start } & \text { Finish } & \text { Average } \\ 752 & 752 & 752 \\ 44 & 43 & 44 \\ 23.3 & 23.4 & 23.4\end{array}$

Uncertainties are calculated using the GUM method of RSS. They are reported at $\mathrm{K}=2,95 \%$ Confidence. Uncertainties do not include the possible effects of magnetism. Magnetism, surface finish and density are not tested. All densities are assumed.

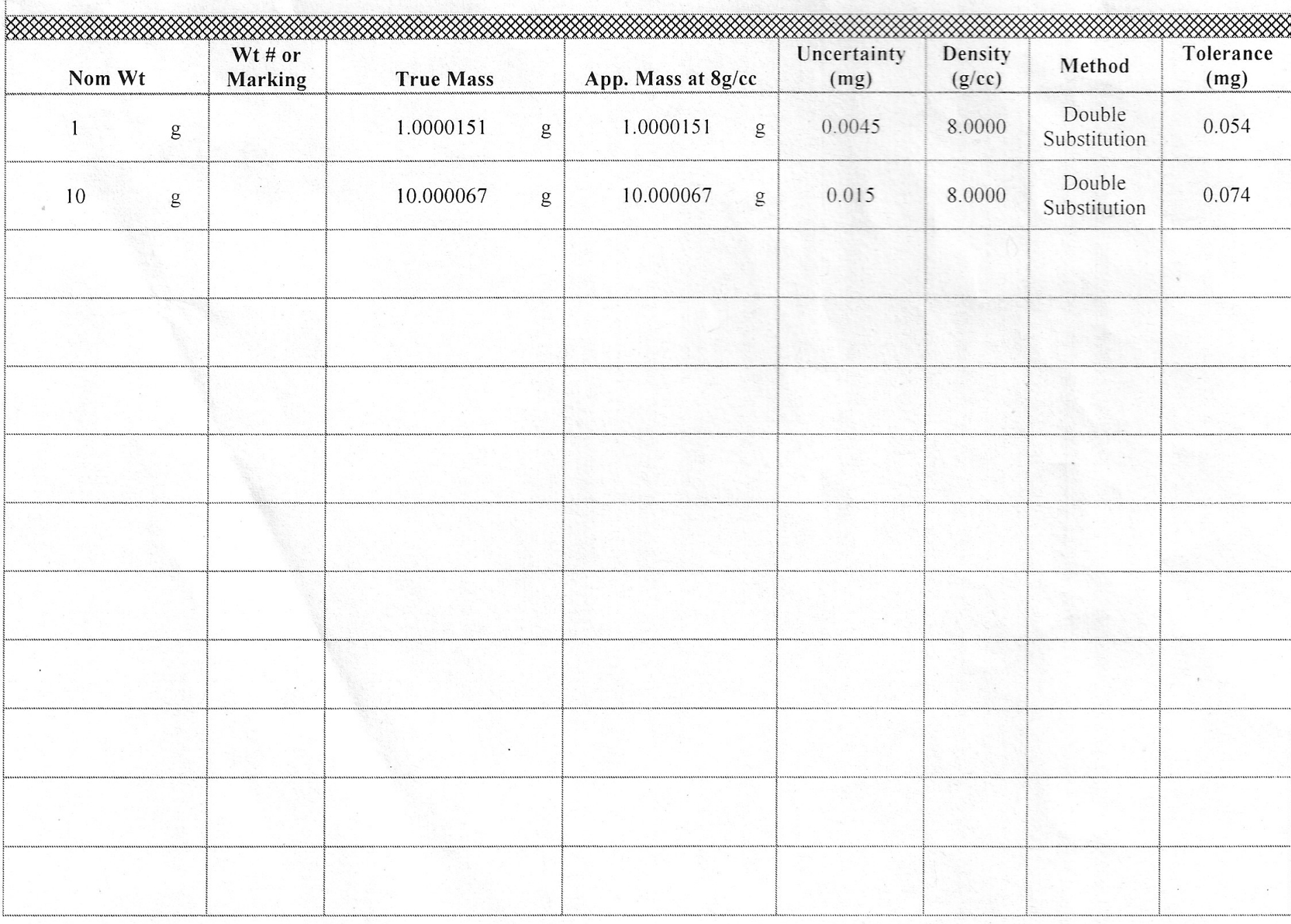




\section{TROEMNER}

Hary romentuc

\section{Calibration Certificate}

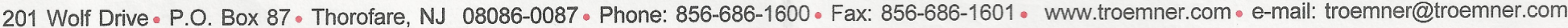

End user

Troemner Inc

201 Wolf Drive

Thorofare NJ 08086
Page 1 of 1

Weight

Certificate Number : JOEWTS-1

Date of Calibration : :09-SEP-2011

Next Due Date : 09-SEP-2012
Description of Weights: ASTM E617-97 Class 1

Order Number $\quad: 0060800$

Construction : One Piece

Material $\quad \vdots$ Stainless Steel $(\mathrm{O} / \mathrm{P})$
Date Received

Date of Issue

Weight Range

ID\#
Pressure: $756.49 \mathrm{~mm} \mathrm{Hg}$

$$
\begin{aligned}
& \text { : N/A } \\
& 09-S E P-2011 \\
& : 50 \mathrm{~g}, 100 \mathrm{~g}
\end{aligned}
$$

Relative Humidity: $48 \%$
Environmental Condition at Time of Test

Temperature: $22.22^{\circ} \mathrm{C}$
Accedled by the

Pertinent Information

This calibration also meets specifications as outlined in ISO 9001, ISO/IEC 17025, ANSI/NCSL Z540-1-1994.

Weights listed in the calibration report have been compared to reference standards that are directly traceable a National Standard under Test No. $822-275872$.

Uncertainty is the standard deviation associated with the results of the measurement that characterizes the dispersion of the values that could reasonably be attributed

to the measureand. The uncertainty is calculated in accordance with NIST Tech Note 1297 using a coverage factor of $k=2$ ( $k=2$ defines the interval having a level

of confidence of approximately $95 \%$ ). Uncertainty does not include possible effects of magnetism.

\begin{tabular}{|c|c|c|}
\hline \multicolumn{3}{|c|}{ Calibration Data } \\
\hline Nominal & Notes & Serial No. \\
\hline $\begin{array}{l}\text { logs value } \\
100 \mathrm{~g}\end{array}$ & $* \mathrm{XF}$ & \\
\hline $100 \mathrm{~g}$ & $2 X F$ & \\
\hline $100 \mathrm{~g}$ & $3 \mathrm{XF}$ & \\
\hline $100 \mathrm{~g}$ & $4 \mathrm{XF}$ & \\
\hline $50 \mathrm{~g}$ & $* \mathrm{XF}$ & \\
\hline $50 \mathrm{~g}$ & $2 \mathrm{XF}$ & \\
\hline $50 \mathrm{~g}$ & $3 \mathrm{XF}$ & \\
\hline $50 \mathrm{~g}$ & $4 X F$ & \\
\hline
\end{tabular}

Conventional mass is defined as a weight taken at $20^{\circ} \mathrm{C}$ with a reference density of $8.0 \mathrm{~g} * \mathrm{~cm}-3$ and balanced in air density of $0.00120 \mathrm{~g} * \mathrm{~cm}-3$.

Notes: * - indicates duplicate weights $\mathbf{F}$ - indicates failed As Found $\mathbf{X}$ - indicates weight out of tolerance not adjustable $\mathbf{N}$-indicates new weight added

Conventiona
As Found
$99.971726 \mathrm{~g}$
$99.953924 \mathrm{~g}$
$99.926428 \mathrm{~g}$
$99.934047 \mathrm{~g}$
$49.984100 \mathrm{~g}$
$49.999962 \mathrm{~g}$
$50.000575 \mathrm{~g}$
$49.998157 \mathrm{~g}$

$49.998157 \mathrm{~g}$

$$
\begin{aligned}
& \text { Uncertainty } \\
& 0.044 \mathrm{mg} \\
& 0.044 \mathrm{mg} \\
& 0.044 \mathrm{mg} \\
& 0.044 \mathrm{mg} \\
& 0.024 \mathrm{mg} \\
& 0.024 \mathrm{mg} \\
& 0.024 \mathrm{mg} \\
& 0.024 \mathrm{mg}
\end{aligned}
$$

Tolerance

$0.250 \mathrm{mg}$
$0.250 \mathrm{mg}$
$0.250 \mathrm{mg}$
$0.250 \mathrm{mg}$
$0.120 \mathrm{mg}$
$0.120 \mathrm{mg}$
$0.120 \mathrm{mg}$
$0.120 \mathrm{mg}$

$0.250 \mathrm{mg}$

$0.250 \mathrm{mg}$

$\mathrm{mg}$

$0.120 \mathrm{mg}$

$0.120 \mathrm{mg}$

\section{Addendum:}

\section{Approved Signatory}

\section{Person Performing Work}

\section{Joseph Moran, Metrology Manager}

\section{Annemarie Love}

This certificate of calibration shall not be reproduced except in full, without the written approval of Henry Troemner, LLC. This certificate of calibration must not be used by the customer to claim product endorsement by NIST/NVLAP or any agency of the U.S. government. 
Calibration Certificate Values for Weights Done in 2011 by Troemner for $50100 \mathrm{~g}$ Weights and SRS Stardard's Lab for 1 and $10 \mathrm{~g}$ weights.

\begin{tabular}{|c|c|c|c|c|c|c|}
\hline Weight Set & Mass & Marking & onventional Wt & & $\begin{array}{c}\text { Uncertainty } \\
\qquad \mathrm{K}=2\end{array}$ & $\begin{array}{c}\text { Tolerance } \\
\text { mg }\end{array}$ \\
\hline \multirow[t]{5}{*}{ * } & $1 \mathrm{~g}$ & 1 & 1.00002 & g & 0.0045 & 0.054 \\
\hline & $10 \mathrm{~g}$ & 10 & 10.00001 & g & 0.015 & 0.074 \\
\hline & $50 \mathrm{~g}$ & * & 49.98410 & g & 0.12 & 0.12 \\
\hline & $100 \mathrm{~g}$ & * & 99.97173 & g & 0.45 & 0.25 \\
\hline & $50+100 \mathrm{~g}$ & * & 149.95583 & g & 0.466 & 0.277 \\
\hline
\end{tabular}

\begin{tabular}{|ccccccc|}
\hline $\mathrm{A}$ & $1 \mathrm{~g}$ & $\mathrm{~A}$ & 1.00003 & $\mathrm{~g}$ & 0.0045 & 0.054 \\
& $10 \mathrm{~g}$ & $\mathrm{~A}$ & 10.00004 & $\mathrm{~g}$ & 0.015 & 0.074 \\
& $50 \mathrm{~g}$ & $* *$ & 49.99996 & $\mathrm{~g}$ & 0.12 & 0.12 \\
& $100 \mathrm{~g}$ & $* *$ & 99.95392 & $\mathrm{~g}$ & 0.45 & 0.25 \\
& $50+100 \mathrm{~g}$ & $* *$ & 149.95389 & $\mathrm{~g}$ & 0.466 & 0.277 \\
& & & & & & \\
\hline
\end{tabular}

\begin{tabular}{|c|c|c|c|c|c|c|}
\hline \multirow[t]{5}{*}{ B } & $1 \mathrm{~g}$ & B & 1.00015 & g & 0.0045 & 0.054 \\
\hline & $10 \mathrm{~g}$ & B & 10.00149 & g & 0.015 & 0.074 \\
\hline & $50 \mathrm{~g}$ & $* \star *$ & 50.00058 & g & 0.12 & 0.12 \\
\hline & $100 \mathrm{~g}$ & $* * *$ & 99.92643 & g & 0.45 & 0.25 \\
\hline & $50+100 \mathrm{~g}$ & $* * *$ & 149.92700 & g & 0.466 & 0.277 \\
\hline \multirow[t]{5}{*}{ C } & $1 \mathrm{~g}$ & $\bar{C}$ & 1.00001 & g & 0.0045 & 0.054 \\
\hline & $10 \mathrm{~g}$ & C & 10.00009 & g & 0.015 & 0.074 \\
\hline & $50 \mathrm{~g}$ & $\star * * *$ & 49.99816 & g & 0.12 & 0.12 \\
\hline & $100 \mathrm{~g}$ & $\star * * *$ & 99.99340 & g & 0.45 & 0.25 \\
\hline & $50+100 \mathrm{~g}$ & **** & 149.99156 & g & 0.466 & 0.277 \\
\hline
\end{tabular}

Environment Conditions are in the Original Calibration Reports 


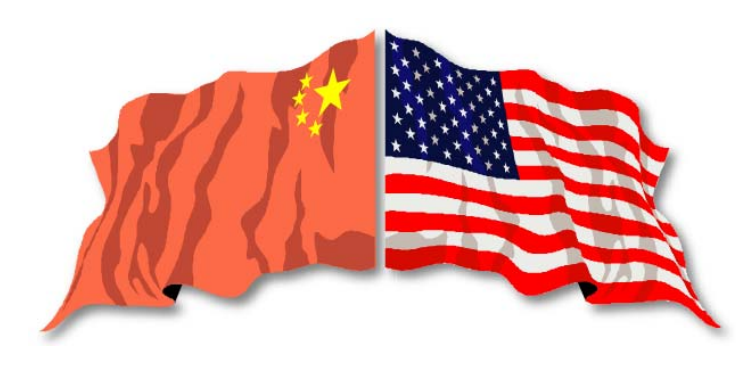

\section{Module 12}

\section{Analytical Chemistry Laboratory Measurement Control Programs}




\section{Objectives}

- Understand laboratory measurements must have uncertainty estimates for accountability of NM

- Review MCP technical \& administrative components of ANSI N15.51

- Discuss laboratory techniques for estimating error

- Discuss uses of measurement control data

- Intro to Laboratory Information Management Systems (LIMS) and review LIMS MCP output for D\&G U method 


\section{Measurement Quality Must Be Known}

Dr. John Keenan Taylor in QA of Chemical Measurements

- "Quantitative measurements are always estimates of the value of the measure and involve some level of uncertainty.

- The measurements must be made so that the limits of uncertainty can be assigned within a stated probability.

- Without such an assignment, no logical use can be made of the data.

- To achieve this, measurements must be made in such a way as to provide statistical predictability." 


\section{Publications on Laboratory QA \& QC}

- ANSI N15.51-2012 "Methods of Nuclear Material Control-Measurement Control Program-Analytical Chemistry Laboratory"

- "Quantifying Uncertainty in Analytical Measurements, ISBN 0-948926-08-2, Eurachem English Publication 1995

- CITAC Guide 1 "International Guide to Quality in Analytical Chemistry--An Aid to Accreditation," ISBN 0948926090 English First Edition 1995.

- *IAEA STR - 368 "International Target Values for Measurement Uncertainties in Safeguarding Nuclear Materials", Vienna, November 2010 (ITVs) 


\section{Analytical Chemistry Laboratory MCP}

- Purpose is to provide reliable measurements for nuclear materials accountability and process control that are fit for purpose

- Has technical and administrative aspects that addresses the basic elements of a MCP that were discussed earlier

- Requires the utilization of a LIMS to facilitate and provide consistency in operation of the laboratory in every aspect of sample handling, personnel qualification, proper procedures, method selection \& qualification, QC for the measurements, reporting, accountability, records, sample management, etc. 


\section{MCP Technical Aspects: Methods}

- A measurement control program provides assurance that data produced from a measurement system are acceptable for meeting established measurement performance requirements.

- For the measurement control program to have any value, it must generate data that represents (or reflects) the quality of measurements performed on process materials.

- Thus, one first selects a measurement method to meet the desired level of performance, and then uses a measurement control program to verify (or detect the lack of) such performance, both initially and on a continuing basis. 


\section{MCP Technical Aspects: Methods cont.}

- A method selected should contains the following characteristics:

- technically sound;

- specific for the property being measured;

- free from interferences (or be correctable for interferences);

- have an acceptable measurement range for the property being measured;

- capable of producing data that will meet established precision and accuracy requirements.

- Target values define the required level of performance of each analytical chemistry laboratory measurement. The IAEA has developed target values for the measurement of nuclear materials

- For individual facilities, the performance-level requirements may exceed or be less than the target values depending on their needs and requirements. These target values provide estimates of the capability that could reasonably and realistically be expected from industrial-type laboratories on a routine basis. 


\section{MCP Technical Aspects: Documentation}

- Document the measurement control program, including descriptions of the statistical tests performed and the minimum acceptable limits

- Measurement and measurement control methods are formally qualified and validated as adequate for their intended use

- Define a standards program to include

- Preparation of control standards

- Determination of standards' values and uncertainties

- Documentation of traceability, storage, and calibration of instruments 


\section{MCP Technical Aspects: Controls}

- Define:

- Limits for measurement control

- Specify the corrective actions and responses to violations of the control limits:

- Responses should provide not only recovery but

- Also either re-measurement of samples or

- Assurance that sample results made just prior to the response condition were acceptable;

- Define conditions for quantifying method performance

- Bias and precision in order to determine measurement uncertainty and to adjust control limits; 


\section{MCP Technical Aspects - Monitoring}

- Collect measurement control data and evaluate it statistically

- Prepare and issue periodic reports on measurement performance

- Review and adjust control limits to reflect the current performance of measurement systems on a routine basis, if corrective action is not indicated

- Monitor and document data from inter-laboratory comparison programs 


\section{MCP Technical Aspects - Monitoring cont.}

- Monitor performance of specifically identified laboratory measurement system components, e.g.,

- Analyst, equipment, standard, etc.

- Require documentation of all performance \& corrective actions

- Train, qualify, and re-qualify analysts, and other personnel associated with measurements, using objective testing methods

- Define a replicate sampling program in order to assure that measurement of the replicate samples is performed in the same manner as measurement of the original (routine) samples 


\section{MCP Administration}

- Define Organization and Management Program to:

- provide for detection and correction of adverse changes;

- maintain the desired level of performance for all measurements conducted in the laboratory;

- quantify the uncertainty associated with each reported measurement;

- quantify the performance of the measurement system.

- Train, qualify, and re-qualify analysts, and other personnel associated with measurements, using objective testing methods

- Review and audit shall consist of a comprehensive examination and evaluation of all aspects of the program. 


\section{MCP Administration continued}

- Documentation is required to ensure a clear understanding of the organization, policies, responsibilities, and procedures, and to enable the review and confirmation of measurement performance (by audit) in a timely manner.

- All procedures shall be controlled and formally reviewed for clarity, consistency, and adequacy before approval. A formal review shall be performed periodically thereafter to ensure that the procedures are still adequate and appropriate.

- Deficiencies and corrective actions

- A facility shall develop a program to promptly detect, correct, and document adverse conditions that affect quality of data.

These adverse conditions are sometimes called deficiencies and include failures, defects, errors, deviations from specific requirements, and other conditions that adversely affect quality. 


\section{Measurement Quality Parameters}

- Accuracy*

- Precision*

- Cost

- Turn around time, and

- Back-up capability limitations

* Most often required by regulators and/or Government Orders. 


\section{Regulations for Measurement Control Vary in USA}

The Environmental Protection Agency:

- is prescriptive in the measurement controls that it requires.

- Establishes "Data Quality Objectives" for laboratory assays

- Specifies methods, MCs and sample handling requirements for the laboratories analyzing samples for their programs

- DOE and Nuclear Regulatory Agency require MCPs that comply with its orders, standards and are in harmony with national and international Standards.

- The contractors have latitude in how they meet requirements. 


\section{Accuracy Definition}

- Accuracy of measurement is closeness of the agreement between the result of a measurement and a reference value of the analyte

- "Accuracy" is a qualitative concept

- The term "precision" should not be used for "accuracy" 


\section{Precision Definitions}

Precision is the closeness of agreement between independent test results obtained under stipulated conditions

- Precision depends only on the distribution of random errors and does not relate to the true value or specified value

- The measure of precision is usually expressed in terms of imprecision and computed as a standard deviation of the test results

- Less precision is reflected by a larger standard deviation

- Quantitative measures of precision depend critically on the stipulated conditions

- Repeatability and reproducibility conditions are particular sets of extreme stipulated conditions 


\section{Uncertainty of Measurement}

- Characterizes the dispersion of the values that could reasonably be attributed to assay

- Defined by standard deviation or confidence interval width

- Comprises many components

- From assumed probability distributions: knowledge based

- From statistical distribution of a series of measurements

- Measurement is the best estimate of the value of the assay and that all components of uncertainty, including those arising from systematic effects, such as components associated with corrections and reference standards contribute to the dispersion 


\title{
Precision Estimates for a Lab Method
}

\author{
SOURCE OF PRECISION RELATIVE STANDARD \\ ESTIMATE \\ DEVIATION
}

Chemist's Value for Method

$\underline{2 \%}$

Monthly QC Report

$4 \%$

Yearly QC Report

$\underline{6 \%}$

- Which is the correct estimate of the method's Precision? 


\section{Chemical Analysis Process With Calibration \& QC Functions}

"Duplicate the Process"

- QC samples should have the same matrix as plant samples

- If not add matrix if required

- Or run a blank through analysis

- Analysis may require sub sampling. Treat QC the same

- Intermediate steps may be required before measurement

- Calibration of method should be done with RM or WRM

- Use appropriate factors

- Calculate \& Report results
Flow Chart of Analysis

\begin{tabular}{|c|c|}
\hline Sample & QC Matrix \\
\hline Sub Sample & Matrix \\
\hline Digestion & Blank \\
\hline Extraction & \\
\hline Derivatization & Spike Sample \\
\hline Separation & \\
\hline Measurement & Calibration RM \\
\hline $\begin{array}{l}\text { Calculation of } \\
\text { Result }\end{array}$ & Factors \\
\hline $\begin{array}{l}\text { Reporting of } \\
\text { Result }\end{array}$ & \\
\hline
\end{tabular}




\section{Techniques \& Parameter Estimated}

Measurement Control Technique Accuracy Precision

1. Bench or check standards Yes Yes

2. Blind standards Yes Yes

3. Split samples No Yes*

4. Replicate measurements No

Yes

5. Inter-laboratory comparisons Yes Yes*

6. Quality control charts No** Yes

7. Spike of known concentration Yes Yes*

* Yes, if several analyses on different samples over time. **No for QC Charts comparing current data with historic data. (Yes, if plotted against a known value) 


\section{Relationships of Bias, Precision \& Uncertainty in a pH Measurement}

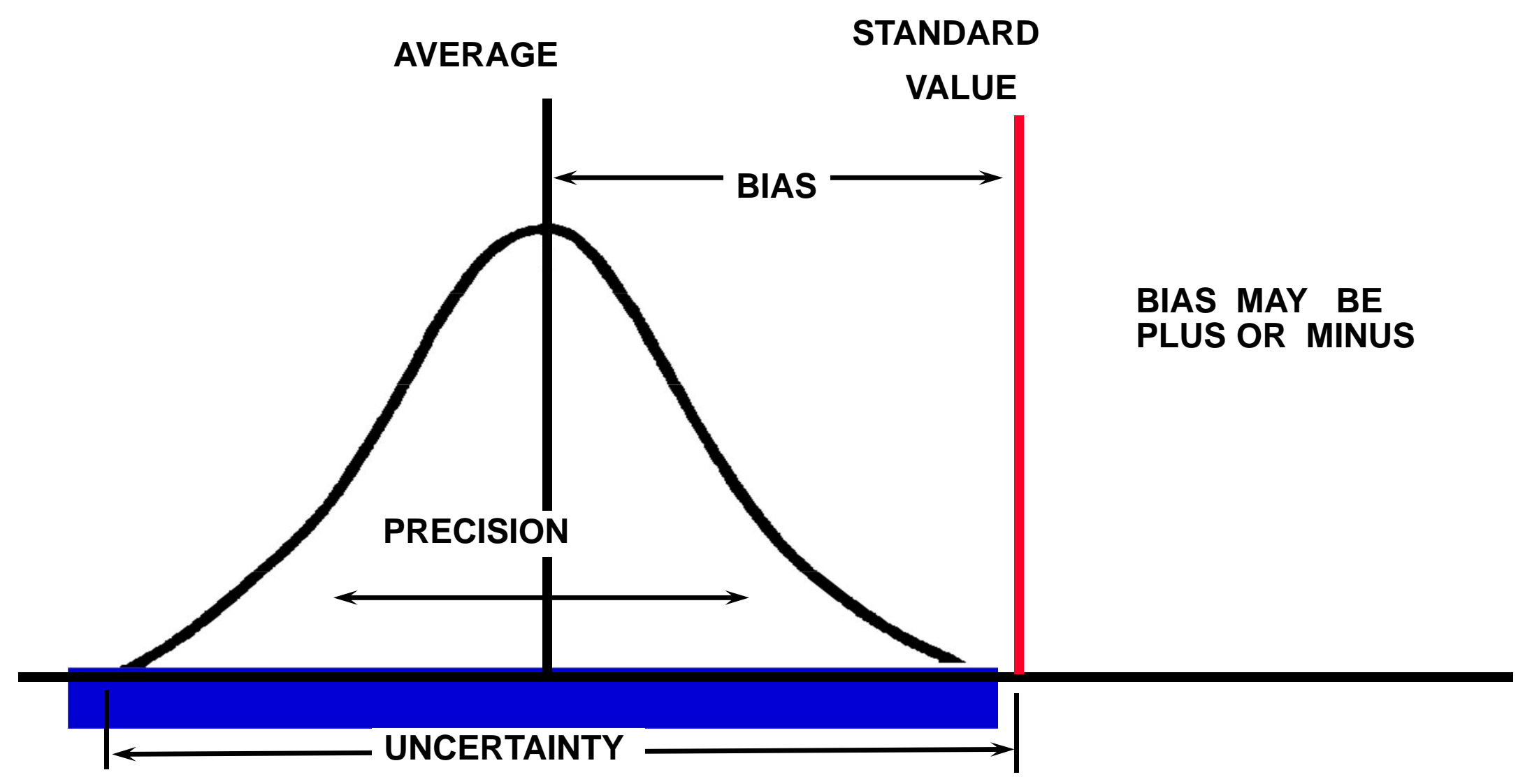




\section{pH Measurement Bias, Precision \& Uncertainty}

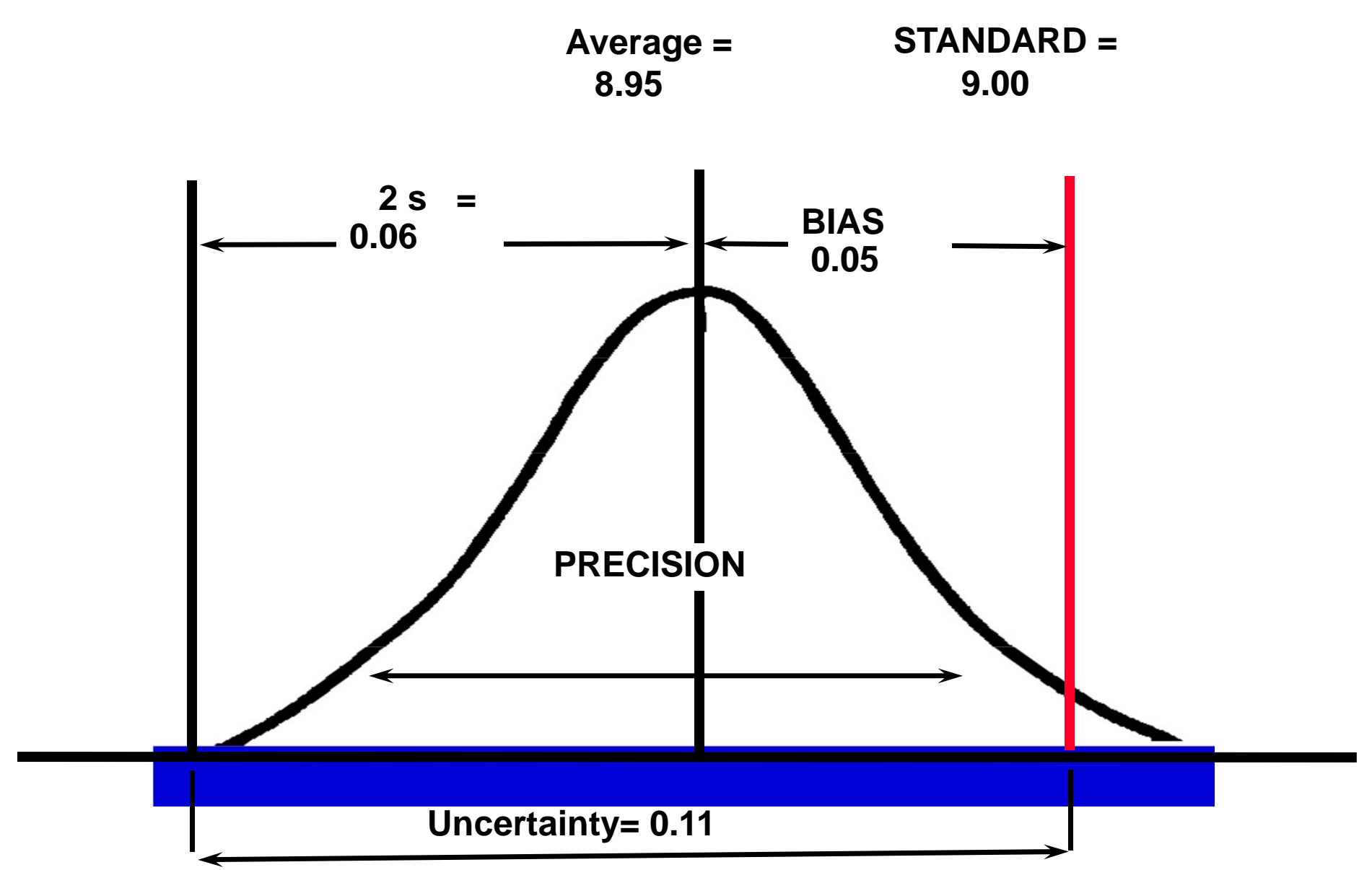

Module 12 - 23 


\section{pH Measurement Uncertainty includes the Uncertainty of the Standard (2s = .02)}

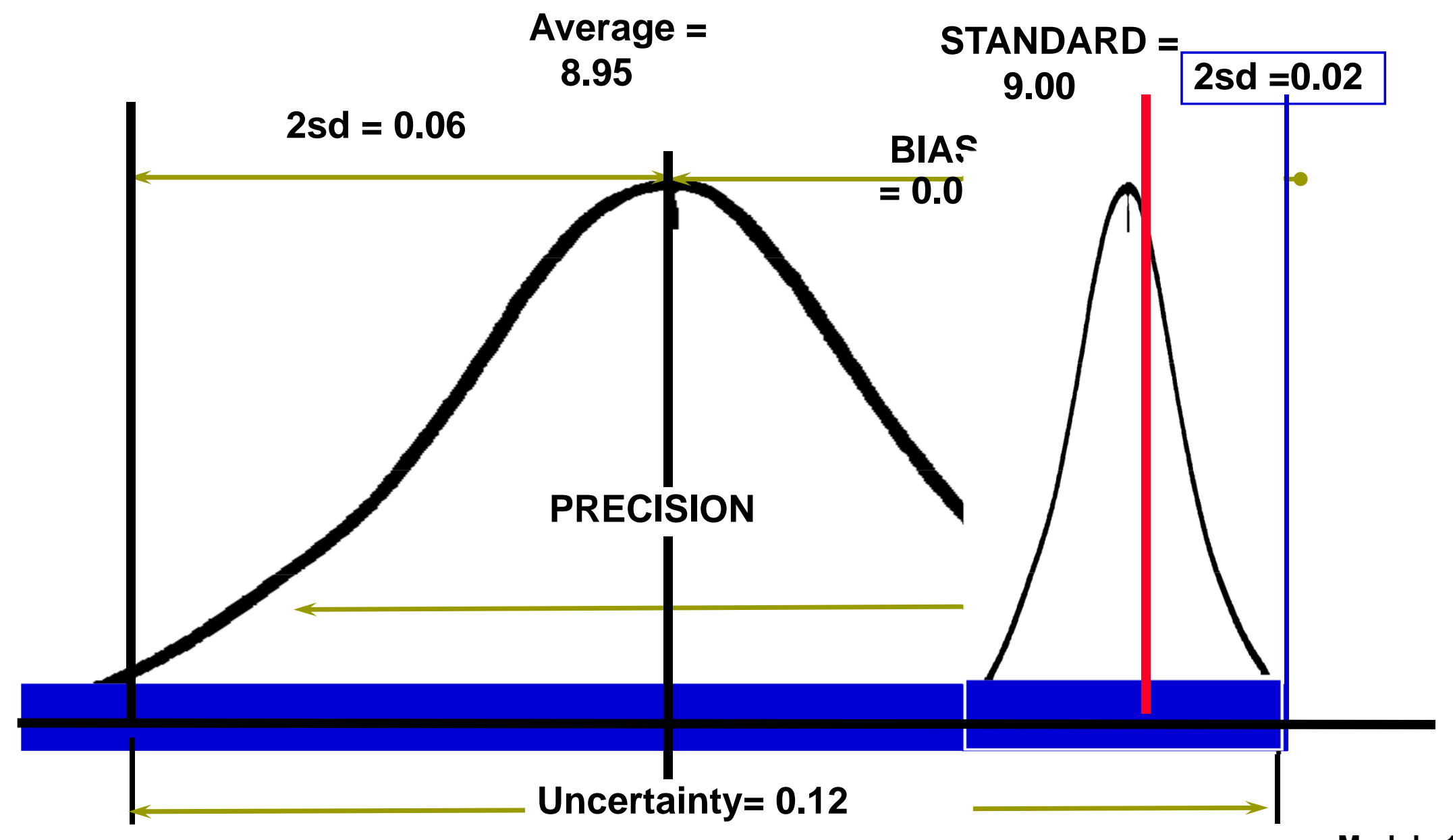




\section{Physical Measurement \& Error models}

- Modeling a Measurement Process

- Controlling a measurement process effectively depends on understanding that process thoroughly

- A thorough understanding, in turn, is gained by determining the significant factors that affect the measurement process and their relationship to the quality of measurement produced

- This relationship usually is expressed in a model

- In general, a measurement process is described by both a physical and a measurement error model

- The GUM method begins with a mathematical formula for the measurement in developing estimates of measurement uncertainty 


\section{Laboratory I nformation Management Systems ( LI MS)}

- Essential in $21^{\text {st }}$ Century to coordinate all the elements of a measurement control program in providing measurements (fit for purpose) for the accountability and control of nuclear materials and the needed statistics for calculating limits of error.

- LIMS have evolved over the years from home grown programs to commercially available systems that can be customized to the specific characteristics of individual laboratories.

- An overview of the information needed for a reliable measurements for a bulk material will be reviewed.

- A LIMS evaluation of D\&G U QC data is presented. 


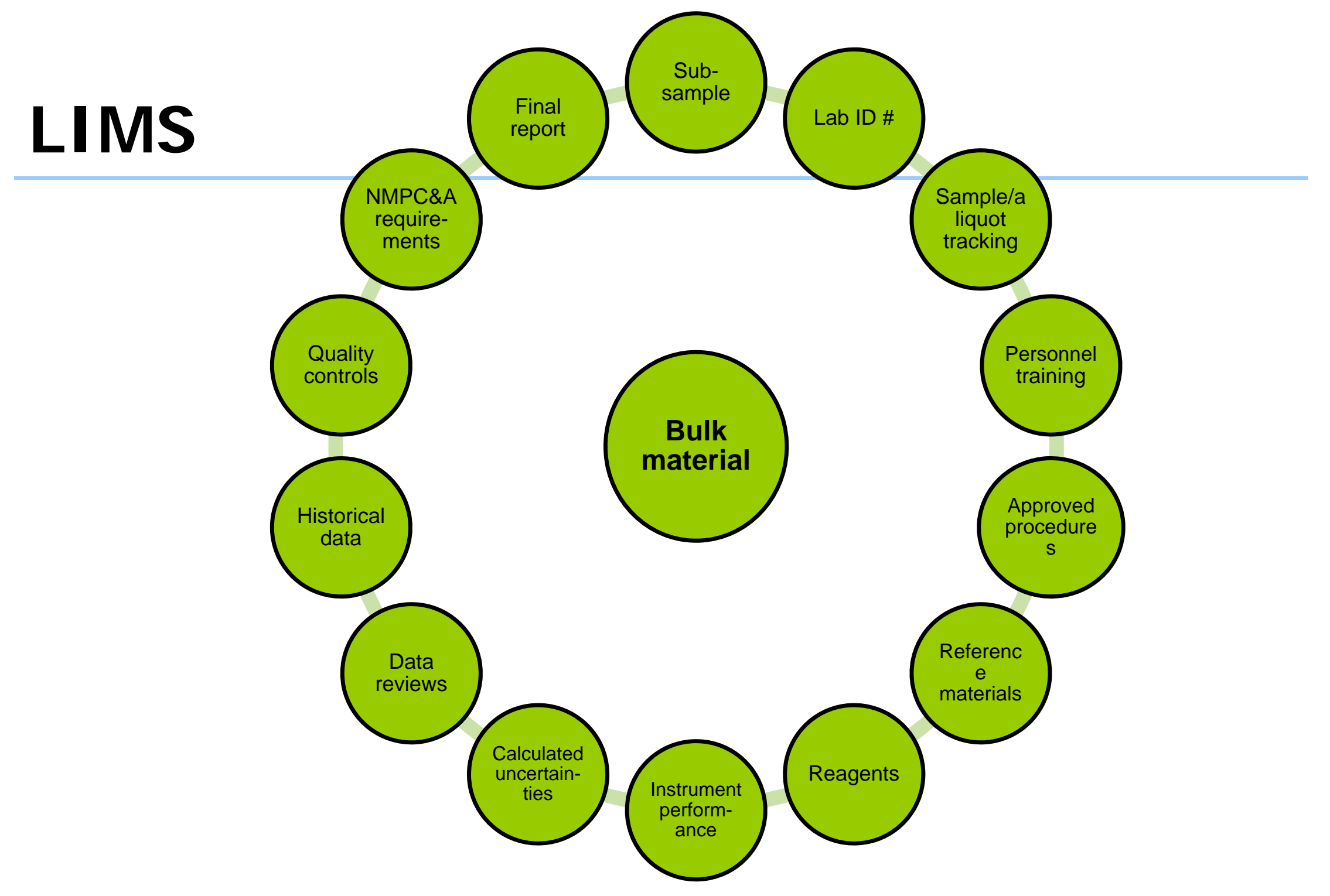

Circle of information associated with the bulk material 


\section{I nformation Associated With Bulk Nuclear Material (Another View)}

- Sub-sample

- Lab ID \#

- Sample/aliquot tracking

- Personnel training

- Approved procedures

- Reference materials and Reagents

- Quality controls and Instrument performance

- Calculated uncertainties

- Data reviews

- Historical data

- NMPC\&A requirements

- Final report 


\section{The LIMS is the interface to all of the laboratory information produced and associated with the bulk material}

- Samples

- Identities and source batch controls (could be for insider protection and security reasons lab personnel do not need the source identity, except for their own created batches and respective analyses)

- Keeps quantity, material type, and other necessary characteristics for proper handling with safety and security

- Tracks locations (lab/room/bin/etc.)

- Tracks lab processing and tracking (splitting, sub-sampling, salvage for recycle, waste disposal, etc.)

- Analyses

- Elemental, isotopic and impurity measurements

- Identification and approval of method, instrument and analyst

- Mean results from multiple aliquots and uncertainties

- Data processing for reporting and historical retention of data

- QA/QC

- Traceability of reference materials used for calibrations and controls (known and blinds)

- Monitoring with control charts for response to anomalous conditions 


\section{MS (continued)}

- There are multiple commercial LIMS packages available for purchase

- Knowing your laboratory processes and what your needs and requirements are is essential when purchasing a LIMS package

- Every analytical lab operates in a unique way

- The LIMS package must be configured for your requirements and your instruments

- The vendor will help with the initial installation but it is up to the lab to configure the details for successful implementation

- For a uranium standards laboratory a LIMS is essential for the defensibility of the measurements you make and the data you produce 
- ORNL is currently considering LIMS vendors for their Nuclear Analytical and Isotopics Laboratories They include:

- STARLIMS ${ }^{\circledR}$

- LABVANTAGE ${ }^{\circledR}$

- LabWare, Inc.

- Thermo Scientific SampleManager LIMS 


\section{MS Provide Measurement Control}

- Use of a computerize information management system allows management to build in control mechanisms for measurement methods

- Limits are set to actually "STOP WORK" when the analysis of the QC sample fails to meet acceptance criteria. Examples will be discussed

- LIMS also can provide QC charts for quick evaluation of the measurement process over specified time intervals

- QC data can be used to evaluate the many variables in the measurement process \& for requalification 
Types of Cases in LMS \& how they are handled

- LIMS compares the result with the reference value. The method status is flagged with 'Active' or 'Locked'

- If the result is within or equal to the \pm 2 standard deviation (sd) limits, it is a Case 1 result, the method is in control and active

- If the result is greater than $2 \mathrm{sd}$ and less than equal to 3 sd limits, it is a Case 2 result. It indicates that the method may be going out of control

- If the previous QCS was a Case 1, samples can be analyzed and data entered into the LIMS system 
Types of Cases in UMS \& how they are handled (continued)

- If two consecutive QCs analyzed on the method are designated Case 2, LIMS locks-out the method. No one can use the method until a Case 1 result is entered

- If the result is between 3 and 6 sd limits, it is a Case 3 "Out-of-Control". The method is "Locked"

- Results greater than 6 sd limits is a Case 4 "Outlier" and the method is "Locked" 


\section{Special Events}

- Run Event

- Any pattern of eight (8) consecutive points that lie on the same side of the control chart center line or no-action band if implemented (runs rule).

- No-action bands: Statistically-denived bands used to expand the target value of an analytical method control chart so that measurement control results falling within these bands do not count towards a runs rule violation

- Two out of three event

- Two out of three consecutive points are outside the warning limits

- Evaluate for non-random data patterns and trends 


\section{Statistics Used}

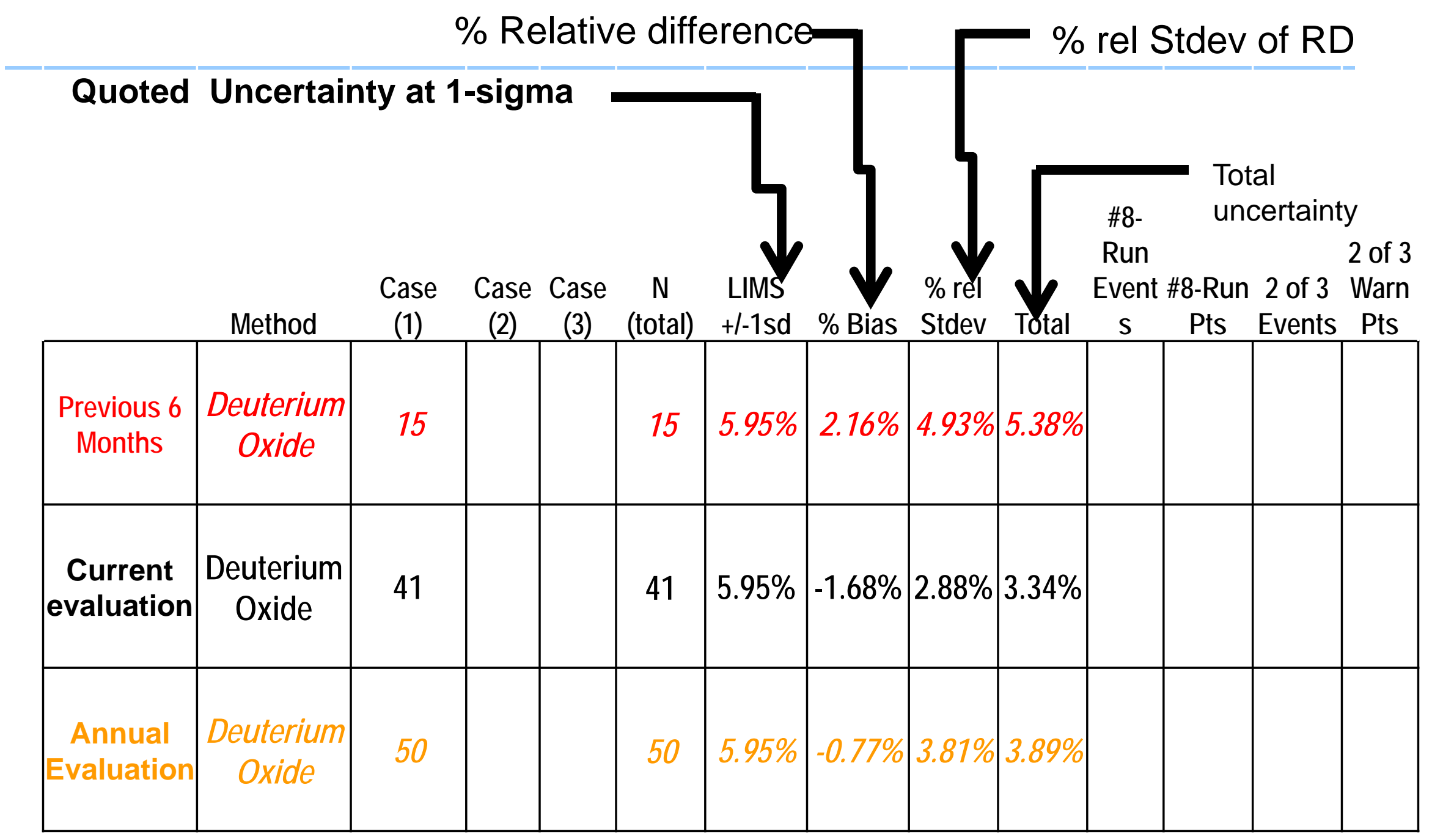

Module 10 - 36 


\section{Summarized Information}

- 16 Available Accountability methods

- 4 Inactive methods

- 12 Active Methods 


\section{Summary of the MC\&A QC Data (12 -16 methods)}

6 Months

953 data points

894

42

17 case 3's

Monthly Average -160
14 Months

- 2859 data points

2686

case 1's

125

case 2's

48

case 3's

- Monthly Average 204 


\section{Chart for Cases (6 and 14 months)}

Case 1 Case $2 \square$ Case 3

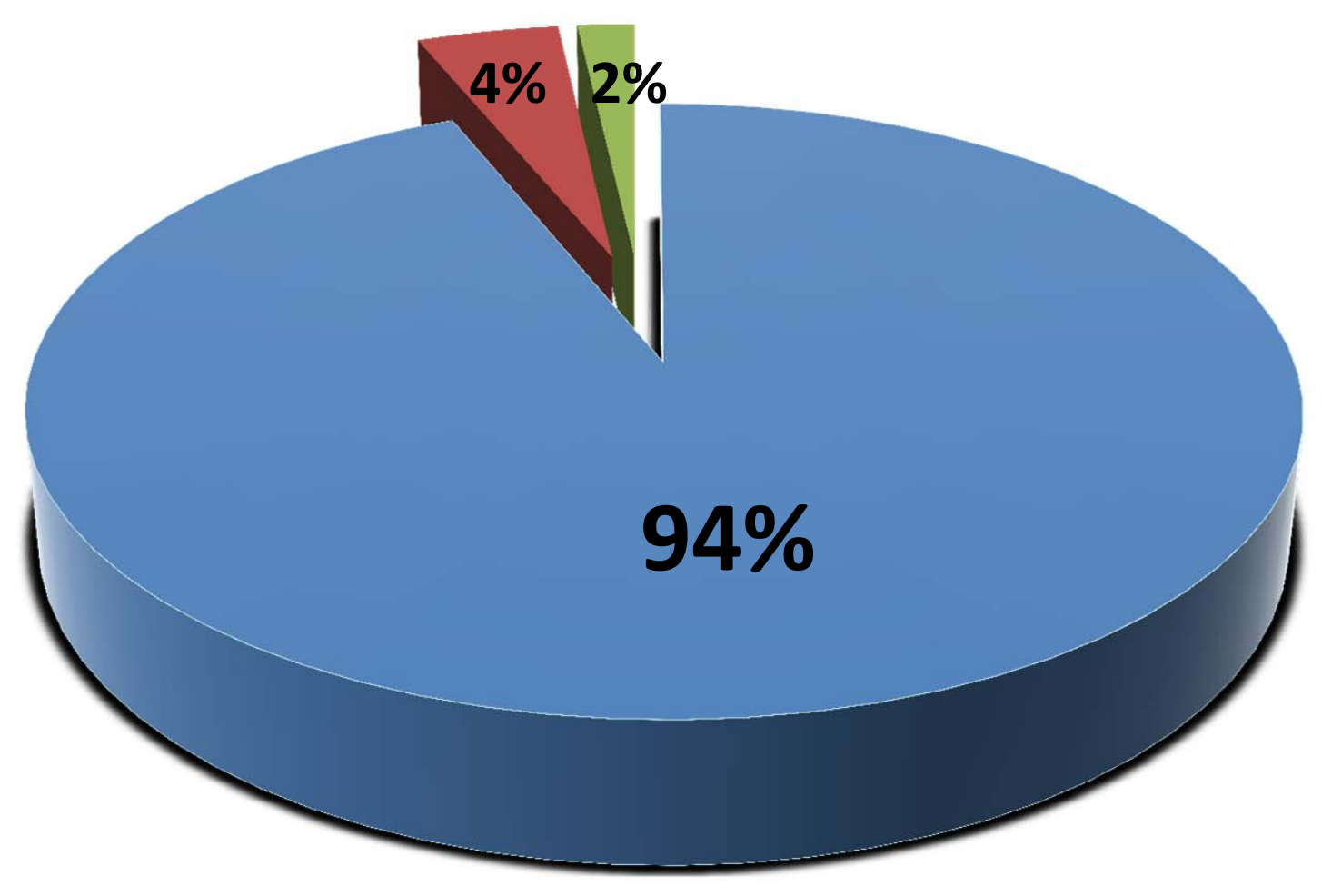

Module 10 - 39 


\section{Davies \& Gray U Method Uncertainty}

- Current method uncertainty

- $0.29 \%$ at 1-sigma

- International Target Value (ITV) $0.1 \%$ for random and systematic. 


\section{Customer Support}

- Customer

- 5 production processes

- Active participant in the NBL SME program

- Internal lab support

- MC\&A for accountable measurements 


\section{Statistics for Uranium by Davies and Gray}

\begin{tabular}{|c|c|c|c|c|c|c|c|c|c|c|c|c|}
\hline Method & $\begin{array}{c}\text { Case } \\
\text { (1) }\end{array}$ & $\begin{array}{c}\text { Case } \\
(2)\end{array}$ & $\begin{array}{c}\text { Case } \\
\text { (3) }\end{array}$ & $\begin{array}{c}\mathbf{N} \\
\text { (total) }\end{array}$ & $\begin{array}{c}\text { UMS } \\
++-15 d \\
\end{array}$ & Bias & Stdev & Total & $\begin{array}{l}\text { \#8-Run } \\
\text { Events }\end{array}$ & $\begin{array}{l}\text { \#8- } \\
\text { Run } \\
\text { Pts }\end{array}$ & $\begin{array}{c}2 \text { of } 3 \\
\text { Events }\end{array}$ & $\begin{array}{c}2 \text { of } 3 \\
\text { Warn } \\
\text { Pts }\end{array}$ \\
\hline $\begin{array}{c}\text { Davies- } \\
\text { Gray } \\
\text { (previous } \\
\text { data) }\end{array}$ & 114 & 4 & 1 & 119 & $0.29 \%$ & $-0.02 \%$ & | $0.33 \%$ & $0.33 \%$ & 4 & 40 & 0 & 0 \\
\hline $\begin{array}{c}\text { Davies- } \\
\text { Gray } \\
\text { (6 months) }\end{array}$ & 61 & 5 & 1 & 67 & $0.29 \%$ & $0.13 \%$ & $0.33 \%$ & $0.35 \%$ & $\mathbf{0}$ & $\mathbf{0}$ & 2 & 4 \\
\hline $\begin{array}{c}\text { Davies- } \\
\text { Gray } \\
(14 \\
\text { months) }\end{array}$ & 198 & 15 & 3 & 216 & $0.29 \%$ & $0.09 \%$ & $0.36 \%$ & $0.37 \%$ & 3 & 47 & 4 & 8 \\
\hline
\end{tabular}

Module 10 - 42 


\section{Control Chart for $U$ by Davies and Gray (6 months)}

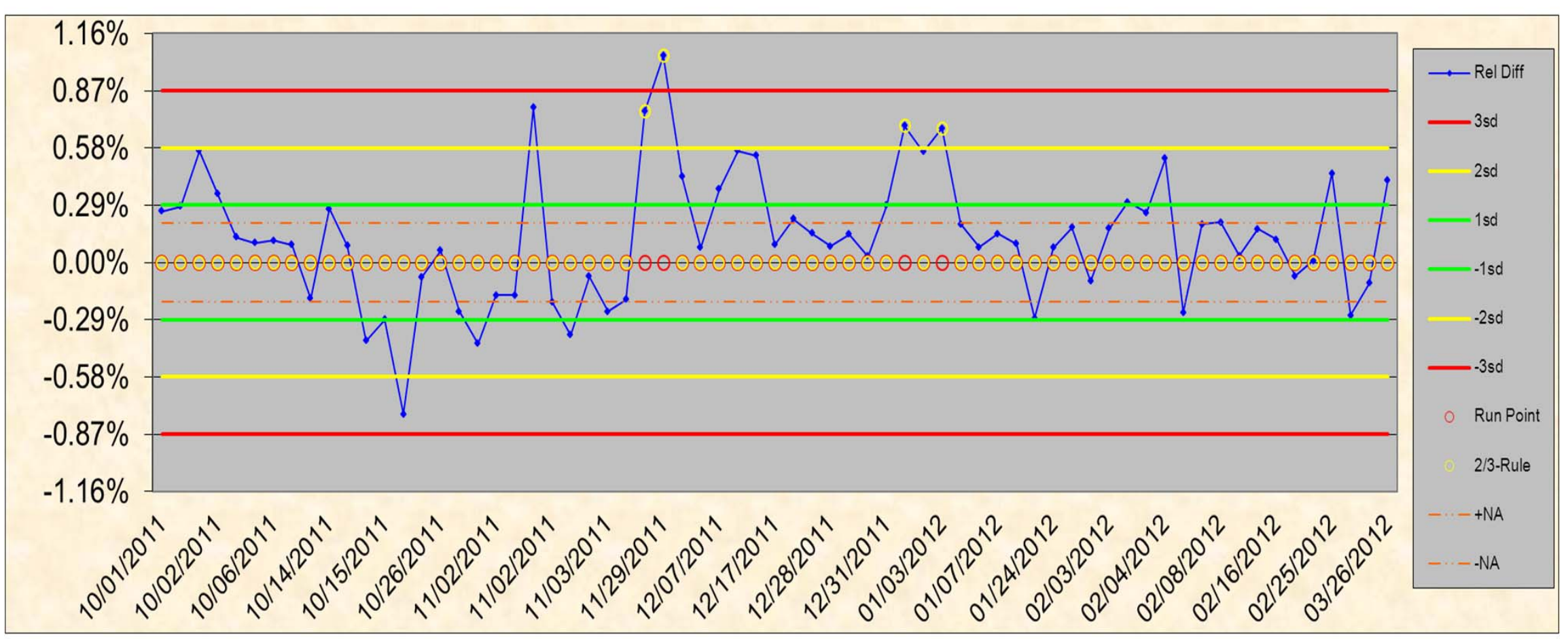




\section{Real Time Estimate of Measurement Uncertainty for an Analytical Method}

- Analysis of the QC data provides an estimate of the measurement process variation that should include all the variables of the process:

- Environmental: Temperature, Humidity \& Barometric Pressure

- Operators/Analysts

- Instruments \& sample preparation

- Standards and reagents

- Other (vibration, time of day, etc.)

- Uncertainty of the standard(s)(goal $<1 / 4$ of method)

- Other major sources (drift?)

- Combine by RSS for standard estimate of uncertainty

- Multiply by appropriate $\mathrm{K}$ value (2 for $95 \% \mathrm{Cl}$ ) 


\section{Precision Estimates for a Lab Method}

- Chemist estimated repeatability of method on one day

- Monthly QC Report estimated intermediate reproducibility over a set of operators, standards, operating conditions, etc. over a month

- Year's QCs captured all variations in operators, standards, operating conditions, etc. It best estimates the total variation that could be expected in Assays. Reproducibility over a year

The correct estimate of the method's PRECISION depends on the assumptions!

$\begin{array}{cc}\text { SOURCE OF } & \text { RELATIVE } \\ \text { PRECISION ESTIMATE } & \text { STANDARD } \\ & \text { DEVIATION } \\ \text { Chemist's Value for } & 2 \% \\ \text { Method } & \\ \text { Monthly QC Report } & 4 \% \\ \text { Yearly QC Report } & 6 \%\end{array}$




\section{Summary}

- Understand laboratory measurements must have uncertainty estimates for accountability of NM

- Reviewed MCP technical \& administrative components of ANSI N15.51

- Discussed laboratory techniques for estimating error

- Discussed uses of measurement control data

- Introduced Laboratory Information Management Systems (LIMS) and reviewed LIMS MCP output for D\&G U method 


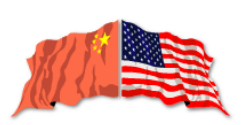

Module 12

\section{Analytical Chemistry Laboratory Measurement Control Programs}

\section{Objectives}

- Review the concept of measurement uncertainty and ways to estimate it

- Review the MCP technical \& administrative components of ANSI N15.51

- Combine \& evaluate uncertainty estimates

- Discuss uses of measurement control data

- Discuss Laboratory Information Management Systems (LIMS)

- Review LIMS MCP output for a D\&G U method

- Briefly consider the use of Kragten Spreadsheets

\section{Uncertainty of Measurement}

- Characterizes the dispersion of the values that could reasonably be attributed to assay

- Defined by standard deviation or confidence interval width

- Comprises many components

- From assumed probability distributions: knowledge based - From statistical distribution of a series of measurements

- Measurement is the best estimate of the value of the assay and that all components of uncertainty, including those arising from systematic effects, such as components associated with corrections and reference standards contribute to the dispersion

\section{Precision Estimates for a Lab Method}

$\begin{array}{cc}\begin{array}{c}\text { SOURCE OF PRECISION } \\ \text { ESTIMATE }\end{array} & \begin{array}{c}\text { RELATIVE STANDARD } \\ \text { DEVIATION }\end{array} \\ \text { Chemist's Value for Method } & \underline{\underline{2 \%}} \\ \text { Monthly QC Report } & \underline{4 \%} \\ \text { Yearly QC Report } & \underline{6 \%}\end{array}$

- Which is the correct estimate of the method's Precision?

\section{Physical Measurement \& Error models}

- Modeling a Measurement Process

- Controlling a measurement process effectively depends on understanding that process thoroughly

- A thorough understanding, in turn, is gained by determining the significant factors that affect the measurement process and their relationship to the quality of measurement produced

- This relationship usually is expressed in a model

- In general, a measurement process is described by both a physical and a measurement error model

- The GUM method begins with a mathematical formula for the measurement in developing estimates of measurement uncertainty

- ANSI N15.51 also stresses importance of both models 


\section{MCP Technical Aspects: Controls}

- Define:

- Measurement control error limits

- Specify the corrective actions and responses to violations of the control limits:

- Responses should provide not only recovery but

- Also either re-measurement of samples or

- Assurance that sample results made just prior to the response condition were acceptable;

- Define conditions for quantifying method performance

- Random and systematic error (Bias \& precision) in order to

- Determine measurement uncertainty

- Adjust control limits and

- Qualify personnel to use the method

Module $12 \cdot 7$

\section{MCP Administration}

- Define Organization and Management Program to:

- Provide for detection and correction of adverse changes;

- Maintain the desired level of performance for all measurements conducted in the laboratory;

- Quantify the uncertainty associated with each reported measurement;

- quantify the performance of the measurement system.

- Train, qualify, and re-qualify analysts, and other personnel associated with measurements, using objective testing methods

- Review and audit shall consist of a comprehensive examination and evaluation of all aspects of the program.

\section{Precision Estimates for a Lab Method}

$$
\begin{array}{cc}
\text { SOURCE OF PRECISION } & \text { RELATIVE STANDARD } \\
\text { ESTIMATE } & \text { DEVIATION } \\
\text { Chemist's RSD for Method } & 2 \% \\
\text { Monthly QC Report } & 4 \% \\
\text { Yearly QC Report } & 6 \%
\end{array}
$$

- Chemist estimated repeatability of method on one day

- Monthly QC Report estimated intermediate reproducibility over a one set of operators, standards, operating conditions, etc. over a month

- Year's QCs captured all variations in operators, standards, operating conditions, etc. It best estimates the total variation that could be expected in Assays. Reproducibility over a year

- The correct estimate of the method's PRECISION depends on the assumptions!

\section{Analytical Chemistry Module Exercise is to use the RSS method to combine Uncertainties}

Alpha Counting method
uses 6 measurements
- This method requires 3
pipettes be used to make 2
dilutions before dispensing
the final dilution onto a
counting dish.
- There are 2 counters
available to make the final
measurement of alpha
concentration.
- Complete the 3 parts of the
exercise and answer the 4
questions.

Instruments with associated tolerances and/or Uncertainties

\begin{tabular}{|c|c|c|c|c|}
\hline Source & Uncertainty & Cal Uncertainty & U Variance & Cal Variance \\
\hline Pipette 1 & 1 & 0.15 & 1 & 0.0225 \\
\hline Flask 1 & 0.16 & 0.16 & 0.0256 & 0.0256 \\
\hline Pipette 2 & 1 & 0.15 & 1 & 0.0225 \\
\hline Flask 2 & 0.16 & 0.16 & 0.0256 & 0.0256 \\
\hline Pipette 3 & 1 & 0.15 & 1 & 0.0225 \\
\hline Counter 1 & 1.2 & 1.2 & 1.44 & 1.44 \\
\hline SUM $=$ & & & 4.49 & 1.56 \\
\hline \multicolumn{3}{|c|}{ Square root= } & 2.12 & 1.25 \\
\hline & ert? & 1.-Ga & & $-41.1 \%$ \\
\hline
\end{tabular}

\begin{tabular}{|c|c|c|}
\hline Instrument & Tolerance & Uncertainty \\
\hline Pipette(s) & $1.00 \%$ & 0.15 \\
\hline $\begin{array}{c}100 \text { ml Flask } \\
\text { Class A }\end{array}$ & $0.08 \%$ & $0.08 \%$ \\
\hline $\begin{array}{c}100 \text { ml Flask } \\
\text { Class B }\end{array}$ & $0.16 \%$ & $0.16 \%$ \\
\hline Counter 1 & $1.20 \%$ & $1.20 \%$ \\
\hline Counter 2 & $0.80 \%$ & $0.80 \%$ \\
\hline
\end{tabular}

RSS Exercise 1 to compare Tolerance and Calibration Uncertainties - Answers

- The uncertainty of an alpha counting method can be reduced by $41.1 \%(2.14 \%$ to $1.28 \%)$ if the calibrated volumes of pipettes are used instead of the nominal values. Then the uncertainties would be used instead of tolerances
RSS Exercise to compare Tolerance and Calibration Uncertainties - Answers -2

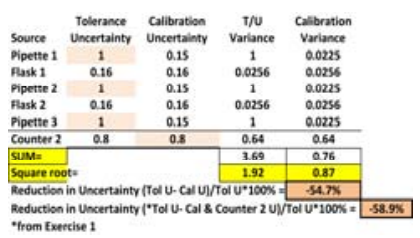

- The uncertainty of an alpha counting method was further reduced by $54.7 \%$ when the calibrated volumes of pipettes are used and a better counter.

- The total uncertainty of an alpha counting method was reduced by $58.9 \%$ by using smaller uncertainties.

Module 12 - 12 


\section{RSS Exercise3 to compare Tolerance and} Calibration Uncertainties - Answers

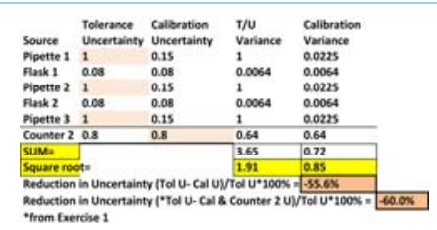

- Using the Class A flask uncertainty only reduced the total uncertainty by $\mathbf{5 5 . 6 \%}$, which is only0.1\% lower than Class B.

- The total uncertainty of an alpha counting method was reduced by $60 \% \%$ by using Calibrated pipettes \& counter 2 as compared to the first T/U using counter 1.

\section{Information Associated With Bulk Nuclear Material}

- Sub-sample

- Lab ID \#

- Sample/aliquot tracking

- Personnel training

- Approved procedures

- Reference materials and Reagents

- Quality controls and Instrument performance

- Calculated uncertainties

- Data reviews

- Historical data

- NMPC\&A requirements

- Final report

\section{Laboratory I nformation Management Systems (LI MS)}

- Essential in $21^{\text {st }}$ Century to coordinate all the elements of a measurement control program in providing measurements (fit for purpose) for the accountability and control of nuclear materials and the needed statistics for calculating limits of error.

- LIMS have evolved over the years from home grown programs to commercially available systems that can be customized to the specific characteristics of individual laboratories.

- An overview of the information needed for a reliable measurements for a bulk material will be reviewed.

- A LIMS evaluation of D\&G U QC data is presented.

\author{
The LI MS is the interface to all of the \\ laboratory information produced and \\ associated with the bulk material \\ - Samples \\ - Identities and source batch controls (could be for insider protection and \\ security reasons lab personnel do not need the source identity, except \\ for their own created batches and respective analyses) \\ - Keeps quantity, material type, and other necessary characteristics for \\ proper handling with safety and security \\ - Tracks locations (lab/room/bin/etc.) \\ Tracks lab processing and tracking (splitting, sub-sampling, salvage for \\ recycle, waste disposal, etc.) \\ - Analyses \\ - Elemental, isotopic and impurity measurements \\ - Identification and approval of method, instrument and analyst \\ - Mean results from multiple aliquots and uncertainties \\ Data processing for reporting and historical retention of data \\ - QA/QC \\ - Traceability of reference materials used for calibrations and controls \\ (known and blinds) \\ - Monitoring with control charts for response to anomalous conditions
} Module 12 - 16

\section{MS (continued)}

- There are multiple commercial LIMS packages available for purchase

- Knowing your laboratory processes and what your needs and requirements are is essential when purchasing a LIMS package

- Every analytical lab operates in a unique way

- The LIMS package must be configured for your requirements and your instruments

- The vendor will help with the initial installation but it is up to the lab to configure the details for successful implementation

- For a uranium standards laboratory a LIMS is essential for the defensibility of the measurements you make and the data you produce

\section{MS (continued)}

- ORNL is currently considering LIMS vendors for their Nuclear Analytical and Isotopics Laboratories They include:

- STARLIMS ${ }^{\circledR}$

- LABVANTAGE ${ }^{\circledR}$

- LabWare, Inc.

- Thermo Scientific SampleManager LIMS 


\section{MS Provide the Control for Analyses}

- Use of a computerize information management system allows management to build in control mechanisms for measurement methods

- Limits are set to actually "STOP WORK" when the analysis of the QC sample fails to meet acceptance criteria. Examples will be discussed

- LIMS also can provide QC charts for quick evaluation of the measurement process over specified time intervals

- QC data can be used to evaluate many variables in a measurement process \& for requalification

\section{Types of Cases in LIMS \& how they are handled}

- LIMS compares the result with the reference value.

- Depending on the evaluation the method status is flagged as 'Active' or 'Locked'

- If the result is within or equal to the \pm 2 standard deviation (sd) limits, it is a Case 1 result, the method is in control and 'active'

- If the result is greater than $2 \mathrm{sd}$ and less than equal to 3 sd limits, it is a Case 2 result. It indicates that the method may be going out of control

- If the previous QCS was a Case 1, samples can be analyzed and data entered into the LIMS system

\section{Special Events}

- Run Event

- Any pattern of eight (8) consecutive points that lie on the same side of the control chart center line or no-action band if implemented (runs rule).

- No-action bands: Statistically-derived bands used to expand the target value of an analytical method control chart so that measurement control results falling within these bands do not count towards a runs rule violation

- Two out of three event

- Two out of three consecutive points are outside the warning limits

- Evaluate for non-random data patterns and trends

Module $12 \cdot 22$

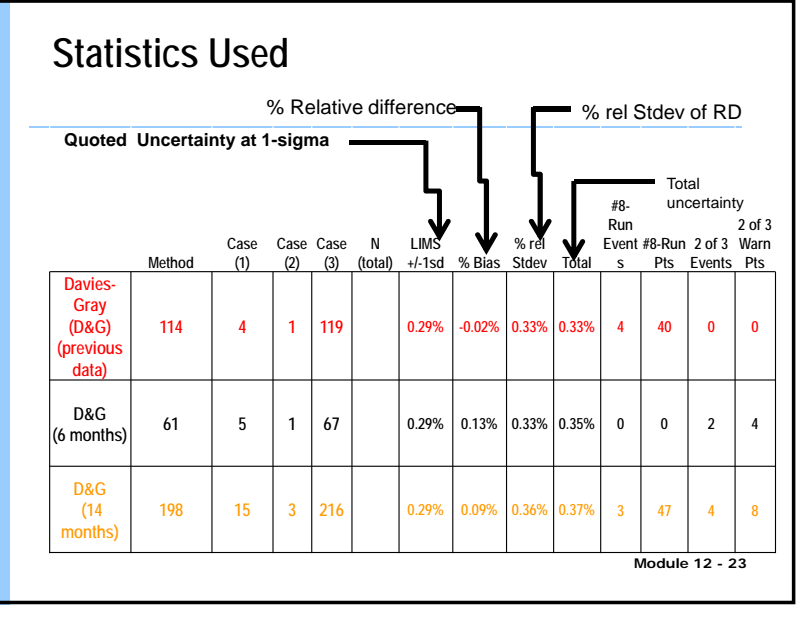

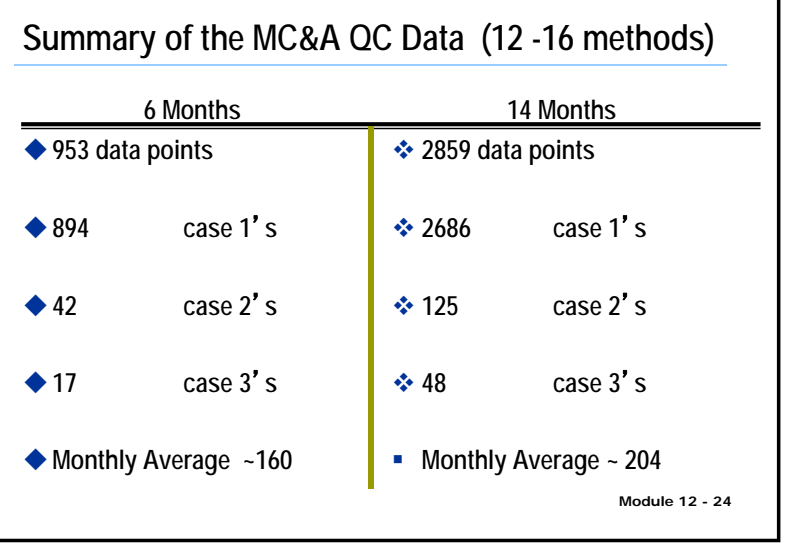


Chart for Cases (6 and 14 months)

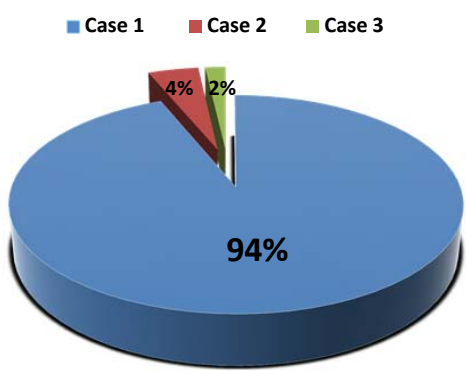

Module 12 - 25

\section{Davies \& Gray U Method Uncertainty}

- Current method uncertainty

- $0.29 \%$ at 1 -sigma

- International Target Value (ITV) $0.1 \%$ for random and systematic.

\section{Customer Support}

\section{- Customer}

\section{- 5 production processes}

- Active participant in the NBL SME program

- Internal lab support

- MC\&A for accountable measurements
Control Chart for U by Davies and Gray (6 months)

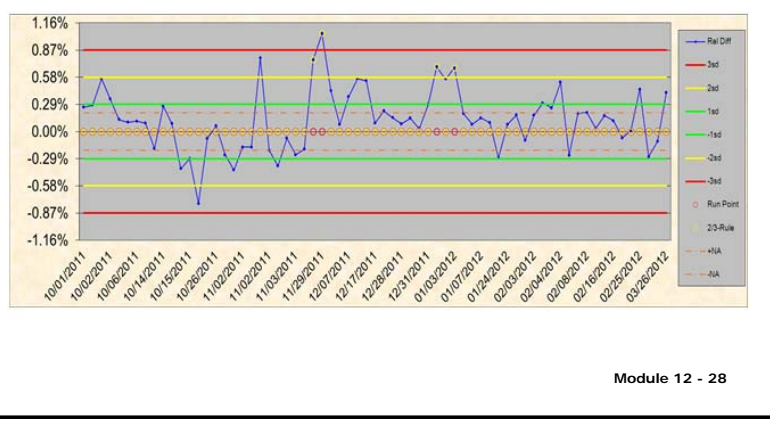

\section{Real Time Estimate of Measurement Uncertainty for an Analytical Method}

- Analysis of the QC data provides an estimate of the measurement process variation that should include all the variables of the process:

- Environmental: Temperature, Humidity \& Barometric Pressure

- Operators/Analysts

- Instruments \& sample preparation

- Standards and reagents

- Other (vibration, time of day, time of year, etc.)

- Uncertainty of the standard(s)(goal $<1 / 4$ of method)

- Other major sources (drift?)

- Combine by RSS for standard estimate of uncertainty

- Multiply by appropriate $\mathrm{K}$ value ( 2 for $95 \% \mathrm{CI}$ )

\section{Measurement Uncertainty Estimates can be analyzed by Kragten Spreadsheets}

- A refinement of the exercise done earlier in the module involves the 8 steps in the GUM method

- J. Kragten uses a spreadsheet to manipulate measurement formula elements' uncertainties to determine the contribution each makes to the combined uncertainty estimate of a measurement.

- NIST scientists have refined the method that is described in the publications below:

$$
\begin{aligned}
& \text { Kragten, J., "Calculating standard deviations and } \\
& \text { confidence intervals with a universally applicable } \\
& \text { spreadsheet technique", Analyst, 119, 1994, 2161-2165. } \\
& \text { Vetter, T.W. "Quantifying measurement uncertainty in } \\
& \text { analytical chemistry - a simplified approach", } \\
& \text { Proceedings of the Measurement Science Conference, } \\
& \text { Paper V-B, January 2001, Anaheim CA; also see } \\
& \text { http://wwww. p2pays.org/ref/18/17628.pdf } \\
& \text { Module 12 - 30 }
\end{aligned}
$$




\section{Uncertainty Components of the Z Factor} used to convert water weight to Volume

- A thermometer reading $1^{\circ} \mathrm{C}$ with $\mathrm{a}+l-1^{\circ}$ Uncertainty was used in this calibration work.

- The graph below shows the large uncertainty of the water temperature contributes $\mathbf{9 8 \%}$.

- Lesson to Learn: Make an uncertainty budget \& calculate the total uncertainty and contribution of each component

- Then reduce the contribution of the largest source.
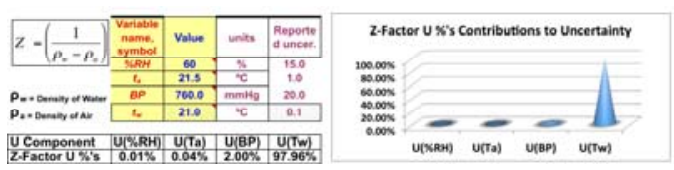

Module 12 - 31

\section{Uncertainty Components of the Z Factor used to convert water weight to Volume}

- The Kragten Spread Sheet can be used to calculate the $\%$ contribution of each measurement used in the calibration of a Pipette.

- The graph below shows the large uncertainty of the barometric pressure contributes $66.6 \%$.

- A thermometer reading $0.1^{\circ} \mathrm{C}$ with a $+l-0.1^{\circ}$ Uncertainty was used in the pipette calibration.

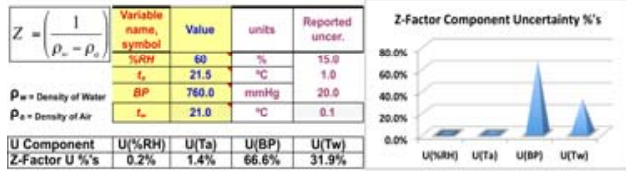

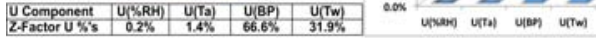

\section{Summary}

- Reviewed the concept of measurement uncertainty and ways to estimate it

- Reviewed MCP technical \& administrative components of ANSI N15.51

- Combined \& evaluated uncertainty estimates

- Discussed uses of measurement control data

- Discussed Laboratory Information Management Systems (LIMS)

- Reviewed LIMS MCP output for a D\&G U method

- Briefly considered the use of Kragten Spreadsheets 


\section{Analytical Chemistry Module Exercise 1}

\section{Session Objectives:}

After completing the exercise the participants will be able to do the following:

1. Combine measurement uncertainties using the root sum square method.

2. Compare the uncertainties of instrument tolerances to the uncertainties of calibrated volumetric equipment.

3. Determine which uncertainty source will have the largest impact on reducing the combined measurement uncertainty estimate.

\section{Estimated Time:}

0.25 hours completing exercise

0.50 hours in large group discussion

0.75 hours total

\section{Materials need:}

1. Exercise Work Sheets for each participant

2. Pen or pencil

3. Scientific Calculators or smart phone that can calculate square roots

\section{Overview:}

An alpha counting method requires two dilutions of a sample before aliquots of the final dilution are dispensed onto counting plates and dried before counting. Two counters are available for the final measurement of the sample dilutions.

Pipettes are periodically sent to the calibration laboratory for tolerance testing. The tolerances are $+/-1 \%$ (conservative estimate of the random and systematic errors). The uncertainty tolerance for the random error is $0.15 \%$. This is the uncertainty value that is used, if the calibrated volumes delivered by specific pipette is used. Class B $100 \mathrm{ml}$ volumetric flasks are used for the dilutions. The Class A and Class B tolerances for $100 \mathrm{ml}$ flasks are $0.08 \%$ and $0.16 \%$ respectively (per ASTM E694 for Volumetric Ware). Each counter has its own uncertainty estimate. Counter 1 has an uncertainty of $+/-1.2 \%$, while Counter 2 's uncertainty is $0.8 \%$.

\section{Instructions:}

The Root Sum Square method is used for combining the uncertainties of several measurements.

1. Complete the calculations required to combine the two sets of uncertainties and compare them in Exercise 1 template below. 
a. Convert the uncertainties to variances by squaring the values in the first and second columns and recording them in the third and forth columns accordingly.

b. Sum the variances in each of these columns and record them in SUM row.

c. Take the square root of the SUM to obtain the combined uncertainty estimates in the spaces below the sum boxes.

d. Determine the magnitude of the reduction of the combined uncertainty by subtracting the Calibrated (Cal) combined uncertainty from the Tolerance (Tol/V) Combined Uncertainty, and then divide by the Tol/V combined uncertainty and multiply by 100 to convert this to a percentage. Record this in the lower right block.

2. Complete the calculations required to combine the two sets of uncertainties and compare them

\begin{tabular}{|l|c|c|c|c|}
\hline Source & $\begin{array}{c}\text { Tolerance } \\
\text { Uncertainty }\end{array}$ & $\begin{array}{c}\text { Cal } \\
\text { Uncertainty }\end{array}$ & $\begin{array}{c}\text { Tol/U } \\
\text { Variance }\end{array}$ & $\begin{array}{c}\text { Cal } \\
\text { Variance }\end{array}$ \\
\hline Pipette 1 & 1 & 0.15 & & \\
\hline Flask 1 & 0.16 & 0.16 & & \\
\hline Pipette 2 & 1 & 0.15 & & \\
\hline Flask 2 & 0.16 & 0.16 & & \\
\hline Pipette 3 & 1 & 0.15 & & \\
\hline Counter 1 & 1.2 & 1.2 & & \\
\hline SUM= & & & & \\
\hline $\begin{array}{l}\text { Square root of SUM= Combined } \\
\text { Uncertainty }\end{array}$ & & & & \\
\hline $\begin{array}{l}\text { Reduction in Uncertainty } \\
\text { (Tol U- Cal U)/Tol U*100\% }\end{array}$ & & & & \\
\hline
\end{tabular}

Exercise 1. Determine the percentage reduction in the combined uncertainty estimate by using the calibrated volumes of the pipettes, instead of the nominal values $\&$ tolerances.

in Exercise 2 template below that use counter 2.

a. Convert the uncertainties to variances by squaring the values in the first and second columns and recording them in the third and forth columns accordingly.

b. Sum the variances in each of these columns and record them in SUM row.

c. Take the square root of the SUM to obtain the combined uncertainty estimates in the spaces below the sum boxes.

d. Determine the magnitude of the reduction of the combined uncertainty by subtracting the Calibrated (Cal) combined uncertainty from the Tolerance (Tol/V) Combined Uncertainty, then divide by the Tol/V combined uncertainty and multiply by 100 to convert this to a percentage. Record this in the next lower right block. 
e. Determine the percentage reduction between the $\mathrm{T} / \mathrm{V}$ combined uncertainty from Exercise 1 above and the Cal combined uncertainty-using counter 2 and record the $\%$ Uncertainty reduction in the final right block.

\begin{tabular}{|l|c|c|c|c|}
\hline Source & $\begin{array}{c}\text { Tolerance / } \\
\text { Uncertainty }\end{array}$ & $\begin{array}{c}\text { Cal } \\
\text { Uncertainty }\end{array}$ & $\begin{array}{c}\text { T/U } \\
\text { Variance }\end{array}$ & $\begin{array}{c}\text { Cal } \\
\text { Variance }\end{array}$ \\
\hline Pipette 1 & 1 & 0.15 & & \\
\hline Flask 1 & 0.16 & 0.16 & & \\
\hline Pipette 2 & 1 & 0.15 & & \\
\hline Flask 2 & 0.16 & 0.16 & & \\
\hline Pipette 3 & 1 & 0.15 & & \\
\hline Counter 2 & 0.8 & 0.8 & & \\
\hline SUM= & & & & \\
\hline $\begin{array}{l}\text { Square } \\
\text { root= }\end{array}$ & & & & \\
\hline Reduction in Uncertainty (Tol U- Cal U)/Tol U*100\% = & \\
\hline
\end{tabular}

Exercise 2. Determine percentage of Uncertainty reduction using

Counter 2 and the Calibration uncertainty estimates. Also

Determine the \% Reduction from the Exercise 1. T/V Uncertainty

*Combined T/U from Exercise 1 in Lower Right block.

3. Complete the calculations required to combine the two sets of uncertainties in Exercise 3 template below, which contains the Class A tolerances for $100 \mathrm{ml}$ flasks and counter 2 .

a. Convert the uncertainties to variances by squaring the values in the first and second columns and recording them in the third and forth columns accordingly.

b. Sum the variances in each of these columns and record them in SUM row.

c. Take the square root of the SUM to obtain the combined uncertainty estimates in the spaces below the sum boxes.

d.Determine the magnitude of the reduction of the combined uncertainty by subtracting the Calibrated (Cal) combined uncertainty from the Tolerance (Tol/V) Combined Uncertainty, and then divide by the Tol/V combined uncertainty and multiply by 100 to convert this to a percentage. Record this in the next lower right block.

e. Determine the percentage reduction between the $\mathrm{T} / \mathrm{V}$ combined uncertainty from exercise 1 and the combined uncertainty using counter 2 and TV combined calibration uncertainties from Exercise 1 and record in last Lower Right block. 


\begin{tabular}{|l|c|c|c|c|}
\hline Source & $\begin{array}{c}\text { Tolerance / } \\
\text { Uncertainty }\end{array}$ & $\begin{array}{c}\text { Cal } \\
\text { Uncertainty }\end{array}$ & $\begin{array}{c}\text { U } \\
\text { Variance }\end{array}$ & $\begin{array}{c}\text { Cal } \\
\text { Variance }\end{array}$ \\
\hline Pipette 1 & 1 & 0.15 & & \\
\hline Flask 1 & 0.08 & 0.08 & & \\
\hline Pipette 2 & 1 & 0.15 & & \\
\hline Flask 2 & 0.08 & 0.08 & & \\
\hline Pipette 3 & 1 & 0.15 & & \\
\hline Counter 2 & 0.8 & 0.8 & & \\
\hline SUM= & \multicolumn{5}{|l|}{} & & \\
\hline $\begin{array}{l}\text { Square } \\
\text { root= }\end{array}$ & \multicolumn{5}{|l}{} & & \\
\hline Reduction in Uncertainty (Tol U- Cal U)/Tol U*100\% = & \\
\hline
\end{tabular}

Exercise 3. Determine the percentage of Uncertainty reduction by using Class A flasks and counter 2. Also determine the total \% reduction by subtracting the combined Cal Uncertainty above, from first Tolerance \& Counter 1 uncertainty.

\section{Discuss the following:}

1. Which single change in the measurements had the most significant change in the final uncertainty estimate?

2. Which change would be the easiest to implement and why?

3. Which change would require the most time and effort?

4. Which change had the least impact on the combined uncertainty and why? 


\begin{tabular}{|c|c|c|c|c|}
\hline $\begin{array}{l}\text { 日期： } \\
\text { Date }\end{array}$ & & & & \\
\hline $\begin{array}{l}\text { Ding } \\
\text { Time }\end{array}$ & & & & \\
\hline Air Temperature & & & & \\
\hline Water Temperature & & & & \\
\hline Barometric Pressure & & & & \\
\hline Humdity & & & & \\
\hline $\begin{array}{l}\text { Pipette ID } \\
\text { Pipetive }\end{array}$ & & & & \\
\hline $\begin{array}{l}\text { 娃石： } \\
\text { Name }\end{array}$ & & & & \\
\hline & $\begin{array}{l}\text { 分析宁-1 } \\
\text { Analyst } 1\end{array}$ & $\begin{array}{l}\text { 分析宁-2 } \\
\text { Analyst } 2\end{array}$ & $\begin{array}{l}\text { 分析宁-3 } \\
\text { Analyst } 3\end{array}$ & $\begin{array}{l}\text { 分析宁 }-4 \\
\text { Analyst } 4\end{array}$ \\
\hline 重量 1 Weight 1 & & & & \\
\hline 重量 2 Weight 2 & & & & \\
\hline 重量 3 Weight 3 & & & & \\
\hline 重量 4 Weight 4 & & & & \\
\hline 重量 5 Weight 5 & & & & \\
\hline 重量 6 Weight 6 & & & & \\
\hline 重量 7 Weight 7 & & & & \\
\hline 重量 8 Weight 8 & & & & \\
\hline 重量 9 Weight 9 & & & & \\
\hline 重量 10 Weight 10 & & & & \\
\hline Average & \#DIV/0! & \#DIV/0! & \#DIV/0! & \#DIV/0! \\
\hline Std. Deviation & \#DIV/0! & \#DIV/0! & \#DIV/0! & \#DIV/0! \\
\hline $\begin{array}{l}\text { Zánis } \\
\text { Z-Factor }\end{array}$ & & & & \\
\hline $\begin{array}{l}\text { Pat } \\
\text { Volume (Z x Avg. Wt.) }\end{array}$ & \#DIV/0! & \#DIV/0! & \#DIV/0! & \#DIV/0! \\
\hline Volume in micro liters $(\mu L)$ & \#DIV/0! & \#DIV/0! & \#DIV/0! & \#DIV/0! \\
\hline 标准偏移 x Z = +/- ml & \#DIV/0! & \#DIV/0! & \#DIV/0! & \#DIV/0! \\
\hline Nominal Volume & 1000 & 1000 & 1000 & 1000 \\
\hline Calculated Volume in $\mu \mathrm{L}$ & \#DIV/0! & \#DIV/0! & \#DIV/0! & \#DIV/0! \\
\hline 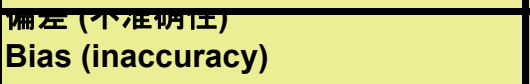 & \#DIV/0! & \#DIV/0! & \#DIV/0! & \#DIV/0! \\
\hline $\begin{array}{l}(+7-8.0 \mu L) \text { 体积容表 }= \\
\text { Volume Tolerance }\end{array}$ & 8 & 8 & 8 & 8 \\
\hline 标准偏移 $\mathrm{ml}$ x 1000= SD in $\mu \mathrm{L}$ & \#DIV/0! & \#DIV/0! & \#DIV/0! & \#DIV/0! \\
\hline $\begin{array}{l}(7 /-1.5 \mu L \text { 标准佩栘谷左) } \\
\text { SD Tolerance }\end{array}$ & 1.5 & 1.5 & 1.5 & 1.5 \\
\hline $\begin{array}{l}\text { 如果称准偏栘和偏寿小于谷寿, 则用 } \\
\text { 过 } \\
\text { Pass if SD \& Bias < Tolerances }\end{array}$ & Pass & Fail & Fail & Fail \\
\hline$\left.U c=\left(U s d^{\wedge} 2+(B / 2)^{\wedge} 2\right)\right)^{\wedge} .5$ & \#DIV/0! & \#DIV/0! & \#DIV/0! & \#DIV/0! \\
\hline
\end{tabular}




\begin{tabular}{|c|c|c|c|c|}
\hline $\begin{array}{l}\text { 打展 } U=U c^{\star} 2 \\
\text { Expanded }\end{array}$ & \#DIV/O! & \#DIV/0! & \#DIV/0! & \#DIV/O! \\
\hline $\begin{array}{l}\text { 惟对 \% 不砽正性 } \\
\text { Relative \% Uncertainty }\end{array}$ & \#DIV/0! & \#DIV/0! & \#DIV/0! & \#DIV/0! \\
\hline & \multicolumn{4}{|c|}{ TEAM AVERAGE EVALUATION OF BIAS \& PRECISION } \\
\hline $\begin{array}{l}\text { 平均 }= \\
\text { Average }\end{array}$ & \#DIV/0! & \#\#\# & \#\#\# & \#\#\# \\
\hline Standard Deviation & \#DIV/O! & \#\#\# & \#\#\# & \#\#\# \\
\hline 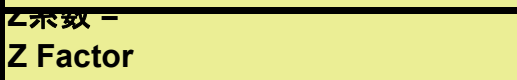 & \#DIV/0! & \#\#\# & \#\#\# & \#\#\# \\
\hline Volume (Z x Avg. Wt.) & \#DIV/O! & \#\#\# & \#\#\# & \#\#\# \\
\hline Volume in micro liters $(\mu L)$ & \#DIV/0! & \#\#\# & \#\#\# & \#\#\# \\
\hline 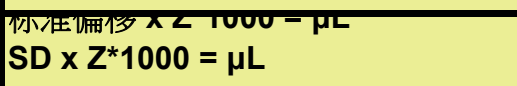 & \#DIV/O! & \#\#\# & \#\#\# & \#\#\# \\
\hline $\begin{array}{l}\text { Vifily } \\
\text { Bias }\end{array}$ & \#DIV/0! & \#\#\# & \#\#\# & \#\#\# \\
\hline$\left.U c=\left(U s d^{\wedge} 2+(B / 2)^{\wedge} 2\right)\right)^{\wedge} .5$ & \#DIV/0! & \#\#\# & \#\#\# & \#\#\# \\
\hline Expanded $U=U c^{*} 2$ & \#DIV/0! & \#\#\# & \#\#\# & \#\#\# \\
\hline $\begin{array}{l}\text { Relative \% Uncertainty } \\
\text { Rella }\end{array}$ & \#DIV/O! & \#\#\# & \#\#\# & \#\#\# \\
\hline $\begin{array}{l}\text { 如果标准偏移和偏差小于容差, 则通 } \\
\text { 过 } \\
\text { Pass if SD \& Bias < Tolerances }\end{array}$ & & \#\#\# & \#\#\# & \#\#\# \\
\hline
\end{tabular}

Sheet Password = CIAE

工作单密码=CIAE 


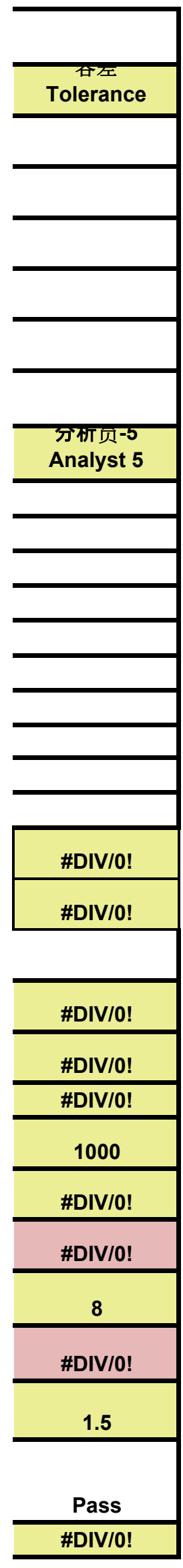




\begin{tabular}{c} 
\#DIV/0! \\
\hline \#DIV/0! \\
\hline \\
\hline \#\#\# \\
\hline \#\# \\
\hline \#\# \\
\hline \#\#\# \\
\hline \#\# \\
\hline \#\# \\
\hline \#\#\# \\
\hline \#\# \\
\hline \#\# \\
\hline \#\# \\
\hline
\end{tabular}




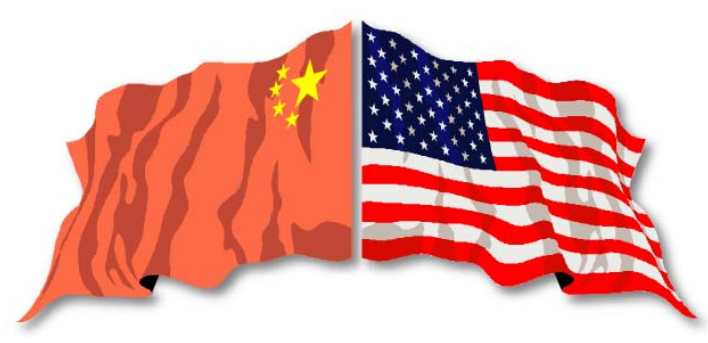

Exercise:

Pipette Calibration 


\section{Objectives}

- Learn the Sources of Error in Volume determinations

- Determine the variation within and between operators

- Estimate the uncertainty of volumetric measurements

- Compare calculated uncertainty estimates with manufacturer's specifications.

- $1000 \mu \mathrm{L}$ Tolerances $=$ Accuracy $+/-0.8 \%$ or $+/-8.0 \mu \mathrm{L}$ Precision $+/-0.15 \%$ or $+/-1.5 \mu \mathrm{L}$ 


\section{Validation of Accuracy and Precision}

- Precision is an agreement between replicate measurements

- Precision is quantified by imprecision

- High precision, i.e. small imprecision means very little variation between repeated measurements

- Accuracy

- It is possible to be very consistent, but consistently wrong

- Inaccuracy is the numerical difference between the mean of a set of replicate measurements and the reference or target value 


\section{Gravimetric Pipette Calibration Exercise:}

- On the data sheet record, name, date and time, water temperature, room temperature, humidity and barometric pressure

- Procedure:

1. Install a new tip on the pipette

2. Tare the balance with a glass flask containing $\mathrm{H} 2 \mathrm{O}$

3. Fill pipette with deionized water or equivalent

4. Dispense water into flask

5. Record the weight of the flask

6. Repeat steps 2 through 5 nine more times

7. Calculate the average, standard deviation \& volume

8. Compare results to manufacturer's specifications. 


\section{Discussion topics for Improving testing: PROPER PIPETTING TECHNIQUES \& TIPS}

Technique -

- Most end users have a tendency to believe that the volume delivery is completely dependent on the setting of the micrometer dial

- Obviously, this is not the case, since many factors associated with pipettes come into play 


\section{PROPER PIPETTING TIPS -1}

Tips - Use Manufacturers' Tips

Temperature -

- The volume delivery performance specifications of pipettes have been referenced by most manufacturers at room temperature which is defined as 20-25ㅇ.C. Any deviation from this specification can affect the amount of liquid dispensed due to the expansion or contraction of the internal components

- Temperature is probably the most important factor that influences pipette performance. In fact, the density of water in a gravimetric analysis is calculated as a function of temperature 


\section{PROPER PIPETTING TIPS -2}

\section{Equilibration Time -}

- It is recommended that the tip, the pipette, the liquid being transferred, and the transfer container itself all be allowed to equilibrate to the same temperature

- This is done to lessen the effects of thermal expansion which can dramatically impact the delivered volume 


\section{PROPER PIPETTING TIPS -3}

Thermal Conductance -

- Thermal energy can be transferred from the operator's hand to the air within the pipette (dead air) or even to the internal components themselves

- This can have a dramatic impact on the amount of liquid dispensed due to the effects of expansion and/or contraction

- To lessen this effect, it is recommended that some type of thermally insulated gloves like latex or cloth be worn 


\section{PROPER PIPETTING TIPS -4}

\section{Position -}

- Pipettes should be held vertical during the aspiration of liquids, however, some end users often hold pipettes at many different angles during a pipetting interval

- Holding a pipette 30o off vertical can cause as much as $0.7 \%$ more liquid to be aspirated due to the impact of hydrostatic pressure

- Always store pipettes in an upright position when not in use 


\section{PROPER PIPETTING TIPS -5}

Pre-Wetting/Pre-Rinsing Tips -

- Failing to pre-wet tips can cause inconsistency between samples since liquid in the initial samples adhere to the inside surfaces of the pipette tip, but liquid from later samples does not

- Also, if a new volume is dialed in on the pipette's micrometer, you will receive better results at the new volume by taking the old tip off and placing a new one on the shaft before you commence pipetting 


\section{PROPER PIPETTING TIPS -6}

\section{Immersion Depth -}

- The pipette tip should only be inserted into the vessel containing the liquid to be transferred about $1-3 \mathrm{~mm}$

- If the tip is immersed beyond this, the results could be erroneously high. This is due to the fact that liquid could adhere to the tip and be transferred along with the aliquot in the tip

- If the tip is not immersed far enough then air could be drawn into the tip which could yield results that are incorrect on the low end 


\section{PROPER PIPETTING TIPS -7}

\section{Release of Plunger -}

- It is recommended that a smooth, consistent pipetting rhythm be employed since it helps to increase both accuracy and precision

- After the liquid has been aspirated into the tip, the pipette should be placed against the wall of the receiving vessel and the plunger slowly depressed. This will help all of the liquid in the tip to be dispensed

- After a pause of about 1 second, depress the plunger to the bottom or blowout position (if equipped) and remove the pipette from the sidewall by utilizing either a sliding action up the wall or a brief movement away from the wall (called "touching off") 


\section{Repeat the Exercise Using the techniques discussed.}

\section{Procedure:}

1. Install a new tip on the pipette

2. Tare the balance with a glass flask containing $\mathrm{H} 2 \mathrm{O}$

3. Fill pipette with deionized water or equivalent

4. Slowly dispense water into flask

5. Record the first stable weight of the flask plus aliquot

6. Repeat steps 2 through 5 nine more times

7. Calculate the average, standard deviation, volume $\&$ bias

8. Compare results to manufacturer's specifications

9. Compare the first and second results

10 . With your team measurements recalculate the same values 11. Determine the addition error caused by different operators 


\section{Gravimetric Pipette Calibration/Validation}

Given a certain mass of water with a known specific gravity, its volume can then be predicted

- Most common calibration method

- Physical Model: $V=\left(X_{i}+e\right) * Z$ - factor

- Z-factor: Conversion factor ( $\mu \mathrm{L} / \mathrm{mg})$ incorporating the density of water when buoyed in air as a function of temperature and pressure.

- Z-factor $=\left(1 /\left(\rho w-\rho_{a}\right)\right) *\left(1-\left(\rho_{a} / \rho_{b}\right)\right)$

- $\rho_{w}=$ density of water

- $\rho_{a}=$ density of air

- $\rho_{b}=$ density of weights 
ISO 8655 gravimetric method

- Given a certain mass of water with a known specific gravity, its volume can then be predicted

- Weighing vessel: Never plastics!!!!

- Thermometer: Uncertainty of $<0,2{ }^{\circ} \mathrm{C}$

- Hygrometer: Uncertainty of $<10 \%$

- Barometer: Uncertainty of $<0,5 \mathrm{kPa}$ 


\section{Z-Factor Values}

Converting Wt. to Volume

- Look up corresponding Zfactor for water temperature

- Multiple the average of the 10 aliquots of water

- Subtract this value from $1,000 \mathrm{ml}$ to determine the bias

- Is it within the $+/-8.0 \mu \mathrm{L}$ tolerance for $1 \mathrm{ml}$ ?

\begin{tabular}{|c|c|}
\hline TEMP ACTUAL & Z-FACTOR \\
${ }^{\circ} \mathrm{C}$ & \\
20.0 & 1.0029 \\
20.5 & 1.0030 \\
21.0 & 1.0031 \\
21.5 & 1.0032 \\
22.0 & 1.0033 \\
22.5 & 1.0034 \\
23.0 & 1.0035 \\
23.5 & 1.0036 \\
24.0 & 1.0038 \\
24.5 & 1.0039 \\
\hline
\end{tabular}




\section{Discussion Topics}

-What variables contributed to the volume uncertainty?

- How was the standard deviation affected by more operators?

- What affect did additional measurements have on the average volume?

- How do your bias and precision estimates compare to the manufacturer's specification?

- What uncertainty value would you assign to the volumes delivered by your pipette?

- What Uncertainty would you assign to any $1 \mathrm{ml}$ volume dispensed by any person in the room? 


\section{Summary}

- There are many variables that affect the uncertainty of volumes, using the gravimetric method

- Training and a comprehensive procedure help minimize variation in volume measurements

- Uncertainty estimates should also include the assumptions made in their determination

- The user of the volume measurements must determine the limit of error that is "fit for purpose" 


\section{Exercise Objectives:}

After the session the participants will be able to do the following:

1. Explain the sources of error in pipette volume measurements

2. Discuss the variation within and between operators

3. Estimate the uncertainty of volumetric measurements

4. Compare calculated uncertainty estimates with manufacturer's specifications.

$1000 \mu \mathrm{L}$ tolerances $=$ Accuracy $+/-0.8 \%$ or $+/-8.0 \mu \mathrm{L}$

$$
\text { Precision }+/-0.15 \% \text { or }+/-1.5 \mu \mathrm{L}
$$

\section{Estimated Time:}

+1.50 hours completing exercise

+0.50 hours in large group discussion

2.00 hours total

\section{Materials needed:}

1. Four laptop computers

2. Work sheets for each person

3. One barometer with calibration certificate or manufacturer specifications

4. Two thermometers with readability to tenth of a degree (0.1C) must have calibration certificates or manufacturers specifications in both English and Chinese. One must be able to be used to read the temperature of water.

5. Humidity meter with calibration certificate or manufacturer specifications in both English and Chinese

6. Four $1 \mathrm{ml}$ fixed volume air displaced pipettes. (Calibration certificates NOT Required)

7. Four boxes of disposable tips from the manufacturer of the pipettes

8. Two liters of distilled water

9. Five $50 \mathrm{ml}$ glass volumetric flask

10. Four $250 \mathrm{ml}$ bottles

11. Flip chart or dry board and colored markers

\section{Instructions:}

On your work sheet record: your name, date, time, water temperature, room temperature, humidity and barometric pressure and the uncertainty or tolerance of each instrument.

Procedure:

1. Install a new tip on the pipette

2. Tare the balance with a glass flask containing $\mathbf{H 2 O}$

3. Fill pipette with deionized water or equivalent

4. Dispense water into flask

5. Record the weight of the dispensed volume of water.

6. Repeat steps b through e $\mathbf{9}$ more times for a total of $\mathbf{1 0}$ dispensing 
7. Calculate the average weight of the water, \& the standard deviation.

\section{Value for Z ( $\mu$ L/Mg), as a Function of Temperature and Pressure, for Distilled Water at 1 atm.}

\begin{tabular}{|cc|}
\hline $\begin{array}{c}\text { TEMP } \\
{ }^{\circ} \mathrm{C}\end{array}$ & $\begin{array}{c}\text { ACTUAL } \\
\text { Z-FACTOR }\end{array}$ \\
\hline 15.0 & 1.0020 \\
15.5 & 1.0020 \\
16.0 & 1.0021 \\
16.5 & 1.0022 \\
17.0 & 1.0023 \\
17.5 & 1.0024 \\
18.0 & 1.0025 \\
18.5 & 1.0026 \\
19.0 & 1.0027 \\
19.5 & 1.0028 \\
& \\
\hline
\end{tabular}

\begin{tabular}{|cc|}
\hline $\begin{array}{c}\text { TEMP } \\
{ }^{\circ} \text { C }\end{array}$ & $\begin{array}{c}\text { ACTUAL } \\
\text { Z-FACTOR }\end{array}$ \\
\hline 20.0 & 1.0029 \\
20.5 & 1.0030 \\
21.0 & 1.0031 \\
21.5 & 1.0032 \\
22.0 & 1.0033 \\
22.5 & 1.0034 \\
23.0 & 1.0035 \\
23.5 & 1.0036 \\
24.0 & 1.0038 \\
24.5 & 1.0039 \\
& \\
\hline
\end{tabular}

\begin{tabular}{|cc|}
\hline $\begin{array}{c}\text { TEMP } \\
{ }^{\circ} \text { C }\end{array}$ & $\begin{array}{c}\text { ACTUAL } \\
\text { Z-FACTOR }\end{array}$ \\
\hline 25.0 & 1.0040 \\
25.5 & 1.0041 \\
26.0 & 1.0043 \\
26.5 & 1.0044 \\
27.0 & 1.0045 \\
27.5 & 1.0047 \\
28.0 & 1.0048 \\
28.5 & 1.0050 \\
29.0 & 1.0051 \\
29.5 & 1.0052 \\
30.0 & 1.0054 \\
\hline
\end{tabular}

Converting Wt. to Volume

1. Look up corresponding Z-factor for water temperature.

2. Multiple the average weight of the 10 aliquots of water by the $Z$ factor.

3. Subtract this value from $1,000 \mathrm{ml}$ to determine the bias.

4. Is it within the $+/-8.0-\mu \mathrm{L}$ tolerances for $1 \mathrm{ml}$ ?

5. Is the standard deviation within the $+/-1.5 \mu \mathrm{L}$ Discussion Topics

6. Combine all of the data from the team and repeat the calculations

Discussion Questions

1. What variables contributed to the volume uncertainty?

2. How did more operators affect the standard deviation?

3. What affect did additional measurements have on the average volume?

4. How do your bias and precision estimates compare to the manufacturer's specification?

5. What uncertainty value would you assign to the volumes delivered by your pipette?

6. What uncertainty would you assign to any $1 \mathrm{ml}$ volume dispensed by any person in the room?

\section{Summary}

1. There are many variables that affect the uncertainty of volumes, using the gravimetric method.

2. Training and a comprehensive procedure help minimize variation in volume measurements.

3. Uncertainty estimates should also include the assumptions made in their determination.

4. The user of the volume measurements must determine the limit of error that is "fit for purpose" 


\begin{tabular}{|c|c|c|c|c|c|}
\hline Date & & Group: & & & Tolerance \\
\hline \multicolumn{6}{|l|}{ Air Temperature= } \\
\hline \multicolumn{6}{|l|}{ Water Temperature $=$} \\
\hline \multicolumn{6}{|l|}{ Barometric Pressure $=$} \\
\hline \multicolumn{6}{|l|}{ Humidity= } \\
\hline \multicolumn{6}{|l|}{ Pipette ID= } \\
\hline \multicolumn{6}{|l|}{ Name: } \\
\hline & Analyst-1 & Analyst-2 & Analyst-3 & Analyst-4 & Analyst-5 \\
\hline \multicolumn{6}{|l|}{1 weight } \\
\hline \multicolumn{6}{|l|}{2 weight } \\
\hline \multicolumn{6}{|l|}{3 weight } \\
\hline \multicolumn{6}{|l|}{4 weight } \\
\hline \multicolumn{6}{|l|}{5 weight } \\
\hline \multicolumn{6}{|l|}{6 weight } \\
\hline \multicolumn{6}{|l|}{7 weight } \\
\hline \multicolumn{6}{|l|}{8 weight } \\
\hline \multicolumn{6}{|l|}{9 weight } \\
\hline \multicolumn{6}{|l|}{10 weight } \\
\hline \multicolumn{6}{|l|}{ Average $=$} \\
\hline \multicolumn{6}{|l|}{ Standard Deviation= } \\
\hline \multicolumn{6}{|l|}{ Z-Factor $=$} \\
\hline \multicolumn{6}{|l|}{ Volume $(\mathrm{Z} \times$ Ave Wt. $)=$} \\
\hline \multicolumn{6}{|c|}{ Volume in micro liters $(\mu \mathrm{L})=$} \\
\hline \multicolumn{6}{|c|}{$\mathrm{SD} \times \mathrm{Z}=+/-\mathrm{ml}$} \\
\hline \multicolumn{6}{|l|}{ SD ml $\times 1000=S D$ in $\mu \mathrm{L}$} \\
\hline \multicolumn{6}{|c|}{ Nominal Volume $=$} \\
\hline Calculated Volume in $\mu$ & & & & & \\
\hline Bias (inaccuracy) & & & & & \\
\hline$(+/-8.0 \mu \mathrm{L})$ Volume Toler & & & & & \\
\hline (+/- 1.5 $\mu \mathrm{L}$ SD Tolera & & & & & \\
\hline Pass if SD \& Bias $<$ Tole & & & & & \\
\hline$\left.U c=\left(U s d^{\wedge} 2+(B / 2)^{\wedge} 2\right)\right)^{\wedge}$ & & & & & \\
\hline Expanded $U=U c^{*} 2$ & & & & & \\
\hline Relative \% Uncertainty & & & & & \\
\hline & TEAM AVEF & GE EVALUA & ON OF BIAS & PRECISION & \\
\hline Average $=$ & & & & & \\
\hline Standard Deviation= & & & & & \\
\hline Z-Factor $=$ & & & & & \\
\hline Volume $(\mathrm{Z} \times$ Ave Wt. $)=$ & & & & & \\
\hline Volume in micro liters ( & & & & & \\
\hline$S D \times Z^{*} 1000=\mu L$ & & & & & \\
\hline Bias & & & & & \\
\hline$\left.U c=\left(U s d^{\wedge} 2+(B / 2)^{\wedge} 2\right)\right)^{\wedge}$ & & & & & \\
\hline Expanded U = Uc*2 & & & & & \\
\hline Relative \% Uncertainty & & & & & \\
\hline
\end{tabular}


Part 2 of the Exercise: Repeat with more detailed instructions and tips for accurate Pipetting;

Discussion topics for Improving testing:

PROPER PIPETTING TECHNIQUES \& TIPS

TECHNIQUES -

1. Most end users have a tendency to believe that the volume delivery is completely dependent on the setting of the micrometer dial.

2. Obviously, this is not the case, since many factors associated with pipettes come into play. TIPS -

1. Use Manufacturers' Tips

Temperature

1. The volume delivery performance specifications of pipettes have been referenced by most manufacturers at room temperature, which is defined as $20-25^{\circ} \mathrm{C}$. Any deviation from this specification can affect the amount of liquid dispensed due to the expansion or contraction of the internal components.

2. Temperature is probably the most important factor that influences pipette performance. In fact, the density of water in a gravimetric analysis is calculated as a function of temperature.

Equilibration Time

1. It is recommended that the tip, the pipette, the liquid being transferred, and the transfer container itself all be allowed to equilibrate to the same temperature.

2. This is done to lessen the effects of thermal expansion, which can dramatically impact the delivered volume.

\section{Thermal Conductance}

1. Thermal energy can be transferred from the operator's hand to the air within the pipette (dead air) or even to the internal components themselves.

2. This can have a dramatic impact on the amount of liquid dispensed due to the effects of expansion and/or contraction.

3. To lessen this effect, it is recommended that some type of thermally insulated gloves like latex or cloth be worn.

\section{Position}

1. Pipettes should be held vertical during the aspiration of liquids; however, some end users often hold pipettes at many different angles during a pipetting interval.

2. Holding a pipette $30^{\circ}$ off vertical can cause as much as $0.7 \%$ more liquid to be aspirated due to the impact of hydrostatic pressure.

3. Always store pipettes in an upright position when not in use.

\section{Pre-Wetting/Pre-Rinsing Tips}

1. Failing to pre-wet tips can cause inconsistency between samples since liquid in the initial samples adhere to the inside surfaces of the pipette tip, but liquid from later samples does not.

2. Also, if a new volume is dialed in on the pipette's micrometer, you will receive better results at the new volume by taking the old tip off and placing a new one on the shaft before you commence pipetting.

\section{Immersion Depth}

1. The pipette tip should only be inserted into the vessel containing the liquid to be transferred about 1$3 \mathrm{~mm}$.

2. If the tip is immersed beyond this, the results could be erroneously high. This is due to the fact that liquid could adhere to the tip and be transferred along with the aliquot in the tip.

3. If the tip is not immersed far enough then air could be drawn into the tip that could yield results that are incorrect on the low end. 


\section{Release of Plunger -}

1. It is recommended that a smooth, consistent pipetting rhythm be employed since it helps to increase both accuracy and precision.

2. After the liquid has been aspirated into the tip, the pipette should be placed against the wall of the receiving vessel and the plunger slowly depressed. This will help all of the liquid in the tip to be dispensed.

3. After a pause of about 1 second, depress the plunger to the bottom or blowout position (if equipped) and remove the pipette from the sidewall by utilizing either a sliding action up the wall or a brief movement away from the wall (called "touching off”).

\section{Repeat the exercise using the techniques given above and discussed.}

\section{- Procedure:}

1. Install a new tip on the pipette

2. Tare the balance with a glass flask containing $\mathrm{H} 2 \mathrm{O}$

3. Fill pipette with deionized water or equivalent

4. Slowly dispense water into flask

5. Record the first stable weight of the flask plus aliquot

6. Repeat steps 2 through 5 nine more times

7. Calculate the average, standard deviation, volume \& bias

8. Compare results to manufacturer's specifications.

9. Compare your first and second results with each other, then

10 . With all of your team measurements recalculate the same values

11. How can you determine the addition error caused by different operators?

\section{Gravimetric Pipette Calibration/Validation}

12. Second validation of a pipette's volume and operator's precision using the "tips and techniques" listed above to reduce operator variation in the use of the pipette to dispense volume measurements. Use another copy of the worksheet for the second attempt to validate the calibration of a pipette and calculate the uncertainty of volume measurements made with the pipette in the laboratory.

13. Discuss the results of the second exercise. 


\begin{tabular}{|c|c|c|c|c|c|}
\hline Date & & Group: & & & Tolerance \\
\hline \multicolumn{6}{|l|}{ Air Temperature= } \\
\hline \multicolumn{6}{|l|}{ Water Temperature $=$} \\
\hline \multicolumn{6}{|l|}{ Barometric Pressure $=$} \\
\hline \multicolumn{6}{|l|}{ Humidity= } \\
\hline \multicolumn{6}{|l|}{ Pipette ID= } \\
\hline \multicolumn{6}{|l|}{ Name: } \\
\hline & Analyst-1 & Analyst-2 & Analyst-3 & Analyst-4 & Analyst-5 \\
\hline \multicolumn{6}{|l|}{1 weight } \\
\hline \multicolumn{6}{|l|}{2 weight } \\
\hline \multicolumn{6}{|l|}{3 weight } \\
\hline \multicolumn{6}{|l|}{4 weight } \\
\hline \multicolumn{6}{|l|}{5 weight } \\
\hline \multicolumn{6}{|l|}{6 weight } \\
\hline \multicolumn{6}{|l|}{7 weight } \\
\hline \multicolumn{6}{|l|}{8 weight } \\
\hline \multicolumn{6}{|l|}{9 weight } \\
\hline \multicolumn{6}{|l|}{10 weight } \\
\hline \multicolumn{6}{|l|}{ Average $=$} \\
\hline \multicolumn{6}{|l|}{ Standard Deviation= } \\
\hline \multicolumn{6}{|l|}{ Z-Factor $=$} \\
\hline \multicolumn{6}{|l|}{ Volume $(\mathrm{Z} \times$ Ave Wt. $)=$} \\
\hline \multicolumn{6}{|c|}{ Volume in micro liters $(\mu \mathrm{L})=$} \\
\hline \multicolumn{6}{|c|}{$\mathrm{SD} \times \mathrm{Z}=+/-\mathrm{ml}$} \\
\hline \multicolumn{6}{|l|}{ SD ml $\times 1000=S D$ in $\mu \mathrm{L}$} \\
\hline \multicolumn{6}{|c|}{ Nominal Volume $=$} \\
\hline Calculated Volume in $\mu$ & & & & & \\
\hline Bias (inaccuracy) & & & & & \\
\hline$(+/-8.0 \mu \mathrm{L})$ Volume Toler & & & & & \\
\hline (+/- 1.5 $\mu \mathrm{L}$ SD Tolera & & & & & \\
\hline Pass if SD \& Bias $<$ Tole & & & & & \\
\hline$\left.U c=\left(U s d^{\wedge} 2+(B / 2)^{\wedge} 2\right)\right)^{\wedge}$ & & & & & \\
\hline Expanded $U=U c^{*} 2$ & & & & & \\
\hline Relative \% Uncertainty & & & & & \\
\hline & TEAM AVEF & GE EVALUA & ON OF BIAS & PRECISION & \\
\hline Average $=$ & & & & & \\
\hline Standard Deviation= & & & & & \\
\hline Z-Factor $=$ & & & & & \\
\hline Volume $(\mathrm{Z} \times$ Ave Wt. $)=$ & & & & & \\
\hline Volume in micro liters ( & & & & & \\
\hline$S D \times Z^{*} 1000=\mu L$ & & & & & \\
\hline Bias & & & & & \\
\hline$\left.U c=\left(U s d^{\wedge} 2+(B / 2)^{\wedge} 2\right)\right)^{\wedge}$ & & & & & \\
\hline Expanded U = Uc*2 & & & & & \\
\hline Relative \% Uncertainty & & & & & \\
\hline
\end{tabular}




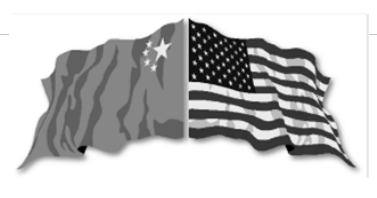

\section{Module 13}

\section{Case Study: Uranium Working Standards Preparation}

\section{Objectives}

- Understand how to produce a standard that has an uncertainty of $1 / 3$ or less of the uncertainty of the measurement it is meant to control

- Review a case study on the production of a Uranium Nitrate Solution to use as working calibration material (WRM) that is traceable to a Certified Reference Material.

- Review the statistical tests used in assigning a concentration value with associated estimate of the uncertainty of the WRM that is traceable to the CRM. 


\section{Introduction:}

- Over $\mathbf{3 0 0 0}$ years ago King Solomon wrote "Differing weights and differing measures -- The Lord detests them both." Proverbs 20:10

- Civilization has recognized the need for standards for millenniums and governments have established special organizations to maintain \& regulate them

- The nuclear industry has its standards \& regulations which which apply to the measurements needed for MC\&A, as well as production and safety.

- Uranium standards are expensive, but can be produced locally at a significant cost savings and still be traceable to CRMs if done correctly.

\section{Overview}

- Personal Experience in Standards Preparation

- Data Quality Objectives

- Measurement Methods Uncertainties

- Available standards

- Uranyl nitrate stock solution preparation

- Characterization Methods and Laboratories

- Data Collection

- Statistical tests used in evaluation characterization of the traceable stock solution

- Assigned Concentrations \& Uncertainties 


\section{Work Experience}

- Chemist at nuclear fuels reprocessing plant

- Qualified by Chemist training \& testing program

- Research Chemist in Standards Lab

- Prepared U and Pu solution standards for QC programs

- Safeguards Analytical Laboratory Evaluation (SALE) program

- Managed Chemist \& Analysts training program \& QC program

- Ran Standards Lab for commercial nuclear fuels reprocessing

- Prepared U standards for calibration, QC \& Training \& Testing

- Prepare $\mathbf{3 0 0}$ liters of $U$ solution stock solution for AGNS

- Savannah River Site -Analytical Services

- Used AGNS stock U solution for QC programs

- Worked on PuO2 standards for NDA MCPs

\section{Standards \& Data Quality Objectives}

- Standard or Certified Reference Material (SRM or CRM)

- Working Reference Material (WRM)

- Working Calibration and Test Material (WCTM)

- Reference Calibration and Test Material (RCTM)

- Data Quality Objectives

- Uncertainty of methods to be calibrated \& monitored

- Target Uncertainty of measurement methods

- Available standards

- NBS SRM-960 normal uranium metal with known purity

- Impurity standards for emission spectroscopy

- Used NUREG-0253 special LANL publication as procedure

- Target Value Uncertainty of Standard is $<1 / 3$ of Method Uncertainty 


\section{WCTM Characteristics}

- Should be stable over extended storage periods

- Closely match the material routinely measured

- Have an uncertainty consistent with its intended use

- It should be convenient to use

- It should be economical

\section{Certified Reference Materials Uranium metal, I sotopic \& I mpurity $U$ oxides}

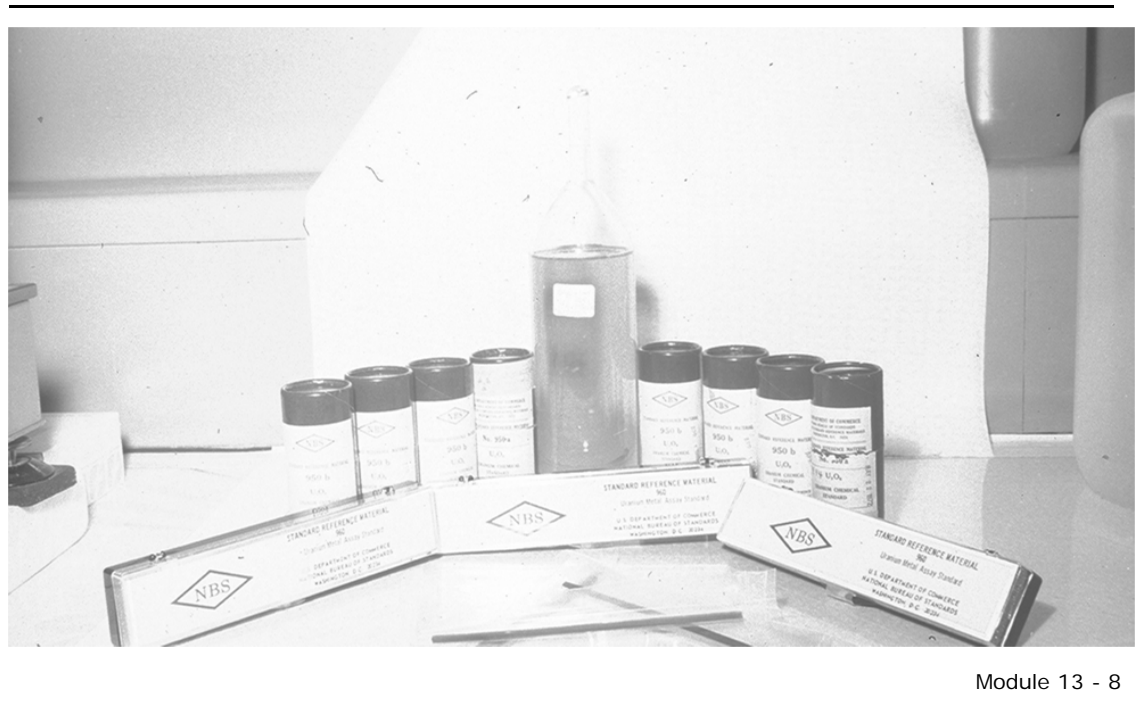




\section{Methods for Preparation of WCTMs}

1. Synthesize from well characterized starting material

- Obtain Standard Reference Material (SRM)

- Prepare using standard gravimetric and volumetric methods

- Integrity of SRM transferred to the WCTM

2. Characterize a plant product by two analysis methods

- Traceability is established using the Reference Material

- This is done by analyzing both solutions concurrently

- Bias correcting the WCTM with biases observed on RCTM

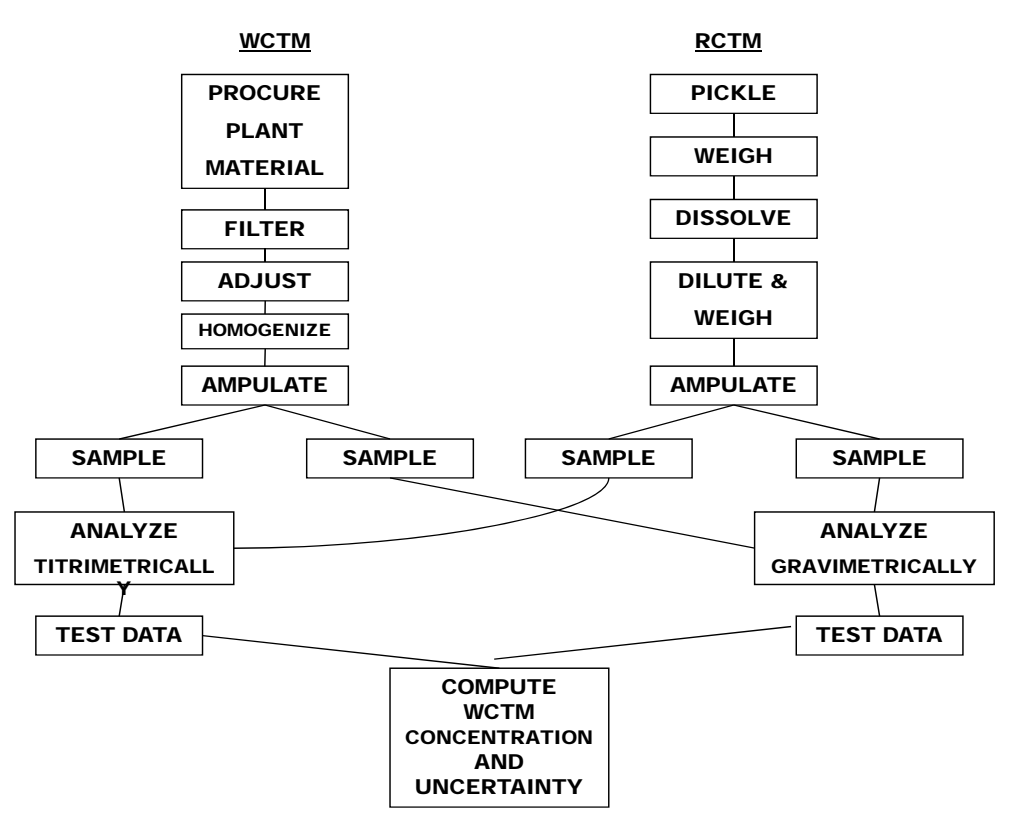

Preparation scheme for calibration and test materials 


\section{Case Study of the Preparation of a Uranyl Nitrate Solution}

- $\sim 80$ Liters of $\mathbf{3 0 0} \mathrm{gU} / \mathrm{L}$ solution obtained from the plant

- Solution filtered, acidity adjusted \& thoroughly mixed.

- Transferred to pre-cleaned glass ampoules \& flame sealed

- one hundred \& eighty $500 \mathrm{ml}$, fifty $50 \mathrm{ml}$ and fifty 10 \& 20 ml ampoules

- SRM-960 U metal used to synthesize a $300 \mathrm{gU} / \mathrm{L}$ solution

- Metal was cleaned, dried, weighed, dissolved, diluted \& weighed

- The concentration and associated uncertainty were calculated

- 50, 20 and $10 \mathrm{ml}$ ampoules were filled and sealed for future use

- Characterization work done from the various ampoules

\section{Characterization}

- Target uncertainty is $1 / 3$ of method uncertainty

- Target for method at that time was $0.25 \%$

- Target for WCTM's uncertainty was $\sim 0.08 \%(0.25 \% / 3)$

- Two accurate and precise methods were selected

- Gravimetric Uranium evaporate and ignite to form U308

- NBL modified Davies and Gray titrimetric method

- Two Laboratories were involved in the program

- The Department of Energy Standards Laboratory (NBL)

- Private commercial laboratory

- A another RCTM was prepared \& the whole characterization repeated in 1978 


\section{Synthesized RCTM Uranyl Nitrate Reference Standard I}

Symbol Component

$A=\quad$ assigned makeup value

$\mathrm{S}_{\mathrm{A}}=\quad$ associated standard deviation

$\mathrm{F}=\quad$ purity of starting material

b = air buoyancy

$\mathrm{w}_{1}=\quad$ Weight of the starting material

$w_{2}=$ tare weight of the flask

$W_{3}=\quad$ gross weight of the solution \& flask

$w_{4}=\left(W_{3}-W_{2}\right)=$ net weight of solution

$\mathrm{S}=\quad$ standard deviation

$\mathrm{S}_{\mathrm{A}}=\quad\left(\mathrm{F}^{*} \mathrm{~b} * \mathrm{~W}_{1}\right) / \mathrm{W}_{4} \quad 0.2268495$

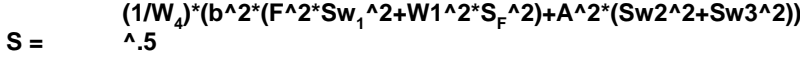

Standard Deviation

Mean Value $g / g$

g/g

\begin{tabular}{rr} 
& 0.000024 \\
0.2268495 & 0.000085 \\
0.99975 & 0 \\
0.99992 & 0.00019 \\
166.45221 & 0.034 \\
176.695 & 0.034 \\
910.209 & 0.048 \\
733.514 & \\
\hline 0.2268495 & \\
\hline +Sw3^2)) & 0.000024
\end{tabular}

Final Concentration in $\mathrm{mg} \mathrm{U} / \mathrm{g}=$

$226.85(+/-0.024 \mathrm{mgU} / \mathrm{g})$

Module $13-13$

\section{Synthesized RCTM Uranyl Nitrate Reference Standard II}

\begin{tabular}{|c|c|c|c|}
\hline Symbol & Component & Mean Value $\mathbf{g} / \mathbf{g}$ & Standard Deviation $\mathrm{g} / \mathrm{g}$ \\
\hline$A=$ & assigned makeup value & 0.216578 & \\
\hline $\mathbf{S}_{\mathrm{A}}=$ & associated standard deviation & & 0.000019 \\
\hline$F=$ & purity of starting material & 0.99975 & 0.000085 \\
\hline $\mathbf{b}=$ & air buoyancy & 0.99992 & 0 \\
\hline$w_{1}=$ & Weight of the starting material & 155.3783 & 0.00011 \\
\hline$w_{2}=$ & tare weight of the flask & 164.858 & 0.0083 \\
\hline$w_{3}=$ & gross weight of the solution \& flask & 882.044 & 0.0132 \\
\hline$w_{4}=$ & $\left(W_{3}-W_{2}\right)=$ net weight of solution & 717.186 & 0.0156 \\
\hline$s=$ & standard deviation & & \\
\hline $\mathbf{S}_{\mathrm{A}}=$ & $\left(F^{*} b^{*} W_{1}\right) / W_{4}$ & 0.216578444 & \\
\hline$S=$ & \multicolumn{2}{|c|}{$\left(1 / W_{4}\right)^{\star}\left(b^{\wedge} 2^{\star}\left(F^{\wedge} 2^{\star} S_{1} w_{1}^{\wedge} 2+W 1^{\wedge} 2^{*} S_{F}^{\wedge} 2\right)+A^{\wedge} 2^{\star}\left(S W 2^{\wedge} 2+S W 3^{\wedge} 2\right)\right)^{\wedge} .5$} & 0.000019 \\
\hline & Final Concentration in $\mathrm{mg} \mathrm{U} / \mathrm{g}=$ & 216.58 & $(+/-0.024 \mathrm{mgU} / \mathrm{g})$ \\
\hline
\end{tabular}


Summary of Uranyl Nitrate WCTM

Plant Material Characterization

\begin{tabular}{|l|c|c|}
\hline LABORATORY & METHOD & CORRECTED MEAN \\
\hline IN-HOUSE - 76 & GRAVIMETRIC & 219.20 \\
\hline OUTSIDE \#1 & & 219.18 \\
\hline IN-HOUSE - 78 & & 219.24 \\
\hline IN-HOUSE - 76a & NBL-MODIFIED & \\
\hline IN-HOUSE - 76b & & 219.17 \\
\hline OUTSIDE \#2 & & 219.20 \\
\hline IN-HOUSE - 78 & & 219.26 \\
\hline
\end{tabular}

THE AVERAGE OF THESE 7 MEANS IS $219.20 \mathrm{mg} / \mathrm{g}$

SOLUTION.

The target RLE of $0.08 \%$ is $>0.014 \%$ of the WCTM, therefore the WCTM characterization is satisfactory

\section{Statistics Used in Characterizing a Working Standard}

Note: See spreadsheet (handout and display)

1. Data collected in confirmation working from 1978 Inhouse Analysis

- The Mean (M) and Standard Deviation (S) of each set of results from each method on both the CRM \& WRM.

2. F-Test of Precision

- Calculated $F$ ratios for both methods are compared to tabulated $F$ values at the $95 \%$ confidence levels.

- If different, pooled estimates of precision may be used

3. Calculation of Method Means Based on CRM

- WRMS is bias corrected by the biases determined on CRM 


\section{Statistics Used in Characterizing a Working Standard(continued)}

4. Calculation of the Equality of the Means

- The approximate variances (V) and degrees of freedom (f) for each mean are calculated.

- These $f$ are used in testing the means using a t-test

5. Compute the $T$ statistic with $f$ degrees of freedom (df) by dividing the difference D\&G and Gravimetric method means by the RMS of the two $S$ estimates and using a special formula to estimate the $f$.

6. Assignment of WRM Concentration Value (A) by weighting the bias corrected means from each method.

\section{Statistics Used in Characterizing a Working Standard(continued)}

7. Calculation of the $S D\left(S_{A}\right)$ Associated with $A$, with $f_{A}$ Degrees of Freedom.

- $f_{A}$ is the df used in the calculation of the limit of error for $A$.

8. Calculation of the Limit of Error (LE) and Relative Limit of Error (RLE)

9. Test to determine if the RLE meets the requirement of $<1 / 3$ of the plant RLE of $0.25 \%$

10. Calculation of the $95 \%$ Confidence Interval $(\mathrm{Cl})$ for the assigned value of the WRMS.

- $\quad \mathrm{Cl}=\mathrm{A}+\mathrm{l}-\mathrm{t}\left(1-\mathrm{a} / 2, \mathrm{n}_{\mathrm{a}}\right)\left(\mathrm{S}_{\mathrm{A}}\right)$

- $t=(0.975,16)=2.120$

- $\mathrm{Cl}=219.22+\mathrm{l}-(2.120 * 0.0318)=219.15$ to $219.29 @ .95 \mathrm{Cl}$ 


\section{Accomplishment}

- A large quantity of Uranyl Nitrate stock solution was prepared from plant material, traceable to a CRM

- Enough material was prepared so it could be used to synthesize standard for all Uranium methods used.

- The WCM uncertainty was less than the target uncertainty

- This standard has been used for 30 years at the Savannah River Site

\section{Uranium Certified Reference Materials}

- CRM metal used to make calibration solutions.

- Depleted Uranium metal used to make synthetic process streams to be used for calibrations, QC, R\&D and training \& testing personnel.

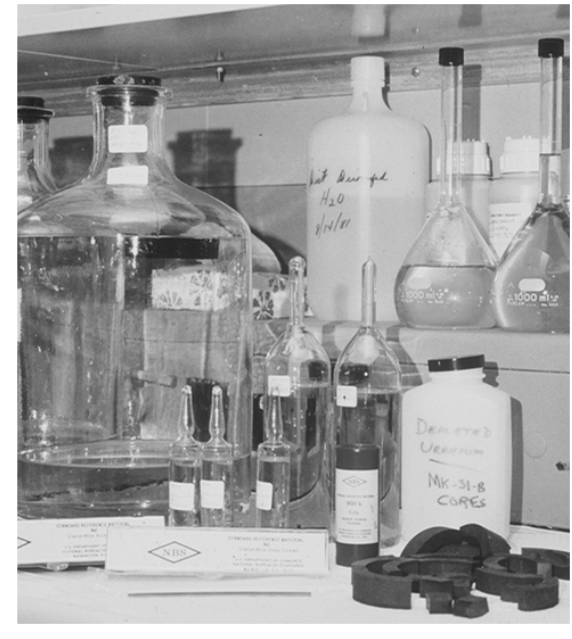


Plutonium Nitrate Standards can be produced the same way, Using a CRM and Plant material

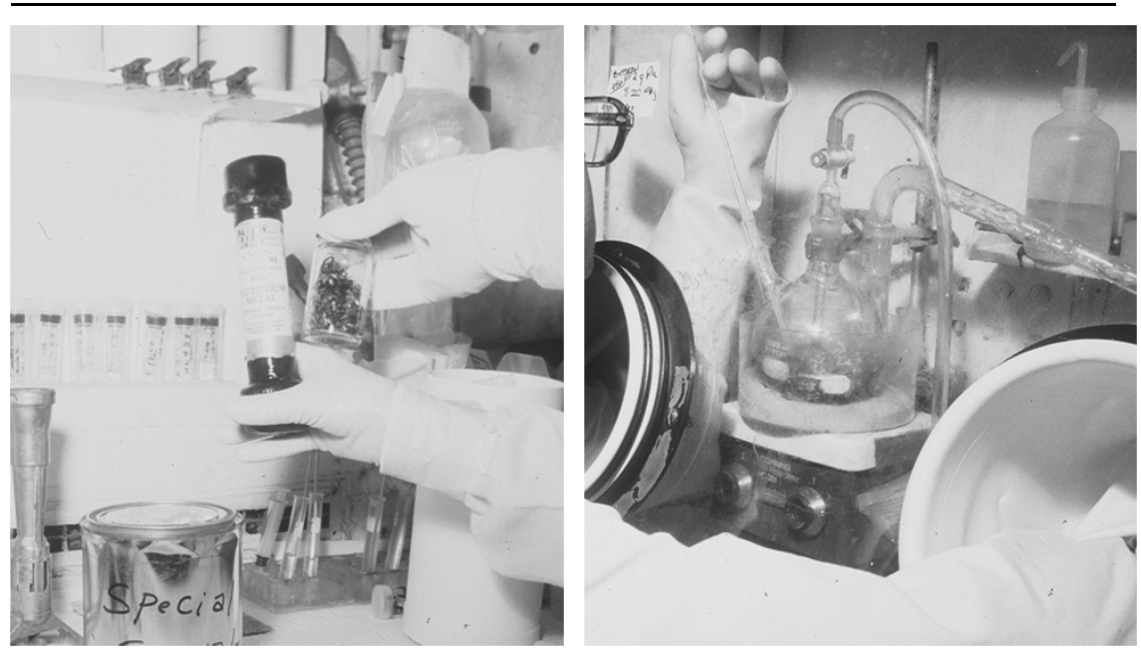

Module $13-21$

\section{Summary}

- Understand how to produce a standard that has an uncertainty of $1 / 3$ or less of the uncertainty of the measurement it is meant to control

- Reviewed a case study on the production of a Uranium Nitrate Solution to use as working calibration material (WRM) that is traceable to a Certified Reference Material.

- Reviewed the statistical tests used in assigning a concentration value with associated estimate of the uncertainty of the WRM that is traceable to the CRM. 
1976 Synthesized RCTM Uranyl Nitrate Reference Standard I

\begin{tabular}{|c|c|c|c|}
\hline $\begin{array}{l}\text { Symbol } \\
A=\end{array}$ & $\begin{array}{l}\text { Component } \\
\text { assigned makeup value }\end{array}$ & Mean Value $\mathbf{g} / \mathbf{g}$ & $\begin{array}{r}\text { Standard Deviation } \mathrm{g} / \mathrm{g} \\
0.000024\end{array}$ \\
\hline$S_{A}=$ & associated standard deviation & 0.2268495 & \\
\hline $\mathbf{F}=$ & purity of starting material & 0.99975 & 0.000085 \\
\hline $\mathbf{b}=$ & air buoyancy & 0.99992 & 0 \\
\hline$W_{1}=$ & Weight of the starting material & 166.45221 & 0.00019 \\
\hline$w_{2}=$ & tare weight of the flask & 176.695 & 0.034 \\
\hline$w_{3}=$ & gross weight of the solution \& flask & 910.209 & 0.034 \\
\hline $\begin{array}{l}W_{4}= \\
S=\end{array}$ & $\begin{array}{l}\left(W_{3}-W_{2}\right)=\text { net weight of solution } \\
\text { standard deviation }\end{array}$ & 733.514 & 0.048 \\
\hline$\overline{S_{A}=}$ & $\left(\bar{c}\left(F^{*} b^{*} W_{1}\right) / W_{4}\right.$ & 0.2268495 & \\
\hline$S=$ & $\left(1 / W_{4}\right)^{*}\left(b^{\wedge} 2^{*}\left(F^{\wedge} 2^{*} S w_{1}^{\wedge} 2+W 1 \wedge 2^{*} S_{F}^{\wedge} 2\right)\right.$ & $\left.2^{\star *}\left(S w 2^{\wedge} 2+S w 3^{\wedge} 2\right)\right)^{\wedge}$ & 0.000024 \\
\hline
\end{tabular}

\section{Synthesized RCTM Uranyl Nitrate Reference Standard II}

\begin{tabular}{|c|c|c|c|}
\hline $\begin{array}{l}\text { Symbol } \\
A=\end{array}$ & $\begin{array}{l}\text { Component } \\
\text { assigned makeup value }\end{array}$ & $\begin{array}{r}\text { Mean Value } \mathrm{g} / \mathrm{g} \\
0.216578\end{array}$ & Standard Deviation $\mathrm{g} / \mathrm{g}$ \\
\hline $\mathbf{S}_{\mathrm{A}}=$ & associated standard deviation & & 0.000019 \\
\hline$F=$ & purity of starting material & 0.99975 & 0.000085 \\
\hline$b=$ & air buoyancy & 0.99992 & 0 \\
\hline$W_{1}=$ & Weight of the starting material & 155.3783 & 0.00011 \\
\hline $\mathbf{w}_{2}=$ & tare weight of the flask & 164.858 & 0.0083 \\
\hline$w_{3}=$ & gross weight of the solution \& flask & 882.044 & 0.0132 \\
\hline $\begin{array}{l}W_{4}= \\
S=\end{array}$ & $\begin{array}{l}\left(W_{3}-W_{2}\right)=\text { net weight of solution } \\
\text { standard deviation }\end{array}$ & 717.186 & 0.0156 \\
\hline $\mathbf{S}_{\mathrm{A}=}$ & 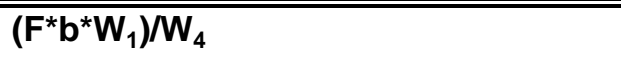 & 0.216578444 & \\
\hline$S=$ & $\left(1 / W_{4}\right) *\left(b^{\wedge} 2 *\left(F^{\wedge} 2 * S^{\prime} w_{1}^{\wedge} 2+W 1 \wedge 2 * S_{F}^{\wedge} 2\right)\right.$ & $\left.2^{*}\left(S w 2^{\wedge} 2+S w 3^{\wedge} 2\right)\right)^{\wedge}$ & 0.000019 \\
\hline
\end{tabular}


Module 14

\section{I nventory Difference (ID) Assessment}




\section{Objectives}

1. Identify how control limits can be used as a method of evaluating ID

2. Identify how various types of errors contributing to the ID affect the ID mean and the ID standard deviation

3. Calculate the loss detection probabilities

4. Identify the characteristics of historic and variance propagation (VP) ID control limits

5. Calculate the uncertainty of the ID 


\section{DOE-STD-1194-2011 Chapter 6.5.5 Evaluating Inventory Programs}

6.5.5.1 - A program for evaluating all special nuclear material (SNM) IDs, including those involving missing items must be developed, documented and implemented...

6.5.5.2 - Procedures for establishing control limits for IDs of SNM must be based on Variance Propagation (VP) using current data.

6.5.5.3 - Assessments of IDs must include statistical tests (for example, tests of trends and biases... 


\section{DOE-STD-1194-2011 Chapter 6.5.5 Evaluating Inventory Programs}

Chapter 6.5.5.2

Other methodologies may be used but they must be approved by the DOE cognizant security authority and must be justified based on factors such as limited data, low transfer rates, and/or material category 


\section{DOE-STD-1194-2011 Performance Requirements MC\&A System Elements}

\subsubsection{4 (7)}

For Category I and II material balance areas (MBAs), limits-of-error (LE) must not exceed two percent of the active inventory during the inventory period and must not exceed a Category II quantity of material 


\section{Objective 1}

\section{I dentify how control limits can be used as a method of evaluating ID}




\section{D Definition}

- ID = Book- EI

- Book inventory - Ending physical inventory (EI)

- Book = Beginning Inventory $(\mathrm{BI})+\mathrm{R}-\mathrm{S}$ where $\mathrm{R}$ represents receipts (or additions) and $S$ represents shipments (or removals)

- The ID is the fundamental indicator of loss of material from the accounting system 


\section{Assessment}

- Construct a control chart for a specific material type for a specific MBA

- X-axis will be an inventory period

- Y-axis will be the value of the ID

- Plot the cumulative ID on the X-axis 


\section{Assessment}

- Calculate the overall mean and standard deviation and the $2 \mathrm{~s}$ and $2.6 \mathrm{~s}$ limits

- The probability exists that an observation from a normal distribution will fall within 2.6 standard deviations from the mean is 0.99 (99\%)

- Many control charts use 3s limits, but the DOE requirement for alarm limits to be set at the $99 \%$ confidence level suggests $2.6 \mathrm{~s}$ be used 


\section{Concerns of the ID Control Chart Methodology}

- All periods are not necessarily independent

- Measurements are correlated

- BI for the nth period is identical to the El of the $\mathrm{n}-1$ period 


\section{Concerns of the I D Control Chart Methodology}

- Since the magnitude of the ID is correlated with the throughput, inventory periods with large or small throughputs will produce erroneous results

- Nevertheless, ID control charts provide a reasonable picture of an MBA's performance 


\section{Objective 2}

\section{I dentify how various types of errors contributing to the ID affect the ID mean, and the ID standard deviation.}




\section{Ds Are Not Zero Because}

- Measurement uncertainty: measurement system effects

- Location of material

- In calorimeter, on scale, etc.

- For example, heat distribution, weight distribution, etc.

- Calibration of scales

- Fluctuations in air pressure, temperature, etc. 


\section{Ds Are Not Zero Because}

- Measurement uncertainty: non-measurementsystem effects

- Power fluctuations

- Electronic functioning of equipment

- Non-homogeneity of material being measured

- Statistical nature of radioactive decay

- Improper or incomplete background measurements 


\section{Ds Are Not Zero Because}

- Sampling effects

- Improper or incomplete blending in a destructive analysis sample

- Nondestructive Analysis (NDA)

limitations

- Material composition of the NDA standards does not match the material composition of the measured items

- Failure to account for background effects 


\section{Ds Are Not Zero Because}

- Accounting system effects

- Better measurement to correct estimates

- Decay, rounding errors, etc.

- Human error

- Clerical mistakes (transcription errors, etc.)

- Failure to follow procedures 


\section{Ds Are Not Zero Because}

- Unmeasured streams or inventories

- Solids entrained in liquid systems settle in tanks

- Holdup can take the form of material associated with specific equipment

- Factors or estimates 


\section{Objective 3}

Calculate the loss detection probabilities 


\section{Evaluating ID}

- In general, recall the following:

- Errors can be total or composed of systematic or random, calculated from standards and/or process materials

- The ID equation contains terms for BI, receipts (or additions/inputs), shipments (removals/outputs), and EI 


\section{Evaluating I D}

- In general, recall:

- ID = BI + R - S - EI

- In this equation, we have "signed" sums (for example, $S$ and El have the minus sign in front of them)

- The impact of the sign is not only on the ID, but also on the systematic error (for example, a bias on an addition and a similar bias on a removal will cancel a systematic error but will not cancel a random error) 


\section{Uncertainty of I D}

- If all terms are random then the following result:

- $\mathbf{I D}=\mathrm{BI}+\mathbf{R}-\mathrm{S}-\mathrm{EI}$

- $\operatorname{Var}(\mathrm{ID})$ = Var (BI) + Var (R) + Var (S) + Var (EI)

- Limit of Error about the ID (LEID) = 2 sqrt (Var(ID))

- We know that many terms are systematic, however, hence a more complicated formula must be used

(Note that variances are additive even though there are minus signs in the ID equation) 


\section{Combining Uncertainties}

The general formula used is the following:

$\operatorname{Var}(M)=\left[\Sigma_{i}\left(s_{r} m_{i}\right)^{2}\right]+\left(s_{s} M\right)^{2}$

Note the

difference

between sum

the squares and

Where:

$m_{i}$ is the content of each of the individual items that are in the same strata

$\mathrm{s}_{\mathrm{r}}=$ Random uncertainty (1s)

$s_{s}=$ Systematic uncertainty (1s)

$M=\Sigma_{i} m_{i}=$ Total for the items within a stratum 


\section{Combining Uncertainties}

- This must be done for all strata:

- For example, you can have an inventory with oxide, nitrate, and scrap

- Each would have random and systematic errors for weighing, sampling, and analytical

- Each of the strata could contain BI, R, S, El terms 


\section{Variance of ID}

\section{$\operatorname{Var}($ ID $)=\left[\Sigma_{\mathrm{i}}\left(\sigma_{\mathrm{r}} \mathrm{m}_{\mathrm{i}}\right)^{2}\right]+\left(\sigma_{\mathrm{s}} \mathrm{M}\right)^{2}$}

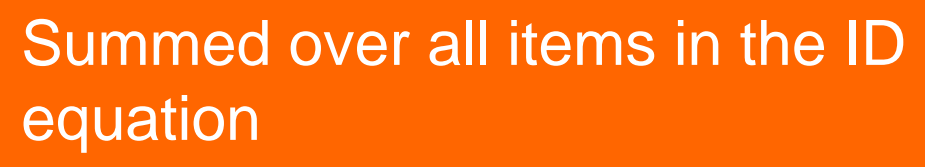

\section{Signed sum over all items in the material balance equation. \\ $\mathrm{BI}$ and $\mathrm{R}$ items are + and El and S are -.}

Example: A site with 10 material types (oxide, metal, etc.) and errors for weight//volume, sampling, analytical, NDA, isotopic could require routine calculation and maintenance of 25-100 uncertainties 


\section{D Uncertainty Considerations}

- Only active inventory items are used in the calculation

- Active inventory is nuclear material contained within the MBA that enters into the calculation of the limit of error and control limit for the MBA

- Noncontributing terms are eliminated from the equation

- Systematic errors 


\section{Example}

\section{Material Balance Report (MBR) format}

\begin{tabular}{|lllc|}
\hline ID Component & $\begin{array}{l}\text { Measurement } \\
\text { Type/Method }\end{array}$ & $\mathrm{kg}$ \\
\hline Beg. Inventory & Feed & 14 Measurements of $2 \mathrm{~kg}$ batches & 28 \\
\hline & In-process & $12 \mathrm{~kg}$ in 1 batch & 12 \\
\hline & Finished Fuel & 75 Measurements of .4 kg batches & 30 \\
\hline Receipt & Feed & 28 Measurements of $2 \mathrm{~kg}$ batches & 56 \\
\hline Shipment & Finished Fuel & 215 Measurements of .4 kg batches & -86 \\
\hline End Inventory & Feed & 18 Measurements of $2 \mathrm{~kg}$ batches & -36 \\
\hline & Scrap & $4 \mathrm{~kg}$ in 1 batch & -4 \\
\hline & & ID is & 0 \\
\hline
\end{tabular}




\section{Example}

\section{Measurement type}

\begin{tabular}{|c|c|c|c|}
\hline ID Component & $\begin{array}{l}\text { Measurement } \\
\text { Type/Method }\end{array}$ & & $\mathrm{kg}$ \\
\hline Beg. Inventory & In-process & $12 \mathrm{~kg} 1$ batch & 12 \\
\hline End Inventory & Scrap & $4 \mathrm{~kg}$ in 1 batch & -4 \\
\hline Beg. Inventory & Finished Fuel & 75 Measurements of $.4 \mathrm{~kg}$ batches & 30 \\
\hline Shipment & Finished Fuel & 215 Measurements of $.4 \mathrm{~kg}$ batches & -86 \\
\hline Beg. Inventory & Feed & 14 Measurements of $2 \mathrm{~kg}$ batches & 28 \\
\hline Receipt & Feed & 28 Measurements of $2 \mathrm{~kg}$ batches & 56 \\
\hline End Inventory & Feed & 18 Measurements of $2 \mathrm{~kg}$ batches & -36 \\
\hline
\end{tabular}




\section{Calculate the LEI D Assuming the Following Uncertainties}

\begin{tabular}{|lcc|}
\hline Measurement Uncertainty & (Relative Standard Deviation in \%) \\
\hline Material Type & Random & Systematic \\
\hline In-process & 0.3 & - \\
\hline Scrap & 0.7 & 0.2 \\
\hline Finished Fuel & 0.25 & 0.05 \\
\hline Feed Material & 0.5 & 0.1 \\
\hline
\end{tabular}




\section{Solution}

- Apply the variance of ID equation to each material type

- Construct a table that summarizes the variances

- Calculate the LEID

- Construct a table where the variances are expressed as a percentage of the total variance to determine where the largest contributors are. 


\section{Finding the I n-Process Variance Contribution}

$\begin{aligned} M_{\text {In-Process }} & =B I_{\text {In-Process }}+R_{\text {In-Process }}-S_{\text {In-Process }}-E I_{\text {In-Process }} \\ & =12 \mathrm{~kg}+0-0-0=12 \mathrm{~kg}\end{aligned}$

$\Sigma\left(\sigma_{r} m_{i}\right)^{2}=(0.003 \times 12 \mathrm{~kg})^{2}+(0.003 \times 0)^{2}+(0.003 \times 0)^{2}+(0.003 \times 0)^{2}$

$$
\begin{aligned}
& =0.001296 k^{2}+0+0+0 \\
& =0.001296 k^{2}
\end{aligned}
$$

$\left(\sigma_{\mathrm{s}} \Sigma \mathrm{m}_{\mathrm{i}}\right)^{2}=0$, since there is no systematic error for in-process

$\operatorname{var}\left(M_{I n-\text { Process }}\right)=\Sigma\left(\sigma_{r} m_{i}\right)^{2}+\left(\sigma_{s} \Sigma m_{i}\right)^{2}$

$=0.001296 \mathrm{~kg}^{2}$ 


\section{Finding the Scrap Variance Contribution}

$\begin{aligned} M_{\text {scrap }} & =B I_{\text {scrap }}+R_{\text {scrap }}-S_{\text {scrap }}-E I_{\text {scrap }} \\ & =0+0-0-4 k g=-4 k g\end{aligned}$

$\Sigma\left(\sigma_{r} m_{i}\right)^{2}=(0.007 \times 0)^{2}+(0.007 \times 0)^{2}+(0.007 \times 0)^{2}+(0.007 \times$ $4 k g)^{2}$

$$
=0.000784 \mathrm{~kg}^{2}
$$

$\left(\sigma_{s} \Sigma m_{i}\right)^{2}=(0.002 \times-4 k g)^{2}=0.000064 k^{2}$

$$
\begin{aligned}
\operatorname{var}\left(M_{\text {scrap }}\right)= & \Sigma\left(\sigma_{r} m_{i}\right)^{2}+\left(\sigma_{s} \Sigma m_{i}\right)^{2} \\
& =0.000784 k^{2}+0.000064 k^{2} \\
& =0.000848 k^{2}
\end{aligned}
$$




\section{Finding the Finished Fuel Variance Contribution}

$$
\begin{aligned}
M_{\text {Final }} & =B I_{\text {Final }}+R_{\text {Final }}-S_{\text {Final }}-E I_{\text {Final }} \\
& =75 \times 0.4 \mathrm{~kg}+0-215 \times 0.4 \mathrm{~kg}-0=-56 \mathrm{~kg}
\end{aligned}
$$

$\Sigma\left(\sigma_{r} m_{i}\right)^{2}=75(0.0025 \times 0.4 \mathrm{~kg})^{2}+0+215(0.0025 \times-0.4 \mathrm{~kg})^{2}+0$

$$
=0.000075 \mathrm{~kg}^{2}+0.000215 \mathrm{~kg}^{2}=0.00029 \mathrm{~kg}^{2}
$$

$\left(\sigma_{s} \Sigma m_{i}\right)^{2}=(0.0005 \times-56 \mathrm{~kg})^{2}=0.000784 \mathrm{~kg}^{2}$

$$
\begin{aligned}
\operatorname{var}\left(M_{\text {Final }}\right)= & \Sigma\left(\sigma_{r} m_{i}\right)^{2}+\left(\sigma_{s} \Sigma m_{i}\right)^{2} \\
& =0.00029 k^{2}+0.000784 k^{2} \\
& =0.001074 k^{2}
\end{aligned}
$$




\section{Finding the Feed Material Variance Contribution}

$M_{\text {Feed }}=B I_{\text {Feed }}+R_{\text {Feed }}-S_{\text {Feed }}-E I_{F e e d}$

$$
=14 \times 2 k g+28 \times 2 k g-0-18 \times 2 k g=48 k g
$$

$\Sigma\left(\sigma_{r} m_{i}\right)^{2}=14(0.005 \times 2 \mathrm{~kg})^{2}+28(0.005 \times 2 \mathrm{~kg})^{2}+0+18(0.005 \times-2 \mathrm{~kg})^{2}$

$$
=0.0014 k^{2}+0.0028 k^{2}+0.0018=0.006 k^{2}
$$

$\left(\sigma_{s} \Sigma m_{i}\right)^{2}=(0.001 \times 48 \mathrm{~kg})^{2}=0.002304 \mathrm{~kg}^{2}$

$$
\begin{aligned}
\operatorname{var}\left(M_{\text {Feed }}\right)= & \Sigma\left(\sigma_{r} m_{i}\right)^{2}+\left(\sigma_{s} \Sigma m_{i}\right)^{2} \\
& =0.006 g^{2}+0.002304 k^{2} \\
& =0.008304 k^{2}
\end{aligned}
$$




\section{Finding the Total Variance and LEI D}

The total variance for the ID is the sum of the variances for all strata.

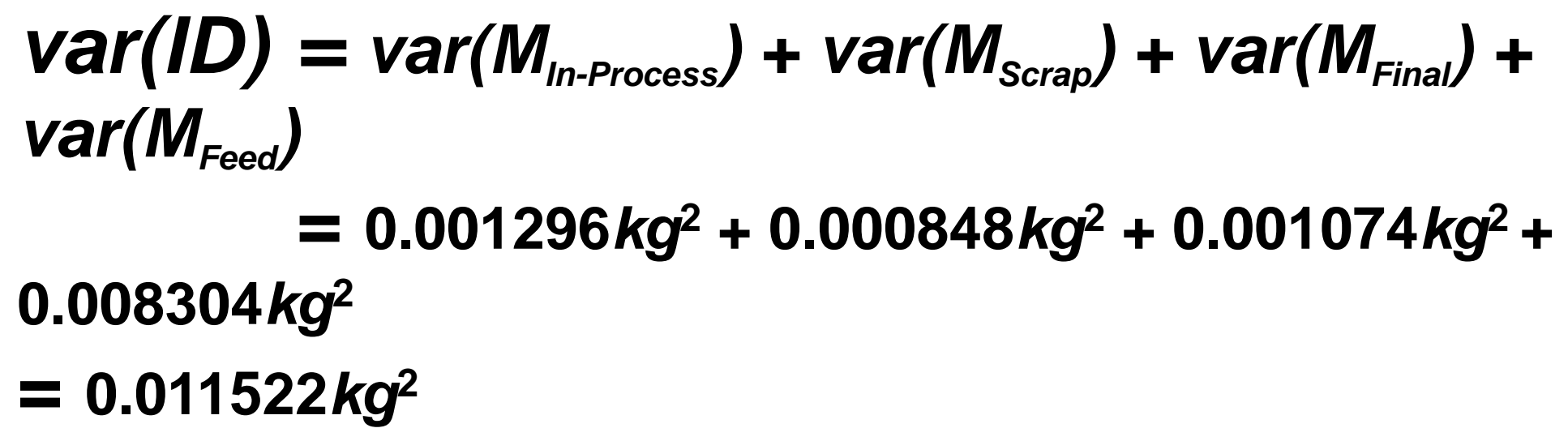

The 2-sigma limit of error about the ID (LEID) is

$$
\begin{aligned}
& \text { LEID }=2 \times \sqrt{\operatorname{var}(I D)}=2 \times \sqrt{0.011522 \mathrm{~kg}^{2}}= \\
& 0.214681 \mathrm{~kg}
\end{aligned}
$$




\section{Table Solution}

\begin{tabular}{|c|c|c|c|c|}
\hline Variances & Random & Systematic & & \\
\hline \multirow[t]{2}{*}{ Feed } & $14^{\star}\left(0.005^{\star} 2\right)^{\wedge} 2+28^{\star}\left(0.005^{\star} 2\right)^{\wedge} 2+0+18^{\star}\left(0.005^{\star}-2\right)^{\wedge} 2$ & {$[(24)(2)(.001)]^{\wedge} 2=$} & & \\
\hline & 0.006000 & 0.002304 & $\rightarrow$ & 0.008304 \\
\hline \multirow[t]{2}{*}{ In Process } & {$[(12)(.003)]^{\wedge} 2=$} & 0 & & \\
\hline & 0.001296 & 0.000000 & $\rightarrow$ & 0.001296 \\
\hline \multirow[t]{2}{*}{ Finished Fuel } & $75^{\star}\left(0.0025^{\star} 0.4\right)^{\wedge} 2+0+215^{\star}\left(0.0025^{\star}(-0.4)\right)^{\wedge} 2+0$ & {$[(140)(.4)(.0005)]^{\wedge} 2=$} & & \\
\hline & 0.000290 & 0.000784 & $\rightarrow$ & 0.001074 \\
\hline \multirow[t]{4}{*}{ Scrap } & {$[(4)(.007)]^{\wedge} 2=$} & {$[(4)(.002)]^{\wedge} 2=$} & & \\
\hline & 0.000784 & 0.000064 & $\rightarrow$ & 0.000848 \\
\hline & $\downarrow$ & $\downarrow$ & & \\
\hline & 0.008370 & 0.003152 & $\rightarrow$ & 0.011522 \\
\hline \multicolumn{2}{|c|}{ Units are typically grams ${ }^{2}$ or kilogram² } & & $\begin{array}{r}\quad s I D= \\
=I D(2 s)\end{array}$ & $\begin{array}{l}0.107341 \\
0.214681\end{array}$ \\
\hline
\end{tabular}




\section{Summarize the Variance Terms $\left(\mathbf{k g}^{2}\right)$}

\begin{tabular}{|l|l|l|r|}
\hline Component & Random & Systematic & Total \\
\hline Feed & 0.00600 & 0.002304 & 0.008304 \\
\hline In-process & 0.001296 & 0 & 0.001296 \\
\hline Finished Fuel & 0.000290 & 0.000784 & 0.001074 \\
\hline Scrap & 0.000784 & 0.000064 & 0.000848 \\
\hline Total & 0.00837 & 0.003152 & 0.011522 \\
\hline & & S ID $^{\mid}$ & 0.107341 \\
\hline & & LEID $\left(2 \mathrm{~s}_{\text {ID }}\right)$ & 0.214681 \\
\hline
\end{tabular}




\section{Solution Percent Contributors}

\begin{tabular}{|l|r|r|r|}
\hline Component & Random & Systematic & Total \\
\hline Feed & 52.1 & 20.0 & 72.1 \\
\hline In-process & 11.2 & 0.0 & 11.2 \\
\hline Finished Fuel & 2.5 & 6.8 & 9.3 \\
\hline Scrap & 6.8 & 0.6 & 7.4 \\
\hline Total & 72.6 & 27.4 & 100.0 \\
\hline
\end{tabular}




\section{Analysis of LEI D}

- What are the largest uncertainty contributors?

- How would you reduce the overall uncertainty?

- If a variance component is very large, is it because the uncertainty was very large or because the amount of material subject to that uncertainty was very large? 


\section{Activity 1}

\section{Material balance}




\section{Activity 1}

- Compute the overall uncertainty of the ID and LEID

- Determine where to allocate efforts to reduce the overall uncertainty

- Discuss the implications of how the nitrate $\mathrm{BI}$, receipts, and $\mathrm{El}$ affect the LEID

- Develop at least three recommendations and be prepared to defend them 


\section{Activity 1}

\section{Analyses Using Variance Contributions}

\begin{tabular}{|lrr|}
\hline Nitrate & $\begin{array}{r}\text { Random } \\
\text { (percent) }\end{array}$ & $\begin{array}{r}\text { Systematic } \\
\text { (percent) }\end{array}$ \\
\hline Oxide & $28.5 \%$ & $28.5 \%$ \\
\hline NDA & $0.1 \%$ & $0.9 \%$ \\
\hline Buttons & $15.6 \%$ & $1.0 \%$ \\
\hline Waste Oxide & $0.3 \%$ & $13.7 \%$ \\
\hline Totals & $0.6 \%$ & $10.8 \%$ \\
\hline
\end{tabular}




\section{Objective 4}

I dentify the characteristics of historic and Variance Propagation I D control limits 


\section{Factors Affecting I D Mean and Standard Deviation}

- What will the following do to the mean and standard deviation of the ID sequence?

- A loss each inventory period of $X$ grams of material

- A large constant systematic error or bias in a flow (S or R) measurement 


\section{Factors Affecting ID Mean and Standard Deviation}

- What will the following do to the mean and standard deviation of the ID sequence?

- A large constant systematic error or bias in an inventory measurement

- A large random error in an inventory or flow measurement 


\section{Characteristics of Historical Limits}

- Easy to compute, reflect real process

- Process stable

- Large uncertainty in standard deviation unless a large quantity of ID data is used 
Characteristics of Historical Limits

- Abnormal conditions can unduly affect control limits

- Do not encourage improvement of practices 


\section{Validating Variance Propagation Calculations}

DOE-STD-1194-2011 6.5.5.2 states: "Significant differences between historical limits and limits based on variance propagation must be investigated for the purpose of validating, revising, and refining the variance propagation model."

- What do you do when they disagree?

- What limits do you use? 


\section{Objective 5}

\section{Calculate the uncertainty of the ID}




\section{Hypothetical MBA Structure}

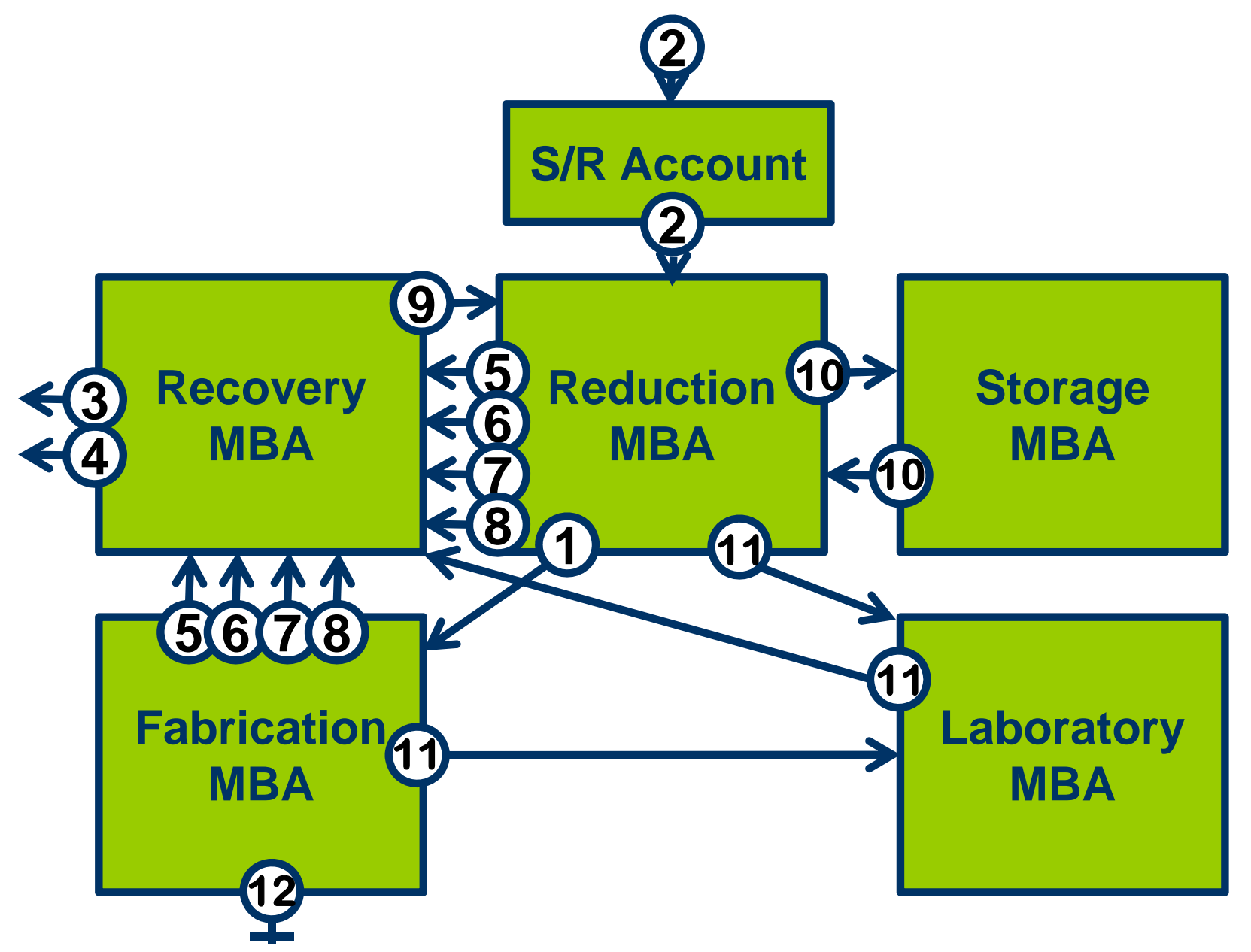

1: Buttons

2: Scrap Oxide

3: LL Solid Waste

4: LL Liquid Waste

5: Solid Waste

6: Liquid Waste

7: Metal Scrap

8: Scrap Powder

9: Recovered Oxide

10: Sweepings

11: Samples

12: Metal Parts 


\section{D Questions}

1. Suppose that the solid waste stream (5) is measured by an NDA instrument that has a positive bias (overstates the amount)

- What will this do to the ID in the reduction MBA and the recovery MBA?

- What will it do to the plant ID?

\begin{tabular}{|l|c|c|c|c|c|}
\hline & BI+ & R+ & El- & S- & ID \\
\hline Reduction MBA & 5 & & & $5+2$ & -2 \\
\hline Recovery MBA & & $5+2$ & & $5+2$ & 0 \\
\hline
\end{tabular}




\section{D Questions}

2. Suppose that an item of metal scrap (7) is removed from the reduction MBA and fed to the recovery MBA, but is not logged in the accounting records through human error

- What is the effect on the IDs?

\begin{tabular}{|l|c|c|c|c|r|}
\hline & BI+ & R+ & El- & S- & ID \\
\hline Reduction MBA & 4 & & 0 & & 4 \\
\hline Recovery MBA & 0 & & 4 & & -4 \\
\hline
\end{tabular}




\section{D Questions}

3. Suppose that the value of an intermediate-product inventory item (for example, a can of off-spec oxide) in the recovery MBA is overestimated on the June 30 physical inventory.

- What does this do to the June ID?

- If it is processed in July, what does it do to the July ID? If it is not processed?

\begin{tabular}{|l|c|c|c|c|c|}
\hline & $\mathrm{BI+}$ & $\mathrm{R}+$ & $\mathrm{EI}-$ & $\mathrm{S}-$ & ID \\
\hline June ID & 6 & & $6+3$ & & -3 \\
\hline July ID & $6+3$ & & 6 & & 3 \\
\hline
\end{tabular}




\section{Activity 2}

Effects of various types of errors on the ID 


\section{Activity 2}

On the Activity 2 worksheet, answer the questions about the effect of various types of errors on the ID 


\section{Hypothetical MBA Structure}

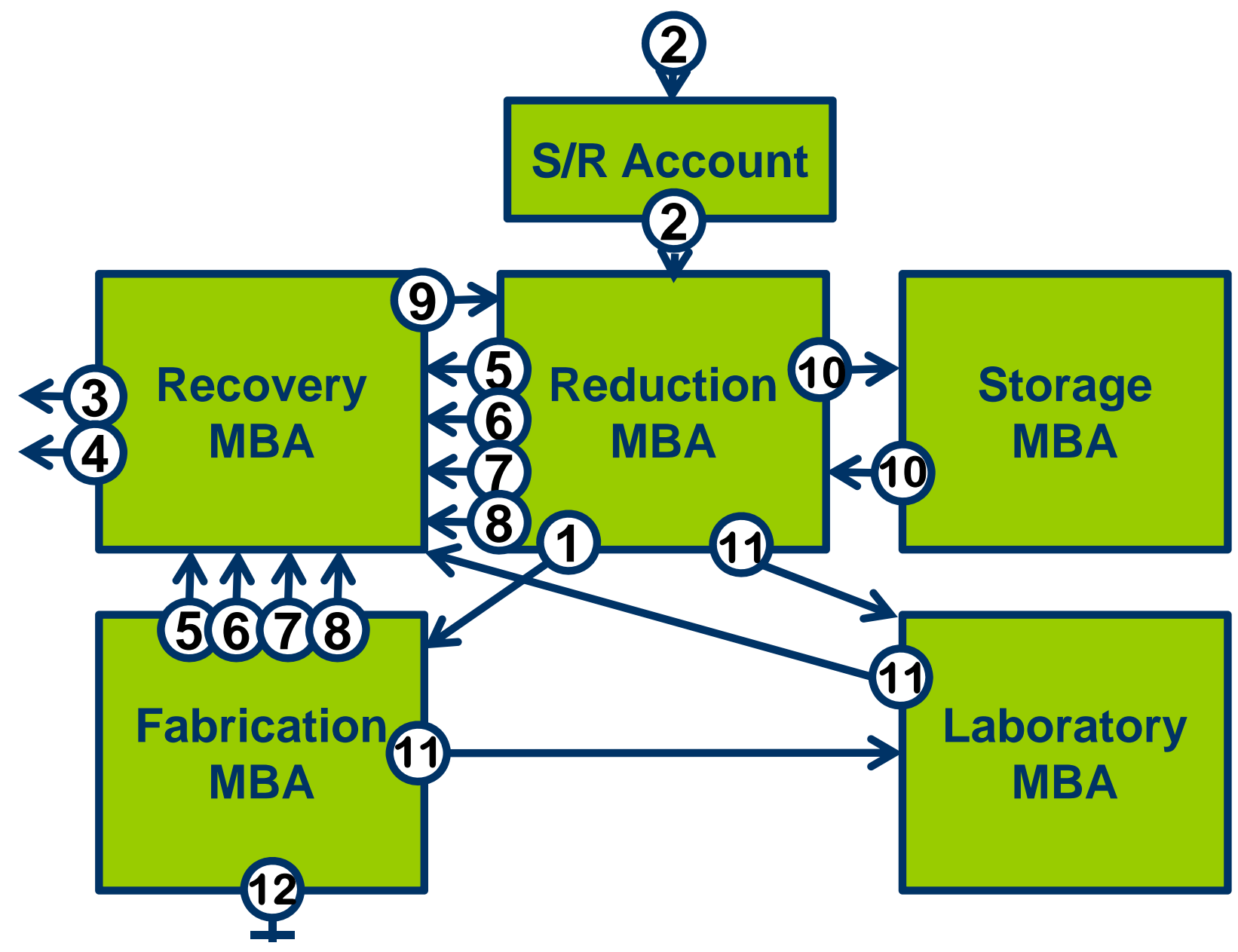

1: Buttons

2: Scrap Oxide

3: LL Solid Waste

4: LL Liquid Waste

5: Solid Waste

6: Liquid Waste

7: Metal Scrap

8: Scrap Powder

9: Recovered Oxide

10: Sweepings

11: Samples

12: Metal Parts 


\section{Lesson Summary}

1. Identify how control limits can be used as a method of evaluating ID

2. Identify how various types of errors contributing to the ID, effect the ID mean and the ID standard deviation

3. Calculate the loss detection probabilities

4. Identify the characteristics of historic and Variance Propagation ID control limits.

5. Calculate the uncertainty of the ID 


\section{Inventory Difference Assessment}

\section{Activity 1 \\ Sample Detailed Material Balance}

Using the material balance, uncertainty information, and variance contributions tables from a facility that produces metal plutonium buttons and has nitrate input, and oxide conversion, complete the following tasks:

1. Compute the overall uncertainty of the inventory difference (ID) and limit of error of the ID (LEID).

- Ignore sample, liquid waste, and air discards.

2. Determine where to allocate efforts to reduce the overall uncertainty.

- Discuss the implications of how the nitrate BI, receipts, and EI impact the LEID.

- Develop at least three recommendations and be prepared to defend them.

Material Balance Table

\begin{tabular}{|c|c|c|c|}
\hline Component & Detail & $\begin{array}{c}\text { Quantity } \\
\text { [Pu-grams (g)] }\end{array}$ & Totals \\
\hline \multicolumn{4}{|c|}{ Beginning Inventory (BI) } \\
\hline Nitrate & 200 liters (L) @ 325 g/L; one batch & 65,000 & \\
\hline Oxide & 8 cans @ 1500 g/can & 12,000 & \\
\hline \multirow[t]{2}{*}{$\begin{array}{l}\text { Equipment Holdup } \\
\text { (NDA) }\end{array}$} & $1850 \mathrm{~g}$; one batch & 1,850 & \\
\hline & & Total BI & 78,850 \\
\hline \multicolumn{4}{|l|}{ Receipts (R) } \\
\hline \multirow[t]{2}{*}{ Nitrate } & 10 batches @ 50 L/batch \& 350 g/L & 175,000 & \\
\hline & & Total Receipts & 175,000 \\
\hline \multicolumn{4}{|l|}{ Shipments (S) } \\
\hline Metal Buttons & 100 buttons @ 2,000 g & 200,000 & \\
\hline \multirow[t]{2}{*}{ Solid Waste } & $\begin{array}{l}\text { Off-specification oxide } 3 \text { cans @ } \\
1,100 \text { g/can }\end{array}$ & 3,300 & \\
\hline & Total Shipments = & Total Shipments & 203,300 \\
\hline \multicolumn{4}{|c|}{ Ending Inventory (EI) } \\
\hline Nitrate & 150 L @ 300 g/L; one batch & 45,000 & \\
\hline \multirow[t]{3}{*}{$\begin{array}{l}\text { Equipment Holdup } \\
\text { (NDA) }\end{array}$} & & 3,500 & \\
\hline & & Total EI & 48,500 \\
\hline & & $B I+R-S-E I=I D$ & 2,050 \\
\hline
\end{tabular}




\section{Uncertainty Information Table}

\begin{tabular}{|l|l|r|r|}
\hline \multicolumn{1}{|c|}{ Material } & \multicolumn{1}{c|}{ Method } & Random & Systematic \\
\hline Nitrate & Calibration & $1.00 \%$ & $0.40 \%$ \\
\hline & Sampling & $0.75 \%$ & $0.50 \%$ \\
\hline & Analytical & $0.60 \%$ & $0.25 \%$ \\
\hline Oxide & Weight & $0.15 \%$ & $0.10 \%$ \\
\hline & Sampling & $1.50 \%$ & $1.75 \%$ \\
\hline & Analytical & $1.25 \%$ & $0.90 \%$ \\
\hline Equipment & NDA & $25.00 \%$ & $15.00 \%$ \\
\hline Metal & Weight & $0.15 \%$ & $0.10 \%$ \\
\hline & Sampling & $0.05 \%$ & $0.06 \%$ \\
\hline & Analytical & $0.70 \%$ & $0.45 \%$ \\
\hline \hline Solid Waste & NDA & $10.00 \%$ & $25.00 \%$ \\
\hline & & &
\end{tabular}

\section{Variance Contributions Table}

\begin{tabular}{|l|r|r|}
\hline Material & \multicolumn{1}{|c|}{ Random } & \multicolumn{1}{c|}{ Systematic } \\
\hline Nitrate & $28.5 \%$ & $28.5 \%$ \\
\hline Oxide & $0.1 \%$ & $0.9 \%$ \\
\hline NDA & $15.6 \%$ & $1.0 \%$ \\
\hline Buttons & $0.3 \%$ & $13.7 \%$ \\
\hline $\begin{array}{l}\text { Waste } \\
\text { Oxide }\end{array}$ & $0.6 \%$ & $10.8 \%$ \\
\hline \hline Totals & $45.0 \%$ & $55.0 \%$ \\
\hline
\end{tabular}


Chinese Measurement Control Workshop

Inventory Difference Assessment Module

Activity 1 LEID Exercise

Student Worksheet

\begin{tabular}{|l|l|c|c|}
\hline \multicolumn{3}{|c|}{ Measurement Uncertainties (Percent Relative Standard Deviations) } \\
\hline Material Type & \multicolumn{1}{|c|}{ Method } & Random & Systematic \\
\hline & Calibration & $1.00 \%$ & $0.40 \%$ \\
\hline & Sampling & $0.75 \%$ & $0.50 \%$ \\
\hline & Analytical & $0.60 \%$ & $0.25 \%$ \\
\hline \hline & Oxide & $0.15 \%$ & $0.10 \%$ \\
\hline & Sampling & $1.50 \%$ & $1.75 \%$ \\
\hline & Analytical & $1.25 \%$ & $0.90 \%$ \\
\hline Equipment & NDA & $25.00 \%$ & $15.00 \%$ \\
\hline Metal & Weight & $0.15 \%$ & $0.10 \%$ \\
\hline & Sampling & $0.05 \%$ & $0.06 \%$ \\
\hline & Analytical & $0.70 \%$ & $0.45 \%$ \\
\hline Solid Waste & NDA & $10.00 \%$ & $25.00 \%$ \\
\hline
\end{tabular}

\begin{tabular}{|c|c|c|}
\hline \multicolumn{3}{|c|}{ Material Balance Table } \\
\hline Component & Detail Description & $\begin{array}{c}\text { Compute Pu } \\
\text { Grams }\end{array}$ \\
\hline \multicolumn{3}{|l|}{ Beginning Inventory (BI) } \\
\hline Nitrate & 200 liters (L) at $325 \mathrm{~g} / \mathrm{L}$; one batch & 0 \\
\hline Oxide & 8 cans at $1500 \mathrm{~g} / \mathrm{can}$ & 0 \\
\hline \multirow[t]{2}{*}{ Equipment Holdup (NDA) } & 1850 grams; one batch & 0 \\
\hline & Total $\mathrm{BI}=$ & 0 \\
\hline \multicolumn{3}{|l|}{ Receipts ( R ) } \\
\hline \multirow[t]{2}{*}{ Nitrate } & 10 batches at $50 \mathrm{~L} / \mathrm{batch}$ and $350 \mathrm{~g} / \mathrm{L}$ & 0 \\
\hline & Total Receipts $=$ & 0 \\
\hline \multicolumn{3}{|l|}{ Shipments (S) } \\
\hline Metal Buttons & 100 buttons at 2,000 grams each & 0 \\
\hline \multirow[t]{2}{*}{ Solid Waste } & Off-specification oxide, 3 cans at $1,100 \mathrm{~g} / \mathrm{can}$ & 0 \\
\hline & Total Shipments = & 0 \\
\hline \multicolumn{3}{|l|}{ Ending Inventory (EI) } \\
\hline Nitrate & 150 liters $(\mathrm{L})$ at $300 \mathrm{~g} / \mathrm{L}$; one batch & 0 \\
\hline \multirow[t]{3}{*}{ Equipment Holdup (NDA) } & 3,500 grams; one batch & 0 \\
\hline & Total El = & 0 \\
\hline & $I D=B I+R-S-E I=$ & 0 \\
\hline
\end{tabular}


Activity 1 LEID Exercise

Student Worksheet

\begin{tabular}{|l|l|r|}
\hline \multicolumn{1}{|c|}{ Component } & \multicolumn{1}{c|}{ Material Balance Table for LEID Calculations } & $\begin{array}{r}\text { Pu Grams } \\
\text { from Above }\end{array}$ \\
\hline Nitrate & \multicolumn{1}{|c|}{ Detail Description } & 0 \\
\hline BI & 200 liters (L) at 325 g/L; one batch & 0 \\
\hline R & 10 batches at 50 L/batch and 350 g/L & 0 \\
\hline El & 150 liters (L) at 300 g/L; one batch & 0 \\
\hline Oxide & & \\
\hline BI & & \\
\hline Metal Buttons & 8 cans at 1500g/can & 0 \\
\hline S & & \\
\hline Solid Waste & & \\
\hline S & 100 buttons at 2,000 grams each & 0 \\
\hline Equipment Holdup (NDA) & & \\
\hline BI & & \\
\hline El & Off-specification oxide, 3 cans at 1,100 g/can & 0 \\
\hline & 1850 grams; one batch & \\
\hline
\end{tabular}

\begin{tabular}{|c|c|c|c|}
\hline \multicolumn{4}{|c|}{ LEID and Percent of Variance Calculations } \\
\hline Component & Random Variance & Systematic Variance & Total \\
\hline \multicolumn{4}{|l|}{ Nitrate } \\
\hline \multicolumn{4}{|l|}{ Formula } \\
\hline Variances & 0 & 0 & 0 \\
\hline Percentage & $0.00 \%$ & $0.00 \%$ & \\
\hline \multicolumn{4}{|l|}{ Oxide } \\
\hline \multicolumn{4}{|l|}{ Formula } \\
\hline Variances & 0 & 0 & 0 \\
\hline Percentage & $0.00 \%$ & $0.00 \%$ & \\
\hline \multicolumn{4}{|l|}{ Metal Buttons } \\
\hline \multicolumn{4}{|l|}{$\begin{array}{r}\text { Formula } \\
\end{array}$} \\
\hline Variances & 0 & 0 & 0 \\
\hline Percentage & $0.00 \%$ & $0.00 \%$ & \\
\hline \multicolumn{4}{|l|}{ Solid Waste } \\
\hline Formula & $3^{\star} 1100^{\wedge} 2^{\star} 0.10^{\wedge} 2$ & $\left(3^{\star} 1100\right)^{\wedge} 2^{\star} 0.25^{\wedge} 2$ & \\
\hline Variances & 36,300 & 680,625 & 716,925 \\
\hline Percentage & $5.06 \%$ & $94.94 \%$ & \\
\hline \multicolumn{4}{|l|}{\begin{tabular}{|l|} 
Holdup (NDA) \\
\end{tabular}} \\
\hline \multicolumn{4}{|l|}{\begin{tabular}{|r|} 
Formula \\
\end{tabular}} \\
\hline Variances & 0 & 0 & 0 \\
\hline Percentage & $0.00 \%$ & $0.00 \%$ & \\
\hline \multirow[t]{4}{*}{ Total } & & & 716,925 \\
\hline & & $\mathrm{ID}=$ & 0 \\
\hline & & $\mathrm{STD}(\mathrm{ID})=$ & 847 \\
\hline & & LEID $=2 * \operatorname{STD}(\mathrm{ID})=$ & 1,693 \\
\hline
\end{tabular}




\section{Inventory Difference Assessment}

\section{Activity 1, Sample Detailed Material Balance}

Using the material balance, uncertainty information, and variance contributions tables from a facility that produces metal plutonium buttons and has nitrate input, and oxide conversion, complete the following tasks:

1. Compute the overall uncertainty of the inventory difference (ID) and limit of error of the ID (LEID).

- Ignore sample, liquid waste, and air discards.

2. Determine where to allocate efforts to reduce the overall uncertainty.

- Discuss the implications of how the nitrate BI, receipts, and EI impact the LEID.

- Develop at least three recommendations and be prepared to defend them.

Material Balance Table

\begin{tabular}{|c|c|c|c|}
\hline \multirow{2}{*}{$\begin{array}{l}\text { Component } \\
\text { Beginning Invento }\end{array}$} & Detail & \multicolumn{2}{|c|}{ Quantity [Pu-grams (g)] $\quad$ Totals } \\
\hline & \\
\hline Nitrate & 200 liters (L) @ 325 g/L; one batch & 65,000 & \\
\hline Oxide & 8 cans @ $1500 \mathrm{~g} / \mathrm{can}$ & 12,000 & \\
\hline \multirow{2}{*}{$\begin{array}{l}\text { Equipment Holdup } \\
\text { (NDA) }\end{array}$} & $1850 \mathrm{~g}$; one batch & 1,850 & \\
\hline & & Total BI & 78,850 \\
\hline \multicolumn{4}{|c|}{ Receipts (Additions) } \\
\hline \multirow[t]{2}{*}{ Nitrate } & 10 batches @ 50 L/batch \& 350 g/L & 175,000 & \\
\hline & & Total Add & 175,000 \\
\hline \multicolumn{4}{|c|}{ Shipments (Removals) } \\
\hline Metal Buttons & 100 buttons @ 2,000 g & 200,000 & \\
\hline \multirow[t]{2}{*}{ Solid Waste } & $\begin{array}{l}\text { Off-specification oxide } 3 \text { cans @ 1,100 } \\
\text { g/can }\end{array}$ & 3,300 & \\
\hline & & Total Removals & 203,300 \\
\hline \multicolumn{4}{|l|}{ Ending Inventory } \\
\hline Nitrate & $150 \mathrm{~L} @ 300 \mathrm{~g} / \mathrm{L} ;$ one batch & 45,000 & \\
\hline \multirow[t]{3}{*}{$\begin{array}{l}\text { Equipment Holdup } \\
\text { (NDA) }\end{array}$} & & 3,500 & \\
\hline & & Total El & 48,500 \\
\hline & & $B I+A-R-E I=I D$ & 2,050 \\
\hline
\end{tabular}




\begin{tabular}{|l|l|r|r|}
\multicolumn{4}{c}{ Uncertainty Information Table } \\
\hline & Method & $\begin{array}{r}\text { Random } \\
\text { (percent) }\end{array}$ & $\begin{array}{r}\text { Systematic } \\
\text { (percent) }\end{array}$ \\
\hline Nitrate & Calibration & 1.000 & 0.400 \\
\hline & Sampling & 0.750 & 0.500 \\
\hline & Analytical & 0.600 & 0.250 \\
\hline Oxide & Weight & 0.150 & 0.100 \\
\hline & Sampling & 1.500 & 1.750 \\
\hline \hline Equipment & Analytical & 1.250 & 0.900 \\
\hline \hline Metal & NDA & 25.000 & 15.000 \\
\hline & Weight & 0.150 & 0.100 \\
\hline & Sampling & 0.050 & 0.060 \\
\hline \hline Solid Waste & NDalytical & 0.700 & 0.450 \\
\hline & NDA & 10.000 & 25.000 \\
\hline
\end{tabular}

\begin{tabular}{|l|r|r|}
\hline \multicolumn{2}{|c}{ Variance Contributions Table } \\
\hline Nitrate & $\begin{array}{r}\text { Random } \\
\text { (percent) }\end{array}$ & $\begin{array}{r}\text { Systematic } \\
\text { (percent) }\end{array}$ \\
\hline Oxide & $0.1 \%$ & $28.5 \%$ \\
\hline NDA & $15.6 \%$ & $0.9 \%$ \\
\hline Buttons & $0.3 \%$ & $1.0 \%$ \\
\hline $\begin{array}{l}\text { Waste } \\
\text { Oxide }\end{array}$ & $0.6 \%$ & $13.7 \%$ \\
\hline \hline Totals & $45.0 \%$ & $10.8 \%$ \\
\hline
\end{tabular}


Chinese Measurement Control Workshop

Inventory Difference Assessment Module

Activity 1 LEID Exercise

Instructor Worksheet

\begin{tabular}{|l|l|c|c|}
\hline \multicolumn{3}{|c|}{ Measurement Uncertainties (Percent Relative Standard Deviations) } \\
\hline Material Type & \multicolumn{1}{|c|}{ Method } & Random & Systematic \\
\hline & Calibration & $1.00 \%$ & $0.40 \%$ \\
\hline & Sampling & $0.75 \%$ & $0.50 \%$ \\
\hline & Analytical & $0.60 \%$ & $0.25 \%$ \\
\hline \hline & Oxide & $0.15 \%$ & $0.10 \%$ \\
\hline & Sampling & $1.50 \%$ & $1.75 \%$ \\
\hline & Analytical & $1.25 \%$ & $0.90 \%$ \\
\hline Equipment & NDA & $25.00 \%$ & $15.00 \%$ \\
\hline Metal & Weight & $0.15 \%$ & $0.10 \%$ \\
\hline & Sampling & $0.05 \%$ & $0.06 \%$ \\
\hline & Analytical & $0.70 \%$ & $0.45 \%$ \\
\hline Solid Waste & NDA & $10.00 \%$ & $25.00 \%$ \\
\hline
\end{tabular}

\begin{tabular}{|c|c|c|}
\hline \multicolumn{3}{|c|}{ Material Balance Table } \\
\hline Component & Detail Description & $\begin{array}{l}\text { Compute Pu } \\
\text { Grams }\end{array}$ \\
\hline \multicolumn{3}{|l|}{ Beginning Inventory (BI) } \\
\hline Nitrate & 200 liters $(\mathrm{L})$ at $325 \mathrm{~g} / \mathrm{L}$; one batch & 65,000 \\
\hline Oxide & 8 cans at $1500 \mathrm{~g} / \mathrm{can}$ & 12,000 \\
\hline \multirow[t]{2}{*}{ Equipment Holdup (NDA) } & 1850 grams; one batch & 1,850 \\
\hline & Total $\mathrm{BI}=$ & 78,850 \\
\hline \multicolumn{3}{|l|}{ Receipts ( R ) } \\
\hline \multirow[t]{2}{*}{ Nitrate } & 10 batches at $50 \mathrm{~L} / \mathrm{batch}$ and $350 \mathrm{~g} / \mathrm{L}$ & 175,000 \\
\hline & Total Receipts $=$ & 175,000 \\
\hline \multicolumn{3}{|l|}{ Shipments (S) } \\
\hline Metal Buttons & 100 buttons at 2,000 grams each & 200,000 \\
\hline \multirow[t]{2}{*}{ Solid Waste } & Off-specification oxide, 3 cans at 1,100 g/can & 3,300 \\
\hline & Total Shipments = & 203,300 \\
\hline \multicolumn{3}{|l|}{ Ending Inventory (EI) } \\
\hline Nitrate & 150 liters $(\mathrm{L})$ at $300 \mathrm{~g} / \mathrm{L}$; one batch & 45,000 \\
\hline \multirow[t]{3}{*}{ Equipment Holdup (NDA) } & 3,500 grams; one batch & 3,500 \\
\hline & Total El = & 48,500 \\
\hline & $\mathrm{ID}=\mathrm{BI}+\mathrm{R}-\mathrm{S}-\mathrm{EI}=$ & 2,050 \\
\hline
\end{tabular}


Chinese Measurement Control Workshop

Inventory Difference Assessment Module

Activity 1 LEID Exercise

Instructor Worksheet

\begin{tabular}{|c|c|c|}
\hline \multicolumn{3}{|c|}{ Material Balance Table for LEID Calculations } \\
\hline Component & Detail Description & $\begin{array}{l}\text { Pu Grams } \\
\text { from Above }\end{array}$ \\
\hline \multicolumn{3}{|l|}{ Nitrate } \\
\hline $\mathrm{BI}$ & 200 liters $(L)$ at $325 \mathrm{~g} / \mathrm{L} ;$ one batch & 65,000 \\
\hline $\mathrm{R}$ & 10 batches at $50 \mathrm{~L} / \mathrm{batch}$ and $350 \mathrm{~g} / \mathrm{L}$ & 175,000 \\
\hline $\mathrm{El}$ & 150 liters $(\mathrm{L})$ at $300 \mathrm{~g} / \mathrm{L} ;$ one batch & 45,000 \\
\hline \multicolumn{3}{|l|}{ Oxide } \\
\hline $\mathrm{BI}$ & 8 cans at $1500 \mathrm{~g} / \mathrm{can}$ & 12,000 \\
\hline \multicolumn{3}{|l|}{ Metal Buttons } \\
\hline $\mathrm{S}$ & 100 buttons at 2,000 grams each & 200,000 \\
\hline \multicolumn{3}{|l|}{ Solid Waste } \\
\hline S & Off-specification oxide, 3 cans at $1,100 \mathrm{~g} / \mathrm{can}$ & 3,300 \\
\hline \multicolumn{3}{|l|}{ Equipment Holdup (NDA) } \\
\hline $\mathrm{BI}$ & 1850 grams; one batch & 1,850 \\
\hline $\mathrm{EI}$ & 3,500 grams; one batch & 3,500 \\
\hline
\end{tabular}

\begin{tabular}{|c|c|c|c|}
\hline \multicolumn{4}{|c|}{ Variance, Percent of Variance and LEID Calculations } \\
\hline Component & Random Variance & Systematic Variance & Totals \\
\hline \multicolumn{4}{|l|}{ Nitrate } \\
\hline Formula & $\left(65000^{\wedge} 2+10^{\star} 17500^{\wedge} 2+45000^{\wedge} 2\right)^{\star}\left(0.01^{\wedge} 2+0.0075^{\wedge} 2+0.006^{\wedge} 2\right)$ & $(65000+175000-45000)^{\wedge} 2^{\star}\left(0.004^{\wedge} 2+0.005^{\wedge} 2+0.0025^{\wedge} 2\right)$ & \\
\hline Variances & $1,790,328$ & \begin{tabular}{|c|}
$1,796,681$ \\
\end{tabular} & $3,587,009$ \\
\hline Percentage & $28.67 \%$ & $28.78 \%$ & \\
\hline \multicolumn{4}{|l|}{ Oxide } \\
\hline Formula & $\left(8^{\star} 1500^{\wedge} 2\right)^{\star}\left(0.0015^{\wedge} 2+0.015^{\wedge} 2+0.0125^{\wedge} 2\right)$ & $\left(8^{\star} 1500^{\wedge} 2\right)^{\star}\left(0.001^{\wedge} 2+0.0175^{\wedge} 2+0.009^{\wedge} 2\right)$ & \\
\hline Variances & 6,903 & 6,989 & 13,892 \\
\hline Percentage & $0.11 \%$ & $0.11 \%$ & \\
\hline \multicolumn{4}{|l|}{ Metal Buttons } \\
\hline Formula & $\left(100^{\star} 2000^{\wedge} 2\right)^{\star}\left(0.0015^{\wedge} 2+0.0005^{\wedge} 2+0.007^{\wedge} 2\right)$ & $\left(100^{\star} 2000\right)^{\wedge} 2^{\star}\left(0.001^{\wedge} 2+0.0006^{\wedge} 2+0.0045^{\wedge} 2\right)$ & \\
\hline Variances & 20,600 & 864,400 & 885,000 \\
\hline Percentage & $0.33 \%$ & $13.84 \%$ & \\
\hline \multicolumn{4}{|l|}{ Solid Waste } \\
\hline Formula & $\left(3^{\star} 1100^{\wedge} 2\right)^{\star}\left(0.10^{\wedge} 2\right)$ & $\left(3^{\star} 1100\right)^{\wedge} 2^{\star}\left(0.25^{\wedge} 2\right)$ & \\
\hline Variances & 36,300 & 680,625 & 716,925 \\
\hline Percentage & $0.58 \%$ & $10.90 \%$ & \\
\hline \multicolumn{4}{|l|}{ Holdup (NDA) } \\
\hline Formula & $\left(1850^{\wedge} 2+3500^{\wedge} 2\right)^{\star}\left(0.25^{\wedge} 2\right)$ & $(1850-3500)^{\wedge} 2^{\star}\left(0.15^{\wedge} 2\right)$ & \\
\hline Variances & 979,531 & 61,256 & $1,040,788$ \\
\hline Percentage & $15.69 \%$ & $0.98 \%$ & \\
\hline \multirow[t]{4}{*}{ Total } & & & $6,243,613$ \\
\hline & & ID $=$ & 2,050 \\
\hline & & STD $(I D)=$ & 2,499 \\
\hline & & LEID $=2 *$ STD $(I D)=$ & 4,997 \\
\hline
\end{tabular}




\section{Activity 1}

\section{Answers}




\section{Activity 1}

Random error of nitrate

$\Sigma_{\mathrm{i}}\left(\sigma_{\mathrm{r}} \mathbf{M}_{\mathrm{i}}\right)^{2}$ :

$\left(0.01^{2}+0.0075^{2}+0.006^{2}\right)^{\star}\left(65,000^{2}+\right.$ $\left.175,000^{2} / 10+45,000^{2}\right)=1,790,328$ grams $^{2}$ 


\section{Activity 1}

- Systematic error of nitrate that has $\mathrm{BI}, \mathrm{A}$, and $\mathrm{El}$ components and three systematic uncertainties

- The term $\sigma_{\mathrm{sj}}{ }^{2}\left(\Sigma_{\mathrm{i}} \boldsymbol{m}_{\mathrm{ij}}\right)^{2}$ is evaluated as $\left(0.004^{2}+0.005^{2}+0.0025^{2}\right) *(65,000+175,000-45,000)^{2}$

$=4.725 \times 10^{-5} * 3.8 \times 10^{10}$

$=1,795,500$ grams $^{2}$ 


\section{Activity 1}

Random and systematic errors of oxide

- Random

$\left(0.0015^{2}+0.015^{2}+0.0125^{2}\right) *\left[12,000^{2} / 8\right]=6,903$

- Systematic

$\left(0.001^{2}+0.0175^{2}+0.009^{2}\right) *[12,000]^{2}=55,908$ 


\section{Activity 1}

Errors for equipment via NDA

- Random

$\left(1,850^{2}+3,500^{2}\right) * 0.25^{2}=979,531$

- Systematic

$(1,850-3,500)^{2 *} 0.15^{2}=61,256$ 


\section{Activity 1}

Random error of the 100 buttons, each button with 2,000 grams of $\mathrm{Pu}$

- $\left(0.0015^{2}+0.0005^{2}+0.007^{2}\right) *\left(2000^{2}+2000^{2}+\ldots\right)=$ 20,600 grams $^{2}$

Or

- $\left(0.0015^{2}+0.0005^{2}+0.007^{2}\right) * 200,000^{2} / 100$

$=20,600$

Systematic error of metal buttons

- $\left(0.0010^{2}+0.0006^{2}+0.0045^{2}\right) * 200,000^{2}$

$=864,400$ grams $^{2}$ 


\section{Activity 1}

Random error of solid waste

- $0.1^{2 *}\left(1,100^{2}+1,100^{2}+1,100^{2}\right)=36,300$ grams $^{2}$ Or

- $0.1^{2 *}\left(3,300^{2} / 3\right)=36,300$ grams $^{2}$

Systematic error of solid waste

- $0.25^{2} * 3,300^{2}=680,625$ 


\section{Activity 1}

\begin{tabular}{|l|c|c|}
\hline \multicolumn{1}{|c|}{ Component } & $\begin{array}{c}\text { Random } \\
\left(\text { grams }^{2}\right)\end{array}$ & $\begin{array}{c}\text { Systematic } \\
\left.\text { (grams }^{2}\right)\end{array}$ \\
\hline Nitrate & $1,790,328$ & $1,795,500$ \\
\hline Oxide & 6,903 & 55,908 \\
\hline NDA & 979,531 & 61,256 \\
\hline Buttons & 20,600 & 864,400 \\
\hline Waste Oxide & 36,300 & 680,625 \\
\hline Total & $2,833,662$ & $3,457,689$ \\
\hline
\end{tabular}

Total Variance: $2,833,662+3,457,689=6,291,351 \mathrm{gram}^{2}$

SEID $=$ sqrt $(6,291,351)=2,508$ grams

LEID $=$ LEMUF $=2 * 2,508=5,016$ grams 


\section{Inventory Difference Assessment - Activity 2}

\section{Effects of Various Types of Errors on the Inventory Difference (ID)}

1. Suppose the measurement of the discard stream (4) of liquid waste from the recovery material balance area (MBA) overestimates the actual amount of material discarded (for example, the actual amount is 10 grams and the measured value is 30 grams). What will this do to the ID in the recovery MBA?

2. Suppose the measurement of recovered oxide (9) from the recovery MBA to the reduction MBA overestimates the actual amount of material. What will this do to the ID in the two MBAs?

3. Suppose there is a tank of material in the reduction MBA whose content is overestimated at the June 30 inventory. What effect does this have on the reduction MBA June ID (the ID computed for the period June 1-30) and the July ID?

4. A solid deposit forms on the walls of a tank in the reduction MBA in June; such a deposit will not be measured by the inventory on June 30 and is not known to have occurred. In July, the deposit dissolves and the material reenters the process stream. What will be the effect on the June and July IDs? (Similar examples occur with filter holdup)

5 Cans of the powder that is an intermediate product in the reduction MBA are measured and removed temporarily to the storage MBA during June. In August, the cans are brought back and processed in the reduction MBA. Suppose the measurement overestimates the amount of material in the cans. What will be the effect on the IDs of the storage and reduction MBAs? 


\section{Inventory Difference Assessment - Activity 2 \\ Effects of Various Types of Errors on the Inventory Difference (ID) (Instructor Key)}

1. Suppose the measurement of the discard stream (4) of liquid waste from the recovery material balance area (MBA) overestimates the actual amount of material discarded (for example, the actual amount is 10 grams and the measured value is 30 grams). What will this do to the ID in the recovery MBA?

It will reduce it by 20 grams.

2. Suppose the measurement of recovered oxide (9) from the recovery MBA to the reduction MBA overestimates the actual amount of material. What will this do to the ID in the two MBAs?

It will reduce the ID in the recovery MBA but increase (loss) in the reduction MBA by the same amount. When one MBA ID runs consistently in the opposite direction from the ID of an MBA for which there is a flow between, it indicates errors in the flow measurement.

3. Suppose there is a tank of material in the reduction MBA whose content is overestimated at the June 30 inventory. What effect does this have on the reduction MBA June ID (the ID computed for the period June 1-30) and the July ID?

It will reduce (gain) the ID in the June ID, but increase (loss) it in the July ID. A month to month up and down behavior of the ID indicates uncertainties in the inventory measurement are dominant.

4. A solid deposit forms on the walls of a tank in the reduction MBA in June; such a deposit will not be measured by the inventory on June 30 and is not known to have occurred. In July, the deposit dissolves and the material reenters the process stream. What will be the effect on the June and July IDs? (Similar examples occur with filter holdup)

In June it will be a loss to ID

In July it will be a gain to ID

5. Cans of the powder that is an intermediate product in the reduction MBA are measured and removed temporarily to the storage MBA during June. In August, the cans are brought back and processed in the reduction MBA. Suppose the measurement overestimates the amount of material in the cans. What will be the effect on the IDs of the storage and reduction MBAs?

Since the material is not remeasured (is static in the storage MBA) there will be no effect on the ID: generally, the ID in such MBAs will be zero. The recovery ID in June will be driven down, in August it will be driven up. 


\section{Inventory Difference Assessment Exam}

Name:

Date:

1. What is one reason why inventory differences (ID) are nonzero.

a. TIDs are not in their proper locations.

b. Sometimes static inventory items are not remeasured.

c. Transfer checks are not performed.

d. Measured values include measurement error.

Given the hypothetical MBA structure, circle the correct answer for the following questions.

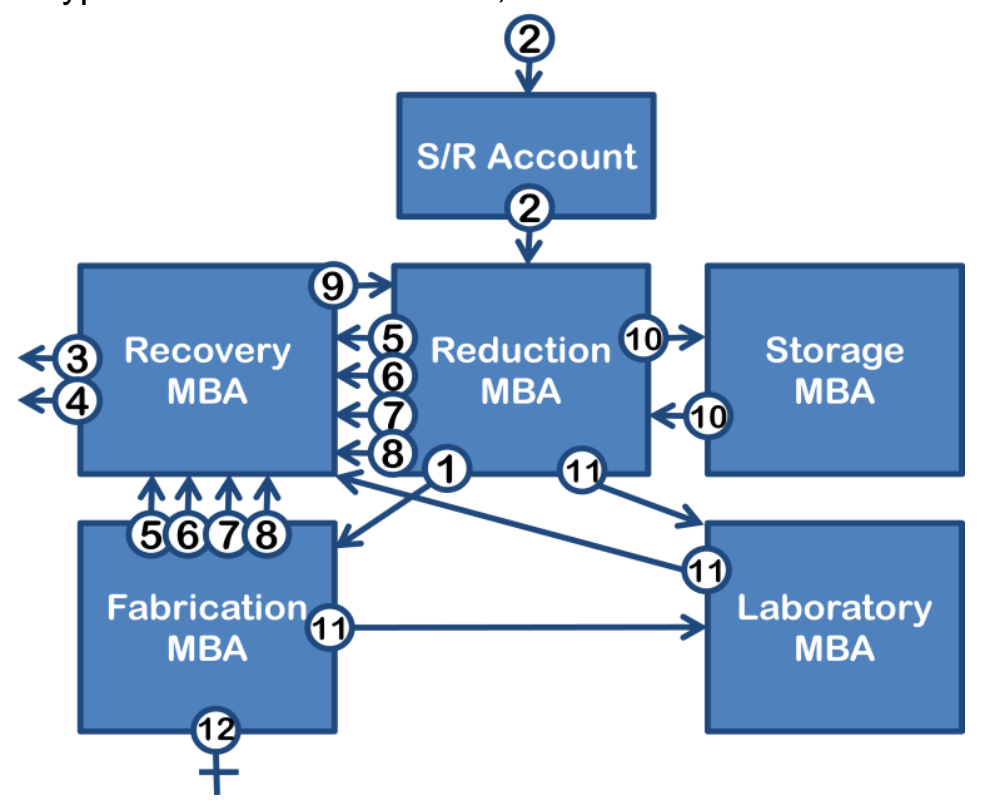

1: Buttons

2: Scrap Oxide

3: LL Solid Waste

4: LL Liquid Waste

5: Solid Waste

6: Liquid Waste

7: Metal Scrap

8: Scrap Powder

9: Recovered Oxide

10: Sweepings

11: Samples

12: Metal Parts

2. Suppose the measurement of the discard stream of liquid waste (4) from the recovery MBA overestimates the actual amount of material discarded: the actual amount is 10 grams, and the measured value is 30 grams. What will this do to the ID in the recovery MBA?

a. The ID will decrease by 20 grams.

b. The ID will increase by 20 grams.

c. The ID will increase by 40 grams.

d. The ID will not be affected by the situation. 
3. Suppose the measurement of recovered oxide (9) from the recovery MBA to the reduction MBA overestimates that actual amount of material: the actual amount is 2.1 $\mathrm{kgs}$ and the measured value is $2.2 \mathrm{kgs}$. What will this do to the ID in the two MBAs.

a. The ID in the recovery MBA will increase by $0.1 \mathrm{kgs}$ and the ID in the reduction MBA will decrease by the same amount.

b. The ID in the recovery MBA will decrease by $0.1 \mathrm{kgs}$ and the ID in the reduction MBA will increase by the same amount.

c. Both IDs will decrease by $0.1 \mathrm{kgs}$.

d. Both IDs will increase by $0.1 \mathrm{kgs}$.

4. Suppose there is a tank of material in the reduction MBA whose content is overestimated at the June 30th inventory. What effect does this have on the reduction MBA June ID (the ID computed for the period June 1-30) and the July ID (the ID computed for the period July 1-31)?

e. Both IDs will decrease.

f. Both IDs will increase.

g. The June ID will decrease and the July ID will increase.

$\mathrm{h}$. The June ID will increase and the July ID will decrease.

5. Suppose that every month for many months the oxide measurements are overstated. This would affect the ID

i. Mean.

j. Standard deviation.

k. Mean and standard deviation.

6. Suppose that the random error of a significant measurement method increased during the month of May. This would affect the ID

a. Mean.

b. Standard deviation.

c. Mean and standard deviation.

Identify the following as characteristics of historical ID limits $(H)$, variance-propagated ID limits $(\mathrm{V})$, or both (B).

7. Relatively easy to calculate

8. Requires assessing measurement errors

9. Requires the use of past ID data

10. Generates an estimate of the standard deviation of the ID

11. Yields standard deviations based on error propagation 


\section{Inventory Difference Assessment Exam}

\section{(Instructor Key)}

Name:

Date:

1. (Objective 7.2) What is one reason why inventory differences (ID) are nonzero.
a. TIDs are not in their proper locations.
b. Sometimes static inventory items are not remeasured.
c. Transfer checks are not performed.
d. Measured values include measurement error.

Given the hypothetical MBA structure, circle the correct answer for the following questions.

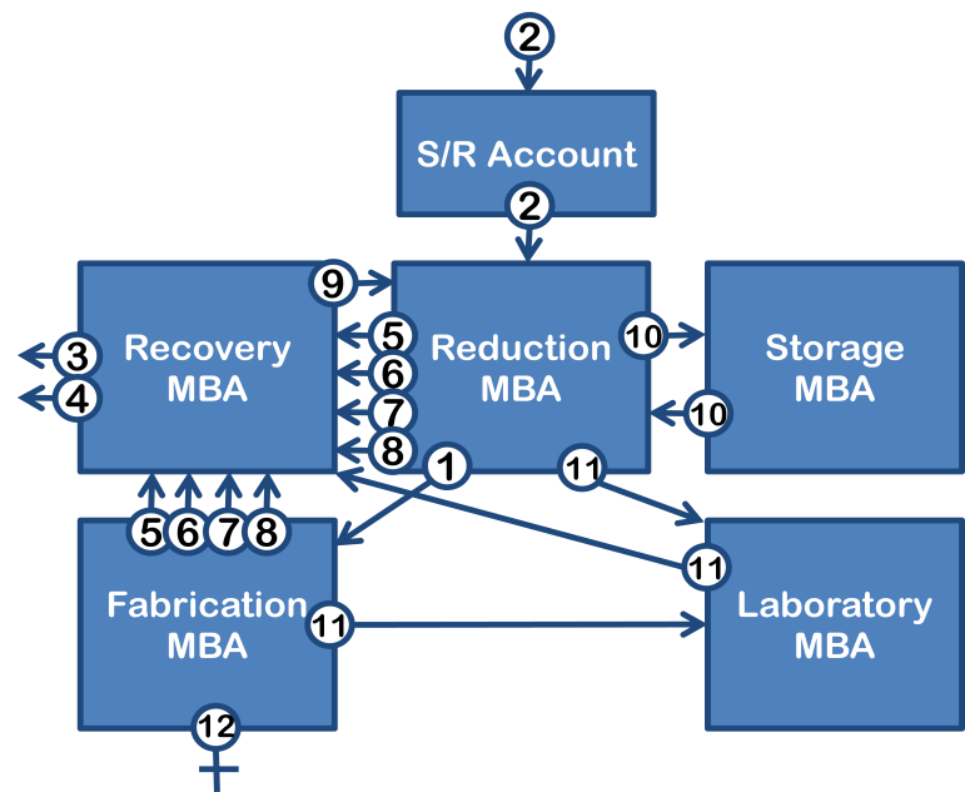

1: Buttons

2: Scrap Oxide

3: LL Solid Waste

4: LL Liquid Waste

5: Solid Waste

6: Liquid Waste

7: Metal Scrap

8: Scrap Powder

9: Recovered Oxide

10: Sweepings

11: Samples

12: Metal Parts

2. (Objective 7.5) Suppose the measurement of the discard stream of liquid waste (4) from the recovery MBA overestimates the actual amount of material discarded: the actual amount is 10 grams, and the measured value is 30 grams. What will this do to the ID in the recovery MBA?
a. The ID will decrease by $\mathbf{2 0}$ grams.
b. The ID will increase by 20 grams.
c. The ID will increase by 40 grams.
d. The ID will not be affected by the situation. 
3. (Objective 7.5) Suppose the measurement of recovered oxide (9) from the recovery MBA to the reduction MBA overestimates that actual amount of material: the actual amount is $2.1 \mathrm{kgs}$ and the measured value is $2.2 \mathrm{kgs}$. What will this do to the ID in the two MBAs.

a. The ID in the recovery MBA will increase by $0.1 \mathrm{kgs}$ and the ID in the reduction MBA will decrease by the same amount.

b. The ID in the recovery MBA will decrease by $0.1 \mathrm{kgs}$ and the ID in the reduction MBA will increase by the same amount.

c. Both IDs will decrease by $0.1 \mathrm{kgs}$.

d. Both IDs will increase by $0.1 \mathrm{kgs}$.

4. (Objective 7.5) Suppose there is a tank of material in the reduction MBA whose content is overestimated at the June 30th inventory. What effect does this have on the reduction MBA June ID (the ID computed for the period June 1-30) and the July ID (the ID computed for the period July 1-31)?
a. Both IDs will decrease.
b. Both IDs will increase.
c. The June ID will decrease and the July ID will increase.
d. The June ID will increase and the July ID will decrease.

5. (Objective 7.1) Suppose that every month for many months the oxide measurements are overstated. This would affect the ID
a. Mean.
b. Standard deviation.
c. Mean and standard deviation.

6. (Objective 7.3) Suppose that the random error of a significant measurement method increased during the month of May. This would affect the ID
a. Mean.
b. Standard deviation.
c. Mean and standard deviation.

(Objective 7.4) Identify the following as characteristics of historical ID limits $(H)$, variancepropagated ID limits (V), or both (B).

7. Relatively easy to calculate $\mathbf{H}$

8. Requires assessing measurement errors $\mathbf{V}$

9. Requires the use of past ID data $\mathbf{H}$

10. Generates an estimate of the standard deviation of the ID $\mathbf{B}$

11. Yields standard deviations based on error propagation $\underline{\mathbf{V}}$ 